\title{
3D Simulation of Magneto-Mechanical Coupling in MRI Scanners Using High Order FEM and POD
}
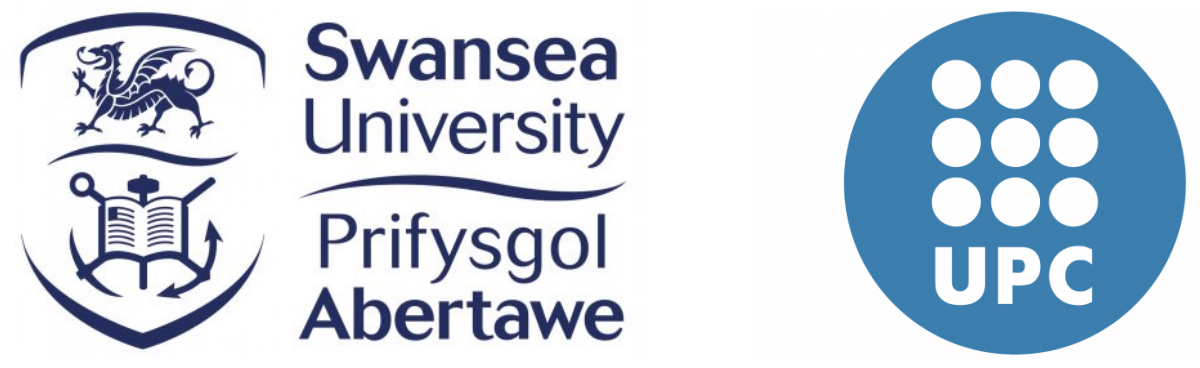

by

Marcos Seoane Chouciño

Submitted to Swansea University in fulfilment of the requirements for the Degree of

Doctor of Philosophy

at

Swansea University

January 24, 2020

Copyright: The author, Marcos Seoane Choucino, 2020. 


\section{Abstract}

Magnetic Resonance Imaging (MRI) scanners have become an essential tool in the medical industry due to their ability to produce high resolution images of the human body. To generate an image of the body, MRI scanners combine strong static magnetic fields with transient gradient magnetic fields. The interaction of these magnetic fields with the conducting components present in superconducting MRI scanners gives rise to an important problem in the design of new MRI scanners. The transient magnetic fields give rise to the appearance of eddy currents in conducting components. These eddy currents, in turn, result in electromagnetic stresses, which cause the conducting components to deform and vibrate. The vibrations are undesirable as they lead to a deterioration in image quality (with image artefacts) and to the generation of noise, which can cause patient discomfort. The eddy currents, in addition, lead to heat being dissipated and deposited into the cryostat, which is filled with helium in order to maintain the coils in a superconducting state. This deposition of heat can cause helium boil off and potentially result in a costly magnet quench. Understanding the mechanisms involved in the generation of these vibrations and the heat being deposited into the cryostat are, therefore, key for a successful MRI scanner design. This involves the solution of a coupled magneto-mechanical problem, which is the focus of this work.

In this thesis, a new computational methodology for the solution of three-dimensional (3D) magneto-mechanical coupled problems with application to MRI scanner design is presented. To achieve this, first an accurate mathematical description of the magnetomechanical coupling is presented, which is based on a Lagrangian formulation and the assumption of small displacements. Then, the problem is linearised using an AC-DC splitting of the fields, and a variational formulation for the solution of the linearised problem in a time-harmonic setting is presented. The problem is then discretised using high order finite elements, where a combination of hierarchical $H^{1}$ and $\boldsymbol{H}$ (curl) basis functions is used. An efficient staggered algorithm for the solution of the coupled system is proposed, which combines the DC and AC stages and makes use of preconditioned iterative solvers when appropriate. This finite element methodology is then applied to a set of challenging academic and industrially relevant problems in order to demonstrate its accuracy and efficiency.

This finite element methodology results in the accurate and efficient solution of the magneto-mechanical problem of interest. However, in the design stage of a new MRI scanner, this coupled problem must be solved repeatedly for varying model parameters such as frequency or material properties. Thus, even if an efficient finite element solver is 
available for the solution of the coupled problem, the need for these repeated simulations result in a bottleneck in terms of computational cost, which leads to an increase in design time and its associated financial implications. Therefore, in order to optimise this process, the application of Reduced Order Modelling (ROM) techniques is considered. A ROM based on the Proper Orthogonal Decomposition (POD) method is presented and applied to a series of challenging MRI configurations. The accuracy and efficiency of this ROM is demonstrated by performing comparisons against the full order or high fidelity finite element software, showing great performance in terms of computational speed-up, which has major benefits in the optimisation of the design process of new MRI scanners. 


\section{DECLARATION}

This work has not previously been accepted in substance for any degree and is not being concurrently submitted in candidature for any degree.

Signed

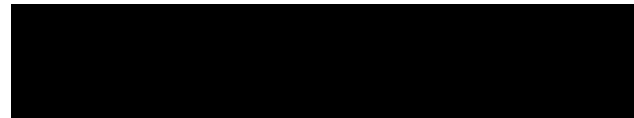

(candidate)

Date ..24/01/2020

\section{STATEMENT 1}

This thesis is the result of my own investigations, except where otherwise stated. When correction services have been used, the extent and nature of the correction is clearly marked in a footnote(s). Other sources are acknowledged by footnotes giving explicit references. A bibliography is appended.

Signed

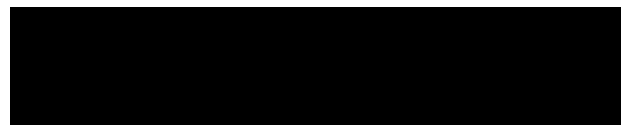

(candidate)

Date ..24/.0.1/20.20

\section{STATEMENT 2}

I hereby give consent for my thesis, if accepted, to be available for photocopying and for inter-library loan, and for the title and summary to be made available to outside organisations.

Signed

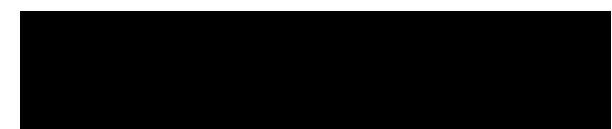

Date .24/01/2020

(candidate) 
To Noelia and my parents... 


\section{Acknowledgements}

Firstly, I would like to thank my supervisors Dr. Ledger and Prof. Gil for their continuous guidance and support throughout the project. They have helped me become a better researcher by sharing with me their wisdom, scientific rigour and their passion for research. I would also like to thank my industrial advisor Dr. Mallett for his continuous support and for sharing with me his wisdom and industrial experience. Without their support and understanding I probably would not have been able to finish this project and for that I will always be grateful to them. Thanks go also to my supervisor at UPC Prof. Zlotnik for his support and guidance during my secondment in Barcelona. I would also like to thank the European Commission for the funding received through the Marie Sklodowska-Curie Innovative Training Network AdMoRe with grant number 675919.

The PhD has been a great experience that gave me the opportunity to meet amazing people that made the road much more enjoyable and worthy. I would like to thank all the colleagues at Siemens Healthineers for many fruitful discussions and for making the time at the office much more enjoyable. Thanks also to all my colleagues from AdMoRe for making all the conferences and meetings much more enjoyable and fun. I would also like to thank all my colleagues at Swansea University for making my time at Swansea much more enjoyable. Particular thanks go to Guillem Barroso for all the time we shared together, including research related discussions, travels, conferences or parties and for hosting me during the last month of my $\mathrm{PhD}$. I would also like to thank my colleagues from "Friday Night Football" for making my life in Eynsham much more enjoyable by sharing many football matches and after match drinks. Particular thanks go to Sam, Shamsa, Aysha and Freya, for welcoming me to their home during the last year of my $\mathrm{PhD}$ and making me feel part of the family. I will always consider it a second home.

I would also like to thank my parents for all their love, support and understanding throughout my life. You made an enormous effort to give me the opportunities that you never had and you always encouraged and supported me, even in the bad moments. I could not have done it without you. Thanks also to my sister, for all her love and support during my life and for always being there in the bad moments. Thanks also to my grandmother for all her love and support and to my nephew for making my holidays and short visits all much more enjoyable and entertaining. Last, but not least, I would like to thank Noelia, the love of my life, for her immense love, support and patience. Thank you for always being there to enjoy the good moments but also for being there to support me in the bad moments. You are my inspiration, the force that drives me to become a better person each day and to achieve all my dreams. I could not have done it without you. 


\section{Agradecementos}

En primeiro lugar gustaríame dar as gracias aos meus supervisores Dr. Ledger e Prof. Gil pola súa continua guía e apoio ó longo do proxecto. Eles axudáronme a converterme nun mellor investigador compartindo comigo a súa sabedoría, rigor científico e paixón pola investigación. Tamén me gustaría agradecer ao meu tutor industrial Dr. Mallett polo seu continuo apoio e por compartir comigo a súa sabedoría e experiencia na industria. Sen o apoio de todos eles probablemente non podería ter rematado este proxecto e, polo tanto, sempre lles estarei agradecido. Gracias tamén ao meu supervisor na UPC Prof. Zlotnik polo seu apoio e guía durante a miña estancia en Barcelona. Gustaríame agradecer tamén á Comisión Europea polo financiamento recibido a través da beca Marie SklodowskaCurie Innovative Training Network AdMoRe, con número 675919.

O doutorado foi unha gran experiencia e deume a oportunidade de coñecer a xente incrible que fixo o camiño moito máis levadeiro e que a experiencia merecese aínda máis a pena. Gustaríame agradecer a todos os meus compañeiros en Siemens Healthineers polas moitas frutíferas discusións e por facer o tempo na oficina moito máis levadeiro. Agradecer tamén a todos os compañeiros de AdMoRe por facer as conferencias e meetings moito máis amenos e divertidos. Gustaríame dar as gracias tamén a todos os meus compañeiros en Swansea University por facer a miña estancia en Swansea moito máis levadeira. Un agradecemento especial vai para Guillem Barroso, por todo o tempo que compartimos, incluíndo discusións sobre ciencia, viaxes, conferencias ou festas, e por acollerme na súa casa durante o último mes do doutorado. Gustaríame agradecer tamén a todos os meus compañeiros de "Friday Night Football" por facer a miña vida en Eynsham moito máis levadeira a través dos moitos partidos que compartimos así como das cervexas de despois do partido. Gustaríame tamén agradecer especialmente a Sam, Shamsa, Aysha e Freya, por acollerme no seu fogar durante o último ano do doutorado e facerme sentir un máis da familia. Para min sempre será unha segunda casa.

Gustaríame tamén dar as gracias aos meus pais polo seu cariño, apoio e comprensión ó longo da miña vida. Fixestes un esforzo enorme para darme as oportunidades que vós nunca tivestes, e sempre me animastes e apoiastes, incluso nos malos momentos. Non o podería ter feito sen vós. Gracias tamén a miña irmá, polo seu cariño e apoio ó longo da miña vida e por estar sempre ahí nos malos momentos. Gracias tamén a miña avóa polo seu cariño e apoio e ao meu afillado por facer as miñas vacacións e escapdas á casa moito máis levadeiras e divertidas. Finalmente, gustaríame dar as gracias a Noelia, o amor da miña vida, polo seu inmenso cariño, apoio e paciencia. Gracias por estar sempre ahí para desfrutar dos bos momentos pero tamén por estar ahí para apoiarme nos malos momentos. 
Eres a miña inspiración, a forza que me guía a intentar converterme nunha mellor persoa cada día e a perseguir todos os meus soños. Non o podería ter feito sen ti. 


\section{Contents}

I Preliminaries $\quad 1$

1 Introduction $\quad 3$

1.1 MRI Scanners . . . . . . . . . . . . . . . . . . . . 3

1.2 Computational Methods . . . . . . . . . . . . . . . . 5

1.2.1 Finite Elements for Electromagnetic Problems . . . . . . . . 6

1.2.2 Finite Elements for Elasticity Problems . . . . . . . . . . . 7

1.2.3 Finite Elements for Coupled Problems . . . . . . . . . . . . . 8

1.2.4 Applications to MRI Scanners . . . . . . . . . . . . . . . . . 8

1.2.5 Reduced Order Modelling . . . . . . . . . . . . . . . . 9

1.3 Aim and Objectives . . . . . . . . . . . . . . . . . . . . . . . . . . . . . . . . . . . .

1.4 Outline of the Thesis . . . . . . . . . . . . . . . . . . 11

1.5 Research Outcomes . . . . . . . . . . . . . . . . . . . . . . . 14

1.5.1 Published and Submitted Journal Publications . . . . . . . . . . . 14

1.5.2 Conference Presentations . . . . . . . . . . . . . . . . . 15

1.5.3 Research Posters . . . . . . . . . . . . . . . . . . . . . . 15

1.5.4 Software Packages . . . . . . . . . . . . . 16

$\begin{array}{lr}\text { II Full Order Model } & 17\end{array}$

2 Governing Physical Laws 19

2.1 Introductory Remarks . . . . . . . . . . . . . . . . . . . . . . 19

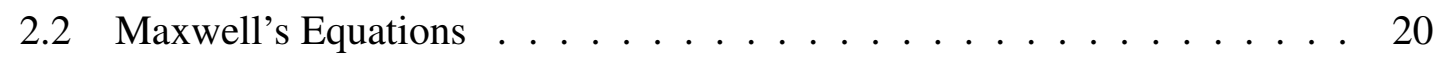

2.3 Moving Bodies: Material and Spatial Descriptions . . . . . . . . . . . . . 20

2.4 Total Lagrangian Formulation . . . . . . . . . . . . . . . . . . . . . . 22

2.5 Updated Lagrangian Formulation . . . . . . . . . . . . . . . . . . . . . . . . . . . . . . . . . 23

2.6 Eulerian Formulation . . . . . . . . . . . . . . . . . . 24

2.7 Interface Conditions . . . . . . . . . . . . . . . . . . . . . . . . . . . . . 25

2.7.1 Total Lagrangian Formulation . . . . . . . . . . . . . . . 25

2.7.2 Updated Lagrangian Formulation . . . . . . . . . . . . . . . . . 26

2.7.3 Eulerian Formulation . . . . . . . . . . . . . . . 26

2.8 Modelling Assumptions . . . . . . . . . . . . . . . . . . . . . 27

2.8.1 Eulerian Formulation for Small Displacements and Velocities . . 27

2.8.2 Lagrangian Formulation for Small Displacements . . . . . . . . . 29 
2.9 Concluding Remarks . . . . . . . . . . . . . . . . . . . 32

3 Linearised Problem: Lagrangian Framework 35

3.1 Introductory Remarks . . . . . . . . . . . . . . . . . . . . . 35

3.2 AC-DC Splitting . . . . . . . . . . . . . . . . 35

3.3 Weak Formulation . . . . . . . . . . . . . . . . 36

3.3.1 Static (DC) Problem . . . . . . . . . . . . . . . . 36

3.3 .2 Dynamic Problem . . . . . . . . . . . . . . . . . 39

3.4 Linearisation . . . . . . . . . . . . . . . . . . 40

3.5 Surface Integral Treatment . . . . . . . . . . . . . . . . . . . 44

3.6 Regularisation . . . . . . . . . . . . . . . . . . . 44

3.6.1 DC Regularised Problem . . . . . . . . . . . . . . . . 45

3.6.2 AC Regularised Problem . . . . . . . . . . . . . . . . . 45

3.7 Chapter Summary . . . . . . . . . . . . . . . . . . . . 46

4 Computational Implementation $\quad 49$

4.1 Introductory Remarks . . . . . . . . . . . . . . . . . . . . . . . . . . 49

4.2 Computational Domain . . . . . . . . . . . . . . . . 50

4.3 Finite Element Discretisation . . . . . . . . . . . . . . . . 51

4.3.1 Reference Element . . . . . . . . . . . . . . . . 53

4.3.2 Hierarchical $H^{1}$ Conforming Basis Functions . . . . . . . . . . . 53

4.3.3 Hierarchical $\boldsymbol{H}$ (curl)-Conforming Basis Functions . . . . . . . . 55

4.3.4 Elemental Mapping . . . . . . . . . . . . . . . . . . 57

4.3.5 Discretisation of the Coupled System . . . . . . . . . . . 59

4.4 Staggered Algorithm . . . . . . . . . . . . . . . . . 67

4.5 Preconditioned Iterative Solvers _ . . . . . . . . . . . . . . . . . 68

4.6 Mechanical Damping . . . . . . . . . . . . . . . . . 72

4.7 Current Source Properties . . . . . . . . . . . . . . . . . . . . 73

4.8 Current Source Mapping . . . . . . . . . . . . . . . . . . 75

4.9 Symmetry Conditions . . . . . . . . . . . . . . . . . . . . 77

4.10 Global Software Structure . . . . . . . . . . . . . . . . . . 79

4.10 .1 Pre-processing Stage . . . . . . . . . . . . . . . 79

4.10 .2 Solver Stage . . . . . . . . . . . . . . . . . . 81

4.10 .3 Post-processing Stage . . . . . . . . . . . . . . . . . 82

4.11 Chapter Summary . . . . . . . . . . . . . . . . . . . . . . . 82

5 Numerical Results for the Full Order Problem 85

5.1 Introductory Remarks . . . . . . . . . . . . . . . . . . . . . 85

5.2 Decoupled Physics . . . . . . . . . . . . . . . . . . 86

5.2.1 DC Stage, Magnetostatics: Permeable Sphere in a Uniform Static

Magnetic Field . . . . . . . . . . . . . . 86

5.2.2 DC stage, Elasticity: Hollow Cylinder Subject to Pressure Field . 91 
5.2.3 AC stage, Eddy Current: Conducting Sphere in a Uniform Alternating Magnetic Field . . . . . . . . . . . . . . . . 94

5.2.4 AC stage, Elasticity: Hollow Cylinder Subject to Body Force . . . 98

5.3 Coupled Physics . . . . . . . . . . . . . . . . . . . . . 100

5.3.1 Conducting and Elastic Sphere in a Uniform Alternating Magnetic Field . . . . . . . . . . . . . . . . . . 101

5.3.2 Test Magnet Problem with $z$ (Longitudinal) Gradient Coil . . . . 104

5.3.3 Test Magnet Problem with $x$ (Transversal) Gradient Coil . . . . . 114

5.3.4 Modified Magnet Problem . . . . . . . . . . . . . . . . . 117

5.4 Chapter Summary . . . . . . . . . . . . . . . . . . . 123

III Reduced Order Modelling 125

6 POD Based Reduced Order Modelling 127

6.1 Introductory Remarks . . . . . . . . . . . . . . . . . . . . . . . 127

6.2 Variational Formulation . . . . . . . . . . . . . . . . . . . . . . . . . . . . . . . . . . . . . . . . . . . . .

6.3 Discrete System . . . . . . . . . . . . . . . . . . . . . . . . . . . 129

6.4 The Singular Value Decomposition (SVD) . . . . . . . . . . . . . . 130

6.4.1 Truncated Singular Value Decomposition . . . . . . . . . . . 131

6.5 The Proper Orthogonal Decomposition . . . . . . . . . . . . . . . . . . 132

6.5.1 Off-line Stage: Construction of the Basis via SVD . . . . . . . 132

6.5.2 On-line Interpolation Based POD (PODI) . . . . . . . . . . . . 134

6.5.3 On-line Projection Based POD (PODP) . . . . . . . . . . . . 135

6.6 Application to Separated Physics . . . . . . . . . . . . . . . . . 137

6.6 .1 Off-line ......................... 137

6.6 .2 On-line . . . . . . . . . . . . . . . 137

6.7 Combined Reduced Order-Full Order Approach . . . . . . . . . . . . . . 138

6.7 .1 Off-line . . . . . . . . . . . . . . . 139

6.7 .2 On-line .......................... 139

6.8 Dissipated Power and Kinetic Energy: POD Approximation . . . . . . . 139

6.9 Chapter Summary . . . . . . . . . . . . . . . . . . . . . 140

7 Reduced Order Model Numerical Results $\quad 141$

7.1 Introductory Remarks . . . . . . . . . . . . . . . . . . . . . . . . . . . . . . 141

7.2 Error Measure . . . . . . . . . . . . . . . . . . . . . . . . 142

7.3 Test Magnet Problem with 1 Parameter . . . . . . . . . . . . . . . . 143

7.3 .1 PODI . . . . . . . . . . . . . . . . . 144

7.3 .2 PODP ........................... 144

7.3.3 Application to Separated Physics Solutions . . . . . . . . . . . 146

7.3.4 Combined Reduced Order-Full Order Approach . . . . . . . . . 151

7.4 Test Magnet Problem with Multiple Parameters . . . . . . . . . . . . . 154

7.4 .1 PODP-Full . . . . . . . . . . . . . . . . . 154 
7.5 Modified Magnet Problem with Multiple Parameters . . . . . . . . . . 161 $7.5 .1 \quad$ PODP-Full . . . . . . . . . . . . . . . 163

7.6 Chapter Summary . . . . . . . . . . . . . . . . 165

$\begin{array}{lll}\text { IV Conclusions } & 167\end{array}$

8 Conclusions and Future Work $\quad 169$

8.1 Concluding Remarks . . . . . . . . . . . . . . . . . . . . . . . . . . . . . . . . . . . . . . . . . . . . .

8.1 .1 Conclusions . . . . . . . . . . . . . . . . . 169

8.2 Recommendations for Future Work . . . . . . . . . . . . . . . 172

V Appendices 175

$\begin{array}{ll}\text { Appendices } & 177\end{array}$

A Proof that $\operatorname{div} \boldsymbol{S}\left(\boldsymbol{A}^{D C}, \mathcal{A}^{A C}\right)=\mathbf{0}$ in $\Omega_{C}^{c} \backslash\left(\operatorname{supp}\left(\boldsymbol{g}^{A C}\right) \cup \operatorname{supp}\left(\boldsymbol{J}^{D C}\right)\right)$

B CAD and Mesh Generation 179

B.1 Introduction . . . . . . . . . . . . . . . . . . . . . . . 179

B.2 CAD Generation . . . . . . . . . . . . . . . . . 179

B.3 Mesh Generation . . . . . . . . . . . . . . . . 180

$\begin{array}{ll}C & \text { Numerical Integration }\end{array}$

D Parallel Implementation 185

D.1 Introduction . . . . . . . . . . . . . . . . . . 185

D.2 Matlab Implementation for Varying Model Parameters . . . . . . . . . . 185

E User Guide $\quad 189$

E.1 Introduction . . . . . . . . . . . . . . . . . . . . . . . 189

E.2 Software Capabilities . . . . . . . . . . . . . . . . . . . 189

E.3 Software Structure . . . . . . . . . . . . . . . . . . . . 190

E.3.1 Pre-processing Stage . . . . . . . . . . . . . . . . . . 190

E.3.2 Solver Stage . . . . . . . . . . . . . . . . . . 192

E.3.3 Post-processing Stage . . . . . . . . . . . . . . . . . 192

E.4 User Interaction . . . . . . . . . . . . . . . . . . . . . . . . 193

E.4.1 Mesh Generation . . . . . . . . . . . . . . . . . . 193

E.4.2 Problem File Definition . . . . . . . . . . . . . . . . 194

E.4.3 Main Functions . . . . . . . . . . . . . . . . . 197

E.4.4 Post-processing . . . . . . . . . . . . . . . . . 200

E.5 Appendix Summary . . . . . . . . . . . . . . . . . 200 


\section{List of Figures}

1.1 MRI scanner. (a) New model Magnetom Lumina 3T. Image Courtesy of Siemens Healthineers. (b) Illustration of the main components: main coils, gradient coils and radiation shields $(4 \mathrm{~K}, 77 \mathrm{~K}$ and Outer Vacuum Chamber $(\mathrm{OVC})) \ldots \ldots \ldots \ldots$. . . . . . . . . . . . . 4

2.1 General motion of a deformable body. . . . . . . . . . . . . . . . . 21

2.2 Magneto-mechanical coupled problem. General representation of the problem illustrating the different components involved. . . . . . . . . . 25

3.1 Current source decomposition, where $\boldsymbol{J}^{s}(t)=\boldsymbol{J}^{D C}+\boldsymbol{J}^{A C}(t) \ldots \ldots 36$

4.1 Computational domain $\Omega=\Omega_{C} \cup \Omega_{N C}$ created by truncating the unbounded free space region $\Omega_{C}^{c}$ at a finite distance away from $\Omega_{C}$, resulting in the bounded non-conducting region $\Omega_{N C} \ldots \ldots \ldots$. . . . . . 50

4.2 Reference tetrahedral element $\hat{\Omega}$. . . . . . . . . . . . . . . . 53

4.3 High order $H^{1}$-conforming basis functions. Illustration of the distribution of elemental DOF into low order vertex and high order edge, face and interior. . . . . . . . . . . . . . . . . . . 54

4.4 Sample $H^{1}$-conforming basis functions plotted over a face of the reference tetrahedron. . . . . . . . . . . . . . . . . . 54

4.5 High order $\boldsymbol{H}$ (curl)-conforming basis functions. Illustration of the distribution of elemental DOF into low and high order edge, face and interior. . 55

4.6 Sample $\boldsymbol{H}$ (curl)-conforming basis functions plotted over a face of the reference tetrahedron. . . . . . . . . . . . . . . 56

4.7 Mapping between the reference $\hat{\Omega}$ and physical $\Omega^{(e)}$ elements. . . . . . . 57

4.8 Orientation of the edges for (a) Type I and (b) Type II reference tetrahedra [10]. . . . . . . . . . . . . . . . . . . . 61

4.9 Schematic representation of the layer of elements $\Omega_{N C}^{1}$ adjacent to $\Omega_{C}$ that is defined in order to improve the efficiency of the assembly process. $\Omega_{C} \cup \Omega_{N C}^{1}$ coloured in red and $\Omega_{N C} \backslash \Omega_{N C}^{1}$ in blue. . . . . . . . . . . 65

4.10 Flow chart illustrating the different steps involved in the solver stage for the Lagrangian and Eulerian formulations. . . . . . . . . . . . . . . . 
4.11 Block Jacobi preconditioner used for the solution of the eddy current problem. Illustration of the general structure of the preconditioning matrix, showing the explicit splitting where the whole block is retained for the lowest order space and only block diagonal contributions are considered for the high order terms, which are split into gradient edge, face and interior functions, $\tilde{\mathbf{D}}_{\mathcal{A} \mathcal{A}}^{g g E}, \tilde{\mathbf{D}}_{\mathcal{A} \mathcal{A}}^{g g, F F}$ and $\tilde{\mathbf{D}}_{\mathcal{A} \mathcal{A}}^{g g, I I}$, respectively and nongradient face $\tilde{\mathbf{D}}_{\mathcal{A} \mathcal{A}}^{n n, F F}$ and interior $\tilde{\mathbf{D}}_{\mathcal{A} \mathcal{A}}^{n n, I I}$ functions. . . . . . . . . .

4.12 Illustration of typical MRI configurations: (a) MRI configuration including longitudinal gradient coils and (b) MRI configuration including transversal gradient coils. . . . . . . . . . . . . . . . . .

4.13 Typical wire patterns of MRI transversal and longitudinal gradient coils [17] . . . . . . . . . . . . . . . . .

4.14 Illustration of a geometrically symmetric domain. The geometry is symmetric with respect to the dashed line $(x$ axis $) \ldots \ldots \ldots \ldots$

4.15 Illustration of a rotationally symmetric (axisymmetric) domain. The geometry is symmetric with respect to the $z$ axis. . . . . . . . . . . 78

4.16 Illustration of physical symmetries; (a) Symmetric distortion and (b) Antisymmetric distortion.

4.17 Schematic representation of the software structure, including the preprocessing, solver and post-processing stages. . . . . . . . . . . . . .

5.1 Permeable sphere in a uniform static magnetic field: problem setup. . . .

5.2 Permeable sphere in a uniform static magnetic field: Convergence of the CG method for: (a) A mesh of 1083 elements without preconditioner, (b) A mesh of 1083 elements using preconditioner, (c) A mesh of 6654 elements without preconditioner and (d) A mesh of 6654 elements using preconditioner.

5.3 Permeable sphere in a uniform static magnetic field: Convergence of PCG for different values of $\varepsilon \ldots \ldots \ldots \ldots \ldots$

5.4 Permeable sphere in a uniform magnetic field: convergence of $\| \mathbf{A}-$ $\boldsymbol{A}_{\text {exact }}\left\|_{\boldsymbol{H}(\mathrm{curl})\left(\Omega_{C}\right)} /\right\| \boldsymbol{A}_{\text {exact }} \|_{\boldsymbol{H}(\mathrm{curl})\left(\Omega_{C}\right)}$ for different polynomial orders of approximation of the geometry under $p$-refinement. . . . . . . . . .

5.5 Permeable sphere in a uniform magnetic field: Convergence of $\| \boldsymbol{A}_{\varepsilon, h q}^{D C}-$ $\boldsymbol{A}_{\text {exact }}^{D C}\left\|_{\boldsymbol{H}(\mathrm{curl})\left(\Omega_{C}\right)} /\right\| \boldsymbol{A}_{\text {exact }}^{D C} \|_{\boldsymbol{H}(\mathrm{curl})\left(\Omega_{C}\right)}$ under $p$-refinement. . . . . . . . .

5.6 Permeable sphere in a uniform magnetic field: Convergence of $\| \boldsymbol{A}_{\varepsilon, h q}^{D C}-$ $\boldsymbol{A}_{\text {exact }}^{D C}\left\|_{\boldsymbol{H}(\mathrm{curl})\left(\Omega_{C}\right)} /\right\| \boldsymbol{A}_{\text {exact }}^{D C} \|_{\boldsymbol{H}(\mathrm{curl})\left(\Omega_{C}\right)}$ under $h$-refinement. . . . . . . . .

5.7 Hollow cylinder subject to pressure field: illustration of a cross section of the cylinder and the applied pressure field. . . . . . . . . . .

5.8 Hollow cylinder subject to pressure field: illustration of the chosen boundary conditions and one of the meshes (7953 elements) used in the computations. $\partial \Omega_{C}^{N}$ in green and $\partial \Omega_{C}^{D}$ in red. 
5.9 Hollow cylinder subject to pressure field: Convergence of the conjugate gradient method for: (a) A mesh of 1366 elements without preconditioner, (b) A mesh of 1366 elements using preconditioner, (c) A mesh of 3144 elements without preconditioner and (d) A mesh of 3144 elements using preconditioner.

5.10 Hollow cylinder subject to pressure field: convergence of $\| \boldsymbol{u}_{h p}^{D C}-$ $\boldsymbol{u}_{\text {exact }}\left\|_{H^{1}\left(\Omega_{C}\right)} /\right\| \boldsymbol{u}_{\text {exact }} \|_{H^{1}\left(\Omega_{C}\right)}$ under $p$-refinement. . . . . . . . . . . .

5.11 Hollow cylinder subject to pressure field: convergence of $\| \boldsymbol{u}_{h p}^{D C}-$ $\boldsymbol{u}_{\text {exact }}\left\|_{H^{1}\left(\Omega_{C}\right)} /\right\| \boldsymbol{u}_{\text {exact }} \|_{H^{1}\left(\Omega_{C}\right)}$ under $h$-refinement. . . . . . . . . . .

5.12 Hollow cylinder subject to pressure field: (a) $\left|\boldsymbol{u}_{h p}^{D C}\right|$, (b) $\left|u_{z, h p}^{D C}\right|$ (c) $\left|u_{x, h p}^{D C}\right|$ on the plane $z=0 \ldots \ldots \ldots \ldots \ldots$. . . . . . . . . . . 95

5.13 Conducting sphere in a uniform alternating magnetic field: problem setup. 95

5.14 Conducting sphere in a uniform alternating magnetic field: Convergence of the iterative solver (GMRES and PGMRES) for: (a) $f=50 \mathrm{~Hz}$ without preconditioner, (b) $f=50 \mathrm{~Hz}$ using preconditioner, (c) $f=1000 \mathrm{~Hz}$ without preconditioner and (d) $f=1000 \mathrm{~Hz}$ using preconditioner. . . . . 96

5.15 Conducting sphere in a uniform alternating magnetic field: convergence

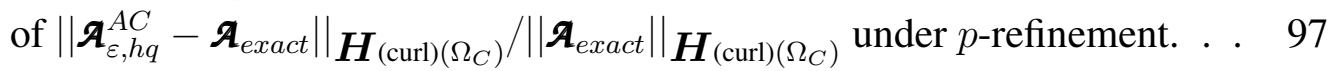

5.16 Conducting sphere in a uniform alternating magnetic field: convergence

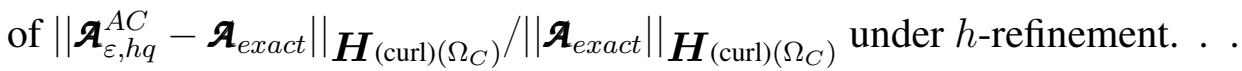

5.17 Conducting sphere in a uniform alternating magnetic field: Magnitude of the eddy current, $\left|\boldsymbol{g}_{h q}^{o}\right|$ in the planes $x=0$ and $z=0$ for different frequencies. . . . . . . . . . . . . . .

5.18 AC elasticity stage, hollow cylinder subject to body force: convergence of $\left\|\boldsymbol{u}_{h p}^{A C}-\boldsymbol{u}_{\text {exact }}^{A C}\right\|_{H^{1}\left(\Omega_{C}\right)} /\left\|\boldsymbol{u}_{\text {exact }}^{A C}\right\|_{H^{1}\left(\Omega_{C}\right)}$ under $p$-refinement. . . . . . .

5.19 AC elasticity stage, hollow cylinder subject to body force: convergence of $\left\|\boldsymbol{u}_{h p}^{A C}-\boldsymbol{u}_{\text {exact }}^{A C}\right\|_{H^{1}\left(\Omega_{C}\right)} /\left\|\boldsymbol{u}_{\text {exact }}^{A C}\right\|_{H^{1}\left(\Omega_{C}\right)}$ under $h$-refinement. . . . . . . 100

5.20 Conducting and elastic sphere in a uniform alternating magnetic field: illustration of the hemisphere used for the computations. . . . . . . . . 101

5.21 Conducting and elastic sphere in a uniform alternating magnetic field: comparison of the solution for different $p, q$ combinations. . . . . . . . 102

5.22 Conducting and elastic sphere in a uniform alternating magnetic field: dissipated power and kinetic energy in the conducting sphere. . . . . . . . 103

5.23 Conducting and elastic sphere in a uniform alternating magnetic field: $\left|\operatorname{Re}\left(\boldsymbol{g}_{h p q}^{o}\right)\right|$ contours in the conducting sphere and $\left|\operatorname{Re}\left(\boldsymbol{B}_{0, h q}^{A C}\right)\right|$ contours and $\operatorname{Re}\left(\mathcal{B}_{0, h q}^{A C}\right)$ streamlines in the air. . . . . . . . . . . . . . . . 104

5.24 Conducting and elastic sphere in a uniform alternating magnetic field: contours of $\left|\operatorname{Re}\left(\boldsymbol{u}_{h p}^{A C}\right)\right|$ on the deformed sphere for different frequencies. Deformation magnitude magnified by a factor of 30 . . . . . . . . . . . 104

5.25 Test magnet problem with $z$ (longitudinal) gradient coil: illustration of the components of the problem in the axisymmetric meridian plane (left) and $3 \mathrm{D}$ view $($ right $) . \ldots \ldots$. . . . . . . . . . . . . . 105 
5.26 Test magnet problem with $z$ (longitudinal) gradient coil: quarter of the geometry used for the simulations exploiting the symmetry of the problem. 106

5.27 Test magnet problem with $z$ (longitudinal) gradient coil: convergence of the dissipated power and kinetic energy in the radiation shields under $p$ refinement. Undamped system $\left(\alpha_{M}=0\right)$.

5.28 Test magnet problem with $z$ (longitudinal) gradient coil: convergence of the dissipated power and kinetic energy in the radiation shields under $p$ refinement. Damped system $\left(\alpha_{M}=20\right) \ldots \ldots \ldots$. . . . . . 108

5.29 Test magnet problem with $z$ (longitudinal) gradient coil: Dissipated power and kinetic energy in the radiation shields. Comparison between different approaches. . . . . . . . . . . . . . . . . . . . 110

5.30 Test magnet problem with $z$ (longitudinal) gradient coil: $\left|\operatorname{Re}\left(\boldsymbol{J}_{h p q}^{o}\right)\right|$ in the deformed $77 \mathrm{~K}$ shield and $\left|\operatorname{Re}\left(\boldsymbol{B}_{0, h q}^{A C}\right)\right|$ contours and $\operatorname{Re}\left(\boldsymbol{B}_{0, h q}^{A C}\right)$ streamlines in the background air for two different frequencies. . . . . . . . . . . . 111

5.31 Test magnet problem with $z$ (longitudinal) gradient coil: snapshots of $\left|\operatorname{Re}\left(\boldsymbol{u}_{h p}^{A C} e^{\mathrm{i} \omega t}\right)\right|$ in the deformed $4 \mathrm{~K}$ shield and $\operatorname{Re}\left(\boldsymbol{B}_{0, h q}^{A C} e^{\mathrm{i} \omega t}\right)$ streamlines in the surrounding air for $f=1000 \mathrm{~Hz}$ and different times. Deformation magnitude magnified by a factor of $10^{4}$ in plot, but not in contours. . . . . 112

5.32 Test magnet problem with $z$ (longitudinal) gradient coils: Dissipated power and kinetic energy in the radiation shields for different $\alpha_{M}=$

5.33 Test magnet problem with $z$ (longitudinal) gradient coils: Illustration of the choice of boundary conditions. Shields fixed at the ends. . . . . . . . 114

5.34 Test magnet problem with $z$ (longitudinal) gradient coils: Dissipated power and kinetic energy in the radiation shields for different $\xi=$ $10^{-4}, 10^{-2}, 10^{-3}$.

5.35 Test magnet problem with $z$ (longitudinal) gradient coils: Dissipated power and kinetic energy in the radiation shields for $q=p=3$ and $q=3, p=4 \ldots \ldots \ldots \ldots \ldots 116 \ldots \ldots \ldots \ldots$

5.36 Test magnet problem with $x$ (transversal) gradient coil: illustration of the geometry including main coils (red), gradient coils (blue) and radiation shields (green).

5.37 Test magnet problem with $x$ (transversal) gradient coil: $\left|\operatorname{Re}\left(\boldsymbol{g}_{h p q}^{o}\right)\right|$ in the OVC shield for $f=500 \mathrm{~Hz}$ compared with the case of $z$ (longitudinal) gradient coil. . . . . . . . . . . . . . . . . . . 118

5.38 Test magnet problem with $x$ (transversal) gradient coil: $\left|\operatorname{Re}\left(\boldsymbol{u}_{h p}^{A C}\right)\right|$ in the 77K shield for $f=500 \mathrm{~Hz}$ compared with the case of $z$ (longitudinal) gradient coil. Deformation magnitude magnified by a factor of $10^{4}$ in plot, but not in contours. . . . . . . . . . . . . . . . 118

5.39 Test magnet problem with $x$ (transversal) gradient coil: Streamlines of $\operatorname{Re}\left(\mathcal{B}_{0, h q}^{A C}\right)$ and contours of $\left|\operatorname{Re}\left(\boldsymbol{J}_{h p q}^{o}\right)\right|$ in the radiation shields for $f=500$ $\mathrm{Hz}$ compared with the case of $z$ (longitudinal) gradient coil. . . . . . . . . 119 
5.40 Test magnet problem with $x$ (transversal) gradient coil: convergence of the dissipated power and kinetic energy in the radiation shields under $p$ refinement. Damped system $\left(\alpha_{M}=200\right)$. . . . . . . . . . . . .

5.41 Test magnet problem: comparison of the dissipated power and kinetic energy in the radiation shields for $z$ gradient coils and $x$ gradient coils. Damped system $\left(\alpha_{M}=200\right) \ldots \ldots \ldots \ldots \ldots$

5.42 Modified magnet problem: Illustration of the geometry for (a) $z$ gradient coil and (b) $x$ gradient coil. . . . . . . . . . . . . . . . . .

5.43 Modified magnet problem with $z$ (longitudinal) gradient coil: comparison of $P_{\Omega_{4 K}}^{0}$ for axisymmetric Eulerian and Lagrangian approaches, 3D Lagrangian approach and experimental data. . . . . . . . . . . . . . 122

5.44 Modified magnet problem with $x$ (transversal) gradient coil: Dissipated power in the 4 K shield . . . . . . . . . . . . . . . . . . 123

6.1 Evolution of (a) $\left\|\boldsymbol{A}_{\varepsilon, h q}^{A C}\right\|_{L^{2}\left(\Omega_{C, n}\right)}$ and (b) $\left\|\boldsymbol{u}_{h p}^{A C}\right\|_{L^{2}\left(\Omega_{C, n}\right)}$ with the frequency for the test magnet problem with $z$ (longitudinal) gradient coil. . . 138

7.1 Test magnet problem with $z$ (longitudinal) gradient coil: illustration of the components of the problem. (a) Axisymmetric meridian plane and (b) 3D view. . . . . . . . . . . . . . . . . . . . . . . 143

7.2 PODI applied to test magnet problem with 1 parameter: Dissipated power and kinetic energy in the radiation shields for different $N_{s}$. Comparison with full order solution. . . . . . . . . . . . . . . . . . . . . . 145

7.3 Test magnet problem with 1 parameter: (a) $E_{\Omega_{77 K}}^{k}\left(2 \pi \mathrm{f}_{i}, \boldsymbol{u}^{P O D I}\right)$ for different $N_{s}$ and (b) $E_{\Omega_{77 K}}^{k}\left(2 \pi \mathrm{f}_{i}, \boldsymbol{u}_{h p}^{A C}\right)$ for different $N_{o} \ldots \ldots \ldots 146$

7.4 PODP applied to test magnet problem with 1 parameter. Dissipated power and kinetic energy in the radiation shields for different $N_{s}$. Comparison with the full order solution. . . . . . . . . . . . . . .

7.5 Test magnet problem with 1 parameter: (a) $E_{\Omega_{77 K}}^{k}\left(2 \pi \mathrm{f}_{i}, \boldsymbol{u}^{P O D P}\right)$ for different $N_{s}$ and (b) $E_{\Omega_{77 K}}^{k}\left(2 \pi \mathrm{f}_{i}, \boldsymbol{u}_{h p}^{A C}\right)$ for different $N_{o} \ldots \ldots \ldots$

7.6 POD applied to separated physics solutions: Decay of the singular values of $\mathbf{D}, \mathbf{D}_{\mathcal{A}}$ and $\mathbf{D}_{\mathcal{U}} \ldots \ldots \ldots \ldots$. . . . . . . . . . . . . . . . . 148

7.7 PODI applied to test magnet problem with 1 parameter: relative error, $e_{2}\left(\mathbf{q}\left(2 \pi \mathrm{f}_{i}\right), \mathbf{q}^{\mathrm{PODI}}\left(2 \pi \mathrm{f}_{i}\right)\right)$ for (a) $\mathbf{q}\left(2 \pi \mathrm{f}_{i}\right)=\mathcal{A}_{\varepsilon}^{\mathrm{AC}}\left(2 \pi \mathrm{f}_{i}\right)$ and (b) $\mathbf{q}\left(2 \pi \mathrm{f}_{i}\right)=$ $\mathcal{U}^{\mathrm{AC}}\left(2 \pi \mathrm{f}_{i}\right) \ldots \ldots \ldots \ldots \ldots \ldots \ldots$

7.8 PODP applied to test magnet problem with 1 parameter: relative error, $e_{2}\left(\mathbf{q}\left(2 \pi \mathrm{f}_{i}\right), \mathbf{q}^{\mathrm{PODI}}\left(2 \pi \mathrm{f}_{i}\right)\right)$ for (a) $\mathbf{q}\left(2 \pi \mathrm{f}_{i}\right)=\mathcal{A}_{\varepsilon}^{\mathrm{AC}}\left(2 \pi \mathrm{f}_{i}\right)$ and (b) $\mathbf{q}\left(2 \pi \mathrm{f}_{i}\right)=$

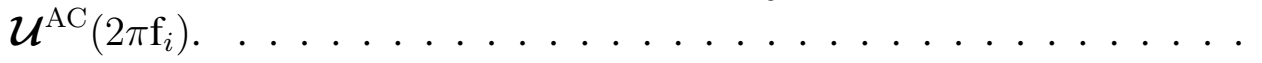

7.9 PODP applied to test magnet problem with 1 parameter: Relative error in the kinetic energy $e_{E_{\Omega_{C, n}}^{k}}^{P O P}$ for each of the radiation shields. . . . . . . .

7.10 PODI-Full applied to test magnet problem with 1 parameter: (a) $P_{\Omega_{4 K}}^{0}$, (b) $E_{\Omega_{4 K}}^{k}$, (c) $P_{\Omega_{77 K}}^{0}$, (d) $E_{\Omega_{77 K}}^{k}$, (e) $P_{\Omega_{O V C}}^{0}$ and (f) $E_{\Omega_{O V C}}^{k}$. Comparison with full order solution. . . . . . . . . . . . . . . . . . . . 
7.11 PODI-Full applied to test magnet problem with 1 parameter: (a) $e_{2}\left(\mathcal{A}_{\varepsilon}^{\mathrm{AC}}\left(2 \pi \mathrm{f}_{i}\right), \mathcal{A}^{\text {PODI-FULL }}\left(2 \pi \mathrm{f}_{i}\right)\right)$ and (b) $e_{2}\left(\mathcal{U}^{\mathrm{AC}}\left(2 \pi \mathrm{f}_{i}\right), \mathcal{U}^{\text {PODI-FULL }}\left(2 \pi \mathrm{f}_{i}\right)\right) .153$

7.12 PODI-Full applied to test magnet problem with 1 parameter: (a) Solver time and (b) speed-up for different values of $N_{s}$ and $N_{o} \ldots \ldots 153$

7.13 PODP-Full applied to test magnet problem with 1 parameter: (a) $P_{\Omega_{4 K}}^{0}$, (b) $E_{\Omega_{4 K}}^{k}$, (c) $P_{\Omega_{77 K}}^{0}$, (d) $E_{\Omega_{77 K}}^{k}$, (e) $P_{\Omega_{O V C}}^{0}$ and (f) $E_{\Omega_{O V C}}^{k}$. Comparison with full order solution. . . . . . . . . . . . . . . . . . . . 155

7.14 PODI-Full applied to test magnet problem with 1 parameter: (a) $\quad e_{2}\left(\mathcal{A}_{\varepsilon}^{\mathrm{AC}}\left(2 \pi \mathrm{f}_{i}\right), \mathcal{A}^{\mathrm{PODP}-\mathrm{FULL}}\left(2 \pi \mathrm{f}_{i}\right)\right) \quad$ and $\quad$ (b)

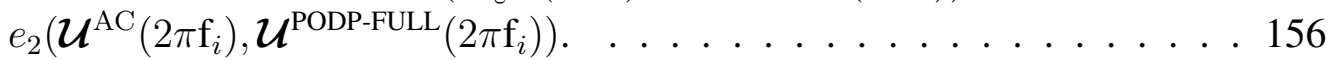

7.15 PODP-Full applied to test magnet problem with 1 parameter. Relative error in the kinetic energy $e_{E_{\Omega_{C, n}}^{K O D P}}^{R}$ for each of the radiation shields. . . . . 156

7.16 PODP-Full applied to test magnet problem with 1 parameter: (a) Solver time and (b) speed-up for different values of $N_{s}$ and $N_{o} \ldots \ldots 157$

7.17 PODP-Full applied to test magnet problem with multiple parameters: Dissipated power and kinetic energy in the radiation shields for different values of $\gamma^{O V C}$. Comparison with full order solution. . . . . . . . . . 158

7.18 PODP-Full applied to test magnet problem with multiple parameters: (a) $e_{2}\left(\mathcal{A}_{\varepsilon}^{\mathrm{AC}}\left(\mathbf{w}_{k}\right), \mathcal{A}^{\mathrm{PODP}-\mathrm{FULL}}\left(\mathbf{w}_{k}\right)\right)$ and (b) $e_{2}\left(\mathcal{U}^{\mathrm{AC}}\left(\mathbf{w}_{k}\right), \mathcal{U}^{\mathrm{PODP}-\mathrm{FULL}}\left(\mathbf{w}_{k}\right)\right) . \quad .159$

7.19 PODP-Full applied to test magnet problem with multiple parameters: (a) $e_{2}\left(\mathcal{A}_{\varepsilon}^{\mathrm{AC}}\left(\mathbf{w}_{k}\right), \mathcal{A}^{\mathrm{PODP}-\mathrm{FULL}}\left(\mathbf{w}_{k}\right)\right)$ and (b) $e_{2}\left(\mathcal{U}^{\mathrm{AC}}\left(\mathbf{w}_{k}\right), \mathcal{U}^{\mathrm{PODP}-\mathrm{FULL}}\left(\mathbf{w}_{k}\right)\right)$ for $\gamma^{O V C}=0.35 \gamma_{r e f}^{O V C}$ and different $N_{\gamma_{s}}=3,5,7 \ldots \ldots \ldots . \ldots 159$

7.20 PODP-Full applied to test magnet problem with multiple parameters: $e_{P_{\Omega_{C, n}}^{O O D P-F U L L}}^{P O}$ and $e_{E_{\Omega_{C, n}}^{P O D P-F U L L}}^{k}$ different values of $\gamma^{O V C}$. Comparison with full order solution. $\ldots \ldots+160$

7.21 PODP-Full applied to test magnet problem with multiple parameters: (a) Solver time and (b) speed-up for different values of $N_{s}$ and $N_{o} \ldots \ldots 161$

7.22 PODP-Full applied to test magnet problem with multiple parameters: Contours of (a) $\left|\operatorname{Re}\left(\boldsymbol{J}_{h p q}^{o}(\boldsymbol{x}, \mathbf{w})\right)\right|$ and (b) $\left|\operatorname{Re}\left(\boldsymbol{g}^{P O D P-F U L L}(\boldsymbol{x}, \mathbf{w})\right)\right|$ in the $77 \mathrm{~K}$ shield for $\mathbf{w}=\left(2000 \pi, 0.35 \gamma_{r e f}^{O V C}\right)$ compared with the full order solution. Deformation exaggerated by a factor of $10^{4}$

7.23 PODP-Full applied to test magnet problem with multiple parameters: Contours of (a) $\left|\operatorname{Re}\left(\boldsymbol{u}_{h p}^{\mathrm{AC}}(\boldsymbol{x}, \mathbf{w})\right)\right|$ and (b) $\left|\operatorname{Re}\left(\boldsymbol{u}^{P O D P-F U L L}(\boldsymbol{x}, \mathbf{w})\right)\right|$ in the $4 \mathrm{~K}$ shield for $\mathrm{w}=\left(2000 \pi, 0.35 \gamma_{r e f}^{O V C}\right) \ldots \ldots \ldots \ldots 2 \ldots \ldots \ldots$

7.24 Modified magnet problem with $x$ (transversal) gradient coil: (a) Illustration of the chosen $\partial \Omega_{C}^{D}$, highlighted in red and (b) illustration of the 3D geometry.

7.25 PODP-Full applied to modified magnet problem with multiple parameters: Dissipated power and kinetic energy in the radiation shields for different values of $\gamma^{77 K}$. Comparison with full order solution. . . . . . . . 164

7.26 PODP-Full applied to modified magnet problem with multiple parameters: (a) Solver time and (b) speed-up for different values of $N_{s}$ and $N_{o} . \quad 165$ 
7.27 PODP-Full applied to modified magnet problem with multiple parameters: Contours of (a) $\left|\operatorname{Re}\left(\boldsymbol{J}_{h p q}^{o}(\boldsymbol{x}, \mathbf{w})\right)\right|$ and (b) $\left|\operatorname{Re}\left(\boldsymbol{g}^{P O D P-F U L L}(\boldsymbol{x}, \mathbf{w})\right)\right|$ in the deformed $4 \mathrm{~K}$ shield for $\mathbf{w}=\left(8000 \pi, 0.5 \gamma_{r e f}^{77 K}\right)$. Deformation exaggerated by a factor of $10^{4} \ldots \ldots \ldots 166 \ldots \ldots \ldots$

B.1 "Healing" the geometry in NetGen [155]. . . . . . . . . . . . . . . 181

D.1 Parallel solution of the system for varying frequencies: code screen shot. . 187

E.1 Schematic representation of the software structure, including the preprocessing, solver and post-processing stages. . . . . . . . . . . . . . . 191

E.2 Problem file definition: illustration of the definition of the material properties. . . . . . . . . . . . . . . . . 195

E.3 Problem file definition: illustration of the definition of the functions used to impose the source terms and analytical solution. . . . . . . . . . 196

E.4 mainParallel function: illustration of the user-defined input parameters. . 197

E.5 mainParallel function: illustration of the user-defined switches that control the different pre-processing, solver and post-processing options. . . . 198

E.6 mainParallel function: illustration of the definition of the parameter problem defining the problem file to be used. . . . . . . . . . . . . . 200

E.7 Screenshot of the main function showing the function to compute the integrated field quantities and the definition of the name for the mat data file. . . . . . . . . . . . . . . . . . . 200

E.8 Screenshot of the main function showing the function to compute the error with respect to the full order solution $e_{2}$ and the definition of the name for 


\section{List of Tables}

4.1 Symmetry conditions for $\boldsymbol{A}_{\varepsilon, h q}^{D C}, \boldsymbol{u}_{h p}^{D C}, \boldsymbol{A}_{\varepsilon, h q}^{A C}$ and $\boldsymbol{u}_{h p}^{A C}$ at the planes $x=0$, $y=0$ and $z=0 \ldots \ldots \ldots \ldots \ldots \ldots \ldots$

4.2 Symmetry conditions for $\boldsymbol{T}$ at the planes $x=0, y=0$ and $z=0$. . . . 79

5.1 Permeable sphere in a uniform static magnetic field: Solver time in seconds for CG and PGC in two different meshes of 1083 and 6654 elements and polynomial orders $q=0,1,2,3,4$. The tolerance was set to $10^{-5}$ and the maximum number of iterations to $5 \times 10^{5} \ldots \ldots \ldots 8$

5.2 Permeable sphere in a uniform magnetic field: rates of convergence for the relative error $\left\|\boldsymbol{A}_{\varepsilon, h q}^{D C}-\boldsymbol{A}_{\text {exact }}^{D C}\right\|_{\boldsymbol{H}(\mathrm{curl})\left(\Omega_{C}\right)} /\left\|\boldsymbol{A}_{\text {exact }}^{D C}\right\|_{\boldsymbol{H}(\mathrm{curl})\left(\Omega_{C}\right)}$ under $h$ refinement ........................ 90

5.3 Hollow cylinder subject to pressure field: Solver time in seconds for CG and PGC in two different meshes of 1366 and 3144 elements and polynomial orders $p=1,2,3,4,5$. The tolerance was set to $10^{-7} \ldots \ldots 92$

5.4 Hollow cylinder subject to pressure field: rates of convergence for the relative error $\left\|\boldsymbol{u}_{h p}^{D C}-\boldsymbol{u}_{\text {exact }}\right\|_{H^{1}(\Omega)} /\left\|\boldsymbol{u}_{\text {exact }}\right\|_{H^{1}(\Omega)}$ under $h$-refinement. . . 93

5.5 Conducting sphere in a uniform alternating magnetic field: Solver time in seconds for GMRES and PGMRES for a mesh 1083 elements, polynomial orders $q=0,1,2,3,4$ and two different frequencies $f=50 \mathrm{~Hz}$ and $f=1000 \mathrm{~Hz}$. The tolerance was set to $10^{-5} \ldots \ldots$. . . . . . . . . 96

5.6 Conducting sphere in a uniform alternating magnetic field magnetic field: rates of convergence for the relative error $\| \mathcal{A}_{\varepsilon, h q}^{A C}$ $\boldsymbol{A}_{\text {exact }}\left\|_{\boldsymbol{H}(\mathrm{curl})\left(\Omega_{C}\right)} /\right\| \boldsymbol{\mathcal { A }}_{\text {exact }} \|_{\boldsymbol{H}(\mathrm{curl})\left(\Omega_{C}\right)}$ under $h$-refinement. . . . . . . . .

5.7 AC elasticity stage, hollow cylinder subject to body force: rates of convergence for the relative error $\left\|\boldsymbol{u}_{h p}^{D C}-\boldsymbol{u}_{\text {exact }}\right\|_{H^{1}(\Omega)} /\left\|\boldsymbol{u}_{\text {exact }}\right\|_{H^{1}(\Omega)}$ under $h$-refinement. 


\section{Part I}

\section{Preliminaries}




\section{Chapter 1}

\section{Introduction}

\subsection{MRI Scanners}

Magnetic Resonance Imaging (MRI) scanners are an essential tool in the medical industry. The high resolution images of the human body that they are able to produce have led to great advances in the diagnosis of many diseases, such as heart and vascular problems [65], tumours [102] or internal bleeding among others, as well as in the area of neuroimaging [25]. As opposed to other medical imaging techniques, such as computed tomography (CT) or X-ray, MRI do not use harmful radiation, which makes it the preferred imaging technique when working with children or patients requiring repeated scans. Furthermore, MRI usually outperforms CT in terms of image quality and, thus, is the preferred methodology when dealing with serious diseases such as cancer $[122,24]$ or cardiac problems [46], as well as in neuroimaging [100].

The most common type used for diagnostic imaging are superconducting magnets, as they allow for high field strength, typically $1.5-3 \mathrm{~T}$, leading to high resolution images. A superconducting MRI scanner, see Figure 1.1, essentially consists of three main components: the cryostat, main coils and gradient coils. The cryostat is formed by several radiation shields and keeps the liquid helium surrounding the superconducting main coils at a temperature of around $4 \mathrm{~K}$. These main coils generate a strong static magnetic field, which aligns the protons of the hydrogen atoms in the patient's body parallel or antiparallel to the field. The (resistive) gradient coils generate time varying pulsed magnetic fields. Three sets of gradient coils are used to generate gradients of the magnetic field in the $x, y$ and $z$ directions, and, by activating combinations of these, a gradient in any desired direction can be obtained. These gradients will disturb the protons bringing them out of alignment by moving them to a higher energy state. As soon as the gradient field is switched off the protons start to return to the lower energy state, in a process called relaxation, and this produces a signal that is then used to reconstruct an image of the body [55].

The interaction between the resulting magnetic fields and the conducting radiation shields gives rise to an important problem in MRI scanner design. The transient magnetic field generated by the gradient coils induces eddy currents in conducting components. These 


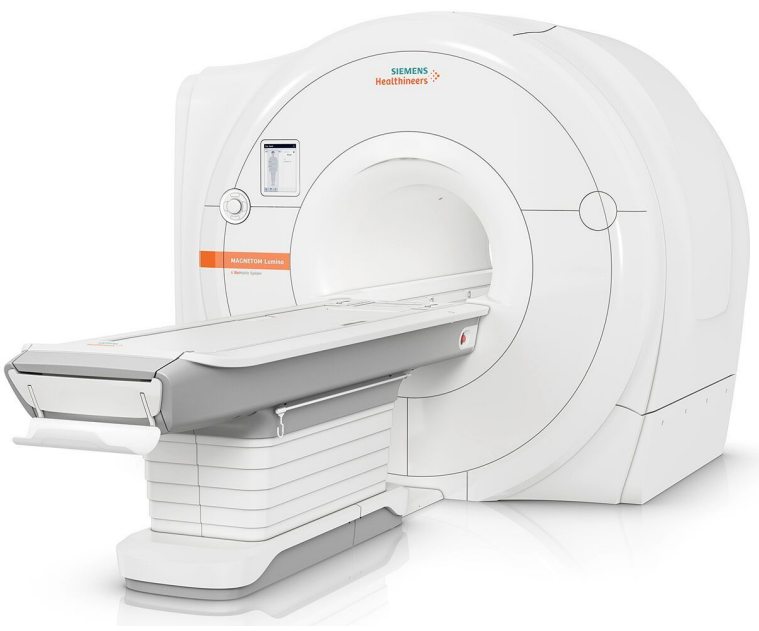

(a) Magnetom Lumina

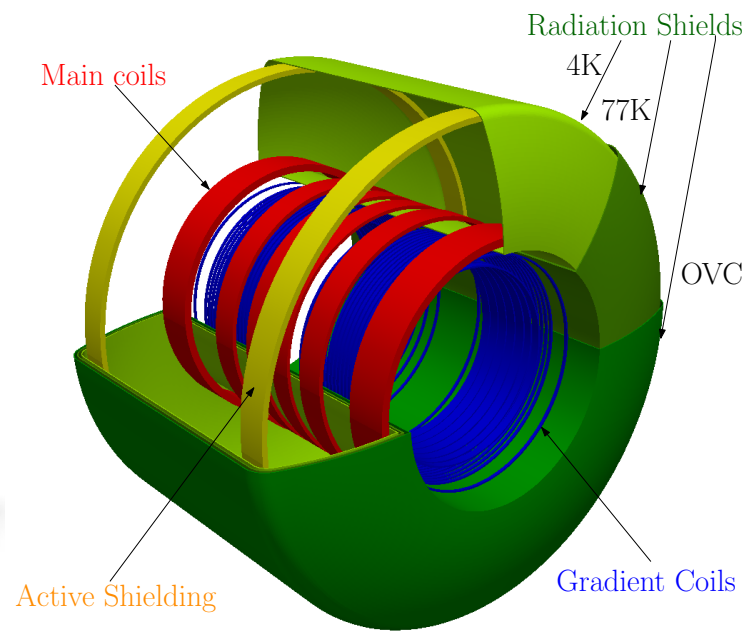

(b) MRI main components

Figure 1.1: MRI scanner. (a) New model Magnetom Lumina 3T. Image Courtesy of Siemens Healthineers. (b) Illustration of the main components: main coils, gradient coils and radiation shields $(4 \mathrm{~K}, 77 \mathrm{~K}$ and Outer Vacuum Chamber (OVC)).

eddy currents, in turn, produce electromagnetic stresses, causing the radiation shields to deform and vibrate $[110,19,18]$. The vibrations are undesirable as they lead to a deterioration in image quality, with image artefacts, as well as associated noise, which can cause patient discomfort. The eddy currents, on the other hand, lead to heat being dissipated and deposited in the cryostat, which can cause helium boil off and potentially result in a costly magnet quench. A quench refers to the sudden loss of superconductivity in the main coils when its temperature is raised. In the superconducting state, the resistance in the main coils is zero and, thus, the current flows freely through the coils. However, when the temperature raises, the resistance in the coils becomes finite, which causes the loss of the strong static magnetic field. Furthermore, when the superconducting state is lost, the current flowing through the coils results in the generation of more heat due to Joule's effect, which can cause an explosive helium boil-off [181]. Thus, understanding the mechanisms involved in the generation of these vibrations and the heat being deposited in the cryostat are key for a successful MRI scanner design. This requires the solution of a coupled physics magneto-mechanical problem, which will be addressed in this thesis.

The latest developments in MRI design have resulted in this problem attracting even more interest. Recently, the Magnetom Terra [162] manufactured by Siemens Healthineers, has become the first 7 T MRI cleared for clinical use [69]. This improvement in field strength has led to a higher image resolution making easier the diagnosis of subtle pathologies which were until then difficult to identify, but it has also brought the problem of larger eddy currents and stronger vibrations. Low helium magnets have also come into production in the last years. Typical MRI scanners require thousands of litres of helium in order to maintain the superconducting coils at a temperature of $4 \mathrm{~K}$. At the time of writing, the availability of helium has become challenging, leading to rising prices and a big impact 
in the production cost of MRI scanners. On the other hand, magnets using low helium technology use only around $1-20$ litres to operate $[132,140]$. For such designs, the minimisation of the heat deposited into the cryostat becomes even more important, as a small amount of helium boil-off could potentially result in a magnet quench.

\subsection{Computational Methods}

Given the issues described in Section 1.1, and considering the high cost of experiments, as well as the large number of iterations in the design process required to develop a new MRI scanner, manufacturers are interested in the development of accurate numerical simulation tools that are able to predict the complex coupling between the electromagnetic and mechanical fields. The development of such tools is challenging due to the complexity involved in accurately describing the interaction between the electromagnetic and mechanical fields, as well as in developing a computational method that is able to accurately resolve the coupling effects in an industrially relevant time.

Different computational methods can be considered for the numerical simulation of physics and engineering problems described by partial differential equations (PDEs). Some of the most popular methods used for the discretisation of PDEs are the finite difference, finite volume and finite element methods, which are all volume based methods, as well as the boundary element method, which is a surface based method.

The boundary element method [12,184] is a surface based method, and as such it only requires the discretisation of the boundary. This method is good for dealing with scattering or wave propagation problems set in unbounded domains, but not for dealing with problems with multiple materials. For this reason, when dealing with unbounded domains involving multiple materials, the boundary element method is often coupled with a domain based approach [23, 120].

The finite difference method [44] is the simplest of the domain based methods discussed above and, as such, has been widely used in the numerical simulation of physical and engineering problems, such as electromagnetics [125, 123], solid mechanics [15, 64] or fluid dynamics $[142,63]$. The method is very simple to implement and can lead to very efficient schemes, but it presents problems when dealing with complex geometries [44] and is, in general, limited to regular grids.

The finite volume method [176] is based on the computation of fluxes across cells and, therefore, becomes very computationally efficient for electromagnetic $[81,82,128]$ and fluid flow applications $[176,93,57,7]$. However, one of the drawbacks of the finite volume method is the non-locality required for high order discretisations [44, 115], leading, therefore, to fine discretisations for practical problems requiring very high accuracy.

The finite element method [187, 89, 171], on the other hand, can be applied to problems with complex geometries and it easily allows high order discretisations to be used. Due to this, the finite element method is widely used for the simulation of physics and engineering problems in many areas, such as solid mechanics [186, 39, 146, 91], fluid dynamics $[66,83]$ and electromagnetism $[177,113,185,129]$. The classical finite element version 
is usually referred to as the $h$-version of the finite element method and, in this version, the accuracy of the solution is improved by refining the mesh ( $h$-refinement). On the other hand, the $p$-version of the finite element method is based on the use of high order elements and, in this version, the accuracy of the solution is improved by increasing the polynomial order of the basis functions ( $p$-refinement). It is well known that, for problems with smooth solutions in a domain formed by linear geometry, $p$-refinement offers an increased rate of convergence of the solution compared to $h$-refinement $[172,171]$. In particular, for this type of problems, $h$-refinement results in an algebraic rate of convergence of the error in the energy norm, while $p$-refinement leads to exponential convergence $[172,171]$. A combination of both versions results in the $h p$-version of the finite element method, where the accuracy of the solution is improved by refining the mesh and increasing the polynomial order of the basis functions. The use of $h p$-finite elements leads to exponential convergence even for problems with a solution that is not smooth, provided that the correct combination of $h$-refinement and $p$-refinement is chosen $[171,172]$.

\subsubsection{Finite Elements for Electromagnetic Problems}

Electromagnetic problems are described by Maxwell's equations. However, there exist a broad range of applications within the field of electromagnetics and, for each of them, different simplifications can be applied to the system of Maxwell equations. Transient applications can be classified into two main groups: low frequency and high frequency. One of the main applications in high frequency electromagnetics is the study of electromagnetic scattering, which has application in areas such as the detection of hidden targets or radar technology [108]. For the simulation of such problems, the finite element method has been widely applied $[60,112,111,108]$. One of the main challenges in these simulations is overcoming problems with dispersion error in wave propagation and the $p$-version of the finite element method is well suited to this.

On the other hand, low frequency electromagnetics has many industrial applications such as the design of electric motors, power generators, transformers or medical imaging devices such as MRI scanners and, thus, this is the relevant simplification of Maxwell's equations for this thesis. In these applications, the eddy current approximation to the Maxwell's equations can be applied [88, 30, 13, 41], where the displacement currents are neglected. The finite element method has also been widely applied for the simulation of eddy current problems $[31,30,113,105]$. In these simulations, one of the main challenges is the accurate resolution of potentially small skin depths [113] in conducting bodies. In practice, this requires localised $h$-refinement of the conducting bodies combined with $p$ refinement for capturing accurate fields.

Furthermore, it is important to note that the electromagnetic field requires only tangential continuity across element interfaces and, therefore, its discretisation using standard $H^{1}$-conforming finite elements is not appropriate, and it has been observed to lead to wrong solutions in the presence of re-entrant corners and edges [53] or to spurious solutions in the case of eigenvalue problems $[34,36]$. To overcome this issue, an alternative set of $\boldsymbol{H}$ (curl)-conforming basis functions must be used. A set of low order $\boldsymbol{H}$ (curl)- 
conforming basis functions was first introduced by Nédélec [131], and these are typically referred to as Nédélec or edge elements, as the degrees of freedom are in this case associated with the edges of the elements. High order $\boldsymbol{H}$ (curl)-conforming basis functions were later proposed [10,156, 185, 58, 175], allowing the use of $p$ - or $h p$-finite elements for the discretisation of the electromagnetic field.

There is also a variety of formulations that can be used for the description of eddy current problems, in terms of different vector and scalar potentials [31, 30]. In [113], the performance of the $\boldsymbol{H}$-based and $\boldsymbol{A}$-based formulations for the solution of problems with multiply connected domains was studied, and it was shown that, for the case of a conductor with a handle, the $\boldsymbol{H}$-based formulation required the insertion of cuts in order to correct the fields, while the $\boldsymbol{A}$-based formulation did not. Therefore, in [113], an $\boldsymbol{A}$-based formulation was used, and an $h p$-finite element discretisation was considered, which was shown to accurately resolve the skin depth effects. The sets of basis functions proposed by Schöberl and Zaglmayr [156] were used for the discretisation and it was shown that with a particular regularisation strategy and the use of a block Jacobi preconditioner this discretisation becomes very efficient.

\subsubsection{Finite Elements for Elasticity Problems}

When considering the deformation of an elastic body different reference systems can be used to describe this deformation. These can be classified in two groups, Lagrangian or material descriptions and Eulerian or spatial descriptions. In a Lagrangian description the focus is on the behaviour of a material particle, which will occupy different spatial positions at different times, while in a spatial description the focus is on a fixed spatial position, that might be occupied by different particles at different times [35]. Lagrangian formulations are very popular in solid mechanics applications, while Eulerian formulations are generally more suitable in fluid dynamics and electromagnetic applications. For small displacements, the updated Lagrangian approach is usually followed and the deformation gradient assumed to be the identity tensor.

Furthermore, several formulations can be considered in order to solve the elasticity equation: displacement-based [52, 32], stress-based [163] and mixed [51, 151] formulations. The advantages of the displacement based formulation include a reduced number of unknowns and an easier treatment of the boundary conditions and external forces. However, when considering the solution to the elasticity equation using a displacement-based formulation and low order finite elements, the problem of volumetric locking arises. This phenomenon appears when dealing with nearly incompressible materials and for static problems it has been shown to result in spurious or inaccurate solutions [48, 169]. One option to overcome this issue would be the use of mixed formulations, but this results in a significant increase of the number of degrees of freedom as well as in the requirement to satisfy the non-trivial Ladyzhenskaya-Babuška-Brezzi (LBB) condition. Another option to overcome this issue is the use of high order elements $[169,182,48,75]$. 


\subsubsection{Finite Elements for Coupled Problems}

Many industrial applications require the solution of coupled multi-physics problems such as fluid-structure interaction [85, 134], thermo-mechanical processes [45], electromechanical [75, 74], electro-thermo-mechanical [138, 137] and magneto-mechanical $[110,19]$ problems. Most coupled problems in these areas are usually dynamic and nonlinear, and require both spatial and temporal integration. To resolve non-linear systems, Newton-Raphson approaches based on a consistent linearisation are often performed, which results in the need to solve monolithic systems, but lead to quadratic convergence of the residual. However, in 3D, computational challenges of inverting the system mean that fixed point solvers are used in which only a single physics need to be solved at a time, but lead to only linear convergence of the residual. In these coupled problems, the resulting linear system of equations can exhibit either a one-way or a two-way coupling. In the first case, one of the physical fields depends on the other fields, but the opposite is not true, while in the second case, both fields depend on the other. For systems with one-way coupling, the system is usually solved in a staggered manner, where the solution for one of the fields is computed first and used then to compute the solution for the second field. On the other hand, if the system exhibits a two-way coupling, the system can be solved either using a monolithic solver, where the whole system of equations is solved at once or a partitioned solver, where the solution to each field is computed independently and the different fields are coupled via source terms that depend on the other fields. The solution is then iterated until convergence is reached. Both, partitioned $[97,71,154]$ and monolithic [75, 19, 95] solvers have been extensively applied for the solution of coupled problems. Another aspect that must be carefully considered is the choice of a consistent frame of reference, e.g. typically in electromagnetic applications the system of Maxwell equations is expressed in an Eulerian setting and in solid mechanics applications the momentum balance equation is usually expressed in a Lagrangian setting, however, when considering coupled magneto-mechanical problems, the complete set of equations must be expressed in the same frame (Eulerian or Lagrangian).

Furthermore, when dealing with coupled problems, different finite elements might be required to discretise the different physical fields. Several sets of compatible $L^{2}, H^{1}$, $\boldsymbol{H}$ (div) and $\boldsymbol{H}$ (curl)-conforming high order finite elements have been proposed [58, 59, $10,156,185]$, that can be used for such discretisations.

\subsubsection{Applications to MRI Scanners}

In the context of MRI scanners, the application of finite difference time domain methods for the prediction of induced eddy currents, but neglecting mechanical effects, was studied in $[76,173,121]$. Methods for the rapid design of MRI coils based on three dimensional electromagnetic simulations have also been considered [101]. Attempts at modelling the magneto-mechanical effects with commercial software, focusing on the structural design of superconducting coils for high field MRI scanners, have also been made, e.g. [117, 56]. However, the currently available commercial software tools capable of solving the 
coupled physics problem of interest, such as Ansys [1], NACS (Numerical Analysis of Coupled Systems) [4] or Opera FEA (Finite Element Analysis) [5], are not specifically designed for MRI scanners and do not offer tailored solution methodologies. In general, even with fine discretisations, they are unable to correctly predict the coupled physics in an MRI scanner with sufficient accuracy and, therefore, manufacturers are interested in specialist solutions.

In the work of Raush et al. [147] a low order temporal finite element scheme for the solution of three-dimensional (3D) magneto-mechanical problems was presented, which was implemented in the finite element/boundary element program CAPA [106]. This was extended in [148] to consider acoustic effects. To describe the coupling, they follow the approach of Kaltenbacher [97], which uses a layer of elements adjacent to the conductor in order to compute the induced electromagnetic force. In [110, 19], the solution in an axisymmetric configuration, using a $h p$-finite element software, a stress tensor formulation and a novel linearised approach using an AC-DC (Alternating Current-Direct Current) splitting was considered, which allows for its time harmonic solution in the frequency domain. This work was based in an Eulerian setting, where the assumption of small displacements and velocities was made. The work was then extended to consider the fully non linear problem in the time domain in $[18,17]$. The use of $h p$-finite elements [58, 59], as opposed to low-order finite element discretisations, was considered as it is known to enhance the resolution of fine scale features such as small skin depths at high frequencies [114], allow volumetric locking phenomena in nearly incompressible materials to be overcome $[170,48,182,86]$ and improve the resolution of acoustic wave propagation [126]. The drawbacks of this axisymmetric approach include that it only allows the rotationally symmetric $z$ gradient coils to be considered and that it assumes small velocities and accelerations. These drawbacks will be addressed in this thesis.

\subsubsection{Reduced Order Modelling}

In the design stage of a new MRI scanner, the same magneto-mechanical problem must be solved repeatedly for varying model parameters, such as frequency or conductivity of the radiation shields. Even if an efficient finite element solver is available for the solution of the coupled problem, the high computational cost required by these multiple simulations results in an increase of the total time required to obtain a new MRI design, which has financial implications.

In order to optimise this process, Reduced Order Models (ROMs) can be considered. ROMs allow to describe a family of solutions (usually arising from parametric partial differential equations) in terms of a reduced basis. The term "reduced" is used here to denote a basis whose size is much smaller than a standard finite element basis. The latter is denoted in this context as "full order" or "high fidelity" model [49, 14, 87, 43]. The reduction of the size of the basis in which the solution is sought has an immediate impact in the size of the matrix to be inverted and, therefore, in the computational cost of the method.

Several numerical techniques can be classified as ROMs. Some examples are Proper 
Generalised Decomposition (PGD) [49, 127, 50] and Proper Orthogonal Decomposition (POD) $[43,119,180,47]$. One key difference between PGD and POD is the complexity of their implementation and the extent to which an existing finite element solver must be modified to implement the corresponding ROM. This is usually called the intrusiveness of the technique; a non-intrusive technique can be used "on top" of an existing finite element solver without any modification. PGD is typically much more intrusive than POD. The intrusiveness, despite being independent of the numerical effectiveness of the technique, is sometimes crucial in the context of industrial applications, when some software might already have been certified for a particular application.

In the context of numerical simulations in engineering, POD has successfully been applied to different areas including mechanics [133, 143], thermal problems [180, 29], fluid flow $[124,139]$ as well as electromagnetic problems with application to integrated circuits [99].

The application of PGD to coupled magneto-mechanical problems with application to MRI scanners but restricted to axisymmetric configurations was also recently considered in $[21,22]$. Furthermore, the application of POD and PGD for the solution of this problem was compared in [22].

\subsection{Aim and Objectives}

The aim of this thesis is the development of a new accurate and efficient methodology for the solution of 3D magneto-mechanical problems with application to MRI scanners. With this in mind, the development of a high fidelity finite element software that allows the computation of accurate solutions is first addressed. Then, in order to optimize the design process of a new MRI scanner, the application of ROM methodologies is considered, which allow fast computations for varying model parameters.

To achieve this aim, the next specific objectives are considered:

1. To accurately describe the complex physics associated with vibrations induced by electromagnetic fields in MRI applications.

2. To develop a rigorous and efficient variational formulation for the complete coupled magneto-mechanical problem in three dimensions.

3. To develop an accurate and efficient high fidelity computational methodology for the solution of the magneto-mechanical problem of interest in three dimensions.

4. To demonstrate the accuracy and efficiency of the high fidelity computational methodology by applying it to challenging academic and industrially relevant problems.

5. To build a new ROM that allows the fast computation of solutions for varying model parameters in order to optimize the design process of new MRI configurations. 
6. To demonstrate the accuracy and efficiency of the ROM by applying it to challenging MRI configurations and performing comparisons against the high fidelity tool.

The research carried out in order to achieve this objectives is split in several stages, which are described in the outline of the thesis. This research was done as part of the AdMoRe project, in collaboration with Swansea University, Universitat Politecnica de Catalunya and the industrial partner Siemens Healthineers and under the supervision of Dr. Paul D. Ledger and Prof. Antonio J. Gil from Swansea University, Prof. Sergio Zlotnik from Universitat Politecnica de Catalunya and Dr. Mike Mallett from Siemens Healthineers. The AdMoRe project was funded by a Marie Sklodowska-Curie Innovative Training Network with grant number 675919.

\subsection{Outline of the Thesis}

This Thesis is formed by 8 chapters and is complemented by 5 appendices, which are organized in 5 parts. The organization of these is as follows:

\section{-PART I- \\ Preliminaries}

- Chapter 1 presents a brief introduction to the physics of MRI scanners and the issues to be addressed in this thesis. Then, a survey of different computational methodologies that can be applied for the numerical simulation of partial differential equation systems is presented, with a special focus on the numerical simulation of electromagnetic and elasticity problems. A review on different methodologies that can be applied for the solution of coupled problems is then presented, followed by a survey of the research that has been presented up to date in the area of magneto-mechanical simulation applied to MRI scanners. A brief introduction to ROM methodologies is also provided, including a brief review of the research in this field. The aim and objectives of this thesis are then stated.

\section{-PART II- \\ Full Order Model}

- Chapter 2 presents the governing equations for the coupled magneto-mechanical problem of interest. For this, the classical system of Maxwell's equations is first presented and, then, the case of problems considering moving or deformable bodies is discussed. For such cases special attention must be paid to obtain a consistent description of the electromagnetic and mechanical fields. For this, an introduction to the motion of a deformable body is provided and the different configurations that can be used to describe this motion, namely Lagrangian or material and Eulerian or spatial configurations, are described. The coupled magneto-mechanical system is then stated in the total Lagrangian, updated Lagrangian and Eulerian configurations, and the transformations between the different configurations are stated. 
Finally, a series of simplifying assumptions are introduced, which together with the introduction of a vector potential formulation and appropriate gauge, transmission and boundary conditions result in the proposed Lagrangian formulation for the description of the magneto-mechanical problem of interest. This constitutes the starting point for the development of the computational methodology presented in this thesis.

- Chapter 3 focuses on the development of a novel variational formulation of the resulting governing equations that lends itself to an efficient computational methodology. This is based on an AC-DC splitting that was first introduced in [19], resulting in a DC problem and an AC problem that can be linearised about the static solution and translated to the frequency domain. The linearisation of the coupled problem as well as a rigorous derivation of its variational form are described. Furthermore, the problem is translated to the frequency domain, where regularisation is applied, which results in the regularised variational formulation of the DC and $\mathrm{AC}$ problems. The main novelties of this chapter are the derivation of a linearised magneto-mechanical problem in a Lagrangian frame and its variational formulation, the translation of the linearised problem to the frequency domain, and the introduction of the regularised DC and AC problems in order to circumvent the Coulomb gauge condition.

- Chapter 4 describes the computational treatment of the linearised and regularised problem presented in Chapter 3. A high order finite element discretisation of the linearised and regularised problem is derived, which combines high order $H^{1}$ and $\boldsymbol{H}$ (curl) basis functions for the discretisation of the mechanical and electromagnetic fields, respectively. The definition of the reference element, a description of the basis functions in the reference element, the approximation of curved boundaries and the mapping from reference to physical domain are first presented, followed by the rigorous derivation of the discrete system of equations. Next, an efficient solver strategy is discussed, which involves the solution to the coupled problem in a staggered manner and the use of preconditioned iterative solvers when appropriate. The chapter presents also the symmetry conditions that can be exploited to reduce the cost of the simulations, as well as a methodology to ensure that the current source is solenoidal at a discrete level, avoiding issues with the compatibility of the equations. The addition of mechanical damping to the system is also discussed. The main novelties of this chapter are the development of a high order finite element discretisation for the linearised and regularised magneto-mechanical problem, which combines $H^{1}$ and $\boldsymbol{H}$ (curl)-conforming finite elements and the development of an efficient staggered algorithm for the solution of the coupled problem, which uses preconditioned iterative solvers when appropriate.

- Chapter 5 focuses on the application of the high fidelity computational methodology to a series of academic and industrially relevant problems. First, the methodology is applied to a series of single physics or decoupled problems with a known 
analytical solution, and the error in the numerical solution is studied in order to verify that the appropriate rates of convergence are obtained. The methodology is then applied to challenging MRI configurations including longitudinal as well as transversal gradient coils. Comparisons against the axisymmetric tool developed in $[110,19,18,17]$, commercial software and experimental data are presented to prove the accuracy and efficiency of the method. The main novelty of this chapter is the application of the developed finite element methodology to the numerical simulation of a series of academic and industrially relevant problems, including MRI configurations with longitudinal and transversal gradient coils.

\section{-PART III- \\ Reduced Order Modelling}

- Chapter 6 focuses on the development of a ROM that can be used in order to optimize the workflow of the MRI design process. The chapter provides a description of the POD methodology, including the off-line and on-line stages. Two options, namely interpolation based and projection based POD, are considered for the online stage. The application of this methodology to the complete coupled problem is first discussed, followed by the application to electromagnetics and mechanics separately and the description of a new reduced order-full order methodology that exploits the staggered nature of the problem. Furthermore, an affine decomposition of the problem in terms of frequency and conductivity is provided, which is key for the efficiency of projection based POD. The main novelty of this chapter is the development of a POD based reduced order-full order methodology for the solution of the 3D magneto-mechanical problem of interest, which exploits the staggered nature of the linearised Lagrangian formulation.

- Chapter 7 presents the application of the ROM techniques described in Chapter 6 to several challenging MRI configurations. First, only the frequency is considered as a model parameter and both interpolation and projection based POD are applied to the complete solution, which shows that a large number of snapshots is required in order to obtain accurate solutions and, thus, no computational gaining can be obtained. Next, POD is applied to the electromagnetic and mechanical solutions separately, showing that it is the mechanical problem that requires a large number of snapshots in order to accurately resolve the solution. Then, the combined reduced order-full order approach is considered and it is shown that it results in accurate results already for a small number of snapshots and, therefore, a big computational speed-up can be achieved. Furthermore a comparison between the interpolation and projection approaches was performed, which showed that the latter results in more accurate results for no significant increase in computational cost. Finally, the most successful approach (projection based reduced order-full order) was applied to different MRI configurations considering as parameters the frequency and the conductivity of the radiation shields. The accuracy and efficiency of the method was assessed by comparing with the full order solver, which showed that very accurate 
results can be obtained as well as an impressive computational speed-up. The main novelty of the chapter is the application of the different POD-based methodologies proposed in Chapter 6 to several challenging MRI configurations, in order to assess their accuracy and efficiency.

\section{-PART IV- \\ Conclusions and Future Work}

- Chapter 8 presents an overview of the work and the achievements of this thesis. The conclusions extracted from each chapter are stated. Furthermore, a list of recommendations for future research, that could further enhance the capabilities of the current methodology and facilitate its adoption as an industry standard, is provided.

\section{- PART V-}

\section{Appendices}

- Appendix A presents the derivation of the proof that $\boldsymbol{S}\left(\boldsymbol{A}^{D C}, \mathcal{A}^{A C}\right)=\mathbf{0}$ in $\Omega_{C}^{c} \backslash\left(\operatorname{supp}\left(\boldsymbol{J}^{A C}\right) \cup \operatorname{supp}\left(\boldsymbol{J}^{D C}\right)\right)$, which is used in Chapter 3 in order to replace the surface integrals by volume integrals.

- Appendix B provides a brief description of issues encountered when generating the CAD geometry using NetGen, AutoCad or AutoCad mechanical and the mesh using NetGen, and how to address them.

- Appendix C presents a description of the process to compute the required integrals using Gaussian quadrature.

- Appendix D provides a brief description of the parallel implementation used to speed-up the computation of the solution for varying frequencies.

- Appendix E presents a user guide to the software package developed as part of the work carried on during this thesis. This user guide is added in order to facilitate the usage of the software by the industrial partner and future students/researchers that might continue the research developed in this project.

\subsection{Research Outcomes}

This section presents a list of the research outcomes from the work performed as part of this thesis.

\subsubsection{Published and Submitted Journal Publications}

- M. Seoane, P.D. Ledger, A.J. Gil and M. Mallett. An accurate and efficient three dimensional high order finite element methodology for the simulation of magnetomechanical coupling in MRI scanners. International Journal for Numerical Methods in Engineering, 119(12):1185-1215, 2019. 
- M. Seoane, P.D. Ledger, A.J. Gil, S. Zlotnik and M. Mallett. A combined reduced order-full order methodology for the solution of 3D magneto-mechanical problems with application to MRI scanners. International Journal for Numerical Methods in Engineering, 121(16):3529-3559, 2020.

- G. Barroso, M. Seoane, A.J. Gil, P.D. Ledger, M. Mallett and A. Huerta. A staggered high-dimensional Proper Generalised Decomposition for coupled magnetomechanical problems with application to MRI scanners. Computer Methods in Applied Mechanics and Engineering, 370:113271, 2020.

\subsubsection{Conference Presentations}

- M. Seoane, P.D. Ledger, A.J. Gil, M. Mallett. Towards an $h p$-finite element approach for the numerical simulation of 3D eddy current problems of relevance to MRI scanners. VIII International Conference on Adaptive Modelling and Simulation, ECCOMAS Thematic Conference, Verbania, Italy, June 2017.

- M. Seoane, P.D. Ledger, A.J. Gil, M. Mallett. Towards an $h p$-finite element approach for the numerical simulation of 3D magneto-mechanical problems of relevance to MRI scanners. VI European Conference on Computational Mechanics-VII European Conference on Computational Fluid Dynamics, ECCM-ECFD, Glasgow, United Kingdom, June 2018.

- G. Barroso, L. Borchini, R. Ibáñez, R. Mena, G. Quaranta, M. Seoane, V. Tsiolakis, S. Vermiglio and M. Giacomini. Empowered decision-making in simulation-based engineering: Advanced Model Reduction for real-time, inverse and optimisation in industrial problems. VI European Conference on Computational Mechanics-VII European Conference on Computational Fluid Dynamics, ECCM-ECFD, Glasgow, United Kingdom, June 2018.

- M. Seoane, P.D. Ledger, A.J. Gil, S. Zlotnik and M. Mallett. A combined reduced order-full order technique for the solution of 3D magneto-mechanical problems with application to MRI scanners. IX International Conference on Adaptive Modelling and Simulation, ECCOMAS Thematic Conference, El Campello (Alicante), Spain, May 2019.

- M. Seoane, P.D. Ledger, A.J. Gil, S. Zlotnik and M. Mallett. A high order finite element formulation for the simulation of 3D magneto-mechanical problems with application to MRI scanners. XXII International Conference on the Computation of Electromagnetic Fields (COMPUMAG), Paris, France, July 2019.

\subsubsection{Research Posters}

- M. Seoane, P.D. Ledger, A.J. Gil, M. Mallett. Towards an $h p$-finite element approach for the numerical simulation of $3 \mathrm{D}$ eddy current problems of relevance to 
MRI scanners. VIII International Conference on Adaptive Modelling and Simulation, ECCOMAS Thematic Conference, Verbania, Italy, June 2017.

- S. Bagwell, P.D. Ledger A.J. Gil, G. Barroso, M. Seoane, M. Kruip and M. Mallett. Acousto-magneto-mechanical simulation using $h p$-finite elements for MRI scanner design. SIAM/UKIE Annual Conference, Glasgow, United Kingdom, January 2017.

- M. Seoane, P.D. Ledger, A.J. Gil and M. Mallett. Towards a 3D magnetomechanical software using $h p$-finite elements for MRI scanner design. ZCCE Postgraduate Student Workshop, Swansea, United Kingdom, January 2018. Awarded prize for second best poster.

- M. Seoane, P.D. Ledger, A.J. Gil and M. Mallett. Towards a 3D magnetomechanical software using $h p$-finite elements for MRI scanner design. Workshop on Numerical Methods in Applied Sciences and Engineering, Castelldefels, Spain, January 2018.

- M. Seoane, P.D. Ledger, A.J. Gil, M. Mallett and S. Zlotnik. A $h p$-finite element software for the numerical simulation of 3D coupled magneto-mechanical problems with application to MRI scanners. ZCCE Postgraduate Student Workshop, Swansea, United Kingdom, January 2019. Awarded prize for third best poster.

\subsubsection{Software Packages}

The methodology described in this thesis has resulted in a new software tool for the simulation of magneto-mechanical coupling in MRI scanners. The proprietary rights of this software belong to the industrial and academic partners of the AdMoRe project and, therefore, open access is not available. However, the methodology in which the software is based is described throughout the thesis, and a simple user guide is provided in Appendix $\mathrm{E}$, which includes a description of the capabilities of the software and a set of instructions on how to use the software. This user guide is added in order to facilitate the usage of the software by the industrial partner and future students/researchers that might continue the research developed in this project. 


\section{Part II}

\section{Full Order Model}




\section{Chapter 2}

\section{Governing Physical Laws}

\subsection{Introductory Remarks}

The purpose of this chapter is to present a consistent system of governing equations for the description of the magneto-mechanical problem of interest. Although the Maxwell equations and the elasticity equations are well known for static problems, challenges arise when dealing with coupled problems involving moving bodies. The chapter will draw together the results from key texts in the field $[174,166,73,35,104]$ and introduce a series of approximations and simplifications that are appropriate for the problems of interest in this thesis. For this, the general system of Maxwell's equations is first introduced, noting that the definition of certain terms becomes challenging for the case of moving bodies. Then, a brief introduction to the theory of deformable bodies is presented, focusing on the different configurations that can be used to describe the motion of the body, namely the material or Lagrangian configuration and the spatial or Eulerian configuration. The sets of equations describing the magneto-mechanical problem of interest in Lagrangian and Eulerian configurations are then presented, and the transformations between both configurations are also stated. A series of assumptions that can be applied to our particular problem are then discussed, which lead to the novel Lagrangian formulation of the magneto-mechanical problem of interest that will be used in this thesis for the development of new computational methodologies.

The structure of the Chapter is as follows. First, in Section 2.2 the system of Maxwell equations is stated in a general format. Then, in Section 2.3 an introduction to the theory of deformable bodies is provided, focusing on the concepts of material and spatial configuration. Then, in Section 2.4, the equations describing the coupled system in a total Lagrangian configuration are presented. Similarly, the equations describing the coupled system in an updated Lagrangian formulation are stated in Section 2.5, together with the transformations of the different fields from the current to the reference frame. The system of equations describing the coupled problem in an Eulerian setting is presented in Section 2.6. The interface conditions describing the behaviour of the fields at the interface between different domains are presented in Section 2.7. Section 2.8 presents a series of simplifications that can be applied to our particular problem that, together with the intro- 
duction of a vector potential and appropriate gauge conditions, results in the final system of equations for the coupled magneto-mechanical problem of interest in a Lagrangian frame. The Chapter finishes with concluding remarks in Section 2.9.

\subsection{Maxwell's Equations}

MRI scanners use static as well as transient magnetic fields in order to generate an image of the patient. This electromagnetic field is physically described by Maxwell's equations. Defining $\boldsymbol{E}, \boldsymbol{H}, \boldsymbol{D}$ and $\boldsymbol{B}$ as the electric field intensity, magnetic field intensity, electric flux density and magnetic flux density, respectively, Maxwell's equations can be stated in differential form as

$$
\left.\begin{array}{rl}
\operatorname{curl} \boldsymbol{E} & =-\frac{\partial \boldsymbol{B}}{\partial t} \\
\operatorname{curl} \boldsymbol{H} & =\frac{\partial \boldsymbol{D}}{\partial t}+\boldsymbol{J} \\
\operatorname{div} \boldsymbol{D} & =\rho_{V} \\
\operatorname{div} \boldsymbol{B} & =0
\end{array}\right\} \text { in } \Omega_{x} \times[0, T]
$$

where the differential operators act with respect to a fixed frame of reference $\Omega_{x}$ (which we will see later corresponds to the Eulerian case), $T$ is the final time of interest, $\rho_{V}$ is the volume charge density and $\boldsymbol{J}$ the electric current density, which, in the case of bodies at rest is composed of an Ohmic current $\boldsymbol{J}^{o}=\gamma \boldsymbol{E}$, being $\gamma$ the electric conductivity, and a source term $\boldsymbol{J}^{s}$. Furthermore, for a wide class of materials, it is generally accepted that the next constitutive equations hold

$$
\boldsymbol{D}=\epsilon_{0} \boldsymbol{E}+\boldsymbol{P}=\epsilon \boldsymbol{E}=\epsilon_{0} \epsilon_{r} \boldsymbol{E}, \quad \boldsymbol{B}=\mu_{0} \boldsymbol{H}+\boldsymbol{M}=\mu \boldsymbol{H}=\mu_{0} \mu_{r} \boldsymbol{H},
$$

where $\epsilon$ is the permittivity, $\boldsymbol{P}$ is the polarisation, $\mu$ is the permeability, $\boldsymbol{M}$ is the magnetisation and the subscripts 0 and $r$ denote the free space and relative values, respectively. Note that $\boldsymbol{P}$ and $\boldsymbol{M}$ depend linearly on $\boldsymbol{E}$ and $\boldsymbol{H}$, respectively, for a linear material and non-linearly on the fields for non-linear materials (e.g. ferroelectric and ferromagnetic materials). In addition, for homogeneous isotropic materials $\epsilon$ and $\mu$ simplify to being constant scalars, while in more general inhomogeneous non-linear anisotropic materials they are rank 2 tensors whose coefficients depend on the position and the fields. In this thesis, restriction will be made to linear homogeneous isotropic materials, which is a reasonable assumption for the components of an MRI scanner.

In the case of moving or deformable bodies, additional terms might arise in the system as a consequence of the movement, and attention must be paid to the reference system used to describe the governing equations. This is discussed in the following sections.

\subsection{Moving Bodies: Material and Spatial Descriptions}

In the study of problems with deformable or moving bodies a proper description of motion is fundamental. Consider the general motion of a deformable body illustrated in Figure 


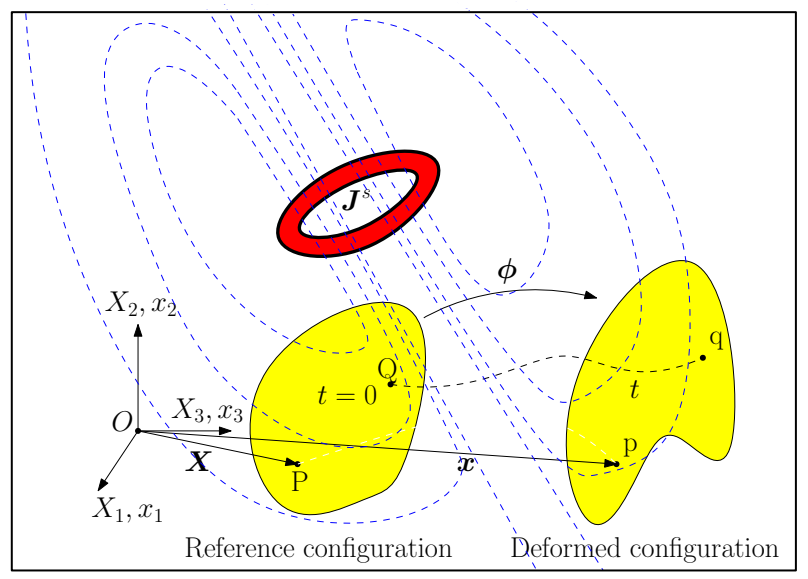

Figure 2.1: General motion of a deformable body.

2.1, where we have a body that is moving inside a region excited by a given current source $\boldsymbol{J}^{s}$ that generates an electromagnetic field. The body can be described as a composition of material particles with coordinates $\boldsymbol{X}$ at the initial or reference configuration, corresponding to time $t=0$. After a certain time $t$, and following a deformation, these particles will have in general different coordinates, denoted as $\boldsymbol{x}$. This motion can be described mathematically by means of a mapping between the initial and current configurations as [35]

$$
\boldsymbol{x}:=\boldsymbol{\phi}(\boldsymbol{X}, t)
$$

where $\boldsymbol{\phi}$ is the mapping function. The deformation (displacement) can then be defined as

$$
\boldsymbol{u}:=\boldsymbol{x}-\boldsymbol{X}
$$

Different coordinate systems can be used for the description of the motion. These can be classified in two groups, Lagrangian or material descriptions and Eulerian or spatial descriptions. In a Lagrangian description, the focus is on the behaviour of a material particle, which will occupy different spatial positions at different times, while, in a spatial description, the focus is on a fixed spatial position that might be occupied by different particles at different times [35]. Furthermore, Lagrangian formulations can be divided in two types; the total Lagrangian and updated Lagrangian formulations. Thus, three different formulations can be used to describe the governing equations: total Lagrangian, updated Lagrangian and Eulerian. Considering again the situation illustrated in Figure 2.1, in a total Lagrangian formulation, the system is described from the point of view of an observer that is placed at a particle $P$ in the reference configuration, while in an updated Lagrangian formulation, the system is described from the point of view of an observer that is mounted in a particle $p$ in the current configuration and, in an Eulerian formulation, the system is described from the point of view of an observer that is placed at a fixed spatial position $O$. Eulerian formulations are typically used in fluid flow applications as well as in electromagnetic applications, while Lagrangian formulations are usually more suitable in the context of solid mechanics [35]. 
To transform quantities from one to another configuration the deformation gradient $\mathcal{F}=$ $\frac{\partial \boldsymbol{\phi}}{\partial \boldsymbol{X}}$ as well as the jacobian $J=\operatorname{det} \mathcal{F}$ and the cofactor $\mathcal{H}=J \mathcal{F}^{-T}$ [35] are used.

For our coupled magneto-mechanical problem of interest, it is not clear, a priori, which approach will be more suitable. In the following sections we state the resulting systems of equations in total Lagrangian, updated Lagrangian and Eulerian formulations.

\subsection{Total Lagrangian Formulation}

In a total Lagrangian form, the governing equations are expressed in terms of the reference configuration $\boldsymbol{X}$. In this configuration, the physical variables are given a subscript 0 and, hence, $\boldsymbol{E}_{0}, \boldsymbol{H}_{0}, \boldsymbol{D}_{0}$ and $\boldsymbol{B}_{0}$ are used to denote the electric field intensity, magnetic field intensity, electric flux density and magnetic flux density, respectively, in this frame. Importantly, $\boldsymbol{J}^{s}$ has to be defined in the reference configuration as does the Ohmic current $\gamma \boldsymbol{E}_{0}$ [73]. On the other hand, the constitutive laws (2.2) must be transformed from the Eulerian form to the reference frame for the total Lagrangian form. The set of Maxwell equations in a total Lagrangian frame can be written as

$$
\left.\begin{array}{rl}
\text { CURL } \boldsymbol{E}_{0} & =-\frac{\mathrm{d} \boldsymbol{B}_{0}}{\mathrm{~d} t} \\
\text { CURL } \boldsymbol{H}_{0} & =\frac{\mathrm{d} \boldsymbol{D}_{0}}{\mathrm{~d} t}+\gamma \boldsymbol{E}_{0}+\boldsymbol{J}^{s} \\
\text { DIV } \boldsymbol{D}_{0} & =\rho_{V_{0}} \\
\text { DIV } \boldsymbol{B}_{0} & =0
\end{array}\right\} \text { in } \Omega_{0} \times[0, T],
$$

where $\Omega_{0}$ denotes the reference or material Lagrangian configuration and $\frac{\mathrm{d}}{\mathrm{d} t}$ denotes the total (material) time derivative [35]. Note that the system has a similar form to (2.1) but now expressed in terms of fields with respect to the reference configuration. Furthermore, the differential operators act now with respect to $\boldsymbol{X}$ (emphasised by the capitol differential operators) and the partial time derivatives $\frac{\partial}{\partial t}$ become, in this frame, material time derivatives $\frac{\mathrm{d}}{\mathrm{d} t}$. The transformations of the electromagnetic fields from the reference to the current configuration are given by $[174,166,73]$

$$
\begin{aligned}
\boldsymbol{D}_{0} & =\mathcal{H}^{T} \boldsymbol{D}, \\
\boldsymbol{B}_{0} & =\mathcal{H}^{T} \boldsymbol{B}, \\
\boldsymbol{E}_{0} & =\mathcal{F}^{T}(\boldsymbol{E}-\boldsymbol{B} \times \boldsymbol{v}), \\
\boldsymbol{H}_{0} & =\mathcal{F}^{T}(\boldsymbol{H}+\boldsymbol{D} \times \boldsymbol{v}) .
\end{aligned}
$$

where $\boldsymbol{v}=\frac{\mathrm{d} \boldsymbol{u}}{\mathrm{d} t}$ is the velocity.

For the case of moving bodies, the Maxwell system (2.5) can no longer be solved in isolation and must be solved in combination with the momentum balance equation, which in the total Lagrangian form is

$$
\frac{\mathrm{d} \boldsymbol{p}_{0}}{\mathrm{~d} t}-\operatorname{DIV} \mathcal{P}=\boldsymbol{f}_{0} \text { in } \Omega_{0} \times[0, T]
$$


where $\mathcal{P}$ is the first Piola-Kirchhoff tensor, $\boldsymbol{f}_{0}$ the body force and $\boldsymbol{p}_{0}$ the linear momentum per unit undeformed volume.

Note that the form of $\mathcal{P}$ depends on the constitutive model, it is certainly a function of the deformation and it can be a function of $\boldsymbol{D}_{0}$ and $\boldsymbol{B}_{0}$ as well as other fields in general $[74,136,135]$.

\subsection{Updated Lagrangian Formulation}

In the updated Lagrangian form, the Maxwell and linear momentum equations are expressed in terms of the current coordinates $\boldsymbol{x}=\phi(\boldsymbol{X}, t)$. In this configuration, the particles $\boldsymbol{X}$ acquire the temporary coordinate labels $\boldsymbol{x}=\phi(\boldsymbol{X}, t)$ at time $t$. The Maxwell system in the updated Lagrangian frame can be written as $[73,174]$

$$
\left.\begin{array}{rl}
\operatorname{curl}(\boldsymbol{E}-\boldsymbol{B} \times \boldsymbol{v}) & =-\mathcal{H}^{-T} \frac{\mathrm{d} \mathcal{H}^{T} \boldsymbol{B}}{\mathrm{d} t} \\
\operatorname{curl}(\boldsymbol{H}+\boldsymbol{D} \times \boldsymbol{v}) & =\mathcal{H}^{-T} \frac{\mathrm{d} \mathcal{H}^{T} \boldsymbol{D}}{\mathrm{d} t}+\mathcal{H}^{-T}\left(\gamma \mathcal{F}^{T}(\boldsymbol{E}-\boldsymbol{B} \times \boldsymbol{v})+\boldsymbol{J}^{s}\right) \\
\operatorname{div} \boldsymbol{D} & =\rho_{V} \\
\operatorname{div} \boldsymbol{B} & =0
\end{array}\right\} \text { in } \Omega(t)
$$

where $\Omega(t)$ denotes the updated Lagrangian configuration. System (2.8) is obtained from (2.5) transforming the fields according to (2.6) and taking into account the relation $\rho_{V}=$ $\frac{1}{J} \rho_{V_{0}}$ as well as the transformations of the differential operators

$$
\begin{aligned}
& \operatorname{CURL}\left(\mathcal{F}^{T} \boldsymbol{b}\right)=\mathcal{H}^{T} \operatorname{curl} \boldsymbol{b}, \\
& \frac{1}{J} \operatorname{DIV}\left(\mathcal{H}^{T} \boldsymbol{b}\right)=\operatorname{div} \boldsymbol{b},
\end{aligned}
$$

where $\boldsymbol{b}$ denotes a general vector field.

Similarly, the elasticity equation can be written in the updated Lagrangian frame as [35, 73]

$$
\rho \boldsymbol{a}-\operatorname{div} \boldsymbol{\sigma}=\boldsymbol{f} \text { in } \Omega(t)
$$

where $\boldsymbol{a}=\frac{\mathrm{d}^{2} \boldsymbol{u}}{\mathrm{d} t^{2}}$ is the acceleration and $\boldsymbol{\sigma}=\boldsymbol{\sigma}^{m}(\boldsymbol{u})+\boldsymbol{\sigma}^{e}\left(\boldsymbol{B}_{0}, \boldsymbol{H}_{0}, \boldsymbol{D}_{0}, \boldsymbol{E}_{0}\right)$ is the total stress tensor, with $\boldsymbol{\sigma}^{m}(\boldsymbol{u})$ the Cauchy stress tensor and $\boldsymbol{\sigma}^{e}\left(\boldsymbol{B}_{0}, \boldsymbol{H}_{0}, \boldsymbol{D}_{0}, \boldsymbol{E}_{0}\right)$ the Maxwell stress tensor. System (2.10) can be obtained from (2.7) using the transformations between fields

$$
\begin{array}{r}
\frac{1}{J} \frac{\mathrm{d} \boldsymbol{p}_{0}}{\mathrm{~d} t}=\rho \boldsymbol{a}, \\
\mathcal{P}=\boldsymbol{\sigma \mathcal { H }}, \\
\frac{1}{J} \boldsymbol{f}_{0}=\boldsymbol{f},
\end{array}
$$

and the transformation for the divergence of a tensor field

$$
\frac{1}{J} \operatorname{DIV}(\boldsymbol{\sigma} \mathcal{H})=\operatorname{div}(\boldsymbol{\sigma})
$$




\subsection{Eulerian Formulation}

In the Eulerian formulation, quantities are expressed in terms of the spatial coordinates $\boldsymbol{x}$. This frame is the usual one used for Maxwell's equations [166, 174]. In this frame the Maxwell system can be written as

$$
\left.\begin{array}{rl}
\operatorname{curl} \boldsymbol{E} & =-\frac{\partial \boldsymbol{B}}{\partial t} \\
\operatorname{curl} \boldsymbol{H} & =\frac{\partial \boldsymbol{D}}{\partial t}+\mathcal{H}^{-T}\left(\gamma \mathcal{F}^{T}(\boldsymbol{E}-\boldsymbol{B} \times \boldsymbol{v})+\boldsymbol{J}^{s}\right) \\
\operatorname{div} \boldsymbol{D} & =\rho_{V} \\
\operatorname{div} \boldsymbol{B} & =0
\end{array}\right\} \text { in } \Omega_{x} \times[0, T],
$$

where $\Omega_{x}$ denotes the Eulerian configuration. System (2.13) can be obtained from (2.5) or (2.8) using the transformations (2.6) and (2.9) as well as the transformation of the material derivative for equations involving the curl of a vector field [73, 174]

$$
\frac{\partial \boldsymbol{b}}{\partial t}=\mathcal{H}^{-T} \frac{\mathrm{d} \boldsymbol{\mathcal { H }}^{T} \boldsymbol{b}}{\mathrm{d} t}-\operatorname{curl}(\boldsymbol{b} \times \boldsymbol{v})
$$

where $\boldsymbol{b}$ denotes a general vector field.

Note that system (2.13) reduces to (2.1) for the case of a body at rest. The term $\gamma \boldsymbol{B} \times \boldsymbol{v}$ which appears in system (2.13) for moving bodies is usually referred to as Lorentz current $[80,166]$. Furthermore, note that for moving bodies (2.13) is often presented for the case of $\mathcal{H} \approx \mathcal{F} \approx \mathbb{I}$, but this is not true in general $[174,166]$.

Similarly, the momentum balance equation, describing the mechanical deformations can be written in Eulerian form as

$$
\frac{\partial \boldsymbol{p}}{\partial t}+\operatorname{div}(\boldsymbol{p} \otimes \boldsymbol{v}-\boldsymbol{\sigma})=\boldsymbol{f} \text { in } \Omega_{x} \times[0, T],
$$

where $\boldsymbol{p}=\rho \boldsymbol{v}$ is the linear momentum per unit deformed volume, $\boldsymbol{\sigma}=\boldsymbol{\sigma}^{e}+\boldsymbol{\sigma}^{m}$ is the total stress tensor, with $\boldsymbol{\sigma}^{e}=\boldsymbol{\sigma}^{e}(\boldsymbol{B}, \boldsymbol{H}, \boldsymbol{D}, \boldsymbol{E}, \mathcal{F})$ and $\boldsymbol{\sigma}^{m}=\boldsymbol{\sigma}^{m}(\mathcal{F})$ the Maxwell and Cauchy stress tensors, and $\boldsymbol{f}$ is a body force. System (2.15) can be obtained from (2.7) or (2.10) using the transformations (2.11) and (2.12) as well as the transformation of the material derivative for equations involving the divergence of a tensor [73, 174]

$$
\frac{\mathrm{d} \boldsymbol{b}}{\mathrm{d} t}=\frac{\partial \boldsymbol{b}}{\partial t}+(\operatorname{grad} \boldsymbol{b}) \boldsymbol{v}
$$

where $\boldsymbol{b}$ denotes a general vector field.

Note that, without considering any extra simplifications, each formulation has advantages and disadvantages. If a total or updated Lagrangian formulation is chosen, the elasticity equation is simple, but the system of Maxwell equations becomes complicated. On the other hand, if an Eulerian formulation is instead chosen, the system of Maxwell's equations becomes simpler, but the elasticity equation becomes much more difficult to solve. In the following sections the interface conditions as well as a series of simplifying assumptions for the case of MRI applications that result in an accurate and efficient set of governing equations in a Lagrangian formulation will be presented. 


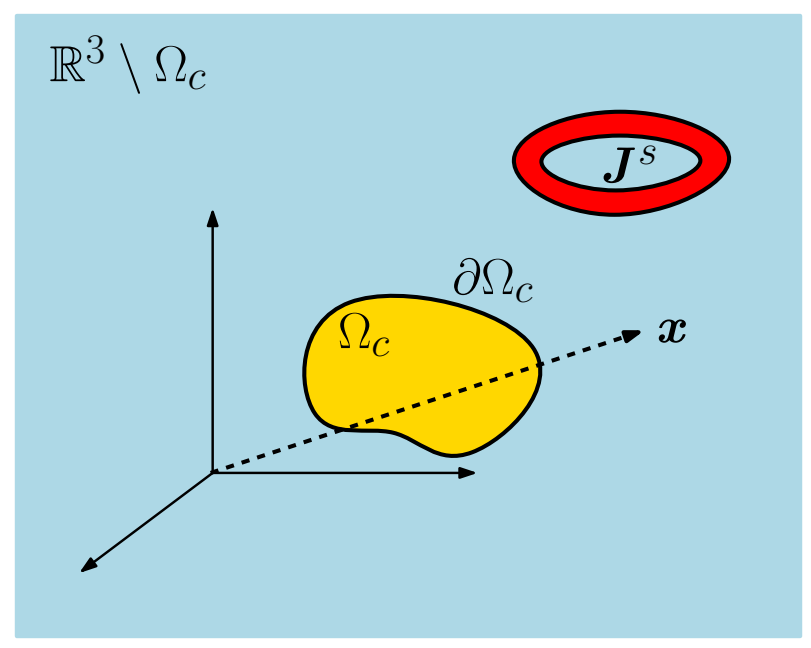

Figure 2.2: Magneto-mechanical coupled problem. General representation of the problem illustrating the different components involved.

\subsection{Interface Conditions}

Consider the case of a magneto-mechanical problem set on an unbounded domain $\mathbb{R}^{3}=$ $\Omega_{C} \cup \Omega_{C}^{c}$ where $\Omega_{C}=\bigcup_{n=1}^{N} \Omega_{C, n}$ denotes the union of $N$ disjoint elastic conducting bodies each with $\gamma \neq 0$ and $\mu$ possibly different from $\mu_{0}$ and $\Omega_{C}^{c}:=\mathbb{R}^{3} \backslash \overline{\Omega_{C}}$ denotes the non-conducting region (Figure 2.2). At the interface between different bodies the electromagnetic and mechanical fields must satisfy some interface or transmission conditions, which can be deduced from the integral form of the governing equations [80, 17, 73]

\subsubsection{Total Lagrangian Formulation}

In the total Lagrangian formulation, the transmission conditions at the interface between different domains are [73]

$$
\begin{aligned}
\boldsymbol{N} \times\left[\boldsymbol{E}_{0}\right]_{\partial \Omega_{C}} & =\mathbf{0}, \\
\boldsymbol{N} \times\left[\boldsymbol{H}_{0}\right]_{\partial \Omega_{C}} & =\boldsymbol{j}_{s_{0}}, \\
\boldsymbol{N} \cdot\left[\boldsymbol{D}_{0}\right]_{\partial \Omega_{C}} & =\rho_{s_{0}}, \\
\boldsymbol{N} \cdot\left[\boldsymbol{B}_{0}\right]_{\partial \Omega_{C}} & =0, \\
\left.\left(\boldsymbol{\mathcal { P }}^{m}+\boldsymbol{\mathcal { P }}^{e}\right)\right|_{\partial \Omega_{C}} ^{-} \boldsymbol{N} & =\left.\mathcal{P}^{e}\right|_{\partial \Omega_{C}} ^{+} \boldsymbol{N},
\end{aligned}
$$

where $[\cdot]_{\partial \Omega_{C}}:=\left.(\cdot)\right|_{\partial \Omega_{C}} ^{+}-\left.(\cdot)\right|_{\partial \Omega_{C}} ^{-}$denotes the jump on the interface $\partial \Omega_{C}$, where $\left.(\cdot)\right|_{\partial \Omega_{C}} ^{+}$ and $\left.(\cdot)\right|_{\partial \Omega_{C}} ^{-}$denote the non-conducting and conducting sides of the interface $\partial \Omega_{C}$, respectively, $\boldsymbol{N}$ is a unit outward normal vector to $\partial \Omega_{C}$ in the reference configuration, $\boldsymbol{j}_{s_{0}}$ is the surface current density in the reference configuration and $\rho_{s_{0}}$ is the surface charge density in the reference configuration. In the absence of surface current and charge density, the 
interface conditions (2.17) reduce to

$$
\begin{aligned}
\boldsymbol{N} \times\left[\boldsymbol{E}_{0}\right]_{\partial \Omega_{C}} & =\mathbf{0}, \\
\boldsymbol{N} \times\left[\boldsymbol{H}_{0}\right]_{\partial \Omega_{C}} & =\mathbf{0} \\
\boldsymbol{N} \cdot\left[\boldsymbol{D}_{0}\right]_{\partial \Omega_{C}} & =0 \\
\boldsymbol{N} \cdot\left[\boldsymbol{B}_{0}\right]_{\partial \Omega_{C}} & =0 \\
\left.\left(\mathcal{P}^{m}+\boldsymbol{P}^{e}\right)\right|_{\partial \Omega_{C}} ^{-} \boldsymbol{N} & =\left.\mathcal{P}^{e}\right|_{\partial \Omega_{C}} ^{+} \boldsymbol{N}
\end{aligned}
$$

which describe the tangential continuity of the electric and magnetic fields and the normal continuity of the electric flux density, magnetic flux density and total stress.

\subsubsection{Updated Lagrangian Formulation}

In the updated Lagrangian formulation, the transmission conditions at the interface between different domains are [73]

$$
\begin{aligned}
\boldsymbol{n} \times[(\boldsymbol{E}-\boldsymbol{B} \times \boldsymbol{v})]_{\partial \Omega_{C}} & =\mathbf{0} \\
\boldsymbol{n} \times[(\boldsymbol{H}+\boldsymbol{D} \times \boldsymbol{v})]_{\partial \Omega_{C}} & =\boldsymbol{j}_{s} \\
\boldsymbol{n} \cdot[\boldsymbol{D}]_{\partial \Omega_{C}} & =\rho_{s} \\
\boldsymbol{n} \cdot[\boldsymbol{B}]_{\partial \Omega_{C}} & =0 \\
\left.\left(\boldsymbol{\sigma}^{m}+\boldsymbol{\sigma}^{e}\right)\right|_{\partial \Omega_{C}} ^{-} \boldsymbol{n} & =\left.\boldsymbol{\sigma}^{e}\right|_{\partial \Omega_{C}} ^{+} \boldsymbol{n},
\end{aligned}
$$

where $\boldsymbol{n}$ is a unit outward normal vector in the deformed configuration, $\boldsymbol{j}_{s}$ is the surface current density in the deformed configuration and $\rho_{s}$ is the surface charge density in the deformed configuration. In the absence of surface current and charge densities (2.19) reduces to

$$
\begin{aligned}
\boldsymbol{n} \times[(\boldsymbol{E}-\boldsymbol{B} \times \boldsymbol{v})]_{\partial \Omega_{C}} & =\mathbf{0}, \\
\boldsymbol{n} \times[(\boldsymbol{H}+\boldsymbol{D} \times \boldsymbol{v})]_{\partial \Omega_{C}} & =\mathbf{0}, \\
\boldsymbol{n} \cdot[\boldsymbol{D}]_{\partial \Omega_{C}} & =0 \\
\boldsymbol{n} \cdot[\boldsymbol{B}]_{\partial \Omega_{C}} & =0, \\
\left.\left(\boldsymbol{\sigma}^{m}+\boldsymbol{\sigma}^{e}\right)\right|_{\partial \Omega_{C}} ^{-} \boldsymbol{n} & =\left.\boldsymbol{\sigma}^{e}\right|_{\partial \Omega_{C}} ^{+} \boldsymbol{n} .
\end{aligned}
$$

\subsubsection{Eulerian Formulation}

In the Eulerian formulation, the transmission conditions at the interface between different domains are [73]

$$
\begin{gathered}
\boldsymbol{n} \times[\boldsymbol{E}]_{\partial \Omega_{C}}=\mathbf{0}, \\
\boldsymbol{n} \times[\boldsymbol{H}]_{\partial \Omega_{C}}=\boldsymbol{j}_{s},
\end{gathered}
$$




$$
\begin{aligned}
\boldsymbol{n} \cdot[\boldsymbol{D}]_{\partial \Omega_{C}} & =\rho_{s}, \\
\boldsymbol{n} \cdot[\boldsymbol{B}]_{\partial \Omega_{C}} & =0 \\
\left.\left(\boldsymbol{\sigma}^{m}+\boldsymbol{\sigma}^{e}\right)\right|_{\partial \Omega_{C}} ^{-} \boldsymbol{n} & =\left.\boldsymbol{\sigma}^{e}\right|_{\partial \Omega_{C}} ^{+} \boldsymbol{n},
\end{aligned}
$$

which in the absence of surface current and charge densities reduces to

$$
\begin{aligned}
\boldsymbol{n} \times[\boldsymbol{E}]_{\partial \Omega_{C}} & =\mathbf{0}, \\
\boldsymbol{n} \times[\boldsymbol{H}]_{\partial \Omega_{C}} & =\mathbf{0}, \\
\boldsymbol{n} \cdot[\boldsymbol{D}]_{\partial \Omega_{C}} & =0, \\
\boldsymbol{n} \cdot[\boldsymbol{B}]_{\partial \Omega_{C}} & =0, \\
\left.\left(\boldsymbol{\sigma}^{m}+\boldsymbol{\sigma}^{e}\right)\right|_{\partial \Omega_{C}} ^{-} \boldsymbol{n} & =\left.\boldsymbol{\sigma}^{e}\right|_{\partial \Omega_{C}} ^{+} \boldsymbol{n} .
\end{aligned}
$$

\subsection{Modelling Assumptions}

In the following we summarize the main assumptions that led to the Eulerian formulation used in $[110,19,18,17]$ as well as the assumptions that lead to the new Lagrangian formulation [73].

\subsubsection{Eulerian Formulation for Small Displacements and Velocities}

In $[110,19,18,17]$ the coupled magneto-mechanical problem of interest was described using an Eulerian approach, which was based in the following assumptions:

1. The eddy current approximation is applied in the Eulerian frame. System (2.13) becomes then

$$
\begin{aligned}
\operatorname{curl} \boldsymbol{E} & =-\frac{\partial \boldsymbol{B}}{\partial t}, \\
\operatorname{curl} \boldsymbol{H} & =\mathcal{H}^{-T}\left(\gamma \mathcal{F}^{T}(\boldsymbol{E}-\boldsymbol{B} \times \boldsymbol{v})+\boldsymbol{J}^{s}\right), \\
\operatorname{div} \boldsymbol{E} & =0 \\
\operatorname{div} \boldsymbol{B} & =0
\end{aligned}
$$

where it has also been assumed that $\rho_{V}=0$, which holds for the application of interest in this thesis.

2. The electromagnetic constitutive laws (2.2) hold in the Eulerian frame.

3. The displacements are assumed to be small so that $\mathcal{F} \approx \mathbb{I}, \mathcal{H} \approx \mathbb{I}$ and $J \approx 1$; the velocities are also assumed to be small so that (2.10) and (2.15) coincide $^{1}$. This

${ }^{1}$ Note that in $[110,19,18,17]$ the term $\boldsymbol{p} \otimes \boldsymbol{v}$, which is quadratic in the velocities, was neglected, but the Lorentz current term in (2.13) was retained. 
also means that elastic bodies have the constitutive behaviour $\boldsymbol{\sigma}^{m}(\boldsymbol{u}):=\boldsymbol{C}: \boldsymbol{\epsilon}(\boldsymbol{u})$, where $\boldsymbol{\epsilon}(\boldsymbol{u}):=\left(\left(\nabla \boldsymbol{u}+(\nabla \boldsymbol{u})^{T}\right) / 2\right.$ is the small strain tensor and $\boldsymbol{C}=\boldsymbol{C}_{i j k \ell} \boldsymbol{e}_{i} \otimes$ $\boldsymbol{e}_{j} \otimes \boldsymbol{e}_{k} \otimes \boldsymbol{e}_{\ell}$ is a rank 4 constitutive tensor with entries

$$
\boldsymbol{C}_{i j k l}=\lambda \delta_{i j} \delta_{k l}+G\left(\delta_{i k} \delta_{j l}+\delta_{j k} \delta_{i l}\right)
$$

with $\delta_{i j}$ the Kronecker delta, $\boldsymbol{e}_{i}$ the $i$ th unit basis vector and $\lambda$ and $G$ the Lamé parameters. Furthermore, the body force can be rigorously described in terms of the divergence of a Maxwell stress tensor in terms of $\boldsymbol{B}$ and $\boldsymbol{H}$.

4. The electromagnetic fields are described in terms of a electric scalar potential and a magnetic vector potential as

$$
\boldsymbol{B}=\operatorname{curl} \boldsymbol{A}_{E u l}, \quad \boldsymbol{E}=-\operatorname{grad} \psi_{E u l}-\frac{\partial \boldsymbol{A}_{E u l}}{\partial t} .
$$

5. A Biot-Savart description of the coils is adopted.

\section{Transmission problem}

Choosing appropriate gauges and adding appropriate boundary and initial conditions, the coupled magneto-mechanical problem of interest in the Eulerian frame can then be described by the transmission problem $[19,17]$ : Find $\left(\boldsymbol{A}_{E u l}, \boldsymbol{u}\right) \in\left(\mathbb{R}^{3} \times \mathbb{R}^{3}\right)([0, T]$ such that

$$
\begin{aligned}
& \operatorname{curl}\left(\mu^{-1} \operatorname{curl} \boldsymbol{A}_{E u l}\right)+\gamma \frac{\partial \boldsymbol{A}_{E u l}}{\partial t}=\gamma \frac{\partial \boldsymbol{u}}{\partial t} \times \operatorname{curl} \boldsymbol{A}_{E u l} \quad \text { in } \Omega_{C}, \\
& \operatorname{curl}\left(\mu_{0}^{-1} \operatorname{curl} \boldsymbol{A}_{E u l}\right)=\boldsymbol{J}_{0} \quad \text { in } \Omega_{C}^{c}, \\
& \operatorname{div} \boldsymbol{A}_{E u l}=0 \quad \text { in } \Omega_{C}^{c}, \\
& \boldsymbol{A}_{\text {Eul }}=O\left(|\boldsymbol{x}|^{-1}\right) \quad \text { as }|\boldsymbol{x}| \rightarrow \infty, \\
& \boldsymbol{n} \times\left[\boldsymbol{A}_{E u l}\right]_{\partial \Omega_{C}}=\mathbf{0} \quad \text { on } \partial \Omega_{C}, \\
& \boldsymbol{n} \times\left[\mu^{-1} \operatorname{curl} \boldsymbol{A}_{E u l}\right]_{\partial \Omega_{C}}=\mathbf{0} \quad \text { on } \partial \Omega_{C}, \\
& \boldsymbol{A}_{\text {Eul }}(t=0)=\mathbf{0} \quad \text { in } \mathbb{R}^{3}, \\
& \operatorname{div}\left(\boldsymbol{\sigma}^{m}(\boldsymbol{u})+\boldsymbol{\sigma}^{e}\left(\boldsymbol{A}_{E u l}\right)\right)=\rho \frac{\partial^{2} \boldsymbol{u}}{\partial t^{2}} \quad \text { in } \Omega_{C}, \\
& \left.\left(\boldsymbol{\sigma}^{e}\left(\boldsymbol{A}_{E u l}\right)+\boldsymbol{\sigma}^{m}(\boldsymbol{u})\right)\right|_{\partial \Omega_{C}} ^{-} \boldsymbol{n}=\left.\left(\boldsymbol{\sigma}^{e}\left(\boldsymbol{A}_{E u l}\right)\right)\right|_{\partial \Omega_{C}} ^{+} \boldsymbol{n} \quad \text { on } \partial \Omega_{C}, \\
& \boldsymbol{\sigma}^{e}\left(\boldsymbol{A}_{E u l}\right):=\mu^{-1}\left(\operatorname{curl} \boldsymbol{A}_{E u l} \otimes \operatorname{curl} \boldsymbol{A}_{E u l}-\frac{1}{2}\left|\operatorname{curl} \boldsymbol{A}_{E u l}\right|^{2} \boldsymbol{I}\right) \quad \text { in } \Omega_{C}, \\
& \boldsymbol{\sigma}^{m}(\boldsymbol{u}):=\boldsymbol{C}: \boldsymbol{\epsilon}(\boldsymbol{u}) \quad \text { in } \Omega_{C}, \\
& \boldsymbol{u}=\boldsymbol{u}_{D} \quad \text { on } \partial \Omega_{C}^{D}, \\
& \boldsymbol{u}(t=0)=\frac{\partial \boldsymbol{u}}{\partial t}(t=0)=\mathbf{0} \quad \text { in } \Omega_{C},
\end{aligned}
$$

where $T$ is the final time of interest, $\boldsymbol{I}$ is the rank 2 identity tensor and the initial conditions to the fields have been chosen to be zero, corresponding to a system at rest at $t=0$. The 
electric and magnetic fields can then be computed once $\boldsymbol{A}_{E u l}$ is known as

$$
\begin{array}{r}
\boldsymbol{E}=-\frac{\partial \boldsymbol{A}_{E u l}}{\partial t}, \\
\boldsymbol{H}=\mu^{-1} \boldsymbol{B}=\mu^{-1} \operatorname{curl} \boldsymbol{A}_{E u l} .
\end{array}
$$

For further details about the Eulerian formulation for small displacements and velocities the reader is referred to $[110,19,18,17]$.

Although displacements are generally small in MRI scanners, the same is not necessarily true of velocities (especially in the case of stronger coupling). Thus, in this work, an alternative formulation based in a Lagrangian formulation and assuming small displacements but not necessarily small velocities is followed [73].

\subsubsection{Lagrangian Formulation for Small Displacements}

For small deformations, but not necessarily small velocities and accelerations, the total Lagrangian and updated Lagrangian descriptions coincide [73] and, henceforth, this will be simply called the Lagrangian approach in this thesis. This approach will be pursued in this thesis and is based on the following key assumptions and steps.

1. The eddy current approximation cannot be applied in the Lagrangian frame and instead must be applied in the Eulerian frame. Thus system (2.13) becomes

$$
\begin{aligned}
\operatorname{curl} \boldsymbol{E} & =-\frac{\partial \boldsymbol{B}}{\partial t} \\
\operatorname{curl} \boldsymbol{H} & =\mathcal{H}^{-T}\left(\boldsymbol{J}^{s}+\gamma \mathcal{F}^{T}(\boldsymbol{E}-\boldsymbol{B} \times \boldsymbol{v})\right) \\
\operatorname{div} \boldsymbol{E} & =0 \\
\operatorname{div} \boldsymbol{B} & =0
\end{aligned}
$$

where we have also assumed that $\rho_{V}=0$, which holds for the application of interest in this thesis.

After applying the eddy current approximation to (2.13), the simplified Maxwell equations (2.28) are then transformed to the Lagrangian frame, but without assuming small displacements ${ }^{2}$

$$
\begin{aligned}
\operatorname{curl}(\boldsymbol{E}-\boldsymbol{B} \times \boldsymbol{v}) & =-\mathcal{H}^{-T} \frac{\mathrm{d} \mathcal{H}^{-T} \boldsymbol{B}}{\mathrm{d} t} \\
\operatorname{curl} \boldsymbol{H} & =\mathcal{H}^{-T}\left(\gamma\left(\mathcal{F}^{T}(\boldsymbol{E}-\boldsymbol{B} \times \boldsymbol{v})\right)+\boldsymbol{J}^{s}\right), \\
\operatorname{div} \boldsymbol{E} & =0 \\
\operatorname{div} \boldsymbol{B} & =0
\end{aligned}
$$

\footnotetext{
${ }^{2}$ Note that under the assumptions of the eddy current approximation $\boldsymbol{H}_{0}=\mathcal{F}^{T}(\boldsymbol{H}+\boldsymbol{D} \times \boldsymbol{v}) \approx \mathcal{F}^{T} \boldsymbol{H}$.
} 
2. The electromagnetic constitutive laws (2.2) hold in a Eulerian frame and are transformed to the Lagrangian frame.

3. In the Lagrangian frame, the displacements are assumed to be small in which case $\mathcal{H} \approx \mathcal{F} \approx \mathbb{I}$ and $J \approx 1$ and, thus,

$$
\boldsymbol{B}_{0} \approx \boldsymbol{B}, \quad \boldsymbol{E}_{0} \approx \boldsymbol{E}-\boldsymbol{B} \times \boldsymbol{v}, \quad \boldsymbol{H}_{0} \approx \boldsymbol{H}, \quad \boldsymbol{X} \approx \boldsymbol{x}
$$

and, therefore, the constitutive relation $\boldsymbol{B}_{0}=\mu \boldsymbol{H}_{0}$ holds in the Lagrangian frame under the assumption of small displacements. This also means that elastic bodies have the constitutive behaviour $\boldsymbol{\sigma}^{m}(\boldsymbol{u}):=\boldsymbol{C}: \boldsymbol{\epsilon}(\boldsymbol{u})$ and that the body force can be rigorously described in terms of the divergence of a Maxwell stress tensor in terms of $\boldsymbol{H}_{0}$ defined as

$$
\boldsymbol{\sigma}^{e}\left(\boldsymbol{H}_{0}\right):=\mu\left(\boldsymbol{H}_{0} \otimes \boldsymbol{H}_{0}-\frac{1}{2}\left|\boldsymbol{H}_{0}\right|^{2} \boldsymbol{I}\right)
$$

Under this assumption, system (2.29) reduces to

$$
\begin{aligned}
\operatorname{curl}(\boldsymbol{E}-\boldsymbol{B} \times \boldsymbol{v}) & =-\frac{\mathrm{d} \boldsymbol{B}}{\mathrm{d} t}, \\
\operatorname{curl} \boldsymbol{H} & =\left(\gamma(\boldsymbol{E}-\boldsymbol{B} \times \boldsymbol{v})+\boldsymbol{J}^{s}\right), \\
\operatorname{div} \boldsymbol{E} & =0 \\
\operatorname{div} \boldsymbol{B} & =0 .
\end{aligned}
$$

4. The magnetic flux density is written in terms of a magnetic vector potential $\boldsymbol{A}$ such that

$$
\boldsymbol{B} \approx \boldsymbol{B}_{0}=\operatorname{curl} \boldsymbol{A}
$$

which ensures that (2.32d) is automatically satisfied. Furthermore, inserting (2.33) in $(2.32 \mathrm{a})$, we can write

$$
\boldsymbol{E}-\boldsymbol{B} \times \boldsymbol{v}=-\operatorname{grad} \psi-\frac{\mathrm{d} \boldsymbol{A}}{\mathrm{d} t}
$$

where the electric scalar potential $\psi$ arises from the freedom in the choice of $\boldsymbol{A}$. That is, $\boldsymbol{A}$ could be replaced by $\boldsymbol{A}+\operatorname{grad} \varphi$ and equation (2.33) would remain unchanged. The system of Maxwell's equations can then be written in terms of $\psi$ and $\boldsymbol{A}$ as

$$
\begin{array}{ll}
\operatorname{curl}\left(\mu^{-1} \operatorname{curl} \boldsymbol{A}\right)+\gamma\left(\operatorname{grad} \psi+\frac{\mathrm{d} \boldsymbol{A}}{\mathrm{d} t}\right)=\boldsymbol{J}^{s} & \text { in } \mathbb{R}^{3} \\
\operatorname{div}\left(-\operatorname{grad} \psi-\frac{\mathrm{d} \boldsymbol{A}}{\mathrm{d} t}+(\operatorname{curl} \boldsymbol{A}) \times \boldsymbol{v}\right)=0 & \text { in } \mathbb{R}^{3} .
\end{array}
$$


5. The Lagrangian system (2.35) is ungauged and, therefore, certain gauge conditions must be imposed in the conducting and non-conducting regions in order to uniquely define the solution of (2.35).

\section{Gauging in the Conducting Region}

In the conducting region, system (2.35) reduces to

$$
\begin{array}{ll}
\operatorname{curl}\left(\mu^{-1} \operatorname{curl} \boldsymbol{A}\right)+\gamma\left(\operatorname{grad} \psi+\frac{\mathrm{d} \boldsymbol{A}}{\mathrm{d} t}\right)=\mathbf{0} & \text { in } \Omega_{C}, \\
\operatorname{div}\left(\left(-\operatorname{grad} \psi-\frac{\mathrm{d} \boldsymbol{A}}{\mathrm{d} t}+(\operatorname{curl} \boldsymbol{A}) \times \boldsymbol{v}\right)=0\right. & \text { in } \Omega_{C} .
\end{array}
$$

In order to uniquely define $\boldsymbol{A}$ in this region, the temporal (Weyl) gauge on the scalar potential

$$
\psi=0,
$$

is considered, which, when applied to system (2.36), results in

$$
\begin{aligned}
\operatorname{curl}\left(\mu^{-1} \operatorname{curl} \boldsymbol{A}\right)+\gamma \frac{\mathrm{d} \boldsymbol{A}}{\mathrm{d} t}=\mathbf{0} & \text { in } \Omega_{C}, \\
\operatorname{div}\left(-\frac{\mathrm{d} \boldsymbol{A}}{\mathrm{d} t}+(\operatorname{curl} \boldsymbol{A}) \times \boldsymbol{v}\right)=0 & \text { in } \Omega_{C},
\end{aligned}
$$

where $\boldsymbol{A}$ is uniquely defined by (2.38a) and, thus, (2.38b) is not required.

\section{Gauging in the Non-Conducting Region}

In the non-conducting region, system (2.35) reduces to

$$
\begin{aligned}
\operatorname{curl}\left(\mu^{-1} \operatorname{curl} \boldsymbol{A}\right) & =\boldsymbol{J}^{s} & & \text { in } \Omega_{C}^{c}, \\
\operatorname{div}\left(-\operatorname{grad} \psi-\frac{\mathrm{d} \boldsymbol{A}}{\mathrm{d} t}\right) & =0 & & \text { in } \Omega_{C}^{c} .
\end{aligned}
$$

In order to ensure the uniqueness of $\boldsymbol{A}$ in this region, the application of the Coulomb gauge

$$
\operatorname{div} \boldsymbol{A}=0
$$

is in this case considered.

If $\boldsymbol{A}$ is divergence free, then grad $\psi$ must also be divergence free in order for (2.39b) to hold. Hence, (2.39b) can be replaced by the Coulomb gauge and so system (2.39) becomes

$$
\begin{aligned}
\operatorname{curl}\left(\mu^{-1} \operatorname{curl} \boldsymbol{A}\right) & =\boldsymbol{J}^{s} & & \text { in } \Omega_{C}^{c}, \\
\operatorname{div} \boldsymbol{A} & =0 & & \text { in } \Omega_{C}^{c} .
\end{aligned}
$$

6. For simplicity, a Biot-Savart description of the coils is assumed, where the current sources are not treated as conductors. However, in general, this choice is not a limitation of the approach presented in this thesis. 


\section{Transmission Problem}

Introducing the elasticity equation in the updated Lagrangian formulation (2.10), rewriting the transmission conditions (2.20) in terms of $\boldsymbol{A}$, and adding appropriate boundary and initial conditions, the magneto-mechanical problem of interest can be described by the transmission problem: Find $(\boldsymbol{A}, \boldsymbol{u})(t) \in\left(\mathbb{R}^{3} \times \mathbb{R}^{3}\right)(0, T]$ such that

$$
\begin{aligned}
\operatorname{curl}\left(\mu^{-1} \operatorname{curl} \boldsymbol{A}\right)+\gamma \frac{\mathrm{d} \boldsymbol{A}}{\mathrm{d} t} & =\mathbf{0} & & \text { in } \Omega_{C}, \\
\operatorname{curl}\left(\mu_{0}^{-1} \operatorname{curl} \boldsymbol{A}\right) & =\boldsymbol{J}_{0} & & \text { in } \Omega_{C}^{c}, \\
\operatorname{div} \boldsymbol{A} & =0 & & \text { in } \Omega_{C}^{c}, \\
\boldsymbol{n} \times[\boldsymbol{A}]_{\partial \Omega_{C}} & =\mathbf{0} & & \text { as }|\boldsymbol{x}| \rightarrow \infty \\
\boldsymbol{n} \times\left[\mu^{-1} \operatorname{curl} \boldsymbol{A}\right]_{\partial \Omega_{C}} & =\mathbf{0} & & \text { on } \partial \Omega_{C}, \\
\boldsymbol{A}(t=0) & =\mathbf{0} & & \text { on } \partial \Omega_{C}, \\
\operatorname{div}\left(\boldsymbol{\sigma}^{m}(\boldsymbol{u})+\boldsymbol{\sigma}^{e}(\boldsymbol{A})\right) & =\rho \frac{\mathrm{d}^{2} \boldsymbol{u}}{\mathrm{d} t^{2}} & & \text { in } \mathbb{R}^{3}, \\
\left.\left(\boldsymbol{\sigma}^{e}(\boldsymbol{A})+\boldsymbol{\sigma}^{m}(\boldsymbol{u})\right)\right|_{\partial \Omega_{C}} ^{-} \boldsymbol{n} & =\left.\left(\boldsymbol{\sigma}^{e}(\boldsymbol{A})\right)\right|_{\partial \Omega_{C}} ^{+} \boldsymbol{n} & & \text { in } \Omega_{C}, \\
\boldsymbol{\sigma}^{e}(\boldsymbol{A}): & =\mu^{-1}\left(\operatorname{curl} \boldsymbol{A} \otimes \operatorname{curl} \boldsymbol{A}-\frac{1}{2}|\operatorname{curl} \boldsymbol{A}|^{2} \boldsymbol{I}\right) & & \text { in } \Omega_{C}, \\
\boldsymbol{\sigma}^{m}(\boldsymbol{u}) & :=\boldsymbol{C}: \boldsymbol{\epsilon}(\boldsymbol{u}) & & \text { in } \Omega_{C}, \\
\mathrm{u} \boldsymbol{u} & =\boldsymbol{u}_{D} & & \text { on } \partial \Omega_{C}^{D}, \\
\boldsymbol{u}(t=0)=\frac{\mathrm{u}}{\mathrm{d} t}(t=0) & =\mathbf{0} & & \text { in } \Omega_{C},
\end{aligned}
$$

where the initial conditions for the fields have been chosen to be zero, corresponding to a system at rest at $t=0$. The Eulerian electric and magnetic fields are coupled to $\boldsymbol{A}$ and $\boldsymbol{v}$ through

$$
\begin{array}{ll}
\boldsymbol{E}=-\frac{\mathrm{d} \boldsymbol{A}}{\mathrm{d} t}+\boldsymbol{B}_{0} \times \boldsymbol{v}=-\frac{\mathrm{d} \boldsymbol{A}}{\mathrm{d} t}+(\operatorname{curl} \boldsymbol{A}) \times \boldsymbol{v} & \text { in } \Omega_{C} \\
\boldsymbol{H}=\mu^{-1} \boldsymbol{B}_{0}=\mu^{-1} \operatorname{curl} \boldsymbol{A} & \text { in } \mathbb{R}^{3},
\end{array}
$$

which can be applied once (2.42) is solved.

\subsection{Concluding Remarks}

This Chapter has presented a Lagrangian formulation for the description of the magnetomechanical problem of interest under the assumption of small displacements. The Chapter started with the definition of the system of Maxwell equations in a general format. Then, an introduction to the motion of moving or deformable bodies was provided, focusing on the different coordinate systems that can be used to describe this motion namely, material or Lagrangian descriptions and spatial or Eulerian descriptions. The system of equations 
describing the coupled problem in total Lagrangian, updated Lagrangian and Eulerian formulations was then presented, as well as the transformations between them. Finally, a series of simplifying assumptions were discussed that, together with the introduction of a magnetic vector potential and the application of appropriate gauges, boundary and transmission conditions, results in the final transmission problem for the description of the magneto-mechanical problem of interest. Notably, this system lends itself to the application of a staggered scheme, as opposed to the approach followed in $[110,19,18,17]$, where a monolithic scheme was required. On top of that, the new approach assumes only small displacements but not necessarily small velocities, as opposed to the approach in [110, 19, 18, 17], where both displacements and velocities were assumed to be small. As will be shown in later chapters, the assumption of small velocities is not necessarily true for MRI applications, especially in the case of strong coupling and, therefore, the Lagrangian approach described in this chapter results in more accurate results compared to the approach in $[110,19,18,17]$. 


\section{Chapter 3}

\section{Linearised Problem: Lagrangian Framework}

\subsection{Introductory Remarks}

This chapter focuses on the development of a novel variational formulation of the transmission problem (2.42) that lends itself to an efficient computational methodology. For this purpose, an AC-DC splitting of the fields will be introduced, and then the variational formulations of the DC and AC problems will be rigorously derived. Furthermore, considering the basic physics of an MRI scanner, the AC problem will be linearised and translated to a time-harmonic setting. The chapter is based on an extended version of the work presented by the author in [158].

The main novelties of the chapter are the derivation of a linearised magneto-mechanical problem in a Lagrangian frame and its variational formulation, the translation of the linearised problem to the frequency domain, and the introduction of the regularised DC and AC problems in order to circumvent the Coulomb gauge condition.

The structure of the chapter is as follows: First, in Section 3.2, the AC-DC splitting of the fields is introduced and the DC (static) transmission problem is stated. Then, in Section 3.3, the weak formulation for both the static and transient problems will be derived. Section 3.4 focuses on the linearisation of the transient problem and its formulation in a time-harmonic setting. The treatment of surface integrals is discussed in Section 3.5. Finally, the regularised DC and AC problems are presented in Section 3.6. The Chapter finishes with concluding remarks in Section 3.7.

\subsection{AC-DC Splitting}

An MRI scanner consists basically of two types of coils: the superconducting main coils that generate a strong static magnetic field and the resistive gradient coils that generate low strength transient magnetic fields. Since these coils have different supports, the current source $\boldsymbol{J}^{s}$ can be decomposed into DC and AC contributions, $\boldsymbol{J}^{s}(t)=\boldsymbol{J}^{\mathrm{DC}}+\boldsymbol{J}^{\mathrm{AC}}(t)$, associated with the main and gradient coils, Figure 3.1, respectively [19]. 


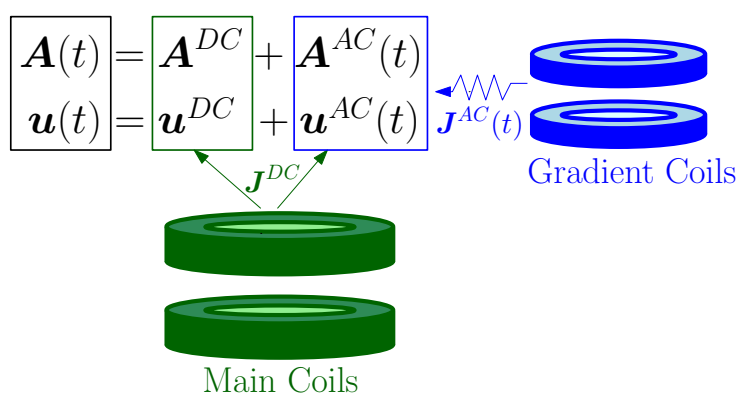

Figure 3.1: Current source decomposition, where $\boldsymbol{J}^{s}(t)=\boldsymbol{J}^{D C}+\boldsymbol{J}^{A C}(t)$.

Based on this decomposition, considering the situation where only the current source $\boldsymbol{J}=\boldsymbol{J}^{D C}$ is excited and neglecting the time dependent terms, the transient transmission problem (2.42) reduces to the following DC (static) problem: Find $\left(\boldsymbol{A}^{D C}, \boldsymbol{u}^{D C}\right) \in \mathbb{R}^{3} \times \mathbb{R}^{3}$ such that

$$
\begin{aligned}
\operatorname{curl}\left(\mu^{-1} \operatorname{curl} \boldsymbol{A}^{D C}\right) & =\boldsymbol{J}^{D C} & & \text { in } \mathbb{R}^{3}, \\
\operatorname{div} \boldsymbol{A}^{D C} & =0 & & \text { in } \mathbb{R}^{3}, \\
\boldsymbol{A}^{D C} & =O\left(|\boldsymbol{x}|^{-1}\right) & & \text { as }|\boldsymbol{x}| \rightarrow \infty, \\
\boldsymbol{n} \times\left[\boldsymbol{A}^{D C}\right]_{\partial \Omega_{C}} & =\mathbf{0} & & \text { on } \partial \Omega_{C}, \\
\boldsymbol{n} \times\left[\mu^{-1} \operatorname{curl} \boldsymbol{A}^{D C}\right]_{\partial \Omega_{C}} & =\mathbf{0} & & \text { on } \partial \Omega_{C}, \\
\operatorname{div}\left(\boldsymbol{\sigma}^{m}\left(\boldsymbol{u}^{D C}\right)+\boldsymbol{\sigma}^{e}\left(\boldsymbol{A}^{D C}\right)\right) & =\mathbf{0} & & \text { in } \Omega_{C}, \\
\boldsymbol{u}^{D C} & =\boldsymbol{u}_{D}^{D C} & & \text { on } \partial \Omega_{C}^{D}, \\
\left.\left(\boldsymbol{\sigma}^{e}\left(\boldsymbol{A}^{D C}\right)+\boldsymbol{\sigma}^{m}\left(\boldsymbol{u}^{D C}\right)\right)\right|_{\partial \Omega_{C}} ^{-} \boldsymbol{n} & =\left.\left(\boldsymbol{\sigma}^{e}\left(\boldsymbol{A}^{D C}\right)\right)\right|_{\partial \Omega_{C}} ^{+} \boldsymbol{n} & & \text { on } \partial \Omega_{C},
\end{aligned}
$$

which would give the response $\boldsymbol{A}=\boldsymbol{A}^{D C}, \boldsymbol{u}=\boldsymbol{u}^{D C}$ in the absence of any time varying fields.

This splitting was first introduced in [19], where the solution to a related magnetomechanical problem in an axisymmetric setting using an Eulerian formulation was considered. Here, the splitting is applied to the novel transmission problem (2.42) in the Lagrangian formulation and the DC problem in this case is given by (3.1).

\subsection{Weak Formulation}

The development of a finite element software requires the problem to be stated in its variational (weak) formulation. Hence, in this section, the variational formulation of both the static (3.1) and dynamic (2.42) transmission problems will be derived.

\subsubsection{Static (DC) Problem}

The weak formulation of problem (3.1) is derived here. In a departure from the approach in [17], where the static problem was linearised and then solved using an iterative NewtonRaphson method, it is noted here that the static problem exhibits only a non-linearity in 
$\boldsymbol{\sigma}^{e}\left(\boldsymbol{A}^{D C}\right)$ in equations (3.1f) and (3.1h). Therefore, linearisation is not required for the solution of this problem and, instead, it has been chosen to retain the non-linearity in $\boldsymbol{\sigma}^{e}\left(\boldsymbol{A}^{D C}\right)$ and solve the problem in a staggered manner, that is, solve first (3.1a)-(3.1e) followed by (3.1f)-(3.1h). This approach was first presented by the author in [158] and is extended here by presenting the complete derivation of the final formulation, including the technical details.

Considering the development of the variational formulation, the following definitions must be stated

$$
\begin{aligned}
X_{\mathbb{R}^{3}} & :=\left\{\boldsymbol{A} \in \boldsymbol{H}\left(\operatorname{curl}, \mathbb{R}^{3}\right): \operatorname{div} \boldsymbol{A}=0 \text { in } \mathbb{R}^{3}, \boldsymbol{A}=O\left(|\boldsymbol{x}|^{-1}\right) \text { as }|\boldsymbol{x}| \rightarrow \infty\right\}, \\
Y(\boldsymbol{g}) & :=\left\{\boldsymbol{u} \in H^{1}\left(\Omega_{C}\right)^{3}: \boldsymbol{u}=\boldsymbol{g} \text { on } \partial \Omega_{C}^{D}\right\}
\end{aligned}
$$

where the spaces $\boldsymbol{H}\left(\right.$ curl, $\left.\mathbb{R}^{3}\right)$ and $H^{1}\left(\Omega_{C}\right)$ are defined, in terms of a general domain $\Omega$ as [129]

$$
\begin{aligned}
\boldsymbol{H}(\operatorname{curl}, \Omega) & :=\left\{\boldsymbol{a} \in\left(L^{2}(\Omega)\right)^{3}: \operatorname{curl} \boldsymbol{a} \in\left(L^{2}(\Omega)\right)^{3}\right\}, \\
H^{1}(\Omega) & :=\left\{a \in L^{2}(\Omega): \nabla a \in\left(L^{2}(\Omega)\right)^{3}\right\},
\end{aligned}
$$

where $L^{2}(\Omega):=\left\{a: \int_{\Omega}|a|^{2} \mathrm{~d} \Omega<\infty\right\}$.

The weak form of the electromagnetic static equation (3.1a) can now be obtained by multiplying the equation by a weighting (test) function and integrating over the domain

$$
\int_{\mathbb{R}^{3}} \operatorname{curl}\left(\mu^{-1} \operatorname{curl} \boldsymbol{A}^{D C}\right) \cdot \delta \boldsymbol{A}^{D C} \mathrm{~d} \Omega=\int_{\operatorname{supp}\left(\boldsymbol{J}^{D C}\right)} \boldsymbol{J}^{D C} \cdot \delta \boldsymbol{A}^{D C} \mathrm{~d} \Omega,
$$

where $\delta \boldsymbol{A}^{D C} \in X_{\mathbb{R}^{3}}$ is the weighting function and the current $\boldsymbol{J}^{D C}$ is only non-zero in the corresponding coils, supp $\left(\boldsymbol{J}^{D C}\right)$. Note that the Coulomb gauge constraint (3.1b) has been embedded in the definition of $X_{\mathbb{R}^{3}}$.

Applying integration by parts $^{1}$ to the left hand side of equation (3.4)

$$
\begin{aligned}
& \int_{\mathbb{R}^{3}} \mu^{-1} \operatorname{curl} \boldsymbol{A}^{D C} \cdot \operatorname{curl} \delta \boldsymbol{A}^{D C} \mathrm{~d} \Omega+\int_{\mathbb{R}^{3}} \operatorname{div}\left(\mu^{-1} \operatorname{curl} \boldsymbol{A}^{D C} \times \delta \boldsymbol{A}^{D C}\right) \mathrm{d} \Omega= \\
& \int_{\operatorname{supp}\left(\boldsymbol{J}^{D C}\right)} \boldsymbol{J}^{D C} \cdot \delta \boldsymbol{A}^{D C} \mathrm{~d} \Omega .
\end{aligned}
$$

Splitting the second term into integrals expressed over subdomains $\Omega_{C}$ and $\mathbb{R}^{3} \backslash \Omega_{C}$

$$
\begin{aligned}
& \int_{\mathbb{R}^{3}} \mu^{-1} \operatorname{curl} \boldsymbol{A}^{D C} \cdot \operatorname{curl} \delta \boldsymbol{A}^{D C} \mathrm{~d} \Omega+\int_{\Omega_{C}} \operatorname{div}\left(\mu^{-1} \operatorname{curl} \boldsymbol{A}^{D C} \times \delta \boldsymbol{A}^{D C}\right) \mathrm{d} \Omega+ \\
& \int_{\mathbb{R}^{3} \backslash \Omega_{C}} \operatorname{div}\left(\mu^{-1} \operatorname{curl} \boldsymbol{A}^{D C} \times \delta \boldsymbol{A}^{D C}\right) \mathrm{d} \Omega=\int_{\operatorname{supp}\left(\boldsymbol{J}^{D C}\right)} \boldsymbol{J}^{D C} \cdot \delta \boldsymbol{A}^{D C} \mathrm{~d} \Omega .
\end{aligned}
$$

\footnotetext{
${ }^{1}$ Recall the vector calculus identity $\operatorname{div}(\boldsymbol{a} \times \boldsymbol{b})=\boldsymbol{b} \cdot \operatorname{curl} \boldsymbol{a}-\boldsymbol{a} \cdot \operatorname{curl} \boldsymbol{b}$
} 
Applying now the divergence theorem ${ }^{2}$

$$
\begin{aligned}
& \int_{\mathbb{R}^{3}} \mu^{-1} \operatorname{curl} \boldsymbol{A}^{D C} \cdot \operatorname{curl} \delta \boldsymbol{A}^{D C} \mathrm{~d} \Omega+\int_{\partial \mathbb{R}^{3}}\left(\boldsymbol{n} \times \mu^{-1} \operatorname{curl} \boldsymbol{A}^{D C}\right) \cdot \delta \boldsymbol{A}^{D C} \mathrm{ds}+ \\
& \left.\int_{\partial \Omega_{C}}\left(\boldsymbol{n} \times \mu^{-1} \operatorname{curl} \boldsymbol{A}^{D C}\right)\right|^{+} \cdot \delta \boldsymbol{A}^{D C} \mathrm{ds}+\left.\int_{\partial \Omega_{C}}\left(\boldsymbol{n} \times \mu^{-1} \operatorname{curl} \boldsymbol{A}^{D C}\right)\right|^{-} \cdot \delta \boldsymbol{A}^{D C} \mathrm{ds}= \\
& \int_{\operatorname{supp}\left(\boldsymbol{J}^{D C}\right)} \boldsymbol{J}^{D C} \cdot \delta \boldsymbol{A}^{D C} \mathrm{~d} \Omega
\end{aligned}
$$

where two surface integrals in the conductor-free space interface $\partial \Omega_{C}$ have appeared, due to the contributions form the conductor side (superscript -) and the free space side (superscript + ). In addition, the normal vectors satisfy $-\boldsymbol{n}^{+}=\boldsymbol{n}^{-}=\boldsymbol{n}$ and noting that the boundary $\partial \mathbb{R}^{3}$ lies at $\infty$ and that since $\boldsymbol{A}^{D C}=O\left(|\boldsymbol{x}|^{-1}\right)$ then $\operatorname{curl} \boldsymbol{A}^{D C}=O\left(|\boldsymbol{x}|^{-1}\right)$, which implies that the far field integral vanishes and (3.7) reduces to

$$
\begin{aligned}
& \int_{\mathbb{R}^{3}} \mu^{-1} \operatorname{curl} \boldsymbol{A}^{D C} \cdot \operatorname{curl} \delta \boldsymbol{A}^{D C} \mathrm{~d} \Omega-\int_{\partial \Omega_{C}} \boldsymbol{n} \times\left[\mu^{-1} \operatorname{curl} \boldsymbol{A}^{D C}\right]_{\partial \Omega_{C}} \cdot \delta \boldsymbol{A}^{D C} \mathrm{ds}= \\
& \int_{\operatorname{supp}\left(\boldsymbol{J}^{D C}\right)} \boldsymbol{J}^{D C} \cdot \delta \boldsymbol{A}^{D C} \mathrm{~d} \Omega .
\end{aligned}
$$

Finally, recalling the interface condition (3.1e) and multiplying by $\mu_{0}$ in order to improve the scaling of the equations, the final expression can be written as: Find $A^{D C} \in X_{\mathbb{R}^{3}}$ such that

$$
\int_{\mathbb{R}^{3}} \mu_{r}^{-1} \operatorname{curl} \boldsymbol{A}^{D C} \cdot \operatorname{curl} \delta \boldsymbol{A}^{D C} \mathrm{~d} \Omega=\mu_{0} \int_{\operatorname{supp}\left(\boldsymbol{J}^{D C}\right)} \boldsymbol{J}^{D C} \cdot \delta \boldsymbol{A}^{D C} \mathrm{~d} \Omega,
$$

for all $\delta \boldsymbol{A}^{D C} \in X_{\mathbb{R}^{3}}$.

The same procedure can be applied to the elasticity equation (3.1f). Consider a test (weighting) function $\delta \boldsymbol{u}^{D C} \in Y(\mathbf{0})$. Multiplying equation (3.1f) by this test function and integrating over the mechanical (conducting) domain $\Omega_{C}$ yields

$$
\int_{\Omega_{C}} \operatorname{div}\left(\boldsymbol{\sigma}^{m}\left(\boldsymbol{u}^{D C}\right)+\boldsymbol{\sigma}^{e}\left(\boldsymbol{A}^{D C}\right)\right) \cdot \delta \boldsymbol{u}^{D C} \mathrm{~d} \Omega=0 .
$$

Applying integration by parts ${ }^{3}$

$$
\begin{aligned}
& \int_{\Omega_{C}} \operatorname{div}\left(\left(\boldsymbol{\sigma}^{m}\left(\boldsymbol{u}^{D C}\right)+\boldsymbol{\sigma}^{e}\left(\boldsymbol{A}^{D C}\right)\right)^{T} \delta \boldsymbol{u}^{D C}\right) \mathrm{d} \Omega- \\
& \int_{\Omega_{C}}\left(\boldsymbol{\sigma}^{m}\left(\boldsymbol{u}^{D C}\right)+\boldsymbol{\sigma}^{e}\left(\boldsymbol{A}^{D C}\right)\right): \operatorname{grad} \delta \boldsymbol{u}^{D C} \mathrm{~d} \Omega=0,
\end{aligned}
$$

where $(\cdot)^{T}$ denotes the transpose.

\footnotetext{
${ }^{2}$ Recall the vector identity $(\boldsymbol{a} \times \boldsymbol{b}) \cdot \boldsymbol{c}=(\boldsymbol{c} \times \boldsymbol{a}) \cdot \boldsymbol{b}$

${ }^{3}$ Note the vector calculus identity for the product of a tensor $\boldsymbol{\sigma}$ and a vector $\boldsymbol{a}: \operatorname{div}\left(\boldsymbol{\sigma}^{T} \boldsymbol{a}\right)=\boldsymbol{\sigma}$ : $\operatorname{grad} \boldsymbol{a}+\boldsymbol{a} \cdot \operatorname{div} \boldsymbol{\sigma}$.
} 
By application of the divergence theorem, noting that $\boldsymbol{\sigma}^{e}$ and $\boldsymbol{\sigma}^{m}$ are symmetric (due to conservation of angular momentum) ${ }^{45}$ and considering the condition $\delta \boldsymbol{u}^{D C}=\mathbf{0}$ on $\partial \Omega_{C}^{D}$

$$
\begin{aligned}
& \int_{\Omega_{C}}\left(\boldsymbol{\sigma}^{m}\left(\boldsymbol{u}^{D C}\right)+\boldsymbol{\sigma}^{e}\left(\boldsymbol{A}^{D C}\right)\right): \operatorname{grad} \delta \boldsymbol{u}^{D C} \mathrm{~d} \Omega \\
& -\left.\int_{\partial \Omega_{C}^{N}} \delta \boldsymbol{u}^{D C} \cdot\left(\boldsymbol{\sigma}^{m}\left(\boldsymbol{u}^{D C}\right)+\boldsymbol{\sigma}^{e}\left(\boldsymbol{A}^{D C}\right)\right)\right|^{-} \boldsymbol{n} \mathrm{ds}=0 .
\end{aligned}
$$

Applying the interface condition (3.1h)

$$
\begin{aligned}
& \int_{\Omega_{C}}\left(\boldsymbol{\sigma}^{m}\left(\boldsymbol{u}^{D C}\right)+\boldsymbol{\sigma}^{e}\left(\boldsymbol{A}^{D C}\right)\right): \operatorname{grad} \delta \boldsymbol{u}^{D C} \mathrm{~d} \Omega \\
& -\int_{\partial \Omega_{C}^{N}} \delta \boldsymbol{u}^{D C} \cdot\left(\left.\boldsymbol{\sigma}^{e}\left(\boldsymbol{A}^{D C}\right)\right|^{+} \boldsymbol{n}\right) \mathrm{ds}=0 .
\end{aligned}
$$

Finally, splitting the first integral, moving the terms involving $\boldsymbol{\sigma}^{e}\left(\boldsymbol{A}^{D C}\right)$ to the right hand side and multiplying by $\mu_{0}$ in order to improve the scaling of the equations

$$
\begin{aligned}
& \mu_{0} \int_{\Omega_{C}} \boldsymbol{\sigma}^{m}\left(\boldsymbol{u}^{D C}\right): \operatorname{grad} \delta \boldsymbol{u}^{D C} \mathrm{~d} \Omega=\mu_{0} \int_{\partial \Omega_{C}^{N}} \delta \boldsymbol{u}^{D C} \cdot\left(\left.\boldsymbol{\sigma}^{e}\left(\boldsymbol{A}^{D C}\right)\right|^{+} \boldsymbol{n}\right) \mathrm{ds} \\
& -\mu_{0} \int_{\Omega_{C}} \boldsymbol{\sigma}^{e}\left(\boldsymbol{A}^{D C}\right): \operatorname{grad} \delta \boldsymbol{u}^{D C} \mathrm{~d} \Omega
\end{aligned}
$$

The weak formulation of the DC problem (3.1) can then be stated as: Find $\left(\boldsymbol{A}^{D C}, \boldsymbol{u}^{D C}\right) \in$ $X_{\mathbb{R}^{3}} \times Y\left(\boldsymbol{u}_{D}^{D C}\right)$ such that

$$
\begin{aligned}
& \int_{\mathbb{R}^{3}} \mu_{r}^{-1} \operatorname{curl} \boldsymbol{A}^{D C} \cdot \operatorname{curl} \delta \boldsymbol{A}^{D C} \mathrm{~d} \Omega=\mu_{0} \int_{\mathbb{R}^{3}} \boldsymbol{J}^{D C} \cdot \delta \boldsymbol{A}^{D C} \mathrm{~d} \Omega, \\
& \mu_{0} \int_{\Omega_{c}} \boldsymbol{\sigma}^{m}\left(\boldsymbol{u}^{D C}\right): \operatorname{grad} \delta \boldsymbol{u}^{D C} \mathrm{~d} \Omega=\left.\mu_{0} \int_{\partial \Omega_{C}^{N}} \boldsymbol{\sigma}^{e}\right|^{+} \boldsymbol{n} \cdot \delta \boldsymbol{u}^{D C} \mathrm{ds} \\
& -\mu_{0} \int_{\Omega_{C}} \boldsymbol{\sigma}^{e}\left(\boldsymbol{A}^{D C}\right): \operatorname{grad} \delta \boldsymbol{u}^{D C} \mathrm{~d} \Omega,
\end{aligned}
$$

for all real valued $\left(\delta \boldsymbol{A}^{D C}, \delta \boldsymbol{u}^{D C}\right) \in X_{\mathbb{R}^{3}} \times Y(\mathbf{0})$.

\subsubsection{Dynamic Problem}

Multiplying equations (2.42a) and (2.42b) by a test function $\delta \boldsymbol{A} \in X_{\mathbb{R}^{3}}$ and equation (2.42h) by a test function $\delta \boldsymbol{u} \in Y(\mathbf{0})$, respectively, and following similar steps to those in Section 3.3.1, the weak formulation of the dynamic problem can be stated as: Find

\footnotetext{
${ }^{4}$ Note the identity $\boldsymbol{\sigma}^{T}=\boldsymbol{\sigma}$ for symmetric tensors.

${ }^{5}$ Note the identity $(\boldsymbol{\sigma} \boldsymbol{a}) \cdot \boldsymbol{b}=(\boldsymbol{\sigma} \boldsymbol{b}) \cdot \boldsymbol{a}$ for symmetric tensors.
} 
$(\boldsymbol{A}, \boldsymbol{u})(t) \in X_{\mathbb{R}^{3}} \times Y\left(\boldsymbol{u}_{D}\right)(0, T]$ such that

$$
\begin{aligned}
R_{A}(\delta \boldsymbol{A} ; \boldsymbol{A}) & :=\int_{\mathbb{R}^{3}} \mu_{r}^{-1} \operatorname{curl} \boldsymbol{A} \cdot \operatorname{curl} \delta \boldsymbol{A} \mathrm{d} \Omega+\mu_{0} \int_{\Omega_{C}} \gamma \frac{\mathrm{d} \boldsymbol{A}}{\mathrm{d} t} \cdot \delta \boldsymbol{A} \mathrm{d} \Omega \\
& -\mu_{0} \int_{\mathbb{R}^{3}} \boldsymbol{J}_{0} \cdot \delta \boldsymbol{A} \mathrm{d} \Omega=0 \\
R_{u}(\delta \boldsymbol{u} ; \boldsymbol{A}, \boldsymbol{u}) & :=\mu_{0} \int_{\Omega_{C}}\left(\boldsymbol{\sigma}^{m}(\boldsymbol{u})+\boldsymbol{\sigma}^{e}(\boldsymbol{A})\right): \operatorname{grad} \delta \boldsymbol{u} \mathrm{d} \Omega+\mu_{0} \int_{\Omega_{C}} \rho \frac{\mathrm{d}^{2} \boldsymbol{u}}{\mathrm{d} t^{2}} \cdot \delta \boldsymbol{u} \mathrm{d} \Omega \\
& -\left.\mu_{0} \int_{\partial \Omega_{C}^{N}} \boldsymbol{\sigma}^{e}(\boldsymbol{A})\right|^{+} \boldsymbol{n} \cdot \delta \boldsymbol{u} \mathrm{ds}=0,
\end{aligned}
$$

for all $(\delta \boldsymbol{A}, \delta \boldsymbol{u}) \in\left(X_{\mathbb{R}^{3}} \times Y(\mathbf{0})\right)$, where the residuals $R_{A}(\delta \boldsymbol{A} ; \boldsymbol{A})$ and $R_{u}(\delta \boldsymbol{u} ; \boldsymbol{A}, \boldsymbol{u})$ have been defined, which must vanish when $\boldsymbol{A}$ and $\boldsymbol{u}$ are the true weak solutions to the system of equations. These residuals are defined with the purpose of constructing a linearised $\mathrm{AC}$ problem in mind. Note that the strong form of this $\mathrm{AC}$ problem is not stated, but an $\mathrm{AC}$ weak form will be obtained by linearising the dynamic problem about the DC solution. The DC problem could also be formulated in terms of residuals, but this would not present any benefit, as the DC problem will be solved in a staggered manner, without linearisation.

\subsection{Linearisation}

In a departure from the Eulerian approach described in Chapter 2, where the Lorentz current term $\gamma \boldsymbol{B} \times \boldsymbol{v}$ leads to a two way coupling and a non linear system of equations [19, 17] making necessary the use of iterative methods (such as fixed point or Newton-Raphson) for its approximate solution, system (3.16) could be solved integrating the equations in time and solving at each time step (3.16a) followed by (3.16b). However, the excitation driving the current in the gradient coils can be decomposed into different frequency modes using a fast Fourier transform (FFT) and, thus, the aim here is to solve the problem in a time harmonic regime in order to analyse the behaviour of the system for the complete range of constituent frequencies. For this purpose, the system must still be linearised due to the non-linearity of $\boldsymbol{\sigma}^{e}(\boldsymbol{A})$ in (3.16b). We focus on a linearisation that exploits the particular properties of $\boldsymbol{J}$ in a MRI context.

Remark 3.1 The directional derivative of a weighted residual $R_{a}$ in the direction of an increment in the solution $\Delta \boldsymbol{a}$ is defined as [35]:

$$
D R_{a}(\boldsymbol{a})[\Delta \boldsymbol{a}]=\left.\frac{\mathrm{d}}{\mathrm{d} \zeta}\right|_{\zeta=0} R(\boldsymbol{a}+\zeta \Delta \boldsymbol{a}),
$$

where $\boldsymbol{a}$ is the solution and $\zeta$ is a parameter used to scale the increment $\Delta \boldsymbol{a}$.

Thus, the directional derivatives of the residuals (3.16) in the direction of the incremental 
variables $\Delta \boldsymbol{A}$ and $\Delta \boldsymbol{u}$ must be computed

$$
\begin{aligned}
D R_{A}(\delta \boldsymbol{A} ; \boldsymbol{A})[\Delta \boldsymbol{A}] & =\int_{\mathbb{R}^{3}}\left(\mu_{r}^{-1} \operatorname{curl} \Delta \boldsymbol{A} \cdot \operatorname{curl} \delta \boldsymbol{A}+\mu_{0} \gamma \frac{\mathrm{d} \Delta \boldsymbol{A}}{\mathrm{d} t} \cdot \delta \boldsymbol{A}\right) \mathrm{d} \Omega \\
D R_{A}(\delta \boldsymbol{A} ; \boldsymbol{A})[\Delta \boldsymbol{u}] & =0 \\
D R_{u}(\delta \boldsymbol{u} ; \boldsymbol{A}, \boldsymbol{u})[\Delta \boldsymbol{A}] & =\int_{\Omega_{C}} \mu_{r}^{-1} S(\boldsymbol{A}, \Delta \boldsymbol{A}): \nabla \delta \boldsymbol{u} \mathrm{d} \Omega \\
& -\left.\int_{\partial \Omega_{C}^{N}} \boldsymbol{S}(\boldsymbol{A}, \Delta \boldsymbol{A})\right|^{+} \boldsymbol{n} \cdot \delta \boldsymbol{u} \mathrm{ds} \\
D R_{u}(\delta \boldsymbol{u} ; \boldsymbol{A}, \boldsymbol{u})[\Delta \boldsymbol{u}] & =\mu_{0} \int_{\Omega_{C}}\left(\boldsymbol{\sigma}^{m}(\Delta \boldsymbol{u}): \nabla \delta \boldsymbol{u}+\rho \frac{\mathrm{d}^{2} \Delta \boldsymbol{u}}{\mathrm{d} t^{2}} \cdot \delta \boldsymbol{u}\right) \mathrm{d} \Omega
\end{aligned}
$$

where

$$
\begin{aligned}
\mu^{-1} \boldsymbol{S}(\boldsymbol{A}, \Delta \boldsymbol{A}) & :=\mu^{-1}(\operatorname{curl} \boldsymbol{A} \otimes \operatorname{curl} \Delta \boldsymbol{A}+\operatorname{curl} \Delta \boldsymbol{A} \otimes \operatorname{curl} \boldsymbol{A} \\
& -(\operatorname{curl} \boldsymbol{A} \cdot \operatorname{curl} \Delta \boldsymbol{A}) \boldsymbol{I})
\end{aligned}
$$

is the linearised Maxwell stress tensor.

System (3.16) can now be rewritten as: Find $(\Delta \boldsymbol{A}, \Delta \boldsymbol{u})(t) \in X_{\mathbb{R}^{3}} \times Y\left(\boldsymbol{u}_{D}\right)(0, T]$ such that

$$
\begin{aligned}
D R_{A}(\delta \boldsymbol{A} ; \boldsymbol{A})[\Delta \boldsymbol{A}] & =-R_{A}(\delta \boldsymbol{A} ; \boldsymbol{A}), \\
D R_{u}(\delta \boldsymbol{u} ; \boldsymbol{A}, \boldsymbol{u})[\Delta \boldsymbol{A}]+D R_{u}(\delta \boldsymbol{u} ; \boldsymbol{A}, \boldsymbol{u})[\Delta \boldsymbol{u}] & =-R_{u}(\delta \boldsymbol{u} ; \boldsymbol{A}, \boldsymbol{u}),
\end{aligned}
$$

for all real valued $(\delta \boldsymbol{A}, \delta \boldsymbol{u}) \in X_{\mathbb{R}^{3}} \times Y(\mathbf{0})$. This system is still non-linear in time and, therefore, cannot be solved in the frequency domain. However, as described in [19, 17], this system can be linearised about the static solution due to the fact that in MRI applications the static DC current source $\boldsymbol{J}^{D C}$ is much bigger than the time varying AC source $\boldsymbol{J}^{A C}$, leading to a DC magnetic field much stronger than the AC magnetic field. Thus, splitting the source into its DC and AC components and evaluating the residuals (3.16) at the static solution

$$
\begin{aligned}
& \tilde{R}_{A}(\delta \boldsymbol{A}):=R_{A}\left(\delta \boldsymbol{A} ; \boldsymbol{A}^{D C}\right)=\int_{\mathbb{R}^{3}} \mu_{r}^{-1} \operatorname{curl} \boldsymbol{A}^{D C} \cdot \operatorname{curl} \delta \boldsymbol{A} \mathrm{d} \Omega \\
& -\mu_{0} \int_{\mathbb{R}^{3}}\left(\boldsymbol{J}^{D C}+\boldsymbol{J}^{A C}\right) \cdot \delta \boldsymbol{A} \mathrm{d} \Omega, \\
& \tilde{R}_{u}(\delta \boldsymbol{u}):=R_{u}\left(\delta \boldsymbol{u} ; \boldsymbol{A}^{D C}, \boldsymbol{u}^{D C}\right)=\mu_{0} \int_{\Omega_{C}}\left(\boldsymbol{\sigma}^{m}\left(\boldsymbol{u}^{D C}\right)+\boldsymbol{\sigma}^{e}\left(\boldsymbol{A}^{D C}\right)\right): \operatorname{grad} \delta \boldsymbol{u} \mathrm{d} \Omega \\
& -\left.\mu_{0} \int_{\partial \Omega_{C}^{N}} \boldsymbol{\sigma}^{e}\left(\boldsymbol{A}^{D C}\right)\right|^{+} \boldsymbol{n} \cdot \delta \boldsymbol{u} \mathrm{ds}
\end{aligned}
$$

where the time derivatives have vanished because the DC fields are independent of time. Furthermore, considering the weak form of the static problem (3.4), the residuals (3.20) can be further reduced to

$$
\begin{aligned}
\tilde{R}_{A}(\delta \boldsymbol{A}) & =-\mu_{0} \int_{\mathbb{R}^{3}} \boldsymbol{J}^{A C} \cdot \delta \boldsymbol{A} \mathrm{d} \Omega \\
\tilde{R}_{u}(\delta \boldsymbol{u}) & =0
\end{aligned}
$$


Continuing with the linearisation about the static solution, the directional derivatives must also be evaluated on the DC fields leading to

$$
\begin{aligned}
D \tilde{R}_{A}(\delta \boldsymbol{A})[\Delta \boldsymbol{A}] & :=D R_{A}\left(\delta \boldsymbol{A} ; \boldsymbol{A}^{D C}\right)[\Delta \boldsymbol{A}] \\
& =\int_{\mathbb{R}^{3}}\left(\mu_{r}^{-1} \operatorname{curl} \Delta \boldsymbol{A} \cdot \operatorname{curl} \delta \boldsymbol{A}+\mu_{0} \gamma \frac{\mathrm{d} \Delta \boldsymbol{A}}{\mathrm{d} t} \cdot \delta \boldsymbol{A}\right) \mathrm{d} \Omega, \\
D \tilde{R}_{A}(\delta \boldsymbol{A})[\Delta \boldsymbol{u}] & :=D R_{A}\left(\delta \boldsymbol{A} ; \boldsymbol{A}^{D C}\right)[\Delta \boldsymbol{u}]=0 \\
D \tilde{R}_{u}\left(\delta \boldsymbol{u} ; \boldsymbol{A}^{D C}\right)[\Delta \boldsymbol{A}] & :=D R_{u}\left(\delta \boldsymbol{u} ; \boldsymbol{A}^{D C}, \boldsymbol{u}^{D C}\right)[\Delta \boldsymbol{A}] \\
& =\int_{\Omega_{C}} \mu_{r}^{-1} \boldsymbol{S}\left(\boldsymbol{A}^{D C}, \Delta \boldsymbol{A}\right): \nabla \delta \boldsymbol{u} \mathrm{d} \Omega \\
& -\left.\int_{\partial \Omega_{C}^{N}} \boldsymbol{S}\left(\boldsymbol{A}^{D C}, \Delta \boldsymbol{A}\right)\right|^{+} \boldsymbol{n} \cdot \delta \boldsymbol{u} \mathrm{ds} \\
D \tilde{R}_{u}(\delta \boldsymbol{u})[\Delta \boldsymbol{u}] & :=D R_{u}\left(\delta \boldsymbol{u} ; \boldsymbol{A}^{D C}, \boldsymbol{u}^{D C}\right)[\Delta \boldsymbol{u}] \\
& =\mu_{0} \int_{\Omega_{C}}\left(\boldsymbol{\sigma}^{m}(\Delta \boldsymbol{u}): \nabla \delta \boldsymbol{u}+\rho \frac{\mathrm{d}^{2} \Delta \boldsymbol{u}}{\mathrm{d} t^{2}} \cdot \delta \boldsymbol{u}\right) \mathrm{d} \Omega
\end{aligned}
$$

The transient system can then be formulated, after linearisation about the DC solution, as: Find $(\Delta \boldsymbol{A}, \Delta \boldsymbol{u})(t) \in X_{\mathbb{R}^{3}} \times Y\left(\boldsymbol{u}_{D}\right)(0, T]$ such that

$$
\begin{aligned}
D \tilde{R}_{A}(\delta \boldsymbol{A})[\Delta \boldsymbol{A}] & =-\tilde{R}_{A}(\delta \boldsymbol{A}), \\
D \tilde{R}_{u}\left(\delta \boldsymbol{u} ; \boldsymbol{A}^{D C}\right)[\Delta \boldsymbol{A}]+D \tilde{R}_{u}(\delta \boldsymbol{u})[\Delta \boldsymbol{u}] & =-\tilde{R}_{u}(\delta \boldsymbol{u})=0,
\end{aligned}
$$

for all real valued $(\delta \boldsymbol{A}, \delta \boldsymbol{u}) \in X_{\mathbb{R}^{3}} \times Y(\mathbf{0})$, which is linear in the time-dependent variables. Hence, the system can be now expressed in the frequency domain by assuming a time harmonic variation of the fields ${ }^{6}$

$$
\begin{aligned}
\Delta \boldsymbol{A} & \rightarrow \Delta \boldsymbol{A} e^{\mathrm{i} \omega t}, \\
\Delta \boldsymbol{u} & \rightarrow \Delta \boldsymbol{U} e^{\mathrm{i} \omega t}, \\
\boldsymbol{J}^{A C} & \rightarrow \boldsymbol{g}^{A C} e^{\mathrm{i} \omega t},
\end{aligned}
$$

where $\mathrm{i}:=\sqrt{-1}$ and $\omega=2 \pi f$ represents the angular frequency of the driving current in the gradient coils in the case of a harmonic excitation. In addition, $\mathcal{A}^{A C}:=\Delta \mathcal{A}$, $\boldsymbol{u}^{A C}:=\Delta \boldsymbol{u}$ and $\boldsymbol{g}^{A C}$ represent the complex amplitudes of the corresponding time varying (update) fields $\Delta \boldsymbol{A}, \Delta \boldsymbol{u}$ and $\boldsymbol{J}^{A C}$, respectively.

Introducing this time harmonic representation in the residuals (3.21) and directional derivatives (3.22), the system can be formulated in the frequency domain as: Find the complex solutions $\left(\boldsymbol{A}^{A C}, \boldsymbol{u}^{A C}\right) \in X_{\mathbb{R}^{3}} \times Y\left(\boldsymbol{u}_{D}^{A C}\right)$ such that

$$
\begin{aligned}
& D \tilde{R}_{\mathcal{A}}\left(\delta \boldsymbol{A}^{A C}\right)\left[\mathcal{A}^{A C}\right]=-\tilde{R}_{\mathcal{A}}\left(\delta \boldsymbol{A}^{A C}\right)=\mu_{0} \int_{\operatorname{supp}(\boldsymbol{g} A C)} \boldsymbol{g}^{A C} \cdot \overline{\delta \boldsymbol{A}^{A C}} \mathrm{~d} \Omega, \\
& D \tilde{R}_{\mathcal{U}}\left(\delta \boldsymbol{u}^{A C} ; \boldsymbol{A}_{\varepsilon}^{D C}\right)\left[\boldsymbol{A}^{A C}\right]+D \tilde{R}_{\mathcal{U}}\left(\delta \boldsymbol{u}^{A C}\right)\left[\boldsymbol{u}^{A C}\right]=-\tilde{R}_{\mathcal{U}}\left(\delta \boldsymbol{U}^{A C}\right)=0,
\end{aligned}
$$

\footnotetext{
${ }^{6}$ Note that $\frac{\mathrm{d}(\cdot)}{\mathrm{d} t}=\left.\frac{\partial(\cdot)}{\partial t}\right|_{\boldsymbol{X}} \neq\left.\frac{\partial(\cdot)}{\partial t}\right|_{\boldsymbol{x}}$.
} 
for all $\left(\delta \boldsymbol{A}^{A C}, \delta \boldsymbol{U}^{A C}\right) \in X_{\mathbb{R}^{3}} \times Y(\mathbf{0})$, where $(\cdot)$ represents the complex conjugate of a given variable ${ }^{7}$ and we have chosen to rename the weights using the same calligraphic font as for the time harmonic trial functions. In the above, in order to allow for possible complex weights, it has been chosen to revisit (3.16) and include a complex conjugate on the weights $\delta \boldsymbol{A}$ and $\delta \boldsymbol{u}$. When this is done and working through the previous steps this leads to the form (3.25) and the directional derivatives of the time harmonic formulation defined as ${ }^{8}$

$$
\begin{aligned}
& D \tilde{R}_{\mathcal{A}_{\varepsilon}}\left(\delta \mathcal{A}^{A C}\right)\left[\boldsymbol{A}^{A C}\right]:=\int_{\mathbb{R}^{3}}\left(\mu_{r}^{-1} \operatorname{curl} \boldsymbol{A}^{A C} \cdot \operatorname{curl} \overline{\delta \mathcal{A}^{A C}}+\mathrm{i} \mu_{0} \omega \gamma \boldsymbol{A}^{A C} \cdot \overline{\delta \boldsymbol{A}^{A C}}\right) \mathrm{d} \Omega, \\
& D \tilde{R}_{\mathcal{U}}\left(\delta \boldsymbol{u}^{A C} ; \boldsymbol{A}^{D C}\right)\left[\boldsymbol{A}^{A C}\right]:=\int_{\Omega_{C}} \mu_{r}^{-1} \boldsymbol{S}\left(\boldsymbol{A}^{D C}, \boldsymbol{A}^{A C}\right): \operatorname{grad} \overline{\delta \boldsymbol{u}^{A C}} \mathrm{~d} \Omega \\
& -\left.\int_{\partial \Omega_{C}^{N}} \boldsymbol{S}\left(\boldsymbol{A}^{D C}, \boldsymbol{A}^{A C}\right)\right|^{+} \boldsymbol{n} \cdot \overline{\delta \boldsymbol{u}^{A C}} \mathrm{ds}, \\
& D \tilde{R}_{\mathcal{u}}\left(\delta \boldsymbol{u}^{A C}\right)\left[\boldsymbol{u}^{A C}\right]:=\mu_{0} \int_{\Omega_{C}}\left(\boldsymbol{\sigma}^{m}\left(\boldsymbol{u}^{A C}\right): \operatorname{grad} \overline{\delta \boldsymbol{u}^{A C}}-\rho \omega^{2} \boldsymbol{u}^{A C} \cdot \overline{\delta \boldsymbol{u}^{A C}}\right) \mathrm{d} \Omega,
\end{aligned}
$$

where for real weights the conjugate could of course be omitted.

The linear system (3.25) exhibits only a one-way coupling and, therefore, can be solved in a staggered manner by solving first (3.25a) followed by (3.25b). Once the solutions to the DC (3.4) and the AC (3.25) systems are computed, the total time dependent fields can be recovered as

$$
\begin{aligned}
\boldsymbol{A}(t) & =\boldsymbol{A}^{D C}+\operatorname{Re}\left(\mathcal{A}^{A C} e^{\mathrm{i} \omega t}\right), \\
\boldsymbol{u}(t) & =\boldsymbol{u}^{D C}+\operatorname{Re}\left(\boldsymbol{u}^{A C} e^{\mathrm{i} \omega t}\right), \\
\boldsymbol{J}^{s}(t) & =\boldsymbol{J}^{D C}+\operatorname{Re}\left(\boldsymbol{g}^{A C} e^{\mathrm{i} \omega t}\right) .
\end{aligned}
$$

The time harmonic physical (Eulerian) electric and magnetic fields for the AC stage are defined by the linearised version of equation (2.43)

$$
\begin{aligned}
\mathcal{E}^{A C} & =-\mathrm{i} \omega \boldsymbol{A}^{A C}+\mathrm{i} \omega \boldsymbol{B}_{0}^{D C} \times \boldsymbol{U}^{A C} & & \text { in } \Omega_{C}, \\
\mathcal{H}^{A C} & =\mu^{-1} \boldsymbol{B}_{0}^{A C} & & \text { in } \mathbb{R}^{3},
\end{aligned}
$$

where $\boldsymbol{B}_{0}^{A C}=\operatorname{curl} \boldsymbol{A}^{A C}$ and $\boldsymbol{B}_{0}^{D C}=\operatorname{curl} \boldsymbol{A}^{D C}$ and the physical Eulerian fields are

$$
\begin{aligned}
\boldsymbol{E} & =\operatorname{Re}\left(\boldsymbol{\mathcal { E }}^{A C} e^{\mathrm{i} \omega t}\right)=\operatorname{Re}\left(\left(-\mathrm{i} \omega \boldsymbol{A}^{A C}+\mathrm{i} \omega \boldsymbol{B}_{0}^{D C} \times \boldsymbol{u}^{A C}\right) e^{\mathrm{i} \omega t}\right) & & \text { in } \Omega_{C}, \\
\boldsymbol{H} & =\mu^{-1} \boldsymbol{B}_{0}=\mu^{-1}\left(\boldsymbol{B}_{0}^{D C}+\operatorname{Re}\left(e^{\mathrm{i} \omega t} \boldsymbol{B}_{0}^{A C}\right)\right) & & \\
& =\mu^{-1}\left(\operatorname{curl} \boldsymbol{A}^{D C}+\operatorname{Re}\left(e^{\mathrm{i} \omega t} \operatorname{curl} \boldsymbol{A}^{A C}\right)\right) & & \text { in } \mathbb{R}^{3} .
\end{aligned}
$$

\footnotetext{
${ }^{7}$ Note that the overbar will be used in this thesis to denote both the complex conjugate and the closure of a domain. However, it will always be clear which definition applies and no confusion should arise

${ }^{8}$ Note the definitions of the first and second derivatives of a time harmonic field: $\frac{\mathrm{d}}{\mathrm{d} t}\left(\boldsymbol{v} e^{\mathrm{i} \omega t}\right)=\mathrm{i} \omega \boldsymbol{v} e^{\mathrm{i} \omega t}$ and $\frac{\mathrm{d}^{2}}{\mathrm{~d} t^{2}}\left(\boldsymbol{v} e^{\mathrm{i} \omega t}\right)=-\omega^{2} \boldsymbol{v} e^{\mathrm{i} \omega t}$
} 


\subsection{Surface Integral Treatment}

In both the DC and AC stages, surface integrals are present which involve $\boldsymbol{\sigma}^{e}$ and $\boldsymbol{S}$, respectively. In the case of the former, integration by parts yields

$$
\left.\int_{\partial \Omega_{C}^{N}} \boldsymbol{\sigma}^{e}\right|^{+} \boldsymbol{n}^{-} \cdot \delta \boldsymbol{u}^{D C} \mathrm{ds}=-\int_{\Omega_{C}^{c}} \boldsymbol{\sigma}^{e}\left(\boldsymbol{A}^{D C}\right): \operatorname{grad} \delta \boldsymbol{u}^{D C} \mathrm{~d} \Omega,
$$

for all real valued $\delta \boldsymbol{u}^{D C} \in Z$ since $\operatorname{div} \boldsymbol{\sigma}^{e}\left(\boldsymbol{A}^{D C}\right)=0$ in $\Omega_{C}^{c}$ where

$$
Z=\left\{\boldsymbol{u} \in\left(H^{1}\left(\mathbb{R}^{3}\right)\right)^{3}: \boldsymbol{u}(\boldsymbol{x})=\mathbf{0} \text { for } \boldsymbol{x} \in\left(\mathbb{R}^{3} \backslash \bar{\Omega}_{C}\right) \cup \partial \Omega_{C}^{D}\right\} .
$$

Note that $\bar{\Omega}_{C}$ is used here to denote the closure of $\Omega_{C}$. In the case of the AC stage, it can be shown that $\operatorname{div}\left(\boldsymbol{S}\left(\boldsymbol{A}^{D C}, \boldsymbol{A}^{A C}\right)\right)=0$ in $\Omega_{C}^{c} \backslash\left(\operatorname{supp}\left(\boldsymbol{J}^{A C}\right) \cup \operatorname{supp}\left(\boldsymbol{J}^{D C}\right)\right)$ (Appendix A) and, thus, it also holds that

$$
\left.\int_{\partial \Omega_{C}^{N}} \boldsymbol{S}\left(\boldsymbol{A}^{D C}, \mathcal{A}^{A C}\right)\right|^{+} \boldsymbol{n} \cdot \overline{\delta \boldsymbol{u}^{A C}} \mathrm{ds}=-\int_{\Omega_{C}^{c}} \boldsymbol{S}\left(\boldsymbol{A}^{D C}, \boldsymbol{A}^{A C}\right): \operatorname{grad} \overline{\delta \boldsymbol{u}^{A C}} \mathrm{~d} \Omega,
$$

for all $\delta \boldsymbol{u} \in Z$, since the current sources are away from the interface. Similar techniques have already been applied in other areas, such as electromagnetic scattering, and this was proven to give an improved convergence of the solution [108, 130]. Furthermore, rewriting the surface integrals in this form enhances an easier and more efficient numerical implementation, as will be discussed in Chapter 4.

\subsection{Regularisation}

In the development of the weak formulation of the DC (3.4) and AC (3.25) problems, the Coulomb gauge constraint was embedded in the definition of the weighted space $X_{\mathbb{R}^{3}}$. However, the methodology to ensure that this condition is actually satisfied was not discussed and is now considered.

One approach to enforce the Coulomb gauge would be to reformulate the problem in terms of a mixed variational formulation through the introduction of a Lagrange multiplier $[114,185,175]$. This approach, however, presents some drawbacks from a computational perspective that will be presented in greater detail in Chapter 4. First, it leads to an increase in the number of degrees of freedom and, second, the resulting saddle point problem is usually more difficult to solve. Furthermore, one has to ensure that not only the correct discrete spaces are chosen, but also that the order of the elements is chosen with care so as to ensure that the LBB condition $[16,40]$ is satisfied. Therefore, with the aim of building an efficient finite element software, we consider instead the regularised version $[114,185]$ of both the DC and AC problems. Note, however, that the application of regularisation brings the problem of having to solve an ill-conditioned system, but this will be mitigated by the application of specialist preconditioned iterative methodologies that will be presented in Chapter 4 . 


\subsubsection{Regularised Problem}

Let $\varepsilon>0$ be a small regularisation (perturbation) parameter. The regularised DC problem can then be stated as: Find $\left(\boldsymbol{A}_{\varepsilon}^{D C}, \boldsymbol{u}^{D C}\right) \in \tilde{X}_{\mathbb{R}^{3}} \times Y\left(\boldsymbol{u}_{D}^{D C}\right)$ such that

$$
\begin{gathered}
\int_{\mathbb{R}^{3}}\left(\mu_{r}^{-1} \operatorname{curl} \boldsymbol{A}_{\varepsilon}^{D C} \cdot \operatorname{curl} \delta \boldsymbol{A}^{D C}+\varepsilon \boldsymbol{A}_{\varepsilon}^{D C} \cdot \delta \boldsymbol{A}^{D C}\right) \mathrm{d} \Omega=\mu_{0} \int_{\mathbb{R}^{3}} \boldsymbol{J}^{D C} \cdot \delta \boldsymbol{A}^{D C} \mathrm{~d} \Omega, \\
\mu_{0} \int_{\Omega_{c}} \boldsymbol{\sigma}^{m}\left(\boldsymbol{u}^{D C}\right): \operatorname{grad} \delta \boldsymbol{u}^{D C} \mathrm{~d} \Omega=-\mu_{0} \int_{\mathbb{R}^{3}} \boldsymbol{\sigma}^{e}\left(\boldsymbol{A}_{\varepsilon}^{D C}\right): \operatorname{grad} \delta \boldsymbol{u}^{D C} \mathrm{~d} \Omega,
\end{gathered}
$$

for all real valued $\left(\delta \boldsymbol{A}^{D C}, \delta \boldsymbol{u}^{D C}\right) \in \tilde{X}_{\mathbb{R}^{3}} \times Z$, where the subscript $\varepsilon$ is used to denote a regularised quantity, the surface integral has been replaced by a volume integral following the approach described in Section 3.5, and the weighted variational space

$$
\tilde{X}_{\mathbb{R}^{3}}:=\left\{\boldsymbol{A} \in \boldsymbol{H}\left(\operatorname{curl}, \mathbb{R}^{3}\right): \boldsymbol{A}=O\left(|\boldsymbol{x}|^{-1}\right) \text { as }|\boldsymbol{x}| \rightarrow \infty\right\}
$$

has been defined.

This approach is discussed in detail in [114] and in [185] it is shown that $\| \boldsymbol{A}_{\varepsilon}^{D C}-$ $\boldsymbol{A}^{D C}\left\|_{\tilde{X}} \leqslant c \mu_{0} \varepsilon\right\| \boldsymbol{J}^{D C} \|_{\tilde{X}^{*}}$ where * denotes the dual space and the constant $c$ is independent of $\varepsilon$, which implies that the error introduced by the regularisation can be controlled by choosing a sufficiently small $\varepsilon[156,185]$.

\subsubsection{AC Regularised Problem}

Following the same methodology as in Section 3.6.1, the AC regularised problem can be derived to be: Find $\left(\boldsymbol{A}_{\varepsilon}^{A C}, \boldsymbol{u}^{A C}\right) \in \tilde{X}_{\mathbb{R}^{3}} \times Y\left(\boldsymbol{u}_{D}^{A C}\right)$ such that

$$
\begin{aligned}
& D \tilde{R}_{\mathcal{A}_{\varepsilon}}\left(\delta \boldsymbol{A}^{A C}\right)\left[\boldsymbol{\mathcal { A }}_{\varepsilon}^{A C}\right]=-\tilde{R}_{\mathcal{A}}\left(\delta \boldsymbol{A}^{A C}\right)=\mu_{0} \int_{\operatorname{supp}\left(\boldsymbol{g}^{A C}\right)} \boldsymbol{g}^{A C} \cdot \overline{\delta \boldsymbol{A}^{A C}} \mathrm{~d} \Omega \\
& D \tilde{R}_{\mathcal{U}}\left(\delta \boldsymbol{u}^{A C} ; \boldsymbol{A}_{\varepsilon}^{D C}\right)\left[\boldsymbol{\mathcal { A }}_{\varepsilon}^{A C}\right]+D \tilde{R}_{\mathcal{U}}\left(\delta \boldsymbol{u}^{A C}\right)\left[\boldsymbol{u}^{A C}\right]=-\tilde{R}_{\mathcal{U}}\left(\delta \boldsymbol{U}^{A C}\right)=0
\end{aligned}
$$

for all $\left(\delta \boldsymbol{A}^{A C}, \delta \boldsymbol{u}^{A C}\right) \in \tilde{X}_{\mathbb{R}^{3}} \times Z$, where the directional derivatives are defined as

$$
\begin{aligned}
D \tilde{R}_{\mathcal{A}_{\varepsilon}}\left(\delta \boldsymbol{A}^{A C}\right)\left[\mathcal{A}_{\varepsilon}^{A C}\right] & :=\int_{\mathbb{R}^{3}}\left(\mu_{r}^{-1} \operatorname{curl} \boldsymbol{A}_{\varepsilon}^{A C} \cdot \operatorname{curl} \overline{\delta \boldsymbol{A}^{A C}}+\kappa \boldsymbol{A}_{\varepsilon}^{A C} \cdot \overline{\delta \boldsymbol{A}^{A C}}\right) \mathrm{d} \Omega, \\
D \tilde{R}_{\mathcal{U}}\left(\delta \boldsymbol{u}^{A C} ; \boldsymbol{A}_{\varepsilon}^{D C}\right)\left[\boldsymbol{\mathcal { A }}_{\varepsilon}^{A C}\right] & :=\int_{\mathbb{R}^{3}} \mu_{r}^{-1} \boldsymbol{S}\left(\boldsymbol{A}_{\varepsilon}^{D C}, \boldsymbol{A}_{\varepsilon}^{A C}\right): \operatorname{grad} \overline{\delta \boldsymbol{u}^{A C}} \mathrm{~d} \Omega \\
D \tilde{R}_{\mathcal{u}}\left(\delta \boldsymbol{u}^{A C}\right)\left[\boldsymbol{u}^{A C}\right] & :=\mu_{0} \int_{\Omega_{C}}\left(\boldsymbol{\sigma}^{m}\left(\boldsymbol{u}^{A C}\right): \operatorname{grad} \overline{\delta \boldsymbol{u}^{A C}}-\rho \omega^{2} \boldsymbol{u}^{A C} \cdot \overline{\delta \boldsymbol{u}^{A C}}\right) \mathrm{d} \Omega,
\end{aligned}
$$


and $\kappa=\left\{\begin{array}{cc}i \mu_{0} \omega \gamma & \text { in } \Omega_{C} \\ \omega \varepsilon & \text { in } \Omega_{C}^{c}\end{array}\right.$. From the definition of $\kappa$, the regularisation parameter $\varepsilon$ can be interpreted as a very small conductivity that is given to the free space region $\Omega_{C}^{c}$.

The regularised systems (3.33) and (3.35) constitute the equations that will be simulated numerically using the finite element software developed in this work and form the starting point of the discretisation process that will be presented in Chapter 4. Once these systems are solved, the time harmonic physical electric and magnetic fields for the AC stage are defined by the regularised version of (3.28)

$$
\begin{aligned}
\mathcal{E}^{A C} & =-\mathrm{i} \omega \boldsymbol{A}_{\varepsilon}^{A C}+\mathrm{i} \omega \boldsymbol{B}_{0}^{D C} \times \boldsymbol{U}^{A C} & & \text { in } \Omega_{C}, \\
\mathcal{H}^{A C} & =\mu^{-1} \boldsymbol{B}_{0}^{A C} & & \text { in } \mathbb{R}^{3},
\end{aligned}
$$

where $\boldsymbol{B}_{0}^{A C}=\operatorname{curl} \mathcal{A}_{\varepsilon}^{A C}$ and $\boldsymbol{B}_{0}^{D C}=\operatorname{curl} \boldsymbol{A}_{\varepsilon}^{D C}$ and the physical Eulerian fields are

$$
\begin{aligned}
\boldsymbol{E} & =\operatorname{Re}\left(\boldsymbol{E}^{A C} e^{\mathrm{i} \omega t}\right)=\operatorname{Re}\left(\left(-\mathrm{i} \omega \boldsymbol{\mathcal { A }}_{\varepsilon}^{A C}+\mathrm{i} \omega \boldsymbol{B}_{0}^{D C} \times \boldsymbol{u}^{A C}\right) e^{\mathrm{i} \omega t}\right) & & \text { in } \Omega_{C}, \\
\boldsymbol{H} & =\mu^{-1} \boldsymbol{B}_{0}=\mu^{-1}\left(\boldsymbol{B}_{0}^{D C}+\operatorname{Re}\left(e^{\mathrm{i} \omega t} \boldsymbol{B}_{0}^{A C}\right)\right) & & \\
& =\mu^{-1}\left(\operatorname{curl} \boldsymbol{A}_{\varepsilon}^{D C}+\operatorname{Re}\left(e^{\mathrm{i} \omega t} \operatorname{curl} \boldsymbol{A}_{\varepsilon}^{A C}\right)\right) & & \text { in } \mathbb{R}^{3} .
\end{aligned}
$$

The procedure to compute the solution can then be summarised in the following steps

1. Solve (3.33) for $\boldsymbol{A}^{D C}, \boldsymbol{u}^{D C}$.

2. Solve (3.35) for $\boldsymbol{A}_{\varepsilon}^{A C}, \boldsymbol{u}^{A C}$.

3. Compute $\boldsymbol{E}$ and $\boldsymbol{H}$ using (3.38).

\subsection{Chapter Summary}

This chapter has presented a novel coupling methodology for the solution of coupled magneto-mechanical problems in MRI scanners. The transmission problem (2.42), which is based in a Lagrangian frame was split into its DC and AC contributions. The variational formulation for both the static and transient problems was derived and the AC problem was obtained by linearising the transient problem about the static solution. A time harmonic variation of the fields was then assumed in order to formulate the problem in the frequency domain. This led to a staggered linear system in the frequency domain, which offers great computational advantages compared to the Eulerian formulation used in $[19,17]$. Furthermore, following a rigorous derivation, the surface integrals were replaced by volume integrals for the purpose of a more efficient computational implementation that will follow in Chapter 4. Finally, the Coulomb gauge was discussed and the

\footnotetext{
${ }^{9}$ Note that from a dimensional analysis, it could be argued that $\varepsilon$ has in this case units of conductivity times permeability, as opposed to the DC problem where from a dimensional analysis it results that $\varepsilon$ is adimensional. However, $\varepsilon$ is not a physical parameter and, as such, it is usually written without units $[113,185]$.
} 
regularised problem was presented in order to avoid the saddle point problem that would arise when imposing the Coulomb gauge constraint using a Lagrange multiplier. An error bound for the solution of the regularised problem in terms of the regularisation parameter was provided and, as will be seen in subsequent chapters, this approach offers significant computational advantages. 


\section{Chapter 4}

\section{Computational Implementation}

\subsection{Introductory Remarks}

This chapter describes the computational treatment of the linearised and regularised problem presented in Chapter 3. With the objective of developing an accurate and efficient methodology, a $h p$-finite element discretisation is considered and a rigorous derivation of the discrete system is provided. Furthermore, the efficient solution of the resulting linear system of equations using preconditioned iterative solvers is discussed. The chapter builds on the work first presented in [158] by the author through an extended discussion of the technical details.

The main novelties of the chapter are as follows: First, a high order finite element discretisation for the solution of the novel linearised magneto-mechanical problem presented in Chapter 3 for three-dimensional configurations is derived, which combines high order $H^{1}$ and $\boldsymbol{H}$ (curl) basis functions for the discretisation of the mechanical displacements and magnetic vector potential, respectively. Second, an efficient solver strategy is discussed, which involves the solution to the coupled problem in a staggered manner and the use of preconditioned iterative solvers when appropriate. Third, a discussion of the geometrical and physical symmetries that can be exploited to reduce the computational cost of the simulations when considering longitudinal as well as transversal gradient coils is presented and the appropriate symmetry conditions to be imposed at each boundary for the AC and DC fields are stated. Fourth, a methodology to ensure that the current source is solenoidal at a discrete level is also discussed.

The structure of this Chapter is as follows: In Section 4.2 a finite computational domain is introduced by truncating the otherwise unbounded domain. Section 4.3 describes the finite element discretisation, including a description of the reference element, the basis functions used to discretise the electromagnetic and mechanical fields, the mapping between physical and reference element, and a rigorous derivation of the discrete system. The staggered algorithm used for the solution of the coupled problem is presented in Section 4.4 and the new solver strategy is compared with the Eulerian approach used in $[19,18,17]$ in order to show the computational advantages of the novel Lagrangian approach. Section 4.5 presents an efficient preconditioning strategy for the solution of the 


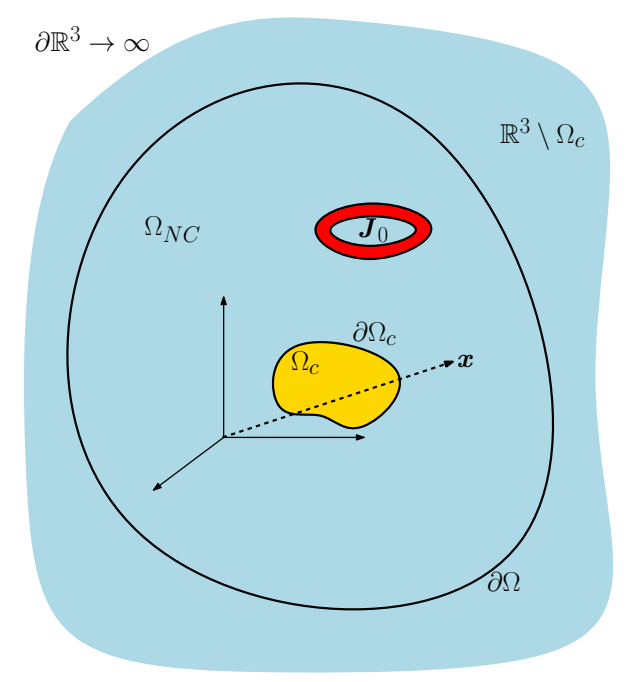

Figure 4.1: Computational domain $\Omega=\Omega_{C} \cup \Omega_{N C}$ created by truncating the unbounded free space region $\Omega_{C}^{c}$ at a finite distance away from $\Omega_{C}$, resulting in the bounded nonconducting region $\Omega_{N C}$.

discrete system using iterative solvers. The introduction of mechanical damping to the system is discussed in Section 4.6. A discussion regarding the properties of the current sources is provided in Section 4.7 and the mapping of the current source to the space of solenoidal functions is described in Section 4.8. The symmetry conditions resulting from the geometrical and physical symmetries are discussed in Section 4.9. Section 4.10 presents a schematic representation of the finite element software, outlining the main stages of the simulation process. The Chapter finishes with concluding remarks in Section 4.11 .

\subsection{Computational Domain}

To allow the introduction of a finite element discretisation, the unbounded free space region $\Omega_{C}^{c}$ must be truncated in order to create a bounded computational domain. Thus, the unbounded free space region $\Omega_{C}^{c}$ is truncated at a finite distance away from the conducting region $\Omega_{C}$ resulting in a truncated non-conducting domain $\Omega_{N C}$, as illustrated in Figure 4.1. The complete bounded domain is then defined as $\Omega:=\Omega_{C} \cup \Omega_{N C}$.

Once the domain is truncated, an appropriate boundary condition must be applied at $\partial \Omega$. Ideally, one would like to know the analytical solution $\boldsymbol{A}_{\text {exact }}$ at the boundary and then apply $\boldsymbol{n} \times \boldsymbol{A}=\boldsymbol{n} \times \boldsymbol{A}_{\text {exact }}$ at $\partial \Omega$. However, analytical solutions are only available for single physics problems consisting of simple geometries. For the coupled problem of interest, where analytical solutions are not available, the decay condition (2.42d) can be approximated by the boundary condition

$$
\boldsymbol{n} \times \boldsymbol{A}=\mathbf{0} \text { on } \partial \Omega,
$$

where the boundary $\partial \Omega$ is assumed to be sufficiently far away from $\Omega_{C}$. Similarly, in the 
DC problem, the decay condition is approximated by

$$
\boldsymbol{n} \times \boldsymbol{A}^{D C}=\mathbf{0} \text { on } \partial \Omega .
$$

Other approaches, such as the use of infinite elements $[27,28]$ or boundary integral methods $[141,20]$, could also be considered, which would allow to truncate the domain at a smaller distance to the conducting bodies. However, these would result in a yet more complex computational implementation and, in this work, it was chosen to approximate the decay condition as described above.

In order to take into account (4.1) and (4.2), the variational space $\tilde{X}_{\mathbb{R}^{3}}$, defined in (3.34), must be replaced by

$$
\tilde{X}_{\Omega}(\boldsymbol{g})=\{\boldsymbol{A} \in \boldsymbol{H}(\operatorname{curl}, \Omega): \boldsymbol{n} \times \boldsymbol{A}=\boldsymbol{n} \times \boldsymbol{g} \text { on } \partial \Omega\},
$$

where $\boldsymbol{g}=\boldsymbol{A}_{\text {exact }}$ in the case of a known analytical solution and $\boldsymbol{g}=\mathbf{0}$ in the general case where the decay condition is approximated as described above.

In the case where $\boldsymbol{A}_{\text {exact }}$ is not known, the accuracy of the approximation improves as the size of $\Omega_{N C}$ increases, but this results also in an increase in computational cost. Thus, the size of $\Omega_{N C}$ must be chosen based on a balance between the accuracy of the approximation and the computational cost.

\subsection{Finite Element Discretisation}

The finite element method $[187,186,89,171]$ is used to discretise the coupled magnetomechanical problem of interest. For this, a non-overlapping unstructured tetrahedral partition of the domain $\Omega=\bigcup_{e=1}^{N_{e}} \Omega^{(e)}$ is introduced, where $\Omega^{(e)}$ is the region corresponding to tetrahedral element $(e)$. Note that unstructured tetrahedral partitions will be considered in this thesis due to the availability of automatic unstructured meshing algorithms for generating meshes around complex configurations and the NetGen mesh generator [155] is used here for this purpose. For a description of the process to create such partitions with NetGen, including the CAD and mesh generation see Appendix B where the issues encountered in this meshing process are summarised. Note that the use of hexahedral elements with high aspect ratio for the discretisation of the thin radiation shields could lead to further computational efficiencies, but this would require the definition of a set of $H^{1}$ and $\boldsymbol{H}$ (curl)-conforming basis functions for hexahedrons and pyramids as well as the use of more sophisticated meshing packages that allow the generation of meshes including tetrahedral, hexahedral and pyramidal high order elements.

Then, a $h p$-finite element framework $[94,58,75,98]$ is considered, which is a combination of the $h$ - and $p$-finite element versions, where the accuracy of the solution is improved by refining the mesh (reducing the size of the elements) and increasing the polynomial order of the basis functions, respectively. This choice of finite element discretisation has been chosen due to its ability to offer exponential convergence of the solution, provided that the correct combination of $h$-refinement and $p$-refinement is chosen. In particular, it 
is well known that, for problems with smooth solution in a domain formed by linear geometry, $h$-refinement results in an algebraic rate of convergence of the error in the energy norm, while $p$-refinement leads to exponential convergence. The a priori estimate for the rate of convergence under $h$-refinement can be defined as $[171,172,11]$

$$
\|\boldsymbol{e}\|_{E(\Omega)} \leqslant \frac{k}{N D O F^{\beta}},
$$

where $e$ denotes the difference between the computational and exact solutions, NDOF denotes the number of degrees of freedom, $k$ is a positive constant and $\beta=p / 3$ for three dimensional problems, with $p$ the polynomial order of the approximation and $\|\boldsymbol{e}\|_{E(\Omega)}$ denotes either the $H^{1}(\Omega)$ or $\boldsymbol{H}(\operatorname{curl}, \Omega)$ norm of $\boldsymbol{e}$, defined as

$$
\begin{aligned}
\|\boldsymbol{e}\|_{H^{1}(\Omega)} & :=\left(\int_{\Omega}|\boldsymbol{e}|^{2}+|\operatorname{grad} \boldsymbol{e}|^{2} \mathrm{~d} \Omega\right)^{1 / 2}, \\
\|\boldsymbol{e}\|_{\boldsymbol{H}_{(\operatorname{curl})(\Omega)}}: & =\left(\int_{\Omega}|\boldsymbol{e}|^{2}+|\operatorname{curl} \boldsymbol{e}|^{2} \mathrm{~d} \Omega\right)^{1 / 2} .
\end{aligned}
$$

Similarly, the a priori estimate for the rate of convergence under $p$-refinement can be defined as [171, 172, 11]

$$
\|\boldsymbol{e}\|_{E(\Omega)} \leqslant \frac{k}{\exp \left(\alpha N D O F^{\theta}\right)}
$$

where $\alpha$ is a positive constant and for three dimensional problems $\theta \geqslant 1 / 3$.

The use of $h p$-finite elements leads to exponential convergence even for problems with a solution that is not smooth, provided that the correct combination of $h$-refinement and $p$-refinement is chosen $[171,172]$. Further advantages of the $h p$-FEM approach have been previously discussed in $[94,58,75,98]$ and, therefore, are not repeated here.

It is important to note that the electromagnetic and mechanical fields have different continuity requirements; more specifically, the mechanical displacements $\boldsymbol{u}$ must be both normal and tangentially continuous across element interfaces, while the magnetic vector potential requires only tangential continuity across element interface. To satisfy this requirements, the mechanical displacements $\boldsymbol{u}^{D C}, \boldsymbol{u}^{A C}$ must be discretised using a set of $H^{1}$ conforming basis functions, while the magnetic vector potential $\boldsymbol{A}_{\varepsilon}^{D C}, \boldsymbol{A}_{\varepsilon}^{A C}$ must be discretised using $\boldsymbol{H}$ (curl) conforming basis functions. Note that the discretisation of three dimensional electromagnetic problems with $H^{1}$ conforming basis functions has been shown to fail [185], leading to wrong solutions in the presence of re-entrant corners and edges [53] or to spurious solutions in the case of eigenvalue problems [34, 36]. Having this in mind, there is a wide variety of $H^{1}$ and $\boldsymbol{H}$ (curl) conforming basis functions that could be considered [58, 59, 10, 156, 185]. However, when considering a $h p$-FEM implementation, it is preferred to allow for easy polynomial refinement, and, therefore, a hierarchic set of basis functions is advantageous for an efficient implementation and also because they lead to better conditioned matrices $[98,171,10]$. Thus, the sets of hierarchical $H^{1}$ and $\boldsymbol{H}$ (curl) conforming basis functions introduced by Schöberl and Zaglmayr [156] are used in this thesis for the discretisation of the coupled problem, which have the advantage of satisfying the complete sequence property

$$
H^{1}(\Omega) \stackrel{\nabla}{\rightarrow} \boldsymbol{H}(\operatorname{curl}, \Omega) \stackrel{\text { curl }}{\longrightarrow} \boldsymbol{H}(\operatorname{div}, \Omega) \stackrel{\operatorname{div}}{\longrightarrow} L^{2}(\Omega)
$$




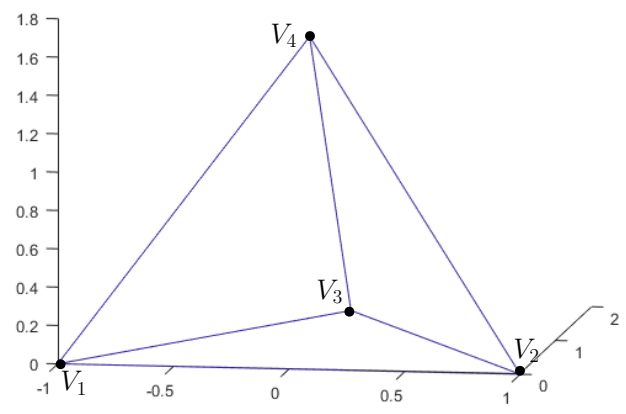

Figure 4.2: Reference tetrahedral element $\hat{\Omega}$.

both globally and locally (each edge, face and element block), which results in the possibility to choose different polynomial degrees for each edge, face and cell. Furthermore, the high order basis functions contain functions that are gradients of the lower order basis, which results in the possibility to neglect these gradients for magnetostatic problems and for non-conducting regions in eddy current problems, reducing the size of the problem and improving the conditioning of the system. With this choice of basis, simple blockJacobi preconditioning becomes efficient $[156,185,114]$. The reference element and the chosen basis functions are described in the following subsections.

\subsubsection{Reference Element}

In the case of tetrahedral elements with flat faces, and a lowest order discretisation, it is possible to explicitly state the basis functions on the physical element. However, for the purpose of specifying the basis functions associated with higher order elements and, in order to deal with meshes with tetrahedral faces that are curved, it is necessary to introduce a reference element on which the $H^{1}$ and $\boldsymbol{H}$ (curl)-conforming basis functions will be defined.

The reference element $\hat{\Omega}$ used in this thesis is the tetrahedron with vertices $V_{1}=$ $(-1,0,0), V_{2}=(1,0,0), V_{3}=(0, \sqrt{3}, 0)$ and $V_{4}=(0,1 / \sqrt{3}, 2 \sqrt{2} / \sqrt{3})$ in the reference coordinate system $(\xi, \eta, \zeta)^{1}$, as illustrated in Figure 4.2.

\subsubsection{Hierarchical $H^{1}$ Conforming Basis Functions}

For the discretisation of $\boldsymbol{u}^{D C}$ and $\boldsymbol{u}^{A C}$ the hierachical $H^{1}$ conforming basis functions proposed by Schöberl and Zaglmayr [156, 185] are used. Their construction is based in a low-order vertex, high-order edge-face-cell splitting as follows

$$
W_{h p}:=W_{h, 1} \oplus \sum_{\text {edges } E \in \mathcal{E}_{h}} W_{p}^{E} \oplus \sum_{\text {faces } F \in \mathcal{F}_{h}} W_{p}^{F} \oplus \sum_{\text {cells } I \in \mathcal{I}_{h}} W_{p}^{I} \subset H^{1}(\Omega)
$$

\footnotetext{
${ }^{1}$ Note that $\xi$ will be used later to denote the damping ratio, where no confusion should arise.
} 


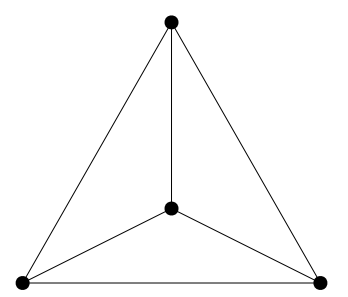

4 Low order vertex

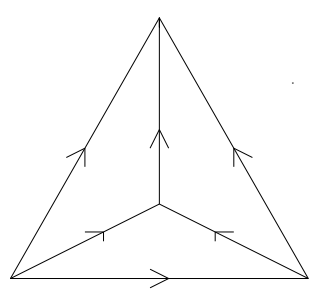

$6(p-1)$ edge

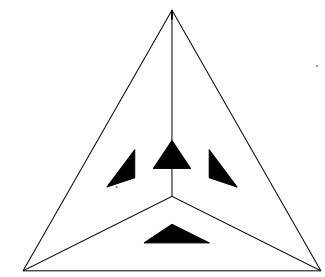

$2\left((p-1)^{2}-(p-1)\right)$ face

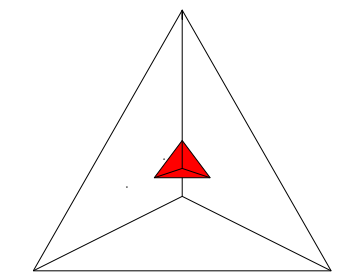

$(p-3)(p-2)(p-1) / 6$

interior

Figure 4.3: High order $H^{1}$-conforming basis functions. Illustration of the distribution of elemental DOF into low order vertex and high order edge, face and interior.

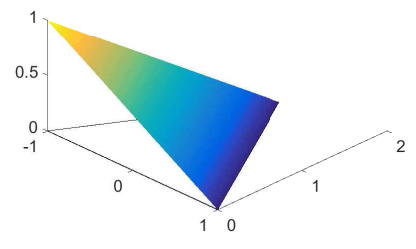

(a) $p=1$ vertex function

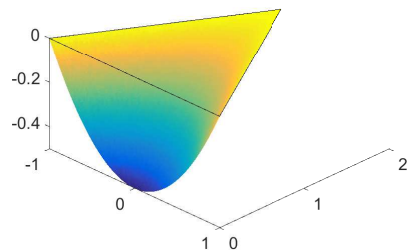

(b) $p=2$ edge function

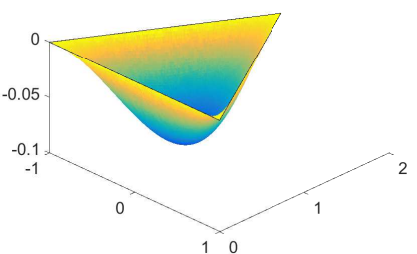

(c) $p=3$ face function

Figure 4.4: Sample $H^{1}$-conforming basis functions plotted over a face of the reference tetrahedron.

where $\mathcal{E}_{h}, \mathcal{F}_{h}, \mathcal{I}_{h}$ denote the sets of edges, faces and cells (or interiors) in our discretisation, respectively, $W_{h, 1}$ is the standard vertex $(\mathrm{V})$ based finite element space of continuous piecewise linear hat functions and $W_{p}^{E}, W_{p}^{F}, W_{p}^{I}$ denote its hierarchic edge (E), face (F) and cell (I) enrichment, respectively.

The total number of degrees of freedom (DOF) in the reference element can be split, for a given polynomial order $p$, into 4 low order vertex $(\mathrm{V}), 6(p-1)$ high order edge (E), $2\left((p-1)^{2}-(p-1)\right)$ high order face $(\mathrm{F})$ and $(p-3)(p-2)(p-1) / 6$ high order cell (I) (or interior) DOF, as illustrated in Figure 4.3. Note that the lowest order case corresponds to $p=1$, in which case there are $4 \mathrm{DOF}$ associated to the vertices of the reference tetrahedron, resulting in a linear approximation.

An illustration of this basis functions over a face of the reference tetrahedron is provided in Figure 4.4. These scalar basis functions are such that the vertex functions associated with vertex $v$ vanish on all other vertices, the edge functions associated with edge $e$ vanish an all other edges and the face functions associated with face $f$ vanish an all other faces and on all the edges of face $f$. Restricted to a given edge, the edge function of order $p$ is an integrated Legendre polynomial of order $p$. The same holds for face and interior functions restricted to a given face or cell, respectively [156, 185]. For further details about these shape functions we refer to $[156,185]$. 


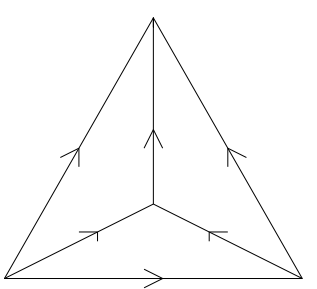

6 Low order edge $(q=0)$

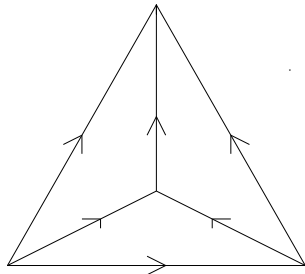

$6 q$ High order edge

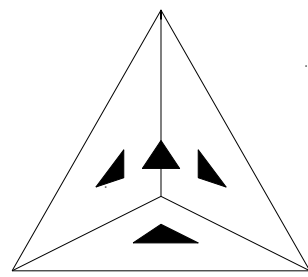

$4\left(q^{2}-1\right)$ face

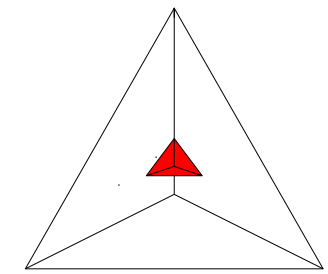

$(q-2)(q-1)(2+(q-$

$3) / 2)$ interior

Figure 4.5: High order $\boldsymbol{H}$ (curl)-conforming basis functions. Illustration of the distribution of elemental DOF into low and high order edge, face and interior.

\subsubsection{Hierarchical $H$ (curl)-Conforming Basis Functions}

As previously discussed, $\boldsymbol{A}_{\varepsilon}^{D C}$ and $\boldsymbol{A}_{\varepsilon}^{A C}$ must be discretised using a set of $\boldsymbol{H}$ (curl) conforming basis functions in order to ensure tangential continuity across element interfaces. Hence, the hierarchical set of $\boldsymbol{H}$ (curl)-conforming basis functions proposed by Schöberl and Zaglmayr [156, 185] are considered. Denoting with $q$ the polynomial order of the $\boldsymbol{H}$ (curl) basis functions, a low-order edge, high-order edge-face-cell based splitting of the $\boldsymbol{H}$ (curl) conforming finite element space can be defined as

$$
X_{h q}:=X_{h, 0} \oplus \sum_{\text {edges } E \in \mathcal{E}_{h}} X_{q}^{E} \oplus \sum_{\text {faces } F \in \mathcal{F}_{h}} X_{q}^{F} \oplus \sum_{\text {cells } I \in \mathcal{I}_{h}} X_{q}^{I} \subset \boldsymbol{H}(\operatorname{curl}, \Omega),
$$

where $X_{h, 0}$ denotes the space of lowest order $(q=0)$ edge $(\mathrm{E})$ or Nédélec elements [131] and $X_{q}^{E}, X_{q}^{F}, X_{q}^{I}$ denote its high order edge (E), face (F) and cell (I) enrichment, respectively.

The DOF can be split in this case into 6 low order edge (E), $6 q$ high order edge (E), $4\left(q^{2}-1\right)$ high order face $(\mathrm{F})$ and $(q-2)(q-1)(2+(q-3) / 2)$ high order interior (I) DOF as illustrated in Figure 4.5. The $\boldsymbol{H}$ (curl) basis are vectorial functions, as opposed to the scalar $H^{1}$ basis. The lowest order $q=0$ edge basis associated with edge $e$ has a constant tangential component on edge $e$ and zero tangential component on other edges. Similarly, the $q=1$ edge basis associated to edge $e$ has a tangential component that varies linearly on edge $e$ and vanishes on all other edges. The same principle holds for face basis functions, where the face basis of order $q=2$ associated with face $f$ varies quadratically on that face and vanishes on all other faces. Restricted to a given edge, face or cell, the edge, face and interior functions can be expressed in terms of products of scaled integrated Legendre polynomials and their gradients [156]. Furthermore, note that the cell functions vanish on all edges and faces, and, therefore, are independent between elements. An illustration of these basis in a face of the reference tetrahedron is provided in Figure 4.6.

Furthermore, splitting the high order terms in (4.10) into gradients and non-gradients of the $H^{1}$ subspaces, (4.10) can also be rewritten as

$$
X_{h q}=X_{h, 0} \oplus \sum_{\text {edges } E \in \mathcal{E}_{h}} \nabla W_{q+1}^{E} \oplus \sum_{\text {faces } F \in \mathcal{F}_{h}} \nabla W_{q+1}^{F} \oplus \tilde{X}_{q}^{F} \oplus \sum_{\text {cells } I \in \mathcal{I}_{h}} \nabla W_{q+1}^{I} \oplus \tilde{X}_{q}^{I},
$$




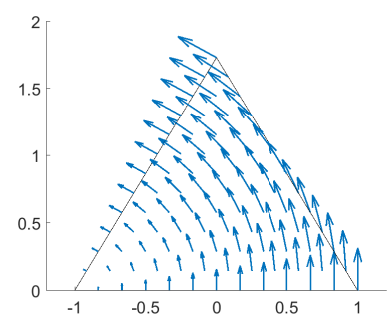

(a) $q=0$ edge function

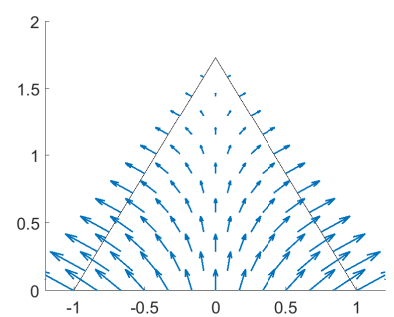

(b) $q=1$ edge function

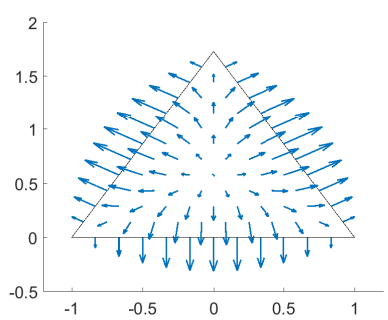

(c) $q=2$ face function

Figure 4.6: Sample $\boldsymbol{H}$ (curl)-conforming basis functions plotted over a face of the reference tetrahedron.

where the high order $\boldsymbol{H}$ (curl) edge basis of order $q$ are gradients of the high order $H^{1}$ edge basis functions of order $q+1$ and the high order face and interior basis functions of order $q$ are split into gradients of the $H^{1}$ basis of order $q+1$ and non gradient face and interior functions, that are added to complete the high order space. The subspaces of nongradient face and interior functions are denoted as $\tilde{X}_{q}^{F}$ and $\tilde{X}_{q}^{I}$, respectively. This splitting of the basis functions into gradients and non-gradients is important for the regularisation strategy adopted in this work and for the construction of an efficient preconditioner, as will be discussed later. For further details about the construction of the $\boldsymbol{H}$ (curl) basis the reader is referred to $[156,185]$.

\section{Reduced basis gauging}

The splitting of $X_{h q}$ offered by (4.11) leads to further efficiencies when combined with the regularisation strategy that was presented in Section 3.6. In particular, those basis functions which are gradients can be skipped in the non-conducting regions (as their curl vanishes) and this leads to a dramatic reduction in the number of unknowns. This reduced basis gauging approach was applied to magnetostatic problems in [156] and to eddy current problems in [114] and its application to both cases is discussed in detail in [185]. Exploiting the splitting (4.11) of the $\boldsymbol{H}$ (curl) space into high order gradient and nongradient functions the main idea of the reduced basis gauging can be summarised as follows:

- For the DC (magnetostatic) problem, the high order gradient subspaces are gauged by skipping the corresponding high order edge, face and cell gradient functions.

- For the AC (eddy current) problem, the high order gradient subspaces are gauged by skipping the corresponding high order edge, face and cell gradient functions in the non-conducting region $\Omega_{N C}$.

- For the lowest order subspace, $X_{h, 0}$, the strategy described in Section 3.6 of adding a small regularisation parameter $\varepsilon$ in order to gauge the system is considered.

This approach results in a big reduction in the size of the discrete system of equations and in a significant improvement of the condition number [185]. 


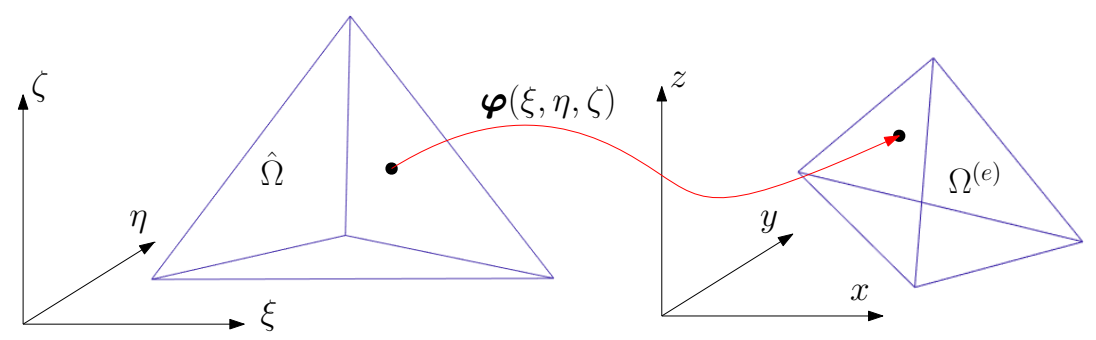

Figure 4.7: Mapping between the reference $\hat{\Omega}$ and physical $\Omega^{(e)}$ elements.

The reduced FE space for the DC problem can then be defined as

$$
X_{\text {reg }}^{D C}=X_{h, 0} \oplus \tilde{X}_{q}^{F} \oplus \tilde{X}_{q}^{I},
$$

where the high order gradient functions have been removed. Similarly, the reduced FE space for the AC problem can be defined as

$$
\begin{aligned}
X_{\text {reg }}^{A C} & =X_{h, 0} \oplus \sum_{\text {edges } E \in \mathcal{E}_{h}^{C}} \nabla W_{q+1}^{E} \oplus \sum_{\text {faces } F \in \mathcal{F}_{h}^{C}} \nabla W_{q+1}^{F} \oplus \sum_{\text {faces } F \in \mathcal{F}_{h}} \tilde{X}_{q}^{F} \\
& \oplus \sum_{\text {cells } I \in \mathcal{I}_{h}^{C}} \nabla W_{q+1}^{I} \oplus \sum_{\text {cells } I \in \mathcal{I}_{h}} \tilde{X}_{q}^{I},
\end{aligned}
$$

where $\mathcal{E}_{h}^{C}, \mathcal{F}_{h}^{C}$ and $\mathcal{I}_{h}^{C}$ denote the sets of edges, faces and cells in the conducting region.

\subsubsection{Elemental Mapping}

The basis functions have been defined in the reference element. However, the basis functions must also be mapped to the physical domain, where the problem is defined. For this, a mapping function describing the transformation from the reference to the physical domain is required, as illustrated in Figure 4.7.

The mapping can be expressed in the general form

$$
\left(\begin{array}{l}
x \\
y \\
z
\end{array}\right)=\boldsymbol{\varphi}(\xi, \eta, \zeta),
$$

where $\boldsymbol{\varphi}$ is the mapping function. Different choices for the mapping function are possible, which are related to the degree of approximation of the geometry.

\section{Linear Mapping}

A linear mapping is typically used in standard finite element solvers, where low order elements are used. The linear mapping can be defined in terms of the barycentric coordinates, which coincide with the lowest order $H^{1}$ basis functions described in Section 4.3.2. 
Denoting these shape functions as $L_{i}$, the linear mapping can be defined as

$$
\left(\begin{array}{l}
x \\
y \\
z
\end{array}\right)=\sum_{i=1}^{4} L^{(e), i}(\xi, \eta, \zeta)\left(\begin{array}{l}
x_{i} \\
y_{i} \\
z_{i}
\end{array}\right)
$$

where $L^{(e), i}, i=1, \ldots, 4$ denote the local evaluation of the 4 lowest order $H^{1}$ basis functions and $x_{i}, y_{i}, z_{i}$ denote the nodal coordinates of the physical element.

\section{Quadratic Mapping}

Curved elements are used typically when considering a geometry with a curved boundary and performing $p$ - or $h p$-refinement, in order to produce a better approximation of the geometry for coarse meshes. One possibility to use such curved elements is the use of Lagrange families of elements [187], where additional nodal points are introduced on the edges and faces of the element. In particular, NetGen allows the generation of quadratic finite elements, where 6 extra nodes are added in the edges of the tetrahedrons. A quadratic approximation of the geometry can then be constructed as

$$
\left(\begin{array}{l}
x \\
y \\
z
\end{array}\right)=\sum_{i=1}^{10} L_{i}^{L}(\xi, \eta, \zeta)\left(\begin{array}{l}
x_{i} \\
y_{i} \\
z_{i}
\end{array}\right)
$$

where $L_{i}^{L}, i=1, \ldots, 10$ denote the set of Lagrange basis functions of order two [89]. Furthermore, $\left(x_{i}, y_{i}, z_{i}\right), i=1, \ldots, 4$ are the coordinates of the vertices of the physical element and $\left(x_{i}, y_{i}, z_{i}\right), i=5, \ldots, 10$ those of the 6 extra nodes added along the edges of the physical element.

\section{The Blending Function Method}

An alternative approach to approximate curved boundaries is the blending function method [171, 98, 77]. The approach described in [77, 54] is considered here, where the mapping is defined as the sum of a linear mapping plus a high order polynomial correction over the faces and edges on the boundary as

$$
\left(\begin{array}{l}
x \\
y \\
z
\end{array}\right)=\sum_{i=1}^{4} L_{i}(\xi, \eta, \zeta)\left(\begin{array}{c}
x_{i} \\
y_{i} \\
z_{i}
\end{array}\right)+\sum_{\text {edges } E \in \Omega^{(e)}}\left(\begin{array}{c}
c_{x}^{E} \\
c_{y}^{E} \\
c_{z}^{E}
\end{array}\right)+\sum_{\text {faces } F \in \Omega^{(e)}}\left(\begin{array}{c}
c_{x}^{F} \\
c_{y}^{F} \\
c_{z}^{F}
\end{array}\right),
$$

where $\left(c_{x}^{E}, c_{y}^{E}, c_{z}^{E}\right)$ and $\left(c_{x}^{F}, c_{y}^{F}, c_{z}^{F}\right)$ denote the high order edge and face corrections, which are functions of $\xi, \eta, \zeta$. The polynomial order of the corrections, $g$, is chosen depending on the curvature of the boundary and the coarseness of the mesh.

Other approaches, such as the NURBS-enhanced finite element method (NEFEM) $[160,161]$ or isogeometric analysis [90] have also been applied in order to obtain accurate geometry representations for problems where the accuracy of the solution is highly dependent on the geometry approximation. 


\section{$H^{1}$ and $\boldsymbol{H}$ (curl)-conforming element transformations}

Once the mapping has been defined, the $H^{1}$ and $\boldsymbol{H}$ (curl) basis functions can be transformed from the reference to the physical element. For this purpose, several transformations must be defined. First, the Jacobian matrix must be defined as

$$
\mathbf{J}=\left[\begin{array}{lll}
\frac{\partial x}{\partial \xi} & \frac{\partial y}{\partial \xi} & \frac{\partial z}{\partial \xi} \\
\frac{\partial x}{\partial \eta} & \frac{\partial y}{\partial \eta} & \frac{\partial z}{\partial \eta} \\
\frac{\partial x}{\partial \zeta} & \frac{\partial y}{\partial \zeta} & \frac{\partial z}{\partial \zeta}
\end{array}\right]
$$

Considering a scalar function $\hat{L} \in H^{1}(\hat{\Omega})$ defined in the reference element, the next $H^{1}$ conforming transformations can be defined [185]

$$
\begin{array}{r}
L(x, y, z)=\hat{L}(\xi, \eta, \zeta), \\
\operatorname{grad} L(x, y, z)=\mathbf{J}^{-T} \hat{\operatorname{grad}} \hat{L}(\xi, \eta, \zeta),
\end{array}
$$

with $L \in H^{1}\left(\Omega^{(e)}\right)$ being the corresponding $H^{1}$-conforming function on the physical element. The hat on grad indicates differentiation with respect to $\xi, \eta, \zeta$.

The transformation of vectorial $\boldsymbol{H}$ (curl)-conforming functions from the reference to the physical element is more complex compared to the case of scalar $H^{1}$-conforming functions, as it must be guaranteed that the degrees of freedom are preserved by the transformation and that gradient fields are mapped onto gradient fields. With these idea in mind, and considering a $\boldsymbol{H}$ (curl)-conforming function $\hat{\boldsymbol{N}} \in \boldsymbol{H}$ (curl, $\hat{\Omega})$ the next transformations can be defined [185]

$$
\begin{array}{r}
\mathbf{N}(x, y, z)=\mathbf{J}^{-T} \hat{\mathbf{N}}(\xi, \eta, \zeta), \\
\operatorname{curl} \mathbf{N}(x, y, z)=|\mathbf{J}|^{-1} \mathbf{J} \operatorname{curl} \hat{\mathbf{N}}(\xi, \eta, \zeta),
\end{array}
$$

with $\boldsymbol{N} \in \boldsymbol{H}$ (curl, $\Omega^{(e)}$ ) being the corresponding $\boldsymbol{H}$ (curl)-conforming function on the physical element. The hat on curl indicates differentiation with respect to $\xi, \eta, \zeta$. In the above $|\mathbf{J}|$ denotes the determinant of $\mathbf{J}$.

\subsubsection{Discretisation of the Coupled System}

This section focuses on the rigorous derivation of the discrete DC and AC linearised and regularised systems that were stated in Sections 3.6.1 and 3.6.2, respectively. The $H^{1}$ conforming basis functions described in Section 4.3.2 are used for the discretisation of $\boldsymbol{u}^{D C}$ and $\boldsymbol{u}^{A C}$, while the $\boldsymbol{H}$ (curl) conforming basis functions described in Section 4.3.3 are used, in conjunction with the reduced basis strategy, for the discretisation of $\boldsymbol{A}_{\varepsilon}^{D C}$ and $\boldsymbol{A}_{\varepsilon}^{A C}$. 


\section{System}

Consider the non-overlapping unstructured tetrahedral partition $\Omega=\bigcup_{e=1}^{N_{e}} \Omega^{(e)}$. The left hand side of equation (3.33a) can then be rewritten as

$$
\begin{aligned}
& \int_{\Omega}\left(\mu_{r}^{-1} \operatorname{curl} \boldsymbol{A}_{\varepsilon}^{D C} \cdot \operatorname{curl} \delta \boldsymbol{A}^{D C}+\varepsilon \boldsymbol{A}_{\varepsilon}^{D C} \cdot \delta \boldsymbol{A}^{D C}\right) \mathrm{d} \Omega= \\
& \sum_{e=1}^{N_{e}} \int_{\Omega^{(e)}}\left(\mu_{r}^{-1} \operatorname{curl} \boldsymbol{A}_{\varepsilon}^{D C} \cdot \operatorname{curl} \delta \boldsymbol{A}^{D C}+\varepsilon \boldsymbol{A}_{\varepsilon}^{D C} \cdot \delta \boldsymbol{A}^{D C}\right) \mathrm{d} \Omega
\end{aligned}
$$

Now let us introduce a discrete Galerkin approximation to the solution $\boldsymbol{A}_{\varepsilon, h q}^{D C} \in X_{\text {reg }}^{D C} \cap$ $\tilde{X}_{\Omega}(\mathbf{0})$ and the test function $\delta \boldsymbol{A}_{h q}^{D C} \in X_{\text {reg }}^{D C} \cap \tilde{X}_{\Omega}(\mathbf{0})$ as

$$
\begin{gathered}
\boldsymbol{A}_{\varepsilon, h q}^{D C}=\sum_{g=1}^{\substack{P_{g l o b a l}^{D C} \\
N^{g}}} \mathrm{~A}_{\varepsilon}^{\mathrm{DC}, g}, \\
\delta \boldsymbol{A}_{h q}^{D C}=\sum_{f=1}^{P_{\text {global }}^{D C}} \boldsymbol{N}^{f} \delta \mathrm{A}^{\mathrm{DC}, f},
\end{gathered}
$$

where $\boldsymbol{N}^{f}, \boldsymbol{N}^{g}$ denote the hierarchical $\boldsymbol{H}$ (curl) conforming basis functions described in Section 4.3.3, $P_{\text {global }}^{D C}$ is the total number of electromagnetic DOF in the DC problem and $\mathrm{A}_{\varepsilon}^{\mathrm{DC}, g}, \delta \mathrm{A}^{\mathrm{DC}, f}$ are the basis functions coefficients, which are constant scalars. Thus, considering a single element $(e)$ with $P_{l o c a l}^{D C}$ degrees of freedom and replacing the continuous variables with their discrete approximation

$$
\begin{aligned}
& \int_{\Omega^{(e)}}\left(\mu_{r}^{-1} \operatorname{curl} \boldsymbol{A}_{\varepsilon}^{D C} \cdot \operatorname{curl} \delta \boldsymbol{A}^{D C}+\varepsilon \boldsymbol{A}_{\varepsilon}^{D C} \cdot \delta \boldsymbol{A}^{D C}\right) \mathrm{d} \Omega \\
& =\sum_{a=1}^{P_{\text {local }}^{D C}} \sum_{b=1}^{P_{\text {local }}^{D C}} \delta \mathrm{A}^{\mathrm{DC}, a}\left(\int_{\Omega^{(e)}}\left(\mu_{r}^{-1} \operatorname{curl} \boldsymbol{N}^{(e), a} \cdot \operatorname{curl} \boldsymbol{N}^{(e), b}+\varepsilon \boldsymbol{N}^{(e), a} \cdot \boldsymbol{N}^{(e), b}\right) \mathrm{d} \Omega\right) \mathrm{A}_{\varepsilon}^{\mathrm{DC}, b} \\
& =\sum_{a=1}^{P_{\text {local }}^{D C}} \sum_{b=1}^{P_{\text {local }}^{D C}} \delta \mathrm{A}^{\mathrm{DC}, a}\left(\left[\mathbf{K}_{\mathrm{AA}}^{\mathrm{DC},(e)}\right]^{a b}+\left[\mathbf{C}_{\mathrm{AA}}^{\mathrm{DC},(e)}\right]^{a b}\right) \mathrm{A}_{\varepsilon}^{\mathrm{DC}, b}
\end{aligned}
$$

where $\boldsymbol{N}^{(e), a}, \boldsymbol{N}^{(e), b}$ denote the local evaluation of the $\boldsymbol{H}$ (curl) basis functions at element $(e)$ and the elemental stiffness and damping matrices have been defined, with components

$$
\begin{aligned}
& {\left[\mathbf{K}_{\mathrm{AA}}^{\mathrm{DC},(e)}\right]^{a b}=\int_{\Omega^{(e)}} \mu_{r}^{-1} \operatorname{curl} \boldsymbol{N}^{(e), a} \cdot \operatorname{curl} \boldsymbol{N}^{(e), b} \mathrm{~d} \Omega,} \\
& {\left[\mathbf{C}_{\mathrm{AA}}^{\mathrm{DC},(e)}\right]^{a b}=\varepsilon \int_{\Omega^{(e)}} \boldsymbol{N}^{(e), a} \cdot \boldsymbol{N}^{(e), b} \mathrm{~d} \Omega .}
\end{aligned}
$$

Note that here and in the following Roman fonts are used for matrices.

Remark 4.1 Note that, as in any typical FE software, the basis functions are defined locally at the reference element, where the numerical integration is performed, after transforming 


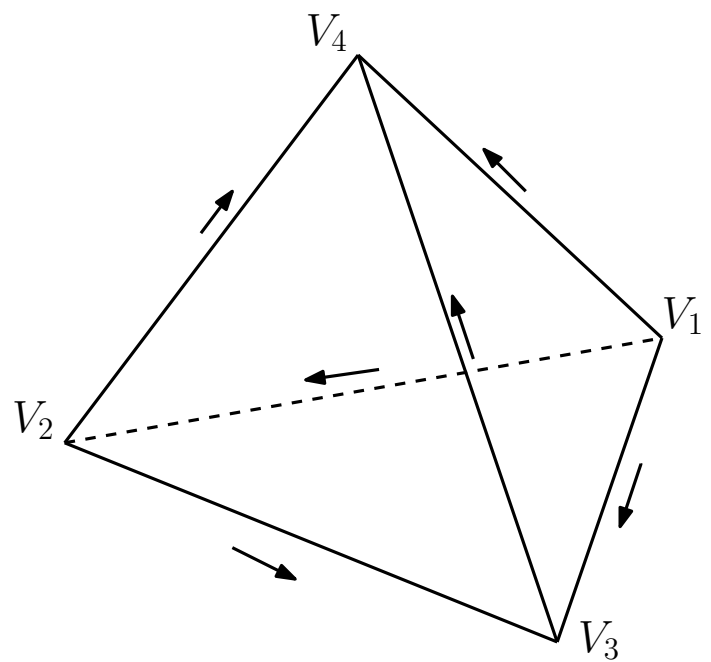

(a) Type I

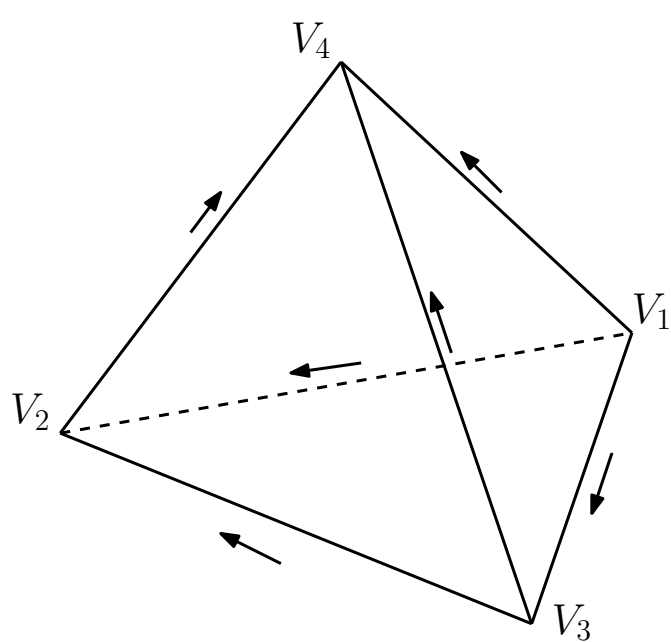

(b) Type II

Figure 4.8: Orientation of the edges for (a) Type I and (b) Type II reference tetrahedra [10].

the integral from $\Omega^{(e)}$ to $\hat{\Omega}$, in order to obtain the corresponding elemental contribution. This is repeated for each element in the partition and the elemental contributions are then assembled to form the global system [171, 89]. The discussion on how the integrals are computed numerically in the reference element is presented in Appendix C.

When assembling elemental contributions from $\boldsymbol{H}$ (curl) conforming discretisations it is important to address the sign conflict problem that arises when imposing tangential continuity between elements due to assigned orientations associated with edges and faces. The approach described in [10], which, through the definition of two reference tetrahedra (Type I and Type II), circumvents this issue, is considered here. This reference elements have the same nodal coordinates as the reference element defined in 4.3.1, and differ only in the orientation of one of the edges, as illustrated in Figure 4.8. The global matrices can then be constructed as

$$
\mathbf{K}_{\mathrm{AA}}^{\mathrm{DC}}=\bigwedge_{e=1}^{N_{e}} \mathbf{K}_{\mathrm{AA}}^{\mathrm{DC},(e)}, \quad \mathbf{C}_{\mathrm{AA}}^{\mathrm{DC}}=\widehat{A}_{e=1}^{N_{e}} \mathbf{C}_{\mathrm{AA}}^{\mathrm{DC},(e)},
$$

where $\mathbb{A}$ is an assembly operator and the global matrices are of size $P_{\text {global }}^{D C} \times P_{\text {global }}^{D C}$. Following the same process, the right hand side of equation (3.33a) can be written as

$$
\mu_{0} \int_{\Omega} \boldsymbol{J}^{D C} \cdot \delta \boldsymbol{A}^{D C} \mathrm{~d} \Omega=\sum_{e=1}^{N_{e}} \mu_{0} \int_{\Omega^{(e)}} \boldsymbol{J}^{D C} \cdot \delta \boldsymbol{A}^{D C} \mathrm{~d} \Omega .
$$

Focusing on a single element and introducing the discrete approximation to the test function

$\mu_{0} \int_{\Omega^{(e)}} \boldsymbol{J}^{D C} \cdot \delta \boldsymbol{A}^{D C} \mathrm{~d} \Omega=\sum_{a=1}^{P_{\text {local }}^{D C}} \delta \mathrm{A}^{\mathrm{DC}, a} \mu_{0} \int_{\Omega^{(e)}} \boldsymbol{J}^{D C} \cdot \boldsymbol{N}^{(e), a} \mathrm{~d} \Omega=\sum_{a=1}^{P_{\text {local }}^{D C}} \delta \mathrm{A}^{\mathrm{DC}, a}\left[\mathbf{F}_{\mathrm{A}}^{\mathrm{DC},(e)}\right]^{a}$, 
where the elemental right hand side vector has been defined, with components

$$
\left[\mathbf{F}_{\mathrm{A}}^{\mathrm{DC},(e)}\right]^{a}:=\mu_{0} \int_{\Omega^{(e)}} \boldsymbol{J}^{D C} \cdot \boldsymbol{N}^{(e), a} \mathrm{~d} \Omega
$$

The global right hand side vector $\mathbf{F}_{\mathrm{A}}^{\mathrm{DC}}$ is then obtained by assembling the elemental contributions using the assembly operator. Once assembled, the Galerkin equation for the electromagnetic DC problem reads

$$
\sum_{f=1}^{P_{\text {global }}^{D C}} \sum_{g=1}^{P_{\text {global }}^{D C}} \delta \mathrm{A}^{\mathrm{DC}, f}\left(\left[\mathbf{K}_{\mathrm{AA}}^{\mathrm{DC}}\right]^{f g}+\left[\mathbf{C}_{\mathrm{AA}}^{\mathrm{DC}}\right]^{f g}\right) \mathrm{A}_{\varepsilon}^{\mathrm{DC}, g}=\sum_{f=1}^{P_{\text {global }}^{D C}} \delta \mathrm{A}^{\mathrm{DC}, f}\left[\mathbf{F}_{\mathrm{A}}^{\mathrm{DC}}\right]^{f}
$$

which must hold for every $\delta \boldsymbol{A}_{h q}^{D C} \in X_{r e g}^{D C} \cap \tilde{X}_{\Omega}(\mathbf{0})$ and, hence, for all $\delta \mathrm{A}^{\mathrm{DC}, f}, f=$ $1,2, \ldots, P_{\text {global }}^{D C}$. Since the $\delta \mathrm{A}^{\mathrm{DC}, f}$ are arbitrary, it necessary follows that

$$
\sum_{g=1}^{P_{\text {global }}^{D C}}\left(\left[\mathbf{K}_{\mathrm{AA}}^{\mathrm{DC}}\right]^{f g}+\left[\mathbf{C}_{\mathrm{AA}}^{\mathrm{DC}}\right]^{f g}\right) \mathrm{A}_{\varepsilon}^{\mathrm{DC}, g}=\left[\mathbf{F}_{\mathrm{A}}^{\mathrm{DC}}\right]^{f} f=1,2, \ldots, P_{\text {global }}^{D C} .
$$

Thus, the discrete system for the electromagnetic DC problem can be written as

$$
\left[\mathbf{K}_{\mathrm{AA}}^{\mathrm{DC}}+\mathbf{C}_{\mathrm{AA}}^{\mathrm{DC}}\right]\left(\mathbf{A}_{\varepsilon}^{\mathrm{DC}}\right)=\left(\mathbf{F}_{\mathrm{A}}^{\mathrm{DC}}\right),
$$

where $\mathbf{A}_{\varepsilon}^{\mathrm{DC}}=\left(\mathrm{A}_{\varepsilon}^{\mathrm{DC}, 1}, \mathrm{~A}_{\varepsilon}^{\mathrm{DC}, 2}, \ldots, \mathrm{A}_{\varepsilon}^{\mathrm{DC}, P_{\text {global }}^{D C}}\right)^{T} \in \mathbb{R}^{P_{\text {global }}}$ is the discrete vector of coefficients, which is the solution to the discrete linear system.

Now, the discretisation of equation (3.33b) is considered, in order to compute $\boldsymbol{u}^{D C}$.

Following the same process as for the discretisation of the electromagnetic problem, the left hand side of equation (3.33b) is rewritten as

$$
\mu_{0} \int_{\Omega_{C}} \boldsymbol{\sigma}^{m}\left(\boldsymbol{u}^{D C}\right): \operatorname{grad} \delta \boldsymbol{u}^{D C} \mathrm{~d} \Omega=\sum_{e=1}^{N_{e}^{C}} \mu_{0} \int_{\Omega^{(e)}} \boldsymbol{\sigma}^{m}\left(\boldsymbol{u}^{D C}\right): \operatorname{grad} \delta \boldsymbol{u}^{D C} \mathrm{~d} \Omega,
$$

where $N_{e}^{C}$ denotes the number of elements in the conducting region $\Omega_{C}$.

Recalling the definition of the strain tensor $\boldsymbol{\epsilon}(\boldsymbol{u}):=\left((\operatorname{grad} \boldsymbol{u})+(\operatorname{grad} \boldsymbol{u})^{T}\right) / 2$, noting that $\boldsymbol{\sigma}^{m}\left(\boldsymbol{u}^{D C}\right)$ is symmetric ${ }^{2}$ and focusing on a single element

$$
\begin{aligned}
a_{u}^{(e)}\left(\boldsymbol{u}^{D C}, \delta \boldsymbol{u}^{D C}\right): & =\mu_{0} \int_{\Omega^{(e)}} \boldsymbol{\sigma}^{m}\left(\boldsymbol{u}^{D C}\right): \operatorname{grad} \delta \boldsymbol{u}^{D C} \mathrm{~d} \Omega \\
& =\mu_{0} \int_{\Omega^{(e)}} \boldsymbol{\sigma}^{m}\left(\boldsymbol{u}^{D C}\right): \boldsymbol{\epsilon}\left(\delta \boldsymbol{u}^{D C}\right) \mathrm{d} \Omega
\end{aligned}
$$

where the elemental bilinear form $a_{u}^{(e)}\left(\boldsymbol{u}^{D C}, \delta \boldsymbol{u}^{D C}\right)$ has been defined for the purpose of an easier presentation. Recalling the definition of the Cauchy stress tensor, $\boldsymbol{\sigma}^{m}(\boldsymbol{u}):=\boldsymbol{C}$ : $\boldsymbol{\epsilon}(\boldsymbol{u})$ and considering the commutative rule for the contraction of second order tensors ${ }^{3}$

$$
a_{u}^{(e)}\left(\boldsymbol{u}^{D C}, \delta \boldsymbol{u}^{D C}\right)=\mu_{0} \int_{\Omega^{(e)}} \boldsymbol{\epsilon}\left(\delta \boldsymbol{u}^{D C}\right):\left(\boldsymbol{C}: \boldsymbol{\epsilon}\left(\boldsymbol{u}^{D C}\right)\right) \mathrm{d} \Omega .
$$

\footnotetext{
${ }^{2}$ Note that given a symmetric tensor $\boldsymbol{A}$ and an arbitrary tensor $\boldsymbol{B}$ the next relation holds: $\boldsymbol{A}: \boldsymbol{B}=\boldsymbol{A}$ : $\boldsymbol{B}^{T}=\boldsymbol{A}: \frac{1}{2}\left(\boldsymbol{B}+\boldsymbol{B}^{T}\right)$.

${ }^{3}$ Note the commutative rule for the contraction of two second order tensors $\boldsymbol{A}: \boldsymbol{B}=\boldsymbol{B}: \boldsymbol{A}$.
} 
Now, let us define $Y_{h p}=\left\{\boldsymbol{u}_{h p}: \boldsymbol{u}_{h p} \in\left(W_{h p}\right)^{3}\right\}$ and introduce a Galerkin approximation to the solution $\boldsymbol{u}_{h p}^{D C} \in Y_{h p} \cap Y\left(\boldsymbol{u}_{D}^{D C}\right)$ and the test function $\delta \boldsymbol{u}_{h p}^{D C} \in Y_{h p} \cap Y(\mathbf{0})$ as

$$
\begin{gathered}
\boldsymbol{u}_{h p}^{D C}=\sum_{d=1}^{Q_{\text {global }}} L^{d} \mathbf{u}^{\mathrm{DC}, d}, \\
\delta \boldsymbol{u}_{h p}^{D C}=\sum_{c=1}^{Q_{\text {global }}} L^{c} \delta \mathbf{u}^{\mathrm{DC}, c},
\end{gathered}
$$

where $L^{d}, L^{c}$ denote typical hierarchical $H^{1}$ basis functions described in Section 4.3.2 and $\mathbf{u}^{\mathrm{DC}, d}, \delta \mathbf{u}^{\mathrm{DC}, c}$ are constant coefficients. Note that the basis functions are in this case scalar functions depending on position, while $\mathbf{u}^{\mathrm{DC}, d}:=\left(\mathrm{u}_{1}^{\mathrm{DC}, d}, \mathrm{u}_{2}^{\mathrm{DC}, d}, \mathrm{u}_{3}^{\mathrm{DC}, d}\right)$ and $\delta \mathbf{u}^{\mathrm{DC}, c}:=$ $\left(\delta \mathrm{u}_{1}^{\mathrm{DC}, c}, \delta \mathrm{u}_{2}^{\mathrm{DC}, c}, \delta \mathrm{u}_{3}^{\mathrm{DC}, c}\right)$ are vectors containing the constant coefficients that multiply the basis functions for each of the three components of the displacement. Thus, the total number of mechanical DOF is $3 Q_{\text {global }}$.

Replacing the continuous trial and test functions $\boldsymbol{u}^{D C}, \delta \boldsymbol{u}^{D C}$ in (4.35) with their discrete approximations $\boldsymbol{u}_{h p}^{D C}, \delta \boldsymbol{u}_{h p}^{D C}$

$$
\begin{aligned}
& a_{u}^{(e)}\left(\boldsymbol{u}_{h p}^{D C}, \delta \boldsymbol{u}_{h p}^{D C}\right)= \\
& \sum_{c=1}^{Q_{\text {local }}} \sum_{d=1}^{Q_{\text {local }}} \int_{\Omega^{(e)}} \frac{1}{2}\left(\left(\delta \mathbf{u}^{\mathrm{DC}, c}\right)_{i} \frac{\partial L^{(e), c}}{\partial x_{j}}+\left(\delta \mathbf{u}^{\mathrm{DC}, c}\right)_{j} \frac{\partial L^{(e), c}}{\partial x_{i}}\right) \boldsymbol{C}_{i j k l} \frac{1}{2}\left(\left(\mathbf{u}^{\mathrm{DC}, d}\right)_{k} \frac{\partial L^{(e), d}}{\partial x_{l}}+\left(\mathbf{u}^{\mathrm{DC}, d}\right)_{l} \frac{\partial L^{(e), d}}{\partial x_{k}}\right) \mathrm{d} \Omega,
\end{aligned}
$$

where $3 Q_{\text {local }}$ is the number of mechanical DOF in an element, $L^{(e), c}$ denotes the local evaluation of the $c$ 'th $H^{1}$ basis function at element $(e)$ and Einstein's summation convention is used to sum over the indices $i, j, k, l$. After suitable index transformations, (4.37) can be rewritten as

$$
\begin{aligned}
& a_{u}^{(e)}\left(\boldsymbol{u}_{h p}^{D C}, \delta \boldsymbol{u}_{h p}^{D C}\right)= \\
& \sum_{c=1}^{Q_{\text {local }}} \sum_{d=1}^{Q_{\text {local }}}\left(\delta \mathbf{u}^{\mathrm{DC}, c}\right)_{i}\left(\int_{\Omega^{(e)}} \frac{\partial L^{(e), c}}{\partial x_{j}} \frac{1}{4}\left(\boldsymbol{C}_{i j k l}+\boldsymbol{C}_{i j l k}+\boldsymbol{C}_{j i k l}+\boldsymbol{C}_{j i l k}\right) \frac{\partial L^{(e), d}}{\partial x_{l}} \mathrm{~d} \Omega\right)\left(\mathbf{u}^{\mathrm{DC}, d}\right)_{k} \\
& =\sum_{c=1}^{Q_{\text {local }}} \sum_{d=1}^{Q_{\text {local }}}\left(\delta \mathbf{u}^{\mathrm{DC}, c}\right)_{i}\left[\int_{\Omega^{(e)}} \frac{\partial L^{(e), c}}{\partial x_{j}} \boldsymbol{C}_{i j k l}^{s y m} \frac{\partial L^{(e), d}}{\partial x_{l}} \mathrm{~d} \Omega\right]\left(\mathbf{u}^{\mathrm{DC}, d}\right)_{k},
\end{aligned}
$$

where

$$
\begin{aligned}
\boldsymbol{C}_{i j k l}^{s y m} & =\frac{1}{4}\left(\boldsymbol{C}_{i j k l}+\boldsymbol{C}_{i j l k}+\boldsymbol{C}_{j i k l}+\boldsymbol{C}_{j i l k}\right) \\
& =\lambda \delta_{i j} \delta_{k l}+\frac{G}{2}\left(\delta_{i k} \delta_{j l}+\delta_{k j} \delta_{i l}+\delta_{i l} \delta_{j k}+\delta_{j k} \delta_{i l}\right),
\end{aligned}
$$

and swapping indices $j$ and $k$

$$
\begin{aligned}
a_{u}^{(e)}\left(\boldsymbol{u}_{h p}^{D C}, \delta \boldsymbol{u}_{h p}^{D C}\right) & =\sum_{c=1}^{Q_{\text {local }}} \sum_{d=1}^{Q_{\text {local }}}\left(\delta \mathbf{u}^{\mathrm{DC}, c}\right)_{i}\left[\int_{\Omega^{(e)}} \frac{\partial L^{(e), c}}{\partial x_{k}} \boldsymbol{C}_{i k j l}^{\text {sym }} \frac{\partial L^{(e), d}}{\partial x_{l}} \mathrm{~d} \Omega\right]\left(\mathbf{u}^{\mathrm{DC}, d}\right)_{j} \\
& =\sum_{c=1}^{Q_{\text {local }}} \sum_{d=1}^{Q_{\text {local }}}\left(\delta \mathbf{u}^{\mathrm{DC}, c}\right)_{i}\left[\mathbf{K}_{u u}^{\mathrm{DC},(e)}\right]_{i j}^{c d}\left(\mathbf{u}^{\mathrm{DC}, d}\right)_{j}
\end{aligned}
$$


where the elemental stiffness matrix has been defined, with components

$$
\left[\mathbf{K}_{u u}^{\mathrm{DC},(e)}\right]_{i j}^{c d}=\int_{\Omega^{(e)}} \frac{\partial L^{(e), c}}{\partial x_{k}} \boldsymbol{C}_{i k j l}^{s y m} \frac{\partial L^{(e), d}}{\partial x_{l}} \mathrm{~d} \Omega,
$$

and the global stiffness matrix $\mathbf{K}_{u u}^{\mathrm{DC}}$ can be constructed as previously using the assembly operator, so that

$$
\mathbf{K}_{u u}^{\mathrm{DC}}=\widehat{A}_{e=1}^{N_{e}^{C}} \mathbf{K}_{u u}^{\mathrm{DC},(e)}
$$

where $N_{e}^{C}$ denotes the number of elements in the conducting region.

Consider now the discretisation of the right hand side of equation (3.33b). Following the same process, this can be expressed as

$$
-\mu_{0} \int_{\Omega} \boldsymbol{\sigma}^{e}\left(\boldsymbol{A}^{D C}\right): \operatorname{grad} \delta \boldsymbol{u}^{D C} \mathrm{~d} \Omega=-\sum_{e=1}^{N_{e}} \mu_{0} \int_{\Omega^{(e)}} \boldsymbol{\sigma}^{e}\left(\boldsymbol{A}_{\varepsilon}^{D C}\right): \operatorname{grad} \delta \boldsymbol{u}^{D C} \mathrm{~d} \Omega .
$$

Furthermore, since $\delta \boldsymbol{u}^{D C} \in Z$ is such that $\delta \boldsymbol{u}^{D C}(\boldsymbol{x})=\mathbf{0}$ for $\boldsymbol{x} \in \Omega \backslash \overline{\Omega_{C}}$, instead of integrating on the whole non-conducting region, it is chosen here to restrict the integration to a layer of elements $\Omega_{N C}^{1}$ adjacent to $\Omega_{C}$, i.e., only those elements that have at least one entity (vertex, edge or face) on $\partial \Omega_{C}$. These elements are identified at a pre-processing stage with a negligible cost, making the subsequent assembly of (4.43) much more efficient. The layer of elements is illustrated in Figure 4.9 for the case of a conducting sphere of radius $1 \mathrm{~cm}$ and a non-conducting sphere of radius $2 \mathrm{~cm}$. Thus, (4.43) can then be expressed as

$$
\begin{aligned}
-\mu_{0} \int_{\Omega} \boldsymbol{\sigma}^{e}\left(\boldsymbol{A}^{D C}\right): \operatorname{grad} \delta \boldsymbol{u}^{D C} \mathrm{~d} \Omega & =-\mu_{0} \int_{\Omega_{C} \cup \Omega_{N C}^{1}} \boldsymbol{\sigma}^{e}\left(\boldsymbol{A}^{D C}\right): \operatorname{grad} \delta \boldsymbol{u}^{D C} \mathrm{~d} \Omega= \\
& -\sum_{e=1}^{N_{e}^{1}} \mu_{0} \int_{\Omega^{(e)}} \boldsymbol{\sigma}^{e}\left(\boldsymbol{A}_{\varepsilon}^{D C}\right): \operatorname{grad} \delta \boldsymbol{u}^{D C} \mathrm{~d} \Omega,
\end{aligned}
$$

where $N_{e}^{1}$ is the number of elements in $\Omega_{C} \cup \Omega_{N C}^{1}$.

Focusing on a single element, and introducing the discrete approximation to $\delta \boldsymbol{u}^{D C}$

$$
\begin{aligned}
-\mu_{0} \int_{\Omega^{(e)}} \boldsymbol{\sigma}^{e}\left(\boldsymbol{A}_{\varepsilon}^{D C}\right): \operatorname{grad} \delta \boldsymbol{u}^{D C} \mathrm{~d} \Omega & =-\sum_{c=1}^{Q_{\text {local }}} \mu_{0} \delta \mathbf{u}^{\mathrm{DC}, c} \int_{\Omega^{(e)}} \boldsymbol{\sigma}^{e}\left(\boldsymbol{A}_{\varepsilon}^{D C}\right)_{i j} \frac{\partial L^{(e), c}}{\partial x_{j}} \mathrm{~d} \Omega \\
& =-\sum_{c=1}^{Q_{\text {local }}} \mu_{0} \delta \mathbf{u}^{\mathrm{DC}, c}\left[\mathbf{F}_{\mathrm{u}}^{\mathrm{DC},(e)}\right]_{i}^{c}
\end{aligned}
$$

where the elemental right hand side vector has been defined, with components

$$
\left[\mathbf{F}_{\mathrm{u}}^{\mathrm{DC},(e)}\right]_{i}^{c}=\mu_{0} \int_{\Omega^{(e)}} \boldsymbol{\sigma}^{e}\left(\boldsymbol{A}_{\varepsilon}^{D C}\right)_{i j} \frac{\partial L^{(e), c}}{\partial x_{j}} \mathrm{~d} \Omega
$$

and the global right hand side vector is constructed as

$$
\mathbf{F}_{\mathrm{u}}^{\mathrm{DC}}=\widehat{A}_{e=1}^{N_{e}^{1}} \mathbf{F}_{\mathrm{u}}^{\mathrm{DC},(e)}
$$




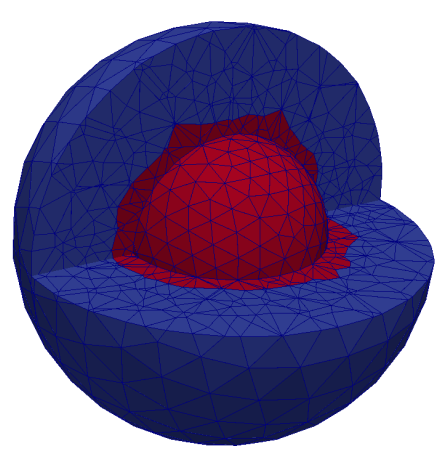

Figure 4.9: Schematic representation of the layer of elements $\Omega_{N C}^{1}$ adjacent to $\Omega_{C}$ that is defined in order to improve the efficiency of the assembly process. $\Omega_{C} \cup \Omega_{N C}^{1}$ coloured in red and $\Omega_{N C} \backslash \Omega_{N C}^{1}$ in blue.

The discrete system for the mechanical DC problem can then be stated as

$$
\left[\mathbf{K}_{\mathrm{uu}}^{\mathrm{DC}}\right]\left(\mathbf{u}^{\mathrm{DC}}\right)=\left(\mathbf{F}_{\mathrm{u}}^{\mathrm{DC}}\right)
$$

where

$$
\mathbf{u}^{\mathrm{DC}}=\left(\begin{array}{c}
\mathbf{u}^{\mathrm{DC}, 1} \\
\mathbf{u}^{\mathrm{DC}, 2} \\
\vdots \\
\mathbf{u}^{\mathrm{DC}, Q_{\text {global }}}
\end{array}\right) \in \mathbb{R}^{3 Q_{\text {global }},}
$$

is the discrete vector of coefficients.

Thus, the complete discrete system for the DC stage can be stated as: Find $\mathbf{A}_{\varepsilon}^{\mathrm{DC}}$ such that

$$
\left[\mathbf{K}_{\mathrm{AA}}^{\mathrm{DC}}+\mathbf{C}_{\mathrm{AA}}^{\mathrm{DC}}\right]\left(\mathbf{A}_{\varepsilon}^{\mathrm{DC}}\right)=\left(\mathbf{F}_{\mathrm{A}}^{\mathrm{DC}}\right)
$$

and then $\mathbf{u}^{\mathrm{DC}}$ such that

$$
\left[\mathbf{K}_{\mathrm{uu}}^{\mathrm{DC}}\right]\left(\mathbf{u}^{\mathrm{DC}}\right)=\left(\mathbf{F}_{\mathrm{u}}^{\mathrm{DC}}\right),
$$

where the global matrices defined above as the assembly of elemental contributions can also be written, for a clearer presentation as:

$$
\begin{aligned}
{\left[\mathbf{K}_{\mathrm{AA}}^{\mathrm{DC}}\right]^{a b} } & =\int_{\Omega} \mu_{r}^{-1}\left(\operatorname{curl} \boldsymbol{N}^{a}\right)_{i}\left(\operatorname{curl} \boldsymbol{N}^{b}\right)_{i} \mathrm{~d} \Omega, \\
{\left[\mathbf{C}_{\mathrm{AA}}^{\mathrm{DC}}\right]^{a b} } & =\varepsilon \int_{\Omega} \boldsymbol{N}_{i}^{a} \boldsymbol{N}_{i}^{b} \mathrm{~d} \Omega, \\
{\left[\mathbf{K}_{\mathrm{uu}}^{\mathrm{DC}}\right]_{i j}^{c d} } & =\mu_{0} \int_{\Omega_{C}} \frac{\partial L^{c}}{\partial x_{k}} \boldsymbol{C}_{i k j l}^{s y m} \frac{\partial L^{d}}{\partial x_{l}} \mathrm{~d} \Omega, \\
{\left[\mathbf{F}_{\mathrm{A}}^{\mathrm{DC}}\right]^{a} } & =\mu_{0} \int_{\Omega} \boldsymbol{J}_{i}^{D C} \boldsymbol{N}_{i}^{a} \mathrm{~d} \Omega, \\
{\left[\mathbf{F}_{\mathrm{u}}^{\mathrm{DC}}\right]_{i}^{c} } & =-\mu_{0} \int_{\Omega_{C} \cup \Omega_{N C}^{1}} \boldsymbol{\sigma}^{e}\left(\boldsymbol{A}_{\varepsilon}^{D C}\right)_{i j} \frac{\partial L^{c}}{\partial x_{j}} \mathrm{~d} \Omega,
\end{aligned}
$$

which are expressed in a global setting and using index notation for all terms. 


\section{AC System}

Let us introduce the discrete Galerkin approximations $\mathcal{A}_{\varepsilon, h q}^{A C} \in X_{\text {reg }}^{A C} \cap \tilde{X}_{\Omega}(\mathbf{0})$ to $\boldsymbol{A}^{A C}$ and $\boldsymbol{u}_{h p}^{A C} \in Y_{h p} \cap Y\left(\boldsymbol{u}_{D}^{A C}\right)$ to $\boldsymbol{u}^{A C}$ such that

$$
\begin{array}{r}
\boldsymbol{A}_{\varepsilon, h q}^{A C}=\sum_{b=1}^{P_{g l o b a l}^{A C}} \boldsymbol{N}^{b} \mathcal{A}_{\varepsilon}^{\mathrm{AC}, b}, \\
\delta \boldsymbol{A}_{\varepsilon, h q}^{A C}=\sum_{a=1}^{P_{\text {global }}^{A C}} \boldsymbol{N}^{a} \delta \mathcal{A}_{\varepsilon}^{\mathrm{AC}, a}, \\
\boldsymbol{u}_{h p}^{A C}=\sum_{d=1}^{Q_{\text {global }}} L^{d} \mathcal{U}^{\mathrm{AC}, d}, \\
\boldsymbol{\delta} \boldsymbol{u}_{h p}^{A C}=\sum_{c=1}^{Q_{g l o b a l}} L^{c} \boldsymbol{\delta} \mathcal{U}^{\mathrm{AC}, c} .
\end{array}
$$

Following similar steps as for the derivation of the DC system, the discrete system of equations for the $\mathrm{AC}$ problem can then be stated as: Find $\mathcal{A}_{\varepsilon}^{\mathrm{AC}}$ and $\mathcal{U}^{\mathrm{AC}}$ such that

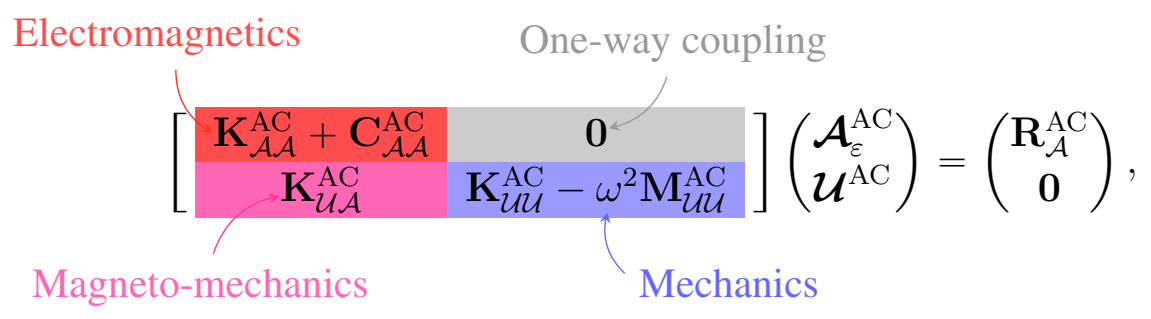

where the purely electromagnetic blocks have been highlighted in red, the purely mechanical blocks in blue and the coupling block in magenta. The different blocks of the system are defined as

$$
\begin{aligned}
{\left[\mathbf{K}_{\mathcal{A} \mathcal{A}}^{\mathrm{AC}}\right]^{a b} } & =\int_{\Omega} \mu_{r}^{-1}\left(\operatorname{curl} \boldsymbol{N}^{a}\right)_{i}\left(\operatorname{curl} \boldsymbol{N}^{b}\right)_{i} \mathrm{~d} \Omega \\
{\left[\mathbf{C}_{\mathcal{A} \mathcal{A}}^{\mathrm{AC}}\right]^{a b} } & =\mathrm{i} \omega \mu_{0} \int_{\Omega_{C}} \gamma \boldsymbol{N}_{i}^{a} \boldsymbol{N}_{i}^{b} \mathrm{~d} \Omega+\omega \varepsilon \int_{\Omega_{N C}} \boldsymbol{N}_{i}^{a} \boldsymbol{N}_{i}^{b} \mathrm{~d} \Omega \\
{\left[\mathbf{K}_{\mathcal{U} \mathcal{U}}^{\mathrm{AC}}\right]_{i j}^{c d} } & =\mu_{0} \int_{\Omega_{C}} \frac{\partial L^{c}}{\partial x_{k}} \boldsymbol{C}_{i k j l}^{s y m} \frac{\partial L^{d}}{\partial x_{l}} \mathrm{~d} \Omega \\
{\left[\mathbf{M}_{\mathcal{U} \mathcal{U}}^{\mathrm{AC}}\right]_{i j}^{c d} } & =\mu_{0} \int_{\Omega_{C}} \rho L^{c} L^{d} \delta_{i j} \mathrm{~d} \Omega \\
{\left[\mathbf{K}_{\mathcal{U} \mathcal{A}}^{\mathrm{AC}}\right]_{i}^{c b} } & =\int_{\Omega_{C} \cup \Omega_{N C}^{1}} \mu_{r}^{-1} \boldsymbol{S}_{i j}^{b} \frac{\partial L^{c}}{\partial x_{j}} \mathrm{~d} \Omega \\
{\left[\mathbf{R}_{\mathcal{A}}^{\mathrm{AC}}\right]^{a} } & =\mu_{0} \int_{\Omega} \boldsymbol{g}_{i}^{A C} \boldsymbol{N}_{i}^{a} \mathrm{~d} \Omega .
\end{aligned}
$$


Mixed approaches arise in the discretisations of linear elasticity problems to avoid mechanical locking [169, 48], Navier-Stokes equations for describing the pressure and velocity fields [157] and in the discretisation of the Maxwell system if a Lagrange multiplier is used to enforce the divergence constraint [175]. In each case, they lead to systems that combine elements of different types and different orders. In the latter two cases, a saddle point problem results and, in such cases, it is not only important that the correct discrete spaces are chosen, but also that the order of the elements be chosen with care so as to ensure that the (discrete) LBB condition is satisfied. The system (4.54) combines two element types, but in common with mixed approaches to elasticity with $\nu \neq 1 / 2$, it does not represent a saddle point problem and, therefore, one can be flexible with the degree approximation $p$ for $\boldsymbol{u}_{h p}^{A C}$ and $q$ for $\boldsymbol{A}_{\varepsilon, h q}^{A C}$.

Furthermore, since the system (4.54) exhibits only a one way coupling, it can be expressed in a form suitable for a staggered scheme as: Find $\mathcal{A}_{\varepsilon}^{\mathrm{AC}}$ such that

$$
\left[\mathbf{K}_{\mathcal{A} \mathcal{A}}^{\mathrm{AC}}+\mathbf{C}_{\mathcal{A} \mathcal{A}}^{\mathrm{AC}}\right]\left(\mathcal{A}_{\varepsilon}^{\mathrm{AC}}\right)=\left(\mathbf{R}_{\mathcal{A}}^{\mathrm{AC}}\right)
$$

and then $\mathcal{U}^{\mathrm{AC}}$ such that

$$
\left[\mathbf{K}_{\mathcal{U} \mathcal{U}}^{\mathrm{AC}}-\omega^{2} \mathbf{M}_{\mathcal{U} \mathcal{U}}^{\mathrm{AC}}\right]\left(\mathcal{U}^{\mathrm{AC}}\right)=\left(\mathbf{R}_{\mathcal{U}}^{\mathrm{AC}}\right)
$$

where

$$
\mathbf{R}_{\mathcal{U}}^{\mathrm{AC}}=-\mathbf{K}_{\mathcal{U} \mathcal{A}}^{\mathrm{AC}} \mathcal{A}_{\varepsilon}^{\mathrm{AC}}
$$

\subsection{Staggered Algorithm}

Unlike the Eulerian approach used in $[19,18]$, the solution vector $\mathcal{A}_{\varepsilon}^{\mathrm{AC}}$ can be computed independently of $\mathcal{U}^{\mathrm{AC}}$ in the $\mathrm{AC}$ stage and, therefore, the simple staggered solver described in algorithmic format in Algorithm 1 is proposed, which combines the DC stage (4.24), (4.51) and the AC stage (4.56), (4.57) as well as the physical field representations (3.37). Notice that steps 1, 2, 4 and 5 are simple linear algebra steps and the steps 3 and 6 follow from using the discrete representation of the fields using the coefficients computed from the solution of the linear systems. This is further emphasised by the different choice of fonts in steps 3 and 6 compared to the others. 


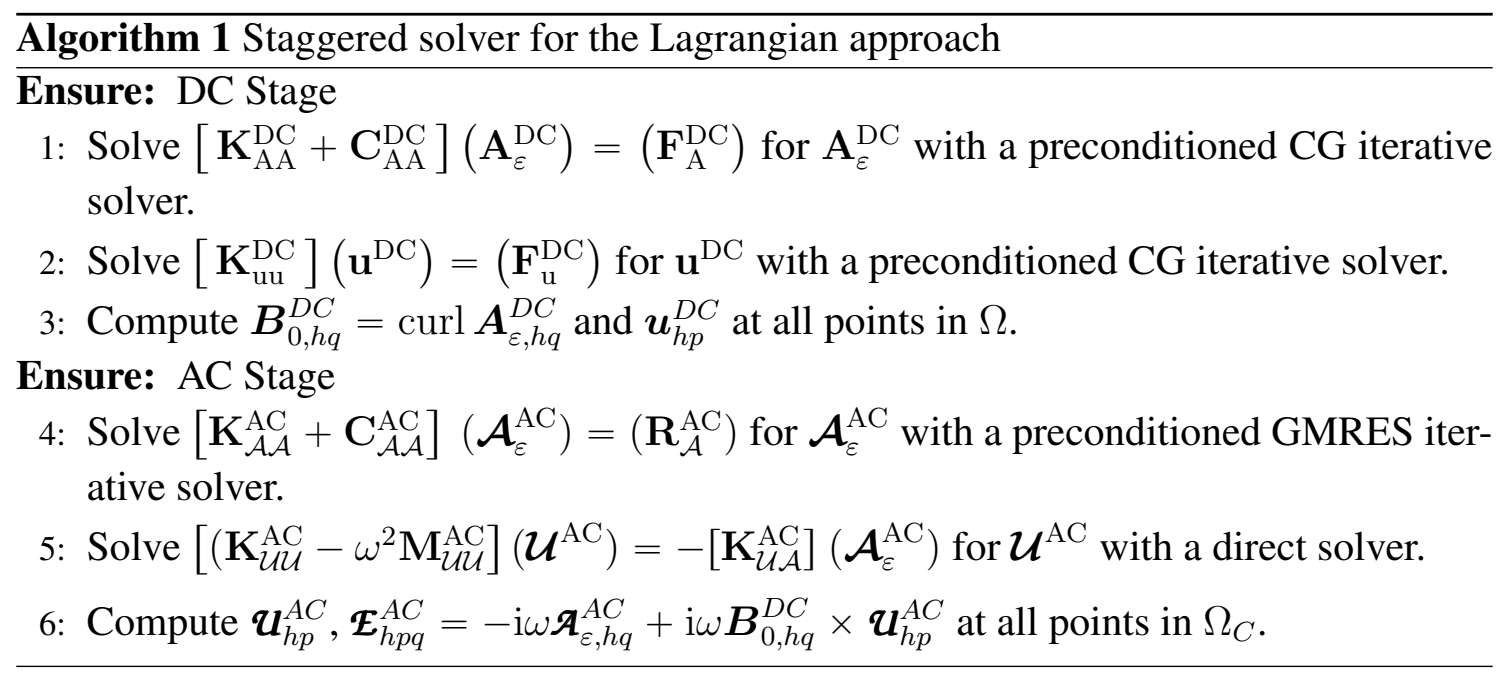

Compared to the Eulerian approach, Algorithm 1 offers considerable advantages when applied to large three-dimensional problems since, in the AC stage, rather than the solution of a single complex indefinite system, the problem has been reduced to the solution of two smaller systems, the larger of which can be solved by preconditioned iterative approach. Specifically, the matrix $\mathbf{K}_{\mathcal{A} \mathcal{A}}^{\mathrm{AC}}+\mathbf{C}_{\mathcal{A} \mathcal{A}}^{\mathrm{AC}}$ is complex symmetric and is positive semi definite. It is of the same form as obtained in the discretisation of eddy current problems by $h p$-finite elements [114]. Therefore, the same preconditioned GMRES technique as described in this work is applied for computing $\mathcal{A}_{\varepsilon}^{\mathrm{AC}}$. The matrix $\mathbf{K}_{\mathcal{U} \mathcal{U}}^{\mathrm{AC}}-\omega^{2} \mathbf{M}_{\mathcal{U} \mathcal{U}}^{\mathrm{AC}}$ is real and indefinite and it is of the form obtained in the discretisation of elastic wave propagation by $h p$-finite elements. For a sufficiently fine grid, an iterative approach to its solution could be applied, but, given its relatively small dimension compared to $\mathbf{K}_{\mathcal{A} \mathcal{A}}^{\mathrm{AC}}+\mathbf{C}_{\mathcal{A} \mathcal{A}}^{\mathrm{AC}}$, a direct solver is instead employed for the computation of $\mathcal{U}^{\mathrm{AC}}$, which is less restrictive.

The AC-stage of the Eulerian approach, which was solved monolithically in [19, 18], can be solved instead using a fixed point strategy. A flow chart comparing the solver structure for the Lagrangian and Eulerian formulations is provided in Figure 4.10, where the advantages of the Lagrangian formulation can be clearly identified.

\subsection{Preconditioned Iterative Solvers}

Due to the large systems of equations that must be solved, iterative solvers must be considered, especially in the case of the elecromagnetic DC and AC systems. However, the number of iterations required by these solvers to converge might become very large, especially in the case of ill-conditioned systems. Thus, in order to accelerate the convergence, these iterative methods must be used in conjunction with an efficient preconditioner.

Consider the solution to the system of equations $\mathbf{K q}=\mathbf{b}$. The main idea is to accelerate the speed of convergence of the iterative solver without substantially increasing the cost of each iteration [153]. An efficient preconditioner is one such that $\mathbf{P}^{-1}$ is a good approximation to $\mathbf{K}^{-1}$ but at the same time the application of $\mathbf{P}^{-1}$ to an arbitrary vector is cheap to compute [153]. Note that the last requirement is due to the fact that most iterative 


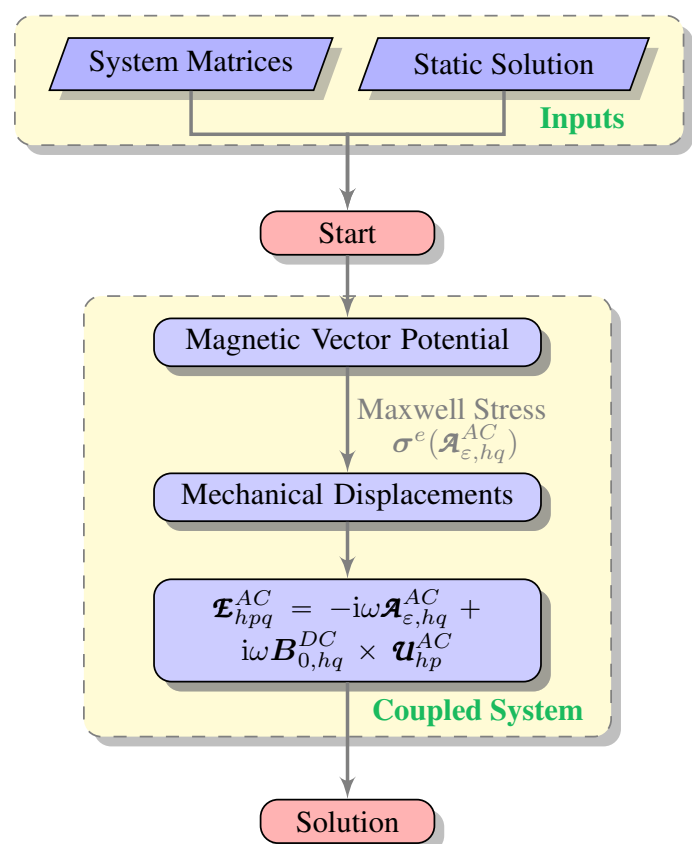

(a) Lagrangian. Staggered algorithm.

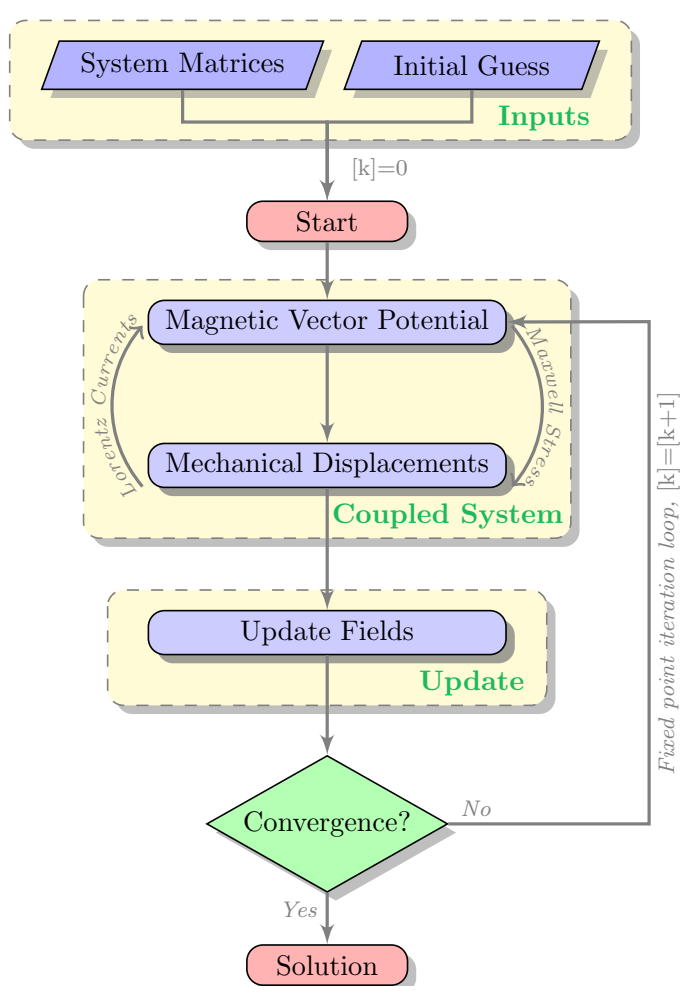

(b) Eulerian. Fixed Point algorithm.

Figure 4.10: Flow chart illustrating the different steps involved in the solver stage for the Lagrangian and Eulerian formulations. 
solvers require only the application of the matrix by vector product $\mathbf{K c}$ and the application of $\mathbf{P}^{-1} \mathbf{d}$ for vectors $\mathbf{c}$ and $\mathbf{d}$ provided during the iterative process. Furthermore, although the preconditioning technique can be loosely thought of applying an iterative solver to the preconditioned system $\mathbf{P}^{-1} \mathbf{K q}=\mathbf{P}^{-1} \mathbf{b}$ this is not how it is applied in practice, since even if $\mathbf{K}$ and $\mathbf{P}^{-1}$ are symmetric and positive definite, $\mathbf{P}^{-1} \mathbf{K}$ might not be symmetric and positive definite, which means that certain iterative solvers could no longer be applied. To overcome this issue, the preconditioning matrix is often chosen to be symmetric positive definite, such that the Cholesky decomposition $\mathbf{P}=\mathbf{E}^{T} \mathbf{E}$ holds and the equivalent system $\left(\mathbf{E}^{-T} \mathbf{K} \mathbf{E}^{-1}\right)(\mathbf{E q})=\mathbf{E}^{-T} \mathbf{b}$ is then solved, where $\left(\mathbf{E}^{-T} \mathbf{K} \mathbf{E}^{-1}\right)$ has the same properties (i.e. positive definiteness) as K [116]. Note, however, that in practice the algorithm for the iterative solver can be written in a way such that it only requires the solution to systems involving $\mathbf{P}^{-1}$, without ever computing $\mathbf{E}$ [116].

The range of preconditioners that can be applied goes from the simplest case of diagonal preconditioning, in which $\mathbf{P}=\operatorname{diag}(\mathbf{K})$, to more complex preconditioners such as incomplete LU factorization (ILU), incomplete Cholesky factorization, block Jacobi, successive over-relaxation (SOR) or algebraic multigrid preconditioners [153, 26]. Similarly, multiple choices are available for the iterative solver, including conjugate gradient (CG), Gauss-Seidel, biconjugate gradient (BiCG), minimal residual (MINRES) or generalized minimal residual methods (GMRES), among others. In this work, we will focus on the preconditioned conjugate gradient method (PCG) and the preconditioned generalized minimal residual method (PGMRES), where the preconditioner will be a block Jacobi preconditioner that exploits the explicit construction of our $H^{1}$ and $\boldsymbol{H}$ (curl) finite element spaces [113]. The application of PCG with a block Jacobi preconditioner to magnetostatic problems was considered in [156]. The same preconditioner was applied, in combination with other iterative solvers, to the solution of eddy current problems in [185]. Later, an improved preconditioner which exploits the explicit construction of the basis functions used in this work was developed by Ledger and Zaglmayr, and applied to eddy current problems using a preconditioned GMRES solver in [113]. Thus, in this work, the preconditioner proposed by Ledger and Zaglmayr for the solution of the magnetostatic (4.50) and eddy current (4.56) problems is considered, and the same idea is then followed to build an efficient preconditioner for the solution of the mechanical DC problem. A summary of the solution strategy for the different discrete systems is as follows:

- The matrix $\mathbf{K}_{\mathrm{AA}}^{\mathrm{DC}}+\mathbf{C}_{\mathrm{AA}}^{\mathrm{DC}}$ is real and positive semi-definite and is of the form obtained in magnetostatic problems discretised by the set of hierarchical basis functions presented in Section 4.3.3. For the solution of this system, a preconditioned conjugate gradient method (PCG) in combination with a block Jacobi preconditioner that exploits the low-order-edge, high-order-edge-face-interior splitting of the $\boldsymbol{H}$ (curl) basis functions becomes effective [158, 113, 185].

- The matrix $\mathbf{K}_{\mathrm{uu}}^{\mathrm{DC}}$ is real and positive definite and is of the form obtained in elasticity problems discretised by the $H^{1}$ basis functions presented in Section 4.3.2. For the solution of this system, a preconditioned conjugate gradient method (PCG) in 
combination with a block Jacobi preconditioner that exploits the low-order-vertex high-order-edge-face-interior splitting of the basis functions becomes effective.

- The matrix $\mathbf{K}_{\mathcal{A} \mathcal{A}}^{\mathrm{AC}}+\mathbf{C}_{\mathcal{A} \mathcal{A}}^{\mathrm{AC}}$ is complex and positive semi-definite and is of the form obtained in eddy current problems discretised by the $\boldsymbol{H}$ (curl) basis functions described in Section 4.3.3. Thus, for the solution of this system the preconditioned GMRES technique proposed by Ledger and Zaglmayr [113] is applied, where the preconditioner exploits the low-order-edge high-order-edge-face-interior splitting of the basis functions.

- The matrix $\mathbf{K}_{\mathcal{U} \mathcal{U}}^{\mathrm{AC}}-\omega^{2} \mathbf{M}_{\mathcal{U} \mathcal{U}}^{\mathrm{AC}}$ is real and indefinite and is of the form obtained in the discretisation of elastic wave propagation by $h p$-finite elements. For a sufficiently fine grid, an iterative solver could be applied for its solution, but, given its relatively small dimension compared to $\mathbf{K}_{\mathcal{A} \mathcal{A}}^{\mathrm{AC}}+\mathbf{C}_{\mathcal{A} \mathcal{A}}^{\mathrm{AC}}$, a direct solver is instead employed for the solution of this system. ${ }^{4}$

In order to illustrate the construction of the block Jacobi preconditioner let us focus on the eddy current problem (for the magnetostatic problem the preconditioner has a similar but simpler structure). The block Jacobi preconditioning matrix takes in this case the form

$$
\mathbf{P}_{\mathcal{A} \mathcal{A}}=\left[\begin{array}{ccc}
\mathbf{K}_{\mathcal{A} \mathcal{A}}^{\mathrm{AC}, \mathcal{N}_{0} \mathcal{N}_{0}}+\mathbf{C}_{\mathcal{A} \mathcal{A}}^{\mathrm{AC}, \mathcal{N}_{0} \mathcal{N}_{0}} & 0 & 0 \\
0 & \tilde{\mathbf{D}}_{\mathcal{A} \mathcal{A}}^{g g} & 0 \\
0 & 0 & \tilde{\mathbf{D}}_{\mathcal{A} \mathcal{A}}^{n n}
\end{array}\right]
$$

where $\mathcal{N}_{0}$ denotes the lowest order Nédélec space and the tilde in $\tilde{\mathbf{D}}_{\mathcal{A} \mathcal{A}}^{g g}, \tilde{\mathbf{D}}_{\mathcal{A} \mathcal{A}}^{n n}$ is used to denote that the blocks are block-diagonal. The $g g$ and $n n$ superscripts refer to the gradient and non-gradient basis functions, respectively. Furthermore, the blocks $\tilde{\mathbf{D}}_{\mathcal{A \mathcal { A }}}^{g g}, \tilde{\mathbf{D}}_{\mathcal{A \mathcal { A }}}^{n n}$ are further split into edge, face and interior contributions. Thus, the preconditioning matrix has the general form illustrated in Figure 4.11. Note that for the lowest order space the whole block is retained, while the high order space is split into gradients and nongradients and into edge, face and interior functions and only the block-diagonal contributions are retained. The lowest order block is solved directly in order to ensure that the preconditioner is robust with respect to $\varepsilon$. Furthremore, note that since the preconditioning matrix is block diagonal, its inverse is also computed block-diagonally. See [113] for further details.

The preconditioner used for the static elasticity problem has a similar, but simpler structure, given that the basis functions are not split into gradients and non-gradients in this case. Thus, the block Jacobi preconditioning matrix for the static elasticity problem takes the form

\footnotetext{
${ }^{4}$ Note also that the MRI configurations considered in this work, consist of several shields which are not connected between them. Thus, instead of solving one large mechanical system, several smaller linear systems are instead solved, one for each of the conductors, which results in a faster solver.
} 


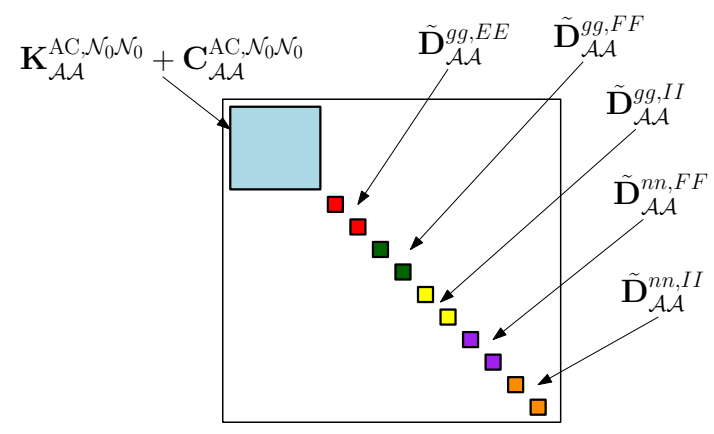

Figure 4.11: Block Jacobi preconditioner used for the solution of the eddy current problem. Illustration of the general structure of the preconditioning matrix, showing the explicit splitting where the whole block is retained for the lowest order space and only block diagonal contributions are considered for the high order terms, which are split into gradient edge, face and interior functions, $\tilde{\mathbf{D}}_{\mathcal{A} \mathcal{A}}^{g, E E}, \tilde{\mathbf{D}}_{\mathcal{A} \mathcal{A}}^{g g, F F}$ and $\tilde{\mathbf{D}}_{\mathcal{A} \mathcal{A}}^{g g, I I}$, respectively and non-gradient face $\tilde{\mathbf{D}}_{\mathcal{A} \mathcal{A}}^{n n, F F}$ and interior $\tilde{\mathbf{D}}_{\mathcal{A} \mathcal{A}}^{n n, I I}$ functions.

$$
\mathbf{P}_{\mathrm{uu}}=\left[\begin{array}{cccc}
\mathbf{K}_{\mathrm{uu}}^{\mathrm{vV}} & 0 & 0 & 0 \\
0 & \tilde{\mathbf{K}}_{\mathrm{uu}}^{\mathrm{EE}} & 0 & 0 \\
0 & 0 & \tilde{\mathbf{K}}_{\mathrm{uu}}^{\mathrm{FF}} & 0 \\
0 & 0 & 0 & \tilde{\mathbf{K}}_{\mathrm{uu}}^{\mathrm{II}}
\end{array}\right],
$$

where the whole block is retained for the lowest order space and only block-diagonal contributions are considered for the high order spaces, which are split into edge, face and interior functions.

\subsection{Mechanical Damping}

In practical MRI scanners, the conducting components are connected together and to other components by materials that absorb and dampen the vibrations. These effects are not taken into account in the mathematical model described previously and, in general, the amount of damping is difficult to quantify precisely. As a first approximation to damping effects, the simple case of mass proportional damping is considered. In this model, a damping block $\mathbf{C}_{\mathfrak{U} \mathfrak{U}}^{\mathrm{AC}}=\alpha_{M} \mathbf{M}_{\mathfrak{U} \mathcal{U}}^{\mathrm{AC}}$ is added to $\mathbf{K}_{\mathcal{U U}}^{\mathrm{AC}}-\omega^{2} \mathbf{M}_{\mathcal{U U}}^{\mathrm{AC}}$ in the system (4.57) so that it becomes $\mathbf{K}_{\mathcal{U U}}^{\mathrm{AC}}+\mathrm{i} \omega \alpha_{M} \mathbf{M}_{\mathcal{U} \mathcal{U}}^{\mathrm{AC}}-\omega^{2} \mathbf{M}_{\mathcal{U} \mathcal{U}}^{\mathrm{AC}}$, where $\alpha_{M}$ is the mass proportional damping coefficient $^{5}$, which can be determined from $[89,8]$

$$
\alpha_{M}=2 \omega \xi
$$

where $\xi$ is a dimensionless factor, called the damping ratio, which is used to attenuate the frequency $\omega$. Note that when adding mechanical damping the system matrix becomes complex but, since a direct solver is used for the AC elasticity stage, this does not affect the solution strategy described in Section 4.4. The damping ratio describes how the

\footnotetext{
${ }^{5}$ Note that from a dimensional analysis $\alpha_{M}$ can be shown to have units of rad/s. However, since $\alpha_{M}$ is not a physical parameter it is usually written without units [8, 97]
} 
amplitude of the oscillations in a system decay to an equilibrium position after a perturbation. According to the damping ratio, a system can be undamped $(\xi=0)$, underdamped $(\xi<1)$, critically damped $(\xi=1)$ and overdamped $(\xi>1)$. If the system is critically damped, it will decay to equilibrium without oscillation in the fastest admissible way. In an overdamped system, an equilibrium position will be reached without oscillation, but in a longer time than in the case of critical damping. If the system is underdamped, it will oscillate to reach equilibrium. The cases of an undamped and underdamped system will be considered in this thesis. Numerical results will be shown later to illustrate the effect of choosing different damping ratios. Note, however, that the development of accurate and realistic damping models is still a very challenging problem, which is out of the scope of this thesis. For a more detailed investigation on different damping models the reader is referred to $[183,8]$.

\subsection{Current Source Properties}

A MRI scanner consists of two sets of coils; the main coils that generate the strong DC field and the gradient coils that generate the low frequency AC field. Furthermore, the gradient coils can be divided in longitudinal and transversal gradient coils. The main coils and the $z$ longitudinal gradient coils are rotationally symmetric around the $z$ axis, both geometrically and regarding the current direction, which has only one component in the angular direction, this is

$$
\boldsymbol{J}^{D C}=J_{\phi}^{D C} \boldsymbol{e}_{\phi}, \quad \boldsymbol{g}^{A C}=g_{\phi}^{A C} \boldsymbol{e}_{\phi},
$$

where $\boldsymbol{e}_{r}, \boldsymbol{e}_{\phi}, \boldsymbol{e}_{z}$ denote the basis vectors in cylindrical coordinates.

On the other hand, the $x$ and $y$ transversal gradient coils have a more complex shape, which cannot be expressed in an axisymmetric setting. To illustrate this, the two main configurations that will be considered in this thesis are illustrated in Figure 4.12. The case of configurations considering only $z$ longitudinal gradient coils is illustrated in $4.12 \mathrm{a}$, while the case of a configuration considering $x$ gradient coils is illustrated in Figure $4.12 b^{6}$. It can be observed that in the first case the geometry is rotationally symmetric around the $z$ axis, while in the second case this is no longer true. Note that the coils shown in this figure represent a simplification of the complex wire pattern of a real gradient coil. The realistic wire patterns for $x, y$ and $z$ gradient coils are illustrated in Figure 4.13. The terms longitudinal and transversal refer to the orientation of the magnetic field gradient generated by these coils. In the case of the $z$ gradient coils, a gradient in the $z$ direction, the same direction as that of the DC field, is generated, while the $x$ and $y$ gradient coils generate a field gradient in the $x$ or $y$ directions, respectively, which are transversal to the direction of the DC field.

\footnotetext{
${ }^{6}$ The case of $y$ gradient coils can be obtained by rotating the $x$ gradient coils 90 degrees around the $z$ axis. Note that $y$ denotes tyically the vertical component, as depicted in Figure 4.13. Figure 4.12b has been rotated so that the vertical direction corresponds to $x$.
} 


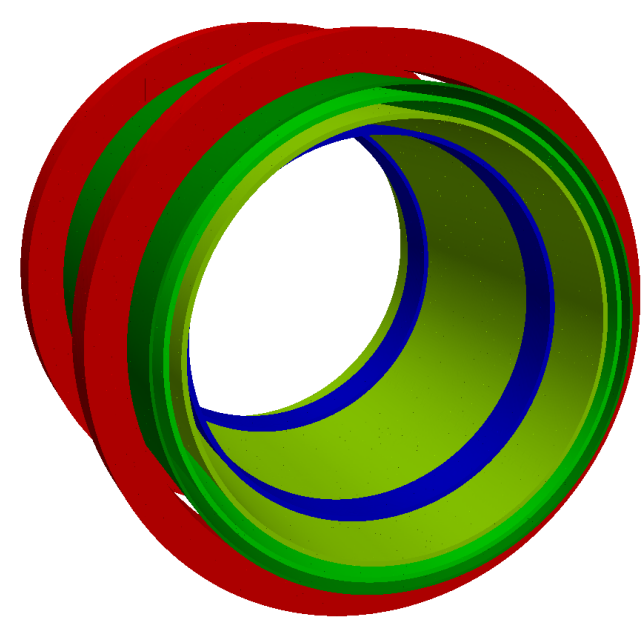

(a) MRI with longitudinal coils

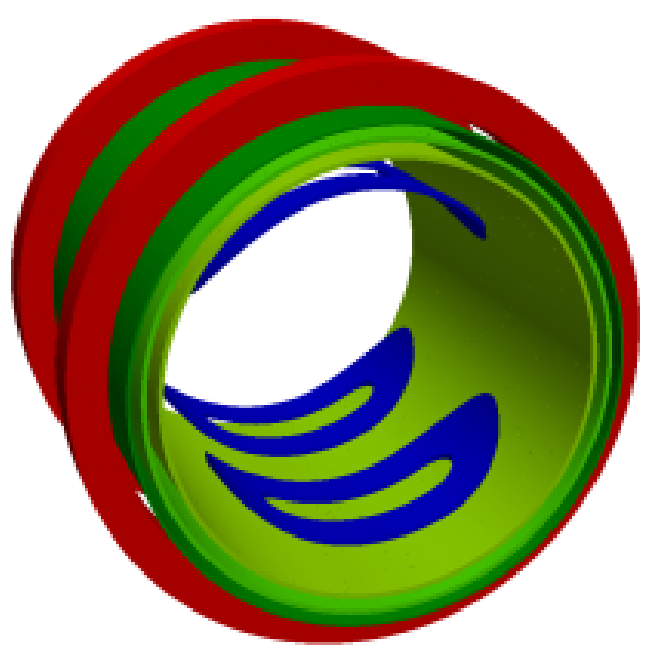

(b) MRI with transversal coils

Figure 4.12: Illustration of typical MRI configurations: (a) MRI configuration including longitudinal gradient coils and (b) MRI configuration including transversal gradient coils.

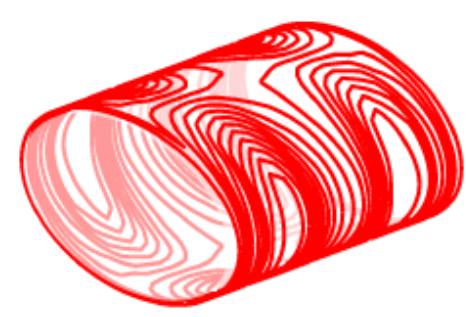

(a) $x$ gradient coil

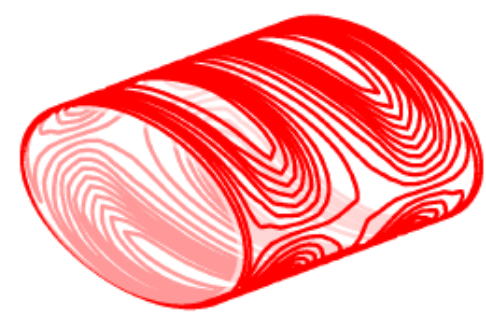

(b) $y$ gradient coil

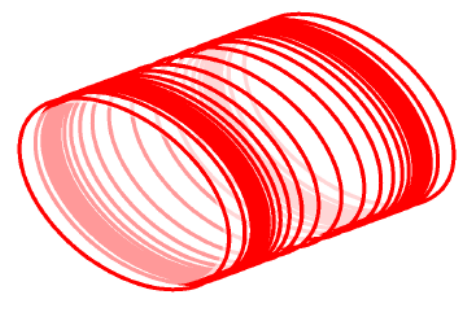

(c) $z$ gradient coil

Figure 4.13: Typical wire patterns of MRI transversal and longitudinal gradient coils [17]. 
Furthermore, a Biot-Savart description of the coils [80] is assumed, where the coils can be treated as non conductors, and thus supp $\left(\boldsymbol{J}^{D C}\right)$ and supp $\left(\boldsymbol{g}^{A C}\right)$ form part of $\Omega_{N C}$. Finally, there are two important conditions that the current source must satisfy; first, the current must be solenoidal, this is, $\operatorname{div} \boldsymbol{J}^{D C}=0$ and $\operatorname{div} \boldsymbol{g}^{A C}=0$, and second, the current must be tangential to the coil's surface, this is, $\boldsymbol{J}^{D C} \cdot \boldsymbol{n}=0$ and $\boldsymbol{g}^{A C} \cdot \boldsymbol{n}=0$.

\subsection{Current Source Mapping}

Clearly, MRI configurations including transversal gradient coils, represent a more challenging problem than configurations including only longitudinal gradient coils, as the latter case can be simulated using the much cheaper axisymmetric formulation, while the former require the use of a 3D formulation. Furthermore, another problem, related to the definition of the current source, arises in the case of transversal gradient coils, which is not present in the case of longitudinal coils. Even if a current source $\boldsymbol{g}^{A C}$ is specified as being solenoidal at a continuous level, this might not be true at a discrete level due to the numerical discretisation, especially in the case of coils with complex shapes, such as the transversal gradient coils showed in Figure 4.12b. If this issue is not addressed, the incompatibility of equation (4.56) leads to the appearance of singularities in the solution and problems with the convergence of the preconditioned GMRES solver in step 4 of Algorithm 1.

To address this issue, the approach proposed in [149] is considered, where the current source is expressed as the curl of a potential $T$, this is

$$
\boldsymbol{g}^{A C}=\operatorname{curl} \boldsymbol{T},
$$

and the next problem must then be solved to find the potential $\boldsymbol{T}$

$$
\begin{aligned}
\operatorname{curl}(\operatorname{curl} \boldsymbol{T}) & =\operatorname{curl} \boldsymbol{g}^{A C} & & \text { in } \Omega, \\
\operatorname{div} \boldsymbol{T} & =0 & & \text { in } \Omega, \\
\boldsymbol{n} \times \boldsymbol{T} & =\mathbf{0} & & \text { on } \partial \Omega .
\end{aligned}
$$

Note that this is equivalent to solving a magnetostatic type problem, except the solution $\boldsymbol{T}$ could possibly be complex valued due to the current source. If the problem was set on an unbounded domain, a decay condition for $\boldsymbol{T}$ of the kind $T=O\left(\frac{1}{|\boldsymbol{x}|}\right)$ as $\boldsymbol{x} \rightarrow \infty$ would hold. However, on a finite computational domain, this condition is approximated by applying the boundary condition $\boldsymbol{n} \times \boldsymbol{T}=\mathbf{0}$ on $\partial \Omega$ in a similar manner to that described in Section 4.2. Note that the condition $\boldsymbol{n} \times \operatorname{curl} \boldsymbol{T}=\mathbf{0}$ on $\partial \Omega$ could be equally applied, provided that the domain $\Omega$ is chosen to be sufficiently large.

To approximate this problem with the developed finite element software, its weak form must first be derived. For the derivation of the variational form, equation (4.64a) is multiplied by a test function $\delta \boldsymbol{T} \in X_{\Omega}:=$ $\left\{\boldsymbol{T} \in \boldsymbol{H}\left(\operatorname{curl}, \mathbb{R}^{3}\right): \operatorname{div} \boldsymbol{T}=0\right.$ in $\Omega, \boldsymbol{n} \times \boldsymbol{T}=0$ on $\left.\partial \Omega\right\}$ and, integrating over the domain,

$$
\int_{\Omega} \operatorname{curl}(\operatorname{curl} \boldsymbol{T}) \cdot \overline{\delta \boldsymbol{T}} \mathrm{d} \Omega=\int_{\Omega} \operatorname{curl} \boldsymbol{g}^{A C} \cdot \overline{\delta \boldsymbol{T}} \mathrm{d} \Omega,
$$


which can be rewritten as ${ }^{7}$

$$
\begin{aligned}
\int_{\Omega} \operatorname{div}(\operatorname{curl} \boldsymbol{T} \times \overline{\delta \boldsymbol{T}}) \mathrm{d} \Omega+\int_{\Omega} \operatorname{curl} \boldsymbol{T} \cdot \operatorname{curl} \overline{\delta \boldsymbol{T}} \mathrm{d} \Omega & =\int_{\Omega} \operatorname{div}\left(\boldsymbol{g}^{A C} \times \overline{\delta \boldsymbol{T}}\right) \mathrm{d} \Omega \\
& +\int_{\Omega} \boldsymbol{g}^{A C} \cdot \operatorname{curl} \overline{\delta \boldsymbol{T}} \mathrm{d} \Omega
\end{aligned}
$$

and applying the divergence theorem ${ }^{8}$

$$
\begin{aligned}
\int_{\Omega} \operatorname{curl} \boldsymbol{T} \cdot \operatorname{curl} \overline{\delta \boldsymbol{T}} \mathrm{d} \Omega & =\int_{\partial \Omega}\left(\boldsymbol{n} \times \boldsymbol{g}^{A C}\right) \cdot \overline{\delta \boldsymbol{T}} \mathrm{ds} \\
& -\int_{\partial \Omega} \operatorname{curl} \boldsymbol{T} \cdot(\boldsymbol{n} \times \overline{\delta \boldsymbol{T}}) \mathrm{ds}+\int_{\Omega} \boldsymbol{g}^{A C} \cdot \operatorname{curl} \overline{\delta \boldsymbol{T}} \mathrm{d} \Omega .
\end{aligned}
$$

Noting that the domain has been chosen such that $\partial \Omega$ is away from the support of $\boldsymbol{g}^{A C}$ and so $\boldsymbol{g}^{A C}=\mathbf{0}$ on $\partial \Omega$ and recalling that $\boldsymbol{n} \times \delta \boldsymbol{T}=\mathbf{0}$ on $\partial \Omega$ we arrive at

$$
\int_{\Omega} \operatorname{curl} \boldsymbol{T} \cdot \operatorname{curl} \overline{\delta \boldsymbol{T}} \mathrm{d} \Omega=\int_{\Omega} \boldsymbol{g}^{A C} \cdot \operatorname{curl} \overline{\delta \boldsymbol{T}} \mathrm{d} \Omega .
$$

Finally, in order to circumvent the Coulomb gauge condition (4.64b), the same approach as for the magnetostatic problem (3.33) is followed and a small regularisation term is added so that the weak form can be stated as: Find $\boldsymbol{T} \in \tilde{X}_{\Omega}(\mathbf{0})$ such that

$$
\int_{\Omega} \operatorname{curl} \boldsymbol{T} \cdot \operatorname{curl} \overline{\delta \boldsymbol{T}} \mathrm{d} \Omega+\varepsilon \int_{\Omega} \boldsymbol{T} \cdot \overline{\delta \boldsymbol{T}} \mathrm{d} \Omega=\int_{\Omega} \boldsymbol{g}^{A C} \cdot \operatorname{curl} \overline{\delta \boldsymbol{T}} \mathrm{d} \Omega
$$

for all $\delta \boldsymbol{T} \in \tilde{X}_{\Omega}(\mathbf{0})$, where the complex conjugate can be omitted if the weights are chosen to be real.

The variational form (4.69) is then discretised using the $\boldsymbol{H}$ (curl) basis functions described in Section 4.3.3 leading to a linear system of equations which is solved using a preconditioned conjugate gradient method. The discretisation process and the preconditioner are analogous to the case of the magnetostatic problem, and therefore are not repeated here. Once the potential $\boldsymbol{T}$ is known, the right hand side of equation (3.35a) can be rewritten as

$$
\mu_{0} \int_{\operatorname{supp}\left(\boldsymbol{g}^{A C}\right)} \boldsymbol{g}^{A C} \cdot \overline{\delta \boldsymbol{A}} \mathrm{d} \Omega=\mu_{0} \int_{\Omega} \operatorname{curl} \boldsymbol{T} \cdot \overline{\delta \boldsymbol{A}} \mathrm{d} \Omega,
$$

which, after applying integration by parts, can be rewritten as ${ }^{9}$

$$
\mu_{0} \int_{\operatorname{supp}(\boldsymbol{g} A C)} \boldsymbol{g}^{A C} \cdot \overline{\delta \boldsymbol{A}} \mathrm{d} \Omega=\mu_{0} \int_{\Omega} \operatorname{div}(\boldsymbol{T} \times \overline{\delta \boldsymbol{A}}) \mathrm{d} \Omega+\mu_{0} \int_{\Omega} \boldsymbol{T} \cdot \operatorname{curl} \overline{\delta \boldsymbol{A}} \mathrm{d} \Omega,
$$

and applying the divergence theorem ${ }^{10}$

$$
\mu_{0} \int_{\operatorname{supp}(\boldsymbol{g} A C)} \boldsymbol{g}^{A C} \cdot \overline{\delta \boldsymbol{A}} \mathrm{d} \Omega=\mu_{0} \int_{\partial \Omega}(\boldsymbol{n} \times \boldsymbol{T}) \cdot \overline{\delta \boldsymbol{A}} \mathrm{ds}+\mu_{0} \int_{\Omega} \boldsymbol{T} \cdot \operatorname{curl} \overline{\delta \boldsymbol{A}} \mathrm{d} \Omega .
$$

\footnotetext{
${ }^{7}$ Recall the vector relation $\operatorname{div}(\boldsymbol{a} \times \boldsymbol{b})=\boldsymbol{b} \cdot \operatorname{curl} \boldsymbol{a}-\boldsymbol{a} \cdot \operatorname{curl} \boldsymbol{b}$.

${ }^{8}$ Recall the vector relation $\boldsymbol{a} \cdot(\boldsymbol{b} \times \boldsymbol{c})=\boldsymbol{b} \cdot(\boldsymbol{c} \times \boldsymbol{a})$ and the relation $\boldsymbol{a} \times \boldsymbol{b}=-\boldsymbol{b} \times \boldsymbol{a}$.

${ }^{9}$ Recall the vector relation $\operatorname{div}(\boldsymbol{a} \times \boldsymbol{b})=\boldsymbol{b} \cdot \operatorname{curl} \boldsymbol{a}-\boldsymbol{a} \cdot \operatorname{curl} \boldsymbol{b}$.

${ }^{10}$ Recall the vector relation $(\boldsymbol{a} \times \boldsymbol{b}) \cdot \boldsymbol{c}=(\boldsymbol{c} \times \boldsymbol{a}) \cdot \boldsymbol{b}$.
} 


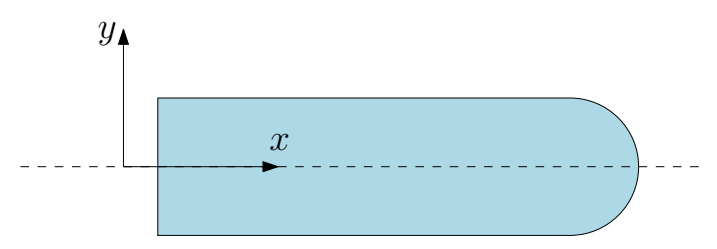

Figure 4.14: Illustration of a geometrically symmetric domain. The geometry is symmetric with respect to the dashed line ( $x$ axis).

Finally, considering the boundary condition $\boldsymbol{n} \times \boldsymbol{T}=\mathbf{0}$ on $\partial \Omega$ it follows that

$$
\mu_{0} \int_{\operatorname{supp}(\boldsymbol{g} A C)} \boldsymbol{g}^{A C} \cdot \overline{\delta \boldsymbol{A}} \mathrm{d} \Omega=\mu_{0} \int_{\Omega} \boldsymbol{T} \cdot \operatorname{curl} \overline{\delta \boldsymbol{A}} \mathrm{d} \Omega,
$$

which is substituted into equation (3.35a) in the case of problems including transversal gradient coils. The discretisation of this problem follows similar lines as those described in Section 4.3 and, therefore, it is not repeated here.

Note that the mapping of $\boldsymbol{g}^{A C}$ to the space of solenoidal functions ensures that equation (3.35a) is compatible and avoids issues with the convergence of the preconditioned iterative solver or the appearance of singularities in the solution. Furthermore, it is important to remark that if one only rewrites (3.35a) as stated in equation (4.70), this does not overcome the issue, and it must be instead written as stated in (4.73). The difference between (4.70) and (4.73) is that in (4.70) the source $\boldsymbol{g}^{A C}$ is projected on the space $X_{\text {reg }}^{A C}$ of $\boldsymbol{H}$ (curl) basis, while in (4.73) $\boldsymbol{g}^{A C}$ is projected on the curl of $X_{\text {reg }}^{A C}$, ensuring that $\operatorname{div} \boldsymbol{g}^{A C}=0$ according to the de Rham sequence $[113,37]$. The same principle holds for the computation of $T$ itself.

\subsection{Symmetry Conditions}

In the context of numerical simulations in engineering, the use of symmetries is typically exploited in order to reduce the computational cost of the simulations. First, a distinction should be made between geometrical and physical symmetries. In order to understand this concept, let us consider the simple domain illustrated in Figure 4.14. The domain is geometrically symmetric with respect to the $x$ axis $(y=0)$, as the half domain with $y>0$ can be obtained as a reflection of the half domain with $y<0$ and viceversa. Other type of geometrical symmetry is the rotational symmetry, where the domain can be obtained by rotating a certain plane around a certain axis. This type of symmetry is illustrated in Figure 4.15 , where the 3D geometry can be obtained by rotating the highlighted plane $\phi$ degrees around the $z$ axis. This is the case of the MRI configuration shown in Figure 4.12a. On the other hand, the physical symmetries are related to a given physical magnitude. For example, consider the deformation of a long filament, as illustrated in Figure 4.16. The deformation showed in Figure 4.16a is said to be symmetric with respect to the point $\mathrm{C}$, while the deformation showed in Figure $4.16 \mathrm{~b}$ is said to be antisymmetric.

The existence of geometrical and physical symmetries can be exploited to reduce the cost of the simulations by cutting the domain at the symmetry planes and adding certain 


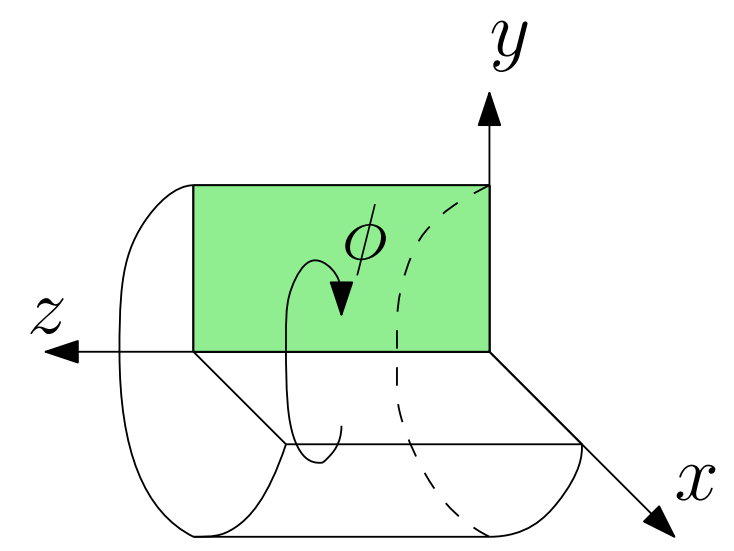

Figure 4.15: Illustration of a rotationally symmetric (axisymmetric) domain. The geometry is symmetric with respect to the $z$ axis.

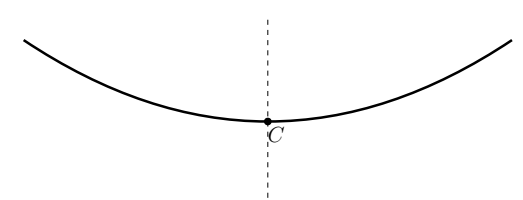

(a) Symmetric

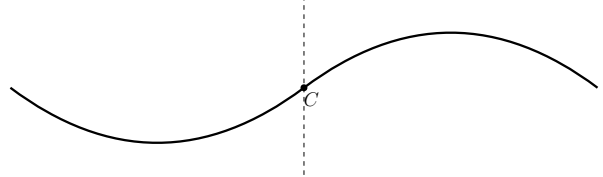

(b) Antisymmetric

Figure 4.16: Illustration of physical symmetries; (a) Symmetric distortion and (b) Antisymmetric distortion.

boundary conditions on the normal or tangential components of the unknown physical fields. The MRI configurations that will be considered in this work can be divided in two types: configurations considering longitudinal coils (Figure 4.12a) and configurations considering transversal coils (Figure 4.12b). In the first case, only the longitudinal $z$ gradient coils are considered and all the non-rotationally symmetric components are neglected, rendering an axisymmetric configuration which will be used to validate the new 3D $h p$-FEM software by performing comparisons against the previously validated axisymmetric software developed in $[19,17]$. In the second case, the longitudinal coils are replaced by transversal $x$ or $y$ gradient coils, thus the rotational symmetry is lost, and a 3D computation is required. However, even in the case of considering transversal coils, certain symmetries are still present that can be used to reduce the computational cost of the simulations. More specifically, such configurations can be reduced to $1 / 8$ of the original size by exploiting the symmetries or anti-symmetries with respect to the Cartesian $x=0, y=0$ and $z=0$ planes. The boundary conditions that must be imposed on the solution fields at each symmetry plane for the DC problem as well as for the AC problem and the different gradient coils are stated in Table 4.1, which have been derived based on the symmetry conditions presented in $[92,186]$. For a review on the application of symmetry conditions in electromagnetics see [92]. Similarly, for a review on the application of symmetry conditions in mechanics see [186].

Similarly, the boundary conditions that must be imposed on the potential $\boldsymbol{T}$ when mapping the current source to the space of solenoidal functions are stated in Table 4.2 for each of 


\begin{tabular}{|c|c|c|c|c|}
\hline & $x$ gradient coil & $y$ gradient coil & $z$ gradient coil & DC field \\
\hline$x=0$ & $\begin{array}{c}\left(\operatorname{curl} \boldsymbol{A}_{\varepsilon, h q}^{A C}\right) \times \boldsymbol{n}=\mathbf{0} \\
\boldsymbol{u}_{h p}^{A C} \times \boldsymbol{n}=\mathbf{0} \\
\left(\boldsymbol{\sigma}^{m}\left(\boldsymbol{u}_{h p}^{A C}\right) \boldsymbol{n}\right) \cdot \boldsymbol{n}=0\end{array}$ & $\begin{array}{c}\boldsymbol{A}_{\varepsilon, h q}^{A C} \times \boldsymbol{n}=\mathbf{0} \\
\boldsymbol{u}_{h p}^{A C} \cdot \boldsymbol{n}=0 \\
\left(\boldsymbol{\sigma}^{m}\left(\boldsymbol{u}_{h p}^{A C}\right) \boldsymbol{n}\right) \times \boldsymbol{n}=\mathbf{0}\end{array}$ & $\begin{array}{c}\boldsymbol{A}_{\varepsilon, h q}^{A C} \times \boldsymbol{n}=\mathbf{0} \\
\boldsymbol{u}_{h p}^{A C} \cdot \boldsymbol{n}=0 \\
\left(\boldsymbol{\sigma}^{m}\left(\boldsymbol{u}_{h p}^{A C}\right) \boldsymbol{n}\right) \times \boldsymbol{n}=\mathbf{0}\end{array}$ & $\begin{array}{c}\boldsymbol{A}_{\varepsilon, h q}^{D C} \times \boldsymbol{n}=\mathbf{0} \\
\boldsymbol{u}_{h p}^{D C} \cdot \boldsymbol{n}=0 \\
\left(\boldsymbol{\sigma}^{m}\left(\boldsymbol{u}_{h p}^{D C}\right) \boldsymbol{n}\right) \times \boldsymbol{n}=\mathbf{0}\end{array}$ \\
\hline$y=0$ & $\begin{array}{c}\boldsymbol{A}_{\varepsilon, h q}^{A C} \times \boldsymbol{n}=\mathbf{0} \\
\boldsymbol{u}_{h p}^{A C} \cdot \boldsymbol{n}=0 \\
\left(\boldsymbol{\sigma}^{m}\left(\boldsymbol{u}_{h p}^{A C}\right) \boldsymbol{n}\right) \times \boldsymbol{n}=\mathbf{0}\end{array}$ & $\begin{array}{c}\left(\operatorname{curl} \boldsymbol{A}_{\varepsilon, h q}^{A C}\right) \times \boldsymbol{n}=\mathbf{0} \\
\boldsymbol{u}_{h p}^{A C} \times \boldsymbol{n}=\mathbf{0} \\
\left(\boldsymbol{\sigma}^{m}\left(\boldsymbol{u}_{h p}^{A C}\right) \boldsymbol{n}\right) \cdot \boldsymbol{n}=0\end{array}$ & $\begin{array}{c}\boldsymbol{A}_{\varepsilon, h q}^{A C} \times \boldsymbol{n}=\mathbf{0} \\
\boldsymbol{u}_{h p}^{A C} \cdot \boldsymbol{n}=0 \\
\left(\boldsymbol{\sigma}^{m}\left(\boldsymbol{u}_{h p}^{A C}\right) \boldsymbol{n}\right) \times \boldsymbol{n}=\mathbf{0}\end{array}$ & $\begin{array}{c}\boldsymbol{A}_{\varepsilon, h q}^{D C} \times \boldsymbol{n}=\mathbf{0} \\
\boldsymbol{u}_{h p}^{D C} \cdot \boldsymbol{n}=0 \\
\left(\boldsymbol{\sigma}^{m}\left(\begin{array}{c}\boldsymbol{u}_{h p}^{D C} \\
) \boldsymbol{n}\end{array}\right) \times \boldsymbol{n}=\mathbf{0}\right.\end{array}$ \\
\hline$z=0$ & $\begin{array}{c}\left(\operatorname{curl} \boldsymbol{A}_{\varepsilon, h q}^{A C}\right) \times \boldsymbol{n}=\mathbf{0} \\
\boldsymbol{u}_{h p}^{A C} \cdot \boldsymbol{n}=0 \\
\left(\boldsymbol{\sigma}^{m}\left(\boldsymbol{u}_{h p}^{A C}\right) \boldsymbol{n}\right) \times \boldsymbol{n}=\mathbf{0}\end{array}$ & $\begin{array}{c}\left(\operatorname{curl} \boldsymbol{A}_{\varepsilon, h q}^{A C}\right) \times \boldsymbol{n}=\mathbf{0} \\
\boldsymbol{u}_{h p}^{A C} \cdot \boldsymbol{n}=0 \\
\left(\boldsymbol{\sigma}^{m}\left(\boldsymbol{u}_{h p}^{A C}\right) \boldsymbol{n}\right) \times \boldsymbol{n}=\mathbf{0}\end{array}$ & $\begin{array}{c}\boldsymbol{A}_{\varepsilon, h q}^{A C} \times \boldsymbol{n}=\mathbf{0} \\
\boldsymbol{u}_{h p}^{A C} \times \boldsymbol{n}=\mathbf{0} \\
\left.\boldsymbol{\sigma}^{m}\left(\boldsymbol{u}_{h p}^{A C}\right) \boldsymbol{n}\right) \cdot \boldsymbol{n}=0\end{array}$ & 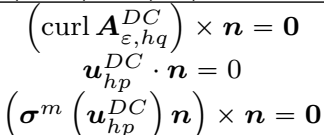 \\
\hline
\end{tabular}

Table 4.1: Symmetry conditions for $\boldsymbol{A}_{\varepsilon, h q}^{D C}, \boldsymbol{u}_{h p}^{D C}, \boldsymbol{A}_{\varepsilon, h q}^{A C}$ and $\boldsymbol{u}_{h p}^{A C}$ at the planes $x=0, y=0$ and $z=0$.

the coil configurations. Note that this mapping was observed to be needed only for the $x$ and $y$ gradient coils. However, for completeness, the symmetry conditions are stated for all the coil configurations.

\begin{tabular}{|c|c|c|c|c|}
\hline & $x$ gradient coil & $y$ gradient coil & $z$ gradient coil & Main coils \\
\hline$x=0$ & $\boldsymbol{T} \times \boldsymbol{n}=\mathbf{0}$ & $(\operatorname{curl} \boldsymbol{T}) \times \boldsymbol{n}=\mathbf{0}$ & $(\operatorname{curl} \boldsymbol{T}) \times \boldsymbol{n}=\mathbf{0}$ & $(\operatorname{curl} \boldsymbol{T}) \times \boldsymbol{n}=\mathbf{0}$ \\
\hline$y=0$ & $(\operatorname{curl} \boldsymbol{T}) \times \boldsymbol{n}=\mathbf{0}$ & $\boldsymbol{T} \times \boldsymbol{n}=\mathbf{0}$ & $(\operatorname{curl} \boldsymbol{T}) \times \boldsymbol{n}=\mathbf{0}$ & $(\operatorname{curl} \boldsymbol{T}) \times \boldsymbol{n}=\mathbf{0}$ \\
\hline$z=0$ & $\boldsymbol{T} \times \boldsymbol{n}=\mathbf{0}$ & $\boldsymbol{T} \times \boldsymbol{n}=\mathbf{0}$ & $(\operatorname{curl} \boldsymbol{T}) \times \boldsymbol{n}=\mathbf{0}$ & $\boldsymbol{T} \times \boldsymbol{n}=\mathbf{0}$ \\
\hline
\end{tabular}

Table 4.2: Symmetry conditions for $\boldsymbol{T}$ at the planes $x=0, y=0$ and $z=0$.

Note that in the case of configurations considering only $z$ gradient coils, the 3D computational domain could be reduced as much as desired, up to the limit of the $2 \mathrm{D}$ axisymmetric plane. For this, the domain can be cut at any two planes with a constant $\phi$ (being $r, \phi, z$ the cylindrical coordinates) and in these planes the same symmetry conditions as in the planes $x=0$ and $y=0$ should be applied. However, for the purpose of an easier implementation and since axisymmetric configurations will only be used in this thesis for the purpose of validation, only symmetries with respect to the Cartesian planes will be considered.

\subsection{Global Software Structure}

To conclude the description of the $h p$-FEM software, a global overview of the software structure is provided and the different steps that must be performed in order to arrive at the desired outputs of interest are summarised. For this purpose, a schematic representation of the software structure is provided in Figure 4.17.

The software can be divided into three main stages: pre-processing, solver and postprocessing. The main steps performed at each of these stages are briefly outlined in the following.

\subsubsection{Pre-processing Stage}

The main steps to be performed at the pre-processing stage are: 


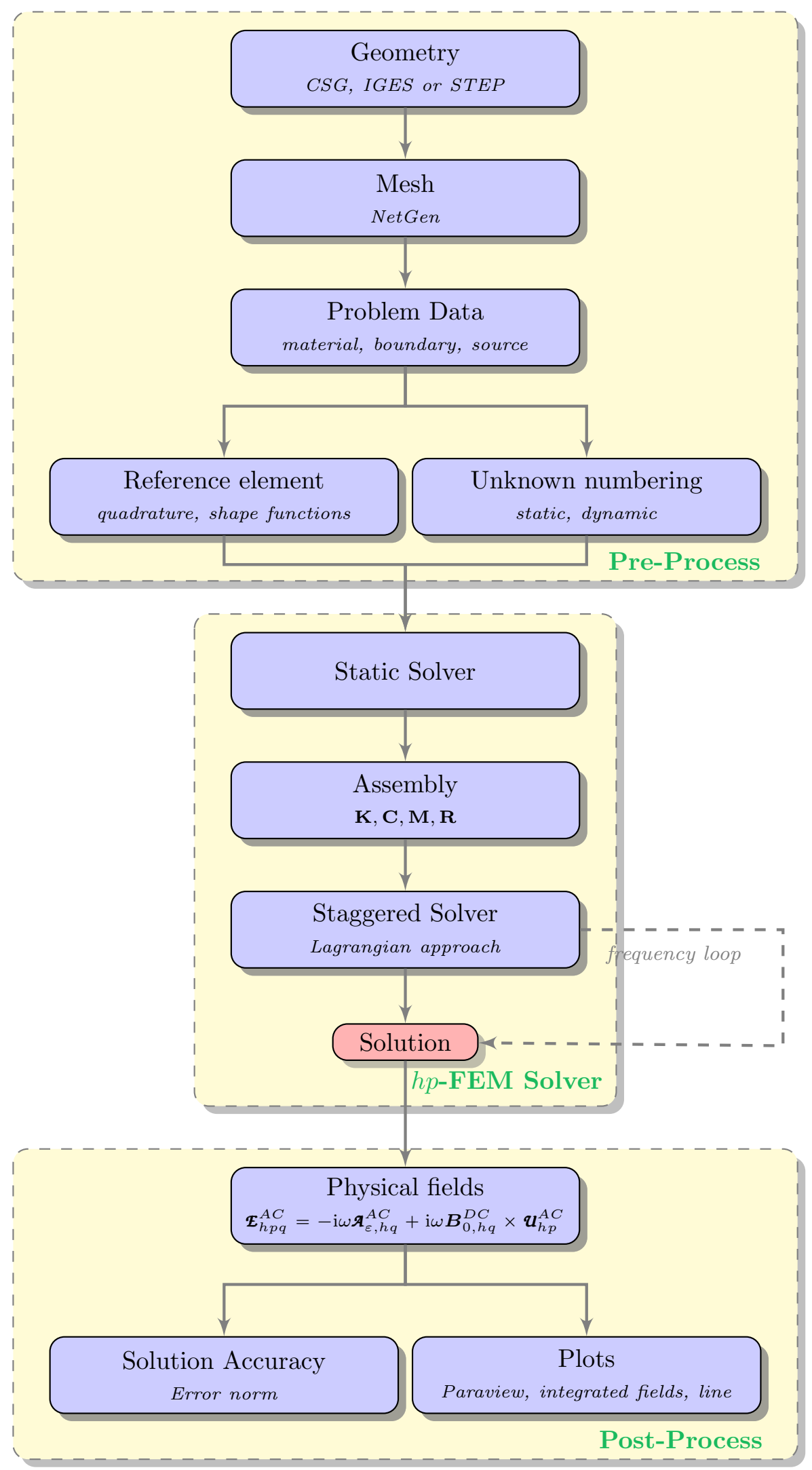

Figure 4.17: Schematic representation of the software structure, including the preprocessing, solver and post-processing stages. 
- Geometry (CAD generation): First a CAD model of the problem geometry must be generated. For very simple geometries, the native NetGen [155] CAD format, named CSG (Constructive Solid Geometry) can be used. This allows the generation of simple geometries by performing basic boolean operations. However, when the complexity of the geometry increases, more sophisticated CAD formats such as IGES (Initial Graphics Exchange Specification) or STEP (Standard for the Exchange of Product Model Data) must be considered. Many commercial and opensource packages are available for the generation of such CAD formats, and in this work AutoCAD [2] and AutoCAD Mechanical [3] have been used.

- Mesh: Once the CAD geometry is available, a mesh must be generated. For this purpose, the open source software NetGen [155] is considered, which allows the generation of complex unstructured tetrahedral meshes. NetGen has the capability of generating linear as well as quadratic elements and allows the local refinement of the mesh. For further details about the geometry and mesh generation the reader is referred to [155] and to Appendix B.

- Problem data definition: Once the mesh is created, it is read into the finite element software and the problem is defined by assigning the material properties, source terms and boundary conditions to each sub-domain or boundary surface.

- Reference element and unknown numbering: The final steps of the preprocessing stage consist in defining the Gauss points (see Appendix C) and the shape functions at the reference element and creating the numbering of degrees of freedom for the DC and AC problems described in Sections 4.3.5 and 4.3.5, respectively.

\subsubsection{Solver Stage}

The main steps to be performed at the solver stage are:

- DC Assembly: The system matrices for the DC problem must first be built by assembling elemental contributions, as described in Section 4.3.5.

- Static solver: The DC problem must then be solved. This step includes the solution of the magnetostatic (4.50) and static elasticity (4.51) problems, which correspond to steps 1 and 2 of Algorithm 1.

- Assembly: In this step, the matrices of the AC system are assembled from elemental contributions as described in Section 4.3.5. Note that the assembled matrices are such that they do not depend on the frequency, and the global system matrices are then computed, for each frequency, by multiplying the assembled matrices by frequency dependent parameters and adding them together. This leads to a much faster computation of the solution for multiple frequencies, as it is not necessary to assemble the system each time the frequency is modified. 
- Staggered solver: The last step of the solver stage is to compute the solution of the AC electromagnetic (4.56) and mechanical (4.57) systems for all the required frequencies. For this, steps 4 and 5 of Algorithm 1 are applied repeatedly for each frequency.

\subsubsection{Post-processing Stage}

The main steps to be performed at the post-processing stage can be divided in two groups:

- Error measure: If the problem under consideration has a known analytical solution, the accuracy of the finite element solution will be benchmarked by computing the appropriate error norms.

- Plots: Finally, the main goal is to produce plots of the outputs of interest that help us understand the solution to the coupled problem. These include the evolution of integrated quantities such as dissipated power or kinetic energy (defined in next chapter, see equation (5.6)) with the frequency, the eddy current, magnetic field or displacement patterns in the radiation shields for selected frequencies or the variation of these magnitudes along a certain line. For the generation of high quality contour plots when computing the solution in coarse meshes using high order elements, the reference element is uniformly subdivided into smaller elements and these are then mapped to the physical domain. The solution in the finer mesh is then exported to Paraview to plot. On the other hand, if the interest lies in the computation of integrated quantities such as dissipated power or kinetic energy, an integral over the domain must be performed and, for this, the developed finite element methodology is used and the integrals are computed using Gauss quadrature as explained in Appendix C, where the solution coefficients $\mathcal{A}_{\varepsilon}^{\mathrm{AC}}, \mathcal{U}^{\mathrm{AC}}$ and the basis functions $\boldsymbol{N}^{a}, L^{b}$ are used to compute the solution at each Gauss point.

\subsection{Chapter Summary}

This chapter has described the $h p$-FEM methodology used to discretise the coupled magneto-mechanical problem of interest and compute a numerical approximation to its solution.

The main novelty of the chapter is the development of a novel $h p$-FEM methodology for the solution of the linearised and regularised magneto-mechanical problem in a Lagrangian frame, which uses a combination of hierarchical $\boldsymbol{H}$ (curl) and $H^{1}$ basis functions and iterative solvers in combination with efficient preconditioners for the solution of the resulting linear systems. Furthermore, a staggered algorithm for the solution of the problem was proposed, the complete set of symmetry conditions to be applied for the different possible configurations was stated and the methodology proposed in [149] to map the current source to the space of solenoidal functions was applied here in combination with high order finite elements. 
The chapter started with the introduction of the truncated domain and the discussion of the boundary conditions to be imposed at the truncation boundary. Then, in Section 4.3 the reference element, the sets of hierarchical $\boldsymbol{H}$ (curl) and $H^{1}$ basis functions used for the discretisation of $\boldsymbol{A}_{\varepsilon}^{A C}$ and $\boldsymbol{U}^{A C}$ and the mapping between physical and reference elements were presented, followed by a brief description of the reduced basis gauging strategy and the rigorous derivation of the the different blocks of the discrete DC and AC systems. Section 4.4 presented the algorithm that is used to obtain the final solution and outlines the computational advantages of the Lagrangian approach over an Eulerian approach. Then, in Section 4.5, the use of preconditioned iterative solvers was discussed and an efficient solver was proposed for each linear system. Section 4.6 presents the approach followed to include a first approximation to the physical damping present in real MRI configurations. The properties of the current sources were discussed in Section 4.7. Next, in Section 4.8 a methodology to ensure that the current source $\boldsymbol{g}^{A C}$ is solenoidal at a discrete level was proposed, avoiding issues with the convergence of the iterative solver and the appearance of singularities in the solution. Then, in Section 4.9, the different symmetries that can be exploited to reduce the computational cost of the simulations were discussed and the symmetry conditions to be imposed at each boundary for the different possible configurations were stated. Finally, in Section 4.10, the global structure of the finite element software was described and the main steps to be performed at the pre-processing, solver and post-processing stages were outlined. 


\section{Chapter 5}

\section{Numerical Results for the Full Order Problem}

\subsection{Introductory Remarks}

This Chapter presents a series of academic and industrially relevant problems in order to benchmark and illustrate the predictive capability of the proposed 3D $h p$-FEM methodology. The chapter is based on an extended version of the work presented by the author in [158].

The main novelties of this chapter are as follows. First, the developed $h p$-FEM methodology is applied to a series of decoupled or single physics problems with a known analytical solution, and the error in the numerical solution is measured in order to verify that the appropriate rates of convergence given by the estimates (4.4)-(4.7) are obtained. Four different decoupled problems will be considered, which correspond to the DC electromagnetic (magnetostatic), DC elasticity, AC electromagnetic (eddy current) and AC elasticty stages. Then, the $h p$-FEM methodology is applied to a series of coupled magneto-mechanical problems in order to validate and illustrate the predictive capability of the proposed coupling methodology. These include an academic problem consisting in a conducting sphere excited by a uniform time harmonic magnetic field, and industrially relevant MRI configurations including longitudinal as well as transversal gradient coils. Comparisons against the previously validated axisymmetric software $[19,17]$, the commercial software NACS [4] and experimental data will be provided.

The structure of the chapter is as follows; First, in Section 5.2, a series of decoupled problems corresponding to the magnetostatic (Section 5.2.1), DC elasticity (Section 5.2.2), eddy current (Section 5.2.3) and AC elasticity (Section 5.2.4) stages are presented. The accuracy of the solution and the efficiency of the proposed solver will be benchmarked. Then, in Section 5.3 a series of magneto-mechanical coupled problems will be presented, which include a conducting and elastic sphere in a uniform alternating magnetic field (Section 5.3.1) and different MRI configurations including longitudinal and transversal gradient coils (Sections 5.3.2, 5.3.3 and 5.3.4). The chapter finishes with concluding remarks in Section 5.4 


\subsection{Decoupled Physics}

A series of examples are presented to illustrate the performance of the DC and AC stages of the finite element scheme for decoupled physics problems. In order to measure the accuracy of the solution and ensure the appropriate rates of convergence are obtained, the $H^{1}$ and $\boldsymbol{H}$ (curl) norms of the error defined in (4.6) will be used.

\subsubsection{Stage, Magnetostatics: Permeable Sphere in a Uniform Static Magnetic Field}

In order to benchmark the magnetostatic stage, a permeable sphere excited by a uniform static magnetic field is considered. An analytical expression for the solution of this problem is available in [164]. A permeable sphere $\Omega_{C}=\left\{\boldsymbol{x}:|\boldsymbol{x}|^{2} \leqslant R^{2}\right\}$ of radius $R=1 \mathrm{~cm}$ and permeability $\mu=1.5 \mu_{0}$ is placed in the region excited by a uniform magnetic field $\boldsymbol{H}_{0}^{D C}=\mu_{0} \boldsymbol{e}_{z}$ (Figure 5.1). The problem is then simulated by solving a simplified version of (3.1a)-(3.1d) with $\boldsymbol{J}^{D C}=\mathbf{0}$ on a truncated domain where $\partial \Omega$ is the surface of a sphere of radius $2 \mathrm{~cm}$. The Dirichlet boundary condition $\boldsymbol{n} \times \boldsymbol{A}_{\varepsilon, h q}^{D C}=\boldsymbol{n} \times \boldsymbol{A}_{\text {exact }}^{D C}$, where $\boldsymbol{A}_{\text {exact }}^{D C}$ is the analytical solution for this problem, is imposed on $\partial \Omega$.

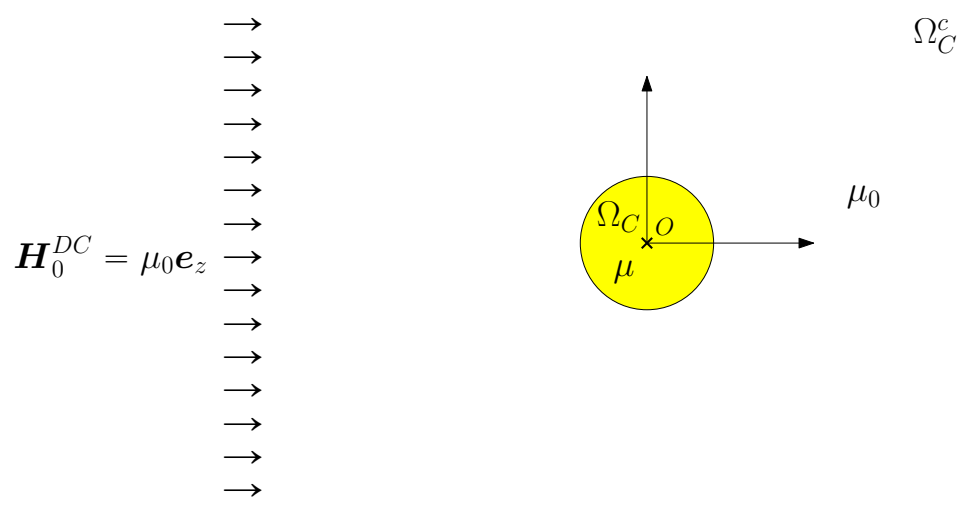

Figure 5.1: Permeable sphere in a uniform static magnetic field: problem setup.

First, the convergence of the preconditioned conjugate gradient method, and the efficiency of the preconditioner was studied. For this, the number of iterations required by the iterative solver to converge with and without preconditioner was measured using two different meshes of 1083 and 6654 elements and polynomial orders $q=0,1,2,3,4$. The regularisation parameter $\varepsilon$ and the tolerance of the iterative solver were set to $10^{-5}$, which means that the iteration will stop when the relative residual is smaller than $10^{-5}$. The result is shown in Figure 5.2, where each line shows the convergence behaviour of the relative residual computed at each iteration for a discretisation using a fixed polynomial degree. It can be observed that the use of the block Jacobi preconditioner described in Section 4.5 results in a massive reduction in iterations, and a monotonic reduction in the relative residual with an algebraic rate of convergence is obtained for the preconditioned system. The increase in iterations for increasing polynomial order is small in the case of PCG, while in the case of $\mathrm{CG}$ the number of iterations increases exponentially with the polynomial 
order. Note that in the case of CG with a mesh of 6654 elements and polynomial order $q=4$ the solution has not converged yet after $5 \times 10^{5}$ iterations, which is the prescribed maximum number of iterations. On the other hand, when using PCG with the same mesh and polynomial order, the solution converges in less than 60 iterations. Finally, to further illustrate the efficiency of the preconditioner, in Table 5.1 the solver time for the different meshes and polynomial orders using CG and PCG is shown. It can be observed that a massive speed-up is obtained by applying the block Jacobi preconditioner, especially as the size of the mesh increases. These are wall-clock times and were computed using a desktop computer with a processor Intel Xeon CPU E5-2687W v2 at $3.4 \mathrm{GHz}$ and 16 cores, and with a RAM of $128 \mathrm{~GB}$. Note that these times include also the construction of the preconditioner. Unless otherwise stated, all the time measurements shown in this Chapter were computed using these settings.

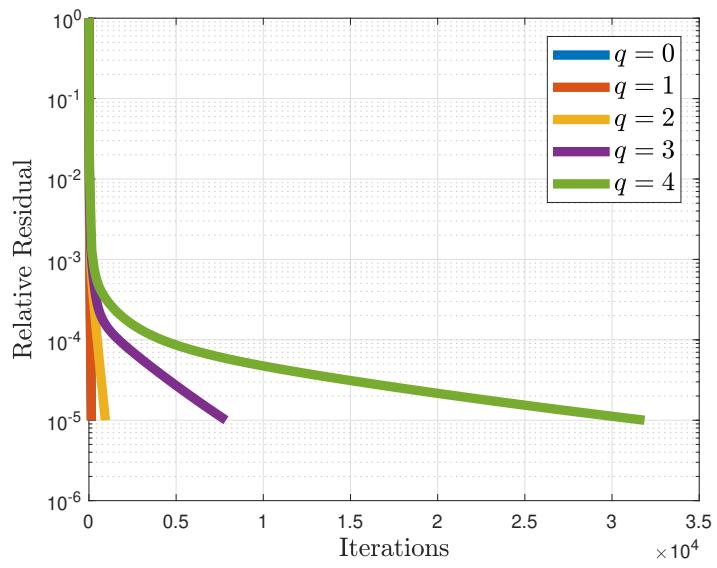

(a) CG, 1083 elements

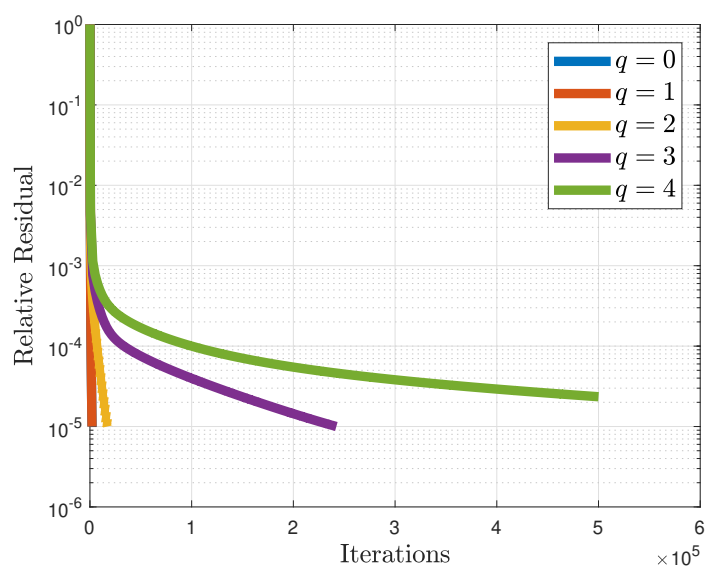

(c) CG, 6654 elements

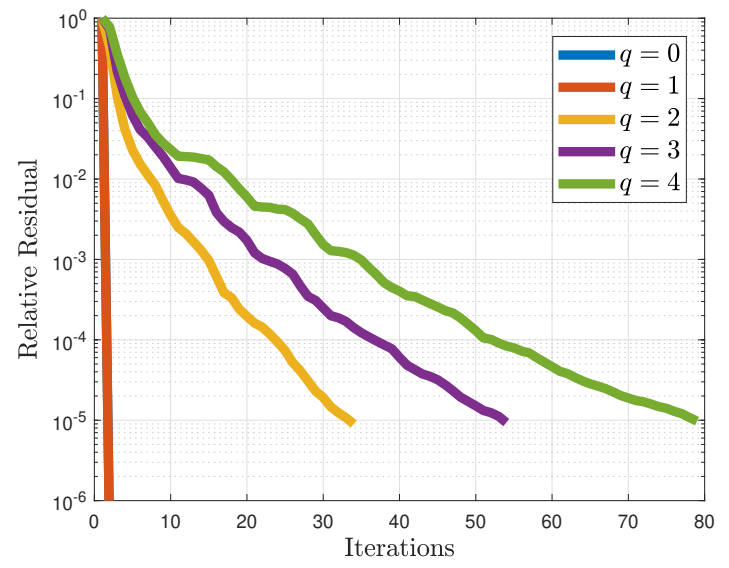

(b) PCG, 1083 elements

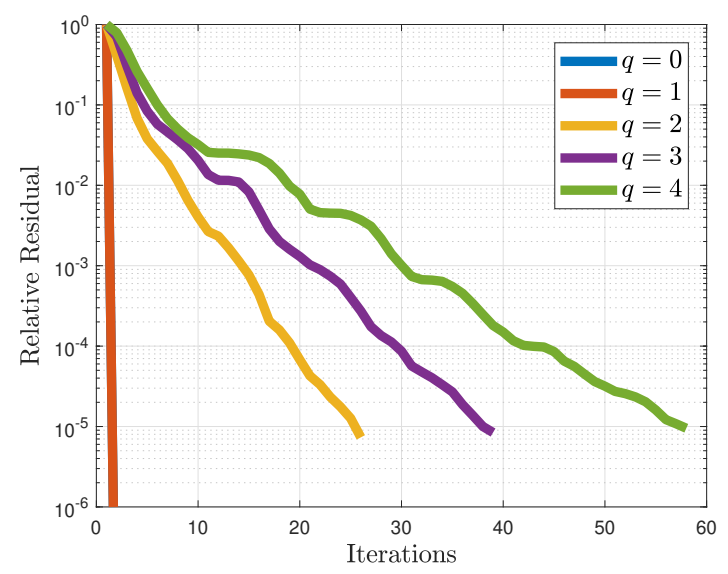

(d) PCG, 6654 elements

Figure 5.2: Permeable sphere in a uniform static magnetic field: Convergence of the CG method for: (a) A mesh of 1083 elements without preconditioner, (b) A mesh of 1083 elements using preconditioner, (c) A mesh of 6654 elements without preconditioner and (d) A mesh of 6654 elements using preconditioner. 


\begin{tabular}{|c|c|c|c|c|c|}
\hline & $q=0$ & $q=1$ & $q=2$ & $q=3$ & $q=4$ \\
\hline CG, 1083 elements & $0.04 \mathrm{~s}$ & $0.04 \mathrm{~s}$ & $0.39 \mathrm{~s}$ & $19.12 \mathrm{~s}$ & $154.43 \mathrm{~s}$ \\
\hline PCG, 1083 elements & $0.01 \mathrm{~s}$ & $0.01 \mathrm{~s}$ & $0.09 \mathrm{~s}$ & $0.53 \mathrm{~s}$ & $1.42 \mathrm{~s}$ \\
\hline CG, 6 654 elements & $1.62 \mathrm{~s}$ & $1.56 \mathrm{~s}$ & $30.45 \mathrm{~s}$ & $2025.81 \mathrm{~s}$ & $10962.10 \mathrm{~s}$ \\
\hline PCG, 6654 elements & $0.28 \mathrm{~s}$ & $0.28 \mathrm{~s}$ & $0.75 \mathrm{~s}$ & $2.46 \mathrm{~s}$ & $7.52 \mathrm{~s}$ \\
\hline
\end{tabular}

Table 5.1: Permeable sphere in a uniform static magnetic field: Solver time in seconds for CG and PGC in two different meshes of 1083 and 6654 elements and polynomial orders $q=0,1,2,3,4$. The tolerance was set to $10^{-5}$ and the maximum number of iterations to $5 \times 10^{5}$.

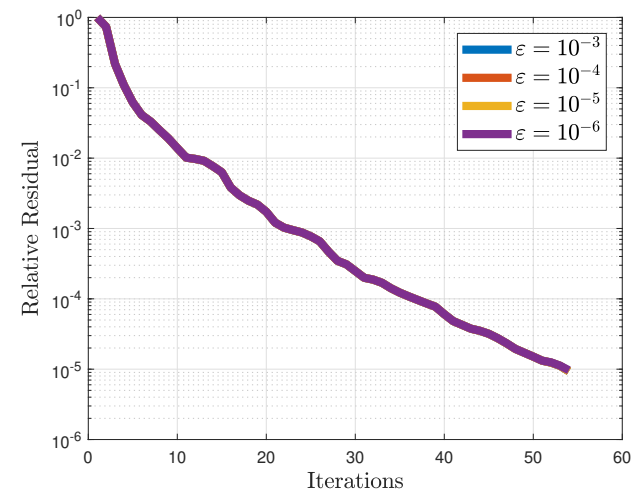

(a) $q=3$

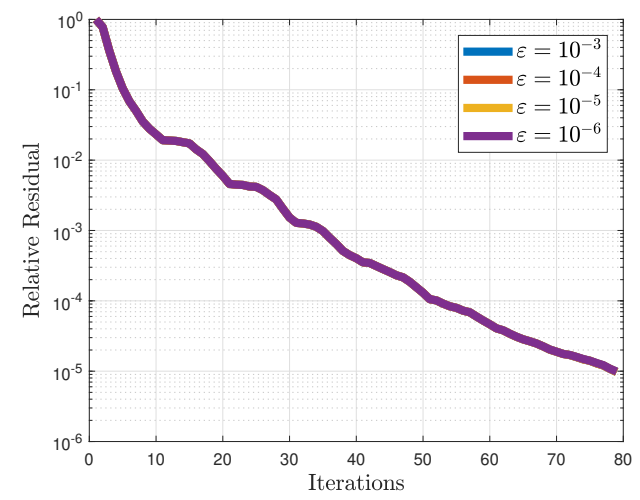

(b) $q=4$

Figure 5.3: Permeable sphere in a uniform static magnetic field: Convergence of PCG for different values of $\varepsilon$.

Furthermore, in order to show the performance of the preconditioner for different values of $\varepsilon$, the convergence of PCG for the cases $\varepsilon=10^{-3}, 10^{-4}, 10^{-5}, 10^{-6}$ and polynomial orders $q=3,4$ was studied. The convergence of the relative residual against the number of iterations is shown in Figure 5.3, where the case $q=3$ is shown on the left (Figure 5.3a) and the case $q=4$ on the right (Figure 5.3b). In these plots each line corresponds to a different value of $\varepsilon$ and, as it can be observed, the different lines are superimposed, which means that the convergence of PCG is robust with respect to $\varepsilon$.

Next, the effect of the geometry resolution is studied. For this, the curved geometry of the sphere is approximated using the blending function method as described in Section 4.3.4, for different polynomial orders of approximation of the geometry $g$. The regularisation parameter was set again to $\varepsilon=10^{-5}$. Figure 5.4 shows the convergence of the relative error $\left\|\boldsymbol{A}_{\varepsilon, h q}^{D C}-\boldsymbol{A}_{\text {exact }}^{D C}\right\|_{\boldsymbol{H}(\mathrm{curl})\left(\Omega_{C}\right)} /\left\|\boldsymbol{A}_{\text {exact }}^{D C}\right\|_{\boldsymbol{H}(\mathrm{curl})\left(\Omega_{C}\right)}$ under $p$-refinement for geometry approximation of degrees $g=1,2,3,4,5$ and a mesh of 195 elements out of which 54 are located in $\Omega_{C}$. Each line of the plot corresponds to a given order of approximation for the geometry $g$, and each point of these lines represents an increasing polynomial order of approximation $q$ for the solution field. It can be observed that for low $g$ the convergence of the error under $p$-refinement stagnates, which shows the need for accurate geometry 
resolution when computing solutions on coarse grids.

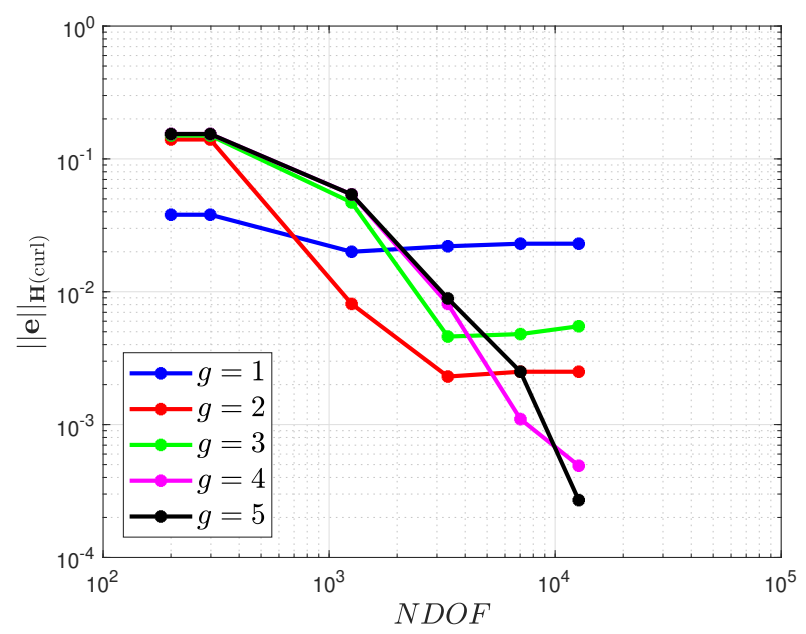

Figure 5.4: Permeable sphere in a uniform magnetic field: convergence of $\| \mathrm{A}-$ $\boldsymbol{A}_{\text {exact }}\left\|_{\boldsymbol{H}(\mathrm{curl})\left(\Omega_{C}\right)} /\right\| \boldsymbol{A}_{\text {exact }} \|_{\boldsymbol{H}(\mathrm{curl})\left(\Omega_{C}\right)}$ for different polynomial orders of approximation of the geometry under $p$-refinement.

Having this in mind, a geometry approximation of order $g=5$ is chosen and $p$ - and $h$ refinement analysis for the convergence of $\left\|\boldsymbol{A}_{\varepsilon, h q}^{D C}-\boldsymbol{A}_{\text {exact }}^{D C}\right\|_{\boldsymbol{H}(\mathrm{curl})\left(\Omega_{C}\right)} /\left\|\boldsymbol{A}_{\text {exact }}^{D C}\right\|_{\boldsymbol{H}(\mathrm{curl})\left(\Omega_{C}\right)}$ in the permeable domain $\Omega_{C}$ are performed. Figure 5.5 shows the convergence of the relative error for a set of meshes of 195, 313, 509, 1 083, 6654, 9957 and 19791 elements and polynomial orders $q=0,1,2,3,4$ under $p$-refinement. These meshes were obtained by setting successively smaller values for the maximum mesh size in NetGen. The predicted exponential rate of convergence can be observed in Figure 5.5a, where the error is plotted against the number of degrees of freedom in a logarithmic scale. Each line corresponds to a different mesh and each point represents a successively higher polynomial order $q$ for the $p$-refinement analysis. This trend is confirmed in Figure 5.5b, where the error is plotted against the number of degrees of freedom to the power of $1 / 3$ and an algebraic rate of convergence is obtained, which agrees with the predicted asymptotic rate given in the estimate (4.7).

Next, a $h$-refinement analysis, using the same set of meshes and polynomial orders, as well as the same order of approximation for the geometry, was performed. The result of this convergence analysis is shown in Figure 5.6, where after a pre-asymptotic region, an algebraic rate of convergence is observed, which agrees with the predicted asymptotic rate given in the estimate (4.4). The numerical value for the slopes of this curves, obtained with a regression analysis and neglecting the first three points of each curve that correspond to the pre-asymptotic region, is shown in Table 5.2. Excellent agreement with the theoretical rates of convergence is obtained. 


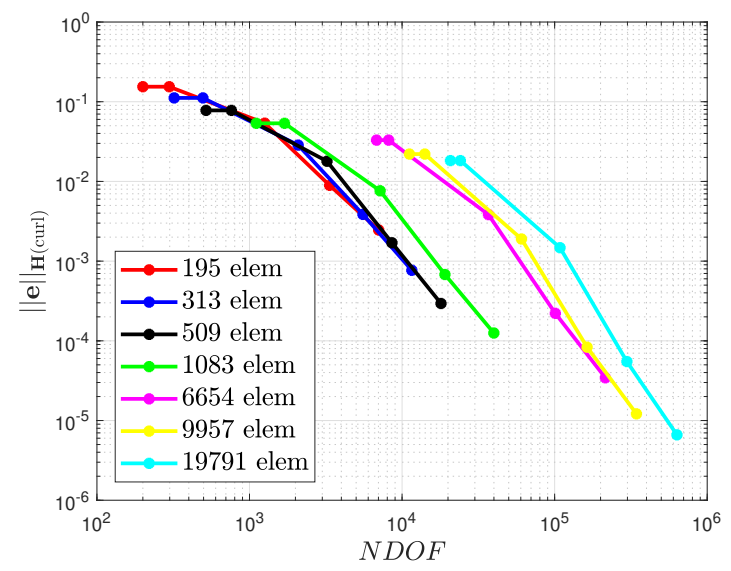

(a) $\log (\mathrm{NDOF})$

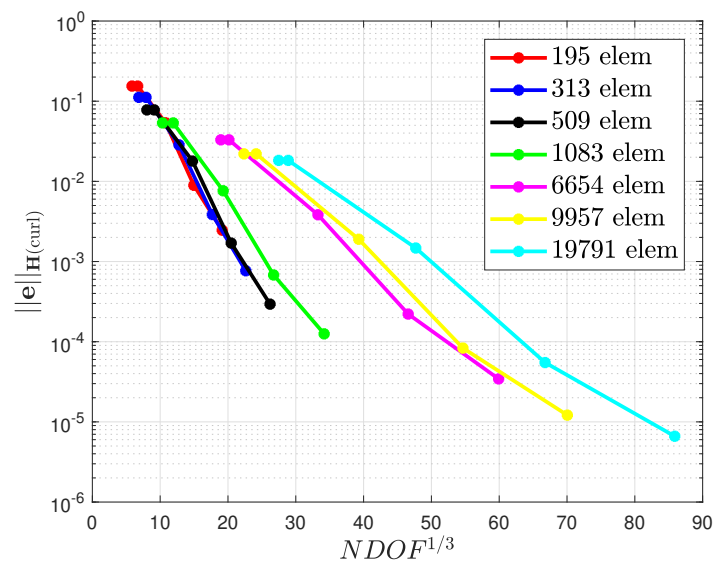

(b) $\mathrm{NDOF}^{1 / 3}$

Figure 5.5: Permeable sphere in a uniform magnetic field: Convergence of $\| \boldsymbol{A}_{\varepsilon, h q}^{D C}-$ $\boldsymbol{A}_{\text {exact }}^{D C}\left\|_{\boldsymbol{H}(\mathrm{curl})\left(\Omega_{C}\right)} /\right\| \boldsymbol{A}_{\text {exact }}^{D C} \|_{\boldsymbol{H}(\mathrm{curl})\left(\Omega_{C}\right)}$ under $p$-refinement.

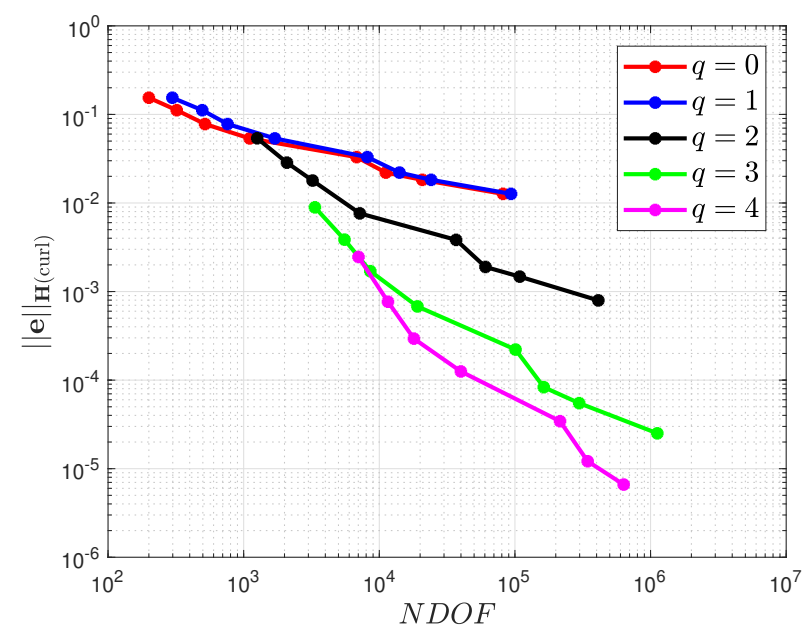

Figure 5.6: Permeable sphere in a uniform magnetic field: Convergence of $\| \boldsymbol{A}_{\varepsilon, h q}^{D C}-$ $\boldsymbol{A}_{\text {exact }}^{D C}\left\|_{\boldsymbol{H}(\mathrm{curl})\left(\Omega_{C}\right)} /\right\| \boldsymbol{A}_{\text {exact }}^{D C} \|_{\boldsymbol{H}(\mathrm{curl})\left(\Omega_{C}\right)}$ under $h$-refinement.

\begin{tabular}{|l|l|l|}
\hline Order & Computed rate & Expected rate \\
\hline \hline 0 & -0.35 & -0.33 \\
\hline 1 & -0.37 & -0.33 \\
\hline 2 & -0.58 & -0.67 \\
\hline 3 & -0.91 & -1 \\
\hline 4 & -1.28 & -1.33 \\
\hline
\end{tabular}

Table 5.2: Permeable sphere in a uniform magnetic field: rates of convergence for the relative error $\left\|\boldsymbol{A}_{\varepsilon, h q}^{D C}-\boldsymbol{A}_{\text {exact }}^{D C}\right\|_{\boldsymbol{H}(\operatorname{curl})\left(\Omega_{C}\right)} /\left\|\boldsymbol{A}_{\text {exact }}^{D C}\right\|_{\boldsymbol{H}(\mathrm{curl})\left(\Omega_{C}\right)}$ under $h$-refinement 


\subsubsection{DC stage, Elasticity: Hollow Cylinder Subject to Pressure Field}

In order to benchmark the DC elasticity stage, a problem consisting of a hollow cylinder $\Omega_{C}:=\{(r, \phi, z): a \leqslant r \leqslant b, 0 \leqslant \phi<2 \pi,-L / 2 \leqslant z \leqslant L / 2\}$ subject to internal and external pressure is considered. A cross section of the scheme of the problem is presented in Figure 5.7. This problem has an analytical solution in terms of $\boldsymbol{u}_{\text {exact }}$, which can be found in [38]. The particular case with internal radius $a=1 \mathrm{~m}$, external radius

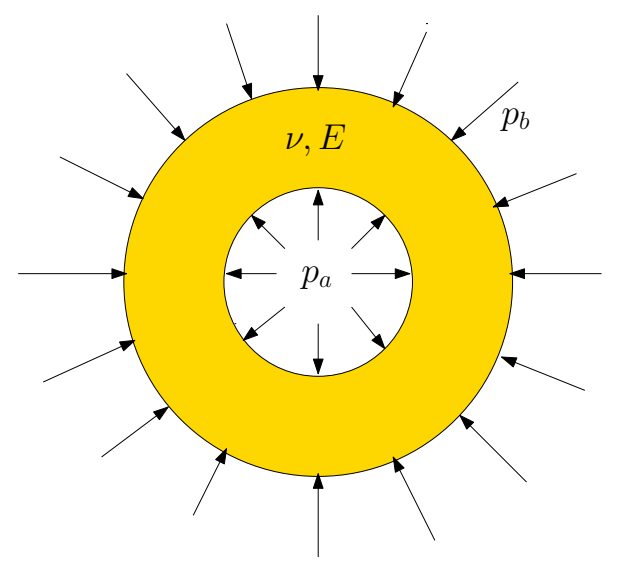

Figure 5.7: Hollow cylinder subject to pressure field: illustration of a cross section of the cylinder and the applied pressure field.

$b=2 \mathrm{~m}$, length $L=5 \mathrm{~m}$, Young Modulus $E=2.1 \times 10^{11} \mathrm{~Pa}$ and Poisson's ratio $\nu=0.33$ is considered here. The internal and external pressure are set to $p_{a}=10^{4} \mathrm{~Pa}$ and $p_{b}=10^{7} \mathrm{~Pa}$, respectively.

This problem is simulated by solving a simplified version of (3.1f)-(3.1h), using appropriate boundary conditions

$$
\begin{aligned}
\boldsymbol{\sigma}^{m}\left(\boldsymbol{u}_{h p}^{D C}\right) \boldsymbol{n} & =\boldsymbol{\sigma}^{m}\left(\boldsymbol{u}_{\text {exact }}\right) \boldsymbol{n} & & \text { on } \partial \Omega_{C}^{N}, \\
\boldsymbol{u}_{h p}^{D C} & =\boldsymbol{u}_{\text {exact }} & & \text { on } \partial \Omega_{C}^{D} .
\end{aligned}
$$

Figure 5.8 shows where the boundary conditions are applied and one of the meshes used for the computations.

First, the convergence of the conjugate gradient method and the efficiency of the preconditioner is studied. For this, in Figure 5.9 the number of iterations required by $\mathrm{CG}$ and PCG to converge to a tolerance of $10^{-7}$ are plotted for two different meshes of 1366 and 3144 elements and polynomial orders $p=1,2,3,4,5$. Furthermore, in Table 5.3 a comparison of solver time between CG and PCG is shown, where the efficiency of the preconditioner is clearly observed. Note that the time for PCG includes the construction of the preconditioner.

Next, a sequence of unstructured tetrahedral meshes with 1366, 3144, 7953, 29637 and 53230 elements (obtained by setting successively smaller values for the maximum mesh size in NetGen) is considered and discretisations with uniform element orders $p=1,2,3,4,5$ are applied, in turn. Quadratic representation of the geometry is used 


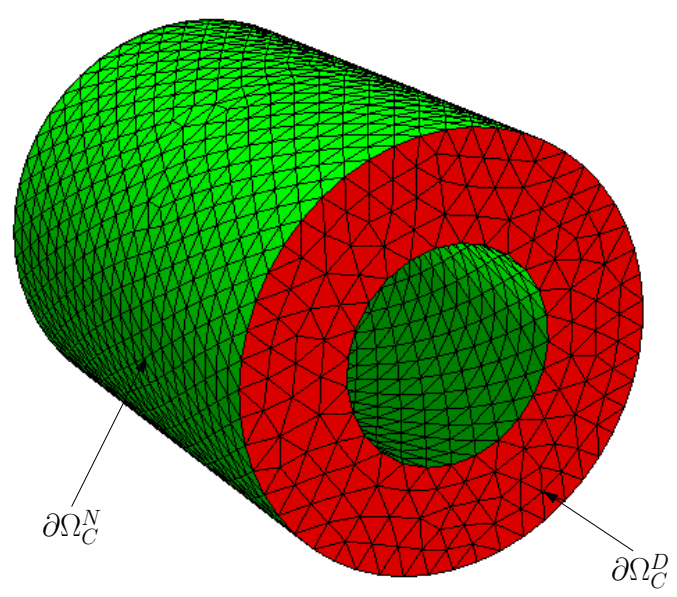

Figure 5.8: Hollow cylinder subject to pressure field: illustration of the chosen boundary conditions and one of the meshes (7953 elements) used in the computations. $\partial \Omega_{C}^{N}$ in green and $\partial \Omega_{C}^{D}$ in red.

\begin{tabular}{|c|c|c|c|c|c|}
\hline & $p=1$ & $p=2$ & $p=3$ & $p=4$ & $p=5$ \\
\hline CG, 1366 elements & $0.1 \mathrm{~s}$ & $0.1 \mathrm{~s}$ & $0.2 \mathrm{~s}$ & $4.2 \mathrm{~s}$ & $42.7 \mathrm{~s}$ \\
\hline PCG, 1366 elements & $0.1 \mathrm{~s}$ & $0.1 \mathrm{~s}$ & $0.4 \mathrm{~s}$ & $1.0 \mathrm{~s}$ & $4.8 \mathrm{~s}$ \\
\hline CG, 3 144 elements & $0.1 \mathrm{~s}$ & $0.1 \mathrm{~s}$ & $2.1 \mathrm{~s}$ & $42 \mathrm{~s}$ & $390.2 \mathrm{~s}$ \\
\hline PCG, 3 144 elements & $0.1 \mathrm{~s}$ & $0.2 \mathrm{~s}$ & $1.4 \mathrm{~s}$ & $6.8 \mathrm{~s}$ & $26.1 \mathrm{~s}$ \\
\hline
\end{tabular}

Table 5.3: Hollow cylinder subject to pressure field: Solver time in seconds for CG and PGC in two different meshes of 1366 and 3144 elements and polynomial orders $p=$ $1,2,3,4,5$. The tolerance was set to $10^{-7}$.

throughout. In each case, the relative error $\left\|\boldsymbol{u}_{h p}^{D C}-\boldsymbol{u}_{\text {exact }}\right\|_{H^{1}\left(\Omega_{C}\right)} /\left\|\boldsymbol{u}_{\text {exact }}\right\|_{H^{1}\left(\Omega_{C}\right)}$ is measured. Figure 5.10 shows this relative error against the number of degrees of freedom for $p$-refinement, where each line represents a single mesh and the points increasing polynomial degree, and Figure 5.11 those for $h$-refinement, where each curve is for a fixed order and the points represent mesh refinement.

In Figure 5.10, the exponential rate of convergence of the relative error with the number of degrees of freedom under a $p$-refinement analysis is observed. This trend is confirmed by the algebraic rate of convergence of the error, which is obtained when the error is plotted against the number of degrees of freedom to the power of $1 / 3$, and agrees with the predicted asymptotic rate stated in equation (4.7). In these plots, each line corresponds to a different mesh, and the points correspond to an increasing polynomial order. After an initial pre-asymptotic region, Figure 5.11 shows the algebraic rate of convergence predicted in (4.4) for the $h$-refinement, where each line corresponds to a different polynomial order $p$ and the points represent successively finer meshes. The obtained rates of convergence for $h$-refinement are in good agreement with the predicted rates, as it can be observed in Table 5.4.

Despite the coarse nature of some of the grids employed, accurate representation of the 


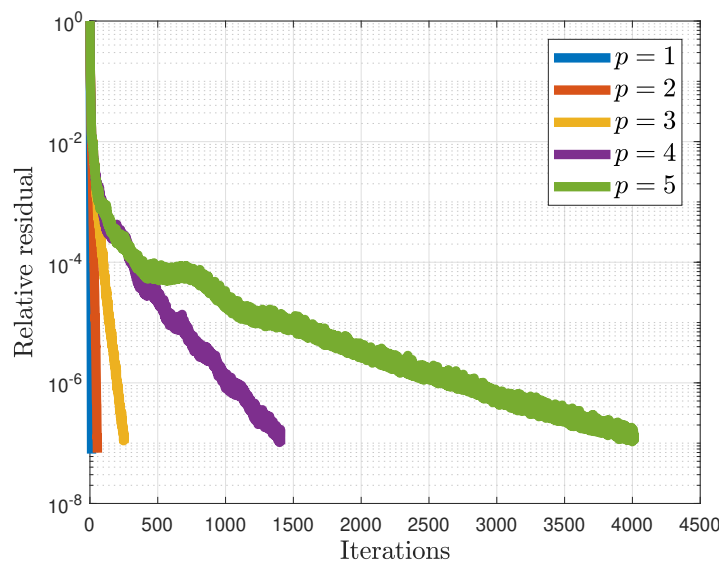

(a) CG, 1366 elements

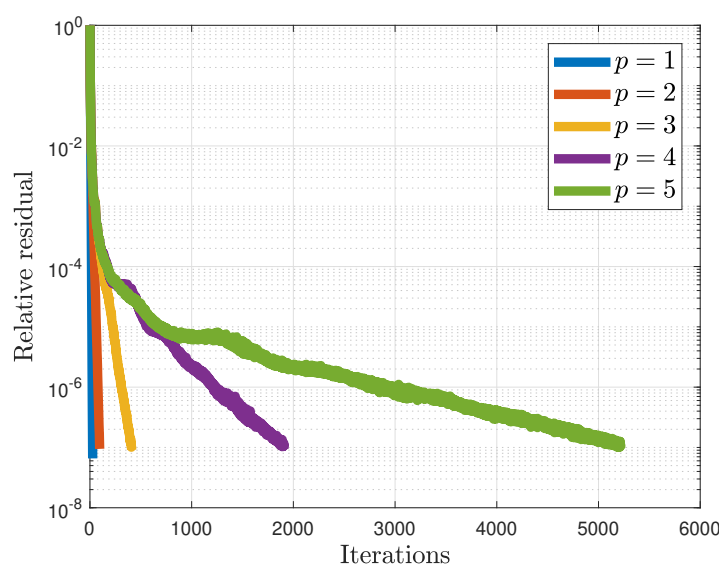

(c) CG, 3144 elements

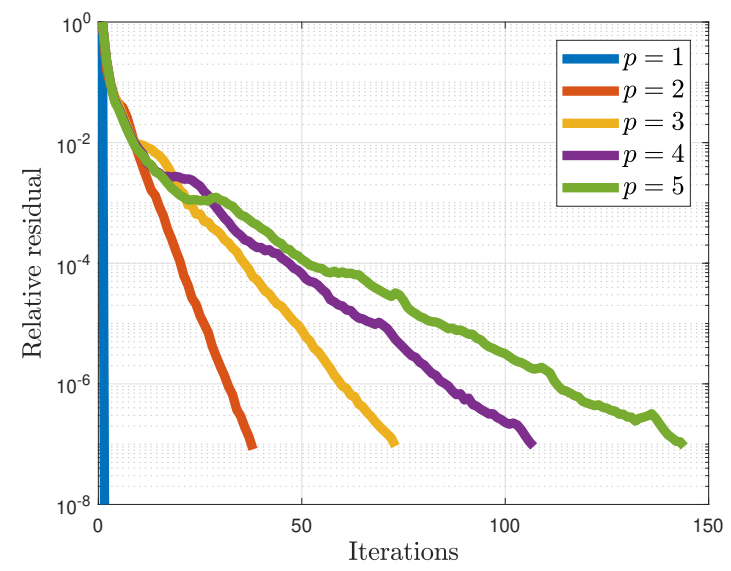

(b) PCG, 1366 elements

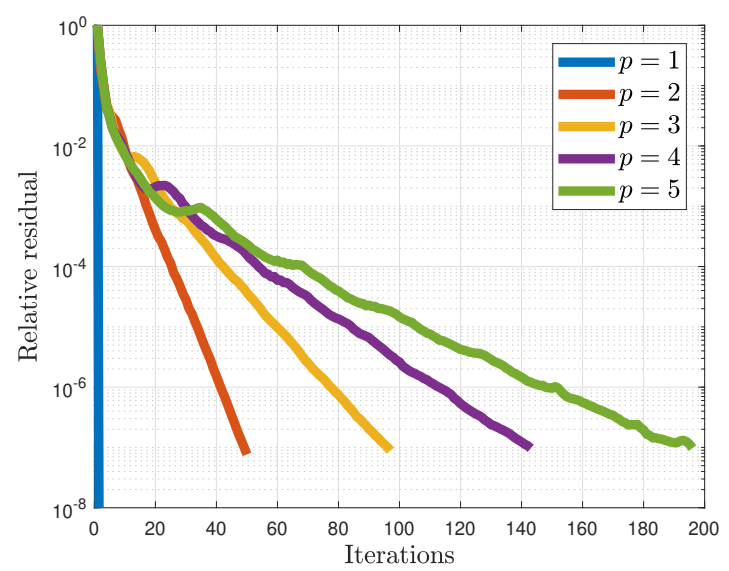

(d) PCG, 3144 elements

Figure 5.9: Hollow cylinder subject to pressure field: Convergence of the conjugate gradient method for: (a) A mesh of 1366 elements without preconditioner, (b) A mesh of 1366 elements using preconditioner, (c) A mesh of 3144 elements without preconditioner and (d) A mesh of 3144 elements using preconditioner.

\begin{tabular}{|l|l|l|}
\hline Order & Computed rate & Expected rate \\
\hline \hline 1 & -0.28 & -0.33 \\
\hline 2 & -0.66 & -0.67 \\
\hline 3 & -1.03 & -1 \\
\hline 4 & -1.37 & -1.33 \\
\hline 5 & -1.58 & -1.67 \\
\hline
\end{tabular}

Table 5.4: Hollow cylinder subject to pressure field: rates of convergence for the relative error $\left\|\boldsymbol{u}_{h p}^{D C}-\boldsymbol{u}_{\text {exact }}\right\|_{H^{1}(\Omega)} /\left\|\boldsymbol{u}_{\text {exact }}\right\|_{H^{1}(\Omega)}$ under $h$-refinement.

solutions can still be achieved with high order elements. For the purpose of plotting, coarse meshes are subdivided and solutions evaluated on the split mesh and then passed 


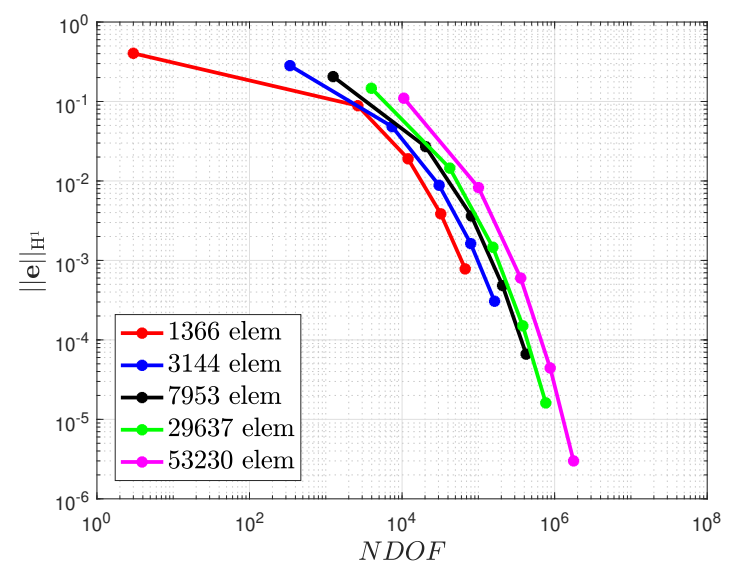

(a) $\log (\mathrm{NDOF})$

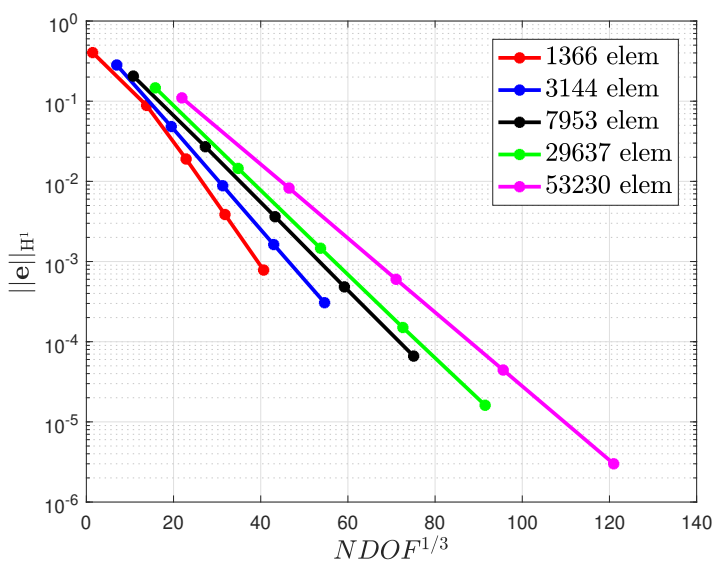

(b) $\mathrm{NDOF}^{1 / 3}$

Figure 5.10: Hollow cylinder subject to pressure field: convergence of $\| \boldsymbol{u}_{h p}^{D C}-$ $\boldsymbol{u}_{\text {exact }}\left\|_{H^{1}\left(\Omega_{C}\right)} /\right\| \boldsymbol{u}_{\text {exact }} \|_{H^{1}\left(\Omega_{C}\right)}$ under $p$-refinement.

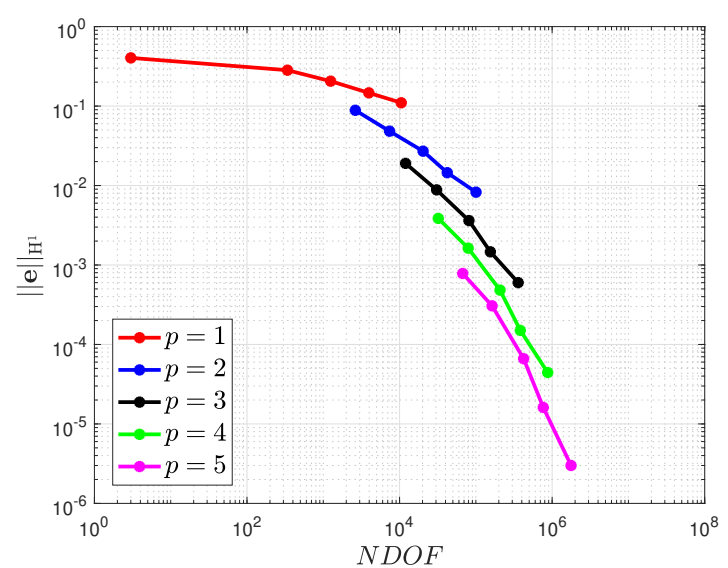

Figure 5.11: Hollow cylinder subject to pressure field: convergence of $\| \boldsymbol{u}_{h p}^{D C}-$ $\boldsymbol{u}_{\text {exact }}\left\|_{H^{1}\left(\Omega_{C}\right)} /\right\| \boldsymbol{u}_{\text {exact }} \|_{H^{1}\left(\Omega_{C}\right)}$ under $h$-refinement.

to Paraview [9] for plotting, as described in Section 4.10.3. Figure 5.12 shows contour plots of the displacements in the cylinder obtained by a mesh of 7953 tetrahedra of order $p=4$.

\subsubsection{AC stage, Eddy Current: Conducting Sphere in a Uniform Al- ternating Magnetic Field}

In order to benchmark the AC eddy current stage, the case of a conducting sphere $\Omega_{C}:=$ $\{\boldsymbol{x}:|\boldsymbol{x}| \leqslant R\}$ placed in an unbounded region of free space $\Omega_{C}^{c}$, where $\mathcal{H}_{0}^{A C}$ tends to a time harmonic uniform field far from the object, is considered. An illustration of the problem is presented in Figure 5.13. This problem has an analytical solution [164] in terms of $\boldsymbol{A}_{\text {exact }}$. The case of the sphere with $R=1 \mathrm{~cm}, \mu=1.5 \mu_{0}, \mu_{0}=4 \pi \times 10^{-7} \mathrm{H} / \mathrm{m}, \sigma=6 \times 10^{6} \mathrm{~S} / \mathrm{m}$ 


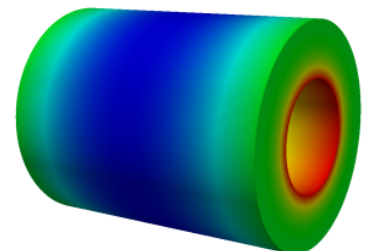

(a) $\left|\boldsymbol{u}_{h p}^{D C}\right|$

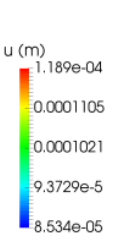

政

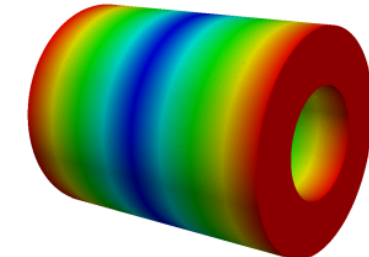

(b) $u_{z, h p}^{D C}$

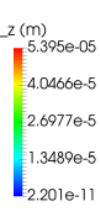

2.201e-11

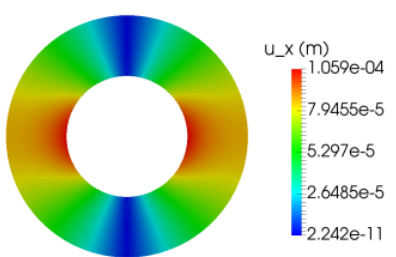

(c) $u_{x, h p}^{D C}$

Figure 5.12: Hollow cylinder subject to pressure field: (a) $\left|\boldsymbol{u}_{h p}^{D C}\right|$, (b) $\left|u_{z, h p}^{D C}\right|$ (c) $\left|u_{x, h p}^{D C}\right|$ on the plane $z=0$.

set in a background field with $\lim _{|\boldsymbol{x}| \rightarrow \infty} \mathcal{H}_{0}^{A C}=\mu_{0} \boldsymbol{e}_{z} \mathrm{~A} / \mathrm{m}$ and a frequency $f=50 \mathrm{~Hz}$ is considered, unless otherwise stated.

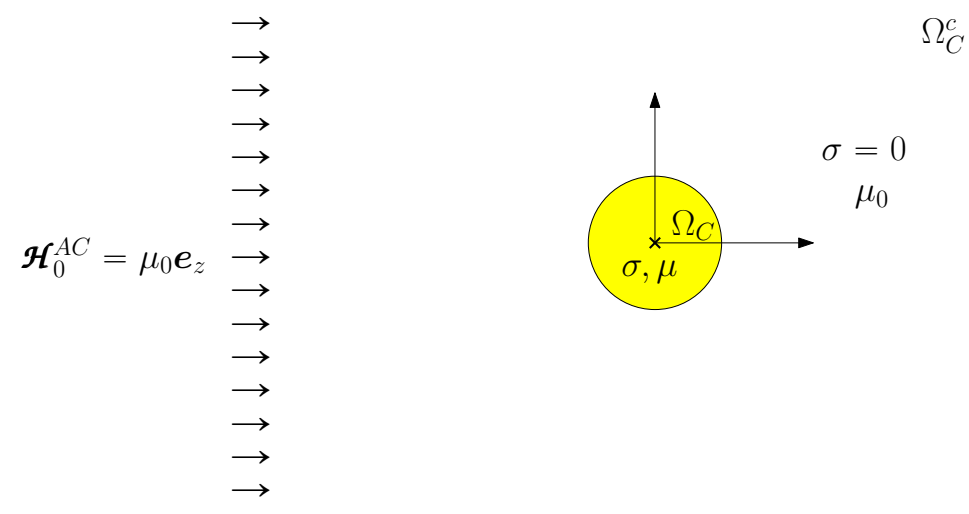

Figure 5.13: Conducting sphere in a uniform alternating magnetic field: problem setup.

This problem is simulated by solving a simplified version of (2.42a)-(2.42g) with $\boldsymbol{g}^{A C}=\mathbf{0}$ on a truncated domain where $\partial \Omega$ is the surface of the sphere of radius $2 \mathrm{~cm}$. On the boundary $\partial \Omega$, the condition $\boldsymbol{n} \times \mathcal{A}_{\varepsilon, h q}^{A C}=\boldsymbol{n} \times \boldsymbol{A}_{\text {exact }}$, where $\boldsymbol{A}_{\text {exact }}$ is the analytical solution for this problem proposed by Smythe [164], is imposed. The regularisation parameter $\varepsilon=10^{-5}$ is applied in all cases. Figure 5.14, shows the convergence of the GMRES and PGMRES solvers for two different frequencies, $f=50 \mathrm{~Hz}$ and $f=1000 \mathrm{~Hz}$, when a $p$-refinement analysis is performed using a mesh of 1083 tetrahedral elements. The tolerance of the iterative solver was set to $10^{-5}$. Similarly to the results showed in Section 5.2.1 for the DC problem, it can be observed that the application of the block Jacobi preconditioner leads to a massive reduction in the number of iterations. The solver time with and without preconditioner was also measured and the result is shown in Table 5.5, which demonstrates once again the efficiency of the preconditioner. Note also, that according to Figure 5.14 and Table 5.5, the number of iterations and solver time of PGMRES exhibits only a very moderate increase with the frequency.

To investigate the accuracy of the approximation, a sequence of unstructured tetrahedral meshes with 195, 313, 509, 1083, 6654, 9957 and 19791 elements is considered and discretisations with uniform element orders $q=0,1,2,3,4$ are applied, in turn. Initially, the geometry is approximated using the blending function method with polynomials of degree $g=5$, as described in Section 4.3.4. In each case, the relative error $\| \mathcal{A}_{\varepsilon, h q}^{A C}-$ 


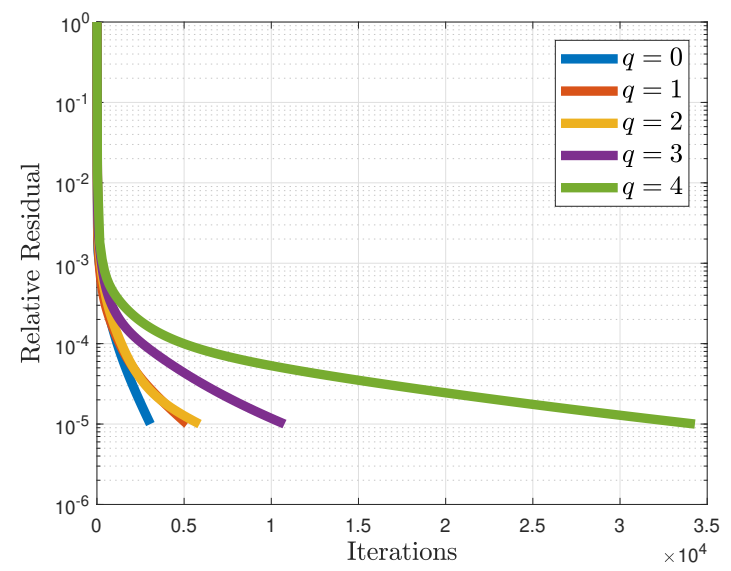

(a) GMRES, $f=50 \mathrm{~Hz}$

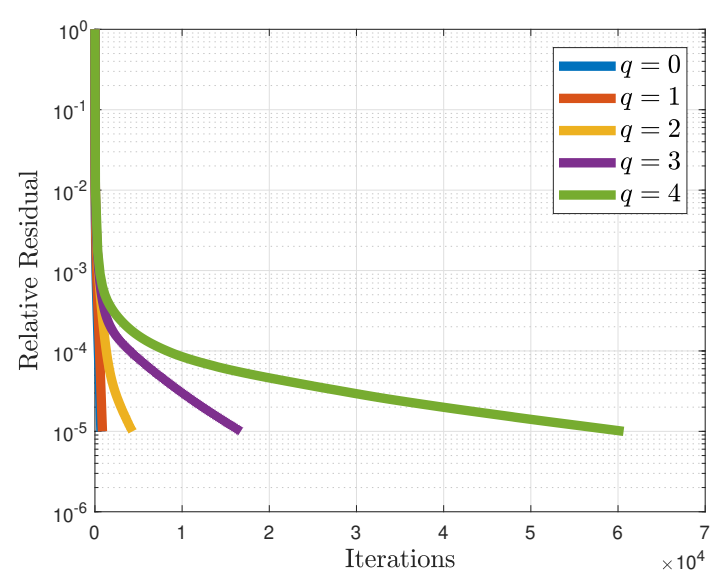

(c) GMRES, $f=1000 \mathrm{~Hz}$

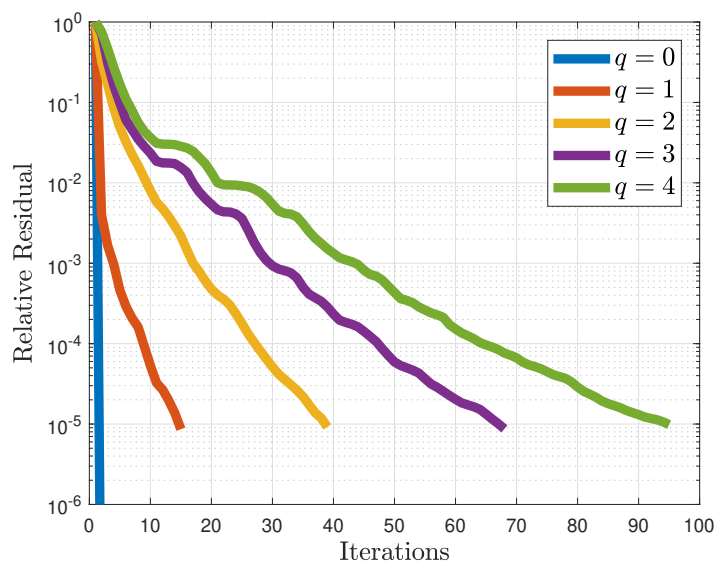

(b) PGMRES, $f=50 \mathrm{~Hz}$

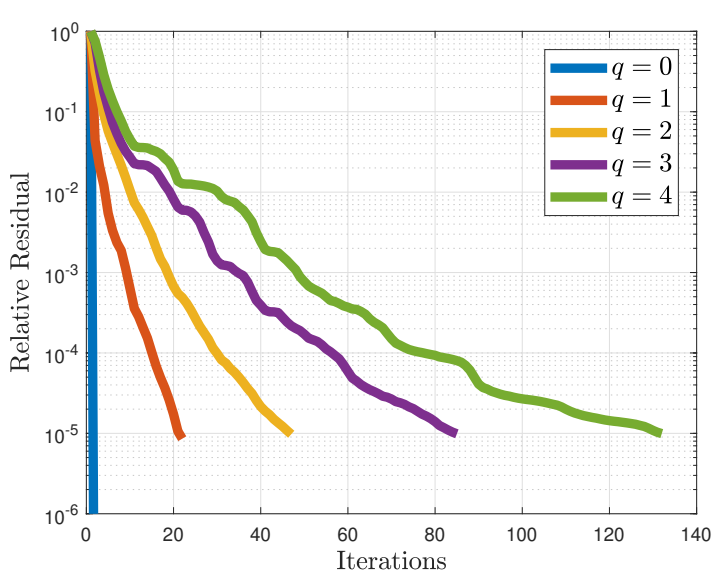

(d) PGMRES, $f=1000 \mathrm{~Hz}$

Figure 5.14: Conducting sphere in a uniform alternating magnetic field: Convergence of the iterative solver (GMRES and PGMRES) for: (a) $f=50 \mathrm{~Hz}$ without preconditioner, (b) $f=50 \mathrm{~Hz}$ using preconditioner, (c) $f=1000 \mathrm{~Hz}$ without preconditioner and (d) $f=1000 \mathrm{~Hz}$ using preconditioner.

\begin{tabular}{|c|c|c|c|c|c|}
\hline & $q=0$ & $q=1$ & $q=2$ & $q=3$ & $q=4$ \\
\hline GMRES, $f=50 \mathrm{~Hz}$ & $1.1 \mathrm{~s}$ & $2.7 \mathrm{~s}$ & $13.2 \mathrm{~s}$ & $82.6 \mathrm{~s}$ & $793.1 \mathrm{~s}$ \\
\hline PGMRES, $f=50 \mathrm{~Hz}$ & $0.07 \mathrm{~s}$ & $0.08 \mathrm{~s}$ & $0.41 \mathrm{~s}$ & $1.3 \mathrm{~s}$ & $4.3 \mathrm{~s}$ \\
\hline GMRES, $f=1000 \mathrm{~Hz}$ & $1.51 \mathrm{~s}$ & $1.58 \mathrm{~s}$ & $9.53 \mathrm{~s}$ & $129.6 \mathrm{~s}$ & $1400 \mathrm{~s}$ \\
\hline PGMRES, $f=1000 \mathrm{~Hz}$ & $0.07 \mathrm{~s}$ & $0.07 \mathrm{~s}$ & $0.21 \mathrm{~s}$ & $1.1 \mathrm{~s}$ & $4.7 \mathrm{~s}$ \\
\hline
\end{tabular}

Table 5.5: Conducting sphere in a uniform alternating magnetic field: Solver time in seconds for GMRES and PGMRES for a mesh 1083 elements, polynomial orders $q=$ $0,1,2,3,4$ and two different frequencies $f=50 \mathrm{~Hz}$ and $f=1000 \mathrm{~Hz}$. The tolerance was set to $10^{-5}$. 


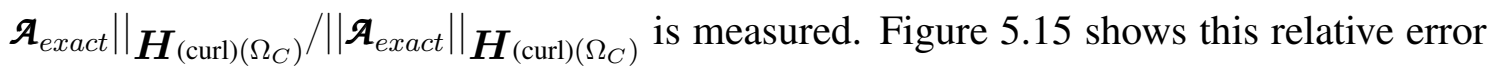
against the number of degrees of freedom for $p$-refinement and Figure 5.16 those for $h$ refinement.

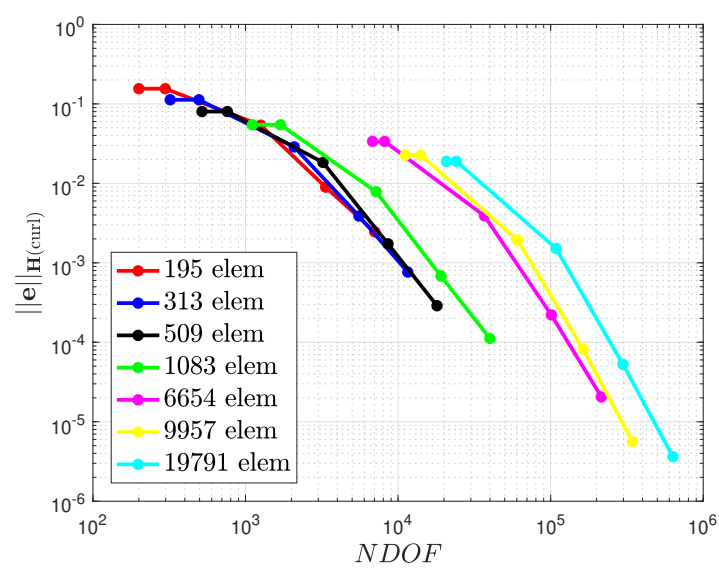

(a) $\log (\mathrm{NDOF})$

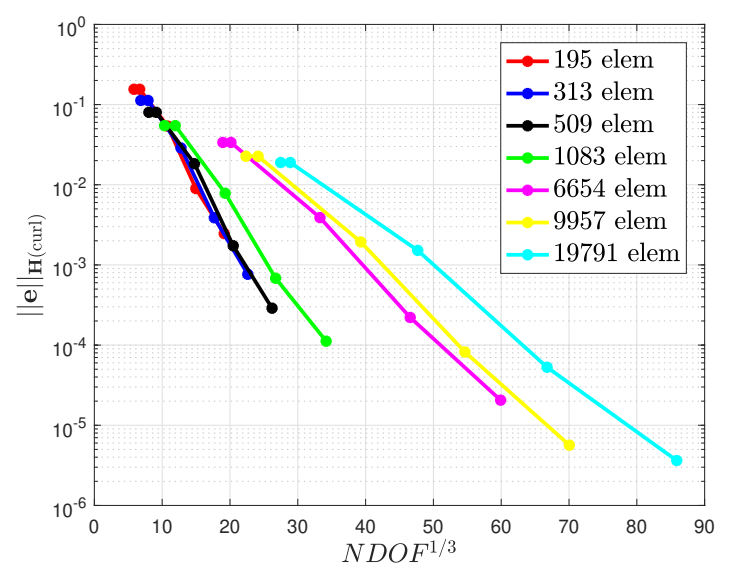

(b) $\mathrm{NDOF}^{1 / 3}$

Figure 5.15: Conducting sphere in a uniform alternating magnetic field: convergence of

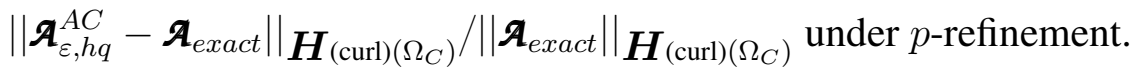

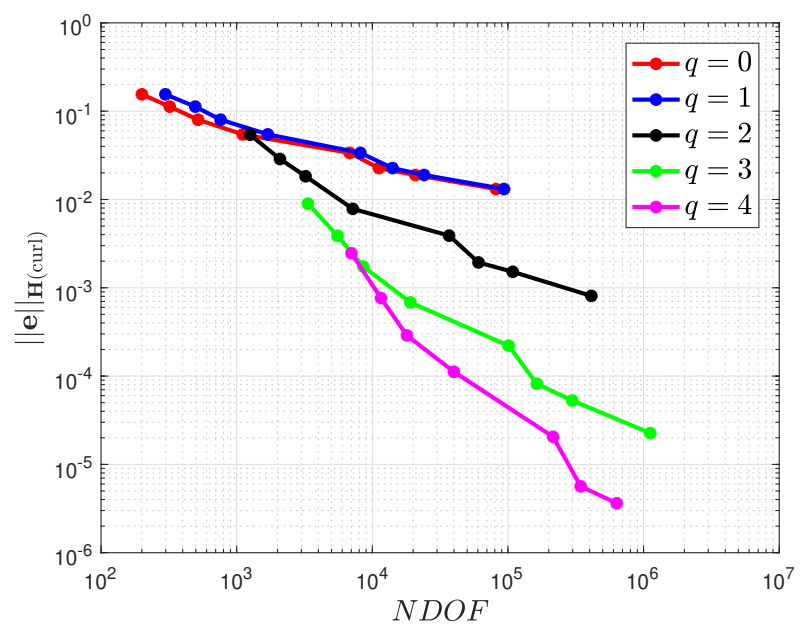

Figure 5.16: Conducting sphere in a uniform alternating magnetic field: convergence of

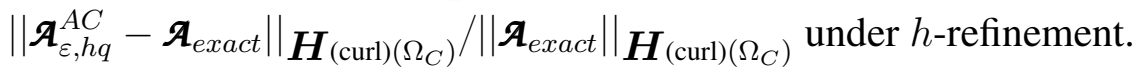

In a similar manner to the DC stage examples, the expected exponential rate of convergence predicted in the estimate (4.7) for the relative error with the number of degrees of freedom under a $p$-refinement analysis can be observed in Figure 5.15, where each line corresponds to a different mesh and the points on each line correspond to an increasing polynomial order $q$. Also, after an initial pre-asymptotic region, Figure 5.16 shows the algebraic rate of convergence predicted in the estimate (4.4) for $h$-refinement. In this plot, 


\begin{tabular}{|l|l|l|}
\hline Order & Computed rate & Expected rate \\
\hline \hline 0 & -0.38 & -0.33 \\
\hline 1 & -0.41 & -0.33 \\
\hline 2 & -0.68 & -0.67 \\
\hline 3 & -0.96 & -1 \\
\hline 4 & -1.27 & -1.33 \\
\hline
\end{tabular}

Table 5.6: Conducting sphere in a uniform alternating magnetic field magnetic field: rates of convergence for the relative error $\left\|\boldsymbol{A}_{\varepsilon, h q}^{A C}-\boldsymbol{A}_{\text {exact }}\right\|_{\boldsymbol{H} \text { (curl) }\left(\Omega_{C}\right)} /\left\|\boldsymbol{\mathcal { A }}_{\text {exact }}\right\|_{\boldsymbol{H} \text { (curl) }\left(\Omega_{C}\right)}$ under $h$-refinement.

each line corresponds to a different polynomial order $q$ and the points in each line correspond to successively finer meshes. Furthermore, in Table 5.6 it can be observed that the obtained rates of convergence are in good agreement with the theoretical prediction in (4.4). Note that, as in the magnetostatic case, the rates of convergence were obtained by performing a linear regression neglecting the first three points corresponding to the pre-asymptotic region.

\section{Skin Effect}

The skin depth is a measure of the penetration of the fields into the conducting object, and is defined as the distance at which the fields decay to $1 / e$ of its value in the conductor's surface [164]. Therefore, for higher frequencies (and hence smaller skin depths), larger gradients in the fields will appear close to the conductor's surface, which makes the accurate numerical simulation of the problem more challenging. This effect is illustrated in Figure 5.17, where a contour plot of the magnitude of the computed eddy currents $\boldsymbol{g}_{h q}^{o}=-\mathrm{i} \omega \gamma \boldsymbol{A}_{\varepsilon, h q}^{A C}$ in the sphere, in particular in the planes $z=0$ and $x=0$, is shown. To obtain these results, sufficiently fine discretisations have bee employed in order to capture the solutions accurately.

\subsubsection{AC stage, Elasticity: Hollow Cylinder Subject to Body Force}

A last single physics problem is now considered in order to study the convergence of the AC elasticity stage. For this, the same cylinder described in Section 5.2.2 is considered and an analytical solution

$$
\boldsymbol{u}_{\text {exact }}^{A C}=\left(\begin{array}{l}
x \sin y \sin z \\
z \sin y \cos x \\
y \sin x \cos z
\end{array}\right)
$$

is defined.

Then, the body force $\boldsymbol{b}$ is computed such that the equation

$$
\operatorname{div} \boldsymbol{\sigma}^{m}\left(\boldsymbol{u}_{\text {exact }}^{A C}\right)-\rho \omega^{2} \boldsymbol{u}_{\text {exact }}^{A C}=\boldsymbol{b},
$$




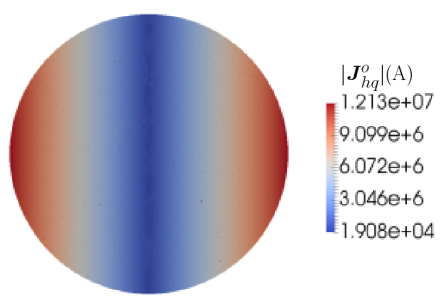

(a) $x=0, f=50 \mathrm{~Hz}$

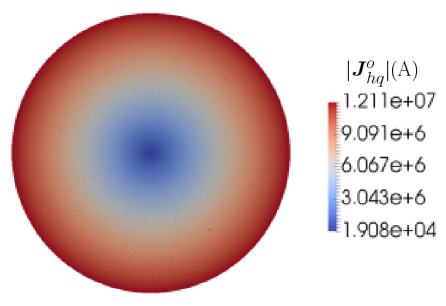

(d) $z=0, f=50 \mathrm{~Hz}$

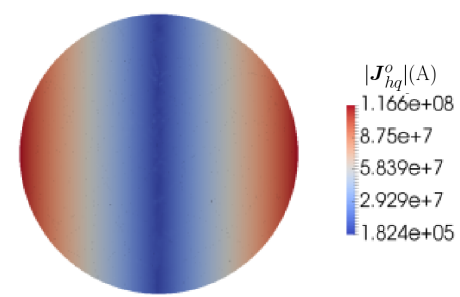

(b) $x=0, f=500 \mathrm{~Hz}$

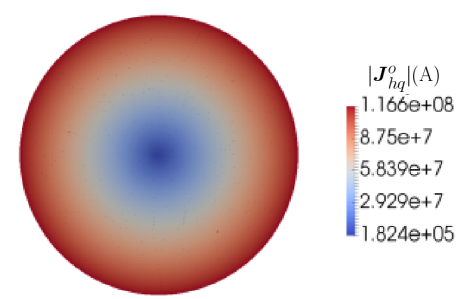

(e) $z=0, f=500 \mathrm{~Hz}$

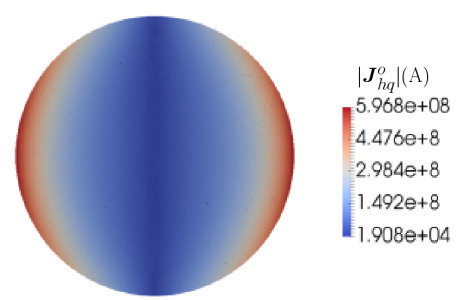

(c) $x=0, f=5000 \mathrm{~Hz}$

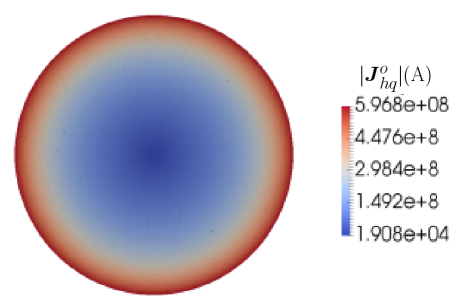

(f) $z=0, f=5000 \mathrm{~Hz}$

Figure 5.17: Conducting sphere in a uniform alternating magnetic field: Magnitude of the eddy current, $\left|\boldsymbol{g}_{h q}^{o}\right|$ in the planes $x=0$ and $z=0$ for different frequencies.

is satisfied, which results in a body force $\boldsymbol{b}=\left(b_{x}, b_{y}, b_{z}\right)^{T}$ with components

$$
\begin{aligned}
b_{x} & =\lambda(-z \cos y \sin x-y \cos x \sin z) \\
& -G(2 \sin y \sin z+z \cos y \sin x+y \cos x \sin z)-\rho \omega^{2}(x \sin y \sin z), \\
b_{y} & =\lambda(\cos y \sin z-z \sin y \cos x-\sin x \sin z) \\
& +G(\cos y \sin z-3 z \sin y \cos x-\sin x \sin z)-\rho \omega^{2}(z \sin y \cos x), \\
b_{z} & =\lambda(\sin y \cos z+\cos y \cos x-y \sin x \cos z) \\
& +G(\sin y \cos z-3 y \sin x \cos z+\cos y \cos x)-\rho \omega^{2}(y \sin x \cos z) .
\end{aligned}
$$

Then, a suitably simplified version of $(2.42 \mathrm{~h})-(2.42 \mathrm{~m})$ is solved, where the electromagnetic induced force is replaced by the body force $\boldsymbol{b}$ and Neumann and Dirichlet boundary conditions are applied as described in Section 5.2.2.

To study the accuracy of the approximation, a sequence of unstructured tetrahedral meshes with $1366,3144,7953,29637$ and 53230 elements is considered and discretisations with uniform element orders $p=1,2,3,4$ are applied, in turn. Quadratic representation of the geometry, as described in Section 4.3.4 is used throughout. In each case, the relative error $\left\|\boldsymbol{u}_{h p}^{A C}-\boldsymbol{u}_{\text {exact }}^{A C}\right\|_{H^{1}\left(\Omega_{C}\right)} /\left\|\boldsymbol{u}_{\text {exact }}^{A C}\right\|_{H^{1}\left(\Omega_{C}\right)}$ is measured, and the results for $p$-refinement and $h$-refinement are shown in Figures 5.18 and 5.19, respetively. Figure 5.18 shows, once again, the expected exponential rate of convergence predicted by the estimate (4.7) for $p$-refinement, where each line corresponds to a different mesh and each point corresponds to an increasing polynomial order $p$. Similarly, in Figure 5.19, the expected algebraic rate of convergence predicted by the estimate (4.4) for $h$-refinement can be observed. Furthermore, the obtained rates of convergence for $h$-refinement are compared with the theoretical rates of convergence predicted by (4.4) in Table 5.7. Analogously as for the 
DC problem studied in Section 5.2.2, the computed rates of convergence are in good agreement with the theoretical prediction.

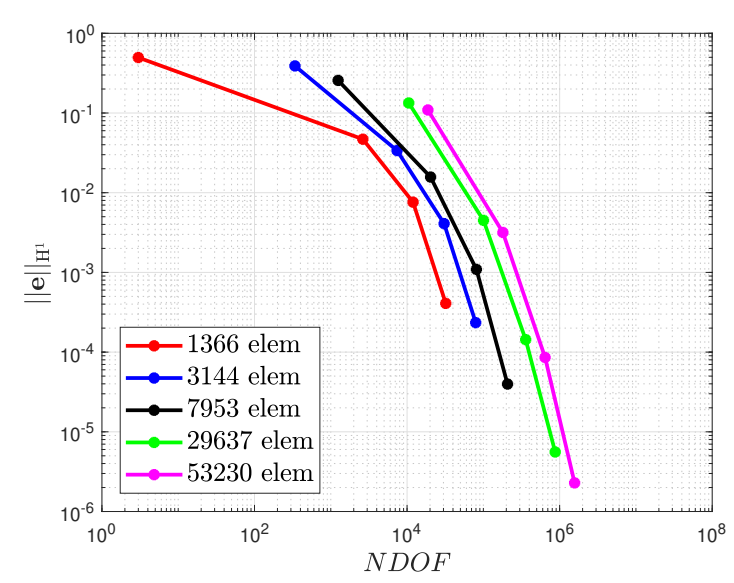

(a) $\log (\mathrm{NDOF})$

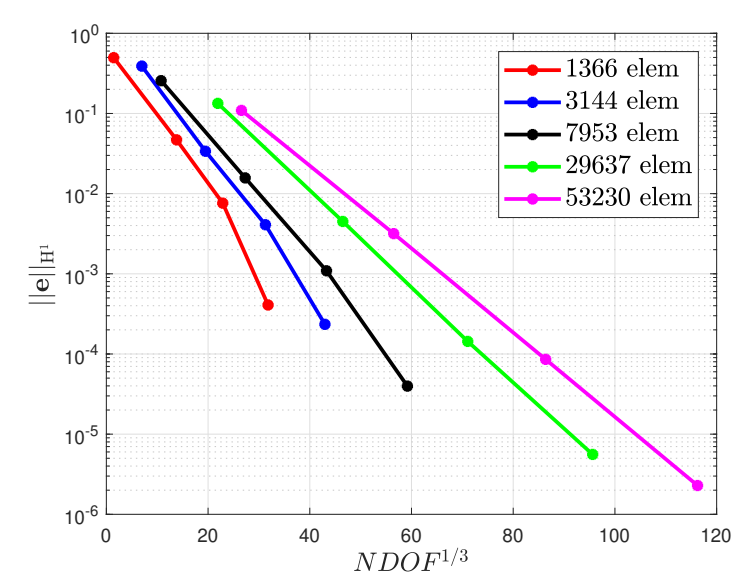

(b) $\mathrm{NDOF}^{1 / 3}$

Figure 5.18: AC elasticity stage, hollow cylinder subject to body force: convergence of $\left\|\boldsymbol{u}_{h p}^{A C}-\boldsymbol{u}_{\text {exact }}^{A C}\right\|_{H^{1}\left(\Omega_{C}\right)} /\left\|\boldsymbol{u}_{\text {exact }}^{A C}\right\|_{H^{1}\left(\Omega_{C}\right)}$ under $p$-refinement.

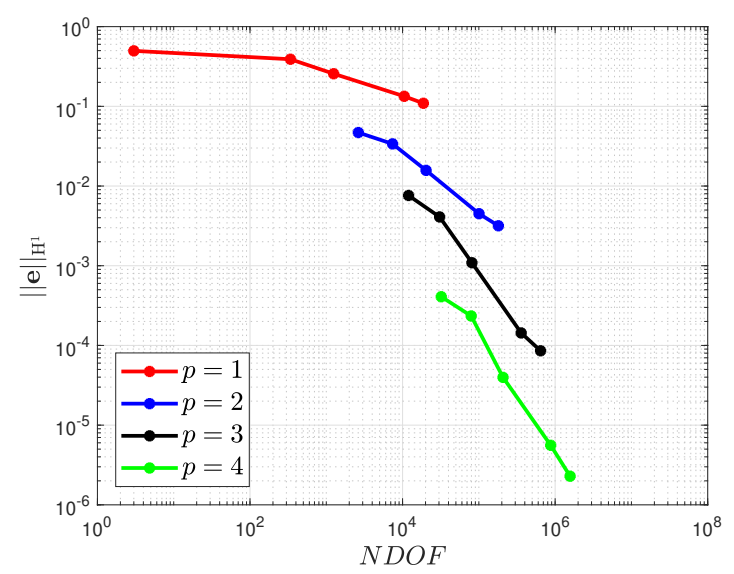

Figure 5.19: AC elasticity stage, hollow cylinder subject to body force: convergence of $\left\|\boldsymbol{u}_{h p}^{A C}-\boldsymbol{u}_{\text {exact }}^{A C}\right\|_{H^{1}\left(\Omega_{C}\right)} /\left\|\boldsymbol{u}_{\text {exact }}^{A C}\right\|_{H^{1}\left(\Omega_{C}\right)}$ under $h$-refinement.

\subsection{Coupled Physics}

A series of more challenging coupled examples are now presented to illustrate the performance of the staggered Lagrangian approach advocated in Algorithm 1 compared to the Eulerian approach and validate the proposed 3D $h p$-FEM methodology. 


\begin{tabular}{|l|l|l|}
\hline Order & Computed rate & Expected rate \\
\hline \hline 1 & -0.31 & -0.33 \\
\hline 2 & -0.74 & -0.67 \\
\hline 3 & -1.25 & -1 \\
\hline 4 & -1.4 & -1.33 \\
\hline
\end{tabular}

Table 5.7: AC elasticity stage, hollow cylinder subject to body force: rates of convergence for the relative error $\left\|\boldsymbol{u}_{h p}^{D C}-\boldsymbol{u}_{\text {exact }}\right\|_{H^{1}(\Omega)} /\left\|\boldsymbol{u}_{\text {exact }}\right\|_{H^{1}(\Omega)}$ under $h$-refinement.

\subsubsection{Conducting and Elastic Sphere in a Uniform Alternating Mag- netic Field}

The case of a conducting elastic sphere $\Omega_{C}:=\{\boldsymbol{x}:|\boldsymbol{x}| \leqslant R\}$, placed in an unbounded region of free space $\Omega_{C}^{c}$, where $\mathcal{H}_{0}^{A C}$ tends to a time harmonic uniform field far from the object, is now considered. Unlike the previous examples this does not have an analytical solution. The sphere has a radius $R=1 \mathrm{~cm}$ and material properties $\mu=\mu_{0}, \gamma=6 \times$ $10^{7} \mathrm{~S} / \mathrm{m}, \rho=7800 \mathrm{~kg} / \mathrm{m}^{3}, E=10^{8} \mathrm{~Pa}$ and $\nu=0.3$.

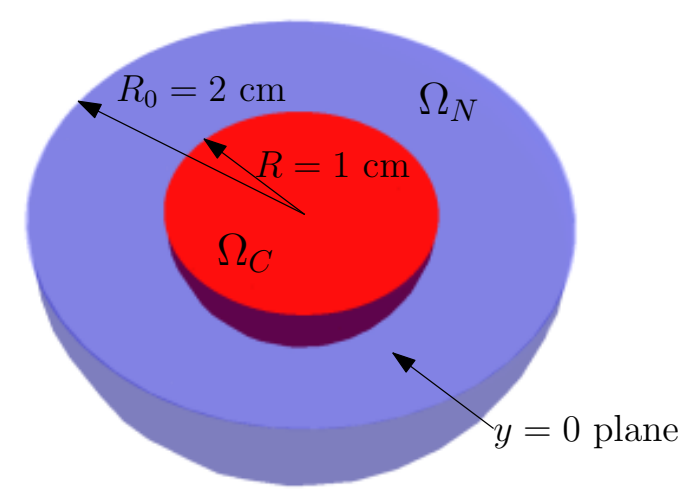

Figure 5.20: Conducting and elastic sphere in a uniform alternating magnetic field: illustration of the hemisphere used for the computations.

For computational purposes, the domain was truncated at a finite distance from the conducting sphere. In particular, the truncation was chosen to be at a radius of $R_{0}=2 \mathrm{~cm}$ from the centre of the sphere. The geometry was further simplified by exploiting the symmetry of the problem about $\boldsymbol{e}_{z}$, which reduces $\Omega$ to a hemisphere with outer radius $R_{0}$. An illustration of the reduced domain is provided in Figure 5.20. On the truncation boundary, the approximate condition $\boldsymbol{n} \times \boldsymbol{A}_{\varepsilon, h q}^{A C}=\boldsymbol{n} \times \mathcal{A}_{\text {exact }}$, where $\boldsymbol{A}_{\text {exact }}$ is the analytical solution for the corresponding non-elastic sphere proposed by Smythe [164], as also used in Section 5.2.3, is imposed. On the symmetry boundaries, the conditions

$$
\begin{aligned}
\left(\boldsymbol{\sigma}^{m}\left(\boldsymbol{u}_{h p}^{A C}\right) \boldsymbol{n}\right) \times \boldsymbol{n} & =\mathbf{0}, \\
\boldsymbol{u}_{h p}^{A C} \cdot \boldsymbol{n} & =0, \\
\boldsymbol{A}_{\varepsilon, h q}^{A C} \times \boldsymbol{n} & =\mathbf{0},
\end{aligned}
$$


are imposed, which follow from the symmetric nature of the problem (see Section 4.9). The regularisation parameter was chosen to be $\varepsilon=10^{-6}$.

As discussed in Section 4.3.5, different combinations for the element orders $p$ and $q$ can be chosen, provided that the discretisation for each field is sufficient to resolve accurately both the electromagnetic and mechanical fields. To illustrate this, a frequency $f=50 \mathrm{~Hz}$ is considered, and the aforementioned sphere is discretised by a mesh of 2319 tetrahedral elements. The converged results for the order combinations $p=q$, $p=q-1$ and $p=q+1$ are then studied (Note that the lowest order combinations, i.e. $p=q=1, p=1, q=2$ and $p=1, q=0$, were considered as the starting polynomial orders and then both $p$ and $q$ were increased uniformly until convergence was reached). In particular, Figure 5.21 shows a plot of $\left|\operatorname{Re}\left(\mathcal{B}_{0, z, h q}^{A C}\right)\right|$ and $\left|\operatorname{Re}\left(\boldsymbol{u}_{h p}^{A C}\right)\right|$ along the line $\{-0.01<x<0.01, y=-0.001, z=0\}$ for the converged solution using the three different combinations resulting in identical results on this scale.

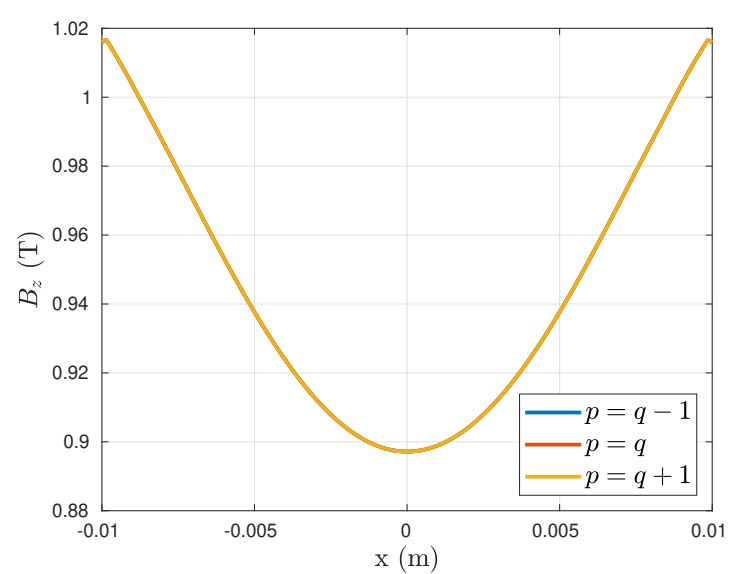

(a) $\left|\operatorname{Re}\left(\mathcal{B}_{0, z, h q}^{A C}\right)\right|$

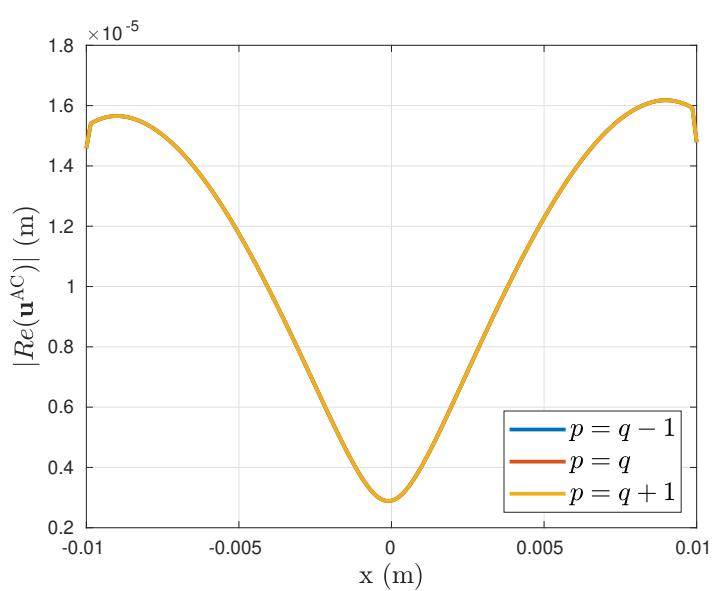

(b) $\left|\operatorname{Re}\left(\boldsymbol{u}_{h p}^{A C}\right)\right|$

Figure 5.21: Conducting and elastic sphere in a uniform alternating magnetic field: comparison of the solution for different $p, q$ combinations.

For the aforementioned conducting sphere, Algorithm 1 is applied over a sweep of frequencies from $f=1 \mathrm{~Hz}$ to $f=4500 \mathrm{~Hz}$. For this sweep, and in anticipation of outputs of interest for MRI configurations, the output (dissipated) power $P_{\Omega}^{0}\left(\omega, \boldsymbol{A}_{\varepsilon, h q}^{A C}, \boldsymbol{B}_{0, h q}^{D C}, \boldsymbol{u}_{h p}^{A C}\right)$ and the kinetic energy $E_{\Omega}^{k}\left(\omega, \boldsymbol{u}_{h p}^{A C}\right)$, which are defined in a form suitable for measurement as

$$
\begin{aligned}
& P_{\Omega}^{0}=\frac{1}{2} \int_{\Omega} \gamma\left|\boldsymbol{E}_{h p q}^{A C}\right|^{2} \mathrm{~d} \Omega=\frac{1}{2} \int_{\Omega} \gamma\left|-\mathrm{i} \omega \boldsymbol{A}_{\varepsilon, h q}^{A C}+\mathrm{i} \omega \boldsymbol{B}_{0, h q}^{D C} \times \boldsymbol{u}_{h p}^{A C}\right|^{2} \mathrm{~d} \Omega, \\
& E_{\Omega}^{k}=\frac{1}{2} \int_{\Omega} \rho \omega^{2}\left|\boldsymbol{u}_{h p}^{A C}\right|^{2} \mathrm{~d} \Omega,
\end{aligned}
$$

were computed, and the results are shown in Figure 5.22 for the aforementioned mesh and elements of order $p=4$ and $q=5$, for all frequencies in the sweep. This combination of $p, q$ is based on the observation that higher order was needed in order to accurately resolve the eddy currents compared to the displacements at high frequencies. At lower 
frequencies it represents an overkill solution. The order of approximation for the geometry was set to $g=2$. The result obtained with the Eulerian formulation proposed in $[19,17]$ (see Section 2.6) is also included to compare both formulations. Note that, for the Eulerian approach, a small mechanical damping has been applied to the system to remove numerical artefacts leading to non physical peaks. To apply the damping, a frequency $f=\omega / 2 \pi=2960 \mathrm{~Hz}$ was selected, corresponding to the resonant peak, and (4.61) was applied with $\xi=5.3 \times 10^{-3}$ to obtain $\alpha_{M}=200$, which is then used for all frequencies in the sweep. The axisymmetric software [18, 19], using a monolithic instead of a fixed point solver for the Eulerian formulation, is also shown as a further means of comparison. This software being applicable, in this case, due to the symmetry of the problem. Furthermore, the previous Eulerian axisymmetric solver [19, 18, 17] has also been adapted to perform the Lagrangian approach, described by Algorithm 1, and these results are included in the figure. For both the Eulerian and Lagrangian approaches, the axisymmetric software is in good agreement with the results of the new 3D solver. In this problem, and for the considered frequency range, no significant differences between both approaches are observed.

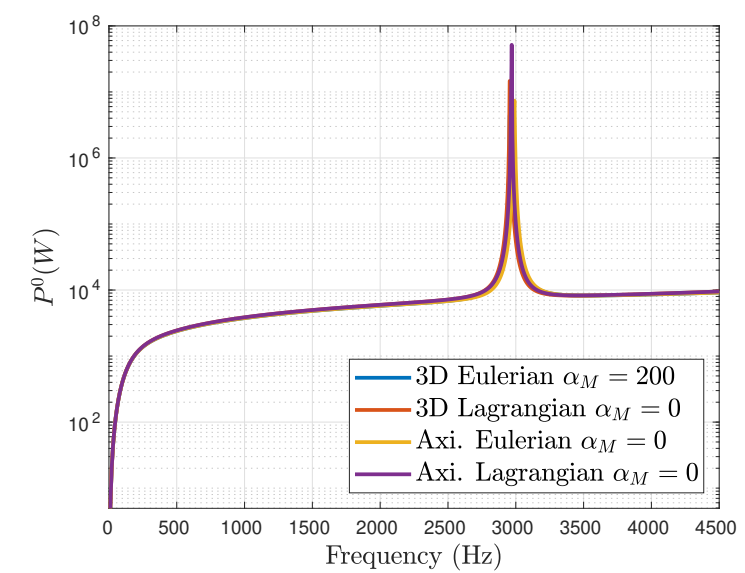

(a) Dissipated Power

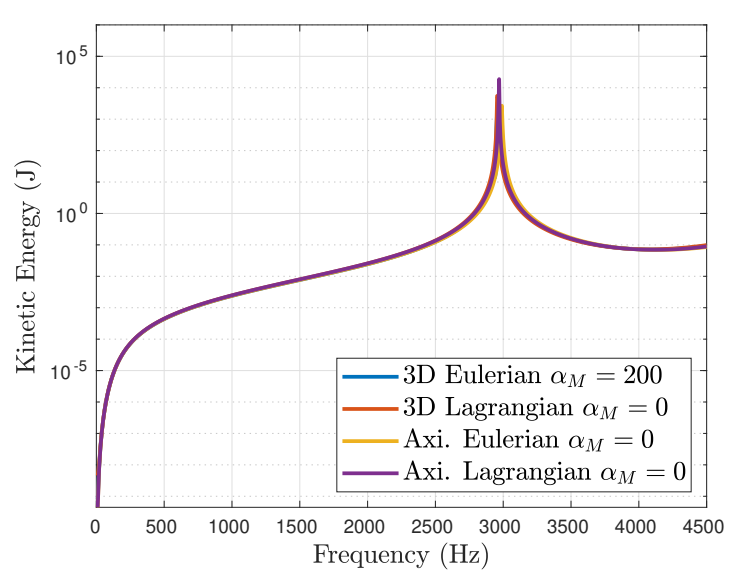

(b) Kinetic Energy

Figure 5.22: Conducting and elastic sphere in a uniform alternating magnetic field: dissipated power and kinetic energy in the conducting sphere.

In order to better illustrate the physical behaviour of the problem, a contour plot of $\left|\operatorname{Re}\left(\boldsymbol{J}_{h p q}^{o}\right)\right|$ in the conducting sphere and $\left|\operatorname{Re}\left(\boldsymbol{B}_{0, h q}^{A C}\right)\right|$ in the surrounding air is presented in Figure 5.23. Here, as in (5.6a), $\boldsymbol{g}^{o}=\gamma \boldsymbol{E}^{A C}$ is approximated as $\boldsymbol{g}_{h p q}^{o}=\gamma\left(-\mathrm{i} \omega \boldsymbol{A}_{\varepsilon, h q}^{A C}+\right.$ $\left.\mathrm{i} \omega \boldsymbol{B}_{0, h q}^{D C} \times \boldsymbol{u}_{h p}^{A C}\right)$, corresponding to Ohmic type currents in an Eulerian frame. Streamlines of the magnetic flux density in the air are also included.

Finally, as a further illustration of the coupled physical behaviour, the deformed sphere for a set of different frequencies $f=\{2000,2500,3500,4000\} \mathrm{Hz}$ is shown in Figure 5.24 . 

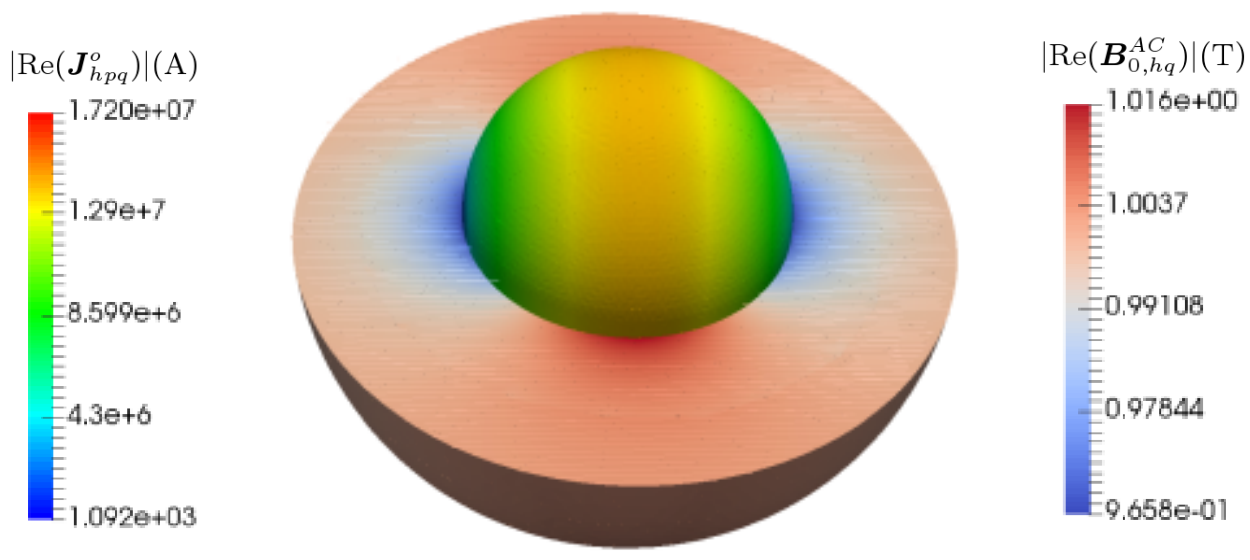

Figure 5.23: Conducting and elastic sphere in a uniform alternating magnetic field: $\left|\operatorname{Re}\left(\boldsymbol{J}_{h p q}^{o}\right)\right|$ contours in the conducting sphere and $\left|\operatorname{Re}\left(\boldsymbol{B}_{0, h q}^{A C}\right)\right|$ contours and $\operatorname{Re}\left(\boldsymbol{B}_{0, h q}^{A C}\right)$ streamlines in the air.

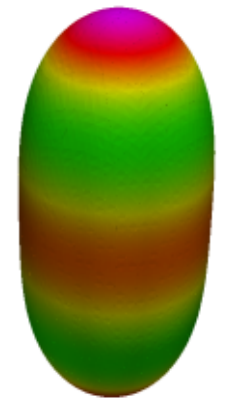

(a) $f=2000 \mathrm{~Hz}$

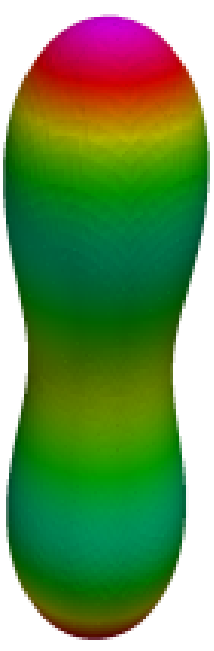

(b) $f=2500 \mathrm{~Hz}$

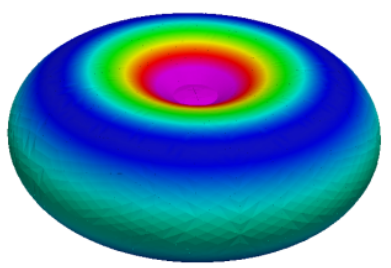

(c) $f=3500 \mathrm{~Hz}$

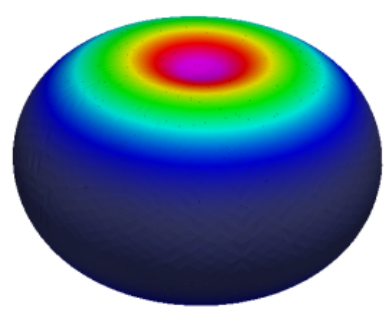

(d) $f=4000 \mathrm{~Hz}$

Figure 5.24: Conducting and elastic sphere in a uniform alternating magnetic field: contours of $\left|\operatorname{Re}\left(\boldsymbol{u}_{h p}^{A C}\right)\right|$ on the deformed sphere for different frequencies. Deformation magnitude magnified by a factor of 30 .

\subsubsection{Test Magnet Problem with $z$ (Longitudinal) Gradient Coil}

The second coupled benchmark problem considered consists of an MRI configuration, where the geometry has been simplified so that it is rotationally symmetric. In addition, the non-rotationally symmetric $x$ and $y$ gradient coils have been removed leaving only the $z$ gradient coil. The configuration of the problem is shown in Figure 5.25, where the main coils are shown in red, the gradient coils in blue, and the different radiation shields $(4 \mathrm{~K}$, $77 \mathrm{~K}$ and OVC) in different green tones. Note that the 3D geometry (Figure 5.25b), can be obtained by rotating the $r-z$ plane (Figure 5.25a), through $0 \leqslant \phi<2 \pi$ rad about the $z$ axis (where $(r, \phi, z)$ are cylindrical coordinates). For confidentiality reasons the exact 
dimensions and material properties cannot be stated. However, the order of magnitude can be given as an indication; the thickness of the radiation shields is of the order of a few millimetres and their length is of the order of a metre. The conductivity of the shields is of order $10^{6}-10^{7} \mathrm{~S} / \mathrm{m}$, the Young modulus of order $10^{10}-10^{11} \mathrm{~Pa}$, the Poisson ratio is $\nu \approx 0.3,\left|\boldsymbol{J}^{D C}\right|$ is of order $10^{8} \mathrm{~A}$ and $\left|\boldsymbol{g}^{A C}\right|$ of order $10^{6} \mathrm{~A}$.

This problem was previously simulated in $[19,17]$ using an axisymmetric software and the Eulerian approach. In this section, the focus is the 3D simulation of this problem using the Lagrangian approach. The same boundary conditions as in $[19,17]$ are considered, and $\boldsymbol{u}_{h p}^{A C}=\mathbf{0}$ is imposed in a small segment of the conductor's surface as illustrated in Figure 5.25a.

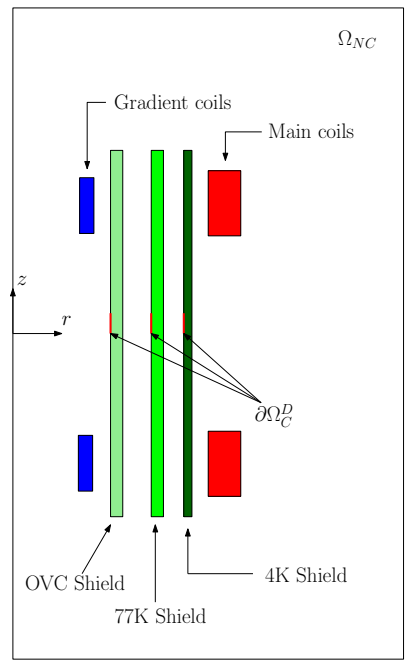

(a) $2 \mathrm{D}$ cross-section

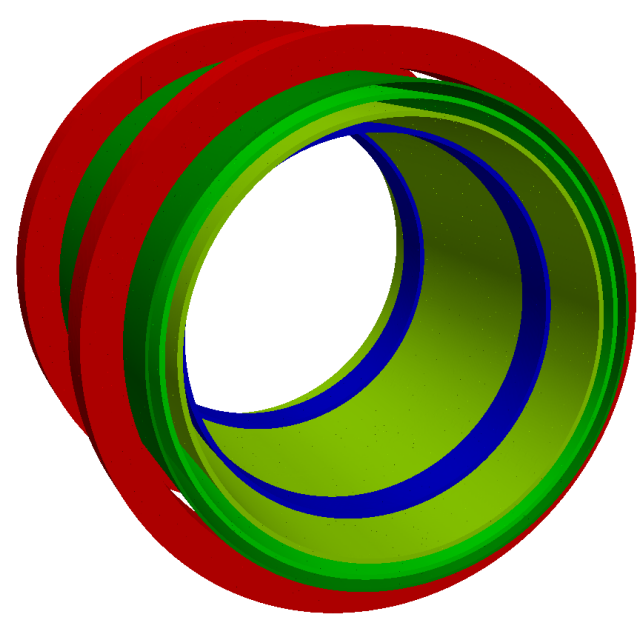

(b) $3 \mathrm{D}$ view

Figure 5.25: Test magnet problem with $z$ (longitudinal) gradient coil: illustration of the components of the problem in the axisymmetric meridian plane (left) and 3D view (right).

The domain $\Omega$ is formed by truncation of $\Omega_{C}^{c}$ by a cylinder of radius $R_{0}=0.9 \mathrm{~m}$ and length $L_{0}=2.4 \mathrm{~m}$ centred about the magnet. This problem is axisymmetric, but in order to illustrate the performance of the 3D solver, an arbitrary choice such that $\Omega$ is reduced to one quarter of the original geometry is made, as shown in Figure 5.26, and the symmetry boundary conditions (5.5) are applied on the symmetry boundaries. Algorithm 1 is then applied to obtain the computational Lagrangian solution.

A frequency sweep from 1 to $5000 \mathrm{~Hz}$ is performed in order to observe the variation of the output power $P_{\Omega}^{0}\left(\omega, \boldsymbol{A}_{\varepsilon, h q}^{A C}, \boldsymbol{B}_{0, h q}^{D C}, \boldsymbol{u}_{h p}^{A C}\right)$ and the kinetic energy $E_{\Omega}^{k}\left(\omega, \boldsymbol{u}_{h p}^{A C}\right)$. For this, a mesh of 33805 tetrahedral elements (with 9974 elements in $\Omega_{C}$ ) is generated and different polynomial orders $q$ and $p=q+1$ applied. This mesh was generated by locally refining the elements in the conducting shields in order to accurately resolve the fields in this region and paying attention to avoid issues with the generation of highly stretched tetrahedrons. A quadratic geometry approximation was used throughout. The case of a system without damping is analysed first, followed by a damped system according to the 


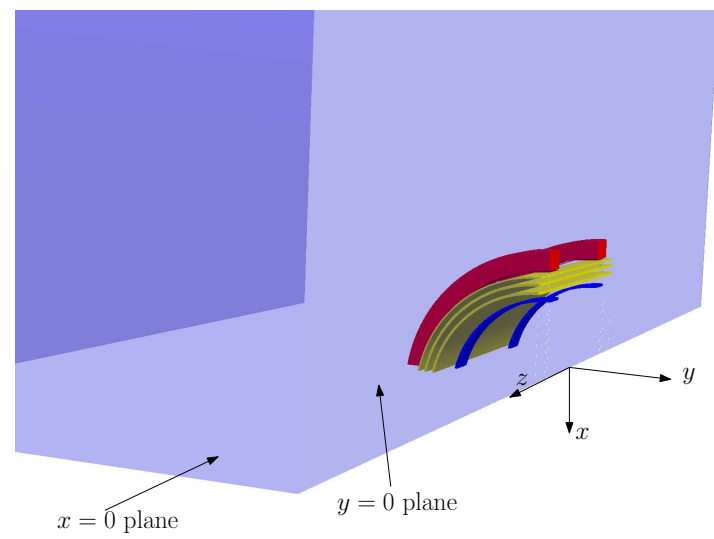

Figure 5.26: Test magnet problem with $z$ (longitudinal) gradient coil: quarter of the geometry used for the simulations exploiting the symmetry of the problem.

approach in Section 4.6. In the latter, $\xi=4.5 \times 10^{-4}$ corresponds to the first resonant frequency $f=3515 \mathrm{~Hz}$ of the system. In a similar way to Section 5.3.1, by applying (4.61) with $\xi=4.5 \times 10^{-4}$ at this resonant peak, an $\alpha_{M}=20$ is obtained, which is then applied for all frequencies in the sweep. The regularisation parameter was chosen to be $\varepsilon=10^{-4}$ and the tolerance of the PGMRES solver was set to $10^{-5}$.

Figure 5.27 shows the convergence of the dissipated power and kinetic energy under $p$ refinement for the undamped system. For this, the lowest possible combination, $q=0$ and $p=1$, was considered as the starting point and the polynomial orders $q$ and $p$ were then uniformly increased until convergence was reached. It can be observed that the solution is effectively converged using $q=3$ elements, but there are still some differences between the results with $q=3$ and $q=4$ in the $4 \mathrm{~K}$ shield. Nevertheless, as it will be shown later, the result achieved with $q=4$ is in perfect agreement with the converged result in the case of a system with damping and that of the axisymmetric software using the Lagrangian approach.

The simulations were then repeated for a system with damping leading to Figure 5.28 for the $p$-refinement analysis. Notice how the addition of a small amount of damping leads to a faster convergence of the frequency sweeps under $p$-refinement compared to the undamped case.

Next, in Figure 5.29, the converged 3D results are compared with those obtained with the axisymmetric code $[19,17]$ employing both the Eulerian and the Lagrangian approaches as well as with the commercial software NACS [4] (only for the dissipated power), which uses low order elements. An excellent agreement between the 3D and axisymmetric results for the Lagrangian approach is observed. The axisymmetric results using the Eulerian approach are in good agreement with the Lagrangian approach for the OVC shield and for the $77 \mathrm{~K}$ shield in the case of small frequencies. But, significant differences between the two approaches are observed in the $77 \mathrm{~K}$ shield for high frequencies and in the $4 \mathrm{~K}$ shield for almost all frequencies. The reason for these differences can be attributed to the stronger coupling and larger velocities and accelerations present in the shields at these frequencies, which mean that the approximations in the Eulerian approach used in 


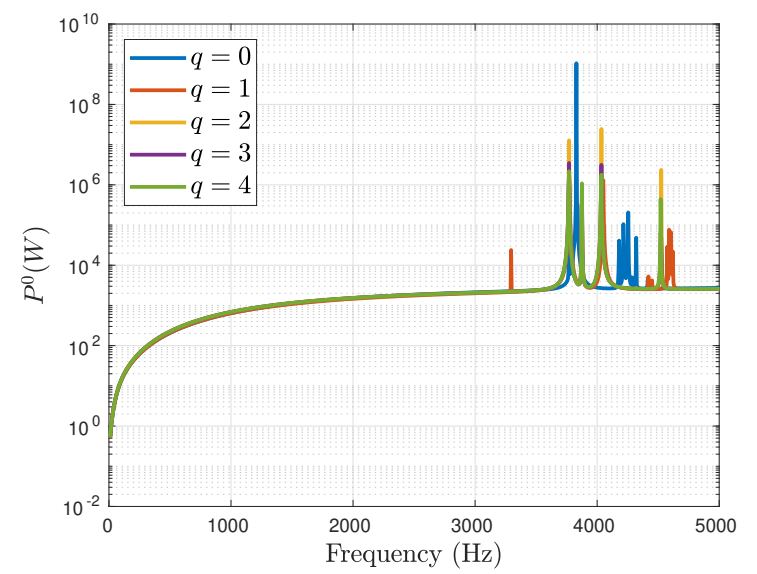

(a) $P_{\Omega_{O V C}}^{0}\left(\omega, \boldsymbol{A}_{\varepsilon, h q}^{A C}, \boldsymbol{B}_{0, h q}^{D C}, \boldsymbol{u}_{h p}^{A C}\right)$

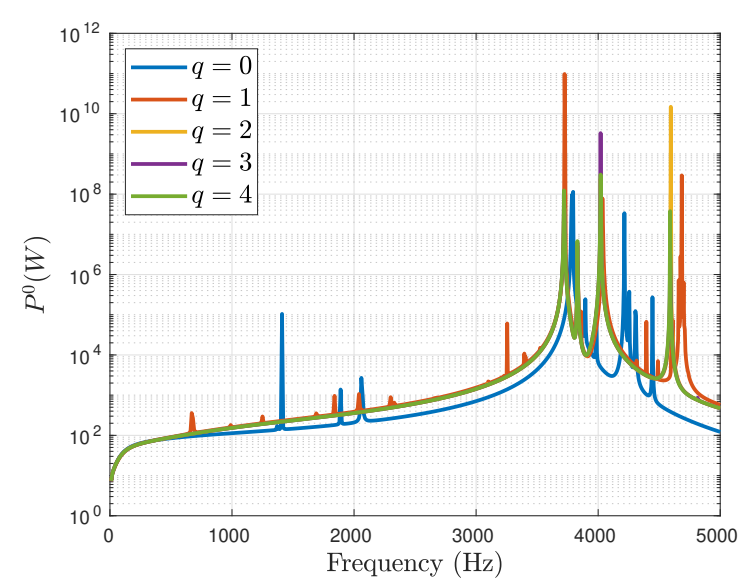

(c) $P_{\Omega_{77 K}}^{0}\left(\omega, \boldsymbol{A}_{\varepsilon, h q}^{A C}, \boldsymbol{B}_{0, h q}^{D C}, \boldsymbol{u}_{h p}^{A C}\right)$

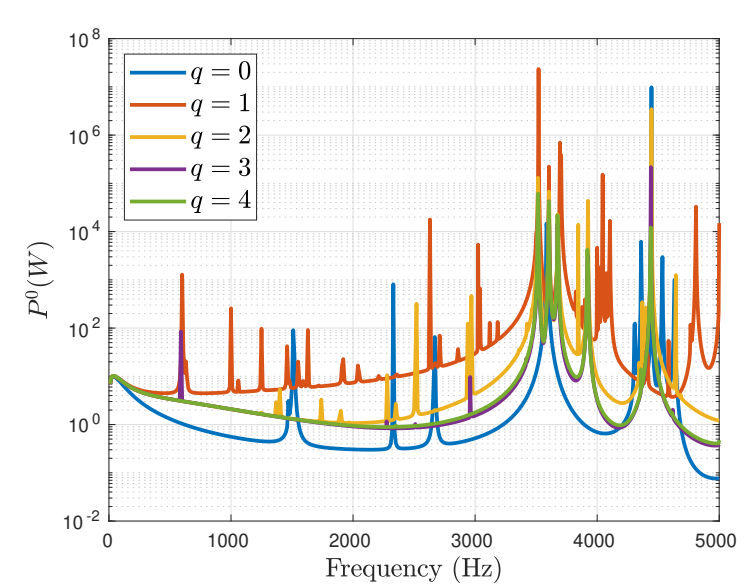

(e) $P_{\Omega_{4 K}}^{0}\left(\omega, \boldsymbol{A}_{\varepsilon, h q}^{A C}, \boldsymbol{B}_{0, h q}^{D C}, \boldsymbol{u}_{h p}^{A C}\right)$

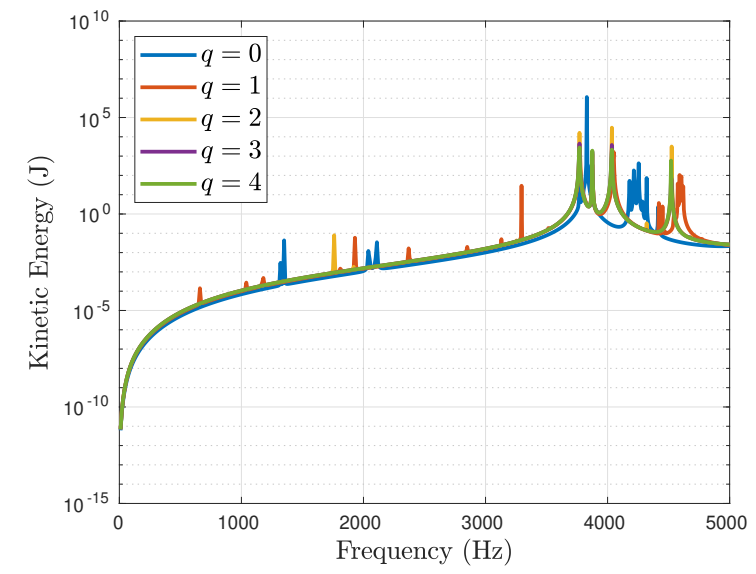

(b) $E_{\Omega_{O V C}}^{k}\left(\omega, \boldsymbol{u}_{h p}^{A C}\right)$

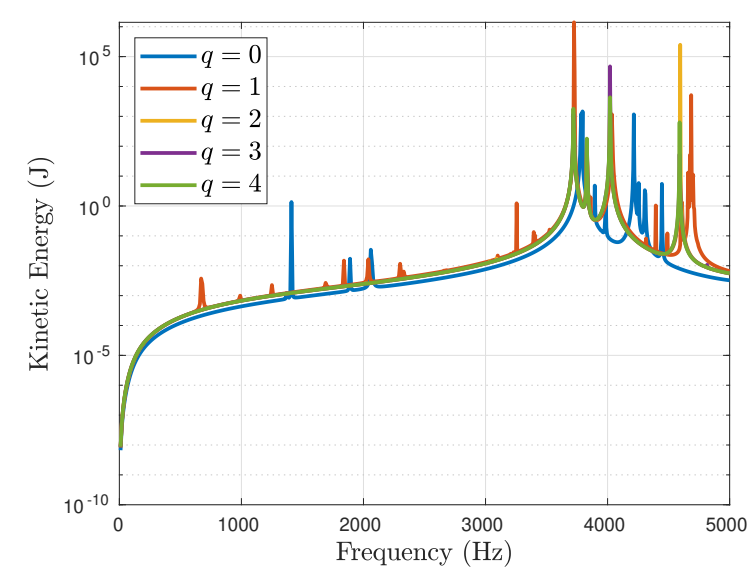

(d) $E_{\Omega_{77 K}}^{k}\left(\omega, \boldsymbol{u}_{h p}^{A C}\right)$

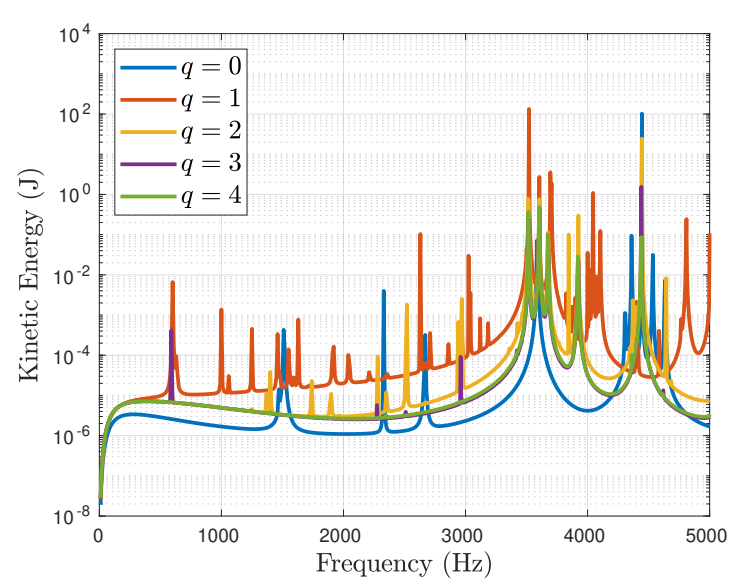

(f) $E_{\Omega_{4 K}}^{k}\left(\omega, \boldsymbol{u}_{h p}^{A C}\right)$

Figure 5.27: Test magnet problem with $z$ (longitudinal) gradient coil: convergence of the dissipated power and kinetic energy in the radiation shields under $p$-refinement. Undamped system $\left(\alpha_{M}=0\right)$. 


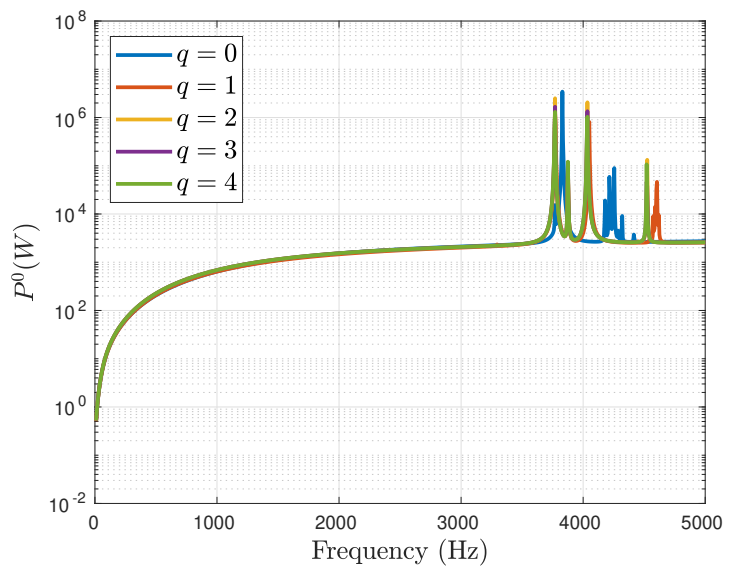

(a) $P_{\Omega_{O V C}}^{0}\left(\omega, \boldsymbol{A}_{\varepsilon, h q}^{A C}, \boldsymbol{B}_{0, h q}^{D C}, \boldsymbol{u}_{h p}^{A C}\right)$

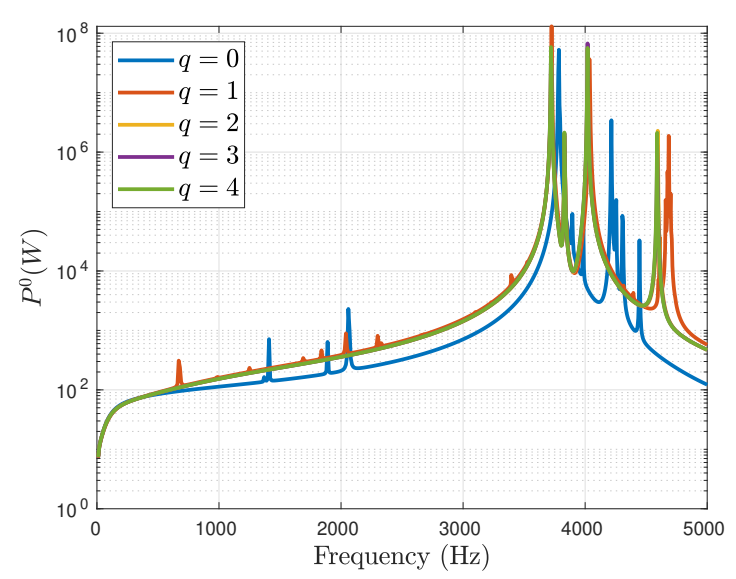

(c) $P_{\Omega_{77 K}}^{0}\left(\omega, \boldsymbol{A}_{\varepsilon, h q}^{A C}, \boldsymbol{B}_{0, h q}^{D C}, \boldsymbol{u}_{h p}^{A C}\right)$

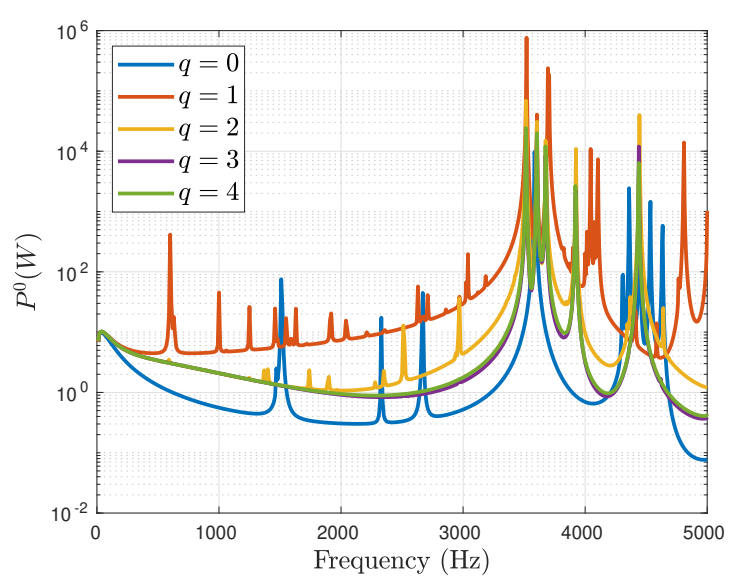

(e) $P_{\Omega_{4 K}}^{0}\left(\omega, \boldsymbol{A}_{\varepsilon, h q}^{A C}, \boldsymbol{B}_{0, h q}^{D C}, \boldsymbol{u}_{h p}^{A C}\right)$

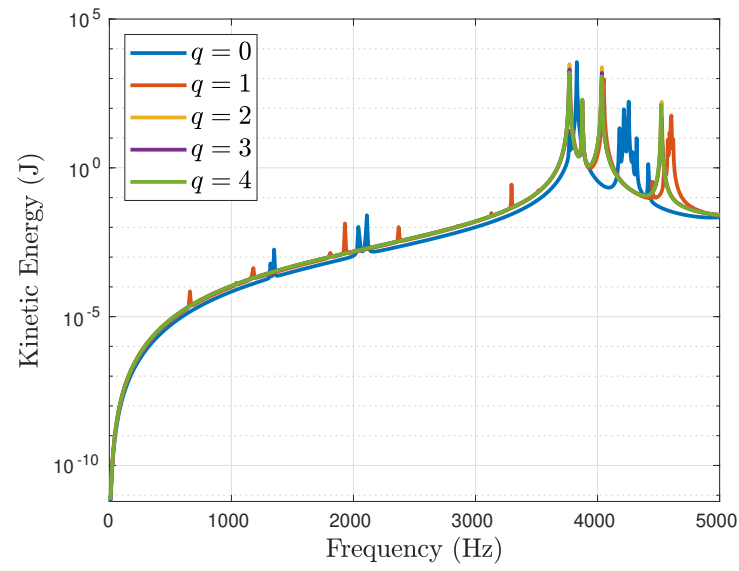

(b) $E_{\Omega_{O V C}}^{k}\left(\omega, \boldsymbol{u}_{h p}^{A C}\right)$

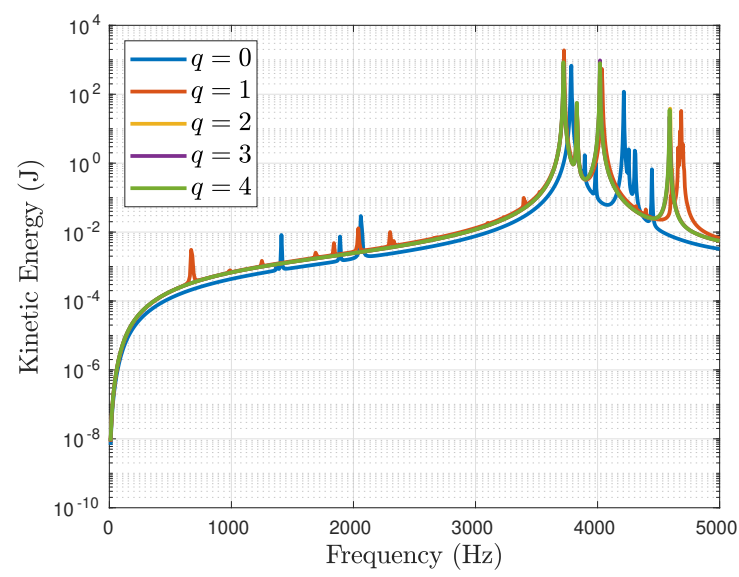

(d) $E_{\Omega_{77 K}}^{k}\left(\omega, \boldsymbol{u}_{h p}^{A C}\right)$

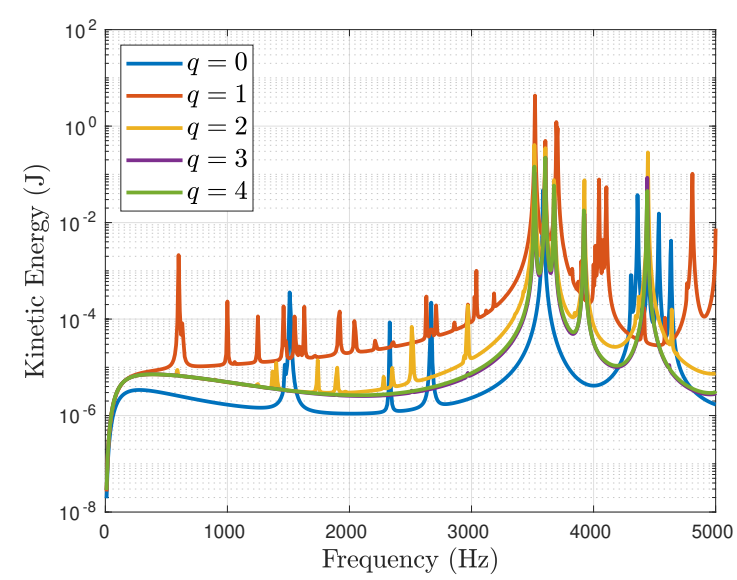

(f) $E_{\Omega_{4 K}}^{k}\left(\omega, \boldsymbol{u}_{h p}^{A C}\right)$

Figure 5.28: Test magnet problem with $z$ (longitudinal) gradient coil: convergence of the dissipated power and kinetic energy in the radiation shields under $p$-refinement. Damped system $\left(\alpha_{M}=20\right)$. 
$[19,17,18]$ break down. The results obtained with NACS software are in good agreement with the Lagrangian approach in the case of the OVC shield, but differ significantly in the $77 \mathrm{~K}$ and $4 \mathrm{~K}$ shields. It can be conjectured that this is due to the inability of low order elements to accurately resolve the complex coupling mechanisms involved in the problem. Note that the $77 \mathrm{~K}$ and $4 \mathrm{~K}$ shields have a bigger conductivity and, therefore, smaller skin depths compared to the OVC shield.

In terms of computational cost, the Lagrangian approach offers considerable advantages over the Eulerian approach since it requires the solution of two small systems as opposed to one larger monolithic system (e.g. for $q=4, p=5 P_{\text {global }}=1,277,370$ and $Q_{\text {global }}=751,245$ compared to an Eulerian which would involve a $P_{\text {global }}+Q_{\text {global }}$ sized system for each frequency). Furthermore, as described in Section 4.3.5, the matrices in the Lagrangian approach have nicer properties compared to the indefinite system that would need to be solved for the Eulerian approach, which allow preconditioned iterative solvers to be applied.

In order to illustrate the physical behaviour of the problem, the eddy currents in the deformed $77 \mathrm{~K}$ shield are shown in Figure 5.30 together with the streamlines of $\operatorname{Re}\left(\boldsymbol{B}_{0, h q}^{A C}\right)$ and contours of its magnitude in the air. These results are shown for two different frequencies, $f=10$ and $f=1000 \mathrm{~Hz}$, to show how the magnitude of the eddy currents increases and the skin depth effect becomes visible for the higher frequency. It can also be observed that the deformation increases with the eddy currents.

Finally, in Figure 5.31, a contour plot of $\left|\operatorname{Re}\left(\boldsymbol{u}_{h p}^{A C}\right)\right|$ in the deformed $4 \mathrm{~K}$ shield is shown, together with the magnetic flux density streamlines in the surrounding air. The result is shown for a frequency $f=1000 \mathrm{~Hz}$ and the different snapshots correspond to different times. Note that for a given frequency, the temporal solution can be recovered from (3.38).

\section{Effect of Damping and Boundary Conditions}

In order to illustrate the effect that the damping and boundary conditions have in the solution, the dissipated power and kinetic energy in the radiation shields are shown in Figure 5.32 for $\alpha_{M}=20,100,200,400$ when the boundary condition $\boldsymbol{u}_{h p}^{A C}=\mathbf{0}$ is imposed at the ends of the shields, as illustrated in Figure 5.33. Note how the position and even the number of peaks have changed from the previous choice of boundary conditions (Figure 5.29) to the current choice. However, the choice of $\alpha_{M}$ does not change the position of the peaks and it only modifies their amplitude.

Note that so far we have only considered a damping model where we use a fixed $\alpha_{M}$ for all the frequencies. A different option would consist in choosing a constant damping ratio $\xi$ and computing the appropriate $\alpha_{M}$ for each frequency according to equation (4.61). The former is a bit more closer to reality, as typically in real systems the damping ratio changes with frequency. However, it may result in some of the peaks (especially at lower frequencies) being overdamped, whilst in the latter, provided that we choose $\xi<1$, all the peaks will be underdamped and, thus, all of them will be visible in the solution. Since in this thesis damping is only considered in order to avoid the solution becoming singular 


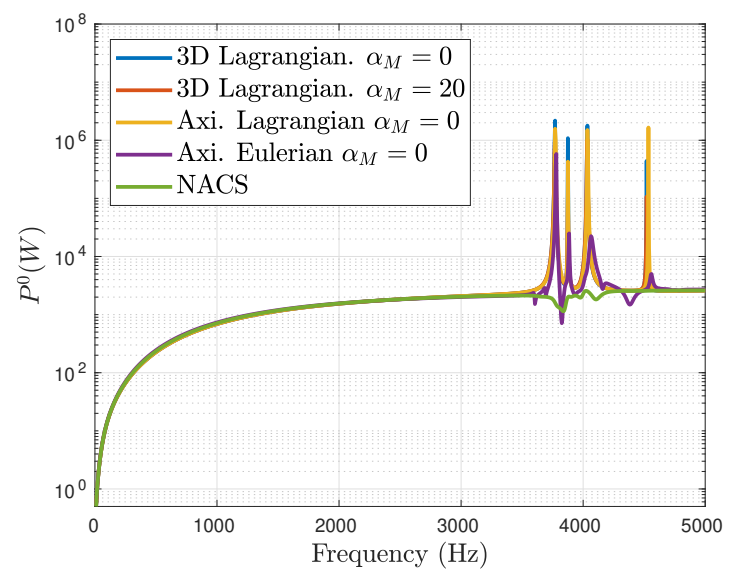

(a) $P_{\Omega_{O V C}}^{0}\left(\omega, \boldsymbol{A}_{\varepsilon, h q}^{A C}, \boldsymbol{B}_{0, h q}^{D C}, \boldsymbol{u}_{h p}^{A C}\right)$

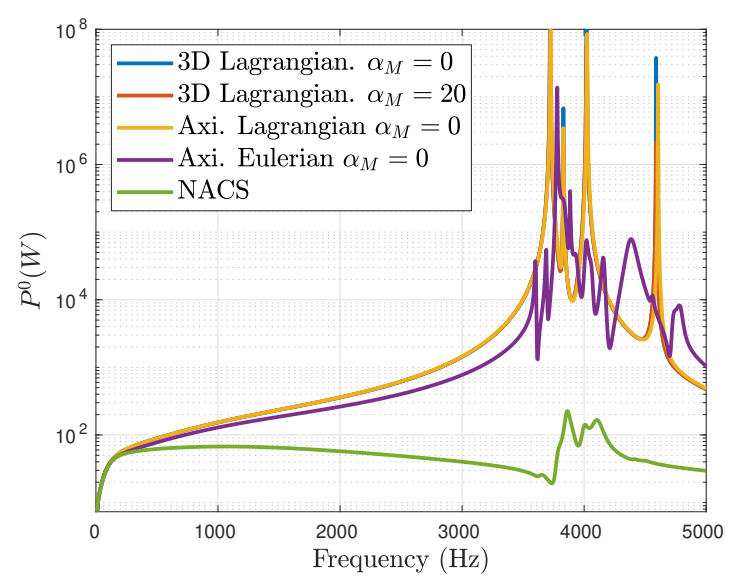

(c) $P_{\Omega_{77 K}}^{0}\left(\omega, \boldsymbol{A}_{\varepsilon, h q}^{A C}, \boldsymbol{B}_{0, h q}^{D C}, \boldsymbol{u}_{h p}^{A C}\right)$

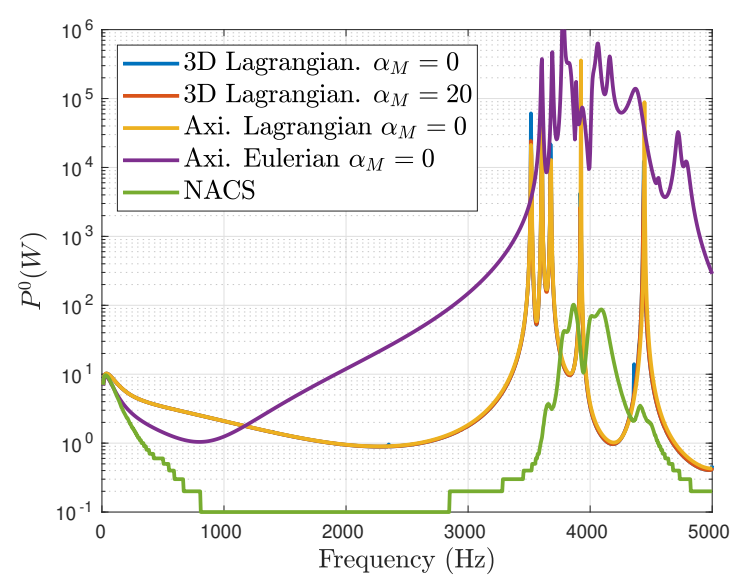

(e) $P_{\Omega_{4 K}}^{0}\left(\omega, \boldsymbol{A}_{\varepsilon, h q}^{A C}, \boldsymbol{B}_{0, h q}^{D C}, \boldsymbol{u}_{h p}^{A C}\right)$

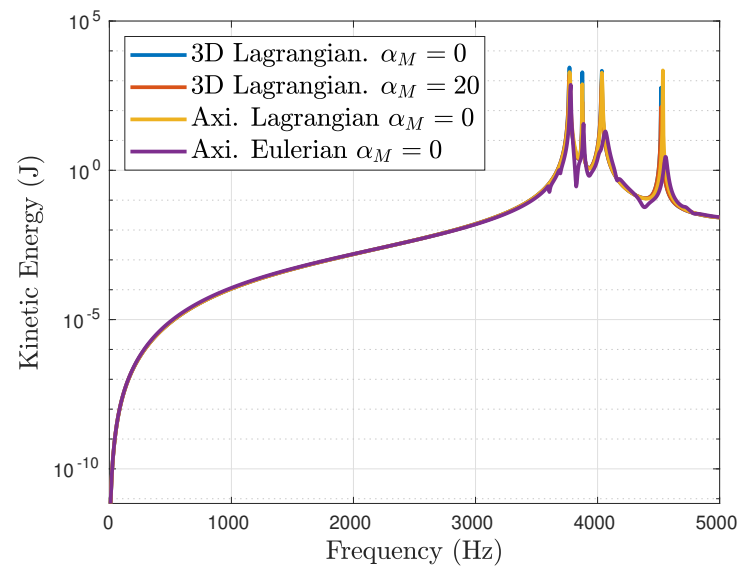

(b) $E_{\Omega_{O V C}}^{k}\left(\omega, \boldsymbol{u}_{h p}^{A C}\right)$

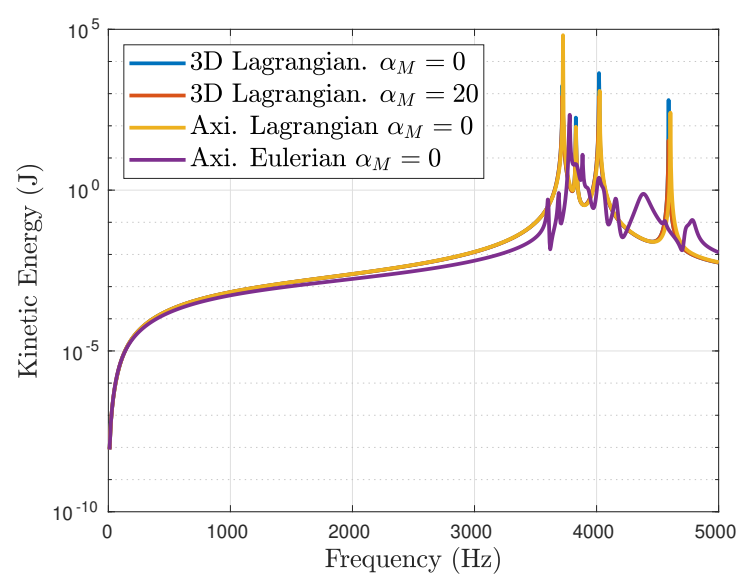

(d) $E_{\Omega_{77 K}}^{k}\left(\omega, \boldsymbol{u}_{h p}^{A C}\right)$

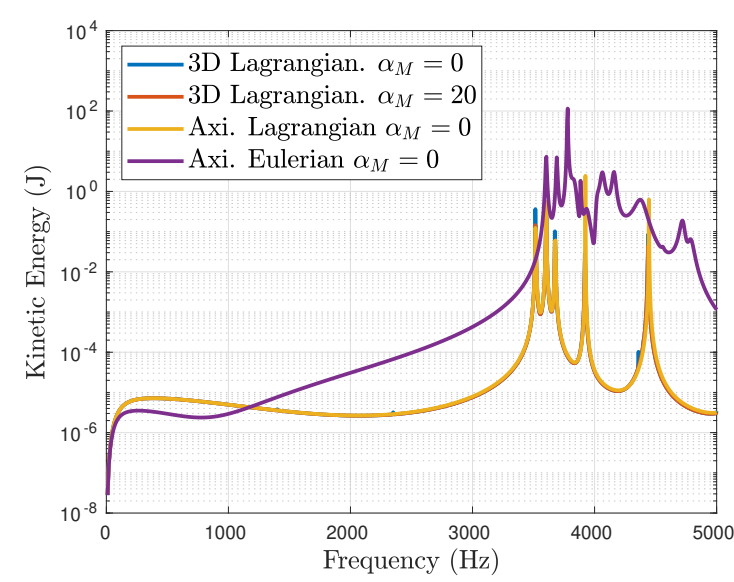

(f) $E_{\Omega_{4 K}}^{k}\left(\omega, \boldsymbol{u}_{h p}^{A C}\right)$

Figure 5.29: Test magnet problem with $z$ (longitudinal) gradient coil: Dissipated power and kinetic energy in the radiation shields. Comparison between different approaches.

at the resonant frequencies (and to enhance a faster convergence), and building a realistic damping model is out of the scope of this work, both options will be considered. To 


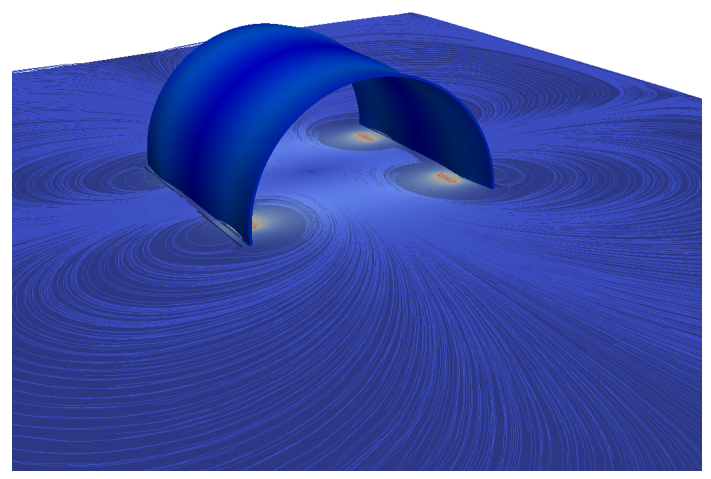

(a) $f=10 \mathrm{~Hz}$

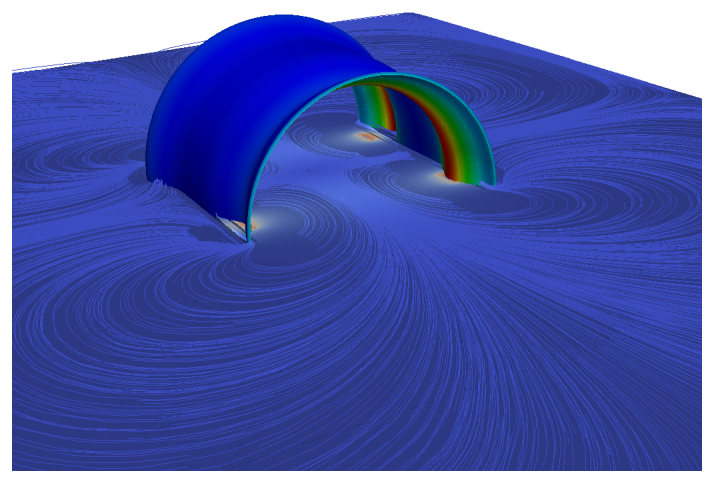

(b) $f=1000 \mathrm{~Hz}$

Figure 5.30: Test magnet problem with $z$ (longitudinal) gradient coil: $\left|\operatorname{Re}\left(\boldsymbol{g}_{h p q}^{o}\right)\right|$ in the deformed $77 \mathrm{~K}$ shield and $\left|\operatorname{Re}\left(\boldsymbol{B}_{0, h q}^{A C}\right)\right|$ contours and $\operatorname{Re}\left(\boldsymbol{B}_{0, h q}^{A C}\right)$ streamlines in the background air for two different frequencies.

illustrate the second option, the plots shown in Figure 5.32 for different constant $\alpha_{M}$ are repeated in Figure 5.34 for different constant damping ratios $\xi=10^{-4}, 10^{-3}, 10^{-2}$. It can be observed that in this case there is no significant difference between both damping models.

\section{Choice of $p, q$ combination}

For the $p$-refinement analysis shown in Figures 5.27 and 5.28, the starting point was the lowest possible order for $p$ and $q$, which corresponds to $q=0, p=1$, and both $p$ and $q$ were then uniformly increased until convergence was reached. However, as discussed in Section 4.3.5, there is freedom in the choice of $p$ and $q$, and the combination $p=q+1$ is not necessarily the optimum choice. To illustrate this, the dissipated power and kinetic energy in the radiation shields are shown in Figure 5.35 for the cases $q=p=3$ and $q=3, p=4$. The shields were fixed in the ends and a constant $\alpha_{M}=400$ was applied for all frequencies in the sweep. It can be observed that the solution is identical for both cases, which means that for this problem $p=q$ is actually a better choice than $p=q+1$ as with the former a converged solution is achieved using less degrees of freedom than with the latter. The optimum choice is problem dependant and it is, in general, difficult to determine a priori. One option to find the best choice is to compute the solution for a reduced number of frequencies (especially for higher frequencies) using different $p, q$ combinations and look at some plots of the solution (eddy currents, displacements or magnetic fields among others) to identify which combination results in a converged solution for the fewest degrees of freedom at the selected frequencies. By doing this, the optimum choice for this problem was found to be $p=q$. 


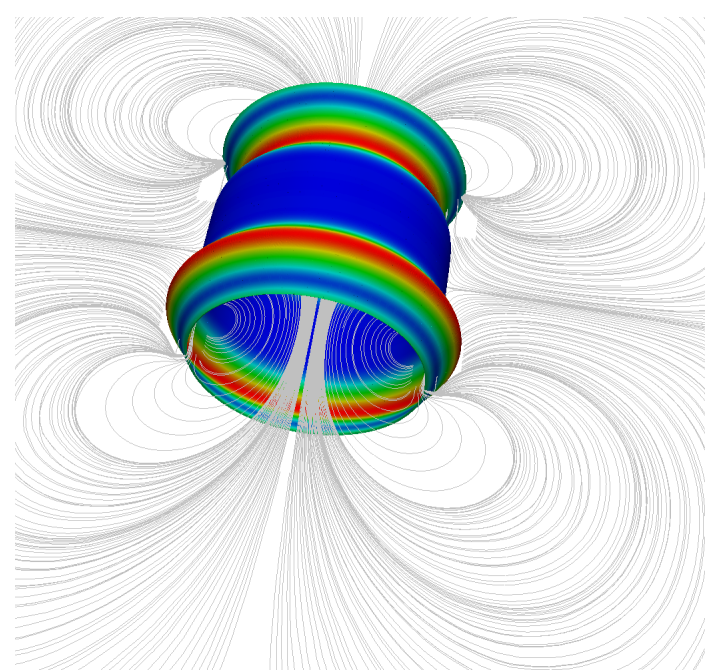

(a) $t=0 \mathrm{~ms}$

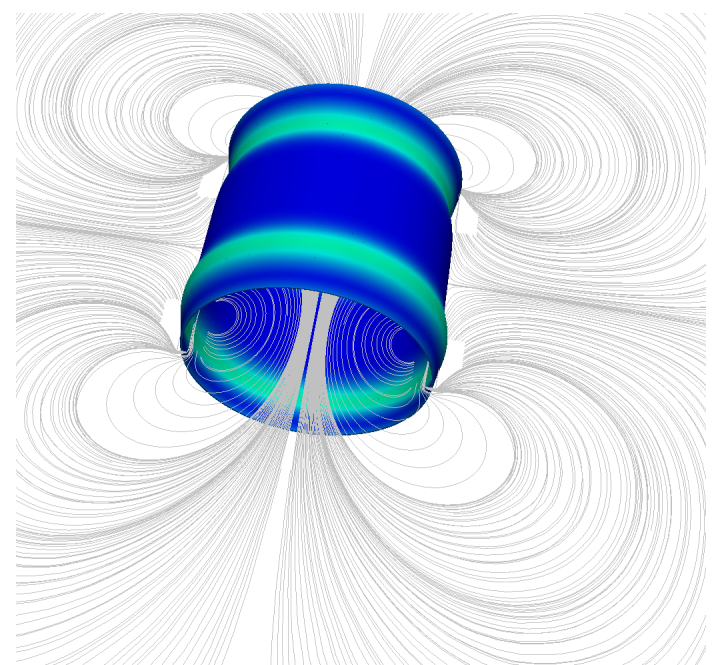

(c) $t=0.2 \mathrm{~ms}$

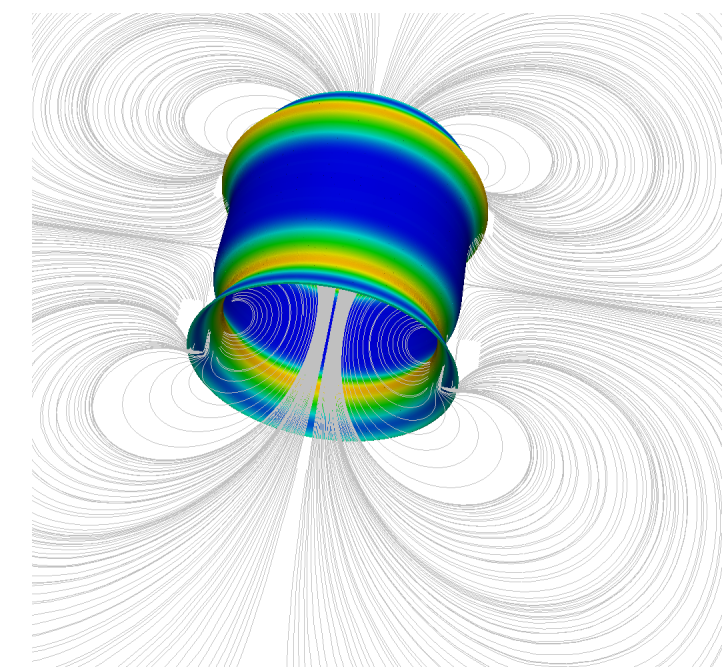

(e) $t=0.4 \mathrm{~ms}$

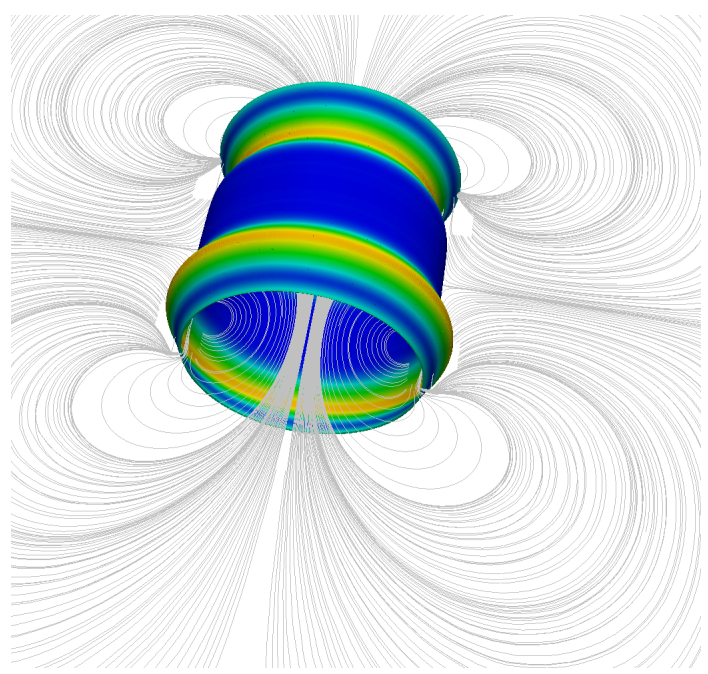

(b) $t=0.1 \mathrm{~ms}$

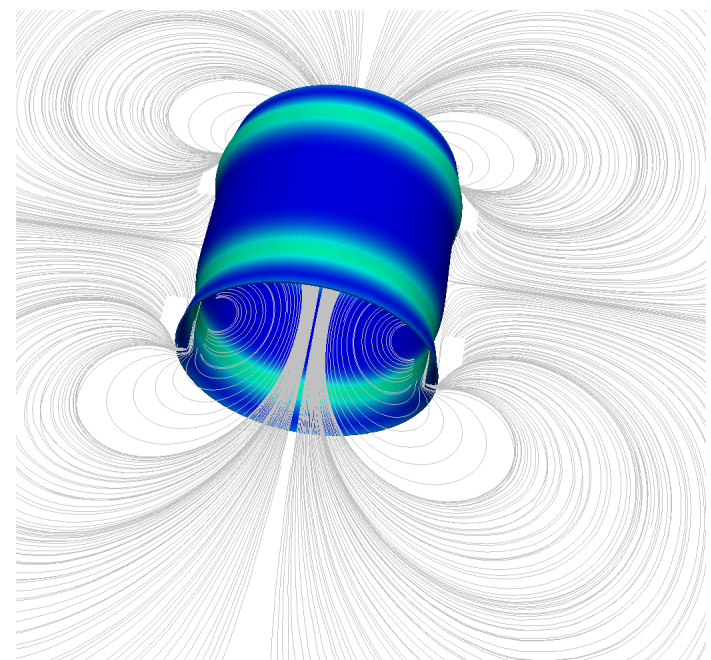

(d) $t=0.3 \mathrm{~ms}$

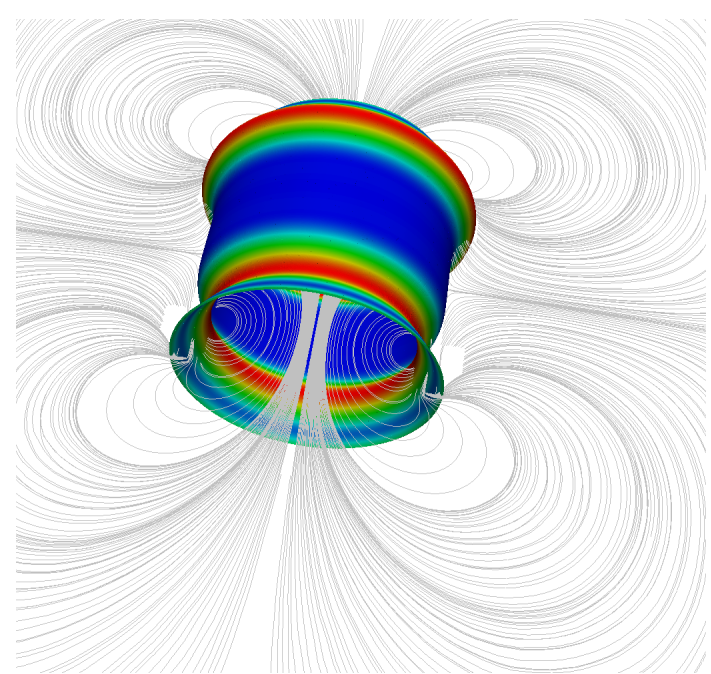

(f) $t=0.5 \mathrm{~ms}$

Figure 5.31: Test magnet problem with $z$ (longitudinal) gradient coil: snapshots of $\left|\operatorname{Re}\left(\boldsymbol{u}_{h p}^{A C} e^{\mathrm{i} \omega t}\right)\right|$ in the deformed $4 \mathrm{~K}$ shield and $\operatorname{Re}\left(\boldsymbol{B}_{0, h q}^{A C} e^{\mathrm{i} \omega t}\right)$ streamlines in the surrounding air for $f=1000 \mathrm{~Hz}$ and different times. Deformation magnitude magnified by a factor of $10^{4}$ in plot, but not in contours. 


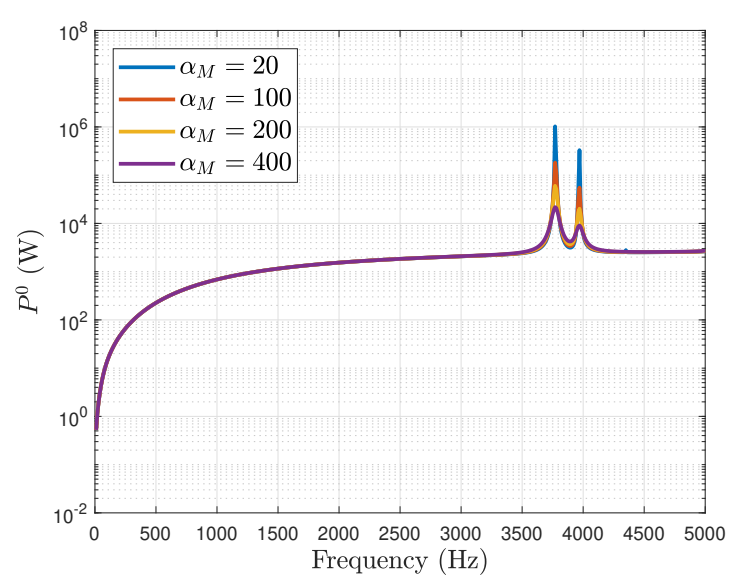

(a) $P_{\Omega_{O V C}}^{0}\left(\omega, \boldsymbol{A}_{\varepsilon, h q}^{A C}, \boldsymbol{B}_{0, h q}^{D C}, \boldsymbol{u}_{h p}^{A C}\right)$

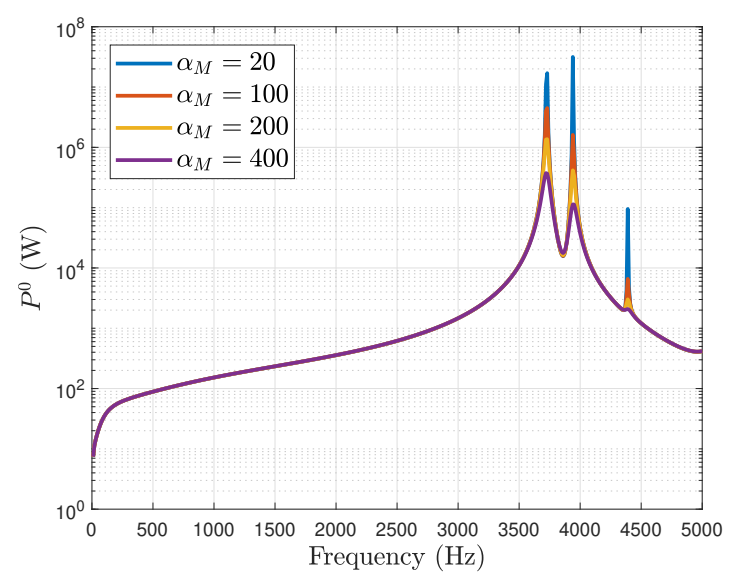

(c) $P_{\Omega_{77 K}}^{0}\left(\omega, \mathcal{A}_{\varepsilon, h q}^{A C}, \boldsymbol{B}_{0, h q}^{D C}, \boldsymbol{u}_{h p}^{A C}\right)$

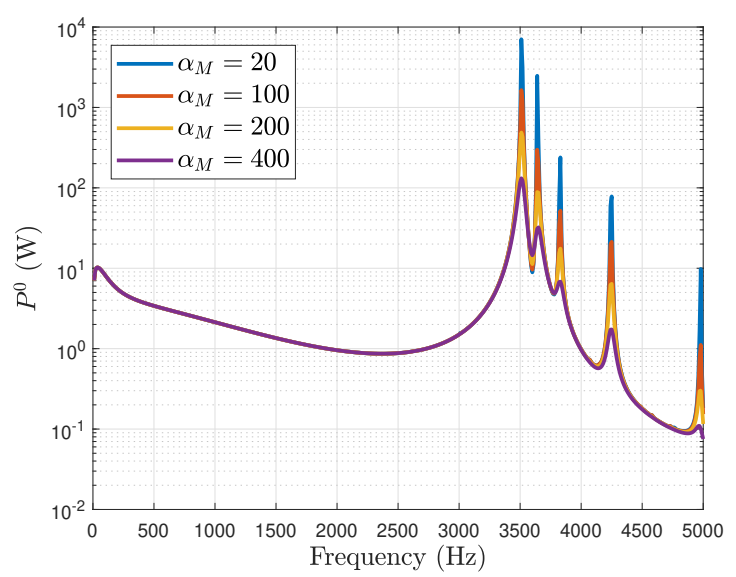

(e) $P_{\Omega_{4 K}}^{0}\left(\omega, \boldsymbol{A}_{\varepsilon, h q}^{A C}, \boldsymbol{B}_{0, h q}^{D C}, \boldsymbol{u}_{h p}^{A C}\right)$

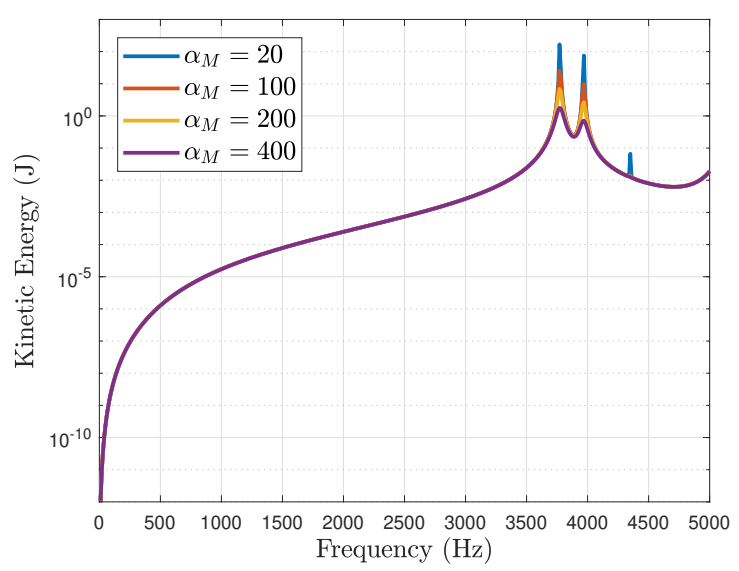

(b) $E_{\Omega_{O V C}}^{k}\left(\omega, \boldsymbol{u}_{h p}^{A C}\right)$

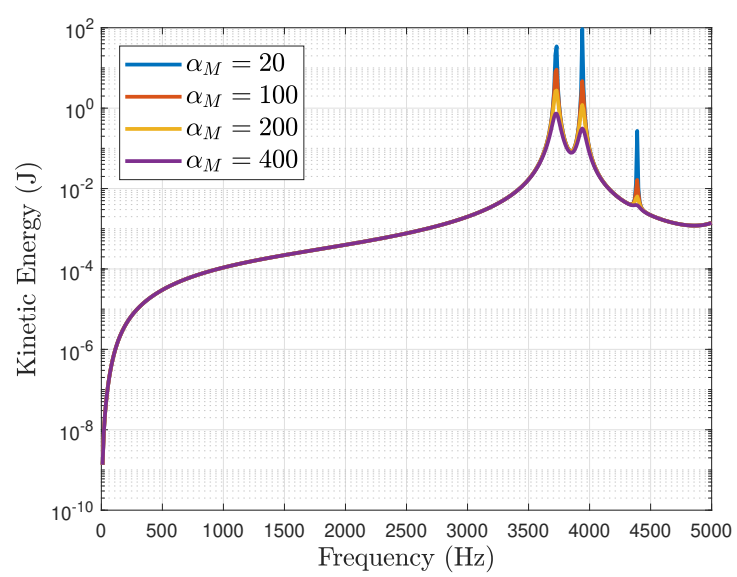

(d) $E_{\Omega_{77 K}}^{k}\left(\omega, \boldsymbol{u}_{h p}^{A C}\right)$

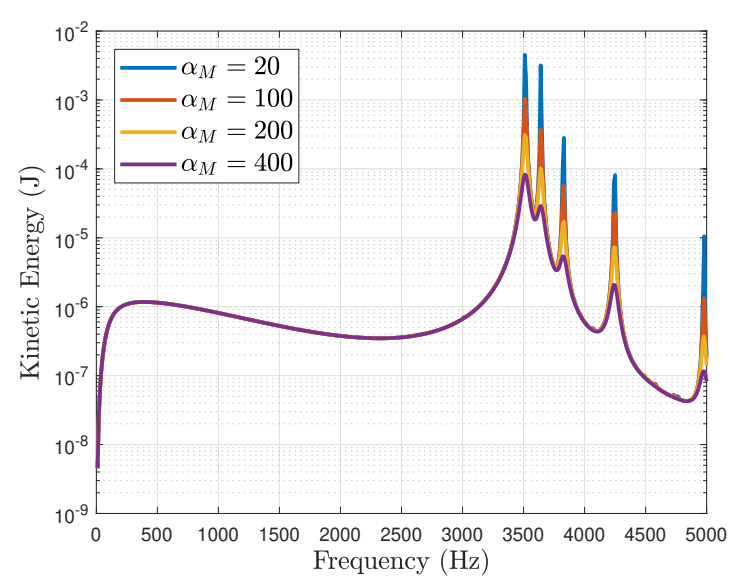

(f) $E_{\Omega_{4 K}}^{k}\left(\omega, \boldsymbol{u}_{h p}^{A C}\right)$

Figure 5.32: Test magnet problem with $z$ (longitudinal) gradient coils: Dissipated power and kinetic energy in the radiation shields for different $\alpha_{M}=20,100,200,400$. 


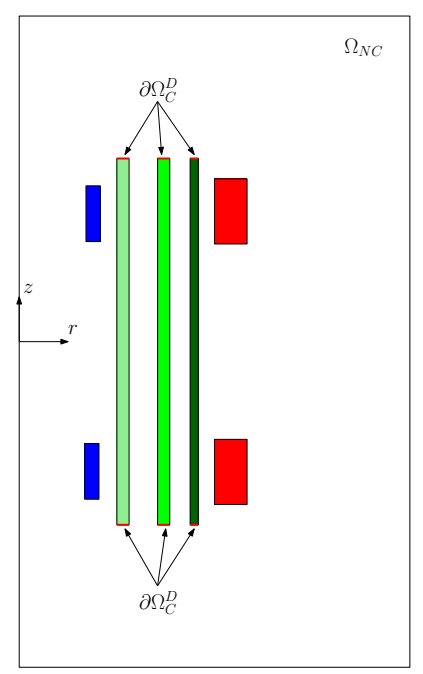

Figure 5.33: Test magnet problem with $z$ (longitudinal) gradient coils: Illustration of the choice of boundary conditions. Shields fixed at the ends.

\subsubsection{Test Magnet Problem with $x$ (Transversal) Gradient Coil}

A new challenging problem, consisting of the configuration described in Section 5.3.2 with the $z$ gradient coils being replaced by a set of $x$ gradient coils, is now considered. Note that the case of only $y$ gradient coils can be obtained by rotating the current situation through $90^{\circ}$. This new set of coils produces a magnetic field gradient in the $x$ direction and, therefore, the problem is no longer rotationally symmetric and cannot be simulated using the axisymmetric tool developed in $[19,18,17]$. Figure 5.36 shows the geometry of the problem, including the new set of gradient coils. These coils represent an approximation to the complex fingerprint shape used in real MRI scanners shown previously in Figure 4.13. The boundary condition $\boldsymbol{u}_{h p}^{A C}=\mathbf{0}$ was imposed at the ends of the shields and mechanical damping with $\alpha_{M}=200$ was added to the system. For computational purposes, the domain was truncated by a cylinder of radius $R_{0}=0.9 \mathrm{~m}$ and length $L_{0}=2.4$ $\mathrm{m}$. The material properties are the same as those in Section 5.3.2.

In the case of transversal gradient coils, the description of the current source $\boldsymbol{g}^{A C}$ becomes more challenging due to increasing complexity in the geometry. This can lead to issues with producing a description of $\boldsymbol{g}^{A C}$ that is both tangential to the coil geometry and that satisfies $\operatorname{div} \boldsymbol{g}^{A C}=0$. Thus, in order to ensure that the current source is divergence free, a mapping of the current source to the space of divergence free functions is performed as described in Section 4.8.

In the new configuration, the rotational symmetry is lost. However, as discussed in Section 4.9 , the problem still exhibits symmetries or anti-symmetries with respect to the Cartesian planes $x=0, y=0$ and $z=0$. Thus, the computational domain is reduced to $1 / 8$ of its original size by exploiting these symmetries and applying the appropriate boundary conditions described in Table 4.1. For the discretisation, a mesh of 27168 tetrahedral elements, with significant refinement in the area of the conducting shields (10290 ele-

\footnotetext{
${ }^{1}$ Note again that the view in Figure 5.36 has been rotated so that the vertical direction corresponds to $x$
} 


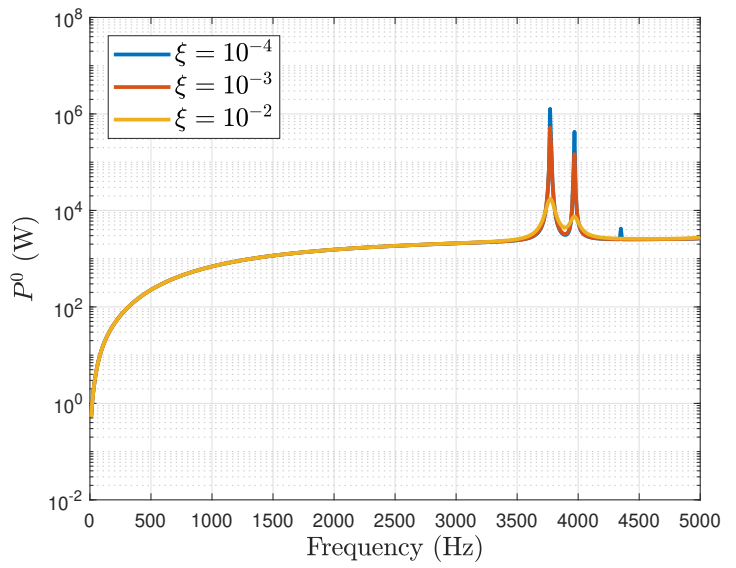

(a) $P_{\Omega_{O V C}}^{0}\left(\omega, \boldsymbol{A}_{\varepsilon, h q}^{A C}, \boldsymbol{B}_{0, h q}^{D C}, \boldsymbol{u}_{h p}^{A C}\right)$

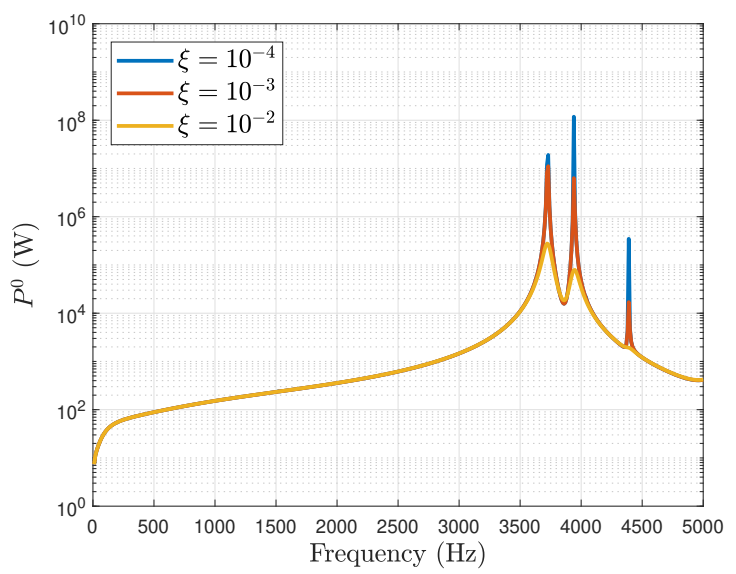

(c) $P_{\Omega_{77 K}}^{0}\left(\omega, \boldsymbol{A}_{\varepsilon, h q}^{A C}, \boldsymbol{B}_{0, h q}^{D C}, \boldsymbol{u}_{h p}^{A C}\right)$

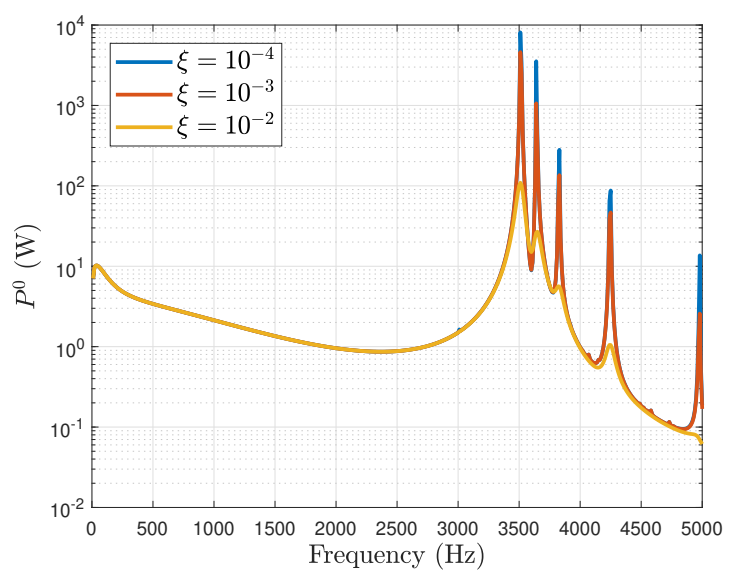

(e) $P_{\Omega_{4 K}}^{0}\left(\omega, \boldsymbol{A}_{\varepsilon, h q}^{A C}, \boldsymbol{B}_{0, h q}^{D C}, \boldsymbol{u}_{h p}^{A C}\right)$

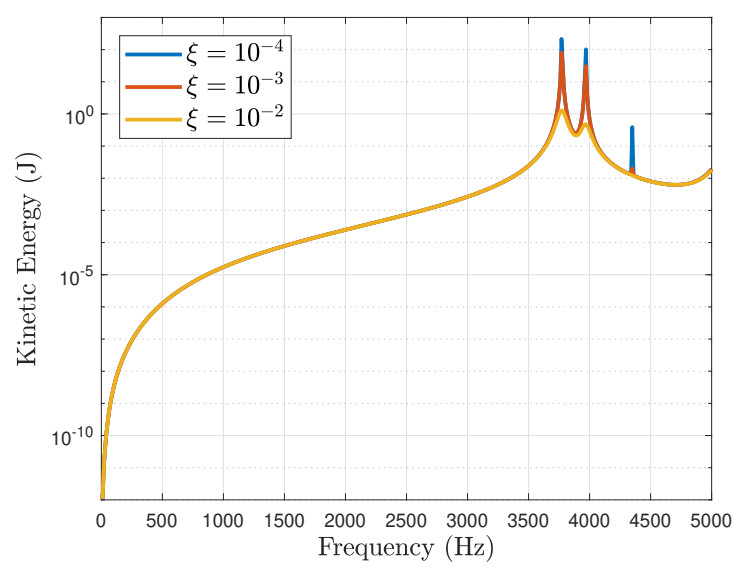

(b) $E_{\Omega_{O V C}}^{k}\left(\omega, \boldsymbol{u}_{h p}^{A C}\right)$

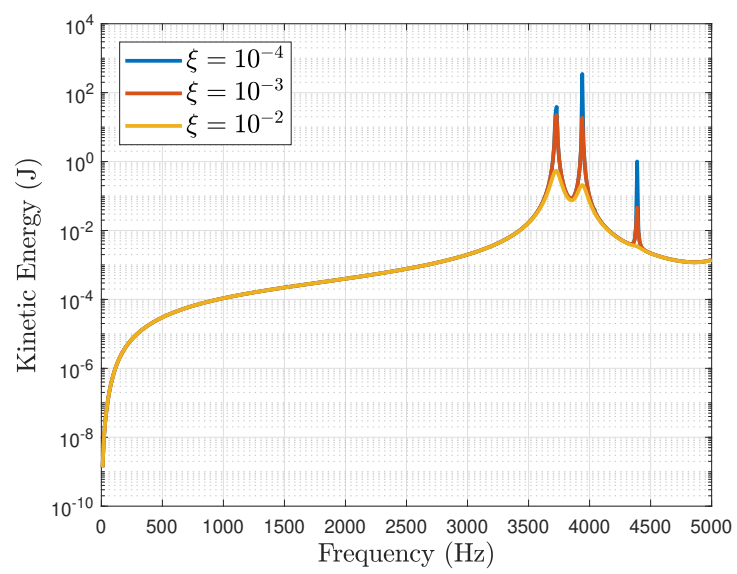

(d) $E_{\Omega_{77 K}}^{k}\left(\omega, \boldsymbol{u}_{h p}^{A C}\right)$

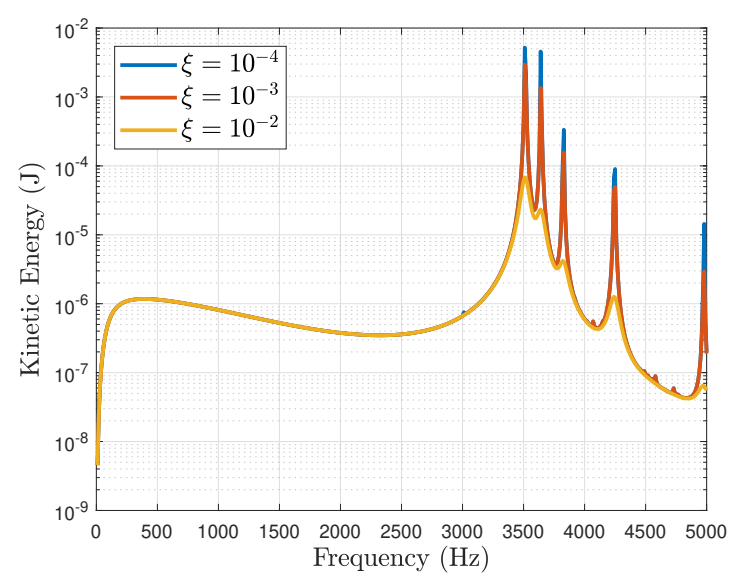

(f) $E_{\Omega_{4 K}}^{k}\left(\omega, \boldsymbol{u}_{h p}^{A C}\right)$

Figure 5.34: Test magnet problem with $z$ (longitudinal) gradient coils: Dissipated power and kinetic energy in the radiation shields for different $\xi=10^{-4}, 10^{-2}, 10^{-3}$. 


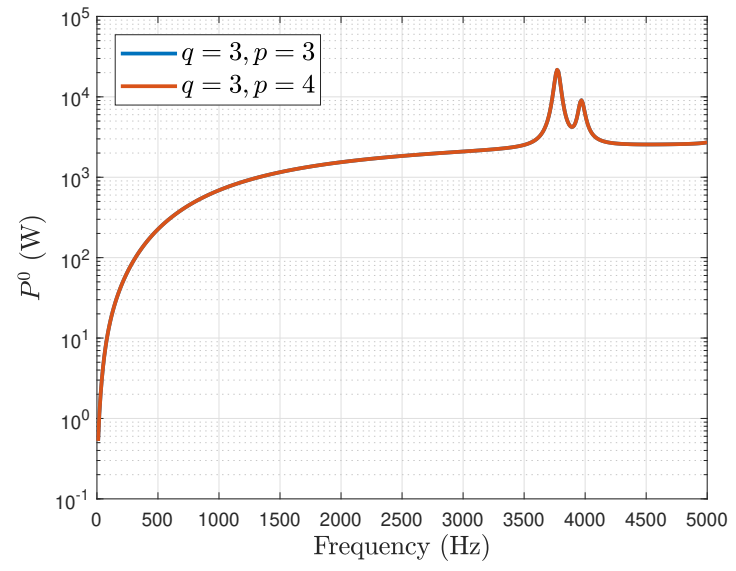

(a) $P_{\Omega_{O V C}}^{0}\left(\omega, \boldsymbol{A}_{\varepsilon, h q}^{A C}, \boldsymbol{B}_{0, h q}^{D C}, \boldsymbol{u}_{h p}^{A C}\right)$

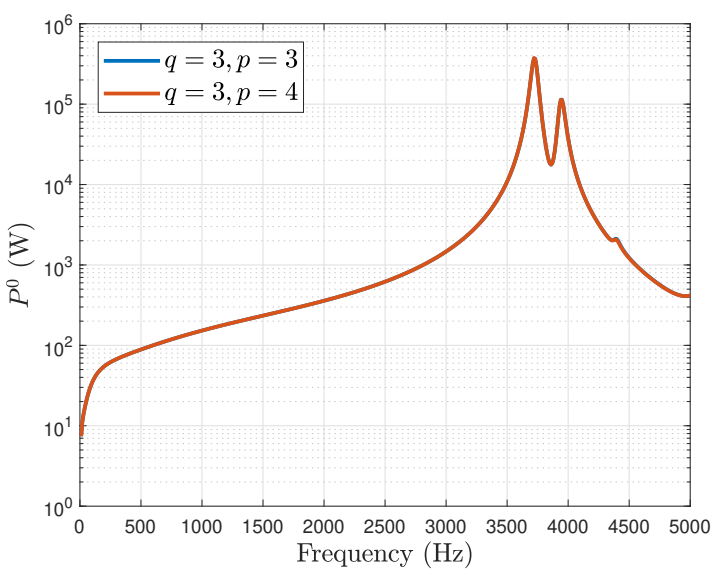

(c) $P_{\Omega_{77 K}}^{0}\left(\omega, \boldsymbol{A}_{\varepsilon, h q}^{A C}, \boldsymbol{B}_{0, h q}^{D C}, \boldsymbol{u}_{h p}^{A C}\right)$

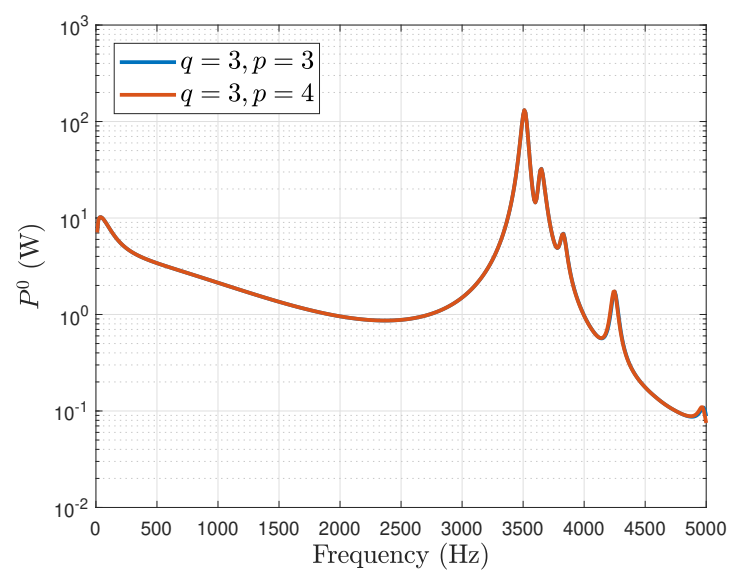

(e) $P_{\Omega_{4 K}}^{0}\left(\omega, \mathcal{A}_{\varepsilon, h q}^{A C}, \boldsymbol{B}_{0, h q}^{D C}, \boldsymbol{u}_{h p}^{A C}\right)$

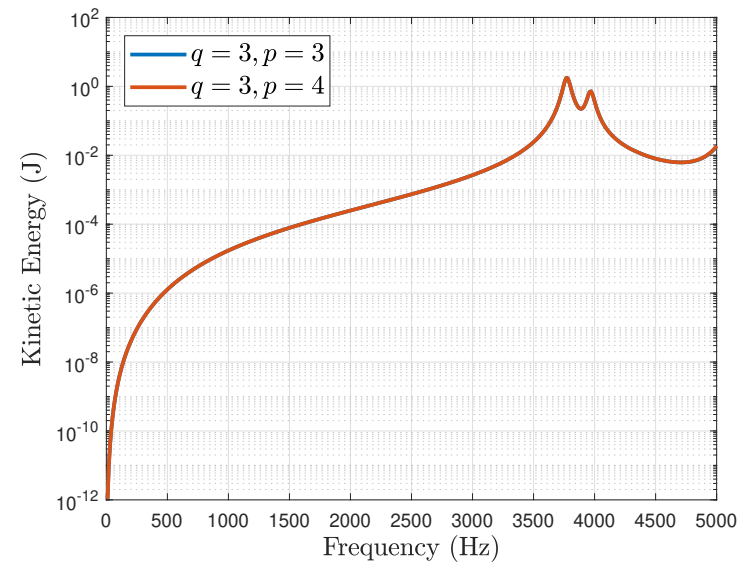

(b) $E_{\Omega_{O V C}}^{k}\left(\omega, \boldsymbol{u}_{h p}^{A C}\right)$

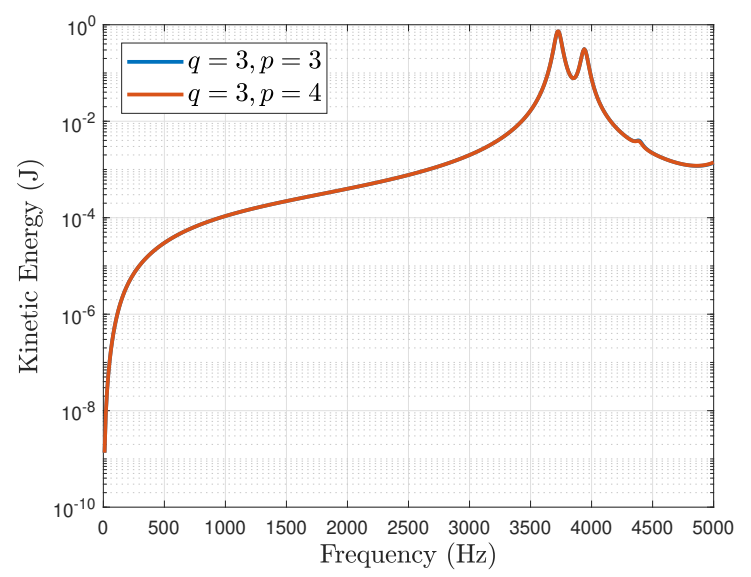

(d) $E_{\Omega_{77 K}}^{k}\left(\omega, \boldsymbol{u}_{h p}^{A C}\right)$

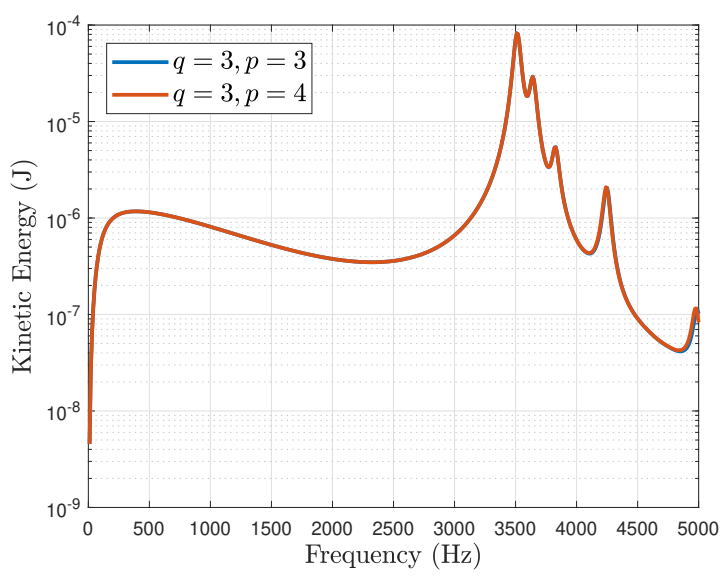

(f) $E_{\Omega_{4 K}}^{k}\left(\omega, \boldsymbol{u}_{h p}^{A C}\right)$

Figure 5.35: Test magnet problem with $z$ (longitudinal) gradient coils: Dissipated power and kinetic energy in the radiation shields for $q=p=3$ and $q=3, p=4$. 


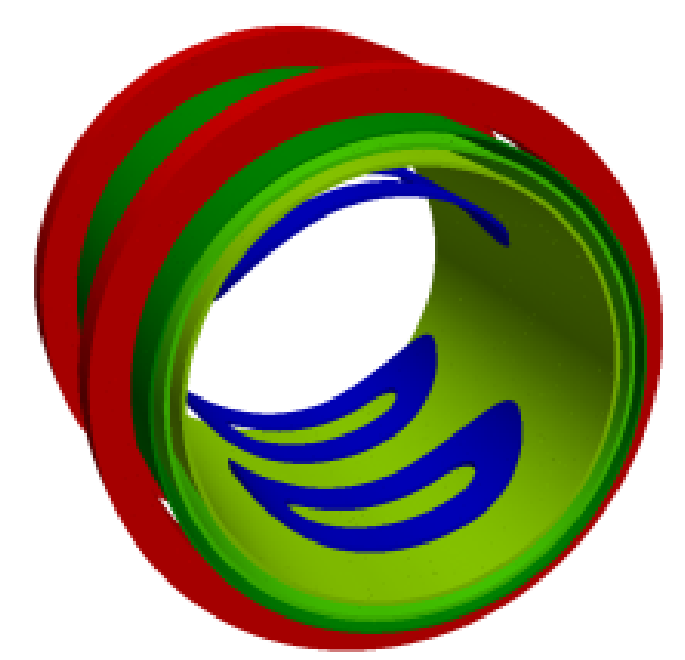

Figure 5.36: Test magnet problem with $x$ (transversal) gradient coil: illustration of the geometry including main coils (red), gradient coils (blue) and radiation shields (green).

ments in the conducting region) was used, and the polynomial order was increased until convergence was reached, which was found to when $q=4$ and $p=4$. The regularisation parameter was set to $\varepsilon=10^{-4}$ and the tolerance of the iterative solver to $10^{-5}$.

In order to illustrate how the solution changes when considering transversal instead of longitudinal coils, a contour plot of $\left|\operatorname{Re}\left(\boldsymbol{J}_{h p q}^{o}\right)\right|$ in the OVC shield for a frequency $f=500$ $\mathrm{Hz}$ is presented in Figure 5.37 comparing both cases. As expected, it can be observed that the solution in the case of $x$ gradient coil is no longer axisymmetric. The same is repeated for the mechanical field in Figure 5.38, where a contour plot of $\left|\operatorname{Re}\left(\boldsymbol{u}_{h p}^{A C}\right)\right|$ on the deformed $77 \mathrm{~K}$ shield is shown.

Next, in Figure 5.39, the streamlines of $\operatorname{Re}\left(\boldsymbol{B}_{0, h q}^{A C}\right)$ around the radiation shields are shown, together with contours of $\left|\operatorname{Re}\left(\boldsymbol{J}_{h p q}^{o}\right)\right|$ in the shields. The main coils and gradient coils are also included and it can be observed that, in the case of $z$ gradient coils, all the streamlines lie in a plane, whilst in the case of $x$ gradient coils, they follow a more complex helical pattern.

Furthermore, the dissipated power and kinetic energy in the radiation shields were also computed and, in Figure 5.40, the convergence of the solution for increasing polynomial order is shown. In this case the optimum choice for the polynomial orders was found to be $q=p$ and the solution converged for $q=p=4$ (effectively for $q=p=3$ ).

Finally, the dissipated power and kinetic energy obtained with the $z$ and $x$ gradient coils are compared in Figure 5.41. Note how the different gradient coils excite different resonant modes of the system. Furthermore, in this problem more modes are excited with the $x$ gradient coil than with the $z$ gradient coil.

\subsubsection{Modified Magnet Problem}

The last problem considered in this chapter consists in a simplified MRI configuration for which experimental data is available. The geometry of the problem is shown in Figure 


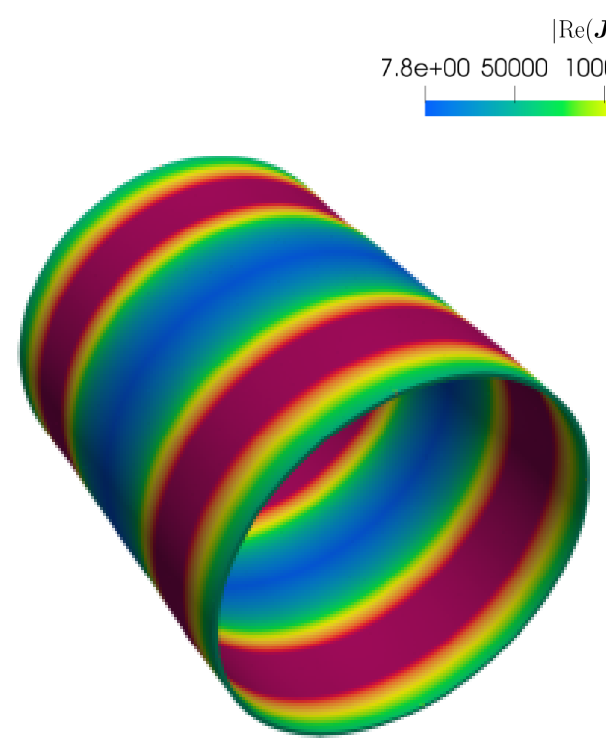

(a) $z$ gradient

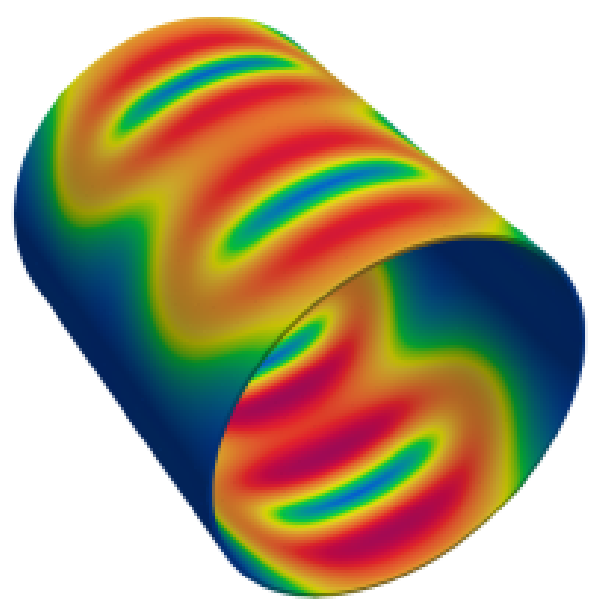

(b) $x$ gradient

Figure 5.37: Test magnet problem with $x$ (transversal) gradient coil: $\left|\operatorname{Re}\left(\boldsymbol{J}_{h p q}^{o}\right)\right|$ in the OVC shield for $f=500 \mathrm{~Hz}$ compared with the case of $z$ (longitudinal) gradient coil.

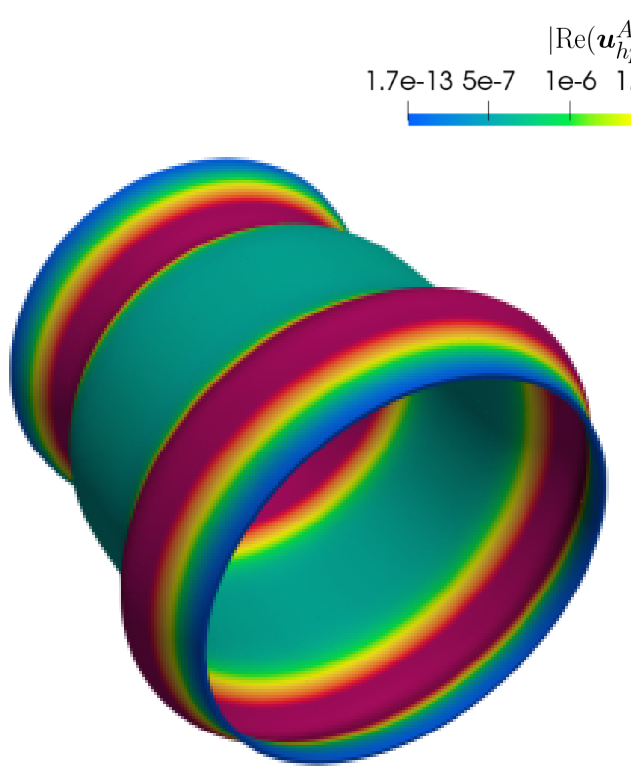

(a) $z$ gradient
$2.8 e-06$

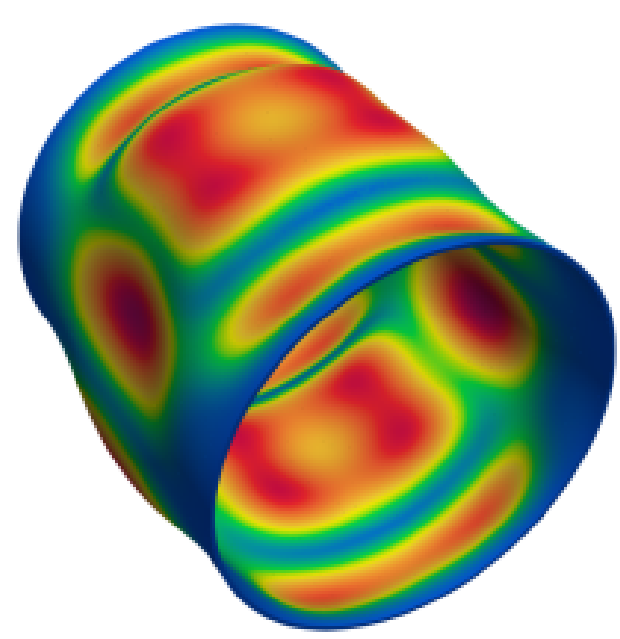

(b) $x$ gradient

Figure 5.38: Test magnet problem with $x$ (transversal) gradient coil: $\left|\operatorname{Re}\left(\boldsymbol{u}_{h p}^{A C}\right)\right|$ in the $77 \mathrm{~K}$ shield for $f=500 \mathrm{~Hz}$ compared with the case of $z$ (longitudinal) gradient coil. Deformation magnitude magnified by a factor of $10^{4}$ in plot, but not in contours. 


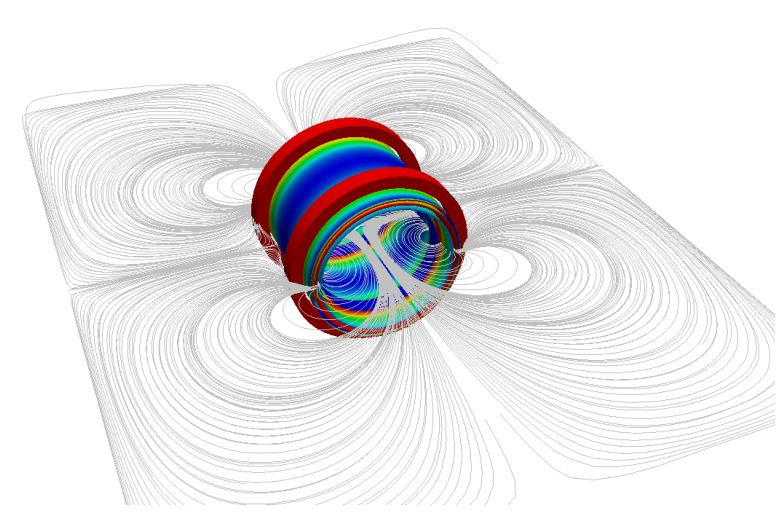

(a) $z$ gradient

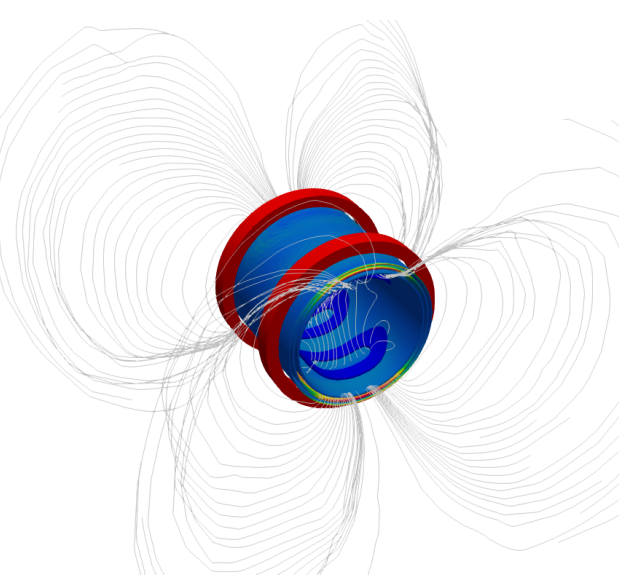

(b) $x$ gradient

Figure 5.39: Test magnet problem with $x$ (transversal) gradient coil: Streamlines of $\operatorname{Re}\left(\boldsymbol{B}_{0, h q}^{A C}\right)$ and contours of $\left|\operatorname{Re}\left(\boldsymbol{J}_{h p q}^{o}\right)\right|$ in the radiation shields for $f=500 \mathrm{~Hz}$ compared with the case of $z$ (longitudinal) gradient coil.

5.42 for the cases of $z$ as well as $x$ gradient coils. Again, the exact geometry and material properties are confidential and, thus, are not stated here. The order of magnitude of these properties is the same as stated in Section 5.3.2.

For this problem, experimental results are available for the dissipated power in the $4 \mathrm{~K}$ shield when considering only $z$ gradient coils. Thus, in Figure 5.43, the experimental results are compared with the results obtained using the axisymmetric software $[19,17]$ for the Eulerian and Lagrangian approaches and the results using the 3D software and the Lagrangian approach. For computational purposes, the domain was truncated by a cylinder of radius $R_{0}=0.9 \mathrm{~m}$ and length $L_{0}=2.4 \mathrm{~m}$. Mechanical damping with a constant $\alpha_{M}=400$ was applied and the Dirichlet boundary condition $\mathfrak{u}_{h p}^{A C}=\mathbf{0}$ was imposed at the ends of the shields. As for the previous problems, a $p$-refinement analysis using a mesh of 41440 elements (with 14192 elements in the conducting region) was performed and the converged solution, corresponding to $q=p=4$ (effectively converged for $q=p=3$ ), is shown. The regularisation term was chosen to be $\varepsilon=10^{-4}$ and the tolerance of the iterative solver was set to $10^{-5}$.

Note that the model has been simplified and many components present in the real configuration have been neglected, thus it is not expected to obtain an excellent agreement. However, it can be observed that the Lagrangian approach leads to an excellent agreement with the experimental data in the non resonant region, corresponding to frequencies $f<3500 \mathrm{~Hz}$, which is not true in the case of the Eulerian approach, where good agreement is obtained only for frequencies $f<1000 \mathrm{~Hz}$. The result in the resonant region is more sensitive to the choice of boundary conditions, mechanical damping or the model simplifications. Thus, in order to obtain good agreement in this region, the model would have to be improved by including all the components present in the real configuration, and choosing a more sophisticated damping model. However, this is out of the scope of this thesis, and given the simplifications considered in our model we can conclude that 


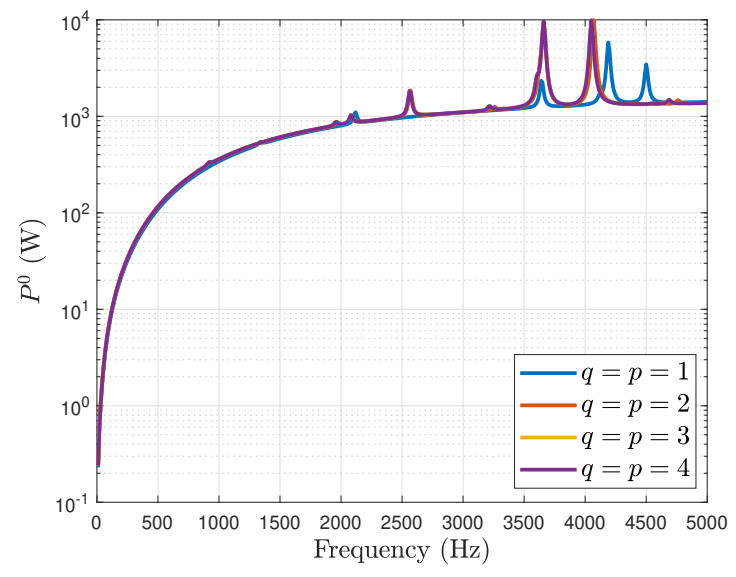

(a) $P_{\Omega_{O V C}}^{0}\left(\omega, \boldsymbol{A}_{\varepsilon, h q}^{A C}, \boldsymbol{B}_{0, h q}^{D C}, \boldsymbol{u}_{h p}^{A C}\right)$

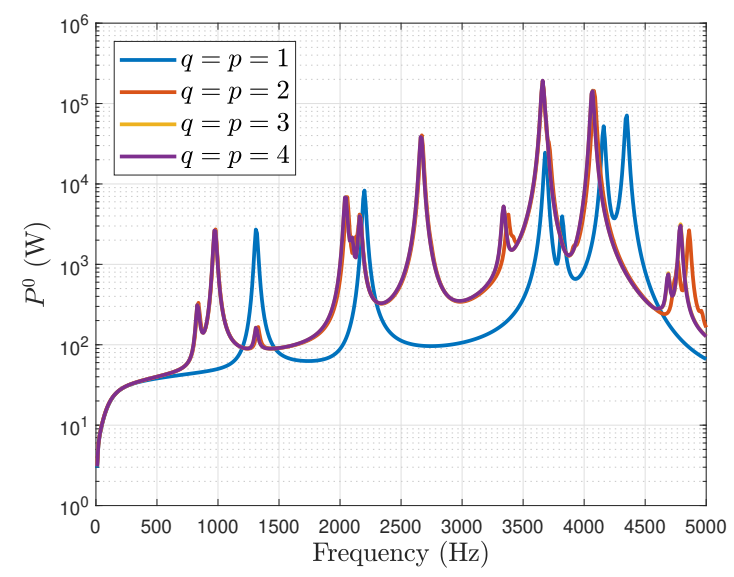

(c) $P_{\Omega_{77 K}}^{0}\left(\omega, \boldsymbol{A}_{\varepsilon, h q}^{A C}, \boldsymbol{B}_{0, h q}^{D C}, \boldsymbol{u}_{h p}^{A C}\right)$

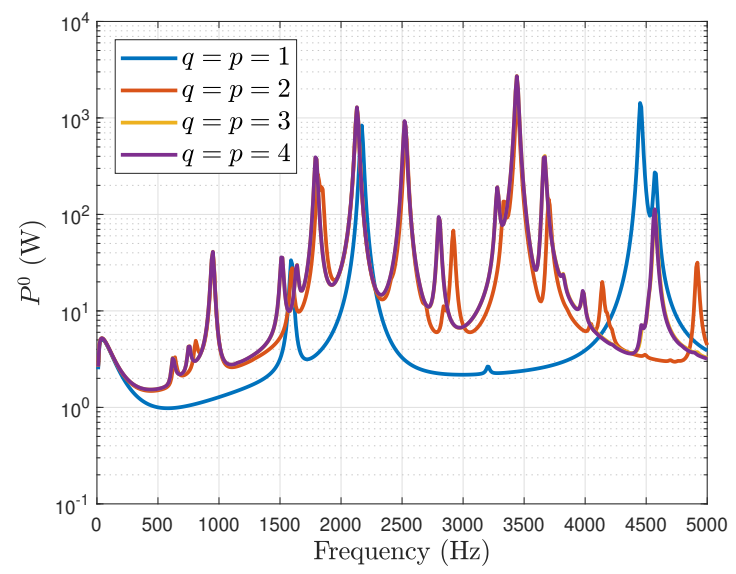

(e) $P_{\Omega_{4 K}}^{0}\left(\omega, \mathcal{A}_{\varepsilon, h q}^{A C}, \boldsymbol{B}_{0, h q}^{D C}, \boldsymbol{u}_{h p}^{A C}\right)$

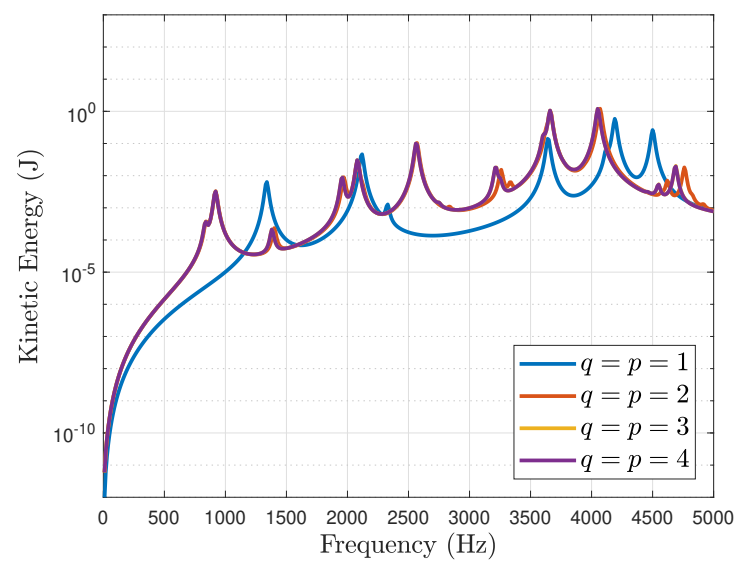

(b) $E_{\Omega_{O V C}}^{k}\left(\omega, \boldsymbol{u}_{h p}^{A C}\right)$

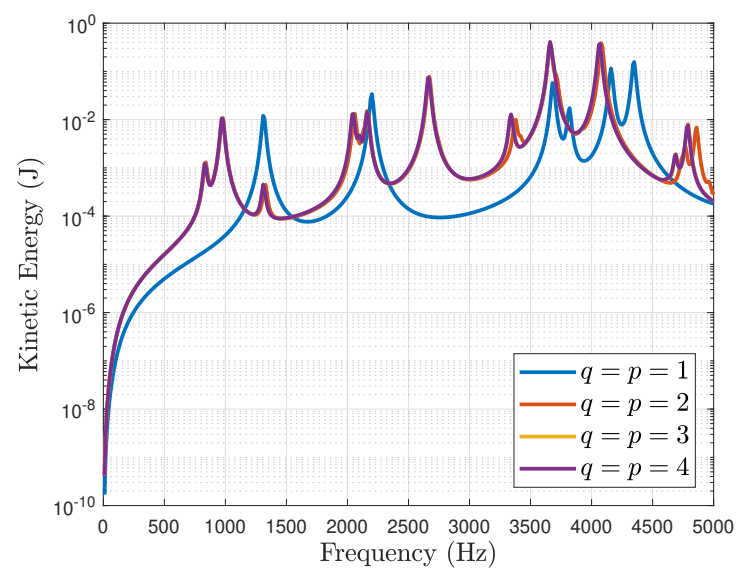

(d) $E_{\Omega_{77 K}}^{k}\left(\omega, \boldsymbol{u}_{h p}^{A C}\right)$

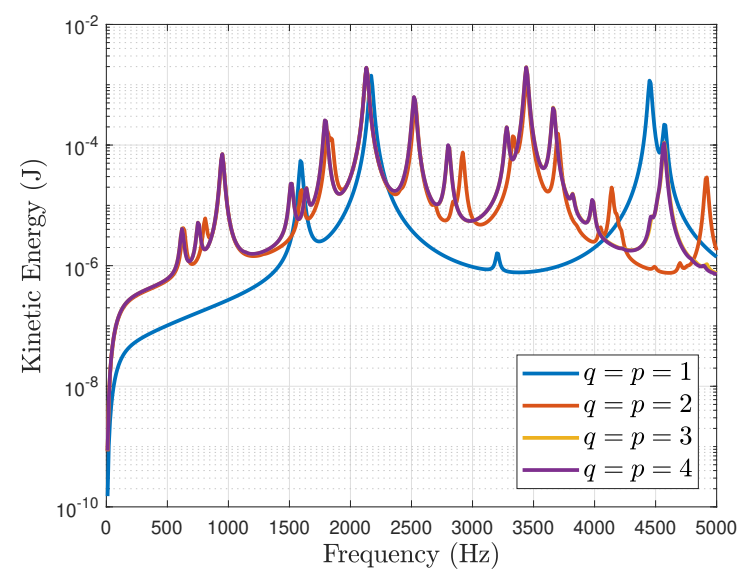

(f) $E_{\Omega_{4 K}}^{k}\left(\omega, \boldsymbol{u}_{h p}^{A C}\right)$

Figure 5.40: Test magnet problem with $x$ (transversal) gradient coil: convergence of the dissipated power and kinetic energy in the radiation shields under $p$-refinement. Damped system $\left(\alpha_{M}=200\right)$. 


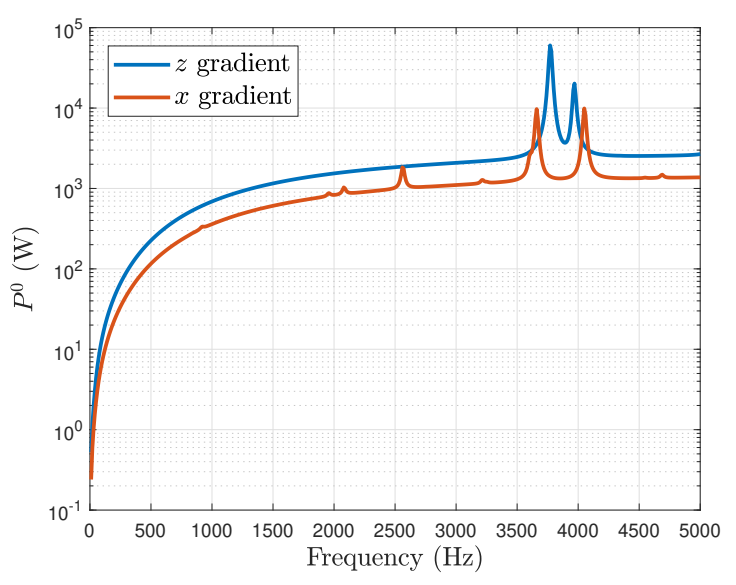

(a) $P_{\Omega_{O V C}}^{0}\left(\omega, \boldsymbol{A}_{\varepsilon, h q}^{A C}, \boldsymbol{B}_{0, h q}^{D C}, \boldsymbol{u}_{h p}^{A C}\right)$

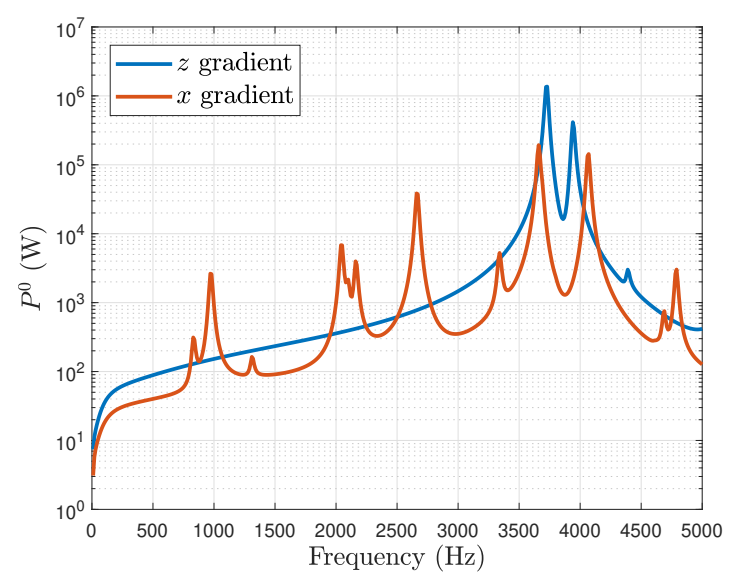

(c) $P_{\Omega_{77 K}}^{0}\left(\omega, \mathcal{A}_{\varepsilon, h q}^{A C}, \boldsymbol{B}_{0, h q}^{D C}, \boldsymbol{u}_{h p}^{A C}\right)$

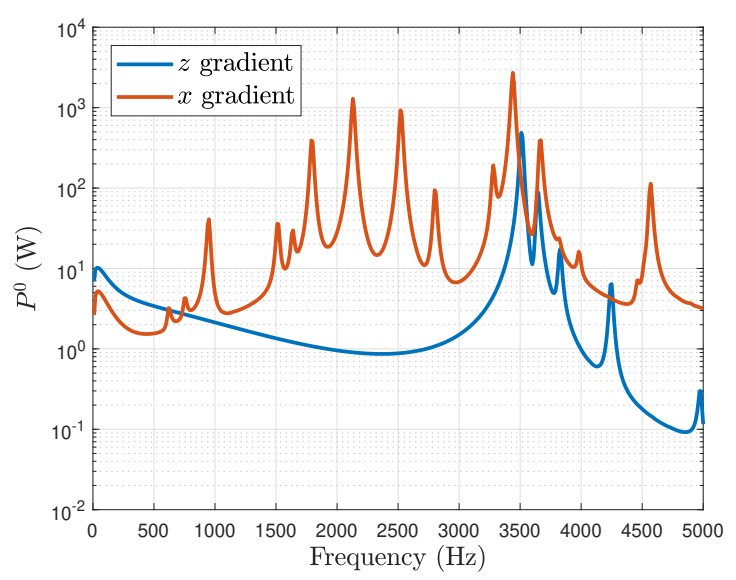

(e) $P_{\Omega_{4 K}}^{0}\left(\omega, \boldsymbol{A}_{\varepsilon, h q}^{A C}, \boldsymbol{B}_{0, h q}^{D C}, \boldsymbol{u}_{h p}^{A C}\right)$

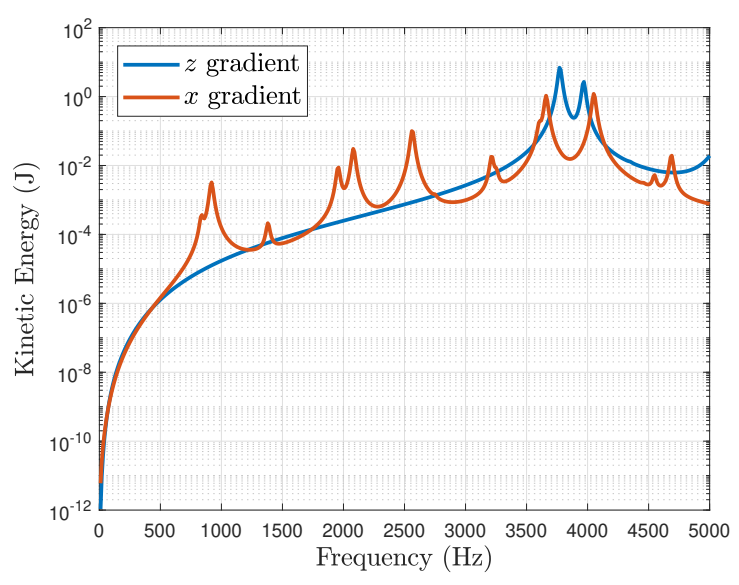

(b) $E_{\Omega_{O V C}}^{k}\left(\omega, \boldsymbol{u}_{h p}^{A C}\right)$

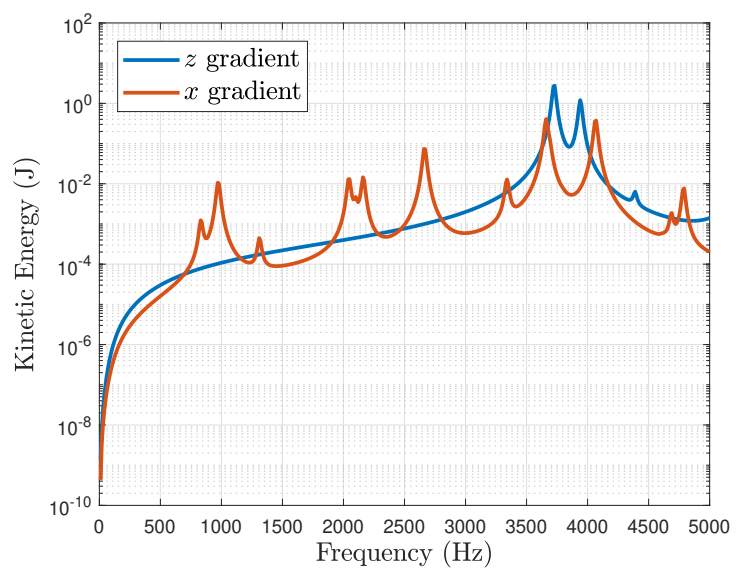

(d) $E_{\Omega_{77 K}}^{k}\left(\omega, \boldsymbol{u}_{h p}^{A C}\right)$

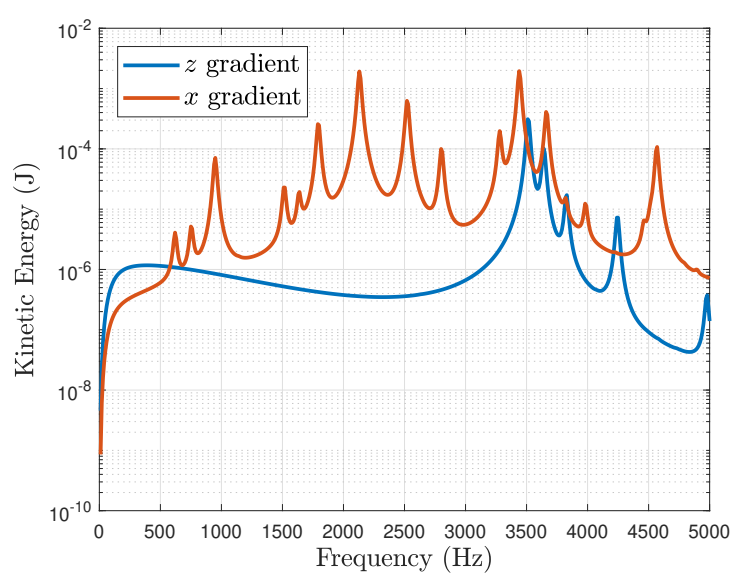

(f) $E_{\Omega_{4 K}}^{k}\left(\omega, \boldsymbol{u}_{h p}^{A C}\right)$

Figure 5.41: Test magnet problem: comparison of the dissipated power and kinetic energy in the radiation shields for $z$ gradient coils and $x$ gradient coils. Damped system $\left(\alpha_{M}=\right.$ 200). 


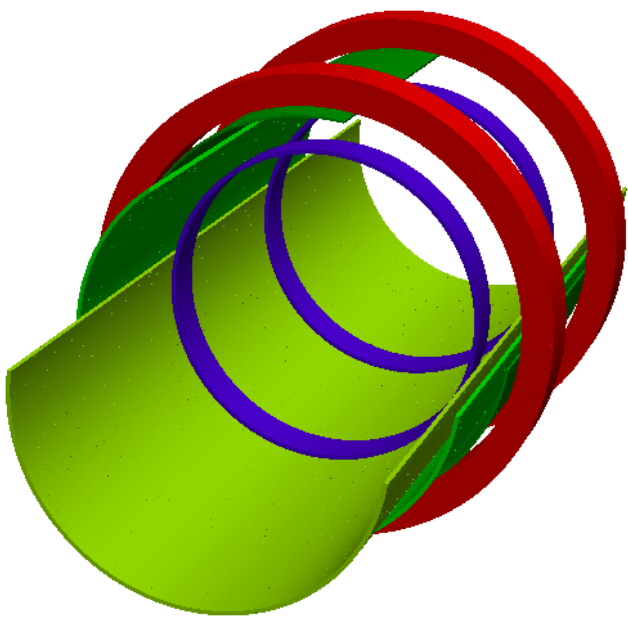

(a) $z$ coils

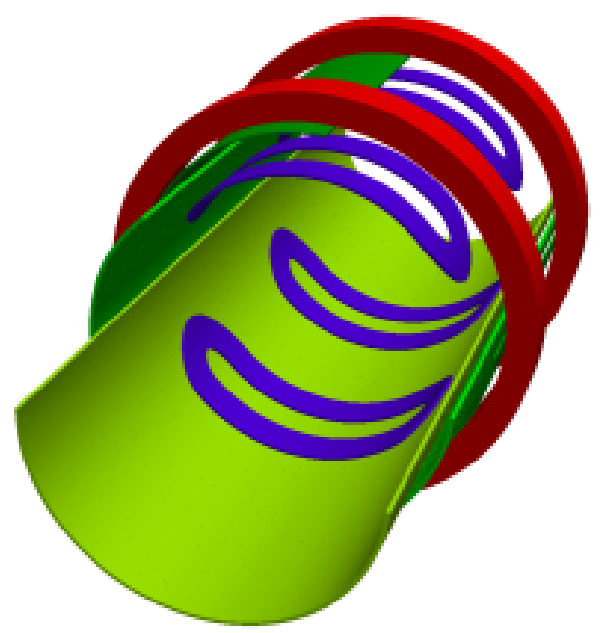

(b) $x$ coils

Figure 5.42: Modified magnet problem: Illustration of the geometry for (a) $z$ gradient coil and (b) $x$ gradient coil.

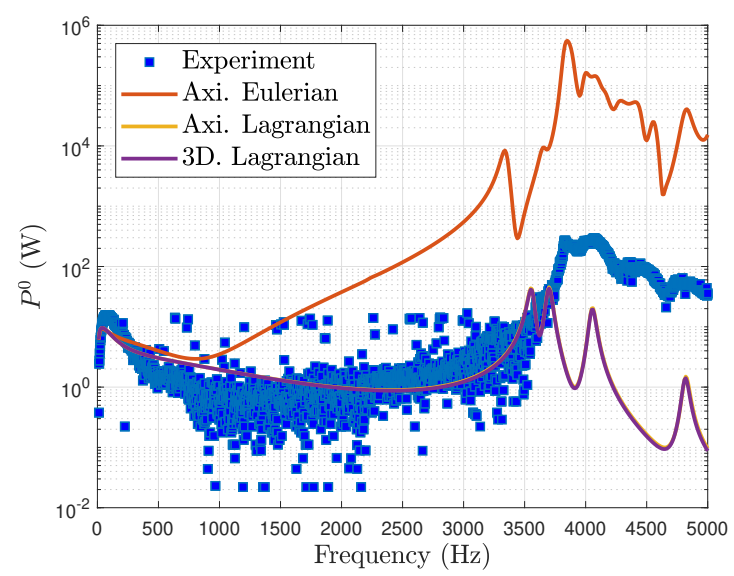

Figure 5.43: Modified magnet problem with $z$ (longitudinal) gradient coil: comparison of $P_{\Omega_{4 K}}^{0}$ for axisymmetric Eulerian and Lagrangian approaches, 3D Lagrangian approach and experimental data.

good agreement is obtained and, importantly, the Lagrangian approach offers a substantial improvement over the previous $[19,17]$ Eulerian approach.

Finally, even though experimental data is not available for the case of $x$-gradient coil, the dissipated power obtained in the $4 \mathrm{~K}$ shield was also computed for this case and the result is shown in Figure 5.44, where it can be observed that more resonant modes are excited by the $x$ gradient coils compared to the $z$ gradient coils. Again, a $p$-refinement analysis, using a mesh of 54796 elements, was performed, and the converged solution, which corresponds to $q=5, p=4$ (effectively converged for $q=4, p=3$ ), is shown. Note that in this case the optimum combination of $p, q$ was found to be $q=p+1$ as it was observed that higher order was needed to resolve the eddy currents compared to 


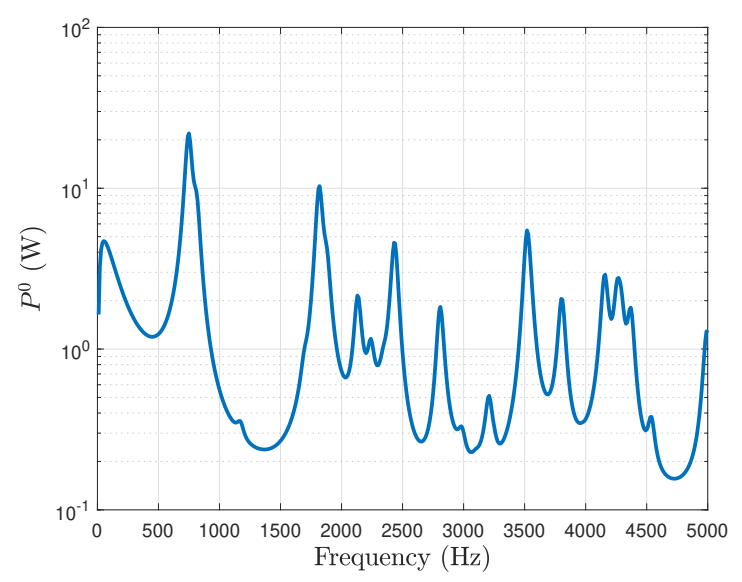

Figure 5.44: Modified magnet problem with $x$ (transversal) gradient coil: Dissipated power in the $4 \mathrm{~K}$ shield.

the displacements, especially at high frequencies. For low frequencies this represents an overkill solution. Note again how the different choice of gradient coils leads to different resonant modes being excited.

\subsection{Chapter Summary}

In this Chapter, the proposed 3D Lagrangian $h p$-FEM methodology has been applied to several academic and industrially relevant problems.

The main novelties of the Chapter are as follows; first, the proposed 3D $h p$-FEM methodology was applied to several single physics problems and it was shown that the expected rates of convergence are obtained for the electromagnetic and mechanical DC and AC stages. Second, the efficiency of the proposed preconditioned iterative solver for each of these stages was assessed. Third, it was shown that there is freedom in the choice of the combination $q, p$ and that different combinations lead to the same converged solution. Fourth, the coupled Lagrangian $h p$-FEM methodology was validated by considering a simplified magnet geometry and performing comparisons against the axisymmetric software [19, 17] and the commercial software NACS [4]. Fifth, the methodology was applied to MRI configurations considering transversal gradient coils and the solution was compared with the case of longitudinal gradient coils. Sixth, comparisons against experimental data were provided for a different simplified MRI configuration. Seventh, the Eulerian and Lagrangian approaches were compared and it was shown that the Lagrangian approach leads to more accurate results. Furthermore, several plots of physically relevant magnitudes were provided for each of the problems considered in order to illustrate the physical behaviour.

These novelties were illustrated as follows. First, in Section 5.2, the methodology was applied to four different single physics or decoupled problems in order to validate the electromagnetic and mechanical DC and AC stages. For each of these problems a conver- 
gence analysis was performed, and the result was compared with the theoretical prediction $[171,172]$ for the rates of convergence under $p$ and $h$-refinement. Furthermore, the efficiency of the preconditioned iterative solver was assessed in each case and different magnitudes of interest were plotted in order to illustrate the physical behaviour. Then, in Section 5.3, the methodology was applied to a series of coupled magneto-mechanical problems. The first coupled problem considered was an academic benchmark problem consisting in a conducting and elastic sphere excited by a time-harmonic magnetic field, and it was shown that different combinations of $q$ and $p$ lead to the same solution. Furthermore, the dissipated power and kinetic energy were computed for a sweep of frequencies from $f=1$ to $f=4500 \mathrm{~Hz}$ and it was shown that for this problem the same solution is obtained with the 3D and axisymmetric softwares and with the Eulerian and Lagrangian approaches. Then, a simplified magnet geometry, considering only $z$ (longitudinal) coils was considered and the dissipated power and kinetic energy in the different radiation shields were computed. Comparisons with the axisymmetric software were again performed in order to validate the $3 \mathrm{D}$ software. In this case, significant differences were observed between the Lagrangian and Eulerian approaches. Comparisons against commercial software were also provided. Furthermore, the effect of the choice of damping and boundary conditions in the final solution was also illustrated. Next, the same problem but replacing the $z$ gradient coils by a set of $x$ gradient coils was considered and it was shown that different resonant modes are excited by different gradient coils. Finally, another problem consisting in a simplified MRI configuration was considered and comparisons against experimental data were provided. 


\section{Part III}

Reduced Order Modelling 


\section{Chapter 6}

\section{POD Based Reduced Order Modelling}

\subsection{Introductory Remarks}

In the design stage of a new MRI scanner, the magneto-mechanical problem (2.42) must be solved multiple times in order to investigate how the solution depends on parameters of interest such as the excitation frequency of the current source or the conductivity of the radiation shields among others. For this, system (4.54) has to be solved repeatedly for each parameter combination, which leads to a bottleneck in terms of computational time. Thus, the main objective of this chapter is the development of a reduced order model (ROM) that can help optimise the workflow of the MRI design process. A ROM can be understood as a method that allows to approximate the solution to a high dimensional parametric problem (the full order problem) by means of a problem of reduced dimensions that captures the dominant components (or modes) of the full order problem [49, 14, 87, 43 and whose solution is computationally much cheaper than the solution to the full order model. To this end, the Proper Orthogonal Decomposition (POD) [43, 119, 180, 47] is considered and, in particular, the focus is on its discrete version, which is also referred to as Principal Component Analysis (PCA) in the literature [43, 133].

The main novelty of this chapter is the development of a POD based reduced order-full order methodology for the solution of the 3D magneto-mechanical problem of interest. This methodology exploits the staggered nature of the linearised Lagrangian approach described in Chapter 3 by building a ROM to approximate the electromagnetic solution and using this to feed the mechanical full order solver. First the methodology to apply POD to the complete problem is described, followed by the application to both problems separately and the description of the reduced order-full order methodology. Furthermore, an affine decomposition of the problem, in terms of frequency and conductivity, is developed, which is key for the efficiency of the projection based POD. The Chapter builds on the work first presented by the author in [159].

The structure of the Chapter is as follows: First, in Sections 6.2 and 6.3, the variational formulation and discrete system of the linearised and regularised problem are rewritten in a form more suitable for the development of a ROM, where the parametric dependency is highlighted. Then, in Section 6.4, the Singular Value Decomposition (SVD), which is a 
key mathematical tool in the application of POD, is introduced. The POD is described in Section 6.5, where the off-line stage is first discussed (Section 6.5.1) followed by the interpolation and projection based on-line stage (Sections 6.5.2 and 6.5.3). The application of POD to electromagnetics and mechanics separately is discussed in Section 6.6 and the combined POD-Full methodology is presented in Section 6.7. The chapter finishes with concluding remarks in Section 6.9.

\subsection{Variational Formulation}

In order to derive a ROM for the magneto-mechanical problem of interest, the variational formulation (3.35) is rewritten here in an alternative format where the dependency on the model parameters is explicitly written. Let $\boldsymbol{w}=\left(w_{1}, w_{2}, \ldots, w_{N_{p}}\right)$ denote a general set of model parameters (frequency or conductivity of each shield among others). Note that in this thesis only parameters on which the solution of the DC problem does not depend upon are considered and, thus, the focus is on the AC problem ${ }^{1}$. The variational formulation of the AC problem (3.35) can then be stated in the alternative form: Find complex solutions $\left(\boldsymbol{A}_{\varepsilon}^{A C}, \boldsymbol{u}^{A C}\right) \in \tilde{X}_{\Omega}(\mathbf{0}) \times Y(\mathbf{0})$ such that

$$
\begin{aligned}
a_{\mathcal{A}_{\varepsilon}}\left(\boldsymbol{A}_{\varepsilon}^{A C}(\boldsymbol{x}, \boldsymbol{w}), \delta \boldsymbol{A}(\boldsymbol{x}, \boldsymbol{w})\right) & =r_{\mathcal{A}_{\varepsilon}}(\delta \boldsymbol{A}(\boldsymbol{x}, \boldsymbol{w})), \\
a_{\mathcal{U}}\left(\boldsymbol{U}^{A C}(\boldsymbol{x}, \boldsymbol{w}), \delta \boldsymbol{u}(\boldsymbol{x}, \boldsymbol{w}) ; \boldsymbol{A}_{\varepsilon}^{D C}(\boldsymbol{x}), \boldsymbol{A}_{\varepsilon}^{A C}(\boldsymbol{x}, \boldsymbol{w})\right) & =0
\end{aligned}
$$

for all $(\delta \boldsymbol{A}, \delta \boldsymbol{u}) \in \tilde{X}_{\Omega}(\mathbf{0}) \times Y(\mathbf{0})$, where

$$
\begin{aligned}
& a_{\mathcal{A}_{\varepsilon}}\left(\mathcal{A}_{\varepsilon}^{A C}(\boldsymbol{x}, \boldsymbol{w}), \delta \boldsymbol{A}(\boldsymbol{x}, \boldsymbol{w})\right):=\int_{\Omega}\left(\mu_{r}^{-1} \operatorname{curl} \boldsymbol{A}_{\varepsilon}^{A C}(\boldsymbol{x}, \boldsymbol{w})\right) \cdot(\operatorname{curl} \overline{\delta \boldsymbol{A}(\boldsymbol{x}, \boldsymbol{w})}) \mathrm{d} \Omega \\
&+\mathrm{i} \omega \mu_{0} \int_{\Omega_{C}} \gamma \boldsymbol{A}_{\varepsilon}^{A C}(\boldsymbol{x}, \boldsymbol{w}) \cdot \overline{\delta \boldsymbol{A}(\boldsymbol{x}, \boldsymbol{w})} \mathrm{d} \Omega \\
&+\omega \varepsilon \int_{\Omega_{N C}} \boldsymbol{A}_{\varepsilon}^{A C}(\boldsymbol{x}, \boldsymbol{w}) \cdot \overline{\delta \boldsymbol{A}(\boldsymbol{x}, \boldsymbol{w})} \mathrm{d} \Omega \\
& a_{\mathcal{U}}\left(\boldsymbol{u}^{A C}(\boldsymbol{x}, \boldsymbol{w}), \delta \boldsymbol{u}(\boldsymbol{x}, \boldsymbol{w}) ; \boldsymbol{A}_{\varepsilon}^{D C}(\boldsymbol{x}), \boldsymbol{A}_{\varepsilon}^{A C}(\boldsymbol{x}, \boldsymbol{w})\right):= \\
& \quad \mu_{0} \int_{\Omega_{C}} \boldsymbol{\sigma}^{m}\left(\boldsymbol{u}^{A C}(\boldsymbol{x}, \boldsymbol{w})\right):(\operatorname{grad} \overline{\delta \boldsymbol{u}(\boldsymbol{x}, \boldsymbol{w})}) \mathrm{d} \Omega \\
&-\mu_{0} \omega^{2} \int_{\Omega_{C}} \rho \boldsymbol{U}^{A C}(\boldsymbol{x}, \boldsymbol{w}) \cdot \overline{\delta \boldsymbol{u}(\boldsymbol{x}, \boldsymbol{w})} \mathrm{d} \Omega \\
&+\int_{\Omega} \mu_{r}^{-1} S\left(\boldsymbol{A}_{\varepsilon}^{D C}(\boldsymbol{x}), \mathcal{A}_{\varepsilon}^{A C}(\boldsymbol{x}, \boldsymbol{w})\right):(\operatorname{grad} \overline{\delta \boldsymbol{u}(\boldsymbol{x}, \boldsymbol{w})}) \mathrm{d} \Omega \\
& r_{\mathcal{A}_{\varepsilon}}(\delta \boldsymbol{A}(\boldsymbol{x}, \boldsymbol{w})):=\mu_{0} \int_{\operatorname{supp}\left(\boldsymbol{g}^{A C}\right)} \boldsymbol{g}^{A C}(\boldsymbol{x}) \cdot \overline{\delta \boldsymbol{A}(\boldsymbol{x}, \boldsymbol{w})} \mathrm{d} \Omega
\end{aligned}
$$

\footnotetext{
${ }^{1}$ Note that the formulation is easily extensible to the case where the solution of the DC problem also depends on the parameters of interest (e.g. permeability). However, typically in MRI applications the biggest interest lies in parameters that affect only the AC problem (frequency, conductivity or shield thickness among others).
} 
Note that $a_{\mathcal{A}_{\varepsilon}}\left(\boldsymbol{A}_{\varepsilon}^{A C}(\boldsymbol{x}, \boldsymbol{w}), \delta \boldsymbol{A}(\boldsymbol{x}, \boldsymbol{w})\right)$ is a sesquilinear form, $r_{\mathfrak{A}_{\varepsilon}}(\delta \boldsymbol{A}(\boldsymbol{x}, \boldsymbol{w}))$ is an antilinear form and $a_{\mathcal{U}}\left(\boldsymbol{u}^{A C}(\boldsymbol{x}, \boldsymbol{w}), \delta \boldsymbol{u}(\boldsymbol{x}, \boldsymbol{w}) ; \boldsymbol{A}_{\varepsilon}^{D C}(\boldsymbol{x}), \boldsymbol{A}_{\varepsilon}^{A C}(\boldsymbol{x}, \boldsymbol{w})\right)$ can be rewritten as

$$
\begin{array}{r}
a_{\mathcal{U}}\left(\boldsymbol{u}^{A C}(\boldsymbol{x}, \boldsymbol{w}), \delta \boldsymbol{u}(\boldsymbol{x}, \boldsymbol{w}) ; \boldsymbol{A}_{\varepsilon}^{D C}(\boldsymbol{x}), \boldsymbol{A}_{\varepsilon}^{A C}(\boldsymbol{x}, \boldsymbol{w})\right)=\tilde{a}_{\mathcal{U}}\left(\boldsymbol{u}^{A C}(\boldsymbol{x}, \boldsymbol{w}), \delta \boldsymbol{u}(\boldsymbol{x}, \boldsymbol{w})\right) \\
-\tilde{r}_{\mathcal{U}}\left(\delta \boldsymbol{u}(\boldsymbol{x}, \boldsymbol{w}) ; \boldsymbol{A}_{\varepsilon}^{D C}(\boldsymbol{x}), \boldsymbol{A}_{\varepsilon}^{A C}(\boldsymbol{x}, \boldsymbol{w})\right),
\end{array}
$$

where the sesquilinear $\tilde{a}_{\mathcal{U}}\left(\boldsymbol{u}^{A C}(\boldsymbol{x}, \boldsymbol{w}), \delta \boldsymbol{u}(\boldsymbol{x}, \boldsymbol{w})\right)$ and antilinear $\tilde{r}_{\mathcal{U}}\left(\delta \boldsymbol{u}(\boldsymbol{x}, \boldsymbol{w}) ; \boldsymbol{A}_{\varepsilon}^{D C}(\boldsymbol{x}), \boldsymbol{A}_{\varepsilon}^{A C}(\boldsymbol{x}, \boldsymbol{w})\right)$ forms are defined as

$$
\begin{aligned}
& \tilde{a}_{\mathcal{U}}\left(\boldsymbol{u}^{A C}(\boldsymbol{x}, \boldsymbol{w}), \delta \boldsymbol{u}(\boldsymbol{x}, \boldsymbol{w})\right):=\mu_{0} \int_{\Omega_{C}} \boldsymbol{\sigma}^{m}\left(\boldsymbol{u}^{A C}(\boldsymbol{x}, \boldsymbol{w})\right):(\operatorname{grad} \overline{\delta \boldsymbol{u}(\boldsymbol{x}, \boldsymbol{w})}) \mathrm{d} \Omega \\
&-\mu_{0} \omega^{2} \int_{\Omega_{C}} \rho \boldsymbol{u}^{A C}(\boldsymbol{x}, \boldsymbol{w}) \cdot \overline{\delta \boldsymbol{u}(\boldsymbol{x}, \boldsymbol{w})} \mathrm{d} \Omega, \quad \text { (6.4a) } \\
& \tilde{r}_{\mathcal{U}}\left(\delta \boldsymbol{u}(\boldsymbol{x}, \boldsymbol{w}) ; \boldsymbol{A}_{\varepsilon}^{D C}(\boldsymbol{x}), \boldsymbol{A}_{\varepsilon}^{A C}(\boldsymbol{x}, \boldsymbol{w})\right):= \\
&-\int_{\Omega} \mu_{r}^{-1} S\left(\boldsymbol{A}_{\varepsilon}^{D C}(\boldsymbol{x}), \boldsymbol{A}_{\varepsilon}^{A C}(\boldsymbol{x}, \boldsymbol{w})\right):(\operatorname{grad} \overline{\delta \boldsymbol{u}(\boldsymbol{x}, \boldsymbol{w})}) \mathrm{d} \Omega . \quad \text { (6.4b) }
\end{aligned}
$$

Furthermore, the abstract weak form (6.1) can be rewritten in a compact form for the solution vector $\boldsymbol{q}(\boldsymbol{x}, \boldsymbol{w}):=\left(\begin{array}{l}\mathcal{A}_{\varepsilon}^{A C}(\boldsymbol{x}, \boldsymbol{w}) \\ \boldsymbol{u}^{A C}(\boldsymbol{x}, \boldsymbol{w})\end{array}\right)$ as: Find complex solutions $\boldsymbol{q} \in \tilde{X}_{\Omega}(\mathbf{0}) \times Y(\mathbf{0})$ such that:

$$
\boldsymbol{a}(\boldsymbol{q}(\boldsymbol{x}, \boldsymbol{w}), \delta \boldsymbol{q}(\boldsymbol{x}, \boldsymbol{w}))=\boldsymbol{r}(\delta \boldsymbol{q}(\boldsymbol{x}, \boldsymbol{w}))
$$

for all $\delta \boldsymbol{q} \in \tilde{X}_{\Omega}(\mathbf{0}) \times Y(\mathbf{0})$.

\subsection{Discrete System}

For parameters of interest $\boldsymbol{w}$, let us define the Galerkin approximation to the AC fields at position $\boldsymbol{x}$ as

$$
\boldsymbol{q}_{h p q}(\boldsymbol{x}, \boldsymbol{w}):=\left(\begin{array}{l}
\mathcal{A}_{\varepsilon, h q}^{A C}(\boldsymbol{x}, \boldsymbol{w}) \\
\boldsymbol{u}_{h p}^{A C}(\boldsymbol{x}, \boldsymbol{w})
\end{array}\right)=\left(\begin{array}{l}
\sum_{i=1}^{P_{\text {global }}} \boldsymbol{N}^{i}(\boldsymbol{x}) \mathcal{A}_{\varepsilon}^{\mathrm{AC}, i}(\boldsymbol{w}) \\
\sum_{i=1}^{Q_{\text {global }}} L^{i}(\boldsymbol{x}) \mathcal{U}^{\mathrm{AC}, i}(\boldsymbol{w})
\end{array}\right) \in S
$$

where $S:=\left(\tilde{X}_{\Omega}(\mathbf{0}) \cap X_{\text {reg }}^{A C}\right) \times\left(Y(\mathbf{0}) \cap Y_{h p}\right)$. Furthermore, $N_{d}=P_{\text {global }}+3 Q_{\text {global }}$ is the total number of DOF, which are split into $P_{\text {global }}$ electromagnetic DOF and $3 Q_{\text {global }}$ mechanical DOF and

$$
\begin{aligned}
\mathbf{N}(\boldsymbol{x}) & :=\left[\boldsymbol{N}^{1}, \boldsymbol{N}^{2}, \ldots, \boldsymbol{N}^{P_{\text {global }}}\right] \\
\mathbf{L}(\boldsymbol{x}) & :=\left[\mathbf{l}_{1}, \mathbf{l}_{2}, \ldots, \mathbf{l}_{3 Q_{\text {global }}}\right]=\left[\begin{array}{ccccccc}
L^{1} & 0 & 0 & \ldots & L^{Q_{\text {global }}} & 0 & 0 \\
0 & L^{1} & 0 & \ldots & 0 & L^{Q_{\text {global }}} & 0 \\
0 & 0 & L^{1} & \ldots & 0 & 0 & L^{Q_{\text {global }}}
\end{array}\right]
\end{aligned}
$$

are the matrices of $\boldsymbol{H}$ (curl) and $H^{1}$ basis functions, where $\mathbf{l}_{1}(\boldsymbol{x}), \mathbf{l}_{2}(\boldsymbol{x}), \ldots, \mathbf{l}_{3 Q_{\text {global }}}(\boldsymbol{x})$ are the columns of $\mathbf{L}(\boldsymbol{x})$, so that

$$
\boldsymbol{q}_{h p q}(\boldsymbol{x}, \boldsymbol{w})=\left(\begin{array}{c}
\mathbf{N}(\boldsymbol{x}) \mathcal{A}_{\varepsilon}^{\mathrm{AC}}(\boldsymbol{w}) \\
\mathbf{L}(\boldsymbol{x}) \mathcal{U}^{\mathrm{AC}}(\boldsymbol{w})
\end{array}\right)=\left[\begin{array}{cc}
\mathbf{N}(\boldsymbol{x}) & \mathbf{0} \\
\mathbf{0} & \mathbf{L}(\boldsymbol{x})
\end{array}\right]\left(\begin{array}{c}
\mathcal{A}_{\varepsilon}^{\mathrm{AC}}(\boldsymbol{w}) \\
\mathcal{U}^{\mathrm{AC}}(\boldsymbol{w})
\end{array}\right)=\mathbf{O}(\boldsymbol{x}) \mathbf{q}(\boldsymbol{w}),
$$


where $\mathbf{o}_{1}(\boldsymbol{x}), \ldots, \mathbf{o}_{N_{d}}(\boldsymbol{x})$ are the columns of $\mathbf{O}(\boldsymbol{x})$ and $\mathbf{q}(\boldsymbol{w}):=\left(\begin{array}{l}\mathcal{A}_{\varepsilon}^{\mathrm{AC}}(\boldsymbol{w}) \\ \mathcal{U}^{\mathrm{AC}}(\boldsymbol{w})\end{array}\right)$ are the solution coefficients. Inserting the discrete approximation to the solution field in the variational form (6.5) results in

$$
\sum_{i=1}^{N_{d}} \sum_{j=1}^{N_{d}} \overline{\delta \mathrm{q}_{i}(\boldsymbol{w})} \boldsymbol{a}\left(\mathbf{o}_{j}(\boldsymbol{x}), \mathbf{o}_{i}(\boldsymbol{x})\right) \mathrm{q}_{j}(\boldsymbol{w})=\sum_{i=1}^{N_{d}} \overline{\delta \mathrm{q}_{i}(\boldsymbol{w})} \boldsymbol{r}\left(\mathbf{o}_{i}(\boldsymbol{x})\right)
$$

and, choosing $\delta \mathrm{q}_{i}(\boldsymbol{w})$ appropriately, this leads to the linear system

$$
\mathbf{A}(\boldsymbol{w}) \mathbf{q}(\boldsymbol{w})=\mathbf{R}(\boldsymbol{w})
$$

This is of size $N_{d}$ and the system matrix and right hand side vector are defined as

$$
\begin{aligned}
& \mathbf{A}(\boldsymbol{w}):=\left[\begin{array}{cc}
\mathbf{A}_{\mathcal{A A}}(\boldsymbol{w}) & \mathbf{0} \\
\mathbf{A}_{\mathcal{U A}}(\boldsymbol{w}) & \mathbf{A}_{\mathcal{U U}}(\boldsymbol{w})
\end{array}\right] \\
& =\left[\begin{array}{cc}
\mathbf{K}_{\mathcal{A} \mathcal{A}}^{\mathrm{AC}}(\boldsymbol{w})+\mathbf{C}_{\mathcal{A \mathcal { A }}}^{\mathrm{AC}}(\boldsymbol{w}) & \mathbf{0} \\
\mathbf{K}_{\mathcal{U} \mathcal{A}}^{\mathrm{AC}}(\boldsymbol{w}) & \mathbf{K}_{\mathcal{U U}}^{\mathrm{AC}}(\boldsymbol{w})+\mathrm{i} \omega \mathbf{C}_{\mathcal{U U}}^{\mathrm{AC}}(\boldsymbol{w})-\omega^{2} \mathbf{M}_{\mathcal{U} \mathcal{U}}^{\mathrm{AC}}(\boldsymbol{w})
\end{array}\right], \\
& \mathbf{R}(\boldsymbol{w}):=\left(\begin{array}{c}
\mathbf{R}_{\mathcal{A}}(\boldsymbol{w}) \\
\mathbf{0}
\end{array}\right)=\left(\begin{array}{c}
\mathbf{R}_{\mathcal{A}}^{\mathrm{AC}}(\boldsymbol{w}) \\
\mathbf{0}
\end{array}\right) \text {. }
\end{aligned}
$$

\subsection{The Singular Value Decomposition (SVD)}

Before proceeding to explain how a ROM for the coupled problem of interest will be constructed, an essential mathematical tool for this construction must be introduced, the so-called SVD.

The SVD [33, 84] states that, given a complex matrix $\mathbf{D} \in \mathbb{C}^{m \times n}$, there exist unitary matrices $\mathbf{H} \in \mathbb{C}^{m \times m}$ and $\mathbf{G} \in \mathbb{C}^{n \times n}$ such that:

$$
\mathbf{D}=\mathbf{H} \boldsymbol{\Sigma} \mathbf{G}^{*}=\sum_{k=1}^{\min (m, n)} \mathbf{h}_{k} \sigma_{k}\left(\mathbf{g}_{k}\right)^{*}
$$

where $\mathbf{H}=\left[\mathbf{h}_{1}, \ldots, \mathbf{h}_{m}\right] \in \mathbb{C}^{m \times m}$ and $\mathbf{G}=\left[\mathbf{g}_{1}, \ldots, \mathbf{g}_{n}\right] \in \mathbb{C}^{n \times n}$ are unitary matrices containing the left and right singular vectors of $\mathbf{D}$, respectively, $\mathrm{G}^{*}$ denotes the complex conjugate transpose of $\mathbf{G}, \boldsymbol{\Sigma} \in \mathbb{R}^{m \times n}$ is the matrix $\boldsymbol{\Sigma}=\left[\begin{array}{cc}\boldsymbol{\Sigma}_{r} & \mathbf{0} \\ \mathbf{0} & \mathbf{0}\end{array}\right]$ with $\boldsymbol{\Sigma}_{r}=\operatorname{diag}\left(\sigma_{1}, \ldots, \sigma_{r}\right), r$ being the rank of $\mathbf{D}$ and $\sigma_{1} \geqslant \sigma_{2} \geqslant \cdots \geqslant \sigma_{r}>0$ are the singular values of $\mathbf{D}$. Note that although the matrix $\Sigma$ can be expressed in terms of $\Sigma_{r}$ for all cases as written above, distinction should be made between the cases $m>n$ and $m<n$. In the case $m>n, \Sigma$ can be written as: 


$$
\left[\begin{array}{cc}
\boldsymbol{\Sigma}_{r} & 0 \\
\mathbf{0} & 0
\end{array}\right]=\left[\begin{array}{cccc}
\sigma_{1} & 0 & \ldots & 0 \\
0 & \sigma_{2} & \ldots & 0 \\
\vdots & \vdots & \ddots & \vdots \\
0 & 0 & \ldots & \sigma_{n} \\
0 & 0 & \ldots & 0 \\
\vdots & \ddots & \ddots & \vdots \\
0 & \ldots & \ldots & 0
\end{array}\right]
$$

with $r \leqslant n$, and for the case $m<n$ :

$$
\left[\begin{array}{cc}
\boldsymbol{\Sigma}_{r} & \mathbf{0} \\
\mathbf{0} & \mathbf{0}
\end{array}\right]=\left[\begin{array}{ccccccc}
\sigma_{1} & 0 & \ldots & 0 & 0 & \ldots & 0 \\
0 & \sigma_{2} & \ldots & 0 & 0 & \ddots & \vdots \\
\vdots & \vdots & \ddots & \vdots & \vdots & \ddots & \vdots \\
0 & 0 & \ldots & \sigma_{m} & 0 & \ldots & 0
\end{array}\right]
$$

where $r \leqslant m$.

Among many other applications, the SVD has successfully been applied to least square problems, such as the minimal least squares solution to rank deficient or ill-conditioned linear systems of equations [33, 84, 109], that arise often in inverse problems such as finding hidden targets and medical imaging, or low rank matrix approximation [33]. This feature of the SVD will constitute the main tool for the construction of the reduced order model presented in this thesis, as will be shown later. Note that some of the singular values can be very small and, thus, the part of the SVD expansion associated with these singular values can in practice be neglected. This is what is called Truncated Singular Value Decomposition (TSVD), that is described in the following.

\subsubsection{Truncated Singular Value Decomposition}

The TSVD [33, 84, 109] can be applied to obtain low rank matrix approximations. Given a matrix $\mathbf{D}$ its TSVD rank $M$ (with $M<r$ ) approximation is given by:

$$
\mathbf{D}^{M}=\sum_{k=1}^{M} \mathbf{h}_{k} \sigma_{k}\left(\mathbf{g}_{k}\right)^{*}=\mathbf{H}^{M} \boldsymbol{\Sigma}^{M}\left(\mathbf{G}^{M}\right)^{*}
$$

where the contributions made by the smallest singular values $\sigma_{k}, M<k \leqslant r$ have been filtered and only the largest $M<r$ singular values are retained. The left singular vectors can then be arranged in a matrix $\mathbf{H}^{M} \in \mathbb{C}^{m \times M}$, the right singular vectors in a matrix $\mathbf{G}^{M} \in \mathbb{C}^{n \times M}$ and the singular values are the diagonal values in the matrix $\Sigma^{M} \in \mathbb{R}^{M \times M}$. This approximation is optimal in a least squares sense [43, 62], this is:

$$
\mathbf{D}^{M}=\arg \min _{\mathbf{W} \in \mathbb{C}^{m \times n} \text { of rank } M}\|\mathbf{D}-\mathbf{W}\|_{F}
$$

where the Frobenius matrix norm is defined as $\|\mathbf{D}\|_{F}^{2}=\mathbf{D}: \mathbf{D}$. 


\subsection{The Proper Orthogonal Decomposition}

As discussed in Chapter 1, several numerical techniques can be classified as ROMs. Some examples are PGD $[49,127,50]$ and POD [43, 119, 180, 47], whose variants include POD with interpolation (PODI) $[145,144,42]$ and projection based POD (in the following PODP), also known as POD based Reduced Basis (RB) or POD-Galerkin [165, 72, 61]. In this thesis the application of POD is considered, due to its relatively low intrusiveness compared to other techniques such as PGD.

In the context of numerical simulations in engineering, POD involves two stages in the solution of the parametric problem. During the first stage, the basis to represent the solution is chosen. To build this basis, many FE solutions need to be obtained and, therefore, this stage is computationally expensive, although this can be mitigated as it is trivially parallelisable and needs only to be done once. From the computed solutions, in this work it is chosen to obtain the basis via a SVD $[33,84]$. The second stage, performed once the basis has been chosen, involves finding the solution for a given set of parameters. In the case of PODI, this second phase consists simply in interpolating the parametric modes (right singular vectors), while in the case of PODP, it involves the solution of a reduced linear system of equations with the same size as the chosen basis. Given that this size is reduced, this second stage is significantly cheaper than the first stage to the extent that the solution can be obtained in real time ${ }^{2}$. This two stage scheme becomes efficient when the second stage is applied many times (multiple queries) or when the second stage has to be solved very fast (fast queries).

\subsubsection{Off-line Stage: Construction of the Basis via SVD}

The solution coefficients $\mathbf{q}(\boldsymbol{w})$ in (6.10) are approximated as

$$
\mathbf{q}(\boldsymbol{w}) \approx \sum_{k=1}^{N} \mathrm{a}_{k}(\boldsymbol{w}) \boldsymbol{\phi}_{k}
$$

for some basis vectors $\boldsymbol{\Phi}_{k}$ and parameter dependent amplitudes $\mathrm{a}_{k}(\boldsymbol{w})$, with a small number of terms $N<<N_{d}$. The first goal is to find a suitable set of $\boldsymbol{\phi}_{k}$ that spans the variation of $\mathbf{q}$ with $\boldsymbol{w}$. To achieve this, a set of snapshots is computed, aiming at sampling the family of solutions $q$ when $\boldsymbol{w}$ changes $[43,47]$. The computation of each snapshot involves the computation of one full order problem. A matrix of snapshots $\mathbf{D} \in \mathbb{C}^{N_{d} \times N_{s}}$ is built in the form ${ }^{3}$

$$
\mathbf{D}=\left[\mathbf{q}\left(\mathbf{w}_{1}\right), \mathbf{q}\left(\mathbf{w}_{2}\right), \ldots, \mathbf{q}\left(\mathbf{w}_{N_{s}}\right)\right],
$$

where $\mathbf{q}\left(\mathbf{w}_{j}\right)$ is a single snapshot obtained as the solution to (6.10) for a given set of parameters $\mathbf{w}_{j}{ }^{4}$ and $N_{s}$ is the number of computed snapshots.

\footnotetext{
${ }^{2}$ Provided most of the assembly of this reduced system can be done off-line as will be the case in the application of interest in this thesis.

${ }^{3}$ Note that for our application $N_{d}>N_{s}$ and thus, for the sake of simplicity, we will restrict our consideration to this case.

${ }^{4}$ Note that the roman font in $\mathbf{w}$ is used to denote a discrete set of sampled parameters.
} 
In order to obtain the optimal basis $\boldsymbol{\phi}_{k}$ to represent this data, a SVD [33, 84] can be applied, leading to the representation of the snapshot matrix

$$
\mathbf{D}=\mathbf{H} \boldsymbol{\Sigma} \mathbf{G}^{*}=\sum_{k=1}^{N_{s}} \mathbf{h}_{k} \sigma_{k}\left(\mathbf{g}_{k}\right)^{*}
$$

The solution for any sampled parameters $\mathbf{w}_{j}$ can then be recovered, if desired, using

$$
\mathbf{q}\left(\mathbf{w}_{j}\right)=\sum_{k=1}^{N_{s}} \mathbf{h}_{k} \sigma_{k} \overline{g_{j k}}=\mathbf{H} \boldsymbol{\Sigma}\left(\mathbf{G}^{*}\right)_{j},
$$

where $\mathrm{g}_{j k}$ is the entry of $\mathbf{G}$ on the $j$-th row and $k$-th column, the optimal set of basis $\boldsymbol{\Phi}_{k}$ have been found to be $\mathbf{h}_{k}$ and the amplitudes $\mathrm{a}_{k}\left(\mathbf{w}_{j}\right)$ have been found to be $\sigma_{k} \overline{g_{j k}}$. Note that the notation $\left(\mathbf{G}^{*}\right)_{j}$ is used to denote the $j$-th column of $\mathbf{G}^{*}$ and the dependency on $\mathbf{w}_{j}$ is emphasized trough the sub-index $j$.

Furthermore, by applying a TSVD, a low rank approximation of D can be obtained

$$
\mathbf{D} \approx \mathbf{D}^{M}:=\sum_{k=1}^{M} \mathbf{h}_{k} \sigma_{k}\left(\mathbf{g}_{k}\right)^{*}=\sum_{k=1}^{M} \mathbf{H}^{M} \boldsymbol{\Sigma}^{M}\left(\mathbf{G}^{M}\right)^{*}
$$

where the left singular vectors are contained in the matrix $\mathbf{H}^{M} \in \mathbb{C}^{N_{d} \times M}$, the right singular vectors in the matrix $\mathbf{G}^{M} \in \mathbb{C}^{N_{s} \times M}$ and the singular values are the diagonal entries in the matrix $\Sigma^{M} \in \mathbb{R}^{M \times M}$. The rank $M$ of the approximated matrix is also referred to as the number of modes.

The solution, for a sampled parameter set, can then be approximated using

$$
\mathbf{q}\left(\mathbf{w}_{j}\right) \approx \mathbf{q}^{\mathrm{POD}}\left(\mathbf{w}_{j}\right):=\sum_{k=1}^{M} \mathbf{h}_{k} \sigma_{k} \overline{\mathrm{g}_{j k}}=\mathbf{H}^{M} \boldsymbol{\Sigma}^{M}\left(\left(\mathbf{G}^{M}\right)^{*}\right)_{j}
$$

where, comparing with (6.17), $\mathrm{a}_{k}\left(\mathbf{w}_{j}\right)=\sigma_{k} \overline{\mathrm{g}_{j k}}, \boldsymbol{\phi}_{k}=\mathbf{h}_{k}$ and $N=M$.

The process of computing the snapshots and applying the TSVD is usually referred to as the off-line stage, as this can be computed and stored at an initial stage independently of sweeps of the parameters of interest $\boldsymbol{w}$. This off-line stage is summarised in Algorithm 2 where $\mathbf{W}_{\mathrm{s}}:=\left(\mathbf{w}_{1}, \mathbf{w}_{2}, \ldots, \mathbf{w}_{N_{s}}\right)$ denotes the chosen samples. Note that the input arguments $\mathbf{A}(\boldsymbol{w}), \mathbf{R}(\boldsymbol{w})$ in the algorithm indicate that a method to compute $\mathbf{A}$ and $\mathbf{R}$ for all $\boldsymbol{w}$ is given to the algorithm.

The aim is to construct an approximated solution $\mathbf{q}^{\mathrm{POD}}(\boldsymbol{w})$ for any $\boldsymbol{w}$, not just for the sampled values. This is done in the so-called on-line stage, where two different approaches, namely PODI and PODP, can be used to obtain $\mathbf{q}^{\mathrm{POD}}(\boldsymbol{w})$ for any value of $\boldsymbol{w}$ [180]. PODI $[145,144,42]$ is less intrusive than PODP $[165,72,61]$ since it does not require any information from the finite element solver, as opposed to PODP, which requires the FE system matrices to be accessible. On the other hand, PODI requires the solution to have a smooth variation with the parameters in order to provide accurate results, while PODP offers an increased accuracy and robustness, especially as the smoothness of the solution decreases $[180,152]$. These approaches are described in the next two subsections. 


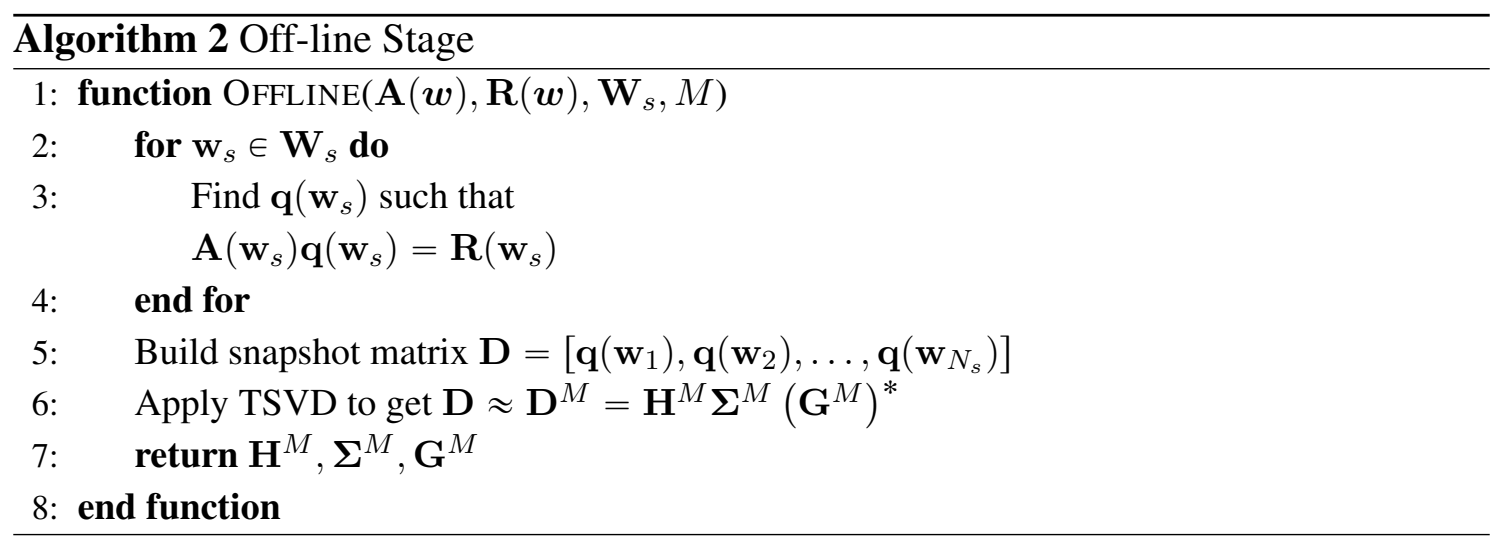

\subsubsection{On-line Interpolation Based POD (PODI)}

Expression (6.22) describes how the POD solution $\mathbf{q}\left(\mathbf{w}_{j}\right)$ can be recovered from a TSVD when $\boldsymbol{w}=\mathbf{w}_{j}$ is a set of parameters that has been sampled. In PODI, the coefficients $\overline{g_{j k}}$ are interpolated in order to obtain the solution at others parameters of interest. This solution takes the form

$$
\mathbf{q}^{\mathrm{PODI}}(\boldsymbol{w}):=\sum_{k=1}^{M} \mathbf{h}_{k} \sigma_{k} \sum_{j=1}^{M} I_{j}(\boldsymbol{w}) \overline{\mathrm{g}_{j k}}
$$

where $I_{j}(\boldsymbol{w})$ denote a certain set of interpolation functions, whose definitions can change depending on $\boldsymbol{w}$. The simple case of Lagrange linear and cubic interpolation will be considered and compared later in this thesis, although (6.23) also holds for arbitrary high order Lagrange interpolation. Such approximations are not considered here as it is well known that this can lead to additional oscillations in the interpolated solution e.g. [168]. Furthermore, when considering arbitrary high dimensional spaces, this interpolation becomes challenging due to the necessity to use dense meshes and ensure a properly posed set of nodes is chosen [118] in order to interpolate the solution vectors. Due to this, it is difficult, in general, to find an interpolation scheme that is both accurate and efficient for the case of an arbitrary high dimensional space [107]. Hence, the application of PODI is, in general, better suited when the number of parameters of interest is small, and becomes more challenging when $N_{p}$ is large.

The on-line PODI stage is summarised in algorithmic format in Algorithm 3, which results in the output $\boldsymbol{q}^{P O D I}(\boldsymbol{x}, \boldsymbol{w}) \in S$.

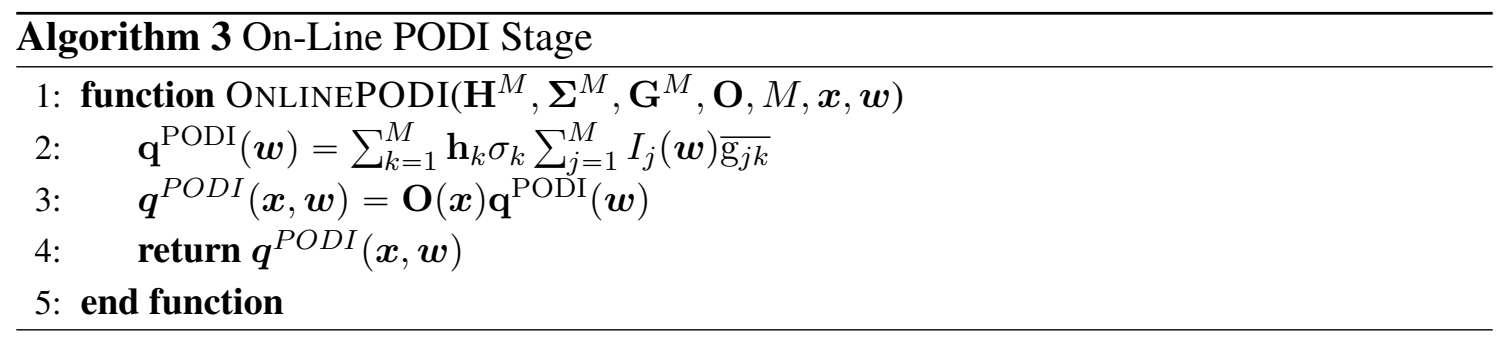




\subsubsection{On-line Projection Based POD (PODP)}

In PODP, the singular values and right singular vectors obtained from the TSVD are neglected, and the solution is approximated as a linear combination of the left singular vectors or POD basis vectors in the form

$$
\mathbf{q}^{\mathrm{PODP}}(\boldsymbol{w}):=\sum_{k=1}^{M} \mathbf{h}_{k} \mathrm{p}_{k}(\boldsymbol{w})=\mathbf{H}^{M} \mathbf{p}(\boldsymbol{w})
$$

where the parametric mode vector $\mathbf{p}(\boldsymbol{w})$ is unknown. Inserting this approximation into expression (6.8), the solution $\boldsymbol{q}_{h p q}(\boldsymbol{x}, \boldsymbol{w})$ can be approximated using

$$
\boldsymbol{q}_{h p q}(\boldsymbol{x}, \boldsymbol{w}) \approx \boldsymbol{q}^{P O D P}(\boldsymbol{x}, \boldsymbol{w}):=\mathbf{O}(\boldsymbol{x}) \mathbf{q}^{\mathrm{PODP}}(\boldsymbol{w})=\mathbf{O}(\boldsymbol{x}) \sum_{k=1}^{M} \mathbf{h}_{k} \mathrm{p}_{k}(\boldsymbol{w}) \in S .
$$

Then, substituting (6.25) into the weak form (6.5) and choosing to approximate the test functions $\delta \boldsymbol{q}$ in the same manner, the next expression is obtained

$$
\sum_{i=1}^{M} \sum_{j=1}^{M} \overline{\delta \mathrm{p}_{i}(\boldsymbol{w})} \boldsymbol{a}\left(\mathbf{O}(\boldsymbol{x}) \mathbf{h}_{j}, \mathbf{O}(\boldsymbol{x}) \mathbf{h}_{i}\right) \mathrm{p}_{j}(\boldsymbol{w})=\sum_{i=1}^{M} \overline{\delta \mathrm{p}_{i}(\boldsymbol{w})} \boldsymbol{r}\left(\mathbf{O}(\boldsymbol{x}) \mathbf{h}_{i}\right)
$$

and, choosing $\delta \mathrm{p}_{i}(\boldsymbol{w})$ appropriately, this leads to the reduced linear system

$$
\left[\left(\mathbf{H}^{M}\right)^{*} \mathbf{A}(\boldsymbol{w}) \mathbf{H}^{M}\right] \mathbf{p}(\boldsymbol{w})=\left(\mathbf{H}^{M}\right)^{*} \mathbf{R}^{\mathrm{q}}
$$

that is of size $M$. Once system (6.27) is solved, the solution $\boldsymbol{q}^{P O D P}(\boldsymbol{x}, \boldsymbol{w})$ can be obtained from expression (6.25).

Defining the reduced matrix of the system, obtained by projection onto the reduced dimension space, as $\mathbf{A}^{M}(\boldsymbol{w}):=\left(\mathbf{H}^{M}\right)^{*} \mathbf{A}(\boldsymbol{w}) \mathbf{H}^{M}$ and the reduced right hand side vector as $\mathbf{R}^{M}(\boldsymbol{w}):=\left(\mathbf{H}^{M}\right)^{*} \mathbf{R}(\boldsymbol{w})$, equation (6.27) becomes

$$
\mathbf{A}^{M}(\boldsymbol{w}) \mathbf{p}(\boldsymbol{w})=\mathbf{R}^{M}(\boldsymbol{w}) .
$$

Note that the coefficients in the solution vector $\mathbf{p}(\boldsymbol{w})$ define the ROM approximation and are obtained by solving a system of size $M$, which is computationally much cheaper than the full order problem when $M<<N_{d}$. The on-line PODP stage is summarised in algorithmic format in Algorithm 4, which has as output $\boldsymbol{q}^{P O D P}(\boldsymbol{x}, \boldsymbol{w}) \in S$.

PODP has the advantage that the POD solution is still forced to obey the equation of the problem in its reduced form (6.27) for all $\boldsymbol{w}$, while in PODI a simple interpolation of parameter dependent amplitudes is considered. This usually leads to a higher accuracy and robustness [180] of PODP compared to PODI, but it also has the increased cost of having to build and solve the small system (6.27). Note that computing the products $\left(\mathbf{H}^{M}\right)^{*} \mathbf{A}(\boldsymbol{w}) \mathbf{H}^{M}$ and $\left(\mathbf{H}^{M}\right)^{*} \mathbf{R}(\boldsymbol{w})$ is expensive and, thus, it is desired to avoid computing it for each new value of $\boldsymbol{w}$. For this purpose, an affine decomposition in terms of the parameters of interest is usually developed [87], where the system matrix is expressed in terms of some modified matrices that are independent of $\boldsymbol{w}$ and parameter dependent 


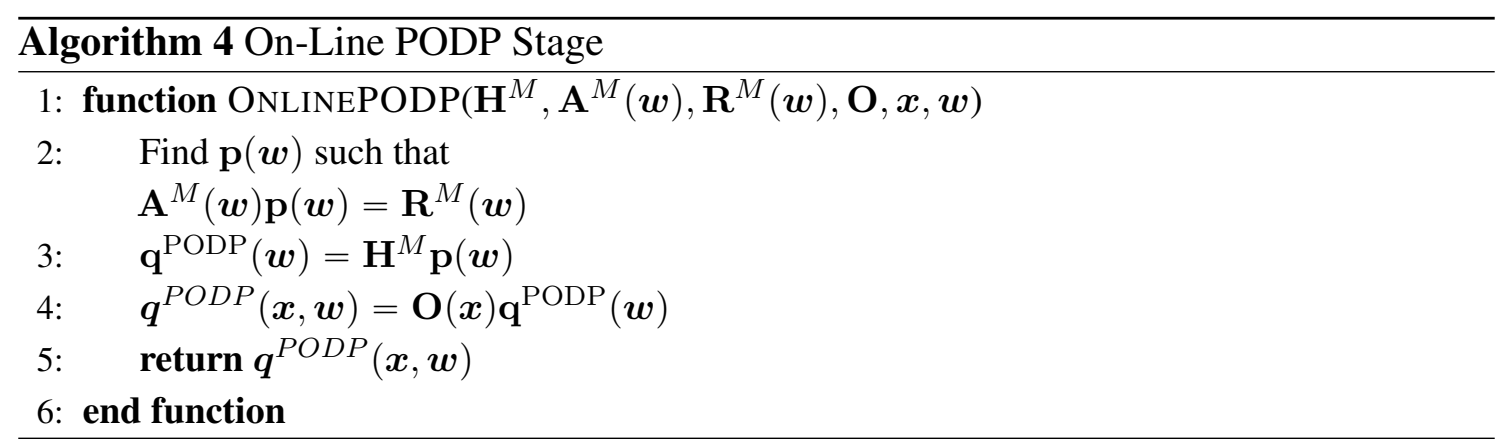

scalar coefficients that multiply these matrices. In particular, the problem of interest in this thesis admits an exact affine decomposition in terms of frequency and conductivity, which leads to a much faster on-line PODP stage. This decomposition is described in the following.

\section{Affine Decomposition in Terms of Frequency and Conductivity}

The only matrix block of $\mathbf{A}$ in (6.11) that depends on frequency and conductivity is $\mathrm{C}_{\mathcal{A} \mathcal{A}}^{\mathrm{AC}}{ }^{5}$ Consider the general case of a problem with $N_{C}$ disjoint conducting subdomains, $\Omega_{C, n}$, $n=1,2, \ldots, N_{C}$ with conductivities $\gamma=\gamma_{n}$ in $\Omega_{C, n}$. An exact affine decomposition in terms of frequency and conductivity can then be achieved by rewriting $\mathrm{C}_{\mathcal{A} \mathcal{A}}^{\mathrm{AC}}$ as

$$
\mathbf{C}_{\mathcal{A} \mathcal{A}}^{\mathrm{AC}}=\mathrm{i} \omega \mu_{0} \sum_{n=1}^{N_{C}} \gamma_{n} \mathbf{C}_{\mathcal{A \mathcal { A }}}^{\mathrm{AC}, \Omega_{C, n}}+\omega \varepsilon \mathbf{C}_{\mathcal{A \mathcal { A }}}^{\mathrm{AC}, \Omega_{C}^{c}}
$$

where the matrices $\mathbf{C}_{\mathcal{A A}}^{\mathrm{AC}, \Omega_{C, n}}$ and $\mathbf{C}_{\mathcal{A} \mathcal{A}}^{\mathrm{AC}, \Omega_{C}^{c}}$ have been defined. Then, the matrix products

$$
\begin{aligned}
& \tilde{\mathbf{K}}_{\mathcal{A A}}^{\mathrm{AC}}:=\left(\mathbf{H}^{M}\right)^{*} \mathbf{K}_{\mathcal{A} \mathcal{A}}^{\mathrm{AC}} \mathbf{H}^{M}, \\
& \tilde{\mathbf{C}}_{\mathcal{A \mathcal { A }}}^{\mathrm{AC}, \Omega_{C}^{l}}:=\left(\mathbf{H}^{M}\right)^{*} \mathbf{C}_{\mathcal{A \mathcal { A }}}^{\mathrm{AC}, \Omega_{C}^{l}} \mathbf{H}^{M}, \\
& \tilde{\mathbf{C}}_{\mathcal{A \mathcal { A }}}^{\mathrm{AC}, \Omega_{C}^{c}}:=\left(\mathbf{H}^{M}\right)^{*} \mathbf{C}_{\mathcal{A \mathcal { A }}}^{\mathrm{AC}, \Omega_{C}^{c}} \mathbf{H}^{M}, \\
& \tilde{\mathbf{K}}_{\mathcal{U A}}^{\mathrm{AC}}:=\left(\mathbf{H}^{M}\right)^{*} \mathbf{K}_{\mathcal{U A}}^{\mathrm{AC}} \mathbf{H}^{M}, \\
& \tilde{\mathbf{K}}_{\mathcal{U U}}^{\mathrm{AC}}:=\left(\mathbf{H}^{M}\right)^{*} \mathbf{K}_{\mathcal{U} \mathcal{U}}^{\mathrm{AC}} \mathbf{H}^{M} \text {, } \\
& \tilde{\mathbf{C}}_{\mathcal{U U}}^{\mathrm{AC}}:=\left(\mathbf{H}^{M}\right)^{*} \mathbf{C}_{\mathcal{U U}}^{\mathrm{AC}} \mathbf{H}^{M} \text {, } \\
& \tilde{\mathbf{M}}_{\mathcal{U} \mathcal{U}}^{\mathrm{AC}}:=\left(\mathbf{H}^{M}\right)^{*} \mathbf{M}_{\mathcal{U} \mathcal{U}}^{\mathrm{AC}} \mathbf{H}^{M},
\end{aligned}
$$

can be precomputed at the off-line stage and the reduced matrix is built in the general case as

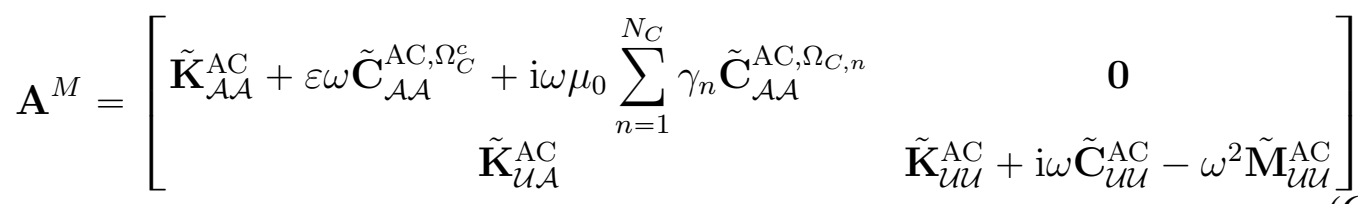

\footnotetext{
${ }^{5}$ Note that the dependency of the mechanical damping and mass blocks on $\omega$ has already been written in an affine format.
} 
where all the costly matrix products have been precomputed and the only operations to be performed at the on-line stage are scalar times matrix products and sum of matrices, where the matrices are of size $M$. Furthermore, the affine decomposition can be used to speedup the off-line stage, or the full order solution for varying parameters, as the parameter independent blocks are assembled only once and the system matrix is then constructed as

$$
\mathbf{A}=\left[\begin{array}{cc}
\mathbf{K}_{\mathcal{A} \mathcal{A}}^{\mathrm{AC}}+\varepsilon \omega \mathbf{C}_{\mathcal{A \mathcal { A }}}^{\mathrm{AC}, \Omega_{C}^{c}}+\mathrm{i} \omega \sum_{n=1}^{N_{C}} \gamma_{n} \mathbf{C}_{\mathcal{A \mathcal { A }}}^{\mathrm{AC}, \Omega_{C, n}} & \mathbf{0} \\
\mathbf{K}_{\mathcal{U} \mathcal{A}}^{\mathrm{AC}} & \mathbf{K}_{\mathcal{U U}}^{\mathrm{AC}}+\mathrm{i} \omega \mathbf{C}_{\mathcal{U} \mathcal{U}}^{\mathrm{AC}}-\omega^{2} \mathbf{M}_{\mathcal{U} \mathcal{U}}^{\mathrm{AC}}
\end{array}\right]
$$

Note that for application to the separated physics, as described in Section 6.6, the methodology can be applied analogously by considering only the relevant blocks of the global matrix.

\subsection{Application to Separated Physics}

POD can also be applied to the electromagnetic or mechanical problems separately. In this section, the steps needed at the off-line and on-line stages when considering application to the separated physics are summarized.

\subsubsection{Off-line}

In the off-line stage, Algorithm 2 is applied to both physics separately. For this, $\boldsymbol{q}(\boldsymbol{x}, \boldsymbol{w})=\boldsymbol{A}_{\varepsilon}^{A C}(\boldsymbol{x}, \boldsymbol{w})$ is first considered and then $\operatorname{OfFLINE}\left(\mathbf{A}_{\mathcal{A A}}(\boldsymbol{w}), \mathbf{R}_{\mathcal{A}}(\boldsymbol{w}), \mathbf{W}_{\mathrm{s}}, M\right)$ is called to obtain the low rank approximation $\mathbf{D}_{\mathcal{A}}^{M}=\mathbf{H}_{\mathcal{A}}^{M} \boldsymbol{\Sigma}_{\mathcal{A}}^{M}\left(\mathbf{G}_{\mathcal{A}}^{M}\right)^{*}$ to the snapshot matrix $\mathrm{D}_{\mathcal{A}}$. Secondly, $\boldsymbol{q}(\boldsymbol{x}, \boldsymbol{w})=\boldsymbol{u}^{A C}(\boldsymbol{x}, \boldsymbol{w})$ is considered and then OF$\operatorname{FLINE}\left(\mathbf{A}_{\mathcal{U U}}(\boldsymbol{w}),-\mathbf{A}_{\mathcal{U} \mathcal{A}}(\boldsymbol{w}) \mathcal{A}_{\varepsilon}^{\mathrm{AC}}(\boldsymbol{w}), \mathbf{W}_{\mathrm{s}}, M\right)$ is called to obtain the low rank approximation $\mathbf{D}_{\mathcal{U}}^{M}=\mathbf{H}_{\mathcal{U}}^{M} \boldsymbol{\Sigma}_{\mathcal{U}}^{M}\left(\mathbf{G}_{\mathcal{U}}^{M}\right)^{*}$ to the snapshot matrix $\mathbf{D}_{\mathcal{U}}$. Note that the application to separated physics offers extra flexibility, as different $N_{s}$ and $M$ can be considered for each physics, if desired.

\subsubsection{On-line}

In the on-line stage, either PODI or PODP can be considered. If it is chosen to apply PODI, the on-line stage for electromagnetics then consists in calling ONLINEPODI $\left(\mathbf{H}_{\mathcal{A}}^{M}, \boldsymbol{\Sigma}_{\mathcal{A}}^{M}, \mathbf{G}_{\mathcal{A}}^{M}, \mathbf{N}, M, \boldsymbol{x}, \boldsymbol{w}\right)$ (Algorithm 3) to get the approximation $\boldsymbol{A}^{P O D I}(\boldsymbol{x}, \boldsymbol{w})$ to $\boldsymbol{A}_{\varepsilon, h q}^{A C}(\boldsymbol{x}, \boldsymbol{w})$. For mechanics, the on-line stage consists in calling ONLINEPODI $\left(\mathbf{H}_{\mathcal{U}}^{M}, \boldsymbol{\Sigma}_{\mathcal{U}}^{M}, \mathbf{G}_{\mathcal{U}}^{M}, \mathbf{L}, M, \boldsymbol{x}, \boldsymbol{w}\right)$ to get the approximation $\boldsymbol{u}^{P O D I}(\boldsymbol{x}, \boldsymbol{w})$ to $\boldsymbol{u}_{h p}^{A C}(\boldsymbol{x}, \boldsymbol{w})$. If it is chosen instead to apply PODP, the on-line stage for electromagnetics then consists in calling $\mathrm{ON}$ $\operatorname{LINEPODP}\left(\mathbf{H}_{\mathcal{A}}^{M},\left(\mathbf{H}_{\mathcal{A}}^{M}\right)^{*} \mathbf{A}_{\mathcal{A A}}(\boldsymbol{w}) \mathbf{H}_{\mathcal{A}}^{M},\left(\mathbf{H}_{\mathcal{A}}^{M}\right)^{*} \mathbf{R}_{\mathcal{A}}(\boldsymbol{w}), \mathbf{N}, \boldsymbol{x}, \boldsymbol{w}\right)$ (Algorithm 4) to obtain the approximation $\boldsymbol{A}^{P O D P}(\boldsymbol{x}, \boldsymbol{w})$ to $\boldsymbol{A}_{\varepsilon, h q}^{A C}(\boldsymbol{x}, \boldsymbol{w})$, and subsequently, for mechan- 
ics ONLinePODP $\left(\mathbf{H}_{\mathcal{U}}^{M},\left(\mathbf{H}_{\mathcal{U}}^{M}\right)^{*} \mathbf{A}_{\mathcal{U} \mathcal{U}}(\boldsymbol{w}) \mathbf{H}_{\mathcal{U}}^{M},\left(\mathbf{H}_{\mathcal{U}}^{M}\right)^{*}\left(-\mathbf{A}_{\mathcal{U} \mathcal{A}}(\boldsymbol{w}) \mathcal{A}_{\varepsilon}^{\mathrm{AC}}(\boldsymbol{w})\right), \mathbf{L}, \boldsymbol{x}, \boldsymbol{w}\right)$ is called to obtain the approximation $\boldsymbol{u}^{P O D P}(\boldsymbol{x}, \boldsymbol{w})$ to $\boldsymbol{u}_{h p}^{A C}(\boldsymbol{x}, \boldsymbol{w}){ }^{6}$

\subsection{Combined Reduced Order-Full Order Approach}

Figures (6.1a) and (6.1b) show the evolution with frequency of $\left\|\mathcal{A}_{\varepsilon, h q}^{A C}\right\|_{L^{2}\left(\Omega_{C, n}\right)}$ and $\left\|\boldsymbol{u}_{h p}^{A C}\right\|_{L^{2}\left(\Omega_{C, n}\right)}$, where $\Omega_{C, n}$ refers to either $\Omega_{4 K}, \Omega_{77 K}$ or $\Omega_{O V C}$, for the test magnet problem with $z$ (longitudinal) gradient coil obtained by performing a full order simulation (see Section 5.3.2 were this problem was simulated using the full order solver). The critical observation is that peaks appear only in $\left\|\boldsymbol{u}_{h p}^{A C}\right\|_{L^{2}\left(\Omega_{C, n}\right)}$, and not in $\left\|\mathcal{A}_{\varepsilon, h q}^{A C}\right\|_{L^{2}\left(\Omega_{C, n}\right)}$, the latter exhibiting a smooth monotonically decay and therefore, being well suited to a POD approximation. Moreover, this typical behaviour will always be the case for the application of interest in this thesis, where the peaks are associated with the resonance modes of the mechanical system, which will be activated depending on the induced electromagnetic stress $\boldsymbol{\sigma}^{e}(\boldsymbol{A})$. This suggests that the application of a novel combined reduced order-full order methodology in which POD is applied to the electromagnetic problem only and the mechanical problem is solved using the full order solver could be beneficial, as the electromagnetic solution should be easier to approximate with fewer snapshots and modes. Furthermore, $3 Q_{\text {global }} \ll P_{\text {global }}$, which means that the cost of the full order mechanical solver is much cheaper than that of the full order electromagnetic solver.

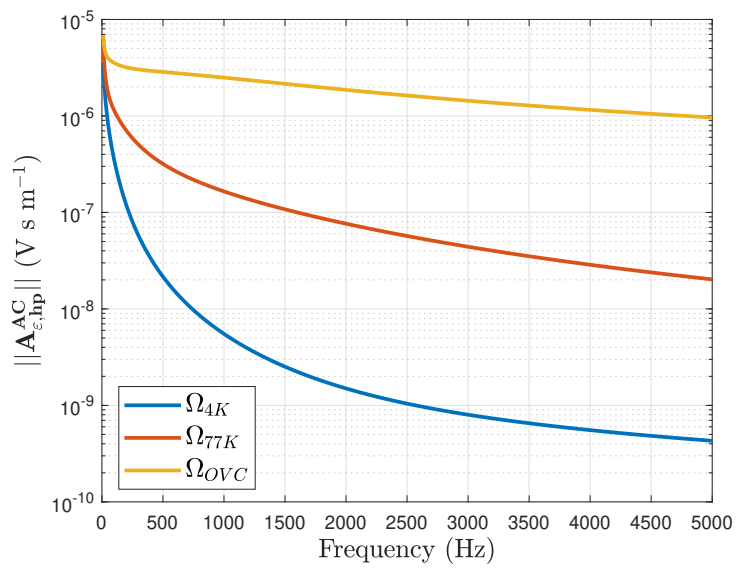

(a) $\left\|\boldsymbol{A}_{\varepsilon, h q}^{A C}\right\|_{L^{2}\left(\Omega_{C, n}\right)}$

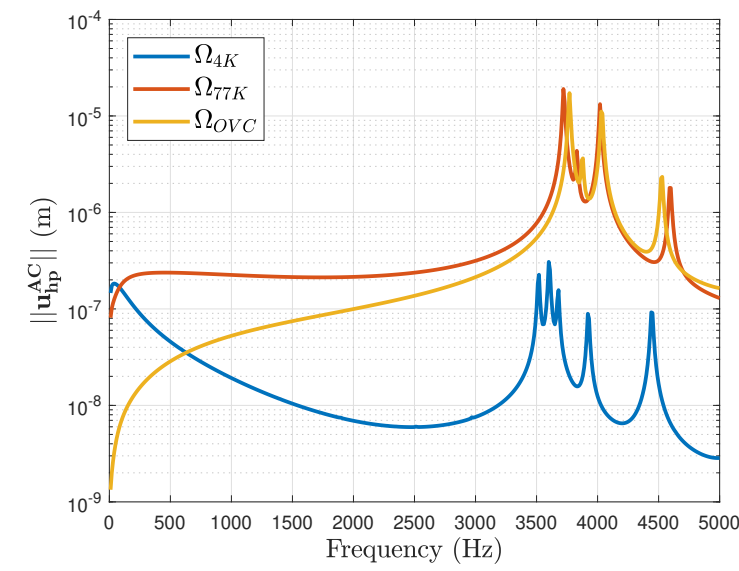

(b) $\left\|\boldsymbol{U}_{h p}^{A C}\right\|_{L^{2}\left(\Omega_{C, n}\right)}$

Figure 6.1: Evolution of (a) $\left\|\mathcal{A}_{\varepsilon, h q}^{A C}\right\|_{L^{2}\left(\Omega_{C, n}\right)}$ and (b) $\left\|\boldsymbol{u}_{h p}^{A C}\right\|_{L^{2}\left(\Omega_{C, n}\right)}$ with the frequency for the test magnet problem with $z$ (longitudinal) gradient coil.

\footnotetext{
${ }^{6}$ Note that PODI can be applied independently to both physics, however in the case of PODP the application to mechanics requires, in practice, the method to be first applied to electromagnetics given that $\tilde{l}_{\mathfrak{u}}$ depends on $\boldsymbol{A}_{\varepsilon}^{A C}(\boldsymbol{x}, \boldsymbol{w})$.
} 


\subsubsection{Off-line}

In the off-line stage, Algorithm 2 is applied to the electromagnetic problem only, i.e. $\boldsymbol{q}(\boldsymbol{x}, \boldsymbol{w})=\boldsymbol{A}_{\varepsilon}^{A C}(\boldsymbol{x}, \boldsymbol{w})$ is considered and then $\operatorname{OFFLINE}\left(\mathbf{A}_{\mathcal{A} \mathcal{A}}(\boldsymbol{w}), \mathbf{R}_{\mathcal{A}}(\boldsymbol{w}), \mathbf{W}_{\mathrm{s}}, M\right)$ is called to obtain the low rank approximation $\mathbf{D}_{\mathcal{A}}^{M}=\mathbf{H}_{\mathcal{A}}^{M} \boldsymbol{\Sigma}_{\mathcal{A}}^{M}\left(\mathbf{G}_{\mathcal{A}}^{M}\right)^{*}$ to the snapshot ma$\operatorname{trix} \mathbf{D}_{\mathcal{A}}$.

\subsubsection{On-line}

In the on-line stage, the solution $\mathcal{A}_{\varepsilon, h q}^{A C}$ for the parameters of interest can be approximated through PODI or PODP, and this is then used to feed the mechanical full order solver. For clarity, the abbreviations PODI-Full and PODP-Full are used to denote the on-line stage using PODI and PODP, respectively. For PODI-Full, Algorithm 5 is applied to obtain an approximation $\boldsymbol{q}^{P O D I-F U L L}(\boldsymbol{x}, \boldsymbol{w})$ to $\boldsymbol{q}_{h p q}(\boldsymbol{x}, \boldsymbol{w})$, and, for PODP-Full, Algorithm 6 is applied to obtain an approximation $\boldsymbol{q}^{P O D P-F U L L}(\boldsymbol{x}, \boldsymbol{w})$ to $\boldsymbol{q}_{h p q}(\boldsymbol{x}, \boldsymbol{w})$.
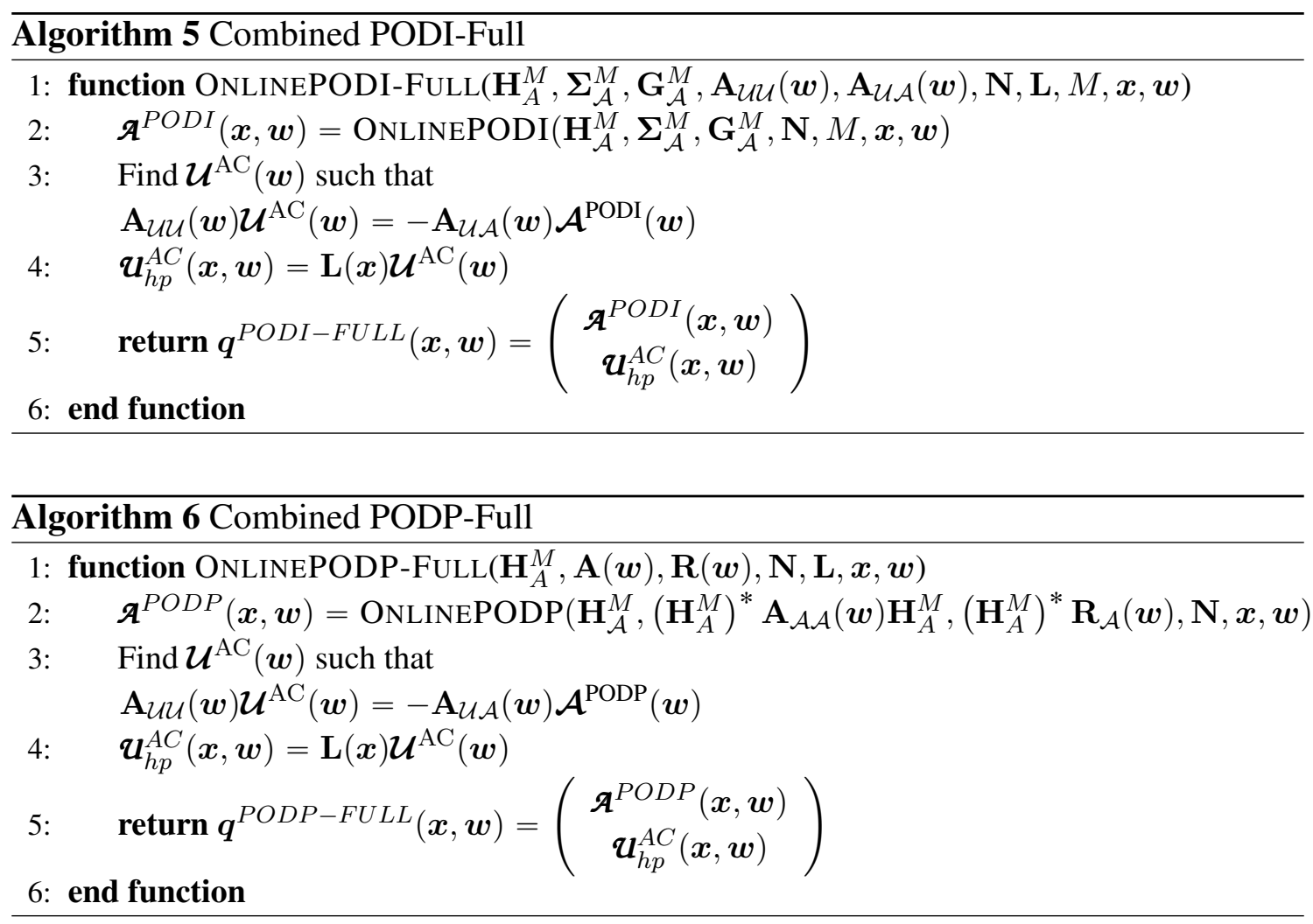

\subsection{Dissipated Power and Kinetic Energy: POD Approx- imation}

For the purpose of an easier presentation, the dissipated power and kinetic energy defined in (5.6) can be rewritten as

$$
P_{\Omega}^{0}\left(\boldsymbol{w}, \boldsymbol{A}_{\varepsilon, h q}^{A C}(\boldsymbol{x}, \boldsymbol{w}), \boldsymbol{B}_{0, h q}^{D C}(\boldsymbol{x}), \boldsymbol{u}_{h p}^{A C}(\boldsymbol{x}, \boldsymbol{w})\right):=\frac{1}{2} \int_{\Omega} \gamma\left|\boldsymbol{E}_{h p q}^{A C}\right|^{2} \mathrm{~d} \Omega
$$




$$
\begin{gathered}
=\frac{1}{2} \int_{\Omega} \gamma\left|-\mathrm{i} \omega \boldsymbol{\mathcal { A }}_{\varepsilon, h q}^{A C}+\mathrm{i} \omega \boldsymbol{B}_{0, h q}^{D C} \times \boldsymbol{u}_{h p}^{A C}\right|^{2} d \Omega \\
E_{\Omega}^{k}\left(\boldsymbol{w}, \boldsymbol{u}_{h p}^{A C}(\boldsymbol{x}, \boldsymbol{w})\right):=\frac{1}{2} \int_{\Omega} \rho \omega^{2}\left|\boldsymbol{u}_{h p}^{A C}\right|^{2} \mathrm{~d} \Omega
\end{gathered}
$$

where the parametric dependency has been explicitly written. The corresponding POD approximations are $P_{\Omega}^{0}\left(\boldsymbol{w}, \boldsymbol{A}^{P O D}(\boldsymbol{x}, \boldsymbol{w}), \boldsymbol{B}_{0, h q}^{D C}(\boldsymbol{x}), \boldsymbol{u}^{P O D}(\boldsymbol{x}, \boldsymbol{w})\right)$ and $E_{\Omega}^{k}\left(\boldsymbol{w}, \boldsymbol{B}_{0, h q}^{D C}, \boldsymbol{u}^{P O D}(\boldsymbol{x}, \boldsymbol{w})\right)$, which reduce to $P_{\Omega}^{0}\left(\boldsymbol{w}, \boldsymbol{A}^{P O D}, \boldsymbol{B}_{0, h q}^{D C}, \boldsymbol{u}^{P O D}\right)$ and $E_{\Omega}^{k}\left(\boldsymbol{w}, \boldsymbol{B}_{0, h q}^{D C}, \boldsymbol{u}^{P O D}\right)$, respectively, where the $(\boldsymbol{x}, \boldsymbol{w})$ dependency on the POD solutions has been dropped for simplicity of notation.

\subsection{Chapter Summary}

In this chapter the application of POD to the coupled 3D magneto-mechanical problem of interest was discussed.

The main novelty of the chapter is the development of a new reduced order-full order methodology for the solution of the 3D magneto-mechanical problem of interest in a Lagrangian frame. This exploits the staggered nature of the problem by applying POD to obtain a ROM approximation to the electromagnetic solution and using this to feed the mechanical full order solver. This ROM is of paramount importance for the industry, given the necessity to run many parameter sweeps in a cost effective manner. The efficiency of the ROM in such scenario will be proven in the next chapter. Furthermore, the application of POD to the complete problem and to both physics separately was discussed, and an affine decomposition of the problem in terms of frequency and conductivity was derived.

The application of the POD to the complete problem was considered followed by the application to electromagnetics and mechanics separately. Then, a new combined reduced order-full order methodology was proposed, where, exploiting the staggered nature of the Lagrangian formulation, POD is applied to the electromagnetic problem and this is then used to feed the mechanical full order solver. Both the off-line and on-line stages were described. The computational advantages of this approach over applying POD to the complete problem will be presented in the next chapter.

First, in Sections 6.2 and 6.3, the variational formulation and discrete system derived in Chapters 3 and 4, respectively, were rewritten in a form more suitable for the description of the ROM, were the parametric dependency was explicitly written. Then, in Section 6.4, the SVD and TSVD were introduced. Section 6.5 focuses on the description of POD, including the off-line and on-line stages. In the on-line stage, both PODI and PODP were considered. The application of POD to the electromagnetic and mechanical problems separately is discussed in Section 6.6. Finally, in Section 6.7, a new reduced order-full order methodology is proposed. 


\section{Chapter 7}

\section{Reduced Order Model Numerical Results}

\subsection{Introductory Remarks}

This chapter focuses on the application of the ROM techniques described in Chapter 6 to several challenging MRI configurations, that have also been solved using the full order model in Chapter 5. First, only one parameter will be considered and the PODI and PODP methodologies will be applied to the complete solution. Then, the application of these methodologies to electromagnetics and mechanics separately will be considered, in order to study the performance of PODI and PODP for each physics. Next, the application of the PODI-Full and PODP-Full methodologies is considered. The most successful approach (PODP-Full) will then be applied to problems considering multiple parameters of interest. Numerous comparisons against the full order solution and different measures of the error will be provided in order to assess the accuracy and efficiency of the methods.

The main novelties of this chapter are as follows; First, PODI and PODP are applied to the magneto-mechanical problem of interest and it is shown that the application of these methodologies to the mechanical problem requires the computation of a large number of snapshots and, thus, no computational gaining with respect to the full order solution can be obtained with this methods. Second, the PODI-Full and PODP-Full methodologies were applied to the problem of interest and their accuracy and efficiency were assessed by performing comparisons against the full order solution and using different error measures. Third, a comparison between PODI-Full and PODP-Full was performed, which showed that PODP-Full results in more accurate approximations for no significant increase in computational cost.

Note that all the time measurements that will be shown in this chapter correspond to wall-clock time and all the computations were made in a cluster node using a machine Bull Sequana X440-E5 2 x Octa-Core (3.2 GHz) Xeon Gold $v 56134$ and using a parallel pool of 8 workers to solve the frequency sweeps in parallel. For details about the implementation using parallel computing see Appendix D. Unless otherwise stated all the computational time measurements in this chapter are based on these settings. 
The structure of the chapter is as follows; First, in Section 7.2, several error measures are defined, which will be used to assess the accuracy of the ROM solution. Then, in Section 7.3, the application of the different ROM methodologies to the test magnet problem with $z$ gradient coils considering only the frequency as a parameter of interest is studied. The PODI and PODP methodologies are studied first, followed by the PODI-Full and PODPFull methodologies. Section 7.4 focuses on the application of PODP-Full to the same problem but considering one extra parameter. The application of PODP-Full to the modified magnet problem with $x$ gradient coils is studied in Section 7.5. The chapter finishes with concluding remarks in Section 7.6.

\subsection{Error Measure}

When considering the solution to the problem of interest using a ROM, an approximation error is introduced. To quantify this error, several measures will be considered. First, the relative truncation error introduced by truncating the SVD after $M$ terms can be quantified using [33]

$$
\frac{\left\|\mathbf{D}-\mathbf{D}^{M}\right\|_{F}}{\|\mathbf{D}\|_{F}}:=\frac{\sqrt{\sum_{i=M+1}^{r} \sigma_{i}^{2}}}{\sqrt{\sum_{i=1}^{r} \sigma_{i}^{2}}},
$$

where $r$ is the rank of $\mathbf{D}$. However, this requires the complete SVD, which is generally not available and instead only a rank $M$ approximation to $\mathbf{D}$ is available for computational efficiency. Thus, an upper bound $e_{1}$ is used [96]

$$
\frac{\left\|\mathbf{D}-\mathbf{D}^{M}\right\|_{F}}{\|\mathbf{D}\|_{F}}=\frac{\sqrt{\sum_{i=M+1}^{r} \sigma_{i}^{2}}}{\sqrt{\sum_{i=1}^{r} \sigma_{i}^{2}}} \leqslant e_{1}:=\frac{\sqrt{(r-M) \sigma_{M}^{2}}}{\sqrt{\sum_{i=1}^{M} \sigma_{i}^{2}}},
$$

where the singular values are sorted in decreasing order and, therefore, $\sigma_{M} \geqslant \sigma_{i}$ for $M+1 \leqslant i \leqslant r$.

The second error measure represents the error of the reduced order solution with respect to the full order solution. To construct this measure, the solution to the problem for parameter values that have not been sampled is computed using both the full order and the ROM and the error is then defined for each parameter set $\boldsymbol{w}$ as

$$
e_{2}\left(\mathbf{q}(\boldsymbol{w}), \mathbf{q}^{\mathrm{POD}}(\boldsymbol{w})\right):=\frac{\left\|\mathbf{q}(\boldsymbol{w})-\mathbf{q}^{\mathrm{POD}}(\boldsymbol{w})\right\|_{N_{d}}}{\|\mathbf{q}(\boldsymbol{w})\|_{N_{d}}},
$$

where $\|\cdot\|_{N_{d}}$ is the $N_{d}$-dimensional Euclidean norm.

Furthermore, the relative error in the dissipated power and kinetic energy can be defined as

$$
\begin{aligned}
e_{P_{\Omega}^{0}}^{\mathrm{POD}}(\boldsymbol{w}) & :=\frac{\left|P_{\Omega}^{0}\left(\boldsymbol{w}, \boldsymbol{A}_{\varepsilon, h q}^{A C}, \boldsymbol{B}_{0, h q}^{D C}, \boldsymbol{u}_{h p}^{A C}\right)-P_{\Omega}^{0}\left(\boldsymbol{w}, \boldsymbol{A}^{P O D}, \boldsymbol{B}_{0, h q}^{D C}, \boldsymbol{u}^{P O D}\right)\right|}{\left|P_{\Omega}^{0}\left(\boldsymbol{w}, \boldsymbol{A}_{\varepsilon, h q}^{A C}, \boldsymbol{B}_{0, h q}^{D C}, \boldsymbol{u}_{h p}^{A C}\right)\right|} \\
e_{E_{\Omega}^{k}}^{P O D}(\boldsymbol{w}) & :=\frac{\left|E_{\Omega}^{k}\left(\boldsymbol{w}, \boldsymbol{u}_{h p}^{A C}\right)-E_{\Omega}^{k}\left(\boldsymbol{w}, \boldsymbol{u}^{P O D}\right)\right|}{\left|E_{\Omega}^{k}\left(\boldsymbol{w}, \boldsymbol{u}_{h p}^{A C}\right)\right|}
\end{aligned}
$$

and will also be used to assess the accuracy of the approximation. 


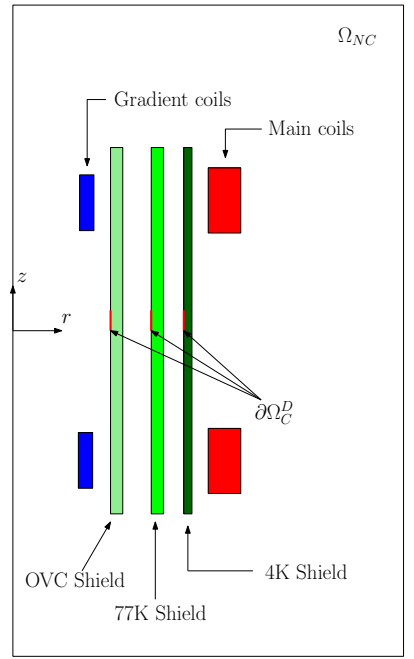

(a) $2 \mathrm{D}$ crosssection

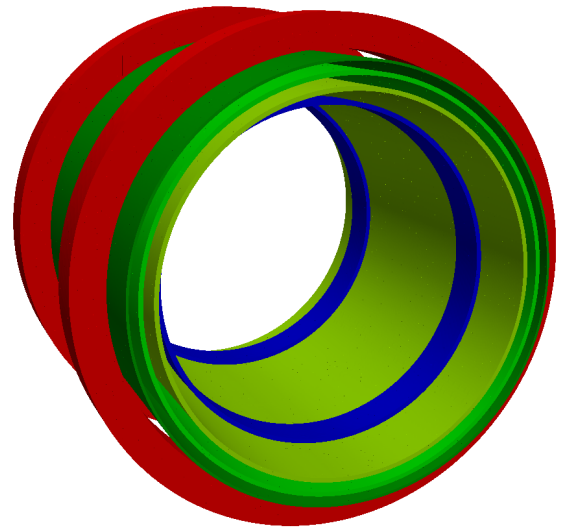

(b) $3 \mathrm{D}$ view

Figure 7.1: Test magnet problem with $z$ (longitudinal) gradient coil: illustration of the components of the problem. (a) Axisymmetric meridian plane and (b) 3D view.

\subsection{Test Magnet Problem with 1 Parameter}

The application of POD to the test magnet problem with $z$ (longitudinal) gradient coils, first presented in Section 5.3.2, is considered here. For the convenience of the reader, the problem geometry is illustrated again in Figure 7.1 as well as the chosen $\partial \Omega_{C}^{D}$, where the condition $\boldsymbol{u}_{h p}^{A C}=\mathbf{0}$ was imposed. Mechanical damping was applied as described in Section 4.6 with a constant damping ratio $\xi=2 \times 10^{-3}$. The same mesh of 33805 tetrahedral elements as used for the full order simulations presented in Section 5.3.2 and polynomial orders $q=3$ and $p=3$ were considered.

As a first study, $\omega=2 \pi f$ is considered as the only parameter. Thus, $N_{p}=1$ and $\boldsymbol{w}$ reduces to $\omega$. Following the methodology described in Section 6.5.1, Algorithm 2 is applied by calling Offline $\left(\mathbf{A}(\boldsymbol{w}), \mathbf{R}(\boldsymbol{w}), \mathbf{W}_{s}, M\right)$ for the sets of samples $\mathbf{W}_{\mathrm{s}}=$ $\left(2 \pi \mathrm{f}_{1}, 2 \pi \mathrm{f}_{2}, \ldots, 2 \pi \mathrm{f}_{N_{s}}\right)$ with $N_{s}=500,250,125,63$, corresponding to $\mathrm{f}_{i}=10+(i-1) \Delta \mathrm{f}$ $\mathrm{Hz}$, with $i=1, \ldots, N_{s}$ and $\Delta \mathrm{f}=10,20,40,80 \mathrm{~Hz}$. The TSVD was truncated with $M=50$ (leading to a maximum upper bound for the truncation error $e_{1}=8.3 \times 10^{-4}$ for the case $N_{s}=500$ ). This offline data is used in the following subsections. It should be remarked that, in this thesis, the snapshots will be chosen either uniformly or based in previous knowledge on the solution. Other approaches such as a greedy basis generation of the basis [87] could be considered. This iterative method starts with the computation of a single snapshot, and at each iteration a new snapshot is added. An error estimator is required, which is evaluated at each iteration in a parametric mesh, and the next snapshot is chosen to correspond to the parameters that maximize this error. For further details about the method the reader is referred to [87]. 


\subsubsection{PODI}

In this section, Algorithm 3 is applied by calling ONLINEPODI $\left(\mathbf{H}^{M}, \boldsymbol{\Sigma}^{M}, \mathbf{G}^{M}, \mathbf{O}, M, \boldsymbol{x}, 2 \pi \mathrm{f}_{i}\right)$ to compute $\boldsymbol{q}^{P O D I}\left(\boldsymbol{x}, 2 \pi \mathrm{f}_{i}\right)$ at the frequencies $\mathrm{f}_{i}=15+(i-1) \Delta \mathrm{f} \mathrm{Hz}$ with $i=1, \ldots, N_{o}, N_{o}=499$ and $\Delta \mathrm{f}=10 \mathrm{~Hz}$. As a means of comparison, the full order solver is then used to compute $\boldsymbol{q}_{h p q}\left(\boldsymbol{x}, 2 \pi \mathrm{f}_{i}\right)$ with $\mathrm{f}_{i}$ as before. The resulting dissipated power and kinetic energy (6.32) for both $\boldsymbol{q}^{P O D I}\left(\boldsymbol{x}, 2 \pi \mathrm{f}_{i}\right)$ and $\boldsymbol{q}_{h p q}\left(\boldsymbol{x}, 2 \pi \mathrm{f}_{i}\right)$ are then compared.

A comparison of $P_{\Omega_{C, n}}^{0}\left(2 \pi \mathrm{f}_{i}, \boldsymbol{A}_{\varepsilon, h q}^{A C}, \boldsymbol{B}_{0, h q}^{D C}, \boldsymbol{u}_{h p}^{A C}\right)$ with $P_{\Omega_{C, n}}^{0}\left(2 \pi \mathrm{f}_{i}, \boldsymbol{A}^{P O D I}, \boldsymbol{B}_{0, h q}^{D C}, \boldsymbol{u}^{P O D I}\right)$ and $E_{\Omega_{C, n}}^{k}\left(2 \pi \mathrm{f}_{i}, \boldsymbol{u}_{h p}^{A C}\right)$ with $E_{\Omega_{77 K}}^{k}\left(2 \pi \mathrm{f}_{i}, \boldsymbol{u}^{P O D I}\right), i=1, \ldots, N_{o}$, where $\Omega_{C, n}$ refers to either the $4 \mathrm{~K}, 77 \mathrm{~K}$ or OVC shields, is provided in Figure 7.2. It can be observed how the resolution of the resonant peaks decreases with $N_{s}$, which leads, in the worst case, to the appearance of non-existent peaks or missing existent peaks. Note that these results correspond to the case where $I_{j}(\omega)$ is chosen to give a linear interpolation and no significant difference is observed in the case of Lagrange cubic interpolation. In order to examine the loss in resolution more clearly, Figure 7.3 shows a comparison between $E_{\Omega_{77 K}}^{k}\left(2 \pi \mathrm{f}_{i}, \boldsymbol{u}^{P O D I}\right), 3400 \leqslant \mathrm{f}_{i} \leqslant 5000 \mathrm{~Hz}$, for different $N_{s}$, and $E_{\Omega_{77 K}}^{k}\left(2 \pi \mathrm{f}_{i}, \boldsymbol{u}_{h p}^{A C}\right)$ for different $N_{o}$ (with $\mathrm{f}_{i}$ appropriately redefined). These results show that the $N_{s}$ that PODI requires in order to accurately reproduce the dissipated power and kinetic energy in the radiation shields must be as large as the $N_{o}$ required by the full order solver. Therefore, PODI does not offer advantages over a full order solution.

\subsubsection{PODP}

The investigation described in Section 7.3.1 is now repeated using PODP by applying Algorithm 4 and calling ONLINEPODP $\left(\mathbf{H}^{M}, \mathbf{A}^{M}\left(2 \pi \mathrm{f}_{i}\right), \mathbf{R}^{M}\left(2 \pi \mathrm{f}_{i}\right), \mathbf{O}, \boldsymbol{x}, 2 \pi \mathrm{f}_{i}\right)$. A comparison of $P_{\Omega_{C, n}}^{0}\left(2 \pi \mathrm{f}_{i}, \boldsymbol{A}^{P O D P}, \boldsymbol{B}_{0, h q}^{D C}, \boldsymbol{u}^{P O D P}\right)$ and $E_{\Omega_{C, n}}^{k}\left(2 \pi \mathrm{f}_{i}, \boldsymbol{u}^{P O D P}\right)$, for $N_{s}=$ $500,250,125,63$, with $P_{\Omega_{C, n}}^{0}\left(2 \pi \mathrm{f}_{i}, \boldsymbol{A}_{\varepsilon, h q}^{A C}, \boldsymbol{B}_{0, h q}^{D C}, \boldsymbol{u}_{h p}^{A C}\right)$ and $E_{\Omega_{C, n}}^{k}\left(2 \pi \mathrm{f}_{i}, \boldsymbol{u}_{h p}^{A C}\right)$ is shown in Figure 7.4. First, it can be observed that an increased accuracy compared to PODI is obtained, as the PODP solution shows an excellent agreement with the full order solution in $\Omega_{O V C}$ and $\Omega_{77 K}$. However, significant differences are still observed in $\Omega_{4 K}$, where the accuracy of the approximation decreases with $N_{s}$ and, similarly to PODI, the $N_{s}$ required by PODP in order to accurately capture the dissipated power and kinetic energy is as large as the $N_{o}$ required by the full order solver, which means that no computational gaining can be obtained by applying PODP. To better illustrate this, consideration is restricted to frequencies $3200 \leqslant \mathrm{f}_{i} \leqslant 5000 \mathrm{~Hz}$ and, in Figure 7.5, a comparison of $E_{\Omega_{4 K}}^{k}\left(2 \pi \mathrm{f}_{i}, \boldsymbol{u}^{P O D P}\right)$ for $N_{s}=500,250,125,63$ with $E_{\Omega_{4 K}}^{k}\left(2 \pi \mathrm{f}_{i}, \boldsymbol{u}_{h p}^{A C}\right)$ for $N_{o}=500,250,125,63$ is shown, in order to show the resonant region of the worst performing shield, where the greatest differences are observed. 


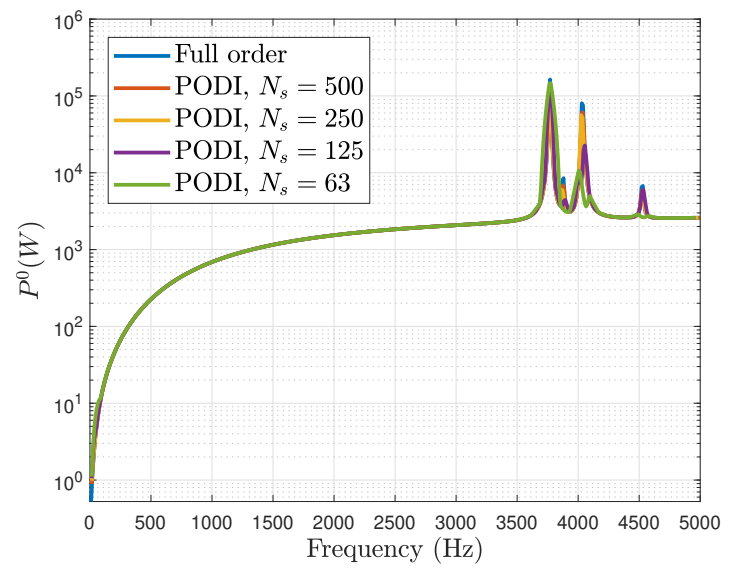

(a) $P_{\Omega_{O V C}}^{0}$

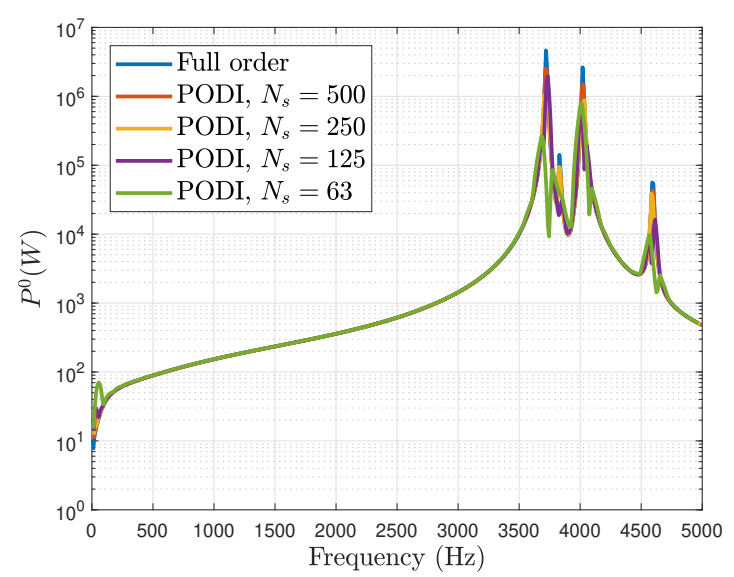

(c) $P_{\Omega_{77 K}}^{0}$

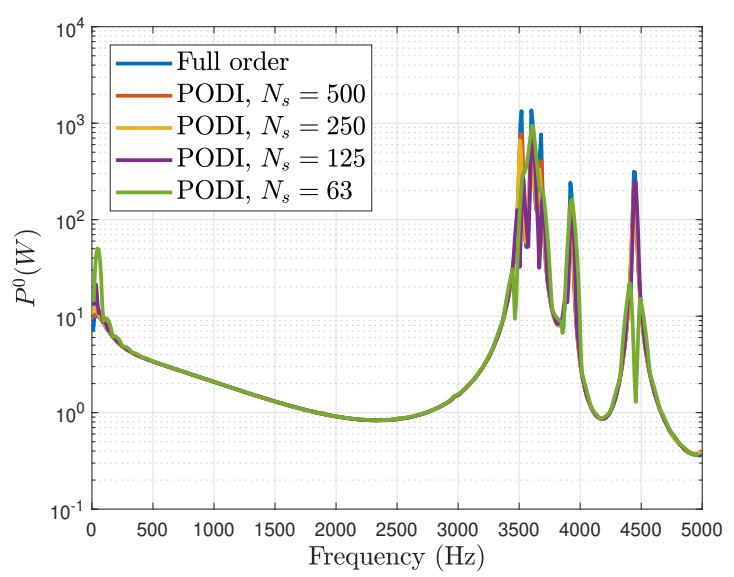

(e) $P_{\Omega_{4 K}}^{0}$

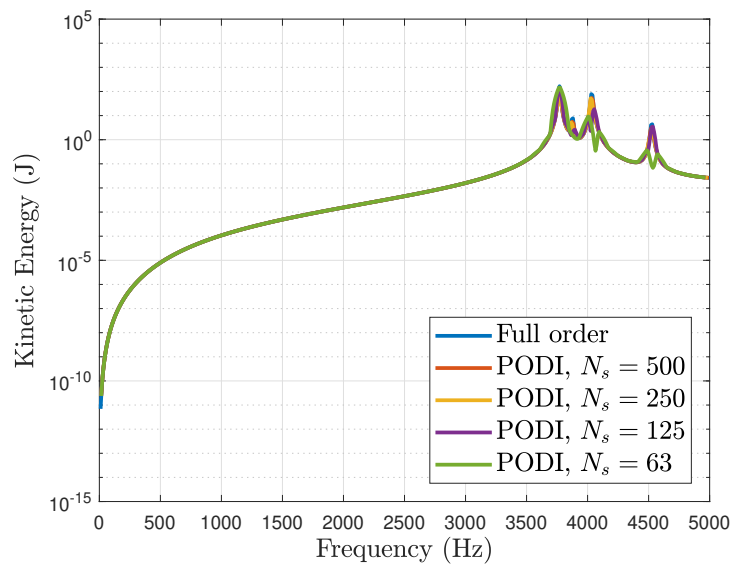

(b) $E_{\Omega_{O V C}}^{k}$

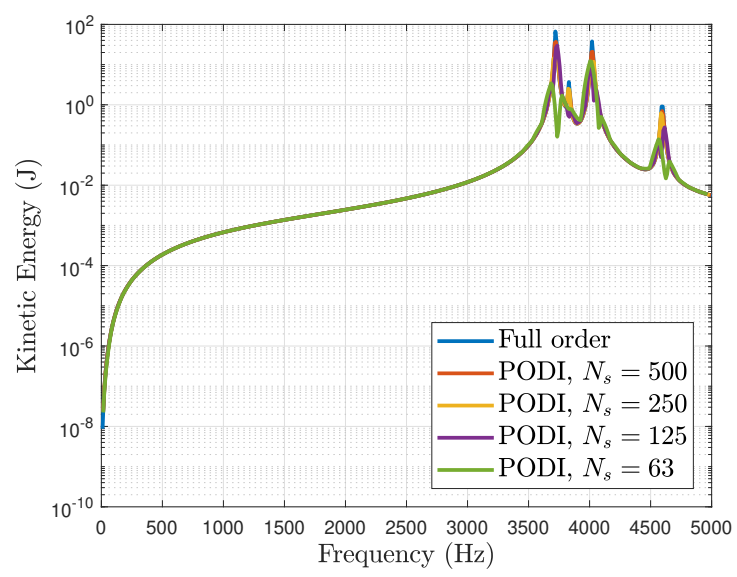

(d) $E_{\Omega_{77 K}}^{k}$

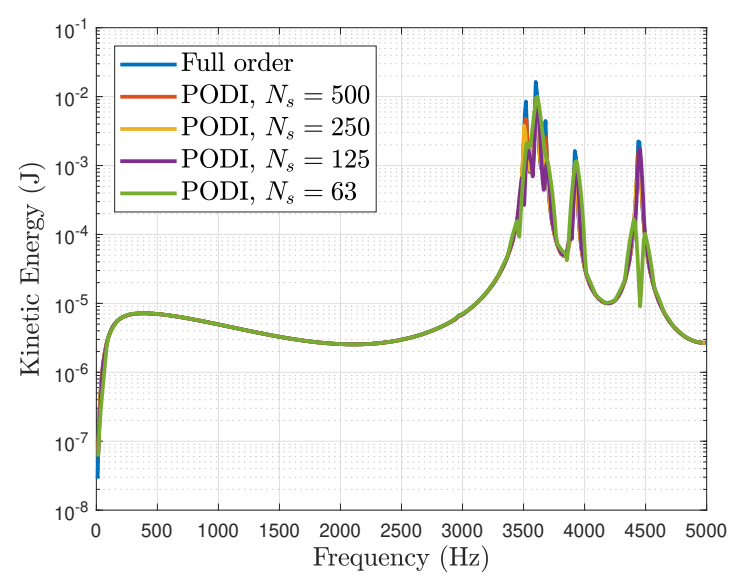

(f) $E_{\Omega_{4 K}}^{k}$

Figure 7.2: PODI applied to test magnet problem with 1 parameter: Dissipated power and kinetic energy in the radiation shields for different $N_{s}$. Comparison with full order solution. 


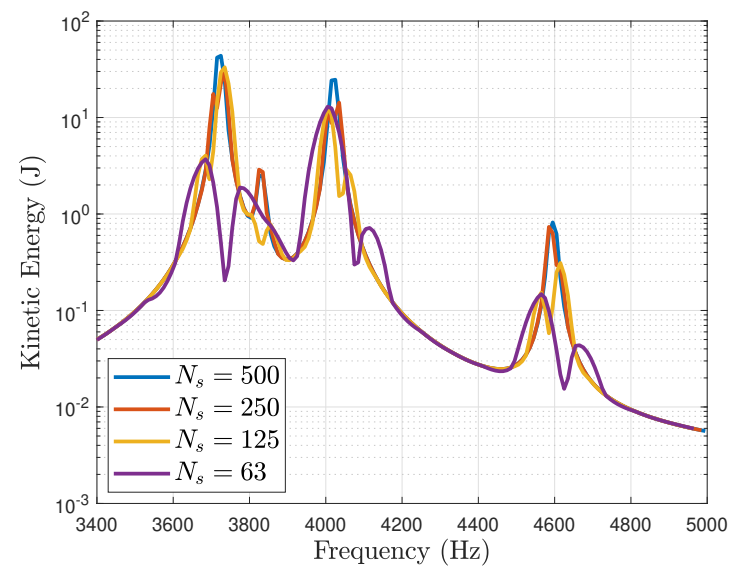

(a) $E_{\Omega_{77 K}}^{k}\left(2 \pi \mathrm{f}_{i}, \boldsymbol{u}^{P O D I}\right)$

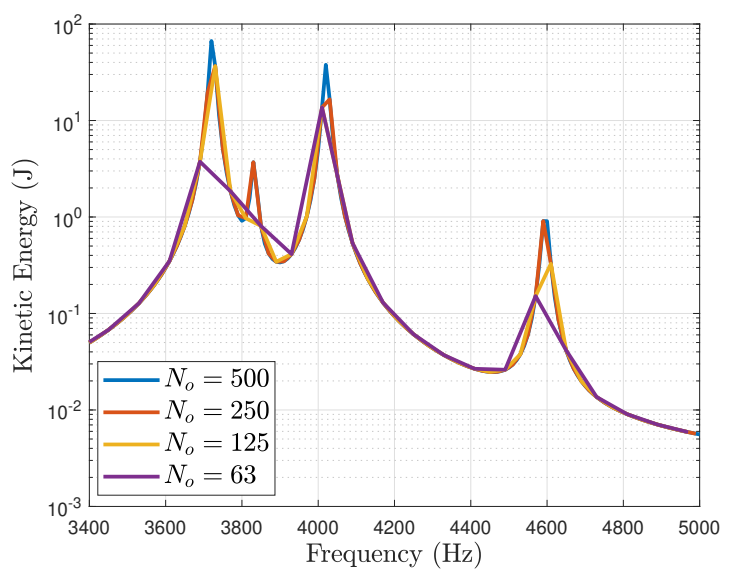

(b) $E_{\Omega_{77 K}}^{k}\left(2 \pi \mathrm{f}_{i}, \boldsymbol{u}_{h p}^{A C}\right)$

Figure 7.3: Test magnet problem with 1 parameter: (a) $E_{\Omega_{77 K}}^{k}\left(2 \pi \mathrm{f}_{i}, \boldsymbol{u}^{P O D I}\right)$ for different $N_{s}$ and (b) $E_{\Omega_{77 K}}^{k}\left(2 \pi \mathrm{f}_{i}, \boldsymbol{u}_{h p}^{A C}\right)$ for different $N_{o}$.

\subsubsection{Application to Separated Physics Solutions}

Since $\left\|\boldsymbol{A}_{\varepsilon, h q}^{A C}\right\|_{L^{2}\left(\Omega_{C, n}\right)}$ has a smoother variation with frequency than $\left\|\boldsymbol{u}_{h p}^{A C}\right\|_{L^{2}\left(\Omega_{C, n}\right)}$, as shown in Figure 6.1, the application of PODI to the electromagnetic, $\boldsymbol{q}(\boldsymbol{x}, \omega)=$ $\boldsymbol{A}_{\varepsilon}^{A C}(\boldsymbol{x}, \omega)$, and mechanical, $\boldsymbol{q}(\boldsymbol{x}, \omega)=\boldsymbol{u}^{A C}(\boldsymbol{x}, \omega)$, solutions separately is now considered, as explained in Section 6.6.

\section{Off-line}

At the off-line stage, OFFLINE $\left(\mathbf{A}_{\mathcal{A A}}(\boldsymbol{w}), \mathbf{R}_{\mathcal{A}}(\boldsymbol{w}), \mathbf{W}_{\mathrm{s}}, M\right)$ is first called followed by OF$\operatorname{FLINE}\left(\mathbf{A}_{\mathcal{U U}}(\boldsymbol{w}),-\mathbf{A}_{\mathcal{U A}}(\boldsymbol{w}) \mathcal{A}_{\varepsilon}^{\mathrm{AC}}(\boldsymbol{w}), \mathbf{W}_{\mathrm{s}}, M\right)$ for $\mathbf{W}_{s}=\left(2 \pi \mathrm{f}_{1}, 2 \pi \mathrm{f}_{2}, \ldots, 2 \pi \mathrm{f}_{N_{s}}\right)$ with $f_{i}=10+(i-1) \Delta \mathrm{f}, i=1, \ldots, N_{s}, N_{s}=500,63$ and $\Delta \mathrm{f}=10,80 \mathrm{~Hz}$. The TSVD was truncated after $M=50$ in both cases, which leads to a maximum upper bound for the truncation error $e_{1}=2.3 \times 10^{-6}$ for the case $\boldsymbol{q}(\boldsymbol{x}, \omega)=\boldsymbol{A}_{\varepsilon}^{A C}(\boldsymbol{x}, \omega)$ and $e_{1}=3.3 \times 10^{-4}$ for the case $\boldsymbol{q}(\boldsymbol{x}, \omega)=\boldsymbol{U}^{A C}(\boldsymbol{x}, \omega)$, corresponding to $N_{s}=500$. Note that $e_{1}$ is smaller in the case $\boldsymbol{q}(\boldsymbol{x}, \omega)=\boldsymbol{A}_{\varepsilon}^{A C}(\boldsymbol{x}, \omega)$ than in the case $\boldsymbol{q}(\boldsymbol{x}, \omega)=\boldsymbol{U}^{A C}(\boldsymbol{x}, \omega)$, which means that given a certain tolerance for $e_{1}$, fewer modes are needed to approximate $\boldsymbol{A}_{\varepsilon, h q}^{A C}(\boldsymbol{x}, \omega)$ compared to $\boldsymbol{u}_{h p}^{A C}(\boldsymbol{x}, \omega)$. To illustrate this, the decay of the singular values for the cases $\boldsymbol{q}(\boldsymbol{x}, \omega)=\boldsymbol{A}_{\varepsilon}^{A C}(\boldsymbol{x}, \omega), \boldsymbol{q}(\boldsymbol{x}, \omega)=\boldsymbol{u}^{A C}(\boldsymbol{x}, \omega)$ and $\boldsymbol{q}(\boldsymbol{x}, \omega)=\left(\begin{array}{l}\boldsymbol{A}_{\varepsilon}^{A C}(\boldsymbol{x}, \omega) \\ \boldsymbol{u}^{A C}(\boldsymbol{x}, \omega)\end{array}\right)$ is shown in Figure 7.6 for the case $N_{s}=500$, where it can be observed that the decay of the singular values is much faster for $\boldsymbol{q}(\boldsymbol{x}, \omega)=\boldsymbol{A}_{\varepsilon}^{A C}(\boldsymbol{x}, \omega)$. Note that $M=50$ was chosen so that $e_{1}$ is sufficiently small for both physics.

\section{On-line PODI}

In the on-line PODI stage, ONLINEPODI $\left(\mathbf{H}_{\mathcal{A}}^{M}, \boldsymbol{\Sigma}_{\mathcal{A}}^{M}, \mathbf{G}_{\mathcal{A}}^{M}, \mathbf{N}, M, \boldsymbol{x}, 2 \pi \mathrm{f}_{i}\right)$ is first called followed by ONLINEPODI $\left(\mathbf{H}_{\mathcal{U}}^{M}, \boldsymbol{\Sigma}_{\mathcal{U}}^{M}, \mathbf{G}_{\mathcal{U}}^{M}, \mathbf{L}, M, \boldsymbol{x}, 2 \pi \mathrm{f}_{i}\right)$, with $\mathrm{f}_{i}$ defined as in Section 


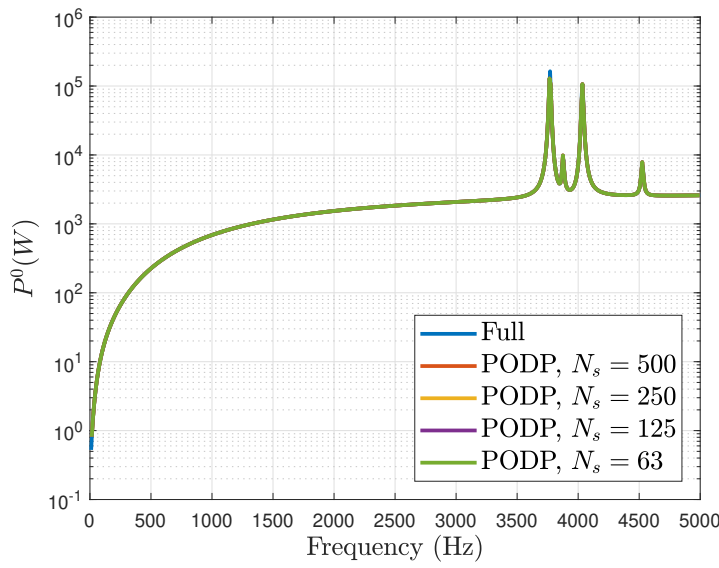

(a) $P_{\Omega_{O V C}}^{0}$

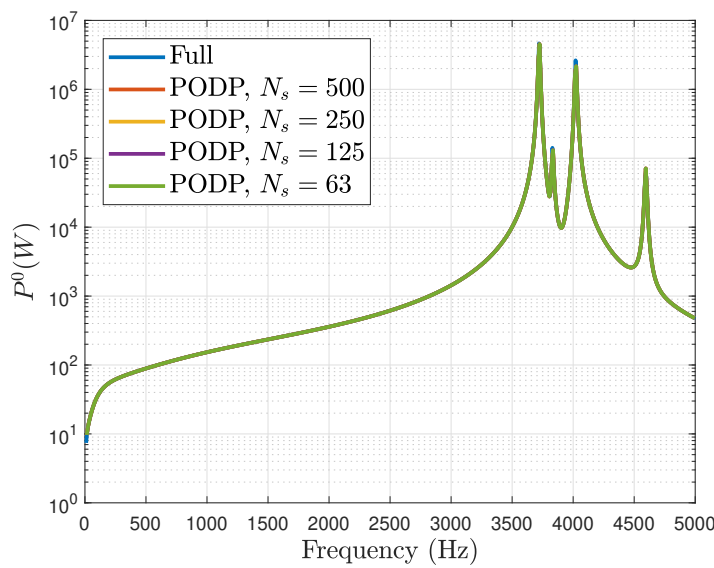

(c) $P_{\Omega_{77 K}}^{0}$

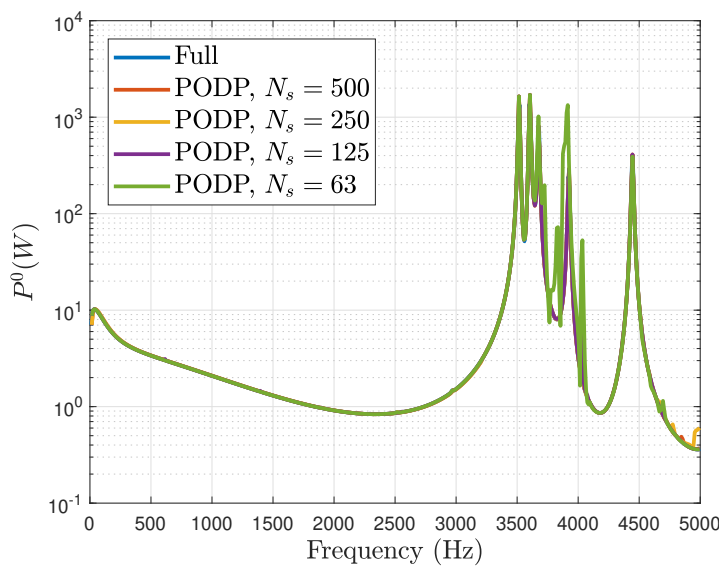

(e) $P_{\Omega_{4 K}}^{0}$

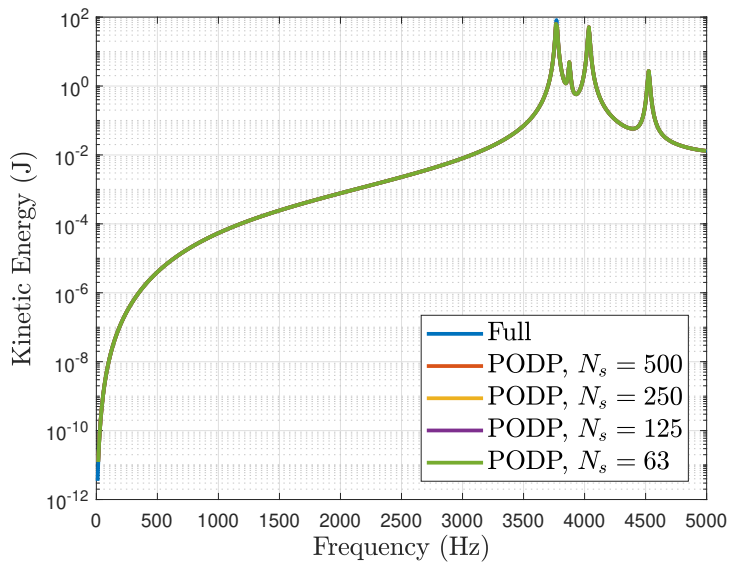

(b) $E_{\Omega_{O V C}}^{k}$

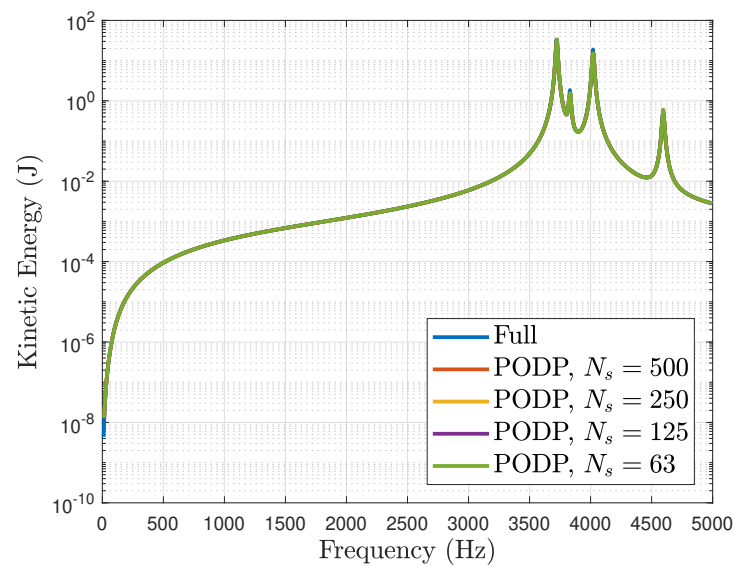

(d) $E_{\Omega_{77 K}}^{k}$

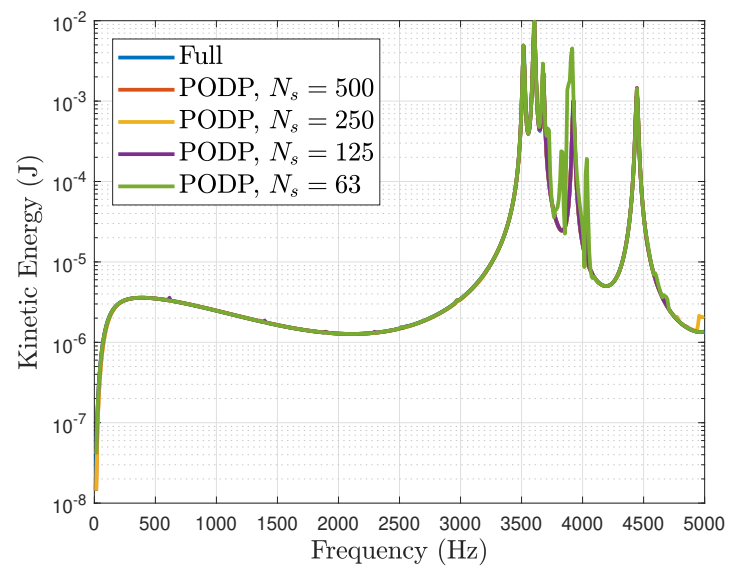

(f) $E_{\Omega_{4 K}}^{k}$

Figure 7.4: PODP applied to test magnet problem with 1 parameter. Dissipated power and kinetic energy in the radiation shields for different $N_{s}$. Comparison with the full order solution. 


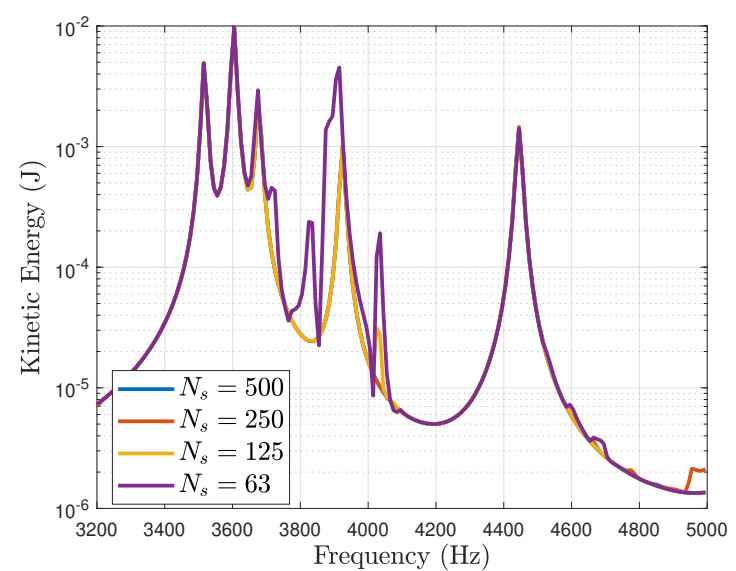

(a) $E_{\Omega_{77 K}}^{k}\left(2 \pi \mathrm{f}_{i}, \boldsymbol{u}^{P O D P}\right)$

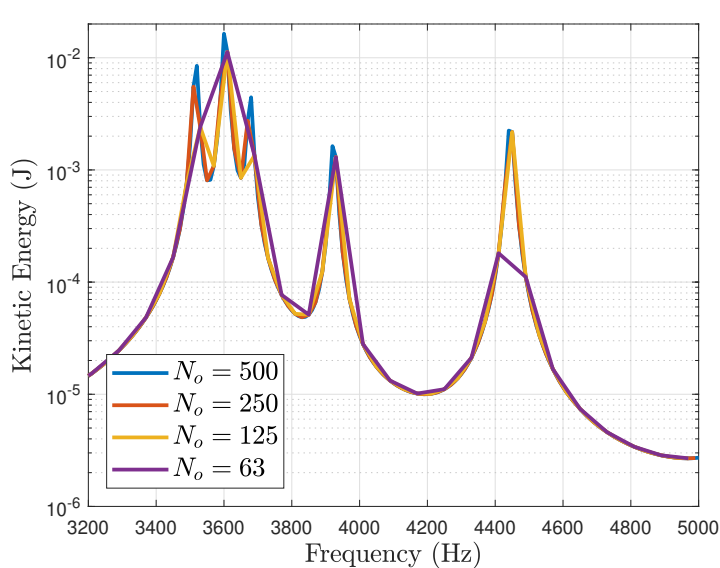

(b) $E_{\Omega_{77 K}}^{k}\left(2 \pi \mathrm{f}_{i}, \boldsymbol{u}_{h p}^{A C}\right)$

Figure 7.5: Test magnet problem with 1 parameter: (a) $E_{\Omega_{77 K}}^{k}\left(2 \pi \mathrm{f}_{i}, \boldsymbol{u}^{P O D P}\right)$ for different $N_{s}$ and (b) $E_{\Omega_{77 K}}^{k}\left(2 \pi \mathrm{f}_{i}, \mathcal{u}_{h p}^{A C}\right)$ for different $N_{o}$.

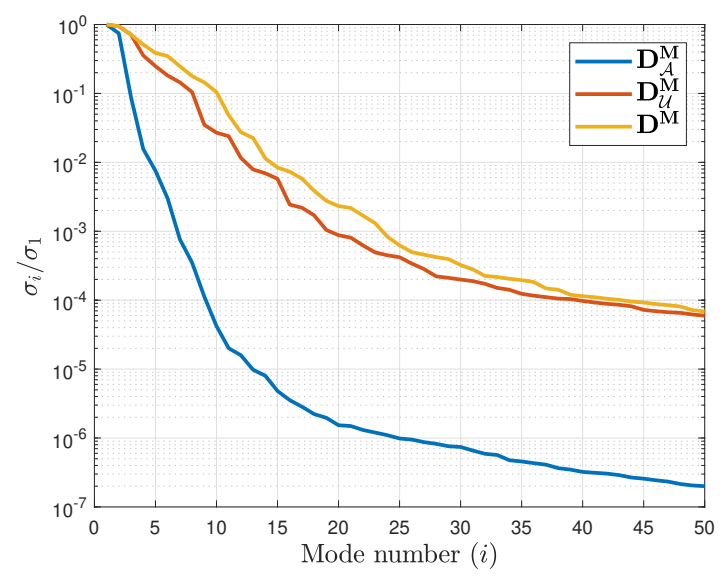

Figure 7.6: POD applied to separated physics solutions: Decay of the singular values of $\mathbf{D}, \mathbf{D}_{\mathcal{A}}$ and $\mathbf{D}_{\mathcal{U}}$. 


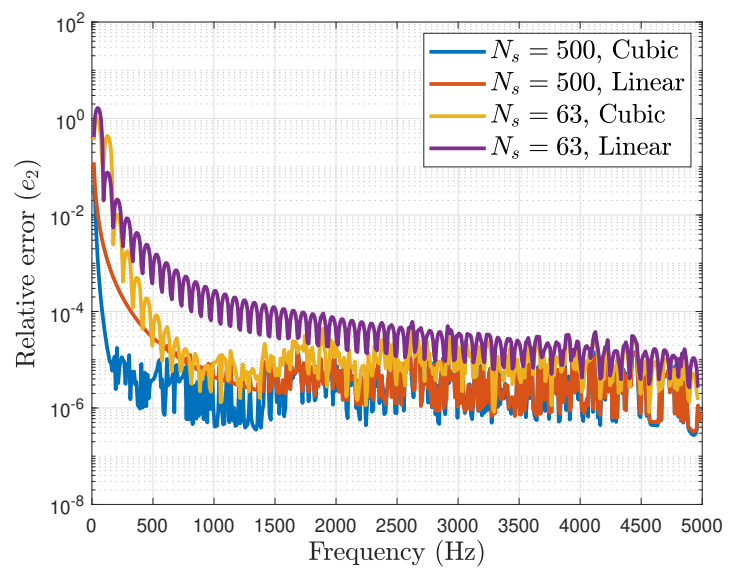

(a) $\mathbf{q}\left(2 \pi \mathrm{f}_{i}\right)=\mathcal{A}_{\varepsilon}^{\mathrm{AC}}\left(2 \pi \mathrm{f}_{i}\right)$

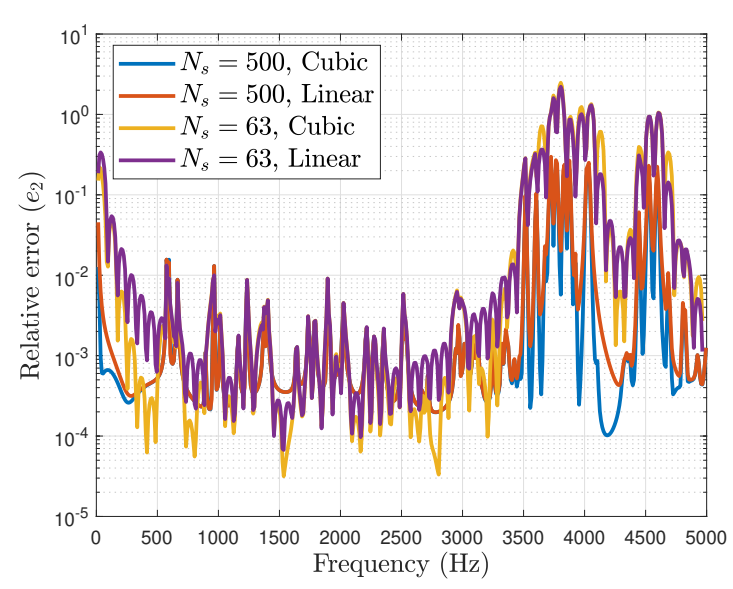

(b) $\mathbf{q}\left(2 \pi \mathrm{f}_{i}\right)=\mathcal{U}^{\mathrm{AC}}\left(2 \pi \mathrm{f}_{i}\right)$

Figure 7.7: PODI applied to test magnet problem with 1 parameter: relative error, $e_{2}\left(\mathbf{q}\left(2 \pi \mathrm{f}_{i}\right), \mathbf{q}^{\mathrm{PODI}}\left(2 \pi \mathrm{f}_{i}\right)\right)$ for (a) $\mathbf{q}\left(2 \pi \mathrm{f}_{i}\right)=\mathcal{A}_{\varepsilon}^{\mathrm{AC}}\left(2 \pi \mathrm{f}_{i}\right)$ and (b) $\mathbf{q}\left(2 \pi \mathrm{f}_{i}\right)=\mathcal{U}^{\mathrm{AC}}\left(2 \pi \mathrm{f}_{i}\right)$.

7.3.1. The cases of $I_{j}\left(2 \pi \mathrm{f}_{i}\right)$ corresponding to linear and cubic Lagrange interpolation were considered. The error $e_{2}\left(\mathbf{q}\left(2 \pi \mathrm{f}_{i}\right), \mathbf{q}^{\mathrm{PODI}}\left(2 \pi \mathrm{f}_{i}\right)\right)$ defined in (7.3) is shown in Figure 7.7, where it can be observed that $e_{2}\left(\mathbf{q}\left(2 \pi \mathrm{f}_{i}\right), \mathbf{q}^{\mathrm{PODI}}\left(2 \pi \mathrm{f}_{i}\right)\right)$ is several orders of magnitude smaller in the case $\mathbf{q}\left(2 \pi \mathrm{f}_{i}\right)=\mathcal{A}_{\varepsilon}^{\mathrm{AC}}\left(2 \pi \mathrm{f}_{i}\right)$ compared to the case $\mathbf{q}\left(2 \pi \mathrm{f}_{i}\right)=\mathcal{U}^{\mathrm{AC}}\left(2 \pi \mathrm{f}_{i}\right)$, especially for $\mathrm{f}_{i}>3000 \mathrm{~Hz}$. The large $e_{2}\left(\mathbf{q}\left(2 \pi \mathrm{f}_{i}\right), \mathbf{q}^{\mathrm{PODI}}\left(2 \pi \mathrm{f}_{i}\right)\right)$ obtained for the case $\mathbf{q}\left(2 \pi \mathrm{f}_{i}\right)=\mathcal{A}_{\varepsilon}^{\mathrm{AC}}\left(2 \pi \mathrm{f}_{i}\right)$ at frequencies $\mathrm{f}_{i}<1000 \mathrm{~Hz}$ is due to its sharp variation in that region, and this can be predicted and taken into account when defining the sampling frequencies. However, the large $e_{2}\left(\mathbf{q}\left(2 \pi \mathrm{f}_{i}\right), \mathbf{q}^{\mathrm{PODI}}\left(2 \pi \mathrm{f}_{i}\right)\right)$ obtained for $\mathbf{q}\left(2 \pi \mathrm{f}_{i}\right)=\mathcal{U}^{\mathrm{AC}}\left(2 \pi \mathrm{f}_{i}\right)$ in the resonant region is due to the singularities (peaks) obtained when the electromagnetic field excites some of the resonant modes of the mechanical system, and cannot be predicted. Thus, in a similar manner to Section 7.3.1, the $N_{s}$ required by PODI in order to accurately capture $\boldsymbol{q}(\boldsymbol{x}, \omega)=\boldsymbol{u}^{A C}(\boldsymbol{x}, \omega)$ is as large as the $N_{o}$ required by the full order solver, which means that no computational gaining can be obtained applying PODI to the mechanical problem. Furthermore, it can be observed that the error decreases as $N_{s}$ increases and also that cubic interpolation offers a slightly improved accuracy compared to linear interpolation. Note that the results for the dissipated power and kinetic energy are identical to those in Figure 7.2 and thus are not repeated here.

\section{On-line PODP}

In the on-line PODP stage, OnlinePODP $\left(\mathbf{H}_{\mathcal{A}}^{M},\left(\mathbf{H}_{A}^{M}\right)^{*} \mathbf{A}_{\mathcal{A A}}\left(2 \pi \mathrm{f}_{i}\right) \mathbf{H}_{A}^{M}\right.$, $\left.\left(\mathbf{H}_{A}^{M}\right)^{*} \mathbf{R}_{\mathcal{A}}\left(2 \pi \mathrm{f}_{i}\right), \mathbf{N}, \boldsymbol{x}, 2 \pi \mathrm{f}_{i}\right)$ is first called, followed by ON$\operatorname{LINEPODP}\left(\mathbf{H}_{\mathcal{U}}^{M},\left(\mathbf{H}_{\mathcal{U}}^{M}\right)^{*} \mathbf{A}_{\mathcal{U} \mathcal{U}}\left(2 \pi \mathrm{f}_{i}\right) \mathbf{H}_{\mathcal{U}}^{M},\left(\mathbf{H}_{\mathcal{U}}^{M}\right)^{*}\left(-\mathbf{A}_{\mathcal{U A}}\left(2 \pi \mathrm{f}_{i}\right) \mathcal{A}_{\varepsilon}^{\mathrm{AC}}(\boldsymbol{w})\right), \mathbf{L}, \boldsymbol{x}, 2 \pi \mathrm{f}_{i}\right)$, with $\mathrm{f}_{i}$ defined as in Section 7.3.1. Again, the results for dissipated power and kinetic energy are practically identical as those in Figure 7.4, and thus are not repeated here. Similarly as for PODI, Figure 7.8 shows the error $e_{2}\left(\mathbf{q}\left(2 \pi \mathbf{f}_{i}\right), \mathbf{q}^{\mathrm{PODI}}\left(2 \pi \mathrm{f}_{i}\right)\right)$ for the cases $N_{s}=63,500$, where again, as for the case of PODI, it can be observed that the error in 


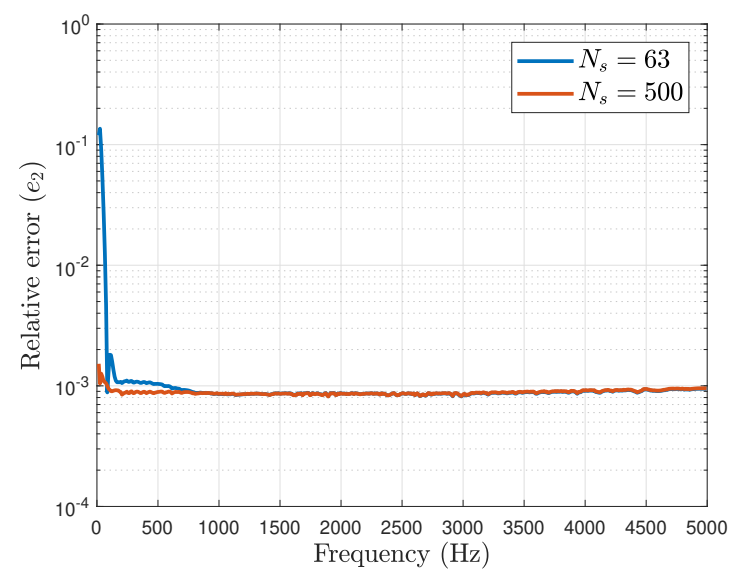

(a) $\mathbf{q}\left(2 \pi \mathrm{f}_{i}\right)=\mathcal{A}_{\varepsilon}^{\mathrm{AC}}\left(2 \pi \mathrm{f}_{i}\right)$

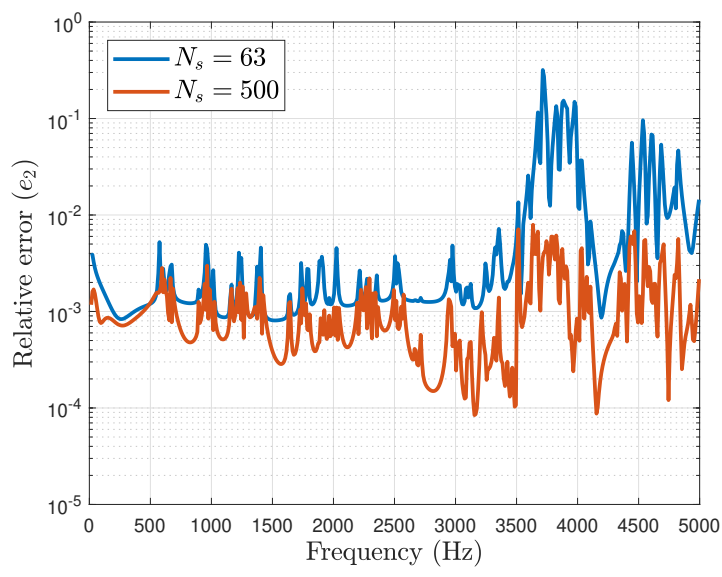

(b) $\mathbf{q}\left(2 \pi \mathrm{f}_{i}\right)=\mathcal{U}^{\mathrm{AC}}\left(2 \pi \mathrm{f}_{i}\right)$

Figure 7.8: PODP applied to test magnet problem with 1 parameter: relative error, $e_{2}\left(\mathbf{q}\left(2 \pi \mathrm{f}_{i}\right), \mathbf{q}^{\mathrm{PODI}}\left(2 \pi \mathrm{f}_{i}\right)\right)$ for (a) $\mathbf{q}\left(2 \pi \mathrm{f}_{i}\right)=\mathcal{A}_{\varepsilon}^{\mathrm{AC}}\left(2 \pi \mathrm{f}_{i}\right)$ and (b) $\mathbf{q}\left(2 \pi \mathrm{f}_{i}\right)=\mathcal{U}^{\mathrm{AC}}\left(2 \pi \mathrm{f}_{i}\right)$.

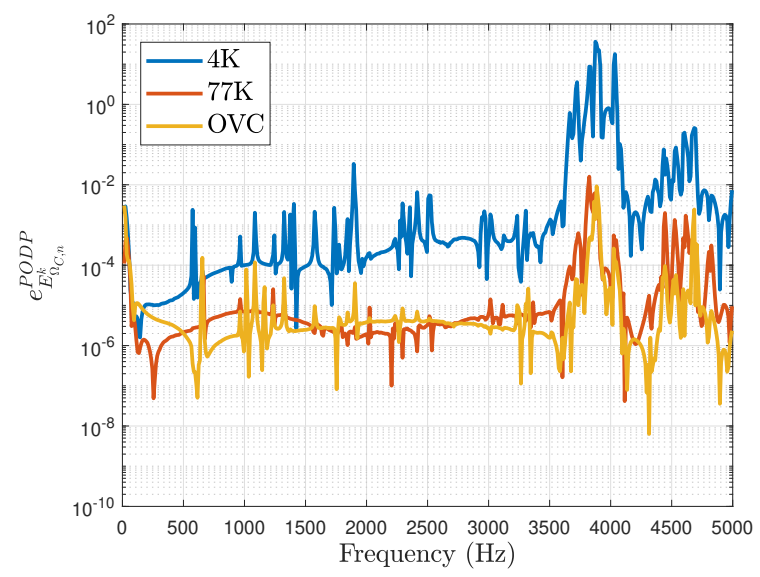

Figure 7.9: PODP applied to test magnet problem with 1 parameter: Relative error in the kinetic energy $e_{E_{\Omega_{C, n}}^{K}}^{P O D P}$ for each of the radiation shields.

the resonant region, $\mathrm{f}_{i}>3000 \mathrm{~Hz}$, is several orders of magnitude smaller for the case $\mathbf{q}\left(2 \pi \mathrm{f}_{i}\right)=\mathcal{A}_{\varepsilon}^{\mathrm{AC}}\left(2 \pi \mathrm{f}_{i}\right)$ compared to the case $\mathbf{q}\left(2 \pi \mathrm{f}_{i}\right)=\mathcal{U}^{\mathrm{AC}}\left(2 \pi \mathrm{f}_{i}\right)$.

Note, however, that the error for $\mathbf{q}\left(2 \pi \mathrm{f}_{i}\right)=\mathcal{U}^{\mathrm{AC}}\left(2 \pi \mathrm{f}_{i}\right)$ is in this case smaller compared to PODI. This is due to the fact that PODP results in an excellent approximation in $\Omega_{O V C}$ and $\Omega_{77 K}$, as shown in Figure 7.4. To further illustrate this, the relative error in the kinetic energy in each of the radiation shields, $e_{E_{\Omega_{C, n}}^{K}}^{P O P}\left(2 \pi \mathrm{f}_{i}\right)$, is shown in Figure 7.9, where it can be observed that the maximum error in the OVC and $77 \mathrm{~K}$ shields is of order $10^{-2}$, while in the case of the $4 \mathrm{~K}$ the maximum error goes up to almost $10^{2}$. 


\subsubsection{Combined Reduced Order-Full Order Approach}

Motivated by the results in Section 7.3.3, where it was shown for separated physics solutions, that the relative error $e_{2}\left(\mathbf{q}(\omega), \mathbf{q}^{\mathrm{POD}}(\omega)\right)$ is several orders of magnitude smaller for the case $\mathbf{q}(\omega)=\mathcal{A}_{\varepsilon}^{\mathrm{AC}}(\omega)$ than for the case $\mathbf{q}(\omega)=\mathcal{U}^{\mathrm{AC}}(\omega)$, the efficiency of the combined reduced order-full order approach described in Section 6.7 is now studied. As it was observed that, in the case $\mathbf{q}(\omega)=\mathcal{A}_{\varepsilon}^{\mathrm{AC}}(\omega)$, the error is higher for $f<1000 \mathrm{~Hz}$, a non uniform $\Delta f$ will be used. In particular, the sampling frequencies are defined as

$$
\mathbf{f}_{i}=\left\{\begin{array}{ccc}
10 \mathrm{~Hz} & \text { if } & i=1 \\
10+(i-1) \Delta \mathrm{f}_{1} \mathrm{~Hz} & \text { if } & \mathrm{f}_{i-1}<1000 \mathrm{~Hz} \text { and } i>1 \\
10+(i-1) \Delta \mathrm{f}_{2} \mathrm{~Hz} & \text { if } & \mathrm{f}_{i-1} \geqslant 1000 \mathrm{~Hz} \text { and } i>1
\end{array}\right.
$$

with $i=1, \ldots, N_{s}$. The frequency steps $\left(\Delta \mathrm{f}_{1}, \Delta \mathrm{f}_{2}\right)=$ $\{(10,50),(20,100),(40,200),(80,400)\} \mathrm{Hz}$ were considered, which results in a set of samples $\mathbf{W}_{\mathrm{s}}=\left\{2 \pi \mathrm{f}_{1}, 2 \pi \mathrm{f}_{2}, \ldots, 2 \pi \mathrm{f}_{N_{s}}\right\}$ with $N_{s}=180,90,45$ and 23 , respectively. Algorithm 2 is applied by calling $\operatorname{OfFLine}\left(\mathbf{A}_{\mathcal{A A}}(\boldsymbol{w}), \mathbf{R}_{\mathcal{A}}(\boldsymbol{w}), \mathbf{W}_{\mathrm{s}}, M\right)$ with $M=20$ (leading to a maximum upper bound for the truncation error $e_{1}=8.9 \times 10^{-6}$ for the case $\left.N_{s}=180\right)$

\section{PODI-Full}

The PODI-Full approach is studied first. For this, Algorithm 5 is applied by calling OnlinePODI-Full $\left(\mathbf{H}_{\mathcal{A}}^{M}, \boldsymbol{\Sigma}_{\mathcal{A}}^{M}, \mathbf{G}_{\mathcal{A}}^{M}, \mathbf{A}_{\mathcal{U} \mathcal{U}}\left(2 \pi \mathrm{f}_{i}\right), \mathbf{A}_{\mathcal{U A}}\left(2 \pi \mathrm{f}_{i}\right), \mathbf{N}, \mathbf{L}, M, \boldsymbol{x}, 2 \pi \mathrm{f}_{i}\right)$ with $\mathrm{f}_{i}=15+(i-1) \Delta \mathrm{f}, i=1, \ldots, N_{o}, N_{o}=499$ and $\Delta \mathrm{f}=10 \mathrm{~Hz}$. A comparison of $P_{\Omega_{C, n}}^{0}\left(2 \pi \mathrm{f}_{i}, \boldsymbol{A}^{P O D I-F U L L}, \boldsymbol{B}_{0, h q}^{D C}, \boldsymbol{u}^{P O D I-F U L L}\right)$ with $P_{\Omega_{C, n}}^{0}\left(2 \pi \mathrm{f}_{i}, \boldsymbol{A}_{\varepsilon, h q}^{A C}, \boldsymbol{B}_{0, h q}^{D C}, \boldsymbol{u}_{h p}^{A C}\right)$ and $E_{\Omega_{C, n}}^{k, n}\left(2 \pi \mathrm{f}_{i}, \boldsymbol{u}^{P O D I-F U L L}\right)$ with $E_{\Omega_{C, n}}^{k}\left(2 \pi \mathrm{f}_{i}, \boldsymbol{u}_{h p}^{A C}\right)$, where $\Omega_{C, n}$ refers to either $\Omega_{4 K}$, $\Omega_{77 K}$ or $\Omega_{O V C}$, is shown in Figure 7.10, with $i=1, \ldots, N_{o}$ in all cases. It can be observed that the results show an excellent agreement with the full order solution and differences are only observed at $f<200 \mathrm{~Hz}$ for $P_{\Omega_{4 K}}^{0}$ and $P_{\Omega_{77 K}}^{0}$ in the case $N_{s}=23$.

To further study the accuracy of the method, the relative error $e_{2}\left(\mathcal{A}_{\varepsilon}^{\mathrm{AC}}\left(2 \pi \mathrm{f}_{i}\right), \mathcal{A}^{\text {PODI-FULL }}\left(2 \pi \mathrm{f}_{i}\right)\right)$ and $e_{2}\left(\mathcal{U}^{\mathrm{AC}}\left(2 \pi \mathrm{f}_{i}\right), \mathcal{U}^{\text {PODI-FULL }}\left(2 \pi \mathrm{f}_{i}\right)\right)$ are shown in Figures 7.11a and 7.11b, respectively.

These results show that $\mathcal{A}^{\text {PODI-FULL }}$ produced by Algorithm 5 is able to accurately reproduce $\mathcal{A}_{\varepsilon, h q}^{A C}$ already by using a small $N_{s}$. The method is much more robust than the application of PODI to the whole problem, as the peaks are very accurately resolved even for only $N_{s}=23$ and the error $e_{2}\left(\mathcal{U}^{\mathrm{AC}}\left(2 \pi \mathrm{f}_{i}\right), \mathcal{U}^{\text {PODI-FULL }}\left(2 \pi \mathrm{f}_{i}\right)\right)$ in the resonant region $\left(f_{i}>3000 \mathrm{~Hz}\right)$ is around three orders of magnitude smaller than when applying PODI to the complete problem. Furthermore, the method offers a great advantage in terms of computational speed. To illustrate this, a comparison of solver time between PODI-Full and the full order solver is provided in Figure 7.12a, where different $N_{s}=180,90,45,23$ and $N_{o}=125,250,500,1000$ have been considered. The time of both the off-line (Algorithm 2) and on-line (Algorithm 5) stages is included in the ROM solver time. Figure $7.12 \mathrm{~b}$ shows the speed-up with respect to the full order solver. Note for example the case 


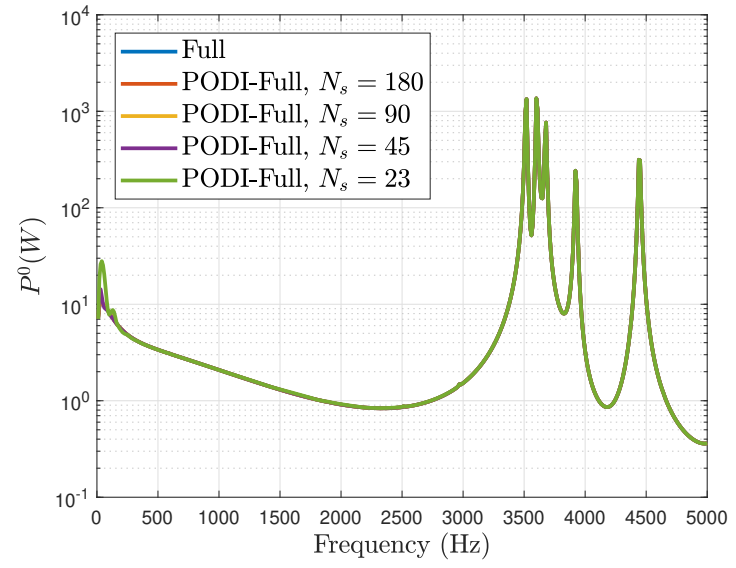

(a) $P_{\Omega_{4 K}}^{0}$

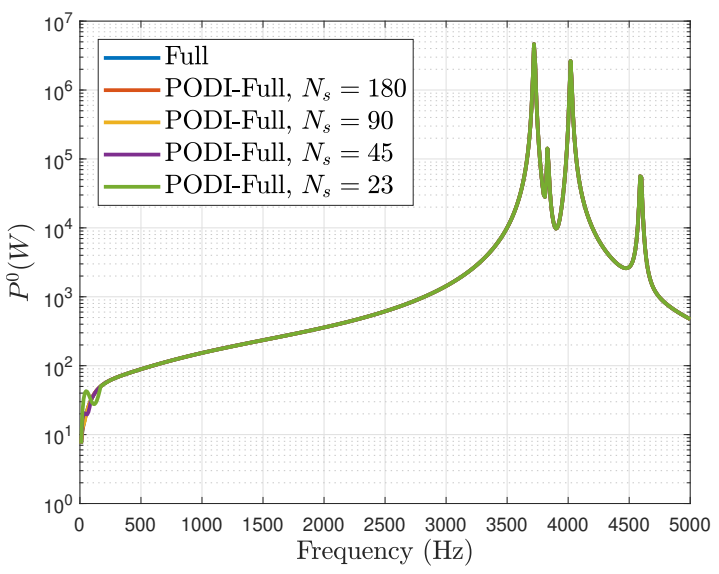

(c) $P_{\Omega_{77 K}}^{0}$

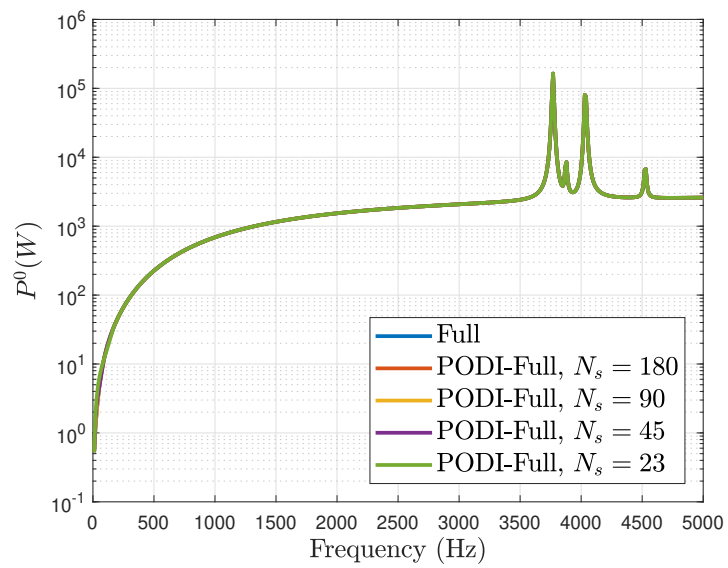

(e) $P_{\Omega_{O V C}}^{0}$

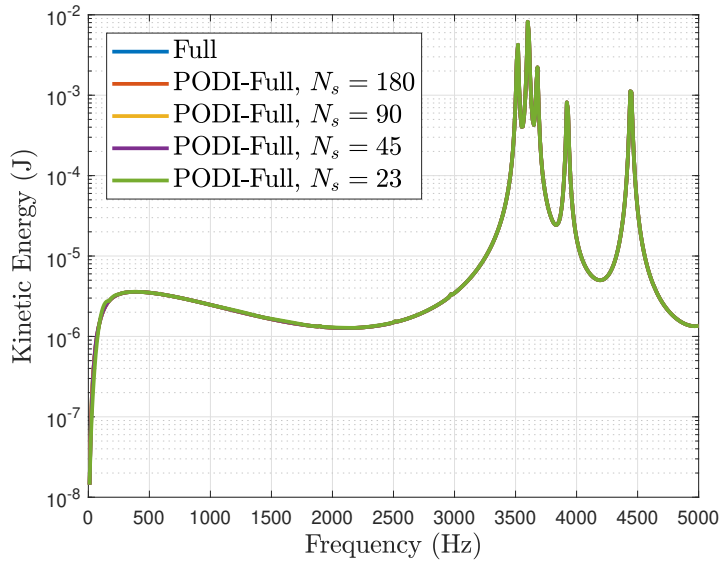

(b) $E_{\Omega_{4 K}}^{k}$

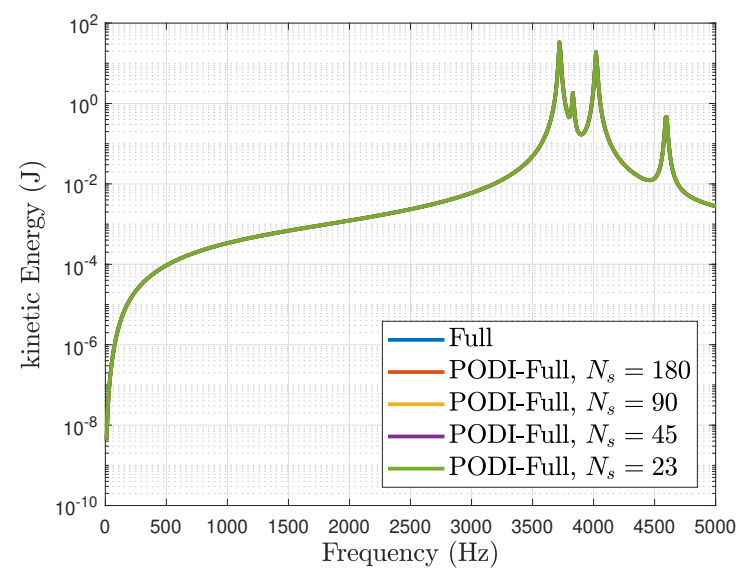

(d) $E_{\Omega_{77 K}}^{k}$

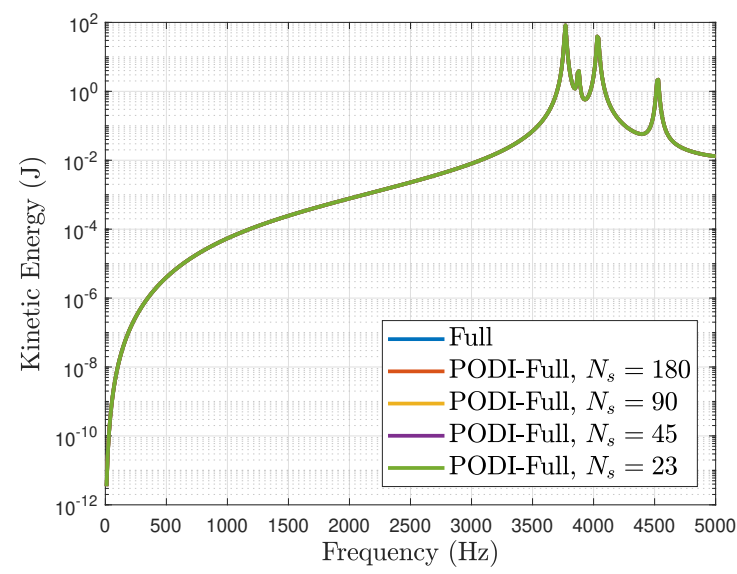

(f) $E_{\Omega_{O V C}}^{k}$

Figure 7.10: PODI-Full applied to test magnet problem with 1 parameter: (a) $P_{\Omega_{4 K}}^{0}$, (b) $E_{\Omega_{4 K}}^{k}$, (c) $P_{\Omega_{77 K}}^{0}$, (d) $E_{\Omega_{77 K}}^{k}$, (e) $P_{\Omega_{O V C}}^{0}$ and (f) $E_{\Omega_{O V C}}^{k}$. Comparison with full order solution. 


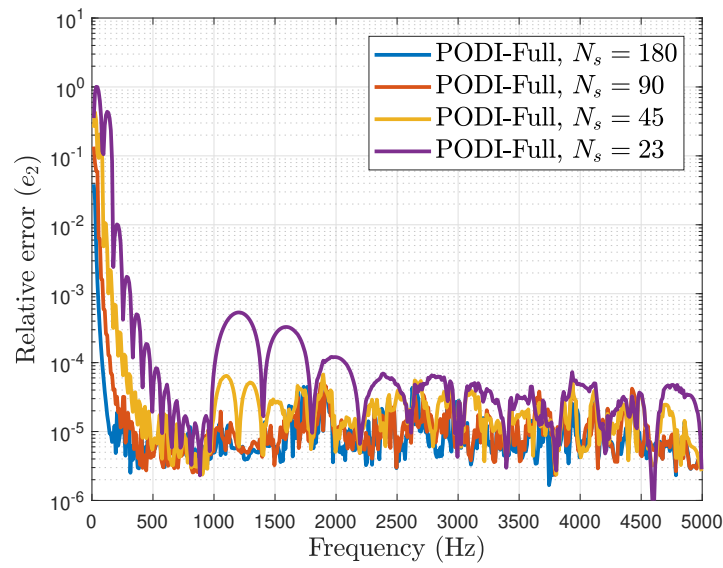

(a) $e_{2}\left(\mathcal{A}_{\varepsilon}^{\mathrm{AC}}\left(2 \pi \mathrm{f}_{i}\right), \mathcal{A}^{\text {PODI-FULL }}\left(2 \pi \mathrm{f}_{i}\right)\right)$

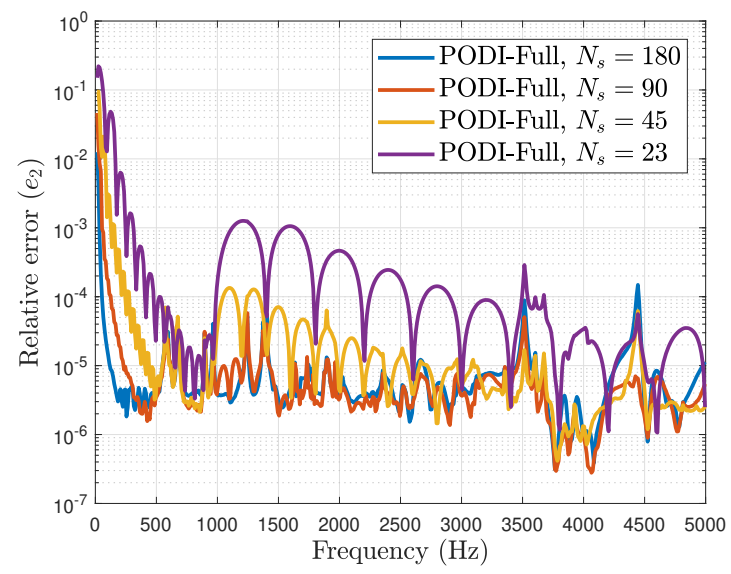

(b) $e_{2}\left(\mathcal{U}^{\mathrm{AC}}\left(2 \pi \mathrm{f}_{i}\right), \mathcal{U}^{\text {PODI-FULL }}\left(2 \pi \mathrm{f}_{i}\right)\right)$

Figure 7.11: PODI-Full applied to test magnet problem with 1 parameter: (a) $e_{2}\left(\mathcal{A}_{\varepsilon}^{\mathrm{AC}}\left(2 \pi \mathrm{f}_{i}\right), \mathcal{A}^{\text {PODI-FULL }}\left(2 \pi \mathrm{f}_{i}\right)\right)$ and $(\mathrm{b}) e_{2}\left(\mathcal{U}^{\mathrm{AC}}\left(2 \pi \mathrm{f}_{i}\right), \mathcal{U}^{\text {PODI-FULL }}\left(2 \pi \mathrm{f}_{i}\right)\right)$.

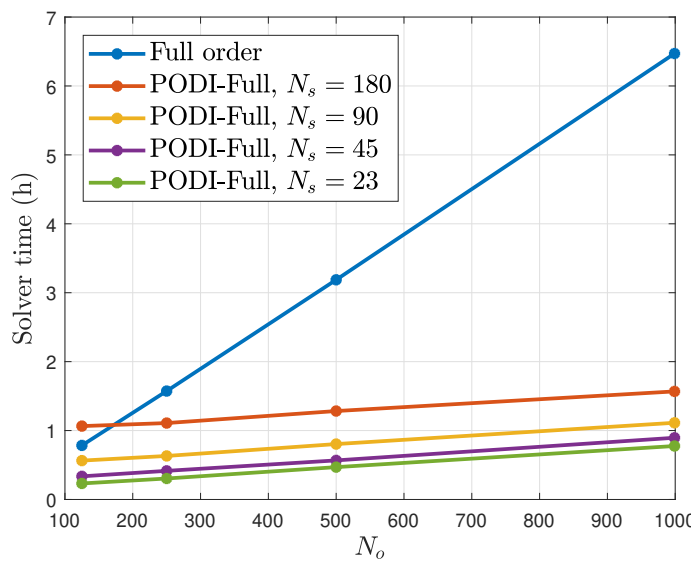

(a) Solver Time

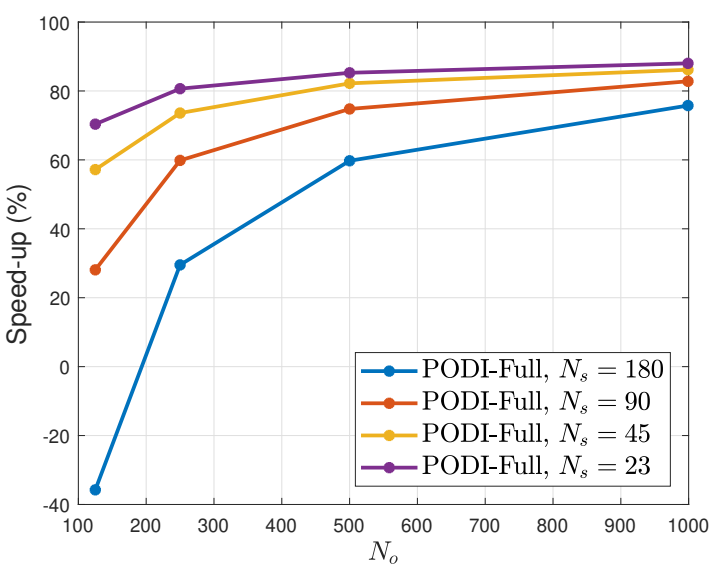

(b) Speed-up

Figure 7.12: PODI-Full applied to test magnet problem with 1 parameter: (a) Solver time and (b) speed-up for different values of $N_{s}$ and $N_{o}$.

$N_{s}=45$ (where there is no appreciable difference in the solution), where the speed up is $57 \%$ for $N_{o}=125$ and grows to $86 \%$ for $N_{o}=1000$.

\section{PODP-Full}

The same study is now repeated for the PODP-Full approach, by applying Algorithm 6 and calling ONLINEPODP-FULL $\left(\mathbf{H}_{\mathcal{A}}^{M}, \mathbf{A}\left(2 \pi \mathrm{f}_{i}\right), \mathbf{R}\left(2 \pi \mathrm{f}_{i}\right), \mathbf{N}, \mathbf{L}, \boldsymbol{x}, 2 \pi \mathrm{f}_{i}\right)$, with $\mathrm{f}_{i}=15+(i-1) \Delta \mathrm{f}, i=1, \ldots, N_{o}, N_{o}=499$ and $\Delta \mathrm{f}=10 \mathrm{~Hz}$. A comparison of $P_{\Omega_{C, n}}^{0}\left(2 \pi \mathrm{f}_{i}, \boldsymbol{A}^{P O D P-F U L L}, \boldsymbol{B}_{0, h q}^{D C}, \boldsymbol{u}^{P O D P-F U L L}\right)$ with $P_{\Omega_{C, n}}^{0}\left(2 \pi \mathrm{f}_{i}, \boldsymbol{A}_{\varepsilon, h q}^{A C}, \boldsymbol{B}_{0, h q}^{D C}, \boldsymbol{u}_{h p}^{A C}\right)$ and $E_{\Omega_{C, n}}^{k}\left(2 \pi \mathrm{f}_{i}, \boldsymbol{u}^{P O D P-F U L L}\right)$ with $E_{\Omega_{C, n}}^{k}\left(2 \pi \mathrm{f}_{i}, \boldsymbol{u}_{h p}^{A C}\right)$, where $\Omega_{C, n}$ refers to either $\Omega_{4 K}$, $\Omega_{77 K}$ or $\Omega_{O V C}$, is shown in Figure 7.13 , with $i=1, \ldots, N_{o}$ in all cases. It can be ob- 
served that the results obtained by applying Algorithm 6 are in excellent agreement with the full order results. With this approach there is no visible difference with the full order results even for $N_{s}=23$.

To further assess the accuracy of the PODP-Full approximation, the relative error $e_{2}\left(\mathcal{A}_{\varepsilon}^{\mathrm{AC}}\left(2 \pi \mathrm{f}_{i}\right), \mathcal{A}^{\text {PODP-FULL }}\left(2 \pi \mathrm{f}_{i}\right)\right)$ and $e_{2}\left(\mathcal{U}^{\mathrm{AC}}\left(2 \pi \mathrm{f}_{i}\right), \mathcal{U}^{\text {PODP-FULL }}\left(2 \pi \mathrm{f}_{i}\right)\right)$ for the cases $N_{s}=23,80$ are shown in Figures $7.14 \mathrm{a}$ and $7.14 \mathrm{~b}$, respectively. Note how the error $e_{2}\left(\mathcal{U}^{\mathrm{AC}}\left(2 \pi \mathrm{f}_{i}\right), \mathcal{U}^{\text {PODP-FULL }}\left(2 \pi \mathrm{f}_{i}\right)\right)$ in the resonance region is several orders of magnitude smaller than when applying PODP to the complete problem. Furthermore, the relative error in the kinetic energy for each of the radiation shields, $e_{E_{\Omega_{C, n}}^{P O D P}}^{P}\left(2 \pi \mathrm{f}_{i}\right)$, is shown in Figure 7.15, where again it can be observed that the error is in this case much smaller than when applying PODP to the complete problem, and even in the $4 \mathrm{~K}$ shield $e_{E_{\Omega_{4 K}}^{k}}^{P O D P}\left(2 \pi \mathrm{f}_{i}\right)<4 \times 10^{-3}$ for all the frequencies in the sweep.

Furthermore, in Figure 7.16, a comparison of solver time between PODP-Full and the full order solver is shown. Similarly to the results for PODI-Full (Figure 7.12), it can be observed that a speed-up higher than $85 \%$ can be obtained. Similarly as before, the time of both the off-line (Algorithm 2) and on-line (Algorithm 6) stages is included in the ROM time. Note also that the speed-up obtained with Algorithm 5 and Algorithm 6 is almost identical, while a higher accuracy is obtained with Algorithm 6.

\subsection{Test Magnet Problem with Multiple Parameters}

The problem described in Section 7.3 is now reconsidered, with both the frequency and the conductivity of the OVC, $\gamma^{O V C}$, considered as parameters, this is, $\boldsymbol{w}=\left(\omega, \gamma^{O V C}\right)$. The same discretisation as in Section 7.3 is used. The conductivity $\gamma^{O V C}$ is assumed to vary in the range $0.1 \gamma_{\text {ref }}^{O V C} \leqslant \gamma^{O V C} \leqslant 10 \gamma_{r e f}^{O V C}$, being $\gamma_{\text {ref }}^{O V C}$ the reference value used for the test magnet problem. The number of sampled conductivities was set to $N_{\gamma_{s}}=3$ and, for each of this conductivities, the number of sampled frequencies is set to $N_{\omega_{s}}=45$, which results in a total number of samples $N_{s}=135$. This means that $\mathbf{W}_{\mathrm{s}}$ has the elements $\mathbf{w}_{k}=\left(2 \pi \mathrm{f}_{i}, \gamma_{j}^{O V C}\right), k=i+(j-1) N_{\omega_{s}}, i=1, \ldots, N_{\omega_{s}}, j=1, \ldots, N_{\gamma_{s}}$ where $\mathrm{f}_{i}$ is defined by (7.5) with $\left(\Delta \mathrm{f}_{1}, \Delta \mathrm{f}_{2}\right)=(40,200) \mathrm{Hz}$ and $\gamma_{j}^{O V C}=0.1\left(10^{j-1}\right) \gamma_{r e f}^{O V C}$. Algorithm 2 is then applied by calling $\operatorname{OfFline}\left(\mathbf{A}_{\mathcal{A A}}(\boldsymbol{w}), \mathbf{R}_{\mathcal{A}}(\boldsymbol{w}), \mathbf{W}_{\mathrm{s}}, M\right)$ where the TSVD was truncated with $M=20$ (leading to an upper bound for the truncation error $e_{1}=1.6 \times 10^{-3}$ ).

Since in Section 7.3.4 it was observed that PODP-Full offers an increased accuracy compared with PODI-Full for almost no increase in computational cost, only PODP-Full will be considered in this multi-parameter case.

\subsubsection{PODP-Full}

Algorithm 6 is applied by calling ONLINEPODP$\operatorname{FULL}\left(\mathbf{H}_{\mathcal{A}}^{M}, \mathbf{A}\left(\mathbf{w}_{k}\right), \mathbf{R}\left(\mathbf{w}_{k}\right), \mathbf{N}, \mathbf{L}, \boldsymbol{x}, \mathbf{w}_{k}\right)$ where $\mathbf{w}_{k}=\left(2 \pi \mathbf{f}_{i}, \gamma_{j}^{O V C}\right), k=i+(j-1) N_{\omega_{o}}$, $i=1, \ldots, N_{\omega_{o}}, j=1, \ldots, N_{\gamma_{o}}, \mathrm{f}_{i}=10+\Delta \mathrm{f}(i-1) \mathrm{Hz}, N_{\omega_{o}}=500$, 


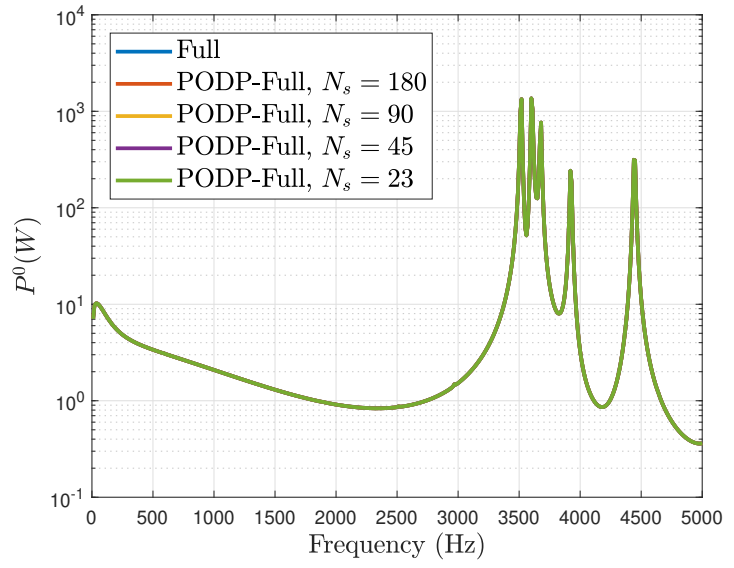

(a) $P_{\Omega_{4 K}}^{0}$

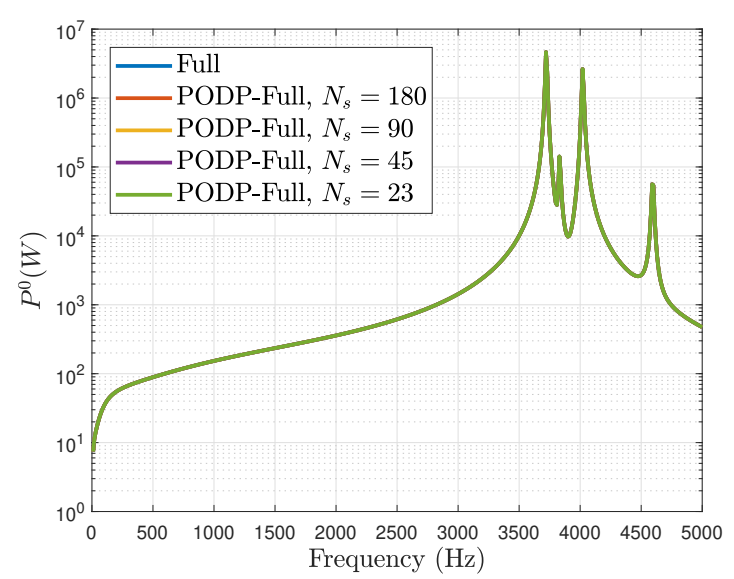

(c) $P_{\Omega_{77 K}}^{0}$

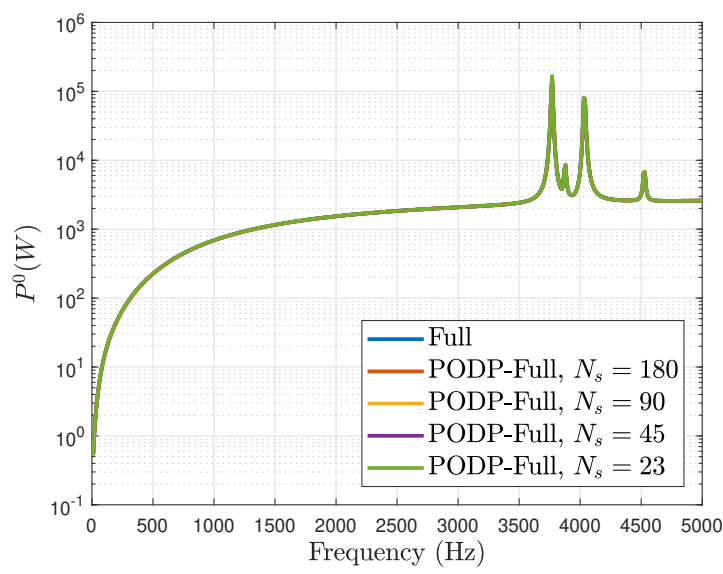

(e) $P_{\Omega_{O V C}}^{0}$

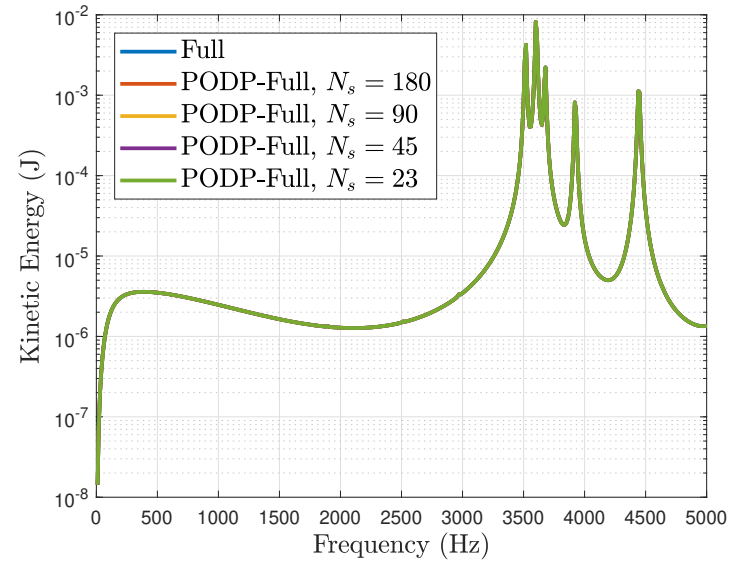

(b) $E_{\Omega_{4 K}}^{k}$

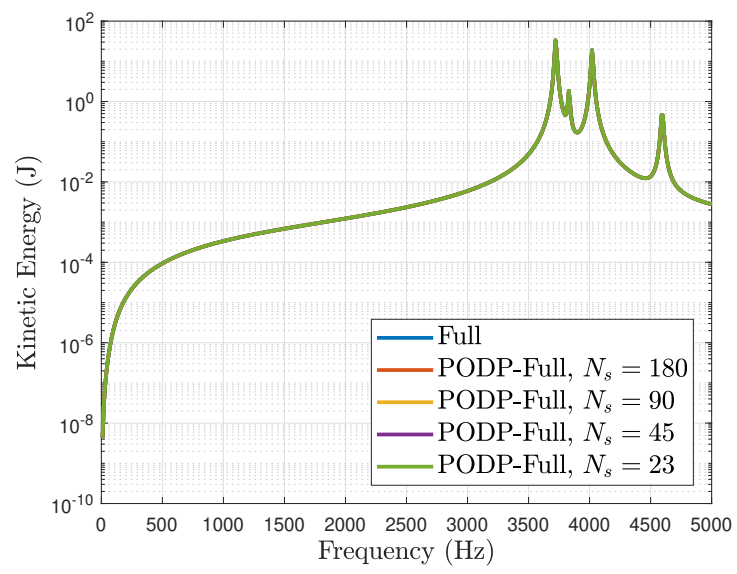

(d) $E_{\Omega_{77 K}}^{k}$

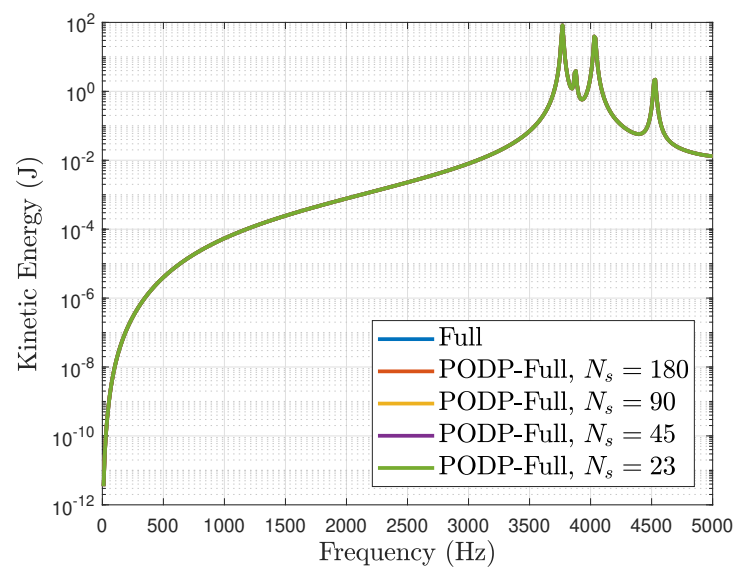

(f) $E_{\Omega_{O V C}}^{k}$

Figure 7.13: PODP-Full applied to test magnet problem with 1 parameter: (a) $P_{\Omega_{4 K}}^{0}$, (b) $E_{\Omega_{4 K}}^{k}$, (c) $P_{\Omega_{77 K}}^{0}$, (d) $E_{\Omega_{77 K}}^{k}$, (e) $P_{\Omega_{O V C}}^{0}$ and (f) $E_{\Omega_{O V C}}^{k}$. Comparison with full order solution. 


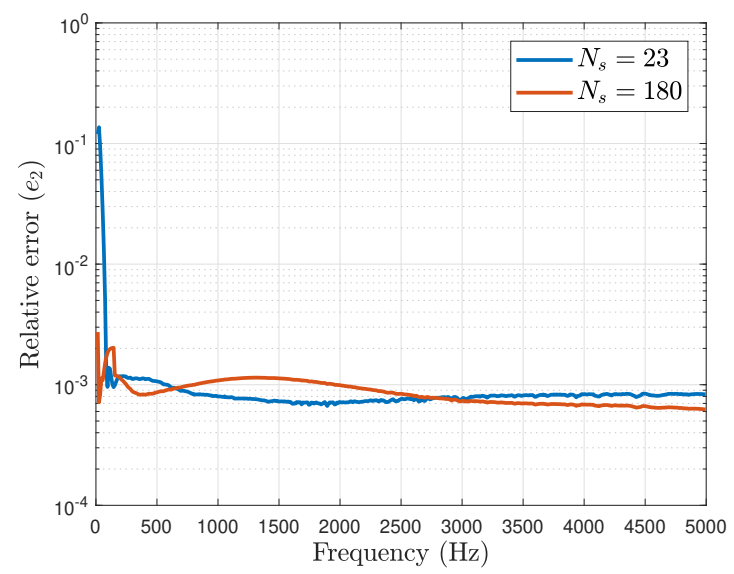

(a) $e_{2}\left(\mathcal{A}_{\varepsilon}^{\mathrm{AC}}\left(2 \pi \mathrm{f}_{i}\right), \mathcal{A}^{\text {PODP-FULL }}\left(2 \pi \mathrm{f}_{i}\right)\right)$

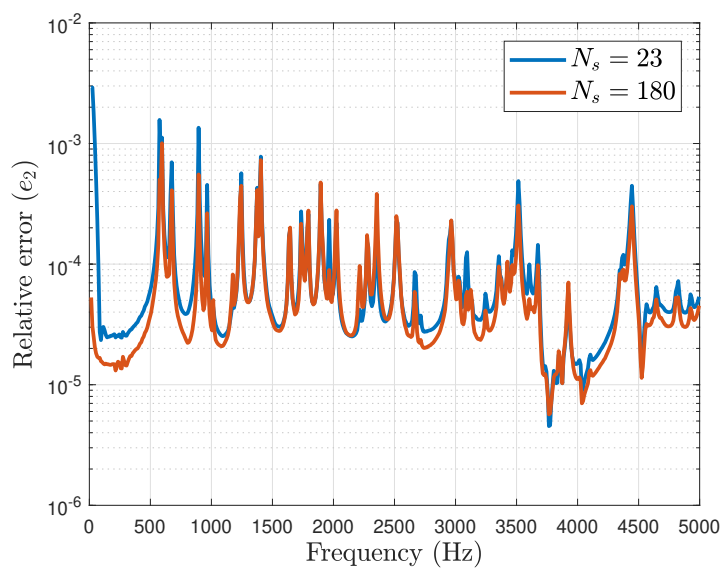

(b) $e_{2}\left(\mathcal{U}^{\mathrm{AC}}\left(2 \pi \mathrm{f}_{i}\right), \mathcal{U}^{\text {PODP-FULL }}\left(2 \pi \mathrm{f}_{i}\right)\right)$

Figure 7.14: PODI-Full applied to test magnet problem with 1 parameter: (a) $e_{2}\left(\mathcal{A}_{\varepsilon}^{\mathrm{AC}}\left(2 \pi \mathrm{f}_{i}\right), \mathcal{A}^{\text {PODP-FULL }}\left(2 \pi \mathrm{f}_{i}\right)\right)$ and $(\mathrm{b}) e_{2}\left(\mathcal{U}^{\mathrm{AC}}\left(2 \pi \mathrm{f}_{i}\right), \mathcal{U}^{\text {PODP-FULL }}\left(2 \pi \mathrm{f}_{i}\right)\right)$.

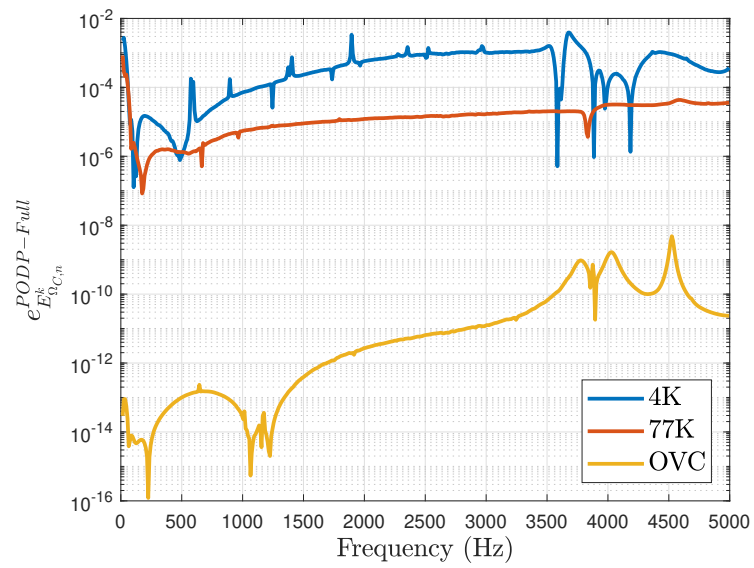

Figure 7.15: PODP-Full applied to test magnet problem with 1 parameter. Relative error in the kinetic energy $e_{E_{\Omega_{C, n}}^{k}}^{P O D P}$ for each of the radiation shields. 


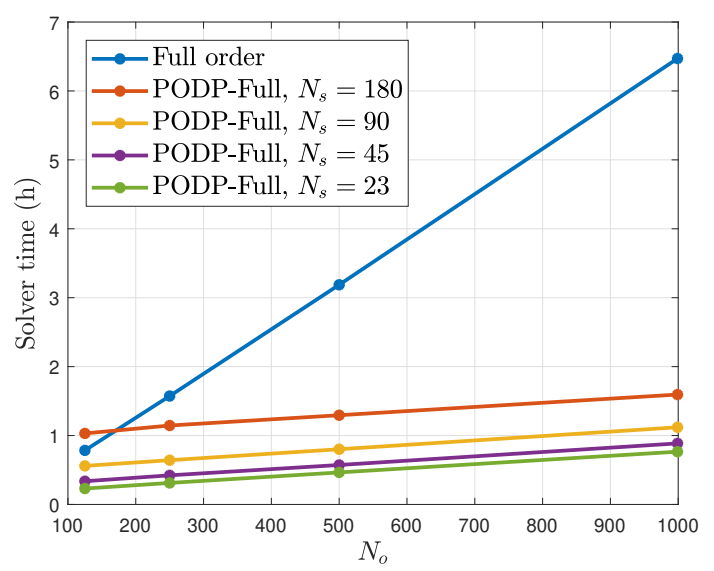

(a) Solver Time

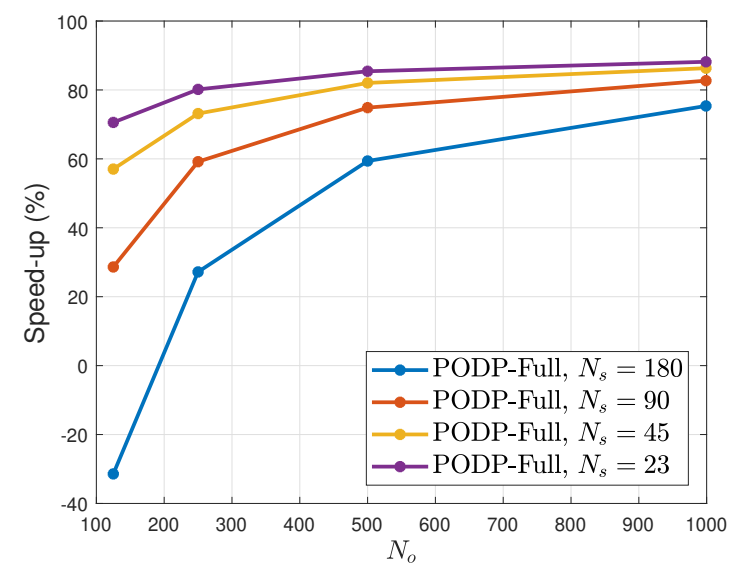

(b) Speed-up

Figure 7.16: PODP-Full applied to test magnet problem with 1 parameter: (a) Solver time and (b) speed-up for different values of $N_{s}$ and $N_{o}$.

$\gamma_{j}^{O V C} \in(0.35,0.75,3.5,7.5) \gamma_{r e f}^{O V C}, N_{\gamma_{o}}=4$ and $\Delta \mathrm{f}=10 \mathrm{~Hz}$. A comparison of $P_{\Omega_{C, n}}^{0}\left(\mathbf{w}_{k}, \boldsymbol{A}^{P O D P-F U L L}, \boldsymbol{B}_{0, h q}^{D C}, \boldsymbol{u}^{P O D P-F U L L}\right)$ with $P_{\Omega_{C, n}}^{0}\left(\mathbf{w}_{k}, \boldsymbol{A}_{\varepsilon, h q}^{A C}, \boldsymbol{B}_{0, h q}^{D C}, \boldsymbol{u}_{h p}^{A C}\right)$ and $E_{\Omega_{C, n}}^{k}\left(\mathbf{w}_{k}, \boldsymbol{u}^{P O D P-F U L L}\right)$ with $E_{\Omega_{C, n}}^{k}\left(\mathbf{w}_{k}, \boldsymbol{u}_{h p}^{A C}\right)$ is shown in Figure 7.17. It can be observed that the PODP-Full result is in perfect agreement with the full order solution.

Next, in order to better benchmark the accuracy of the method, $e_{2}\left(\mathcal{A}_{\varepsilon}^{\mathrm{AC}}\left(\mathbf{w}_{k}\right), \mathcal{A}^{\mathrm{PODP}-\mathrm{FULL}}\left(\mathbf{w}_{k}\right)\right)$ and $e_{2}\left(\mathcal{U}^{\mathrm{AC}}\left(\mathbf{w}_{k}\right), \mathcal{U}^{\mathrm{PODP}-\mathrm{FULL}}\left(\mathbf{w}_{k}\right)\right)$ are computed, and the result is shown in Figure 7.18. The maximum $e_{2}\left(\mathcal{A}_{\varepsilon}^{\mathrm{AC}}\left(\mathbf{w}_{k}\right), \mathcal{A}^{\mathrm{PODP}-\mathrm{FULL}}\left(\mathbf{w}_{k}\right)\right)$ is $13 \%$ for $f<50 \mathrm{~Hz}$ and is below $8 \%$ for $f>50 \mathrm{~Hz}$. Note that $e_{2}\left(\mathcal{U}^{\mathrm{AC}}\left(\mathbf{w}_{k}\right), \mathcal{U}^{\text {PODP-FULL }}\left(\mathbf{w}_{k}\right)\right)$ is smaller than $e_{2}\left(\mathcal{A}_{\varepsilon}^{\mathrm{AC}}\left(\mathbf{w}_{k}\right), \mathcal{A}^{\text {PODP-FULL }}\left(\mathbf{w}_{k}\right)\right)$, and that even though $e_{2}\left(\mathcal{A}_{\varepsilon}^{\mathrm{AC}}\left(\mathbf{w}_{k}\right), \mathcal{A}^{\mathrm{PODP}-\mathrm{FULL}}\left(\mathbf{w}_{k}\right)\right)$ is smaller at $f>1000 \mathrm{~Hz}$, this is not true for the case $e_{2}\left(\mathcal{U}^{\mathrm{AC}}\left(\mathbf{w}_{k}\right), \mathcal{U}^{\text {PODP-FULL }}\left(\mathbf{w}_{k}\right)\right)$ due to the effect of the peaks in the resonant region. Furthermore, the accuracy can be improved if desired by increasing $N_{s}$. To show this, consideration is restricted to the case $\gamma^{O V C}=0.35 \gamma_{\text {ref }}^{O V C}$ and, in Figure $7.19 e_{2}\left(\mathcal{A}_{\varepsilon}^{\mathrm{AC}}\left(\mathbf{w}_{k}\right), \mathcal{A}^{\text {PODP-FULL }}\left(\mathbf{w}_{k}\right)\right)$ and $e_{2}\left(\mathcal{U}^{\mathrm{AC}}\left(\mathbf{w}_{k}\right), \mathcal{U}^{\text {PODP-FULL }}\left(\mathbf{w}_{k}\right)\right)$ are shown for different numbers of sampled conductivities, $N_{\gamma_{s}}=3,5,7$, where it can be observed that as expected the error decreases as $N_{\gamma_{s}}$ increases. Note that the error could also be reduced by increasing $N_{\omega_{s}}$. Recall however, that even for the case $N_{\gamma_{s}}=3$ and $N_{\omega_{s}}=45$ an excellent agreement with the full order solution is obtained.

As a further assessment of the accuracy, $e_{P_{\Omega_{C, n}}^{0}}^{P O D P-F U L L}\left(\mathbf{w}_{i}\right)$ and $e_{E_{\Omega_{C, n}}^{k}}^{P O D P-F U L L}\left(\mathbf{w}_{i}\right)$ were computed, and the result is shown in Figure 7.20, where it can be observed that the maximum error for both the dissipated power and kinetic energy is of order $10^{-2}$.

The computational speed-up obtained with this method is also assessed comparing the time taken by the full order solver for different $N_{o}=N_{\gamma_{o}} N_{\omega_{o}}$ with the time taken by PODP-Full for different $N_{o}$ and $N_{s}=N_{\gamma_{s}} N_{\omega_{s}}$, with $\mathbf{W}_{s}$ and $\mathbf{w}_{k}, k=1, \ldots N_{o}$, appropriately redefined. Note that both the off-line (Algorithm 2) and on-line (Algorithm 6) stages are taken into account to compute the total ROM time. The result is shown in Figure 


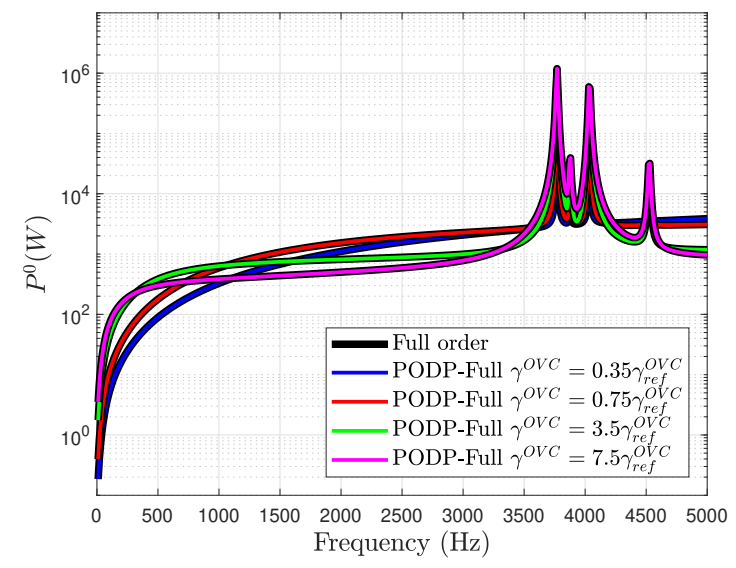

(a) $P_{\Omega_{O V C}}^{0}$

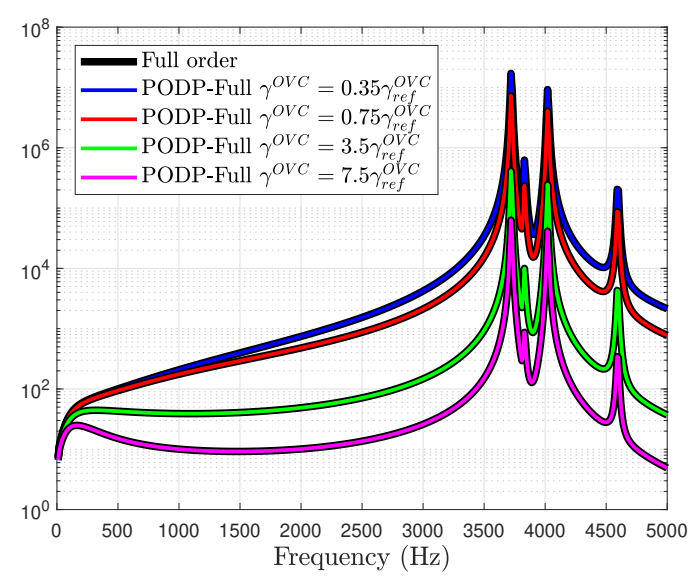

(c) $P_{\Omega_{77 K}}^{0}$

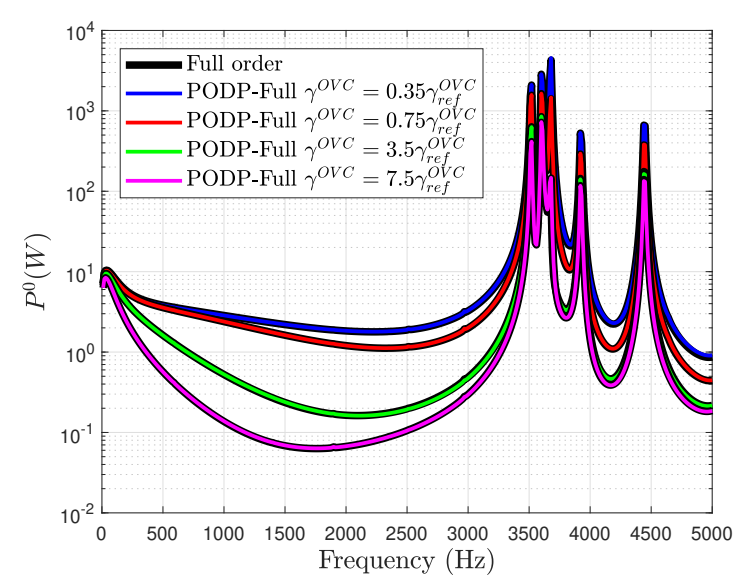

(e) $P_{\Omega_{4 K}}^{0}$

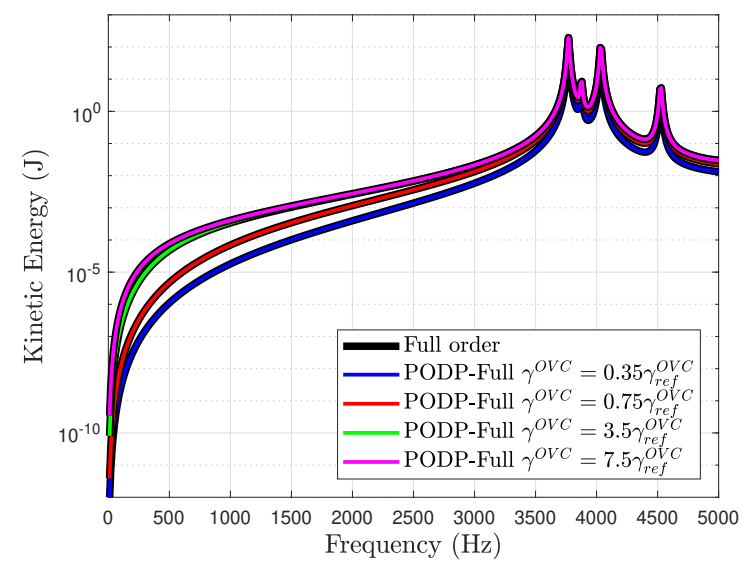

(b) $E_{\Omega_{O V C}}^{k}$

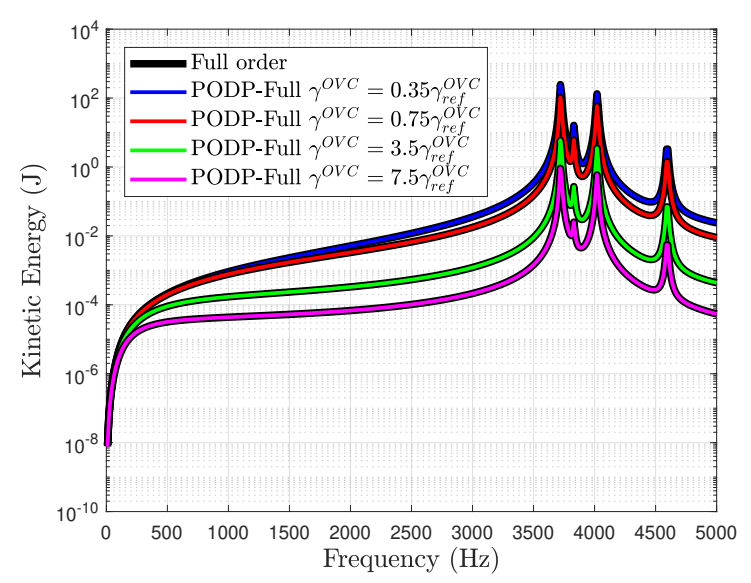

(d) $E_{\Omega_{77 K}}^{k}$

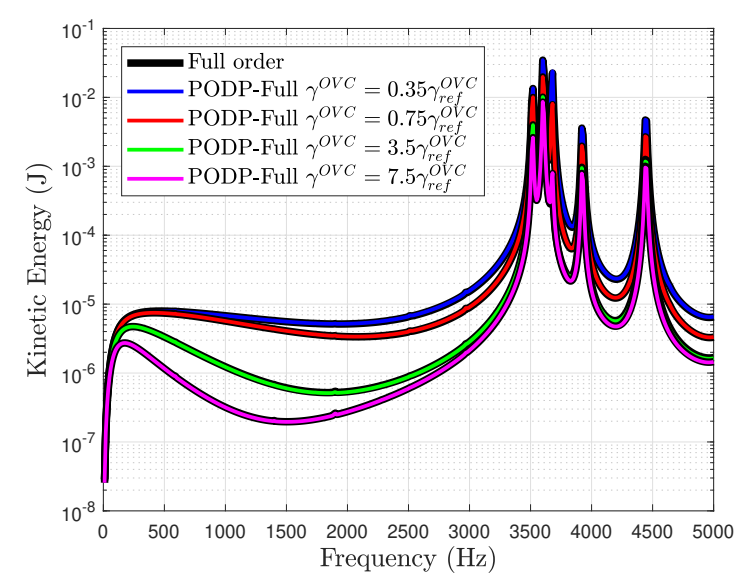

(f) $E_{\Omega_{4 K}}^{k}$

Figure 7.17: PODP-Full applied to test magnet problem with multiple parameters: Dissipated power and kinetic energy in the radiation shields for different values of $\gamma^{O V C}$. Comparison with full order solution. 


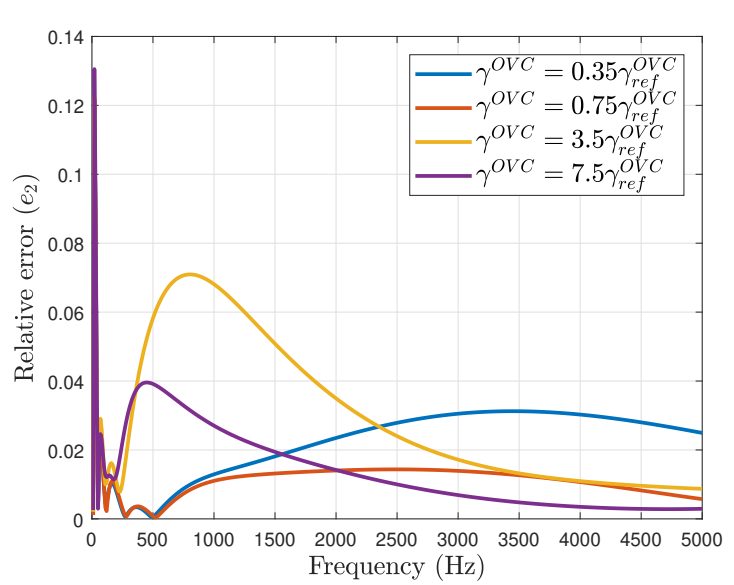

(a) $e_{2}\left(\mathcal{A}_{\varepsilon}^{\mathrm{AC}}\left(\mathbf{w}_{k}\right), \mathcal{A}^{\mathrm{PODP}-\mathrm{FULL}}\left(\mathbf{w}_{k}\right)\right)$

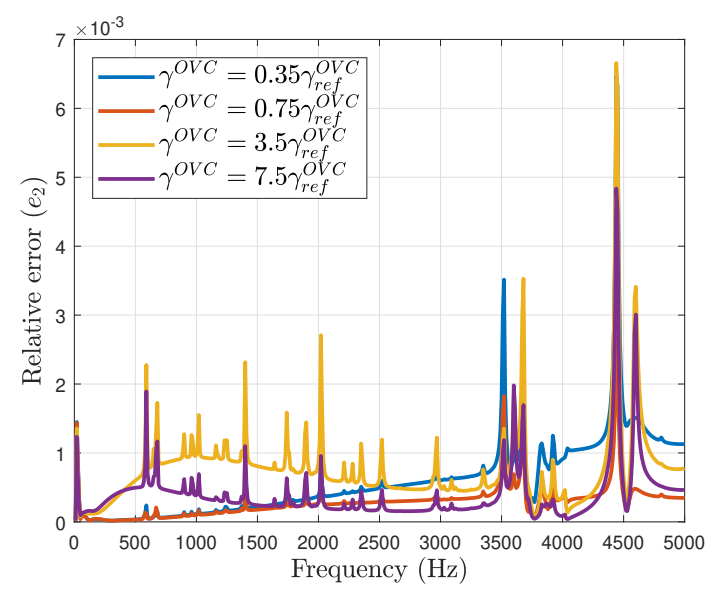

(b) $e_{2}\left(\mathcal{U}^{\mathrm{AC}}\left(\mathbf{w}_{k}\right), \mathcal{U}^{\mathrm{PODP}-\mathrm{FULL}}\left(\mathbf{w}_{k}\right)\right)$

Figure 7.18: PODP-Full applied to test magnet problem with multiple parameters: (a) $e_{2}\left(\mathcal{A}_{\varepsilon}^{\mathrm{AC}}\left(\mathbf{w}_{k}\right), \mathcal{A}^{\mathrm{PODP}-\mathrm{FULL}}\left(\mathbf{w}_{k}\right)\right)$ and $(\mathrm{b}) e_{2}\left(\mathcal{U}^{\mathrm{AC}}\left(\mathbf{w}_{k}\right), \mathcal{U}^{\mathrm{PODP}-\mathrm{FULL}}\left(\mathbf{w}_{k}\right)\right)$.

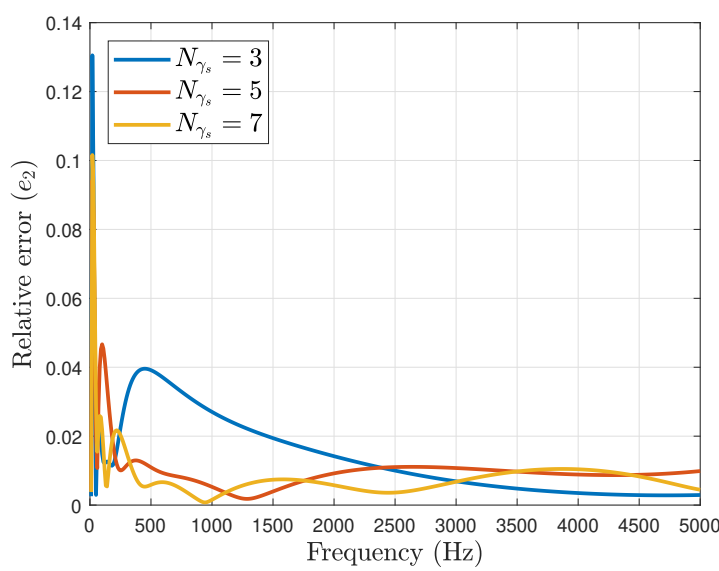

(a) $e_{2}\left(\mathcal{A}_{\varepsilon}^{\mathrm{AC}}\left(\mathbf{w}_{k}\right), \mathcal{A}^{\mathrm{PODP}-\mathrm{FULL}}\left(\mathbf{w}_{k}\right)\right)$

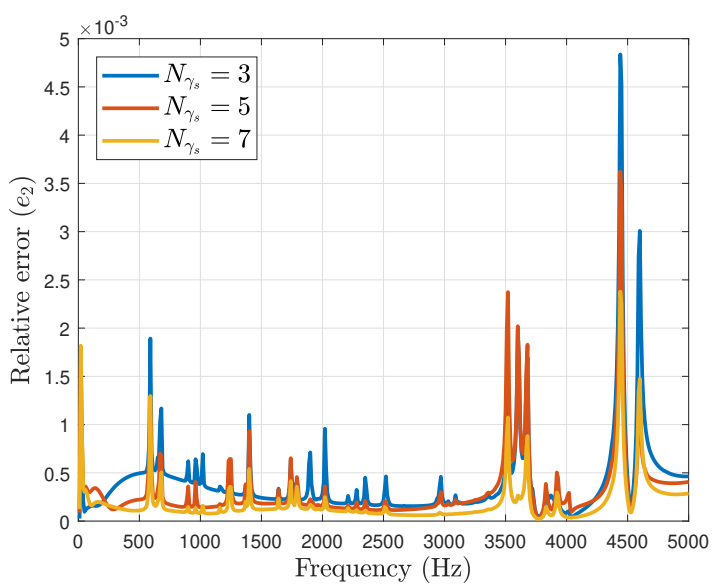

(b) $e_{2}\left(\mathcal{U}^{\mathrm{AC}}\left(\mathbf{w}_{k}\right), \mathcal{U}^{\mathrm{PODP}-\mathrm{FULL}}\left(\mathbf{w}_{k}\right)\right)$

Figure 7.19: PODP-Full applied to test magnet problem with multiple parameters:

(a) $e_{2}\left(\mathcal{A}_{\varepsilon}^{\mathrm{AC}}\left(\mathbf{w}_{k}\right), \mathcal{A}^{\mathrm{PODP}-\mathrm{FULL}}\left(\mathbf{w}_{k}\right)\right)$ and (b) $e_{2}\left(\mathcal{U}^{\mathrm{AC}}\left(\mathbf{w}_{k}\right), \mathcal{U}^{\text {PODP-FULL }}\left(\mathbf{w}_{k}\right)\right)$ for $\gamma^{O V C}=$ $0.35 \gamma_{\text {ref }}^{O V C}$ and different $N_{\gamma_{s}}=3,5,7$. 


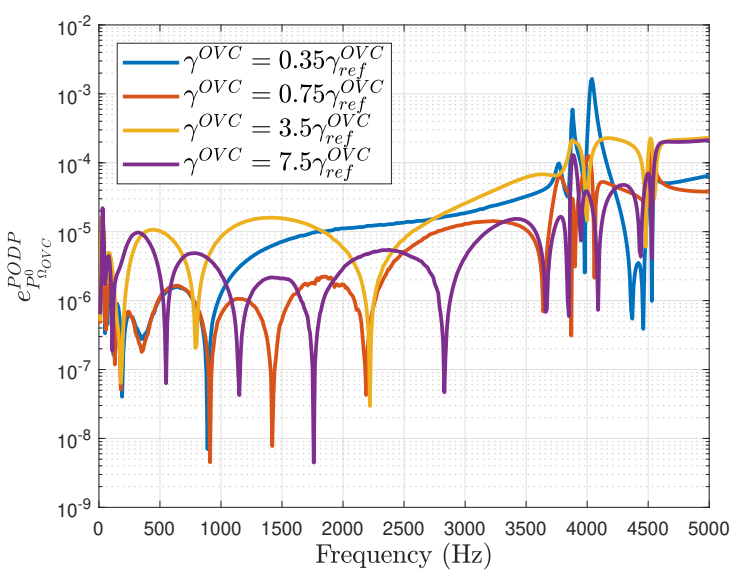

(a) $e_{P_{\Omega_{O V C}}^{\mathrm{O} O D P}-F U L L}$

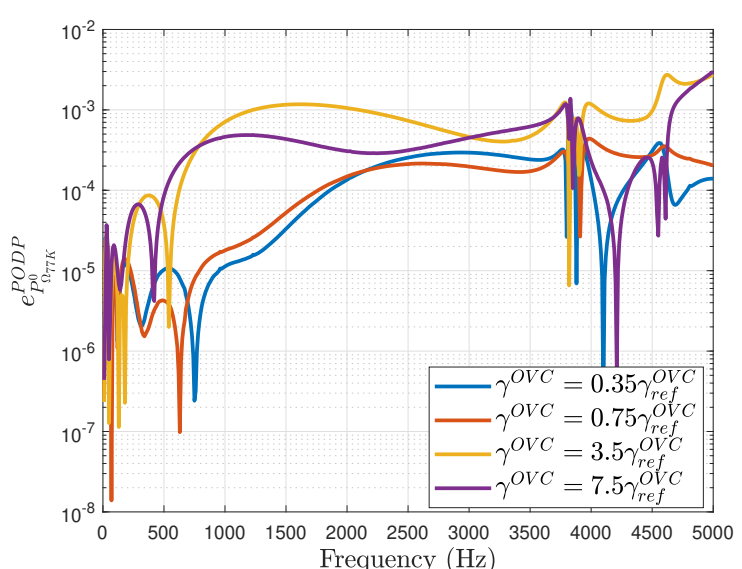

(c) $e_{P_{\Omega_{77 K}}^{O}}^{P O D P-F U L L}$

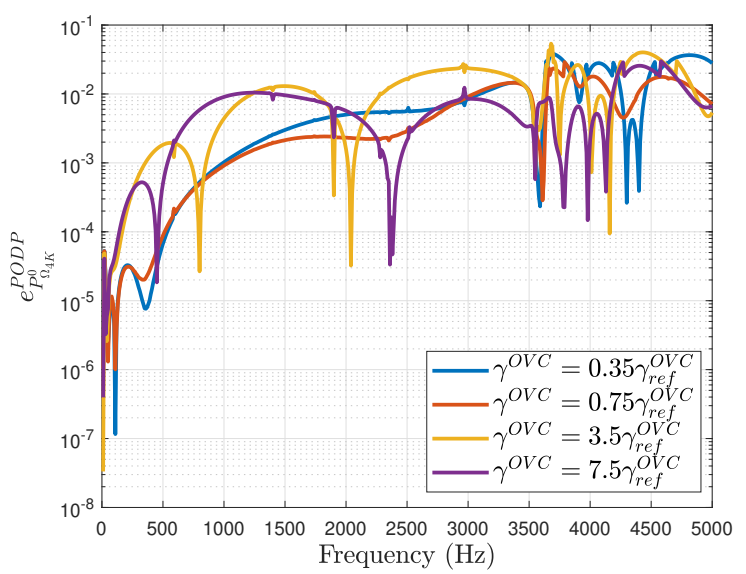

(e) $e_{P_{\Omega_{H K}}^{0}}^{P O D P-F U L L}$

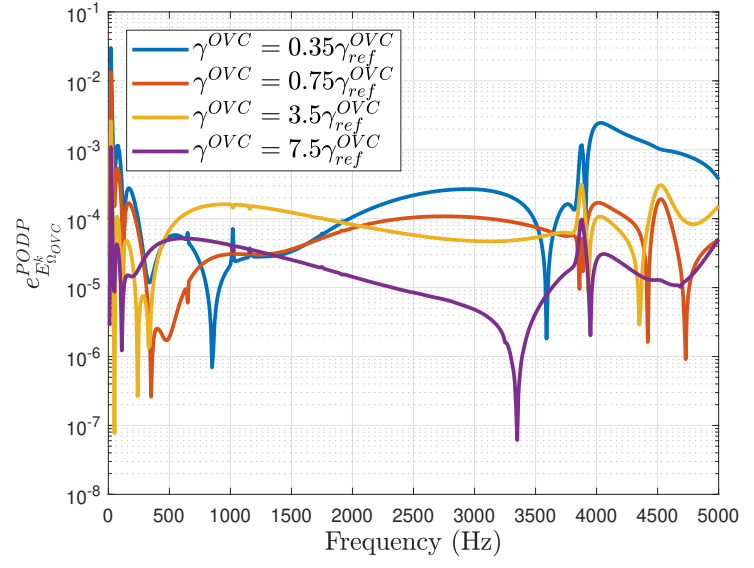

(b) $e_{E_{\Omega_{O V C}}^{k}}^{P O P-F U L L}$

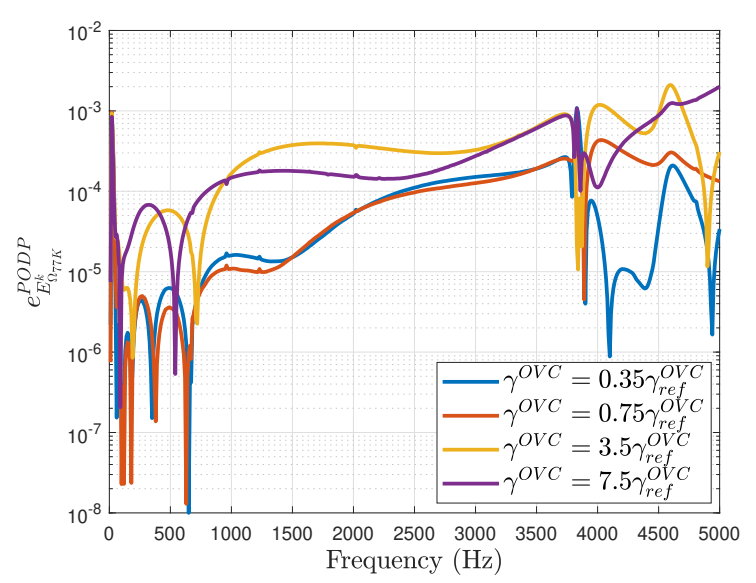

(d) $e_{E_{\Omega_{77 K}}^{k}}^{P O D P-F U L L}$

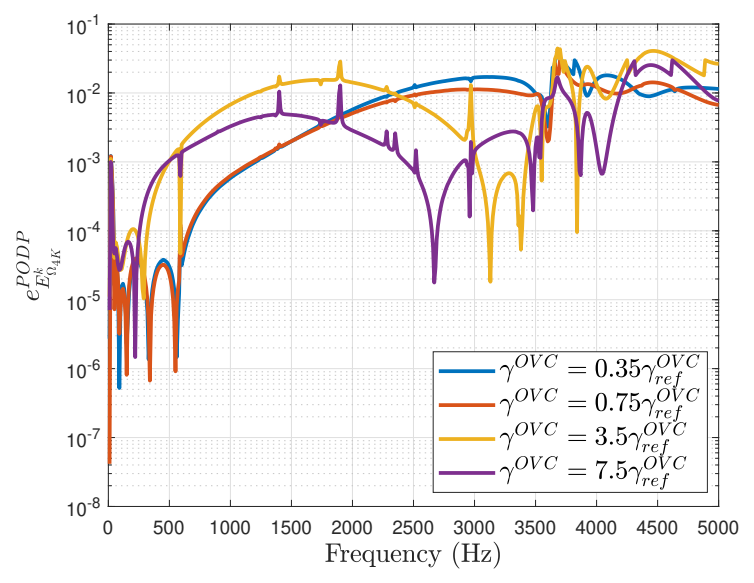

(f) $e_{E_{\Omega_{4 K}}^{R}}^{P O D P-F U L L}$

Figure 7.20: PODP-Full applied to test magnet problem with multiple parameters: $e_{P_{\Omega_{C, n}}^{P O D P-F U L L}}^{O D}$ and $e_{E_{\Omega_{C, n}}^{R O D P-F U L L}}^{P}$ different values of $\gamma^{O V C}$. Comparison with full order solution. 


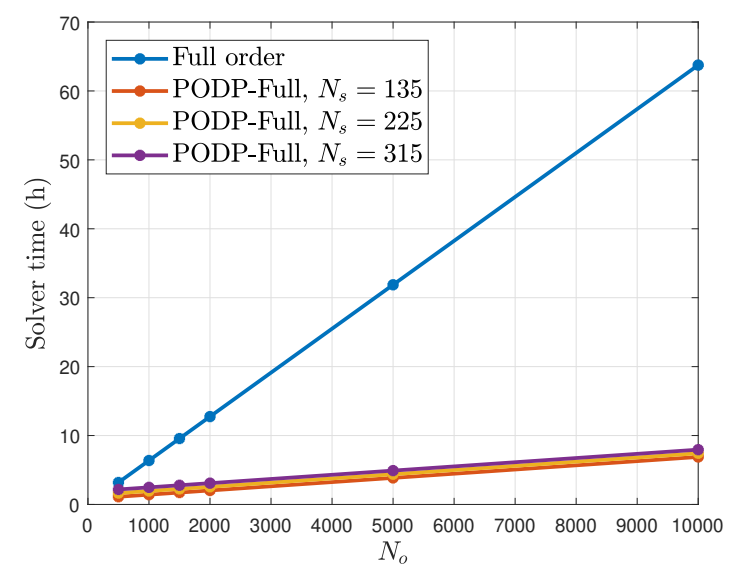

(a) Solver Time

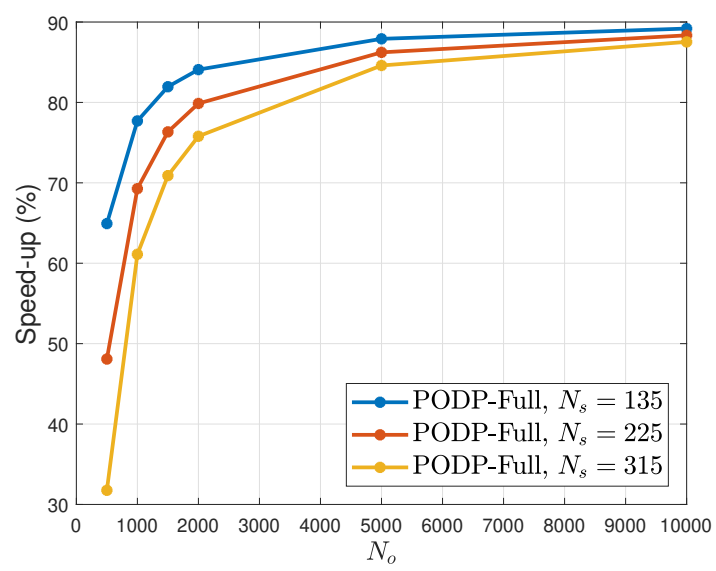

(b) Speed-up

Figure 7.21: PODP-Full applied to test magnet problem with multiple parameters: (a) Solver time and (b) speed-up for different values of $N_{s}$ and $N_{o}$.

7.21, where it can be observed that a massive speed-up of up to $89 \%$ can be obtained ${ }^{1}$. As an example, consider the case $N_{\gamma_{s}}=3, N_{\omega_{s}}=45, N_{\omega_{o}}=500$ and $N_{\gamma_{o}}=10$, this is, it is desired to compute a frequency sweep using a step $\Delta \mathrm{f}=10 \mathrm{~Hz}$ for 10 different conductivities. The time required by the full order solver would be $31.8 \mathrm{~h}$, while using the ROM, this reduces to only $3.8 \mathrm{~h}$ and of course the time saving increases as either $N_{\gamma_{o}}$ or $N_{\omega_{o}}$ increase.

Furthermore, in order to further assess the accuracy of the ROM, Figure 7.22 shows contours of the eddy currents $\boldsymbol{g}_{h p q}^{o}(\boldsymbol{x}, \mathbf{w})=\gamma \boldsymbol{E}_{h p q}^{\mathrm{AC}}(\boldsymbol{x}, \mathbf{w})=\gamma\left(-\mathrm{i} \omega \boldsymbol{A}_{\varepsilon, h q}^{A C}(\boldsymbol{x}, \mathbf{w})+\right.$ $\left.\mathrm{i} \omega \boldsymbol{B}_{0, h q}^{D C} \times \boldsymbol{U}_{h p}^{A C}(\boldsymbol{x}, \mathbf{w})\right)$ and its approximation $\boldsymbol{g}^{P O D P-F U L L}(\boldsymbol{x}, \mathbf{w})$ in the deformed $77 \mathrm{~K}$ shield for the case where $\mathbf{w}=\left(2000 \pi, 0.35 \gamma_{\text {ref }}^{O V C}\right)$. It can be observed that an excellent agreement is obtained. Furthermore, a contour plot of $\left|\operatorname{Re}\left(\boldsymbol{U}_{h p}^{\mathrm{AC}}(\boldsymbol{x}, \mathbf{w})\right)\right|$ and $\left|\operatorname{Re}\left(\boldsymbol{u}^{P O D P-F U L L}(\boldsymbol{x}, \mathbf{w})\right)\right|$ in the $4 \mathrm{~K}$ shield for the same parameters is shown in Figure 7.23 , where again an excellent agreement is observed.

\subsection{Modified Magnet Problem with Multiple Parameters}

The application of the PODP-Full methodology to the modified magnet problem with $x$ (transversal) gradient coils presented in Section 5.3.4 is now considered. The problem geometry is illustrated again in Figure 7.24 as well as the boundary conditions used in this case. On the Dirichlet part of the boundary, $\partial \Omega_{C}^{D}$ the condition $\boldsymbol{u}_{h p}^{A C}=\mathbf{0}$ was imposed. A mesh of 54796 elements (the same as in Section 5.3.4) and polynomial orders $p=3$ and $q=4$ were considered for the finite element discretisation.

In this case, the frequency and the conductivity of the $77 \mathrm{~K}$ shield, $\gamma^{77 K}$, are considered as parameters, this is, $\boldsymbol{w}=\left(\omega, \gamma^{77 K}\right)$. The conductivity $\gamma^{77 K}$ is assumed to vary in

\footnotetext{
${ }^{1}$ Note that in the case of computational time for the full order solver only the first 4 points were measured, and the last two were extrapolated.
} 


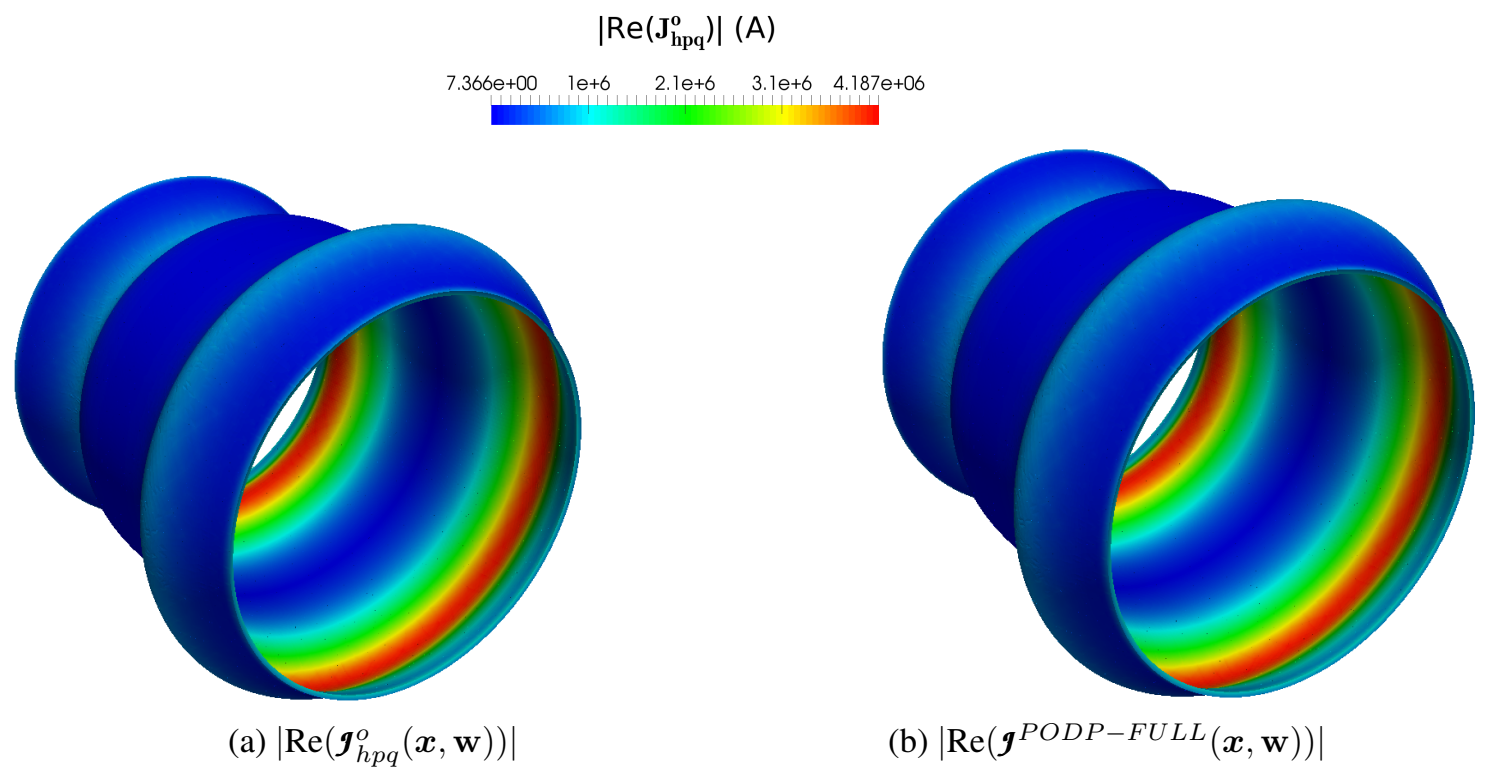

Figure 7.22: PODP-Full applied to test magnet problem with multiple parameters: Contours of (a) $\left|\operatorname{Re}\left(\boldsymbol{J}_{h p q}^{o}(\boldsymbol{x}, \mathbf{w})\right)\right|$ and (b) $\left|\operatorname{Re}\left(\boldsymbol{g}^{P O D P-F U L L}(\boldsymbol{x}, \mathbf{w})\right)\right|$ in the $77 \mathrm{~K}$ shield for $\mathbf{w}=\left(2000 \pi, 0.35 \gamma_{\text {ref }}^{O V C}\right)$ compared with the full order solution. Deformation exaggerated by a factor of $10^{4}$.

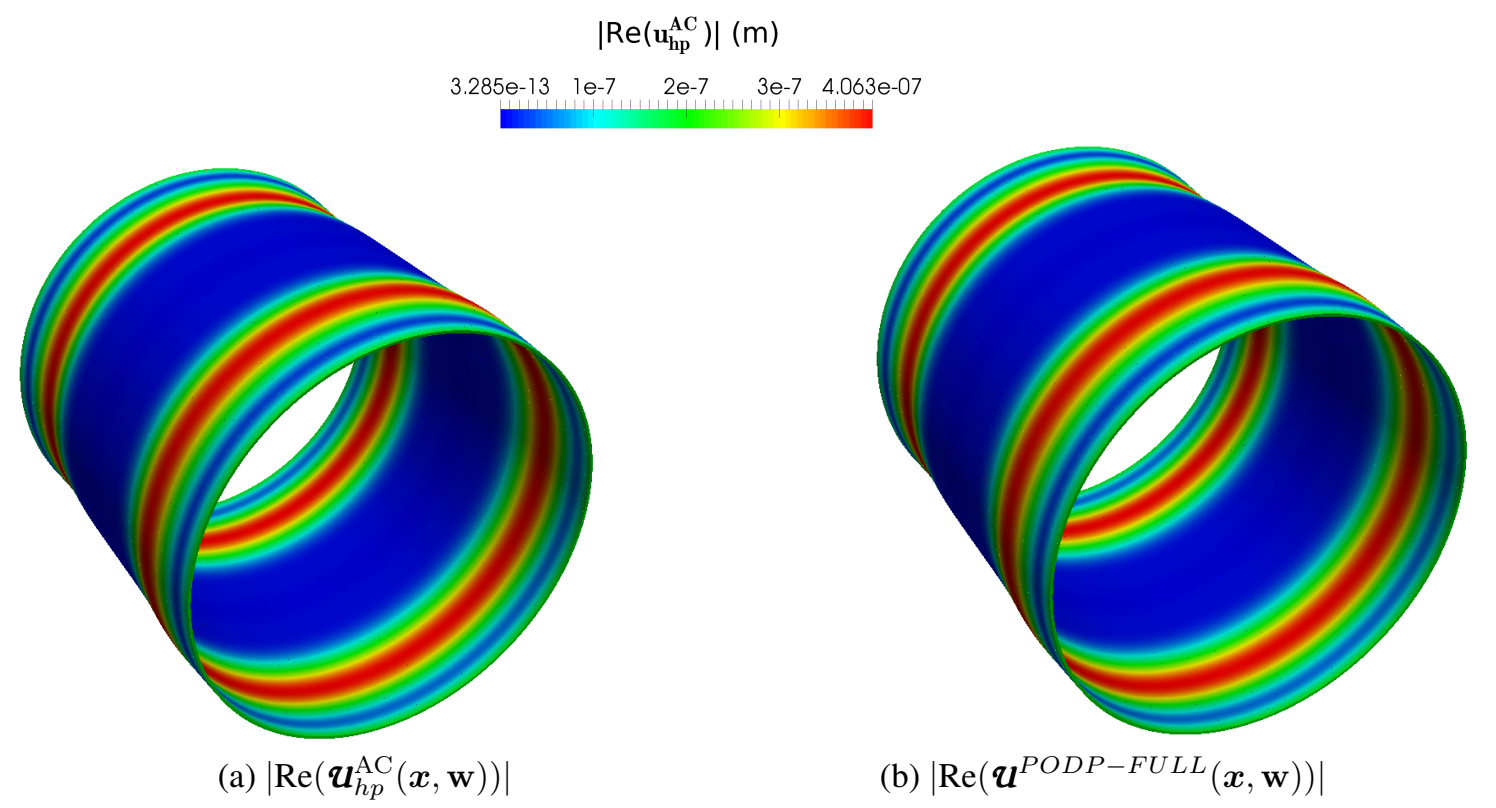

Figure 7.23: PODP-Full applied to test magnet problem with multiple parameters: Contours of (a) $\left|\operatorname{Re}\left(\boldsymbol{u}_{h p}^{\mathrm{AC}}(\boldsymbol{x}, \mathbf{w})\right)\right|$ and (b) $\left|\operatorname{Re}\left(\boldsymbol{u}^{P O D P-F U L L}(\boldsymbol{x}, \mathbf{w})\right)\right|$ in the $4 \mathrm{~K}$ shield for $\mathbf{w}=\left(2000 \pi, 0.35 \gamma_{\text {ref }}^{O V C}\right)$. 


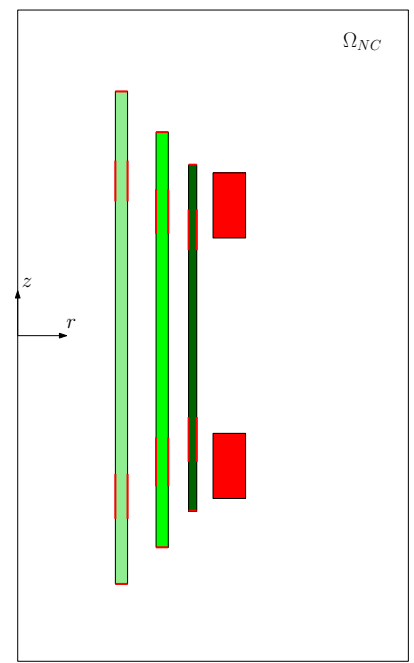

(a) Illustration of $\partial \Omega_{C}^{D}$

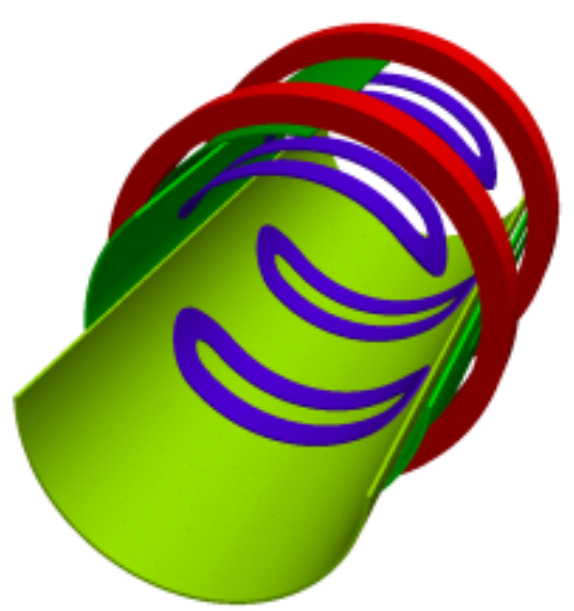

(b) 3D geometry

Figure 7.24: Modified magnet problem with $x$ (transversal) gradient coil: (a) Illustration of the chosen $\partial \Omega_{C}^{D}$, highlighted in red and (b) illustration of the 3D geometry.

the range $0.4 \gamma_{\text {ref }}^{77 K} \leqslant \gamma^{77 K} \leqslant 2.5 \gamma_{\text {ref }}^{77 K}$, being $\gamma_{\text {ref }}^{77 K}$ the reference value used for the test magnet problem. The number of sampled conductivities was set to $N_{\gamma_{s}}=3$ and, for each conductivity, the number of sampled frequencies is set to $N_{\omega_{s}}=45$, which results in a total number of samples $N_{s}=135$. This means that $\mathbf{W}_{\mathrm{s}}$ has the elements $\mathbf{w}_{k}=$ $\left(2 \pi \mathrm{f}_{i}, \gamma_{j}^{77 K}\right), k=i+(j-1) N_{\omega_{s}}, i=1, \ldots, N_{\omega_{s}}, j=1, \ldots, N_{\gamma_{s}}$ where $\mathrm{f}_{i}$ is defined by (7.5) with $\left(\Delta \mathrm{f}_{1}, \Delta \mathrm{f}_{2}\right)=(40,200) \mathrm{Hz}$ and $\gamma_{j}^{77 K} \in(0.4,1,2.5) \gamma_{r e f}^{77 K}$. Algorithm 2 is then applied by clling $\operatorname{OfFLine}\left(\mathbf{A}_{\mathcal{A A}}(\boldsymbol{w}), \mathbf{R}_{\mathcal{A}}(\boldsymbol{w}), \mathbf{W}_{\mathrm{s}}, M\right)$ where the TSVD was truncated with $M=20$ (leading to an upper bound for the truncation error $e_{1}=6.8 \times 10^{-3}$ ).

\subsubsection{PODP-Full}

Algorithm 6 is now applied by calling ONLINEPODP$\operatorname{FULL}\left(\mathbf{H}_{\mathcal{A}}^{M}, \mathbf{A}\left(\mathbf{w}_{k}\right), \mathbf{R}\left(\mathbf{w}_{k}\right), \mathbf{N}, \mathbf{L}, \boldsymbol{x}, \mathbf{w}_{k}\right)$ where $\mathbf{w}_{k}=\left(2 \pi \mathbf{f}_{i}, \gamma_{j}^{77 K}\right), k=i+(j-1) N_{\omega_{o}}$, $i=1, \ldots, N_{\omega_{o}}, j=1, \ldots, N_{\gamma_{o}}, \mathrm{f}_{i}=10+\Delta \mathrm{f}(i-1), \Delta \mathrm{f}=10 \mathrm{~Hz}, N_{\omega_{o}}=500$, $\gamma_{j}^{77 K} \in(0.5,0.75,1.5,2) \gamma_{\text {ref }}^{77 K}$ and $N_{\gamma_{o}}=4$.

A comparison of $P_{\Omega_{C, n}}^{0}\left(\mathbf{w}_{k}, \mathcal{A}^{P O D P-F U L L}, \boldsymbol{B}_{0, h q}^{D C}, \boldsymbol{u}^{P O D P-F U L L}\right) \quad$ with $P_{\Omega_{C, n}}^{0}\left(\mathbf{w}_{k}, \mathcal{A}_{\varepsilon, h q}^{A C}, \boldsymbol{B}_{0, h q}^{D C}, \boldsymbol{u}_{h p}^{A C}\right) \quad$ and $E_{\Omega_{C, n}}^{k}\left(\mathbf{w}_{k}, \boldsymbol{u}^{P O D P-F U L L}\right) \quad$ with $E_{\Omega_{C, n}}^{k}\left(\mathbf{w}_{k}, \boldsymbol{u}_{h p}^{A C}\right)$ is shown in Figure 7.25. It can be observed that an excellent agreement with the full order solution is obtained. Note that the biggest variations in the dissipated power and kinetic energy for varying $\gamma^{77 K}$ are observed in the $4 \mathrm{~K}$ shield. This is due to the variation in shielding effect of the $77 \mathrm{~K}$ shield with a changing conductivity. Or in other words, when the conductivity of the $77 \mathrm{~K}$ shield increases its skin depth reduces, thus the AC magnetic field at the $4 \mathrm{~K}$ shield decreases.

The solver time was again measured and compared with the full order solver in order to study the efficiency of the method. The result is shown in Figure 7.26. It can be observed that the speed-up is in this case even higher than for the test magnet problem with $z$ 


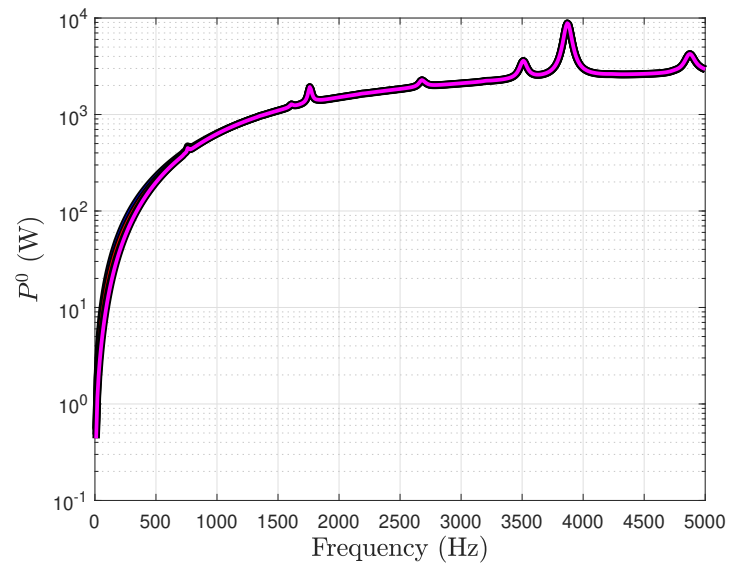

(a) $P_{\Omega_{O V C}}^{0}$

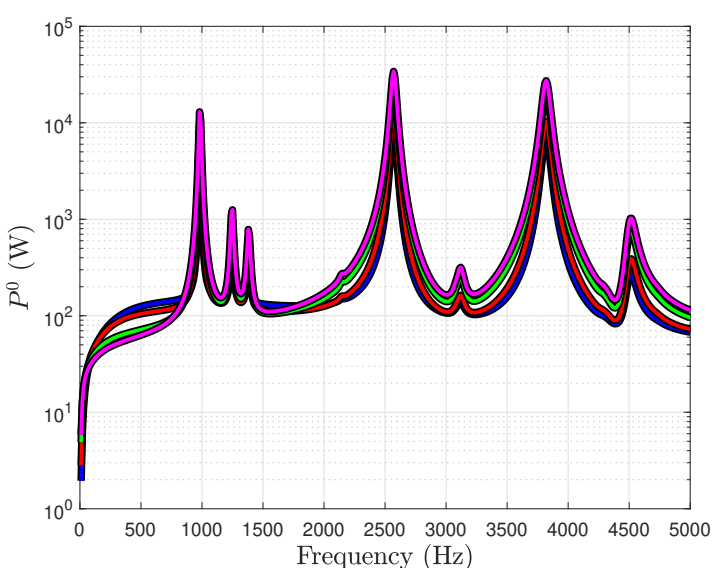

(c) $P_{\Omega_{77 K}}^{0}$

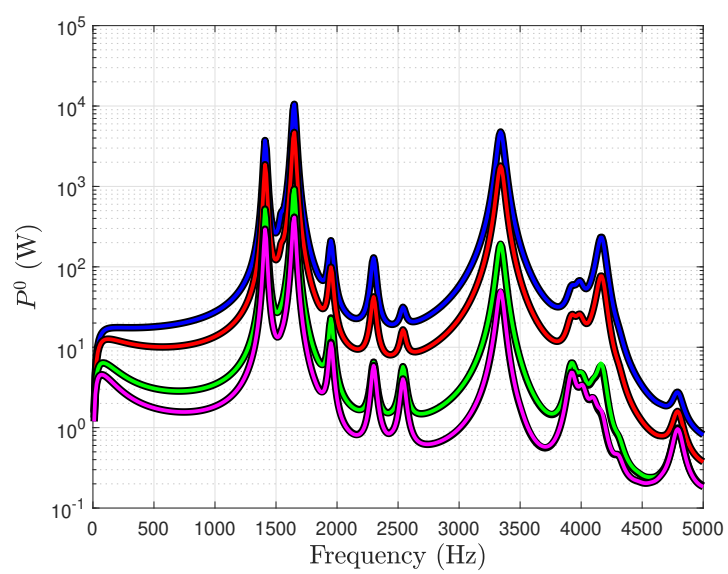

(e) $P_{\Omega_{4 K}}^{0}$

Full order

PODP-Full $\gamma^{77 K}=0.5 \gamma_{\text {rf }}^{77 K}$ PODP-Full $\gamma^{77 K}=0.75 \gamma_{r e f}^{77 K}$

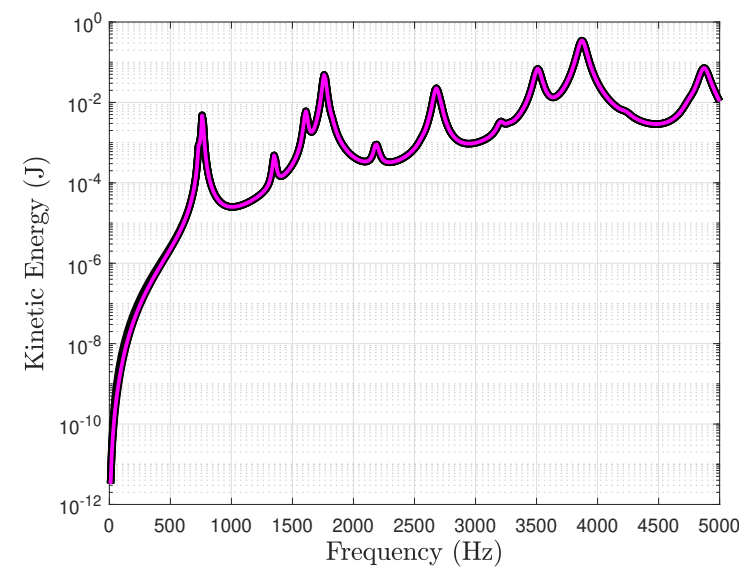

(b) $E_{\Omega_{O V C}}^{k}$

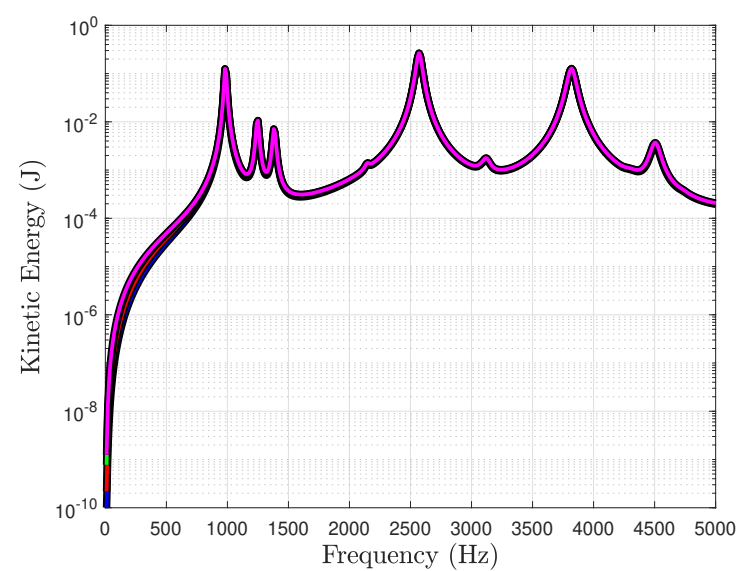

(d) $E_{\Omega_{77 K}}^{k}$

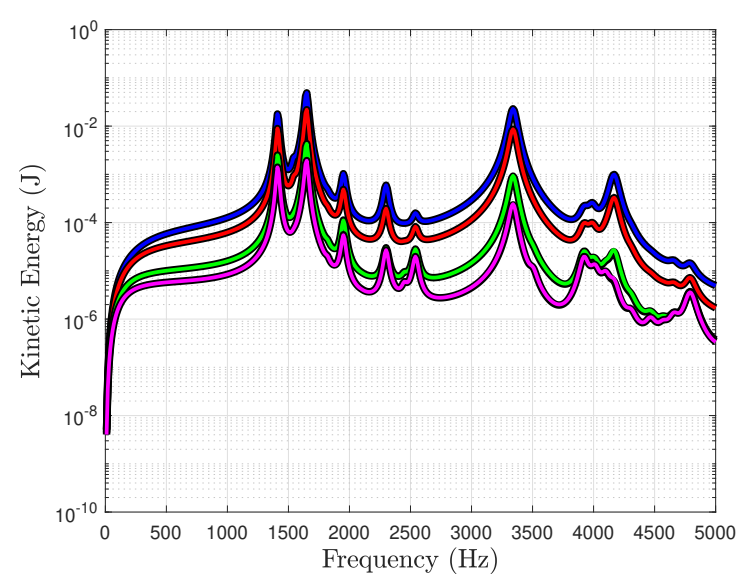

(f) $E_{\Omega_{4 K}}^{k}$

PODP-Full $\gamma^{77 K}=1.5 \gamma_{r e f}^{77 K}$ PODP-Full $\gamma^{77 K}=2 \gamma_{r e f}^{77 K}$

Figure 7.25: PODP-Full applied to modified magnet problem with multiple parameters: Dissipated power and kinetic energy in the radiation shields for different values of $\gamma^{77 K}$. Comparison with full order solution. 


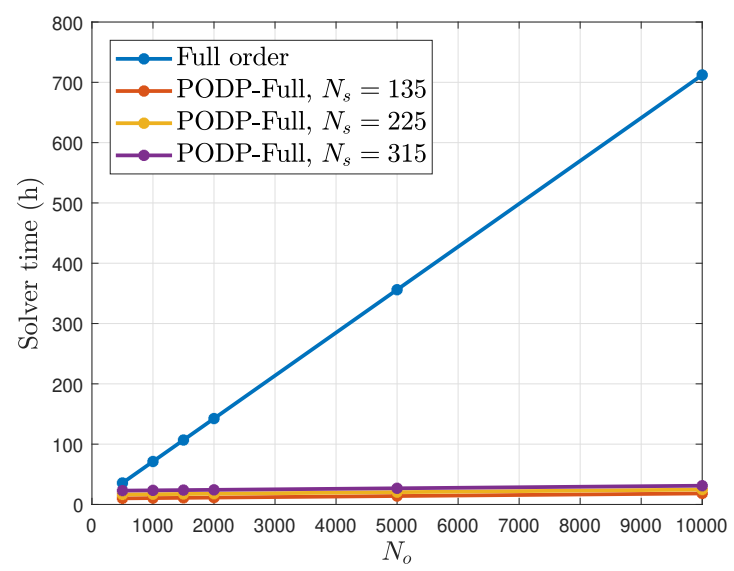

(a) Solver Time

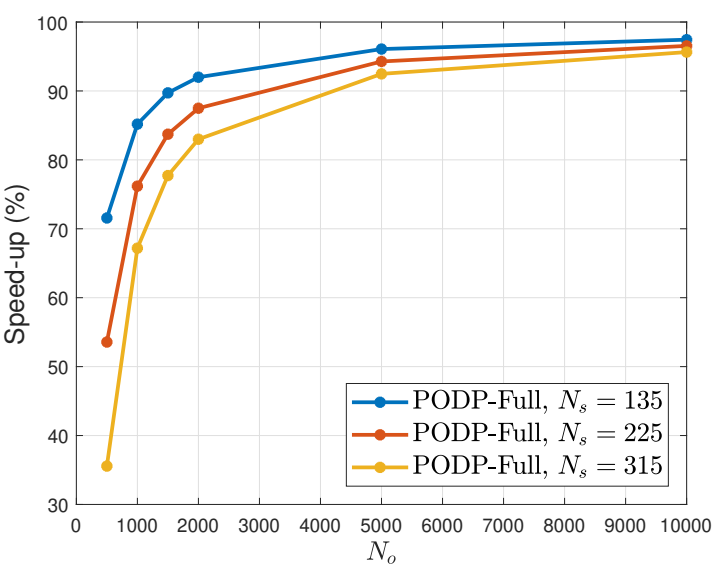

(b) Speed-up

Figure 7.26: PODP-Full applied to modified magnet problem with multiple parameters: (a) Solver time and (b) speed-up for different values of $N_{s}$ and $N_{o}$.

gradient coil and it grows to values above $95 \%{ }^{2}$. This is due to the increased complexity of the electromagnetic problem which results in the need to increase the polynomial order of the $\boldsymbol{H}$ (curl) basis functions to $q=4$ in order to reach a converged solution. On the other hand, the order of the $H^{1}$ basis functions can be kept as $p=3$ which is the same used for the test magnet problem.

Finally, in order to further assess the accuracy of the method, a contour plot of $\left|\operatorname{Re}\left(\boldsymbol{g}_{h p q}^{o}(\boldsymbol{x}, \mathbf{w})\right)\right|$ and $\left|\operatorname{Re}\left(\boldsymbol{g}^{P O D P-F U L L}(\boldsymbol{x}, \mathbf{w})\right)\right|$ in the deformed $4 \mathrm{~K}$ shield for $\mathbf{w}=$ $\left(8000 \pi, 0.5 \gamma_{r e f}^{77 K}\right)$ is presented in Figure 7.27. It can be observed that an excellent agreement is obtained.

\subsection{Chapter Summary}

In these Chapter, the application of POD to different challenging MRI configurations has been considered.

The main novelties of this Chapter are as follows; first, it was shown that the application of POD to the mechanical problem requires the computation of a large number of snapshots in order to give accurate approximations and, thus, no computational speed-up with respect to the full order solver can be obtained with this method. Second, it was shown that both PODI-Full and PODP-Full lead to accurate approximations, but PODP-Full offers an increased accuracy compared to PODI-Full for no significant increase in computational cost. Third, the accuracy and efficiency of the PODP-Full approach was demonstrated by applying it to different MRI configurations and considering different parameters of interest.

To illustrate these novelties, POD was first applied to the test magnet problem with $z$

\footnotetext{
${ }^{2}$ Note again that for the time of the full order solver only the first 4 points of the curve were measured and the last two were extrapolated.
} 


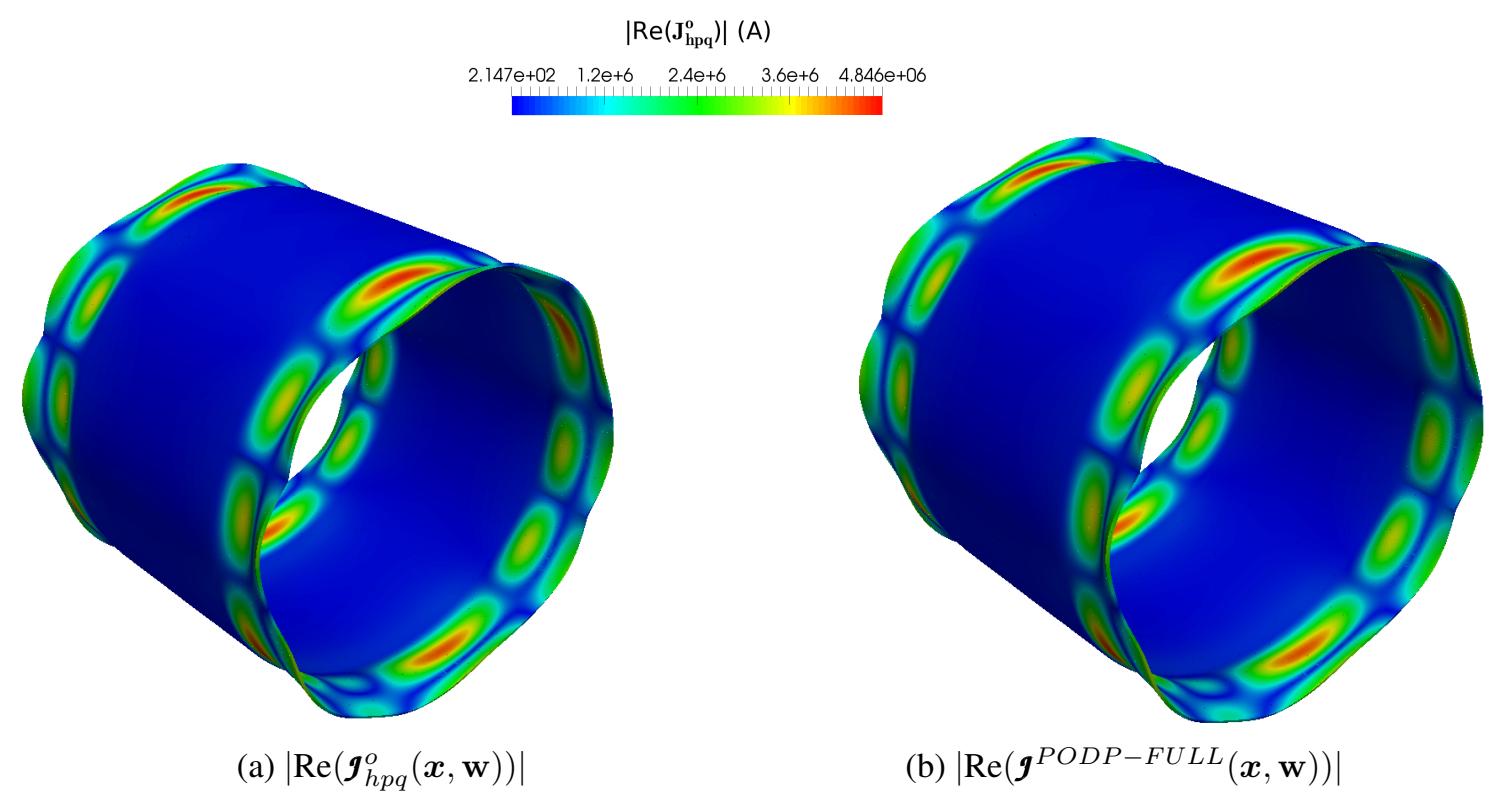

Figure 7.27: PODP-Full applied to modified magnet problem with multiple parameters: Contours of (a) $\left|\operatorname{Re}\left(\boldsymbol{g}_{h p q}^{o}(\boldsymbol{x}, \mathbf{w})\right)\right|$ and (b) $\left|\operatorname{Re}\left(\boldsymbol{g}^{P O D P-F U L L}(\boldsymbol{x}, \mathbf{w})\right)\right|$ in the deformed $4 \mathrm{~K}$ shield for $\mathbf{w}=\left(8000 \pi, 0.5 \gamma_{\text {ref }}^{77 K}\right)$. Deformation exaggerated by a factor of $10^{4}$.

gradient coils considering only the frequency as a parameter. For this problem it was shown that both PODI and PODP require a large number of snapshots in order to accurately approximate the mechanical solution and therefore no computational gaining with respect to the full order solver can be obtained by applying these methods to the mechanical problem. Thus, the application of the PODI-Full and PODP-Full methodologies was considered, and it was shown that in this case accurate results are obtained even with a very small number of snapshots. Furthermore, the speed-up obtained by PODI-Full and PODP-Full was measured, and found to be almost identical for both approaches, while a higher accuracy was obtained through PODP. Then, the PODP-Full approach was applied to the same problem but considering both $\omega$ and $\gamma^{O V C}$ as parameters, and it was shown that accurate results were also obtained in this case. Furthermore, the speed-up was also measured, resulting in time savings of up to $90 \%$. Finally, the PODP-Full approach was applied to the modified magnet problem with $x$ gradient coils, and the accuracy and efficiency of the method were again assessed, resulting in this case in time savings higher than $95 \%$. 


\section{Part IV}

\section{Conclusions}




\section{Chapter 8}

\section{Conclusions and Future Work}

\subsection{Concluding Remarks}

This thesis has presented a new accurate and efficient methodology for the numerical simulation of magneto-mechanical coupling in MRI scanners. First, a high fidelity software for the simulation of the coupled problem was presented, which is based in the accurate description of the problem using a Lagrangian formulation and its discretisation using high order finite elements. The accuracy and efficiency of the high fidelity software was assessed by applying it to challenging academic and industrially relevant problems. Then, a POD based ROM was developed with the objective of making the design process of new MRI configurations more efficient by allowing the rapid solution of the coupled problem for varying model parameters, such as frequency and conductivity. The accuracy and efficiency of the ROM was assessed by applying it to challenging MRI configurations and comparing with the high fidelity software, which showed that the ROM results in accurate solutions and in a speed-up higher than $95 \%$ with respect to the full order solver. Therefore, it can be concluded that the aim and objectives stated in Chapter 1 have been achieved. In the following a more detailed description of the conclusions that can be extracted from each of the chapters of the thesis is provided.

\subsubsection{Conclusions}

The complex physics associated with vibrations induced by electromagnetic fields in MRI applications have been accurately described. The complete 3D configuration was considered and a rigorous formulation for the coupled magneto-mechanical problem of interest was presented.

- In Chapter 2 a new formulation for the coupled magneto mechanical problem of interest was presented. This is based in a Lagrangian formulation and the assumption of small displacements. As opposed to the Eulerian formulation adopted in $[110,19,18,17]$, the new Lagrangian formulation does not assume small velocities and, therefore, it results in a more accurate description of the coupled problem. Furthermore, the Lagrangian formulation results in a transmission problem that can 
be solved in a staggered manner, as opposed to the Eulerian approach where the system had to be solved monolithically and, therefore, the Lagrangian approach is computationally much more efficient, especially for large three dimensional problems. This chapter addresses the objective "To accurately describe the complex physics governing the physical issues to be addressed in this work for MRI applications".

An accurate and efficient variational formulation for the 3D coupled magneto-mechanical problem in a Lagrangian frame was rigorously derived.

- In Chapter 3 the variational formulation of the problem was developed. For this, the problem was first linearised using the AC-DC splitting first presented in [19]. Then, the weak formulation of the DC and AC problem was rigorously derived, and regularisation was applied to the system in order to avoid having to explicitly impose the Coulomb gauge. This chapter addresses the objective "To develop a rigorous and efficient variational formulation for the complete coupled magnetomechanical problem in three dimensions".

An accurate and efficient high fidelity computational methodology for the solution of the $3 \mathrm{D}$ magneto-mechanical problem was developed, which is based in the application of high order $H^{1}$ and $\boldsymbol{H}$ (curl)-conforming finite elements.

- Chapter 4 presented the discretisation of the linearised and regularised DC and AC problems using high order finite elements. The sets of hierarchical $H^{1}$ and $\boldsymbol{H}$ (curl)conforming basis functions used for the discretisation of the mechanical and electromagnetic fields, respectively, were presented. The rigorous discretisation of the DC and AC systems was then developed and the mapping between reference and physical elements was briefly described. The discrete DC and AC systems were stated and an efficient algorithm for the solution of the full coupled problem was proposed, which includes the use of preconditioned iterative solvers when appropriate. A discussion on the use of preconditioned iterative solvers was presented, and the block Jacobi preconditioner used in this work was described. Then, the introduction of mechanical damping was discussed, followed by a discussion on the properties of the current source and the methodology to ensure that the current is solenoidal at a discrete level. The symmetry conditions used to reduce the cost of the 3D simulations were also presented. This chapter addresses the objective "To develop an accurate and efficient high fidelity computational methodology for the solution of the magneto-mechanical problem of interest in three dimensions".

The accuracy and efficiency of the proposed finite element methodology were demonstrated by applying it to challenging academic and industrially relevant problems, including different MRI configurations.

- In Chapter 5 a series of academic and industrially relevant problems were considered in order to assess the accuracy and efficiency of the proposed high order finite 
element methodology. First, a series of single physics or decoupled problems were considered in order to verify that the predicted rates of convergence of the solution under $h$ - and $p$-refinement are obtained. Then, a series of academic and industrially relevant problems were considered in order to demonstrate the accuracy and efficiency of the methodology, which were proven by performing comparisons against commercial software, the axisymmetric software developed in $[110,19,18,17]$ and experimental results. This chapter addresses the objective "To prove the accuracy and efficiency of the high fidelity computational methodology by applying it to challenging academic and industrially relevant problems".

A ROM which allows the rapid solution of the coupled magneto-mechanical problem for varying model parameters was presented. The ROM is based in POD and is of paramount importance for the optimisation of new MRI configurations.

- In Chapter 6 a new ROM for the fast solution of coupled magneto-mechanical problems in MRI scanners was presented, which is based in the POD. First, an introduction to POD was presented including a description of the off-line and on-line stages. In the latter, two options, namely PODI and PODP were considered. The methodology to apply PODI and PODP to the complete solutions as well as to each physics separately was then presented, followed by a novel reduced order-full order methodology that exploits the staggered nature of the system by applying POD to approximate the electromagnetic solution and using this approximation to feed the mechanical full order solver and compute the mechanical solution. An affine decomposition of the system in terms of frequency and conductivity of each shield was then presented, which is key for the efficiency of PODP. This chapter addresses the objective "To build a new ROM that allows the fast computation of solutions for varying model parameters in order to optimize the design process of new MRI configurations".

The accuracy and efficiency of the ROM were demonstrated by applying it to challenging MRI configurations and comparing against the high fidelity methodology.

- In Chapter 7 a series of industrially relevant MRI configurations were considered in order to assess the accuracy and efficiency of the proposed ROM. First, the application of PODI and PODP to the complete solution was considered which showed that the number of snapshots required to obtain accurate solutions is very large and, thus, no computational gaining could be obtained with this method. Next, PODI and PODP were applied to the electromagnetic and mechanical solutions separately, which showed that a large number of snapshots is required in order to accurately approximate the mechanical solution while the electromagnetic solution can be approximated with a reduced number of snapshots. The application of the PODI-Full and PODP-Full methodologies was then considered, which showed that they lead to accurate approximations of the solution for varying frequencies and conductivities. Furthermore, it was shown that PODP-Full results in an increased accuracy compared to PODI-Full for no significant increase in computational cost, and that 
a speed-up higher than $95 \%$ with respect to the full order solver can be obtained with the combined reduced order-full order approach. This result is of paramount importance for the industry as it enables the possibility of studying the performance of MRI configurations with different model parameters almost in real time during the design process. This chapter addresses the objective "To demonstrate the accuracy and efficiency of the ROM by applying it to challenging MRI configurations and performing comparisons against the high fidelity tool".

In summary, a consistent formulation for the description of 3D coupled magnetomechanical problems in MRI applications was presented, which is based in a Lagrangian frame and the assumption of small displacements. This formulation presents several advantages compared to the Eulerian formulation proposed in $[19,18,17]$ as it does not assume small velocities and accelerations and results in a efficient staggered scheme, which has very positive computational implications, critical for 3D simulations. Furthermore, the extension to the case of 3D problems allows the non-rotationally symmetric $x$ and $y$ gradient coils to be considered and, therefore, enable the numerical simulation of general 3D designs. A rigorous variational formulation for the coupled magneto-mechanical problem in a time-harmonic setting was then developed, which involved the linearisation of the coupled problem by means of an AC-DC splitting of the fields and the application of regularisation in order to circumvent the Coulomb gauge. Then, the problem was discretised using high order hierarchical $H^{1}$ and $\boldsymbol{H}$ (curl)-conforming finite elements. For the solution of the resulting system of equations an efficient algorithm was proposed, which involves the application of preconditioned iterative solvers when appropriate. The accuracy and efficiency of the finite element methodology was demonstrated by applying it to challenging academic and industrially relevant 3D problems. A series of decoupled problems with a known analytical solution were used in order to verify that the computed convergence rates under $h$ - and $p$-refinement agreed with the a priori estimates (4.4), (4.7). Several challenging MRI configurations were also considered and comparisons against commercial software, the axisymmetric software developed in $[19,17]$ and experimental data were presented. Finally, a POD based ROM was presented, which combines the application of POD to the electromagnetic problem with the solution to the mechanical problem using the full order solver. The accuracy and efficiency of the ROM was demonstrated by applying it to challenging MRI configurations and performing comparisons against the full order solver, which showed that accurate results can be obtained with a computational speed-up higher than $95 \%$, which is of paramount importance in order to optimize the design of new MRI configurations.

\subsection{Recommendations for Future Work}

The achievements accomplished in this thesis open several research lines that can be considered in order to enhance the capabilities of the current methodology. A list of these is as follows: 
- Mesher: In this thesis, the open source software NetGen [155] was used to generate a mesh of the problems of interest. However, from an industrial point of view it could be beneficial to consider the use of commercial tools used by the manufacturer in order to embed the developed software into the current design process. Several tools, such as Ansys, Opera FEA or Siemens NX could be considered for this purpose.

- Geometry approximation: Another area of improvement is in the approximation of curved geometries. Currently, the software is able to produce linear and quadratic approximations for any arbitrary shape, and higher order approximations via the blending function method for problems where an analytical expression for the representation of the curved boundary is available. Other approaches, such as the use of NURBS [161, 160] or isogeometric analysis [90] could be considered in order to produce a more accurate approximation of the boundary with a smaller number of elements.

- $\boldsymbol{H}$ (div) basis functions: A further enhancement to the current methodology would be the inclusion of $\boldsymbol{H}$ (div)-conforming basis functions. Using these basis to discretise the current source would ensure that this is tangential to the surface of the coil $^{1}$. Furthermore, these basis could be used in order to assess to which degree the Coulomb gauge is approximated using regularisation.

- Hybrid meshes: A further improvement to the current methodology would be to allow the use of hybrid meshes formed by tetrahedrons, hexahedrons and pyramids. The use of hexahedral elements would be advantageous in order to allow the use of elements with high aspect-ratio in the discretisation of the thin radiation shields. This could result in a significant decrease in the number of elements required to reach a certain accuracy and, therefore, in a significant increase in computational efficiency. However, this would require the definition of high order $H^{1}$ and $\boldsymbol{H}$ (curl)-conforming basis functions for hexahedrons and pyramids to be implemented in the software, which might require a significant effort.

- Solver efficiency: Although the developed software is able to solve challenging MRI configurations in an accurate and efficient manner, there is still room to further optimize the software. For this, a possibility would be to translate key parts of the software, such as the assembly routine, from Matlab to a more efficient language especially in terms of memory usage and speed, such as $\mathrm{C}++$. Furthermore, the use of alternative direct solvers, such as PARDISO [6], could be considered which might lead to faster simulations, especially for problems involving the solution of a large mechanical system. Furthermore, a greater use of parallel computations could be considered in order to further optimise the software. In the current implementation the parallel computation of solutions for different parameters (frequency and

\footnotetext{
${ }^{1}$ Note, however, that this would not be enough to ensure that the current is solenoidal at a discrete level and, thus, the mapping of the current source to the space of solenoidal functions would still be required.
} 
conductivity) is considered but, to further optimize the computations, the assembly of the system matrices could also be parallelised.

- Acoustic and thermal effects: The methodology developed in this thesis could be further enhanced by considering acoustic and thermal effects. Due to the vibrations induced in the radiation shields, sound waves are generated, which can potentially result in high levels of noise during an MRI scanner examination and, therefore, in the patient experiencing discomfort. These acoustic effects were studied in [19, $18,17]$ for the case of axisymmetric problems. The methodology could also be enhanced by considering thermal effects in order to determine the thermal energy being deposited into the helium vessel, which is directly related with the dissipated power and has a direct impact in helium boil off effects and, thus, in a potential magnet quench.

- POD snapshot selection: The developed ROM could potentially be improved by considering a more sophisticated selection of the snapshots. At the moment these are chosen based on previous knowledge about the solution. However, other techniques such as a greedy basis generation [87] could be considered, which might reduced the number of snapshots needed to reach a certain accuracy, and could even result in the mechanical solution being approximated with a reduced number of snapshots. As briefly described in Chapter 7, this iterative method starts with the computation of a single snapshot, and at each iteration a new snapshot is added. An error estimator is required, which is evaluated at each iteration in a parametric mesh, and the next snapshot is chosen to correspond to the parameters that maximize this error. The development of reliable a posteriori error estimators could also be considered in order to provide a certified error control, ensuring the reliability and efficiency of the ROM approximation [87].

- Alternative ROM techniques: A further area of research would consist in studying the application of different ROM methodologies. First, the application of PGD could be considered, as this has already been applied to the magneto-mechanical problem of interest in axisymmetric configurations [21, 22]. However, based on the comparison between POD and PGD performed in [21] for axisymmetric problems and having into account that preconditioned iterative solvers are required to solve the 3D electromagnetic problem, a new ROM where POD is applied to approximate the electromagnetic solution and PGD is applied to approximate the mechanical solution is suggested. 


\section{Part V}

Appendices 


\section{Appendix A}

\section{Proof that $\operatorname{div} \boldsymbol{S}\left(\boldsymbol{A}^{D C}, \boldsymbol{A}^{A C}\right)=\mathbf{0}$ in $\Omega_{C}^{C} \backslash\left(\operatorname{supp}\left(\boldsymbol{g}^{A C}\right) \cup \operatorname{supp}\left(\boldsymbol{J}^{D C}\right)\right)$}

First, $\boldsymbol{S}\left(\boldsymbol{A}^{D C}, \mathcal{A}^{A C}\right)$ (defined in (3.18)) is rewritten in the form

$$
\boldsymbol{S}=\boldsymbol{B}_{0}^{D C} \otimes \boldsymbol{B}_{0}^{A C}+\boldsymbol{B}_{0}^{A C} \otimes \boldsymbol{B}_{0}^{D C}-\left(\boldsymbol{B}_{0}^{A C} \cdot \boldsymbol{B}_{0}^{D C}\right) \mathbb{I}
$$

where $\boldsymbol{B}_{0}^{A C}:=\operatorname{curl} \boldsymbol{A}^{A C}$. For simplicity, the subscript 0 is dropped in the following. Then, using index notation, this can be written as

$$
\boldsymbol{S}_{i j}=\boldsymbol{B}_{i}^{D C} \boldsymbol{B}_{j}^{A C}+\boldsymbol{B}_{i}^{A C} \boldsymbol{B}_{j}^{D C}-\left(\boldsymbol{B}_{k}^{A C} \boldsymbol{B}_{k}^{D C}\right) \delta_{i j}
$$

Thus

$(\operatorname{div} \boldsymbol{S})_{i}=\boldsymbol{S}_{i j, j}=\boldsymbol{B}_{i, j}^{D C} \boldsymbol{B}_{j}^{A C}+\boldsymbol{B}_{i}^{D C} \boldsymbol{B}_{j, j}^{A C}+\boldsymbol{B}_{i, j}^{A C} \boldsymbol{B}_{j}^{D C}+\boldsymbol{B}_{i}^{A C} \boldsymbol{B}_{j, j}^{D C}-\boldsymbol{B}_{k, i}^{A C} \boldsymbol{B}_{k}^{D C}-\boldsymbol{B}_{k}^{A C} \boldsymbol{B}_{k, i}^{D C}$ and by renaming the index $k$ as $j$

$$
\begin{aligned}
\boldsymbol{S}_{i j, j} & =\left(\boldsymbol{B}_{i, j}^{D C}-\boldsymbol{B}_{j, i}^{D C}\right) \boldsymbol{B}_{j}^{A C}+\left(\boldsymbol{B}_{i, j}^{A C}-\boldsymbol{B}_{j, i}^{A C}\right) \boldsymbol{B}_{j}^{D C}+\boldsymbol{B}_{i}^{D C} \boldsymbol{B}_{j, j}^{A C}+\boldsymbol{B}_{i}^{A C} \boldsymbol{B}_{j, j}^{D C} \\
& =\left(\boldsymbol{B}_{i, j}^{D C}-\boldsymbol{B}_{j, i}^{D C}\right) \boldsymbol{B}_{j}^{A C}+\left(\boldsymbol{B}_{i, j}^{A C}-\boldsymbol{B}_{j, i}^{A C}\right) \boldsymbol{B}_{j}^{D C}
\end{aligned}
$$

where, in the final step, the last two terms vanish due to $\operatorname{div} \boldsymbol{B}_{0}^{D C}=0$ and $\operatorname{div} \boldsymbol{B}_{0}^{A C}=$ 0 in $\mathbb{R}^{3}$. Next, using the property that $\left(\left(\nabla \boldsymbol{u}-(\nabla \boldsymbol{u})^{T}\right) \boldsymbol{v}\right)_{i}=\left(\boldsymbol{u}_{i, p}-\boldsymbol{u}_{p, i}\right) \boldsymbol{v}_{p}=$ $\epsilon_{i \ell p}\left(\epsilon_{\ell j k} \boldsymbol{u}_{k, j}\right) \boldsymbol{v}_{p}=((\operatorname{curl} \boldsymbol{u}) \times \boldsymbol{v})_{i}$ then

$$
\operatorname{div} \boldsymbol{S}=\left(\operatorname{curl} \boldsymbol{B}_{0}^{D C}\right) \times \boldsymbol{B}_{0}^{A C}+\left(\operatorname{curl} \boldsymbol{B}_{0}^{A C}\right) \times \boldsymbol{B}_{0}^{D C},
$$

so that $\operatorname{div} \boldsymbol{S}=\boldsymbol{J}^{D C} \times \boldsymbol{B}_{0}^{A C}+\boldsymbol{g}^{A C} \times \boldsymbol{B}_{0}^{D C}$ in $\operatorname{supp}\left(\boldsymbol{g}^{A C}\right) \cup \operatorname{supp}\left(\boldsymbol{J}^{D C}\right)$ and $\operatorname{div} \boldsymbol{S}=\mathbf{0}$,

in $\Omega_{C}^{c} \backslash\left(\operatorname{supp}\left(\boldsymbol{g}^{A C}\right) \cup \operatorname{supp}\left(\boldsymbol{J}^{D C}\right)\right)$. 


\section{Appendix B}

\section{CAD and Mesh Generation}

\section{B.1 Introduction}

In this thesis, NetGen [155] has been used to generate unstructured tetrahedral meshes of complex domains. For the generation of the CAD geometry, the CSG (NetGen native format), IGES and STEP formats have been considered. This appendix presents a simple user guide summarizing the issues encountered when trying to generate these CAD geometries and mesh them using NetGen. The aim of the appendix is to help future users of the software in generating different meshes using NetGen.

\section{B.2 CAD Generation}

For the generation simple CAD geometries, the NetGen native format CSG can be considered, which is based in the combination of simple primitive objects using boolean operators. During the work carried out in this thesis, it was observed that this format could be used to generate simple geometries such as those corresponding to the conducting sphere in a uniform magnetic field (Section 5.2.3), the hollow cylinder subject to pressure field (Section 5.2.2) or even the test magnet problem with $z$ gradient coils (Section 5.3.2). However, issues were encountered when trying to generate a CSG file for the geometry corresponding to the test magnet problem with $x$ gradient coils (Section 5.3.3) due to a higher complexity in the shape of the coils. These issues involved the appearance of nonconforming meshes as well as the NetGen software not being able to read the geometry file.

To overcome these issues, the use of alternative CAD format, such as IGES and STEP, was considered. For this, the AutoCad and AutoCad Mechanical packages were used, the latter allowing the generation of IGES and STEP formats as opposed to the former which does not allow the generation of STEP files. With both of these formats the CAD geometry corresponding to the test magnet problem with $x$ gradient coils could be generated without issues. However, some issues were encountered in the meshing process when considering the IGES format, as will be discussed in the next section. 


\section{B.3 Mesh Generation}

As previously discussed, the NetGen software was used in this thesis to generate complex unstructured tetrahedral meshes around complex geometries. The software allows the generation of linear as well as quadratic tetrahedral elements, and offers control over the mesh size through a series of parameters such as the mesh granularity (ranging from very coarse to very fine), the maximum and minimum mesh size or a mesh-size grading. Furthermore, it allows the use of mesh size files, where different mesh sizes can be applied to elements around specified points and lines. For a more complete description on the NetGen capabilities the user is referred to [155].

In spite of these advantages, some issues were also encountered when trying to generate meshes of complex geometries as that corresponding to the test magnet problem with $x$ gradient coils. It was observed that when trying to generate meshes using a IGES geometry format, the software was not robust and could result in the appearance of undesired non-conforming meshes. To overcome this, the use of STEP geometry files was considered, which allowed this issue to be overcome. This required, however, one additional and non-trivial step in order to ensure that a conforming tetrahedral mesh is obtained, which consists in "healing" the geometry so that NetGen can mesh it appropriately. NetGen offers an option called "IGES/STEP Topology Explorer/Doctor" which allows to analyse the geometry and fix small defects so that NetGen can handle the geometry appropriately. Several options can be selected, which include: "Fix small edges", "Fix spot/strip edges", "Sew faces", "Make solids" and "split partitions". Selecting the options shown in Figure B.1 in order to fix small defects in the edges and faces and clearly identify the interfaces between subdomains, and pressing the heal option, the geometry could then be meshed without issues, resulting in a conforming tetrahedral mesh.

This appendix aimed only at highlighting issues encountered in the CAD and mesh generation for the problems considered in this thesis and how to fix them. The appendix does not constitute a complete guide on how to use NetGen, and for this the reader is referred to [155]. 


\begin{tabular}{l}
\hline IGES/STEP Topology Explorer/Doctor \\
\hline Topology
\end{tabular}

\begin{tabular}{l}
\hline Zoom to highlighted entity \\
$\square$ Show \\
\hline Mealing tolerance: \\
$\square$ Fix small edges \\
$\square$ Fix spot/strip faces \\
$\square$ Sew faces \\
$\square$ Make solids \\
$\square$ Split partitions: \\
\hline Heal geometry \\
\hline
\end{tabular}

Figure B.1: "Healing" the geometry in NetGen [155]. 


\section{Appendix C}

\section{Numerical Integration}

As explained in Section 4.3, the DC and AC system matrices are computed by assembling elemental contributions, which are defined as the integral over a given element of the different terms in the system; see for instance (4.26) where the elemental stiffness and damping matrices for the DC electromagnetic problem are defined. These integrals are computed numerically by performing Gauss quadrature [89] on the reference element. For this, the integrals on the physical element must first be transformed to the reference element. In order to do so, let us define the transformation for the differential of volume from the physical to the reference domain, which can be written as

$$
\mathrm{d} \Omega=|\mathbf{J}| \mathrm{d} \hat{\Omega}
$$

where $\mathrm{d} \Omega=\mathrm{d} x \mathrm{~d} y \mathrm{~d} z$ is the differential of volume in the physical element and $\mathrm{d} \hat{\Omega}=$ $\mathrm{d} \xi \mathrm{d} \eta \mathrm{d} \zeta$ the differential of volume in the reference element. Considering this transformation and the $H^{1}$ and $\boldsymbol{H}$ (curl)-conforming transformations defined in Section 4.3.4 all the integrals can be transformed to the reference element, where Gauss quadrature is to be performed. In order to illustrate this process, let us consider the elemental stiffness matrix $\mathbf{K}_{\mathrm{AA}}^{\mathrm{DC},(e)}$, defined in (4.26) as

$$
\left[\mathbf{K}_{\mathrm{AA}}^{\mathrm{DC},(e)}\right]^{a b}=\int_{\Omega^{(e)}} \mu_{r}^{-1} \operatorname{curl} \boldsymbol{N}^{(e), a} \cdot \operatorname{curl} \boldsymbol{N}^{(e), b} \mathrm{~d} \Omega
$$

Using the previous transformations, the integral can be written in the reference element as

$$
\begin{aligned}
& \int_{\Omega^{(e)}} \mu_{r}^{-1} \operatorname{curl} \boldsymbol{N}^{(e), a} \cdot \operatorname{curl} \boldsymbol{N}^{(e), b} \mathrm{~d} \Omega \\
& \approx \int_{\hat{\Omega}} \mu_{r}^{-1}\left(|\mathbf{J}|^{-1} \mathbf{J} \operatorname{curl} \hat{\mathbf{N}}^{(e), a}\right) \cdot\left(|\mathbf{J}|^{-1} \mathbf{J} \operatorname{curl} \hat{\boldsymbol{N}}^{(e), b}\right)|\mathbf{J}| \mathrm{d} \hat{\Omega},
\end{aligned}
$$

where the approximation becomes exact when the true geometry is represented exactly. The integral in the reference domain is then computed numerically using Gauss quadra- 
ture as

$$
\begin{aligned}
& \int_{\hat{\Omega}} \mu_{r}^{-1}\left(|\mathbf{J}|^{-1} \mathbf{J} \operatorname{curl} \hat{\mathbf{N}}^{(e), a}\right) \cdot\left(|\mathbf{J}|^{-1} \mathbf{J} \operatorname{curl} \hat{\mathbf{N}}^{(e), b}\right)|\mathbf{J}| \mathrm{d} \hat{\Omega} \\
& \approx \sum_{i=1}^{n i p} \mu_{r}^{-1}\left(|\mathbf{J}|^{-1} \mathbf{J} \operatorname{curl} \hat{\boldsymbol{N}}^{(e), a}\right) \cdot\left(|\mathbf{J}|^{-1} \mathbf{J} \operatorname{curl} \hat{\boldsymbol{N}}^{(e), b}\right)|\mathbf{J}| \alpha_{i}(\xi, \eta, \zeta),
\end{aligned}
$$

where nip is the number of integration (Gauss) points and $\alpha_{i}(\xi, \eta, \zeta)$ the corresponding integration weights. Many Gauss quadrature formulas can be considered and, in this thesis, the canonical product formula proposed by Stroud and Secrest [167] has been used.

Note that if a linear mapping is considered, the Jacobian matrix of the transformation is constant and, thus, the integrands are polynomials and the integrals can be computed exactly, provided that a sufficient number of integration points is chosen. However, when considering higher order geometry approximations this is no longer true, since the inverse of the Jacobian will be a rational function. The number of integration points is chosen, in practice, depending on the polynomial order of the $H^{1}$ and $\boldsymbol{H}$ (curl) basis functions used to approximate the mechanical and electromagnetic fields.

In a similar manner, other volume integrals, and surface integrals such as those in (4.52), (4.55), can be approximated using an analogous process and the transformations stated in Section 4.3.4.

In general, the application of Gauss quadrature and geometry approximation for $I=$ $\left[\mathbf{K}_{\mathrm{AA}}^{\mathrm{DC},(e)}\right]^{a b}$ in (C.2) leads to

$$
I=\sum_{i=1}^{n i p} \mu_{r}^{-1}\left(|\mathbf{J}|^{-1} \mathbf{J} \operatorname{curl} \hat{\mathbf{N}}^{(e), a}\right) \cdot\left(|\mathbf{J}|^{-1} \mathbf{J} \operatorname{curl} \hat{\mathbf{N}}^{(e), b}\right)|\mathbf{J}| \alpha_{i}(\xi, \eta, \zeta)+e_{\text {int }}+e_{\text {geom }},
$$

where $e_{\text {int }}$ is the approximation error associated with the numerical integration and $e_{\text {geom }}$ that associated with the geometry.

The error $e_{\text {geom }}$ can be reduced by refining the mesh or increasing the order of approximation of the geometry, $g$. The error $e_{\text {int }}$ can be decreased by increasing the number of integration points. These errors will depend, in practice, on the curvature of the boundary and the coarseness of the mesh. The rates of convergence of these errors are problem dependent, and, thus, it is not possible to quantify them for a general problem. In idealised cases, bounds on these errors can be found [39]. However, in practice, provided that $e_{\text {geom }}$ and $e_{i n t}$ are smaller than the error associated with the finite element approximation, the a priori rates of convergence stated in (4.4)-(4.7) still hold for $h$ - and $p$-refinement. 


\section{Appendix D}

\section{Parallel Implementation}

\section{D.1 Introduction}

Parallel computing $[79,103]$ has been widely used in the area of numerical simulations in engineering and physics problems due to its great cost benefit. Many applications have benefit from the use of parallel computing, including aerodynamic shape optimization [150], the simulation of thermo-mechanical processes [179] and electro-mechanical coupled problems [70] among others.

Some tasks are easily parallelisable, meaning that little manipulation is needed to separate a big problem into a number of smaller parallel tasks. This is usually the case when these tasks are mostly independent between them and, therefore, there is no need for communication between them. In other cases, the parallelization becomes more challenging due to the need to interchange information between tasks, but many advances have been made in the past years in order to facilitate this communication, such as the development of the Message Passing Interface (MPI) protocol [79], which facilitates the exchange of information between different processors or nodes.

The application considered in this thesis offers some easily parallelisable tasks such as the solution to the coupled system for varying model parameters and, therefore, the parallelisation of this tasks has been implemented in order to reduce the computational cost. In the following, a brief summary of this parallel implementation in Matlab is provided. Note that a rigorous analysis of parallel performance is outside the scope of this thesis. However, the MATLAB parallel computing toolbox has been used to accelerate aspects of the computation and this appendix relates to observations and implementation details of this.

\section{D.2 Matlab Implementation for Varying Model Parame- ters}

As seen in Chapter 6, the solution to the coupled magneto-mechanical problem of interest for varying model parameters is of paramount importance in order to optimize the design 
of new MRI scanners. In order to optimize this repeated simulations, ROM have been considered, but the cost can be further reduced by computing the solution for different parameters in parallel. These can be applied to the numerical simulation using the full order solver but also to further decrease the cost of the ROM, especially in the off-line stage summarised in Algorithm 2 in Chapter 6, where the computation of the snapshots can be done in parallel.

The Matlab parallel computing toolbox offers several functionalities that enable the parallelization of this tasks in an easy manner. First, the parpool command, allows the generation of a pool of cores or workers that will be used for the computation. The number of cores to be used can be passed as an argument. Once a pool of workers is available, the solution to the linear system of equations for varying model parameters can be computed in parallel using the parfor Matlab command, which can be seen as a parallel for loop. The parfor loop, however, presents some restrictions compared to the for loop and, therefore, a for loop cannot, in general, be converted into a parfor loop without any modifications. These restrictions include that the body of the parfor loop must be independent, that is, one iteration cannot depend on previous iterations and also that two parfor loops cannot be nested.

For the application of interest in this thesis, the for loop iterating over the different model parameters for which the system must be solved can be converted into a parfor loop with a limited number of minor modifications. It was observed, however, that if only the strictly necessary modifications for the parfor loop to run were applied, the parallel implementation was not efficient. For instance, considering a parallel pool formed by 8 cores only a speed-up of around 2 was obtained ${ }^{1}$. This is due to the data transfer overhead, which refers to the cost of sending the necessary data to each core in order to perform the computations. Sometimes the cost of data transfer can even exceed the cost of the computations. Thus, in order to optimize the parallel implementation, the necessary data (or at least the larger pieces of data) must be copied to each worker before starting the parallel loop. For this Matlab offers the command parallel.pool.Constant which allows to create a copy of a given object into each worker. Note of course that this object cannot change during the execution of the parallel loop.

In this particular application, several parameter independent matrices must be stored to then construct the global system matrix for each parameter combination, as described in the affine decomposition presented in Chapter 6. For fine discretisations, these matrices contain a large amount of data and, therefore they must be copied to each worker in order to optimize the parallel implementation. The speed-up obtained when these matrices were copied into the workers grows to 6 for a parallel pool of 8 workers, which is a great result having in mind that not all the data has been copied as some data changes between iterations and that there exist other overhead costs, such as the cost of starting and terminating tasks or the reduction of implicit multi-threading in algebraic operations [103].

\footnotetext{
${ }^{1}$ Note that a detailed analysis of the performance of the parallel implementation is out of the scope of this appendix and only some rough figures are discussed in order to illustrate the main bottlenecks in this implementation.
} 


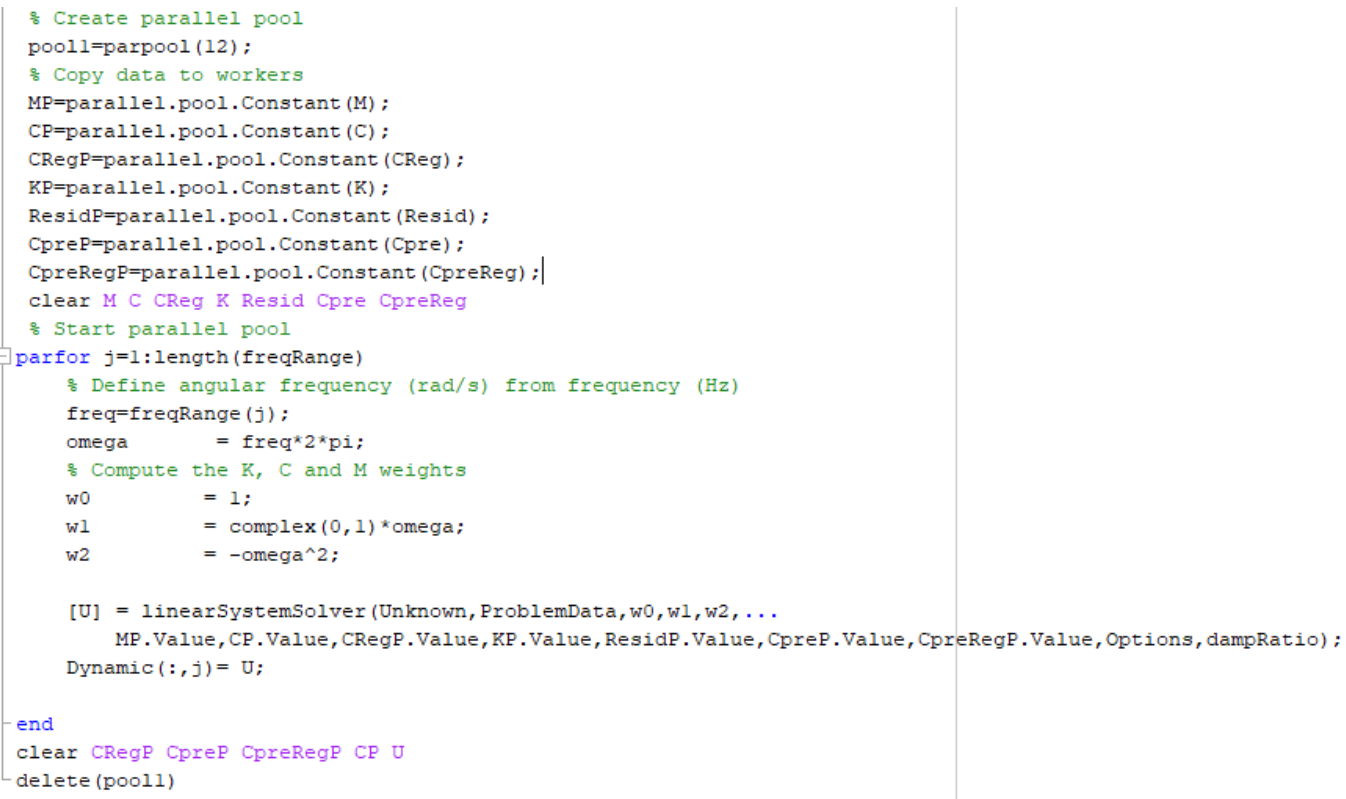

Figure D.1: Parallel solution of the system for varying frequencies: code screen shot.

In order to illustrate this parallel implementation, a screen shot of the software corresponding to a script used to solve the system for different frequencies in parallel is provided in Figure D.1. As it can be observed, first a parallel pool consisting of 12 workers is created. Then, the system matrices are copied to the workers before starting the parallel loop to iterate over frequencies and solve the linear system. Once the iteration finishes, the matrices copied to the workers are removed in order to free memory and the parallel pool is closed.

Note also that parallel computing could be used to further optimize the computations. For instance, the assembly process, which in the current implementation is done in serial could potentially be parallelized, although this would not be as simple given that a given entry of the global matrix might be formed by contributions from several elemental matrices and, therefore, the different workers need to communicate between them. However, such implementation is certainly possible and would lead to an increased efficiency of the software. To parallelise the assembly process many authors have considered the use of domain decomposition methods [68, 78, 67], while others have proposed alternative methods where the assembly is done degree of freedom by degree of freedom rather than element by element [178]. 


\section{Appendix E}

\section{User Guide}

\section{E.1 Introduction}

This appendix presents an user guide for the coupled magneto-mechanical solver developed during this thesis, which is based on the high order version of the finite element method and a POD based ROM. The solution to the full order model using high order FEM was first presented in [158] and the model order reduction via POD was presented in [159].

The aim of the appendix is to provide a simple guide on how to use the software. For a discussion on the methodologies and the technical details the reader is referred to [158, $159]$ and to the main body of this thesis.

The structure of the document is as follows. First, in Section E.2, the capabilities of the software are stated. Second, in Section E.3, the general structure of the software is described and then, in Section E.4, the scripts with which the user must interact to run a given problem are described. The document finishes with concluding remarks in Section E.5.

\section{E.2 Software Capabilities}

The developed software has the following capabilities:

1. Full Order Solution: The software can be used to obtain the solution to single physics or coupled magneto-mechanical problems.

(a) Decoupled problems: The software can be used to solve different decoupled or single physics problems [158], which include:

- Magnetostatics

- Static elasticity

- Eddy current

- Transient (time-harmonic) elasticity 
(b) Coupled problems. The software can also be used to solve coupled-magneto mechanical problems, which include:

- Static magneto-mechanical problems

- Transient (time-harmonic) magneto-mechanical problems in frequency domain.

2. POD: The software can also be used to find the solution to a high dimensional parametric problem via POD, as described in [159] and in the main body of this thesis. In particular, the combined reduced order-full order methodology is used. The following options can be considered:

(a) PODI: POD with interpolation (PODI) has been implemented for the case of a single parameter of interest.

(b) PODP: Projection based POD (PODP) can be used to obtain a ROM for an arbitrary number of parameters. An affine decomposition has been implemented for frequency and electric conductivity.

Note that in the case of MRI applications, the transient magneto-mechanical problem of interest [158], is solved by using an AC-DC splitting and solving first a DC problem followed by a linearised AC problem [158].

Finally, it should also be noted that results for different examples, ranging from academic single physics problems to industrially relevant MRI configurations have been presented in $[158,159]$ and in Chapters 5 and 7 of this thesis.

\section{E.3 Software Structure}

In this section we provide a global overview of the software structure and summarise the different steps that must be performed in order to arrive at the desired outputs of interest. For this purpose, a schematic representation of the software structure is provided in Figure E.1.

The software can be divided into three main stages: pre-processing, solver and postprocessing. The main steps performed at each of these stages are briefly outlined in the following.

\section{E.3.1 Pre-processing Stage}

The main steps to be performed at the pre-processing stage are:

- Geometry (CAD) and Mesh generation: This step is performed at an initial stage using available open source packages. First a CAD model of the problem geometry must be generated. For very simple geometries, the native NetGen [155] CAD format, named CSG (Constructive Solid Geometry) can be used. This allows us to create simple geometries by performing basic boolean operations. However, when 


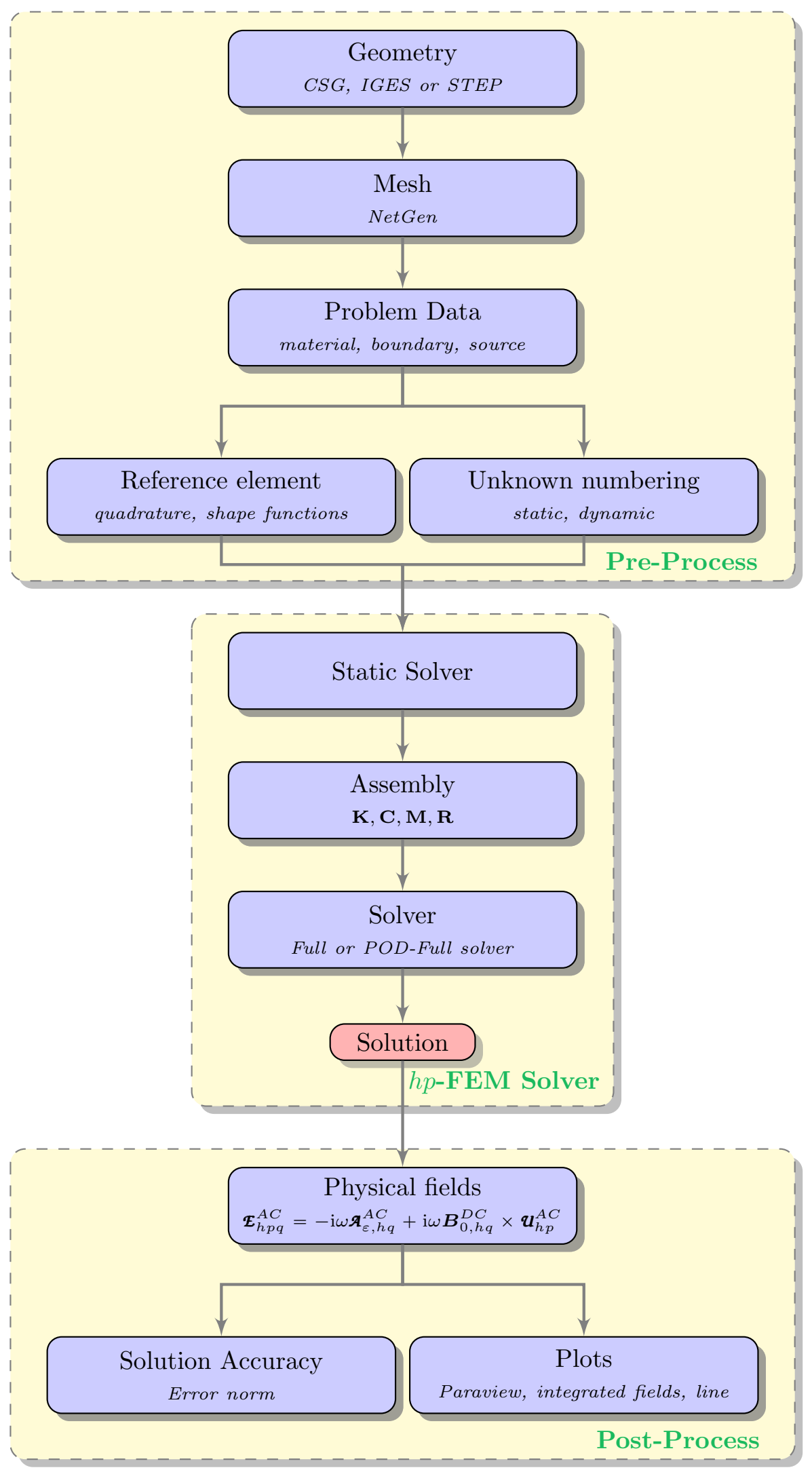

Figure E.1: Schematic representation of the software structure, including the preprocessing, solver and post-processing stages. 
the complexity of the geometry increases, more sophisticated CAD formats such as IGES (Initial Graphics Exchange Specification) or STEP (Standard for the Exchange of Product Model Data) must be considered. Many commercial and opensource packages are available for the generation of such CAD formats, and in this work AutoCAD [2] and AutoCAD Mechanical [3] have been used. Once the CAD geometry is available, a mesh must be generated. For this purpose we conisder the open source software NetGen [155], which allows the generation of complex unstructured tetrahedral meshes. NetGen has the capability of generating linear as well as quadratic elements and allows the local refinement of the mesh.

- Problem data definition: Once the mesh is created, it is read into our software and the problem is defined by assigning the material properties, source terms and boundary conditions to each sub-domain or boundary surface.

- Reference element and unknown numbering: The final steps of the preprocessing stage consist in defining the Gauss points and the shape functions at the reference element and creating the numbering of degrees of freedom for the DC and $\mathrm{AC}$ problems.

\section{E.3.2 Solver Stage}

The main steps to be performed at the solver stage are:

- Static solver: First, the DC problem must be solved. This step includes the assembly of the DC system and the solution of the magnetostatic and static elasticity problems.

- Assembly: In this step, the matrices of the AC system are assembled from elemental contributions. Note that the assembled matrices are such that they do not depend on the frequency, and the global system matrices are then computed, for each frequency, by multiplying the assembled matrices by frequency dependent parameters and adding them together. This leads to a much faster computation of the solution for multiple frequencies, as it is not necessary to assemble the system each time the frequency is modified.

- Staggered solver: The last step of the solver stage is to compute the so.lution of the AC electromagnetic and mechanical systems for all the required parameters. For this purpose, either the full order [158] or the reduced order-full order solver [159] can be used.

\section{E.3.3 Post-processing Stage}

The main steps to be performed at the post-processing stage can be divided in two groups: 
- Error measure: If the problem under consideration has a known analytical solution, the accuracy of the finite element solution can be benchmarked by computing the appropriate error norms.

- Plots: Finally, the main goal is to produce plots of the outputs of interest that help us understand the solution to the coupled problem. These include the evolution of integrated quantities such as dissipated power or kinetic energy with the frequency, the eddy current, magnetic field or displacement patterns in the radiation shields for selected frequencies or the variation of these magnitudes along a certain line. For the generation of high quality contour plots when computing the solution in coarse meshes using high order elements, the reference element is split into smaller elements and these are then mapped to the physical domain. The solution in the finer mesh is then exported to Paraview to plot. On the other hand, if we are interested in the computation of integrated quantities such as dissipated power or kinetic energy, an integral over the domain must be performed and for this we use the developed finite element methodology and compute the integrals using Gauss quadrature.

\section{E.4 User Interaction}

This section describes the steps where user interaction is required. This basically consist in four steps: Mesh generation, problem file definition, main function and postprocessing.

\section{E.4.1 Mesh Generation}

As previously described, the mesh is generated beforehand using the open source software NetGen. For instructions on how to use this software, the reader is referred to [155] and Appendix B of this thesis. However, it is important to note here that the different boundaries of the domain must be labelled appropriately in NetGen, before reading the mesh into the magneto-mechanical software. This boundary conditions are then assigned to the corresponding entities (vertices, edges, faces) of the mesh.

The numbering of the boundaries for the different scenarios is as follows:

- Decoupled electromagnetics: The far field boundary $\partial \Omega$ must be labelled as 1 or 9. If labelled as 1 , the boundary will be defined as Dirichlet or Neumann in the problem file. If numbered as 9 , the boundary will be defined as Neumann. The last option is useful if the user wishes to split $\partial \Omega$ into $\partial \Omega^{D}$ and $\partial \Omega^{N}$. The first option is useful if all the boundary is considered as either Dirichlet or Neumann, as the type can be changed in the problem file, without reading the mesh again. The interfaces conductor-air or coil-air must be labelled as 2 .

- Decoupled mechanics: To apply a Dirichlet condition the boundary must be labelled as 4 . To apply a Neumann condition the boundary must be labelled as 9 . 
- Coupled problems: The far field boundary $\partial \Omega$ must be numbered identically as for decoupled electromagnetic problems. The part of the conductor's surface where a Dirichlet boundary condition will be applied $\left(\partial \Omega_{C}^{D}\right)$ must be labelled as 4 . The Neumann part must be labelled as 3 (zero Neumann BC type). Alternatively the Neumann part could be numbered as 9 if a non-zero Neumann condition was required, but this was not necessary for any of the problems considered in this thesis.

- Symmetry boundaries: Different symmetry boundaries have been considered in $[158,159, ?]$ in order to reduce the cost of the computations. In particular, three different situations must be distinguished:

- Problems with rotational symmetry around $z$ axis: The $x=0$ plane must be labelled as 7 and the $y=0$ plane as 8 .

- MRI configurations considering longitudinal $z$ gradient coils: The $x=0$ plane must be labelled as 7 , the $y=0$ plane as 8 and the $z=0$ plane as 12 .

- MRI configurations considering transversal $x$ gradient coils: The $x=0$ plane must be labelled as 10 , the $y=0$ plane must be labelled as 8 and the $z=0$ plane must be labelled as 13 .

Note that the symmetry conditions for the case of $y$ gradient coils have not been implemented in the software. However, this implementation could be done very easily by following the symmetry conditions presented in Chapter 4.

\section{E.4.2 Problem File Definition}

For the problems presented in $[158,159]$ and in this thesis, the problem files are already available and thus no action from the user is required in order to solve these problems. However, if the user wants to solve a different problem, a new problem file must be defined. Thus, in this Section, a discussion of the variables that need to be defined in the problem file is presented.

The most relevant variables to be defined in the problem file are:

- job: String defining the name of the mesh to be loaded.

- regopt: Variable defining the solver option; (1) for iterative solver with action of preconditioner on gradient blocks computed iteratively. (2) for iterative solver with action of preconditioner on gradient blocks computed directly (3) Direct solver. The default option is (2).

- TOL_GMRES: Tolerance of the iterative solver.

- JDC and JAC: Current density of the main and gradient coils, respectively.

- regterm: Value assigned to the regularisation parameter $\varepsilon$. 


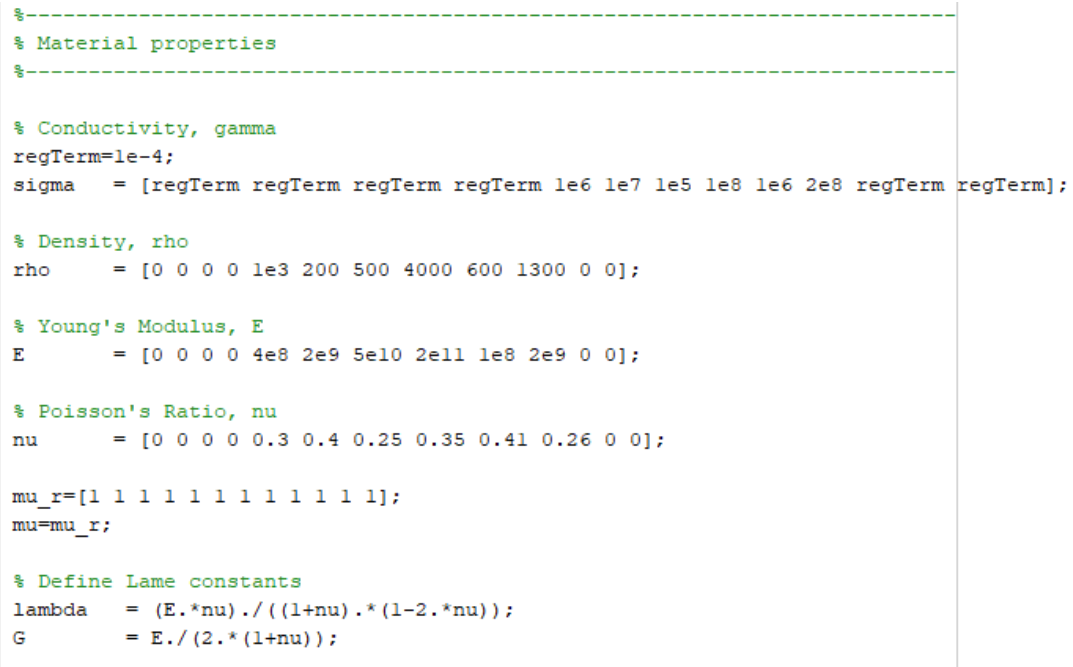

Figure E.2: Problem file definition: illustration of the definition of the material properties.

- sigma, rho, E, nu and mu: vectors containing the values of conductivity, material density, Young's modulus, Poisson's ratio and magnetic permeability, respectively, for each subdomain in the mesh.

- D: Cell array containing the elasticity matrix for each subdomain.

- matcond: vector containing the subdomain numbers where the high order $\boldsymbol{H}$ (curl) basis functions should be included. This means conducting components and gradient coils.

- matmech: vector containing the subdomain numbers of the conducting components that conform $\Omega_{C}$.

- mat4K, mat77K and matOVC: vectors containing the subdomain numbers for the $4 \mathrm{~K}, 77 \mathrm{~K}$ and OVC shields in the case of MRI configurations. Note that this is only used to customize the output for the case of MRI configurations, obtaining the dissipated power and kinetic energy in each shield instead of for each subdomain.

- NmechBodies: Number of conducting subdomains.

- gorder: Order of approximation for the geometry. (0) linear, (1) quadratic or (>1) for the case of higher order approximations using blending functions.

As an illustration, a screen shot of the definition of the material properties for a typical MRI configuration is shown in Figure E.2. Note that the material properties used for the MRI configurations in $[158,159]$ and in this thesis are confidential and, therefore, the values that we show here are random.

Furthermore, the functions used to define the Dirichlet and Neumann boundary conditions, the source terms as well as the analytical solution (if available), are defined in the problem file. A screen shot of the definition of these functions is shown in Figure E.3. 


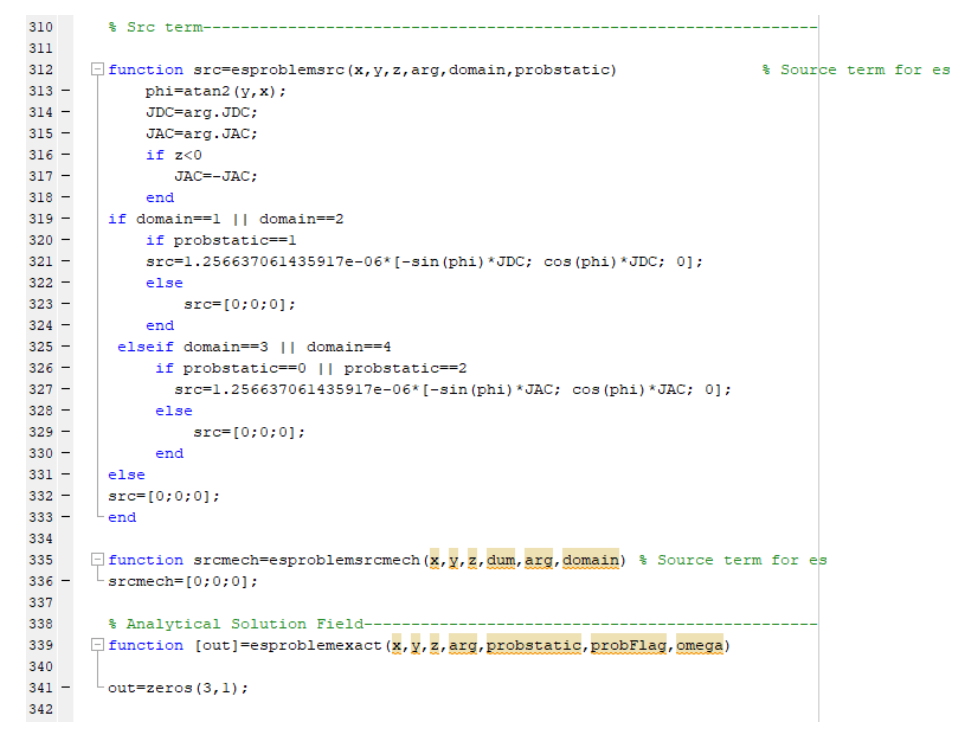

Figure E.3: Problem file definition: illustration of the definition of the functions used to impose the source terms and analytical solution.

Finally, it should be remarked that several problem files are already defined and available to use, which correspond to the problems presented in $[158,159]$ and in Chapters 5 and 7 of this thesis. The list of the problem files available is in the problemFiles folder is:

- problem1: Conducting and elastic sphere in a uniform alternating magnetic field.

- problemCylinder: Hollow cylinder subject to pressure field.

- problemToy: Test magnet problem with $z$ gradient coils.

- problemToyGrad_Symmetry: Test magnet problem with $x$ gradient coils.

- problemMHI: Modified magnet problem with $z$ gradient coils. Shields constrained at the ends.

- problemMHIGradZSplit: Modified magnet problem with $z$ gradient coils. Shields constrained at the ends and at extra inner bands.

- problemMHIGradXNewCoilOnlyEnds: Modified magnet problem with $x$ gradient coil. Shields constrained at the ends.

- problemMHIGradXSplitNewCoil: Modified magnet problem with $x$ gradient coils. Shields constrained at the ends and at extra inner bands.

Furthermore, it should be noted that the mesh files for all these problems are also already available in the folder meshes. 


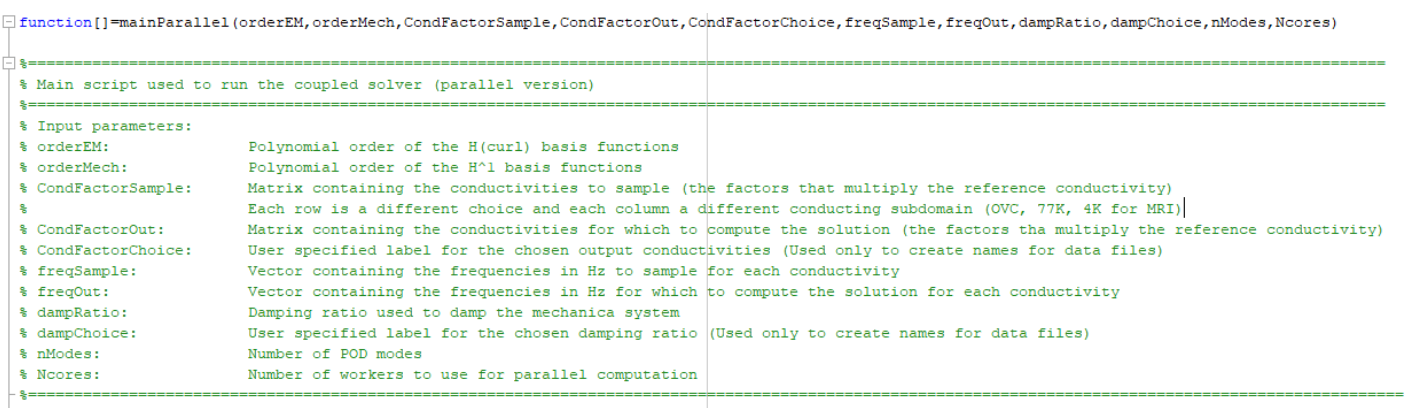

Figure E.4: mainParallel function: illustration of the user-defined input parameters.

\section{E.4.3 Main Functions}

Once the mesh and the problem file are available, the software can be run from one of the two main functions, namely mainSerial and mainParallel. Both scripts are almost identical, with the difference that mainParallel has one extra parameter named Ncores, that will be used in the solver stage to solve the frequency sweeps in parallel. Note that to run mainParallel the Matlab Parallel Computing Toolbox must be installed. In the following the different parameters that the user must define in order to run the software are discussed.

\section{User-Defined Input Parameters}

The mainSerial and mainParallel functions have several input parameters that the user must define when calling these functions from the command line. A screenshot of the first lines of the mainParallel function, where these parameters are defined is shown in Figure E.4. Note that mainSerial has the same input parameters excluding Ncores.

The complete list of input parameters is:

- orderEM: Polynomial order of the $\boldsymbol{H}$ (curl) basis functions.

- orderMech: Polynimial order of the $H^{1}$ basis functions.

- CondFactorSample: Matrix containing the list of multiplying factors defining the conductivities to sample for POD. These factors multiply the reference value for the conductivity $\gamma_{r e f}$ and each row of the matrix corresponds to a different choice, while each column corresponds to a different conducting subdomain. This parameter is only used when running the ROM.

- CondFactorOut: Matrix containing the list of multiplying factors defining the conductivities for which the solution must be computed. These factors multiply the reference value for the conductivity $\gamma_{r e f}$ and each row of the matrix corresponds to a different choice, while each column corresponds to a different conducting subdomain.

- CondFactorChoice: User specified label for the chosen output conductivities, used only to define the names of the output data files. 


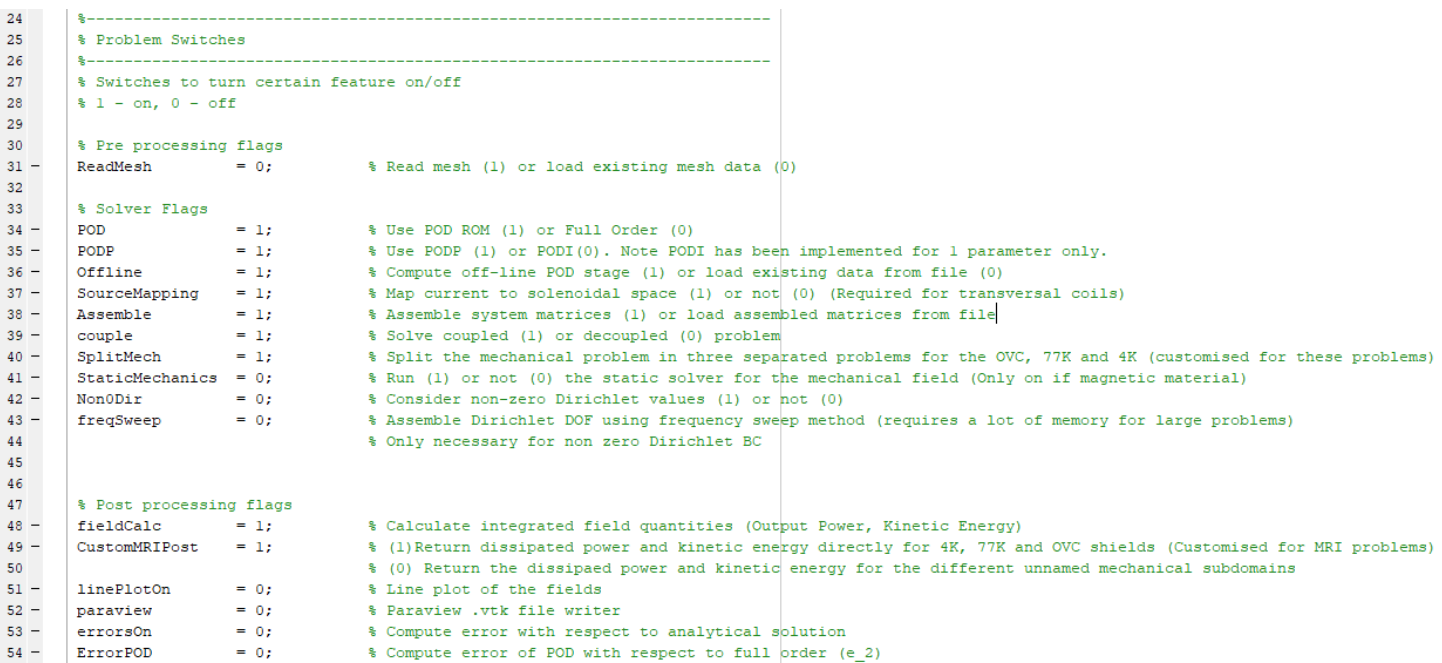

Figure E.5: mainParallel function: illustration of the user-defined switches that control the different pre-processing, solver and post-processing options.

- freqSample: Vector containing the frequencies $f$ in $\mathrm{Hz}$ that must be sampled for each conductivity, when running POD. This parameter is only used when running the ROM.

- freqOut: Vector containing the frequencies $f$ in $\mathrm{Hz}$ for which the solution must be computed.

- dampRatio: Damping ration $\xi$ used to define the mechanical damping.

- dampChoice: Label for the choice of damping, used only to define the names of the output data files.

- nModes: Number of modes $M$ at which the TSVD is truncated.

- Ncores: Number of workers used for parallel computation.

\section{Problem Switches}

In the main functions, different problem switches are defined in order to choose between different pre-processing, solver and post-processing options. A screenshot of the definition of these switches shown in Figure E.5.

The complete list of switches and is:

- Pre-processing switches

- ReadMesh: Read mesh from external file (1) or load existing mesh data in .mat format (0).

- Solver switches

- POD: Use POD-Full ROM (1) or full order (0). 
- PODP: Use PODP (1) or PODI (0). Note that PODI has only been implemented for the case of considering frequency as the only parameter. This switch is only used if the POD switch is set to 1 .

- Offline: Compute off-line POD stage (1) or load existing data from saved file (0).

- SourceMapping: Map current source to solenoidal space (1) or not (0). This option is required when considering transversal gradient coils.

- Assemble: Assemble system matrices (1) or load assembled matrices from data file (0).

- couple: Activate (1) or deactivate (0) coupling blocks.

- SplitMech: Split the mechanical problem in several smaller problems for each conducting subdomain (1) or solve as one single problem (0).

- StaticMechanics: Solve (1) or not (0) the static mechanical problem. Note that for the MRI applications considered in this work the static displacement is zero and thus we do not want to solve the mechanical problem in such cases.

- Non0Dir: Consider non-zero Dirichlet values (1) or not (0). This switch will be used to determine how the assembly is done.

- freqSweep: Assemble Dirichlet contributions using frequency sweep method. This option allows to run frequency sweeps assembling the matrices only once even if we solve for multiple frequencies. Requires a large amount of memory for big problems. It is only needed if there are non-zero Dirichlet boundary conditions.

- Post-processing switches

- fieldCalc: Calculate integrated field quantities (dissipated power, kinetic energy) (1) or not (0).

- CustomMRIPost: Return dissipated power and kinetic energy directly for $4 \mathrm{~K}, 77 \mathrm{~K}$ and OVC (1) or for each conducting subdomain (0). Only activated when considering MRI configurations in order to obtain a customised output.

- LinePlotOn: Compute line plots of the fields (1) or not (0). The lines where the plot is required must be defined in the problem file.

- paraview: Write .vtu files in order to produce 3D plots in Paraview (1) or not $(0)$.

- errorsOn: Compute error with respect to analytical solution if available (1) or not (0).

- ErrorPOD: Compute error $e_{2}$ of POD with respect to full order (1) or not (0).

Finally, the last parameter that the user must define in the main function is problem, which defines the name of the problem file to be used. For example, problem='Toy' to use the problem file problem Toy (see Figure E.6). 


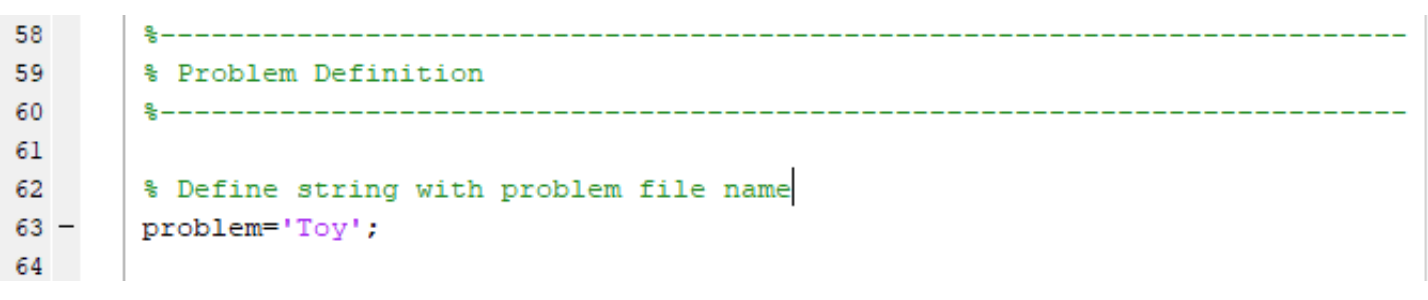

Figure E.6: mainParallel function: illustration of the definition of the parameter problem defining the problem file to be used.

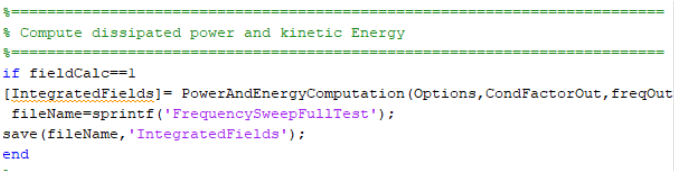

Figure E.7: Screenshot of the main function showing the function to compute the integrated field quantities and the definition of the name for the mat data file.

\section{E.4.4 Post-processing}

Depending on the post-processing switches that were activated, the software will return different outputs that can be used for post-processing purpose. These outputs can be summarised as follows:

- If the switch linePlotOn was activated, a figure (or figures) showing the desired plots at the specified line will be generated.

- If the witch errorsOn was activated, the $\boldsymbol{H}$ (curl) and $H^{1}$ norm of the error with respect to the exact solution will be printed on the screen.

- If the switch paraview was activated, the software will generate the .vtu files that can then be read into paraview for post-processing. The files will be named using the name of the mesh.

- If the switch fieldCalc was activated, a .mat file containing, for each parameter set, the dissipated power and the displacement norm $\left\|\boldsymbol{u}_{h p}^{A C}\right\|_{L^{2}(\Omega)}=$ $\left(\int_{\Omega}\left|\boldsymbol{u}_{h p}^{A C}\right|^{2} \mathrm{~d} \Omega\right)^{1 / 2}$, from which the kinetic energy can be trivially computed (see $[158, ?])$. The name of the .mat file is defined in the main script (see Figure E.7).

- If the switch ErrorPOD was activated, the error $e_{2}$ of the POD-Full with respect to the full order solution for each parameter set will be computed and saved to a .mat file. The name of the .mat file is defined in the main script (see Figure E.8).

\section{E.5 Appendix Summary}

This appendix provides a simple user guide on how to use the coupled magnetomechanical software developed in during this thesis. The steps that are needed to run 


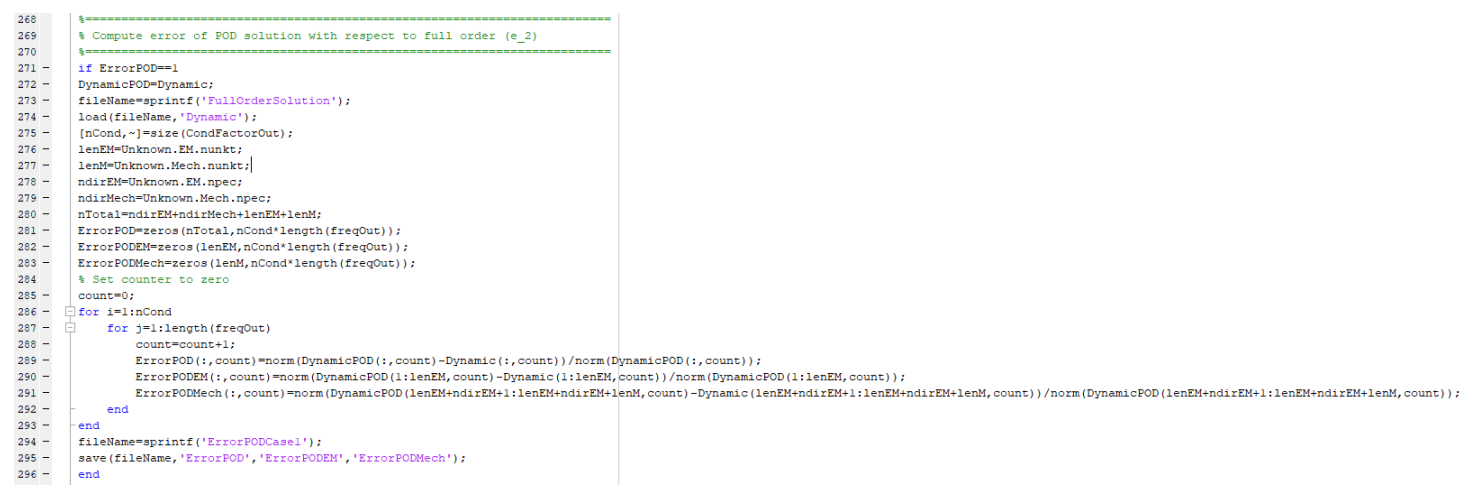

Figure E.8: Screenshot of the main function showing the function to compute the error with respect to the full order solution $e_{2}$ and the definition of the name for the .mat data file.

the code were described, including the definition of a mesh with appropriate boundary condition labels, the definition of the problem file and the input parameters and switches that must be defined in the main functions. The output of the code, depending on the activated switches was also described. The appendix constitutes a simple user guide, and it is recommended to use it in conjunction with the work presented in $[158,159]$ and in the main body of this thesis to have a better understanding of the problems under consideration and the different methods used for their solution. 


\section{Bibliography}

[1] Ansys software. Available at: http://www.ansys.com. Accessed: 13/11/2018.

[2] AutoCAD. Available at: http://www.autodesk.com. Accessed 29/07/2019.

[3] AutoCAD Mechanical. Available at: http://www.autodesk.com. Accessed 29/07/2019.

[4] Numerical analysis of coupled systems (NACS). Available at: http://www.simetris.de. Accessed 15/11/2018.

[5] Opera FEA simulation software. Available at: https://operafea.com. Accessed: $13 / 11 / 2018$.

[6] PARDISO project. https://www.pardiso-project.org/. Accessed 24/10/2019.

[7] S. Acharya, B. R. Baliga, K. Karki, J. Y. Murthy, C. Prakash, and S. P. Vanka. Pressure-based finite-volume methods in computational fluid dynamics. Journal of Heat Transfer, 129(4):407-424, 2007.

[8] S. Adhikari. Damping Models for Structural Vibration. PhD thesis, University of Cambridge, UK., 2001.

[9] J. Ahrens, B. Geveci, and C. Law. Paraview: An end-user tool for large data visualization. In Visualization Handbook. Elsevier, 2005.

[10] M. Ainsworth and J. Coyle. Hierarchic finite element bases on unstructured tetrahedral meshes. International Journal for Numerical Methods in Engineering, 58(14):2103-2130, 2003.

[11] M. Ainsworth and K. Pinchedez. hp-Approximation theory for BDFM and RT finite elements on quadrilaterals. SIAM Journal on Numerical Analysis, 40(6):20472068, 2002.

[12] M. H. Aliabadi. The Boundary Element Method: Applications in Solids and Structures, volume 2. John Wiley \& Sons, 2002.

[13] A. Alonso and A. Valli. Eddy Current Approximation of Maxwell Equations: Theory, Algorithms and Applications, volume 4. Springer Science \& Business Media, Milano, 2010. 
[14] D. Amsallem and C. Farhat. Interpolation method for adapting reduced-order models and application to aeroelasticity. AIAA Journal, 46(7):1803-1813, 2008.

[15] S. N. Atluri, H. T. Liu, and Z. D. Han. Meshless local Petrov-Galerkin (MLPG) mixed finite difference method for solid mechanics. Computer Modeling in Engineering and Sciences, 15(1):1, 2006.

[16] I. Babuška. The finite element method with Lagrangian multipliers. Numerische Mathematik, 20(3):179-192, 1973.

[17] S. Bagwell. A Numerical Multi-Physics Approach to Understanding MRI Scanners and their Complex Behaviour. PhD thesis, Swansea University, UK., 2018.

[18] S. Bagwell, P. D. Ledger, A. J. Gil, and M. Mallett. Transient solutions to nonlinear acousto-magneto-mechanical coupling for axisymmetric MRI scanner design. International Journal for Numerical Methods in Engineering, 115:209-237, 2018.

[19] S. Bagwell, P.D. Ledger, A.J. Gil, M. Mallett, and M. Kruip. A linearised $h p$ finite element framework for acousto- magneto-mechanical coupling in axisymmetric MRI scanners. International Journal for Numerical Methods in Engineering, 112:1323-1352, 2017.

[20] P. K. Banerjee and R. Butterfield. Boundary Element Methods in Engineering Science, volume 17. McGraw-Hill, London, 1981.

[21] G. Barroso, A. J. Gil, P. D. Ledger, M. Mallett, and A. Huerta. A regularisedadaptive Proper Generalised Decomposition implementation for coupled magnetomechanical problems with application to MRI scanners. Computer Methods in Applied Mechanics and Engineering, 358:112640, 2019.

[22] G. Barroso, M. Seoane, A. J. Gil, P. D. Ledger, M. Mallett, and A. Huerta. A staggered high-dimensional Proper Generalised Decomposition for coupled magnetomechanical problems with application to MRI scanners. Computer Methods in Applied Mechanics and Engineering, 370:113271, 2019.

[23] G. Beer. Finite element, boundary element and coupled analysis of unbounded problems in elastostatics. International Journal for Numerical Methods in Engineering, 19(4):567-580, 1983.

[24] R. G. H. Beets-Tan, G. L. Beets, A. C. W. Borstlap, T. K. Oei, T. M. Teune, M. F. Von Meyenfeldt, and J. M. A. Van Engelshoven. Preoperative assessment of local tumor extent in advanced rectal cancer: CT or high-resolution MRI? Abdominal Imaging, 25(5):533-541, 2000.

[25] J. W. Belliveau, D. N. Kennedy, R. C. McKinstry, B. R. Buchbinder, R. M. Weisskoff, M. S. Cohen, J. M. Vevea, T. J. Brady, and B. R. Rosen. Functional mapping of the human visual cortex by magnetic resonance imaging. Science, 254(5032):716-719, 1991. 
[26] M. Benzi. Preconditioning techniques for large linear systems: a survey. Journal of Computational Physics, 182(2):418-477, 2002.

[27] P. Bettess. Infinite elements. International Journal for Numerical Methods in Engineering, 11(1):53-64, 1977.

[28] P. Bettess. Infinite Elements. Penshaw Press, UK, 1992.

[29] R. A. Bialecki, A. J. Kassab, and A. Fic. Proper orthogonal decomposition and modal analysis for acceleration of transient FEM thermal analysis. International Journal for Numerical Methods in Engineering, 62(6):774-797, 2005.

[30] O. Bíró. Edge element formulations of eddy current problems. Computer Methods in Applied Mechanics and Engineering, 169(3-4):391-405, 1999.

[31] O. Bíró and K. Preis. On the use of the magnetic vector potential in the finiteelement analysis of three-dimensional eddy currents. IEEE Transactions on Magnetics, 25(4):3145-3159, 1989.

[32] J. E. Bishop. A displacement-based finite element formulation for general polyhedra using harmonic shape functions. International Journal for Numerical Methods in Engineering, 97(1):1-31, 2014.

[33] A. Bjorck. Numerical Methods for Least Squares Problems, volume 51. SIAM, Philadelphia, USA, 1996.

[34] D. Boffi, P. Fernandes, L. Gastaldi, and I. Perugia. Computational models of electromagnetic resonators: analysis of edge element approximation. SIAM Journal on Numerical Analysis, 36(4):1264-1290, 1999.

[35] J. Bonet, A. J. Gil, and R. D. Wood. Nonlinear Solid Mechanics for Finite Element Analysis: Statics. Cambridge University Press, 2016.

[36] A. Bossavit. Solving Maxwell equations in a closed cavity, and the question of “spurious modes". IEEE Transactions on Magnetics, 26(2):702-705, 1990.

[37] A. Bossavit. Computational Electromagnetism: Variational Formulations, Complementarity, Edge elements. Academic Press, 1998.

[38] A. Bower. Applied Mechanics of Solids. CRC Press, Florida, 2009.

[39] D. Braess. Finite Elements: Theory, Fast Solvers, and Applications in Solid Mechanics. Cambridge University Press, Cambridge, United Kingdom, 2007.

[40] F. Brezzi. On the existence, uniqueness and approximation of saddle-point problems arising from lagrangian multipliers. Publications Mathématiques et Informatique de Rennes, (S4):1-26, 1974. 
[41] A. Buffa, H. Ammari, and J. C. Nédélec. A justification of eddy currents model for the Maxwell equations. SIAM Journal on Applied Mathematics, 60(5):1805-1823, 2000 .

[42] T. Bui-Thanh, M. Damodaran, and K. Willcox. Proper orthogonal decomposition extensions for parametric applications in compressible aerodynamics. In 21 st AIAA Applied Aerodynamics Conference, DOI:10.2514/6.2003-4213, 2003.

[43] V. Buljak. Inverse Analyses with Model Reduction: Proper Orthogonal Decomposition in Structural Mechanics. Springer Science \& Business Media, Berlin, Germany, 2011.

[44] D. M. Causon and C. G. Mingham. Introductory Finite Difference Methods for PDEs. Bookboon, Frederiksberg, Denmark, 2010.

[45] M. Cervera, C. Agelet De Saracibar, and M. Chiumenti. Thermo-mechanical analysis of industrial solidification processes. International Journal for Numerical Methods in Engineering, 46(9):1575-1591, 1999.

[46] J. A. Chalela, C. S. Kidwell, L. M. Nentwich, M. Luby, A. M. Butman, J. A .and Demchuk, M. D. Hill, N. Patronas, L. Latour, and S. Warach. Magnetic resonance imaging and computed tomography in emergency assessment of patients with suspected acute stroke: a prospective comparison. The Lancet, 369(9558):293-298, 2007.

[47] A. Chatterjee. An introduction to the Proper Orthogonal Decomposition. Current Science, 78(7):808-817, 2000.

[48] L. Chilton and M. Suri. On the selection of a locking-free hp element for elasticity problems. International Journal for Numerical Methods in Engineering, 40(11):2045-2062, 1997.

[49] F. Chinesta, R. Keunings, and A. Leygue. The Proper Generalized Decomposition for Advanced Numerical Simulations: A Primer. Springer Science \& Business Media, Berlin, Germany, 2013.

[50] F. Chinesta and P. Ladevèze. Separated Representations and PGD-Based Model Reduction: Fundamentals and Applications. International Centre for Mechanical Siences, Courses and Lectures. Springer, Vienna, Austria, 2014.

[51] M. Chiumenti, Q. Valverde, C. A. De Saracibar, and M. Cervera. A stabilized formulation for incompressible elasticity using linear displacement and pressure interpolations. Computer Methods in Applied Mechanics and Engineering, 191(46):5253-5264, 2002.

[52] L. Corradi. A displacement formulation for the finite element elastic-plastic problem. Meccanica, 18(2):77-91, 1983. 
[53] M. Costabbel and M. Dauge. Singularities of electromagnetic fields in polyhedral domains. Archive for Rational Mechanics and Analysis, 151(3):221-276, 2000.

[54] J. Coyle and P. D. Ledger. Evidence of exponential convergence in the computation of Maxwell eigenvalues. Computer Methods in Applied Mechanics and Engineering, 194(2-5):587-604, 2005.

[55] S. Currie, N. Hoggard, I. J. Craven, M. Hadjivassiliou, and I. D. Wilkinson. Understanding MRI: Basic MR physics for physicians. Postgraduate Medical Journal, 89(1050):209-223, 2013.

[56] Y. Dai, Q. Wang, C. Wang, L. Li, H. Wang, Z. Ni, S. Song, S. Chen, B. Zhao, H. Wang, Y. Li, X. Hu, C. Cui, J. Cheng, Y. Lei, and L. Yan. Structural design of a 9. $4 \mathrm{~T}$ whole-body MRI superconducting magnet. IEEE Transactions on Applied Superconductivity, 22(3):4900404-4900404, 2012.

[57] I. Demirdžić and M. Perić. Finite volume method for prediction of fluid flow in arbitrarily shaped domains with moving boundaries. International Journal for $\mathrm{Nu}$ merical Methods in Fluids, 10(7):771-790, 1990.

[58] L. Demkowicz. Computing with hp-adaptive Finite Elements: Volume 1. One and Two Dimensional Elliptic and Maxwell Problems. Chapman and Hall/CRC, New York, USA, 2006.

[59] L. Demkowicz, J. Kurtz, D. Pardo, M. Paszynski, W. Rachowicz, and A. Zdunek. Computing with Hp-Adaptive Finite Elements, Vol. 2: Frontiers Three Dimensional Elliptic and Maxwell Problems with Applications. Chapman \& Hall/CRC, 1st edition, 2007.

[60] L. Demkowicz and L. Vardapetyan. Modeling of electromagnetic absorption/scattering problems using $h p$-adaptive finite elements. Computer Methods in Applied Mechanics and Engineering, 152(1-2):103-124, 1998.

[61] N. Demo, N. Tezzele, G. Gustin, G. Lavini, and G. Rozza. Shape optimisation by means of proper orthogonal decomposition and dynamic mode decomposition. In Technology and Science for the Ships of the Future: Proceedings of NAV 2018: 19th International Conference on Ship \& Maritime Research, pages 212219, 2018.

[62] P. Díez, S. Zlotnik, A. García-González, and A. Huerta. Algebraic PGD for tensor separation and compression: An algorithmic approach. Comptes Rendus Mécanique, 346(7):501-514, 2018.

[63] H. Ding, C. Shu, K. S. Yeo, and D. Xu. Simulation of incompressible viscous flows past a circular cylinder by hybrid FD scheme and meshless least square-based finite difference method. Computer Methods in Applied Mechanics and Engineering, 193(9-11):727-744, 2004. 
[64] J. O. Dow, M. S. Jones, and S. A. Harwood. A new approach to boundary modelling for finite difference applications in solid mechanics. International Journal for Numerical Methods in Engineering, 30(1):99-113, 1990.

[65] J. P. Earls, V. B. Ho, T. K. Foo, E. Castillo, and S. D. Flamm. Cardiac MRI: recent progress and continued challenges. Journal of Magnetic Resonance Imaging: An Official Journal of the International Society for Magnetic Resonance in Medicine, 16(2):111-127, 2002.

[66] H. C. Elman, D. J. Silvester, and A. J. Wathen. Finite Elements and Fast Iterative Solvers: With Applications in Incompressible Fluid Dynamics. Oxford University Press, USA, 2014.

[67] C. Farhat and F. Roux. A method of finite element tearing and interconnecting and its parallel solution algorithm. International Journal for Numerical Methods in Engineering, 32(6):1205-1227, 1991.

[68] C. Farhat and F. Roux. An unconventional domain decomposition method for an efficient parallel solution of large-scale finite element systems. SIAM Journal on Scientific and Statistical Computing, 13(1):379-396, 1992.

[69] Food and Drug Administration (FDA). FDA clears first $7 \mathrm{~T}$ magnetic resonance imaging device. Available at: https://www.fda.gov/NewsEvents/Newsroom/PressAnnouncements/ucm580154. Accessed: 13/11/2018.

[70] P. C. Franzone, L. F. Pavarino, and S. Scacchi. Parallel multilevel solvers for the cardiac electro-mechanical coupling. Applied Numerical Mathematics, 95:140$153,2015$.

[71] N. Galopin, X. Mininger, F. Frederic, and L. Daniel. Finite element modeling of magnetoelectric sensors. IEEE Transactions on Magnetics, 44(6):834-837, 2008.

[72] S. Georgaka, G. Stabile, G. Rozza, and M. J. Bluck. Parametric PODGalerkin model order reduction for unsteady-state heat transfer problems. arXiv:1808.05175v3, 2018.

[73] A. J. Gil. Lagrangian and Eulerian formulation of magneto-mechanical coupled problems. Technical report, Swansea University, 2019.

[74] A. J. Gil and R. Ortigosa. A new framework for large strain electromechanics based on convex multi-variable strain energies: variational formulation and material characterisation. Computer Methods in Applied Mechanics and Engineering, 302:293-328, 2016.

[75] A.J. Gil and P.D. Ledger. A coupled hp-finite element scheme for the solution of two-dimensional electrostrictive materials. International Journal for Numerical Methods in Engineering, 91(11):1158-1183, 2012. 
[76] G. Giovannetti, V. Hartwig, V. Viti, P. Zadaricchio, L. Meini, L. Landini, and A. Benassi. Low field elliptical MR coil array designed by FDTD. Concepts in Magnetic Resonance Part B: Magnetic Resonance Engineering: An Educational Journal, 33(1):32-38, 2008.

[77] W. J. Gordon and C. A. Hall. Construction of curvilinear coordinate systems and applications to mesh generation. International Journal for Numerical Methods in Engineering, 7(4):461-477, 1973.

[78] P. Gosselet and C. Rey. Non-overlapping domain decomposition methods in structural mechanics. Archives of Computational Methods in Engineering, 13(4):515, 2006.

[79] A. Grama, V. Kumar, A. Gupta, and G. Karypis. Introduction to Parallel Computing. Pearson Education, 2003.

[80] D. J. Griffiths. Introduction to Electrodynamics. Pearson Education, 2014.

[81] E. Haber and U. M. Ascher. Fast finite volume simulation of 3D electromagnetic problems with highly discontinuous coefficients. SIAM Journal on Scientific Computing, 22(6):1943-1961, 2001.

[82] E. Haber, U. M. Ascher, D. A. Aruliah, and D. W. Oldenburg. Fast simulation of 3D electromagnetic problems using potentials. Journal of Computational Physics, 163(1):150-171, 2000.

[83] P. Hansbo and A. Szepessy. A velocity-pressure streamline diffusion finite element method for the incompressible Navier-Stokes equations. Computer Methods in Applied Mechanics and Engineering, 84(2):175-192, 1990.

[84] P. C. Hansen. Rank-Deficient and Discrete Ill-Posed Problems: Numerical Aspects of Linear Inversion, volume 4. SIAM, Philadelphia, USA, 2005.

[85] M. Heil. An efficient solver for the fully coupled solution of large-displacement fluid-structure interaction problems. Computer Methods in Applied Mechanics and Engineering, 193(1-2):1-23, 2004.

[86] U. Heisserer, S. Hartmann, A. Düster, and Z. Yosibash. On volumetric locking-free behaviour of p-version finite elements under finite deformations. Communications in Numerical Methods in Engineering, 24(11):1019-1032, 2008.

[87] J. S. Hesthaven, G. Rozza, B. Stamm, et al. Certified Reduced Basis Methods for Parametrized Partial Differential Equations. Springer, Cham, Switzerland, 2016.

[88] R. Hiptmair and O. Sterz. Current and voltage excitations for the eddy current model. International Journal of Numerical Modelling: Electronic Networks, Devices and Fields, 18(1):1-21, 2005. 
[89] J.R.T. Hughes. The Finite Element Method: Linear Static and Dynamic Finite Element Analysis. Courier Corporation, 2012.

[90] T. J. R. Hughes, J. A. Cottrell, and Y. Bazilevs. Isogeometric analysis: CAD, finite elements, NURBS, exact geometry and mesh refinement. Computer Methods in Applied Mechanics and Engineering, 194(39-41):4135-4195, 2005.

[91] A. Ibrahimbegovic. Nonlinear Solid Mechanics: Theoretical Formulations and Finite Element Solution Methods, volume 160. Springer Science \& Business Media, New Jersey, USA, 2009.

[92] N. Ida and J. P. Bastos. Electromagnetics and calculation of fields. Springer Science \& Business Media, New York, USA, 2013.

[93] P. Jenny, S. H. Lee, and H. A. Tchelepi. Multi-scale finite-volume method for elliptic problems in subsurface flow simulation. Journal of Computational Physics, 187(1):47-67, 2003.

[94] D. Jin. An hp-Finite Element Computational Framework for Nonlinear MagnetoFluid Problems Including Magnetostriction. PhD thesis, Swansea University, 2015.

[95] D. Jin, P. D. Ledger, and A. J. Gil. $h p$-Finite element solution of coupled stationary magnetohydrodynamics problems including magnetostrictive effects. Computers \& Structures, 164:161-180, 2016.

[96] D. Kalman. A singularly valuable decomposition: the SVD of a matrix. The College Mathematics Journal, 27(1):2-23, 1996.

[97] M. Kaltenbacher. Numerical Simulation of Mechatronic Sensors and Actuators. Springer, 2004.

[98] G. Karniadakis and S. Sherwin. Spectral/hp Element Methods for Computational Fluid Dynamics. Oxford University Press, Oxford, United Kingdom, 2013.

[99] J. Kerler-Back and T. Stykel. Model reduction for linear and nonlinear magnetoquasistatic equations. International Journal for Numerical Methods in Engineering, 111(13):1274-1299, 2017.

[100] C. S. Kidwell, J. A. Chalela, J. L. Saver, S. Starkman, M. D. Hill, A. M. Demchuk, J. A. Butman, N. Patronas, J. R. Alger, L. L. Latour, et al. Comparison of MRI and CT for detection of acute intracerebral hemorrhage. Jama, 292(15):1823-1830, 2004.

[101] M. Kozlov and R. Turner. Fast MRI coil analysis based on 3-D electromagnetic and RF circuit co-simulation. Journal of Magnetic Resonance, 200(1):147-152, 2009. 
[102] P. Kozlowski, S. D. Chang, E. C. Jones, K. W. Berean, H. Chen, and S. L. Goldenberg. Combined diffusion-weighted and dynamic contrast-enhanced MRI for prostate cancer diagnosis - Correlation with biopsy and histopathology. Journal of Magnetic Resonance Imaging: An Official Journal of the International Society for Magnetic Resonance in Medicine, 24(1):108-113, 2006.

[103] V. Kumar. Introduction to Parallel Computing. Addison-Wesley Longman Publishing Co., Inc., 2002.

[104] S. Kurz, J. Fetzer, G. Lehner, and W. M. Rucker. A novel formulation for 3D eddy current problems with moving bodies using a Lagrangian description and BEMFEM coupling. IEEE Transactions on Magnetics, 34(5):3068-3073, 1998.

[105] R.M. Kynch and P.D. Ledger. Resolving the sign conflict problem for hphexahedral Nédélec elements with application to eddy current problems. Computers \& Structures, 181:41-54, 2017.

[106] H. Landes, M. Kaltenbacher, and R. Lerch. CAPA User Manual, Release 4.1. WisSoft, Erlangen, 2003, 1994.

[107] D. Lazzaro and L. B. Montefusco. Radial basis functions for the multivariate interpolation of large scattered data sets. Journal of Computational and Applied Mathematics, 140(1-2):521-536, 2002.

[108] P. D. Ledger. An hp-Adaptive Finite Element Procedure for Electromagentic Scattering Problems. PhD thesis, University of Wales, Swansea, 2000.

[109] P. D. Ledger. hp-Finite element discretisation of the electrical impedance tomography problem. Computer Methods in Applied Mechanics and Engineering, 225:154-176, 2012.

[110] P. D. Ledger, A. J. Gil, R. Poya, M. Kruip, I. Wilkinson, and S. Bagwell. Solution of an industrially relevant coupled magneto-mechanical problem set on an axisymmetric domain. Applied Mathematical Modelling, 40:1959-1971, 2016.

[111] P. D. Ledger, K. Morgan, O. Hassan, and N. P. Weatherill. Arbitrary order edge elements for electromagnetic scattering simulations using hybrid meshes and a PML. International Journal for Numerical Methods in Engineering, 55(3):339358, 2002.

[112] P. D. Ledger, K. Morgan, J. Peraire, O. Hassan, and N. P. Weatherill. The development of an $h p$-adaptive finite element procedure for electromagnetic scattering problems. Finite Elements in Analysis and Design, 39(8):751-764, 2003.

[113] P. D. Ledger and S. Zaglmayr. $h p$-Finite element simulation of three-dimensional eddy current problems on multiply connected domains. Computer Methods in Applied Mechanics and Engineering, 199(49):3386-3401, 2010. 
[114] P. D. Ledger and S. Zaglmayr. $h p$-Finite element simulation of three-dimensional eddy current problems on multiply connected domains. Computer Methods in Applied Mechanics and Engineering, 199:3386-3401, 2010.

[115] R. J. LeVeque. Finite Volume Methods for Hyperbolic Problems, volume 31. Cambridge university press, Cambridge, United Kingdom, 2002.

[116] R. J. LeVeque. Finite Difference Methods for Ordinary and Partial Differential Equations: Steady-State and Time-Dependent Problems, volume 98. SIAM, Philadelphia, USA, 2007.

[117] L. Li, J. Cheng, C. Cui, Y. Li, Y. Dai, X. Hu, J. Liu, L. Wang, and Q. Wang. Numerical analysis of mechanical behavior for a 9.4-T whole-body MRI magnet. IEEE Transactions on Applied Superconductivity, 27(4):1-5, 2017.

[118] X. Liang, J. Zhang, M. Zhang, and L. Cui. Multivariate Lagrange interpolation and an application of Cayley-Bacharach theorem for it. arXiv:math/0608115v2, 2006.

[119] Y. C. Liang, H. P. Lee, S. P. Lim, W. Z. Lin, K. H. Lee, and C. G. Wu. Proper Orthogonal Decomposition and its applications-Part I: Theory. Journal of Sound and vibration, 252(3):527-544, 2002.

[120] C. Lin, E. C. Lawton, J. A. Caliendo, and L. R. Anderson. An iterative finite element-boundary element algorithm. Computers \& Structures, 59(5):899-909, 1996.

[121] F. Liu and S. Crozier. An FDTD model for calculation of gradient-induced eddy currents in MRI system. IEEE transactions on applied superconductivity, 14(3):1983-1989, 2004.

[122] R. N. Low, R. M. Barone, and J. Lucero. Comparison of MRI and CT for predicting the Peritoneal Cancer Index (PCI) preoperatively in patients being considered for cytoreductive surgical procedures. Annals of Surgical Oncology, 22(5):1708-1715, 2015.

[123] R. J. Luebbers, K. S. Kunz, M. Schneider, and F. Hunsberger. A finite-difference time-domain near zone to far zone transformation (electromagnetic scattering). IEEE Transactions on Antennas and Propagation, 39(4):429-433, 1991.

[124] Z. Luo, J. Du, Z. Xie, and Y. Guo. A reduced stabilized mixed finite element formulation based on proper orthogonal decomposition for the non-stationary NavierStokes equations. International Journal for Numerical Methods in Engineering, 88(1):31-46, 2011.

[125] R. L. Mackie, J. T. Smith, and T. R. Madden. Three-dimensional electromagnetic modeling using finite difference equations: The magnetotelluric example. Radio Science, 29(4):923-935, 1994. 
[126] C. Michler, L. Demkowicz, J. Kurtz, and D. Pardo. Improving the performance of perfectly matched layers by means of hp-adaptivity. Numerical Methods for Partial Differential Equations, 23:832-858, 2007.

[127] D. Modesto, S. Zlotnik, and A. Huerta. Proper generalized decomposition for parameterized Helmholtz problems in heterogeneous and unbounded domains: Application to harbor agitation. Computer Methods in Applied Mechanics and Engineering, 295:127-149, 2015.

[128] A. H. Mohammadian, V. Shankar, and W. F. Hall. Computation of electromagnetic scattering and radiation using a time-domain finite-volume discretization procedure. Computer Physics Communications, 68(1-3):175-196, 1991.

[129] P. Monk. Finite element methods for Maxwell's equations. Oxford University Press, Oxford, UK, 2003.

[130] P. Monk and K. Parrott. Phase-accuracy comparisons and improved far-field estimates for 3D edge elements on tetrahedral meshes. Journal of Computational Physics, 170(2):614-641, 2001.

[131] J. C. Nédélec. Mixed finite elements in $\mathbb{R}^{3}$. Numerische Mathematik, 35:315-341, 1980.

[132] Imaging Technology News. GE introduces freelium technology to reduce MRI helium needs. https://www.itnonline.com/content/ge-introduces-freeliumtechnology-reduce-mri-helium-needs. Accessed 07/11/2018.

[133] S. Niroomandi, I. Alfaro, E. Cueto, and F. Chinesta. Model order reduction for hyperelastic materials. International Journal for Numerical Methods in Engineering, 81(9):1180-1206, 2010.

[134] L. G. Olson and K. Bathe. Analysis of fluid-structure interactions. A direct symmetric coupled formulation based on the fluid velocity potential. Computers \& Structures, 21(1-2):21-32, 1985.

[135] R. Ortigosa and A. J. Gil. A new framework for large strain electromechanics based on convex multi-variable strain energies: Conservation laws, hyperbolicity and extension to electro-magnetomechanics. Computer Methods in Applied Mechanics and Engineering, 309:202-242, 2016.

[136] R. Ortigosa and A. J. Gil. A new framework for large strain electromechanics based on convex multi-variable strain energies: Finite element discretisation and computational implementation. Computer Methods in Applied Mechanics and Engineering, 302:329-360, 2016.

[137] R. Palma, E. Moliner, and J. L. Pérez-Aparicio. Elasto-thermoelectric beam formulation for modeling thermoelectric devices. Finite Elements in Analysis and Design, 129:32-41, 2017. 
[138] J. L. Pérez-Aparicio, R. Palma, and P. Moreno-Navarro. Elasto-thermoelectric nonlinear, fully coupled, and dynamic finite element analysis of pulsed thermoelectrics. Applied Thermal Engineering, 107:398-409, 2016.

[139] C. L. Pettit and P. S. Beran. Application of proper orthogonal decomposition to the discrete Euler equations. International Journal for Numerical Methods in Engineering, 55(4):479-497, 2002.

[140] Philips. Helium free MR magnets. https://www.philips.co.uk/healthcare/resources/landing/sealedmr-technology. Accessed: 01/11/2018.

[141] C. Pozrikidis. Boundary Integral and Singularity Methods for Linearized Viscous Flow. Cambridge University Press, Cambride, United Kingdom, 1992.

[142] T. H. Pulliam and J. L. Steger. Implicit finite-difference simulations of threedimensional compressible flow. AIAA Journal, 18(2):159-167, 1980.

[143] A. Radermacher and S. Reese. POD-based model reduction with empirical interpolation applied to nonlinear elasticity. International Journal for Numerical Methods in Engineering, 107(6):477-495, 2016.

[144] R. R. Rama. Proper Orthogonal Decomposition with Interpolation-Based RealTime Modelling of the Heart. PhD thesis, University of Cape Town, South Africa, 2017.

[145] R. R. Rama, S. Skatulla, and C. Sansour. Real-time modelling of the heart using the proper orthogonal decomposition with interpolation. In VI International Conference on Computational Bioengineering ICCB, pages 1-12, Barcelona, Spain, 2015.

[146] E. Ramm, E. Rank, R. Rannacher, K. Schweizerhof, E. Stein, W. Wendland, G. Wittum, P. Wriggers, and W. Wunderlich. Error-Controlled Adaptive Finite Elements in Solid Mechanics. John Wiley \& Sons, Chichester, United Kingdom, 2003.

[147] M. Rausch, M. Gebhardt, M. Kaltenbacher, and H. Landes. Magnetomechanical field computations of a clinical magnetic resonance imaging (MRI) scanner. The International Journal for Computation and Mathematics in Electrical and Electronic Engineering (COMPEL), 22:576-588, 2003.

[148] M. Rausch, M. Gebhardt, M. Kaltenbacher, and H. Landes. Computer-aided design of clinical magnetic resonance imaging scanners by coupled magnetomechanicalacoustic modeling. IEEE Transactions on Magnetics, 41:72-81, 2005.

[149] Z. Ren. Influence of the RHS on the convergence behaviour of the curl-curl equation. IEEE Transactions on Magnetics, 32(3):655-658, 1996. 
[150] J. J. Reuther, A. Jameson, J. J. Alonso, M. J. Rimlinger, and D. Saunders. Constrained multipoint aerodynamic shape optimization using an adjoint formulation and parallel computers, part 1. Journal of Aircraft, 36(1):51-60, 1999.

[151] T. Rong and A. Lu. Generalized mixed variational principles and solutions of ill-conditioned problems in computational mechanics: Part I. Volumetric locking. Computer Methods in Applied Mechanics and Engineering, 191(3-5):407422, 2001.

[152] G. Rozza, H. Malik, N. Demo, M. Tezzele, M. Girfoglio, G. Stabile, and A. Mola. Advances in reduced order methods for parametric industrial problems in computational fluid dynamics. arXiv preprint arXiv:1811.08319, 2018.

[153] Y. Saad. Iterative Methods for Sparse Linear Systems, volume 82. SIAM, Philadelphia, 2003.

[154] M. Schinnerl, J. Schoberl, M. Kaltenbacher, and R. Lerch. Multigrid methods for the three-dimensional simulation of nonlinear magnetomechanical systems. IEEE Transactions on Magnetics, 38(3):1497-1511, 2002.

[155] J. Schöberl. Netgen - an advancing front 2D/3D-mesh generator based on abstract rules. Computing and Visualization in Science, 1(1):41-52, 1997.

[156] J. Schöberl and S. Zaglmayr. High order Nédélec elements with local complete sequence properties. The International Journal for Computation and Mathematics in Electrical and Electronic Engineering (COMPEL), 24:374-384, 2005.

[157] C. Schwab. $p$ and hp- Finite Element Methods: Theory and Applications in Solid and Fluid Mechanics. Oxford University Press, 1998.

[158] M. Seoane, P. D. Ledger, A. J. Gil, and M. Mallet. An accurate and efficient three dimensional high order finite element methodology for the simulation of magnetomechanical coupling in MRI scanners. International Journal for Numerical Methods in Engineering, 119(12):1185-1215, 2019.

[159] M. Seoane, P. D. Ledger, A. J. Gil, S. Zlotnik, and M. Mallet. A combined reduced order-full order methodology for the solution of 3D magneto-mechanical problems with application to MRI scanners. International Journal for Numerical Methods in Engineering, 121(16):3529-3559, 2019.

[160] R. Sevilla. NURBS-enhanced finite element method (NEFEM). PhD thesis, Universitat Politècnica de Catalunya (UPC), Barcelona, Spain, 2009.

[161] R. Sevilla, S. Fernández-Méndez, and A. Huerta. NURBS-enhanced finite element method (NEFEM). International Journal for Numerical Methods in Engineering, 76(1):56-83, 2008. 
[162] Siemens. Magnetom Terra: translate $7 \mathrm{~T}$ research power into clinical care. https://www.halthcare.siemens.co.uk/magnetic-resonance-imaging/7t-mriscanner/magnetom-terra. Accessed: 13/11/2018.

[163] J. C. Simo and J. W. Ju. Strain-and stress-based continuum damage models-I. Formulation. International Journal of Solids and Structures, 23(7):821-840, 1987.

[164] W.R. Smythe. Static and Dynamic Electricity. McGraw-Hill, 1968.

[165] G. Stabile, S. Hijazi, A. Mola, S. Lorenzi, and G. Rozza. POD-Galerkin reduced order methods for CFD using Finite Volume Discretisation: vortex shedding around a circular cylinder. Communications in Applied and Industrial Mathematics, 8(1):210-236, 2017.

[166] J. A. Stratton. Electromagnetic theory. John Wiley \& Sons, New York, USA, 1941.

[167] A. Stroud and D. Secrest. Gaussian quadrature formulas. Prentice-Hall, London, United Kingdom, 1966.

[168] E. Süli and D. Mayers. An Introduction to Numerical Analysis. Cambridge University Press, Cambridge, UK., 2003.

[169] M. Suri. Analytical and computational assessment of locking in the $h p$ finite element method. Computer Methods in Applied Mechanics and Engineering, 133:347-371, 1996.

[170] M. Suri. Analytical and computational assessment of locking in the hp finite element method. Computer Methods in Applied Mechanics and Engineering, 133(34):347-371, 1996.

[171] B. Szabó and I. M. Babuška. Finite Element Analysis. John Wiley and Sons, 1991.

[172] B. Szabó, A. Düster, and E. Rank. The $p$-version of the finite element method. Encyclopedia of Computational Mechanics, 2004.

[173] A. Trakic, H. Wang, F. Liu, H. Lopez, and S. Crozier. Analysis of transient eddy currents in MRI using a cylindrical FDTD method. IEEE Transactions on Applied Superconductivity, 16(3):1924-1936, 2006.

[174] C. Trimarco. A Lagrangian approach to electromagnetic bodies. Technische Mechanik, 22(3):175-180, 2002.

[175] L. Vardapetyan and L. Demkowicz. $h p$-adaptive finite elements in electromagnetics. Computer Methods in Applied Mechanics and Engineering, 169:331-344, 1999.

[176] H. K. Versteeg and W. Malalasekera. An Introduction to Computational Fluid Dynamics: The finite Volume Method. Pearson Education, Harlow, United Kingdom, 2007. 
[177] J. L. Volakis, A. Chatterjee, and L. C. Kempel. Finite Element Method Electromagnetics: Antennas, Microwave Circuits, and Scattering Applications, volume 6. John Wiley \& Sons, London, United Kingdom, 1998.

[178] C. Vollaire, L. Nicolas, and A. Nicolas. Parallel computing for the finite element method. The European Physical Journal-Applied Physics, 1(3):305-314, 1998.

[179] W. Wang, G. Kosakowski, and O. Kolditz. A parallel finite element scheme for thermo-hydro-mechanical (THM) coupled problems in porous media. Computers \& Geosciences, 35(8):1631-1641, 2009.

[180] Y. Wang, B. Yu, Z. Cao, W. Zou, and G. Yu. A comparative study of POD interpolation and POD projection methods for fast and accurate prediction of heat transfer problems. International Journal of Heat and Mass Transfer, 55(17-18):4827-4836, 2012.

[181] Z. Wang, J. M. van Oort, and M. X. Zou. Development of superconducting magnet for high-field MR systems in China. Physica C: Superconductivity and its Applications, 482:80-86, 2012.

[182] G. N. Wells, L. J. Sluys, and R. De Borst. A p-adaptive scheme for overcoming volumetric locking during plastic flow. Computer Methods in Applied Mechanics and Engineering, 191(29-30):3153-3164, 2002.

[183] J. Woodhouse. Linear damping models for structural vibration. Journal of Sound and Vibration, 215(3):547-569, 1998.

[184] L. C. Wrobel. The Boundary Element Method: Applications in Thermo-Fluids and Acoustics, volume 1. John Wiley \& Sons, USA, 2002.

[185] S. Zaglmayr. High Order Finite Element Methods for Electromagnetic Field Computation. PhD thesis, Institut für Numerische Mathematik, Johannes Kepler Univerität Linz, Austria. 2006.

[186] O. C. Zienkiewicz and R. L. Taylor. The Finite Element Method: Solid Mechanics, volume 2. Butterworth-Heinemann, London, United Kingdom, 2000.

[187] O. C. Zienkiewicz, R. L. Taylor, P. Nithiarasu, and J. Z. Zhu. The Finite Element Method, volume 3. McGraw-Hill, London, United Kingdom, 1977. 